Visible Light-Mediated Metal-Free Photocatalytic Oxidative Reactions and Cycloadditions

Dissertation

for the award of the degree

"Doctor rerum naturalium"

of the Georg-August-Universität Göttingen

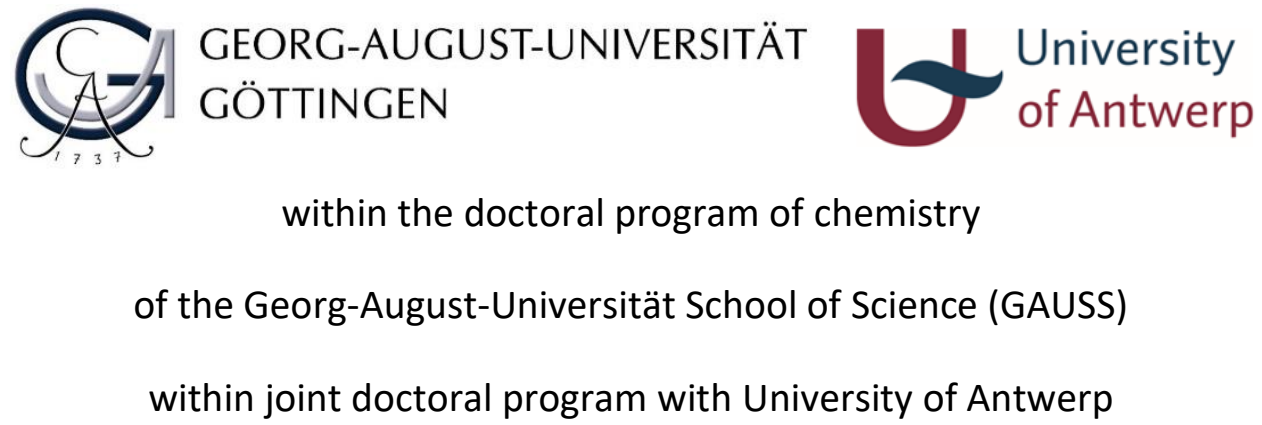

Submitted by

Yu Zhang

From Shanxi (China)

Göttingen, 2020 



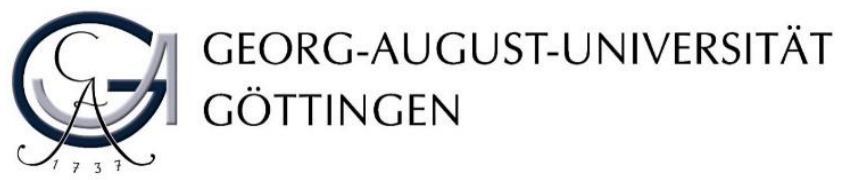

Organic Synthesis (ORSY)

Department of Chemistry

\section{Visible Light-Mediated Metal-Free Photocatalytic Oxidative Reactions and Cycloadditions}

Metaalvrije Fotokatalytische Oxidatieve Reacties en Cycloaddities onder invloed van Zichtbaar Licht

Proefschrift voorgelegd tot het behalen van de graad van Doctor in de Wetenschappen: chemie aan de Universiteit Antwerpen, te verdedigen door within joint doctoral program with Georg-August-Universität Göttingen

\section{Yu Zhang}

\section{Supervisor}

Prof. Dr. Shoubhik Das

Supervisor

Antwerp

Prof. Dr. Konrad Koszinowski 



\section{Thesis Committee}

Prof. Dr. Konrad Koszinowski, Institute of Organic and Bimolecular Chemistry, GeorgAugust-Universität Göttingen, Göttingen, Germany

Prof. Dr. Shoubhik Das, ORSY Division, Department of Chemistry, University of Antwerp, Antwerp, Belgium

\section{Members of the Examination Board}

Reviewer: Prof. Dr. Konrad Koszinowski, Institute of Organic and Bimolecular Chemistry, Georg-August-Universität Göttingen, Göttingen, Germany

Second Reviewer: Prof. Dr. Shoubhik Das, ORSY Division, Department of Chemistry, University of Antwerp, Antwerp, Belgium

\section{Further members of the Examination Board}

Prof. Dr. Dietmar Stalke, Institute of Inorganic Chemistry, Georg-August-Universität Göttingen, Göttingen, Germany

Dr. Michael John, Institute of Organic and Biomolecular Chemistry, Georg-AugustUniversität Göttingen, Göttingen, Germany

Prof. Dr. Bert Maes, ORSY Division, Department of Chemistry, University of Antwerp, Antwerp, Belgium

Prof. Dr. Vera Meynen, Department of Chemistry, University of Antwerp, Antwerp, Belgium

Date of the oral examination: 03.11 .2020 



\section{Acknowledgements}

At the outset, I would like to extend my sincere gratitude to my supervisor Prof. Dr. Shoubhik Das during my graduate study. Without his constant encouragement, excellent guidance, patience in the past three years, I would never be able to accomplish my doctoral degree. He always shares his ideas and discusses with me about the projects which sped up my projects a lot.

I am very grateful to Prof. Dr. Konrad Koszinowski for his kindly accepting to be the second supervisor for my first two-year study and becomes my first supervisor in Göttingen during my joint PhD program study. He always gives me highly valuable suggestions and comments in last three years. I am also really appreciated for his kind corrections, suggestions, and polish of my thesis. I benefited and learned much from his rigorous spirit.

I deeply thank my lab colleagues Daniel Riemer, Waldemar Schilling, Jiri Kollmann, Tong Zhang, Shaowei Qin, Nareh Hatami, Prakash Sahoo etc. for their kind help during my PhD study. I am also appreciated for the kind help of Prof. Dr. Maes and all ORSY members during the study in the University of Antwerp.

I would like to thank the analytical departments especially the Dr. Michael John and Dr. Holm Frauendorf at the IOBC, for their strong support and offered possibility to operate several machines by myself.

I gratefully acknowledge China Scholarship Council (CSC) and BOF International Joint PhD Fellowships for the financial support during my research stay in Germany and Belgium.

Special thanks should give to my wife, Mrs. Na Zhang who always encourages me to face and overcome challenges. She is always there for me.

At last, I will thank my parents, sister and other families for their continuous support and encouragement. I love you all forever. 


\section{Contents}

1. Introduction 1

1.1 Visible-Light-Mediated Homogeneous Photocatalysis ......................4

1.1.1 General Mechanism of Homogeneous Photocatalysis. .4

1.1.2 Merger of Homogeneous Photoredox Catalysis with Other Catalytic Systems

1.2 Visible-Light-Mediated Heterogeneous Photocatalysis

1.2.1 General Mechanism of Visible-Light-Mediated Photocatalysis Using Polymeric Carbon Nitride

1.2.2 Recent Advances of Visible-Light-Mediated Photocatalysis Using Polymeric Carbon Nitride

1.3 Metal-free Photocatalytic Benzylic C(sp $\left.{ }^{3}\right)-\mathrm{H}$ Oxygenation Using Oxygen as an Oxidant. 23

1.3.1 Advantages of Photocatalytic Oxygenation Using Oxygen 23

1.3.2 Homogeneous Photocatalytic Benzylic $\mathrm{C}\left(\mathrm{sp}^{3}\right)-\mathrm{H}$ Oxygenation

Using Oxygen 25

1.3.3 Heterogeneous Photocatalytic $\mathrm{C}\left(\mathrm{sp}^{3}\right)-\mathrm{H}$ Oxygenation Using Oxygen 29

1.4 Metal-free Photocatalytic Oxidative $\mathrm{C}=\mathrm{C}$ Bonds Cleavage 30

1.4.1 Earlier Examples of Oxidative $\mathrm{C}=\mathrm{C}$ Bonds Cleavage 30

1.4.2 Recent Advances of Oxidative $\mathrm{C}=\mathrm{C}$ Bonds Cleavage 30

1.5 Metal-free Photocatalytic Cycloadditions 33

1.5.1 Examples of Photocatalytic [2+2] Cycloadditions 34

1.5.2 Examples of Photocatalytic [4+2] Cycloadditions 39

2. Objectives 44

3. Results and Discussion

3.1 Visible-Light-Mediated Efficient Metal-Free $\alpha$-Oxygenation of

Tertiary Amines to Amides ${ }^{1}$ .46

3.1.1 Optimization for the Synthesis of 1-Benzoylpiperidine 46

3.1.2 Scope of $\alpha$-Oxygenation of Tertiary Amines to Amides 48

3.1.3 Mechanistic Studies 52

3.1.4 Proposed Mechanism 56 
3.1.5 Unsuccessful Substrates Scope 57

3.2 Heterogeneous Photocatalytic Benzylic $\mathrm{C}\left(\mathrm{sp}^{3}\right)-\mathrm{H}$ Hydroxylation......60

3.2.1 Optimization for Synthesis of 2-Phenyl-2-propanol.................60

3.2.2 Substrate Scope for the Hydroxylation under Blue LED .............61

3.2.3 Recycling Experiments and ${ }^{18} \mathrm{O}$-Labelling Experiments..............63

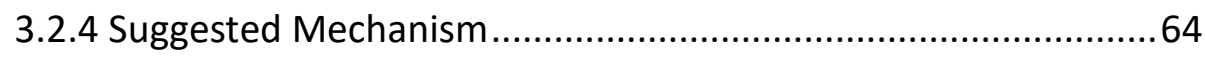

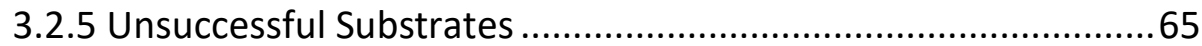

3.3 A Metal-Free Heterogeneous Photocatalyst for the Selective

Oxidative Cleavage of $\mathrm{C}=\mathrm{C}$ Bonds via Harvesting Direct Solar Energy ${ }^{2}$

3.3.1 Optimization of the $\mathrm{C}=\mathrm{C}$ Bonds Cleavage ................................69

3.3.2 Scope of the C=C Bonds Cleavage ….....................................70

3.3.3 Recycling Experiments and Spectroscopic Analyses .................76

3.3.4 Mechanistic Studies ...............................................................79

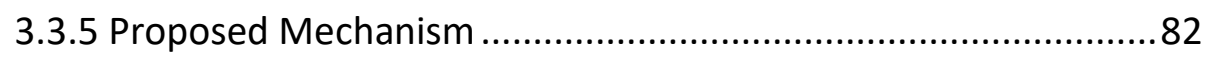

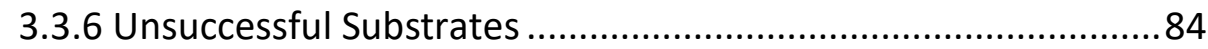

3.4 A Simple Ketone as An Efficient Metal-free Catalyst for VisibleLight-Mediated Cycloadditions ${ }^{3}$

3.4.1 Optimization of Diels-Alder Reactions.

3.4.2 Scope of the Diels-Alder Reactions under the Irradiation of

Visible-Light. 88

3.4.3 Optimization of aza-Diels-Alder Reactions 90

3.4.4 Scope of aza-Diels-Alder Reactions under the Irradiation of Visible-light. 91

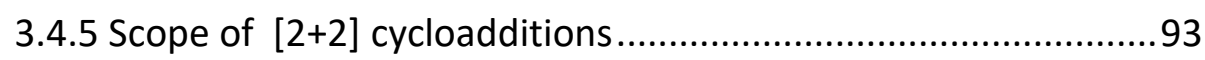

3.4.6 Mechanistic Studies and Proposed Mechanism........................94

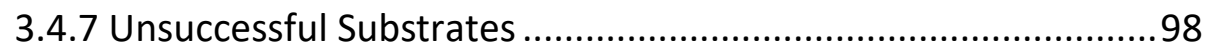

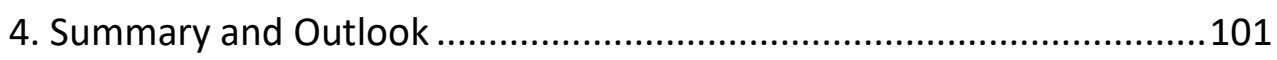

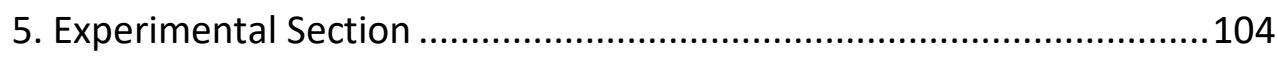

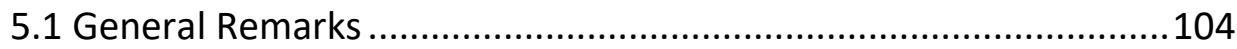

5.2 Reaction Procedures of $\alpha$-Oxygenation of Tertiary Amines ............108

5.3 Reaction Procedures of Blue LED-Mediated Hydroxylation ...........115 
5.4 Reaction Procedures for $\mathrm{C}=\mathrm{C}$ Bond Cleavage Reactions 118

5.5 Reaction Procedures of Blue LED-Mediated (aza)-Diels-Alder Reactions .................................................................................... 121

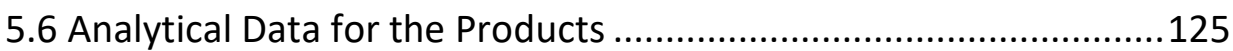

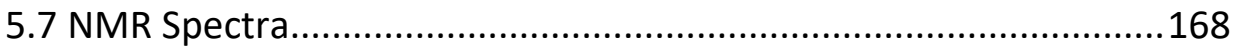

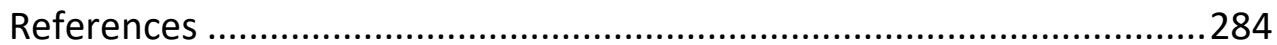




\section{List of Abbreviations}

\begin{tabular}{|c|c|}
\hline Ac & acetyl \\
\hline$A$ & accept \\
\hline Alk & alkyl \\
\hline AQY & apparent quantum yield \\
\hline $\operatorname{Ar}$ & aryl \\
\hline BHT & 2,6-di-tert-butyl-4-methylphenol \\
\hline $\mathrm{Bn}$ & benzyl \\
\hline Boc & tert-butyloxycarbonyl \\
\hline BNAH & 1-benzyl-1,4-dihydronicotinamide \\
\hline $\mathrm{Bu}$ & butyl \\
\hline $\mathrm{Bz}$ & benzoyl \\
\hline calc. & calculated \\
\hline cat. & catalytic \\
\hline CFL & compact fluorescent lamp \\
\hline$\delta$ & chemical shift \\
\hline$d$ & doublet \\
\hline D & donor \\
\hline DCE & 1,2-dichloroethane \\
\hline $\mathrm{dd}$ & \\
\hline DMF & $N, N$-dimethylformamide \\
\hline DMSO & dimethyl sulfoxide \\
\hline$d t$ & doublet of triplet \\
\hline $\mathrm{e}^{-}$ & electron \\
\hline E-factors & environmental factor \\
\hline El & electron ionization \\
\hline
\end{tabular}




\begin{tabular}{|c|c|}
\hline Ent & energy transfer \\
\hline Equiv (e.q.) & equivalent \\
\hline ESI & electro spray ionization \\
\hline ESR & electron spin resonance \\
\hline Et & ethyl \\
\hline FG & functional group \\
\hline FMO & frontier molecular orbital \\
\hline g & gram \\
\hline GC & gas chromatography \\
\hline GC-MS & gas chromatography-mass spectrometry \\
\hline $\mathrm{h}$ & hour \\
\hline$h^{+}$ & positive hole \\
\hline Hal & halogen \\
\hline HAT & hydrogen atom transfer \\
\hline Het & hetero atom \\
\hline HPLC & high performance liquid chromatography \\
\hline HR-MS & high resolution mass spectrometry \\
\hline $\mathrm{Hz}$ & Hertz \\
\hline HOMO & highest occupied molecular orbital \\
\hline LED & Light-emitting diode \\
\hline$i$ & iso \\
\hline KIE & kinetic isotope effect \\
\hline $\mathrm{L}$ & ligand \\
\hline LUMO & lowest unoccupied molecular orbital \\
\hline $\mathrm{m}$ & multiplet \\
\hline$M$ & molar \\
\hline
\end{tabular}




\begin{tabular}{|c|c|}
\hline$[\mathrm{M}]^{+}$ & molecular ion peak \\
\hline SET & single electron transfer \\
\hline TPP & meso-tetraphenylporphine \\
\hline $\mathrm{Me}$ & methyl \\
\hline Mes & mesityl \\
\hline $\mathrm{mg}$ & milligram \\
\hline $\mathrm{MHz}$ & megahertz \\
\hline $\min$ & minute \\
\hline $\mathrm{mL}$ & milliliter \\
\hline $\mathrm{mmol}$ & millimol \\
\hline MS & mass spectrometry \\
\hline$m / z$ & mass-to-charge ratio \\
\hline NHPI & $N$-hydroxyphthalimide \\
\hline $\mathrm{NHC}$ & $N$-heterocyclic carbene \\
\hline NHS & $N$-hydroxysuccinimide \\
\hline NMR & nuclear magnetic resonance \\
\hline 0 & ortho \\
\hline OPV & oil pump vacuum \\
\hline $\mathrm{OQ}$ & oxidative quencher \\
\hline$p$ & para \\
\hline PC & photocatalyst \\
\hline $\mathrm{Ph}$ & phenyl \\
\hline $\mathrm{PCN}$ & polymeric carbon nitride \\
\hline $\mathrm{ppm}$ & parts per million \\
\hline $\operatorname{Pr}$ & propyl \\
\hline$q$ & quartet \\
\hline
\end{tabular}




\begin{tabular}{|c|c|}
\hline RT & room temperature \\
\hline $\mathrm{RQ}$ & reductive quencher \\
\hline SCS & spin-center shift \\
\hline s & singlet \\
\hline$S$ & substrate \\
\hline sat. & saturated \\
\hline Set. & selectivity \\
\hline $\mathrm{t}$ & tert \\
\hline $\mathrm{t}$ & triplet \\
\hline $\mathrm{T}$ & temperature \\
\hline t. a. & trace amount \\
\hline THF & tetrahydrofuran \\
\hline TLC & thin layer chromatography \\
\hline TM & transition metal \\
\hline TMP & 2,2,6,6-tetramethylpiperidine \\
\hline TMS & trimethylsilyl \\
\hline Ts & para-toluene sulfonyl \\
\hline TS & transition state \\
\hline UV & ultraviolet \\
\hline UCNF & nitrogen deficient carbon nitride \\
\hline VB & valence band \\
\hline$w t \%$ & weight by volume \\
\hline
\end{tabular}




\section{Introduction}

Sustainable development becomes now a mainstream accepted by industry, academics and public due to the increasing environmental pollutions and other related global crises. Within this, there is always an imperious demand for the sustainable development of the chemical industry by reducing or eliminating the use or generation of hazardous substances and using less energy in the production. Hence, 'Green chemistry' or 'Sustainable chemistry' emerges as the key topic. ${ }^{[1]}$ In the 1990s, the 12 principles of green chemistry were established, which assist chemists to discover and design new synthetic pathways for reducing or eliminating the use and generation of hazardous substances. ${ }^{[2]}$ These principles revolve around the utilization of less or non-hazardous chemicals and solvents, the elimination of chemical waste, and atom or energy efficient transformations, which are definitely important for solving progressive challenges. ${ }^{[3]}$

Visible-light-mediated organic syntheses experienced a blooming renaissance due to their numerous strengths such as cleanliness, low energy consumption compared to higher energy-consuming UV-light mediated reactions and safe handling in comparison to thermal reactions. Moreover, UV-lamps generate a lot of heat, which can induce unwanted thermal reactions. Visible light with designable and narrow wavelength range enables productive photoreactions of compounds possessing weak bonds that are sensitive toward UV photodegradation. Parallel to this, development of solar-energy mediated organic transformation is also highly attractive for the augmentation of sustainable approach in the organic synthesis. ${ }^{[4]}$ In fact, sunlight is renewable and available throughout the entire world. The average intensity of the total solar irradiance is about $1366.1 \mathrm{~W} \mathrm{~m}^{-2}$ which provides roughly $4.3 \times 10^{20} \mathrm{~J}$ energy only in $1 \mathrm{~h} .{ }^{[4]}$ Therefore, if it is harvested and utilized in organic transformations, could bring a vision to solve the sustainable issues.

As a key factor in the recent rapid growth of this field has been recognized the fact that readily accessible metal complexes and organic dyes can facilitate the conversion of visible light into chemical energy under exceptionally mild conditions. Among them, ruthenium- and iridium-based organometallic complexes stand at the forefront of this 
class. These species are mostly employed as radical sources: they exhibit excellent visible-light absorption and long-lived excited states (Scheme 1.1). ${ }^{[5]}$

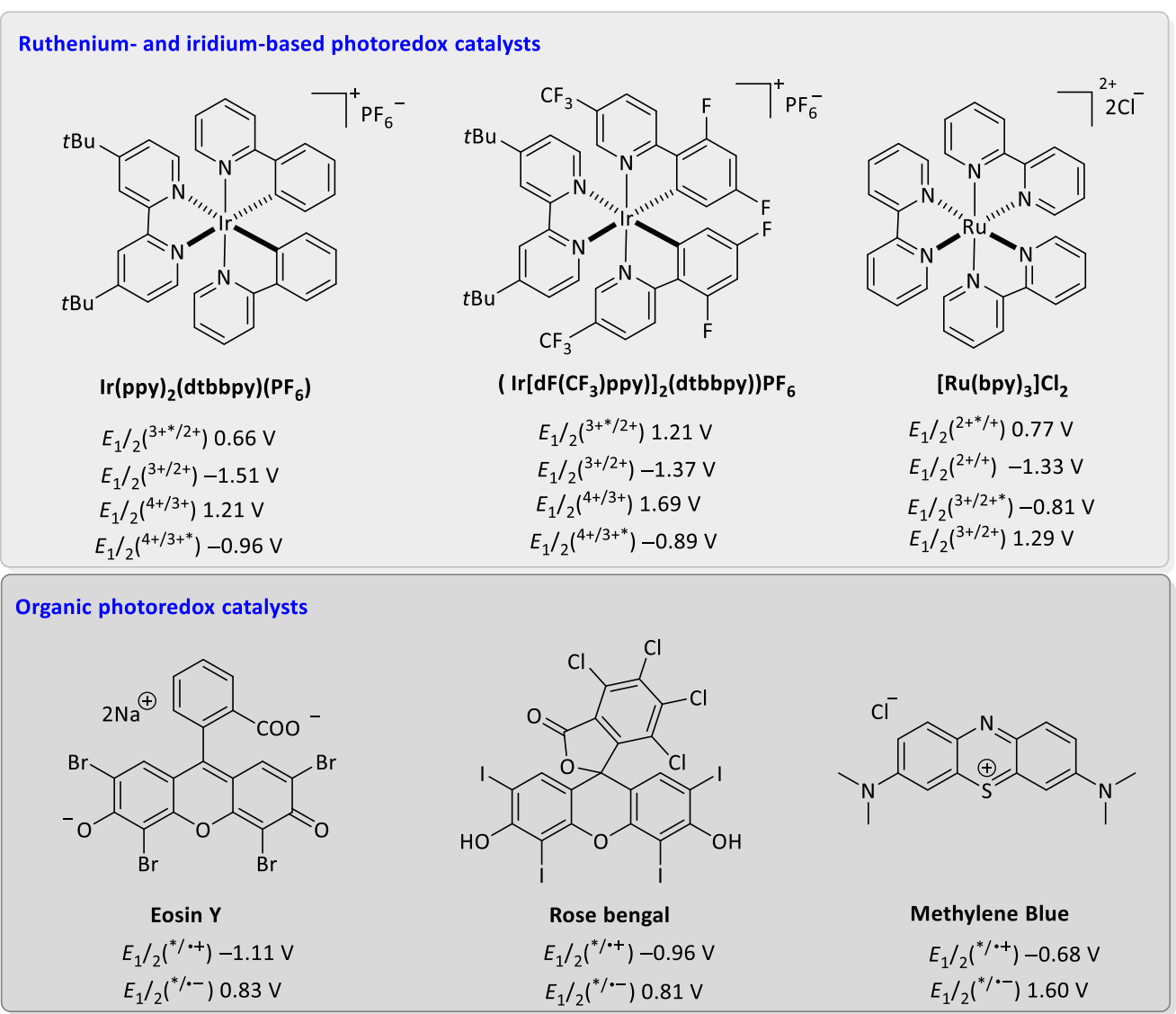

Scheme 1.1 Chemical structures of typical homogeneous photocatalysts. ${ }^{[4][6 a]}$

Even though these species are not strong oxidants or reductants in their ground states, in their excited state they are potent single electron transfer reagents with suitable redox potentials. The redox potential is a measure of the tendency of a chemical species to gain electrons from an electrode and thereby get reduced. In principle, a higher positive value of the redox potential signifies a greater affinity for electrons to be gained, thereby a higher tendency to be reduced. Hence, the redox potential of the photocatalyst must be matched to the reagents of the reaction. Compared to the transition metal complexes, metal-free organic dyes (e.g. eosin $Y$, rose bengal, 9flourenone, methylene blue and rhodamine B) have recently emerged as organic photoredox catalysts since they enable to obviate the necessity of metal complexes in synthetic transformations (Scheme 1.1). ${ }^{[6]}$ According to the comprehensive review published by the group of Nicewicz, ${ }^{[6 a]}$ organic photoredox catalysis could also offer more opportunities than transition metal-catalysed reactions. Importantly, the potent 
reactivity afforded by many organic catalysts allows access to unique reactivity and a broad range of substrates that are unreactive in most synthetic contexts. In addition to these assets, organic compounds also provide more choices in the discovery and optimization of new synthetic methodologies.

The first application of photoredox catalysis in organic synthesis was reported over 40 years ago, which built the foundations for the recent development of modern photoredox catalysis. Kellogg reported the photo-mediated reduction of sulfonium ions to the corresponding ketone (4) and thioethers using $N^{-}$substituted 1,4dihydropyridines as the terminal reductant in 1978 (Table 1.1). It worked even without catalyst with longer time. However, the reaction was accelerated by the addition of catalytic amount of photocatlysts including TPP, eosin and $\left[\mathrm{Ru}(\mathrm{bpy})_{3}\right] \mathrm{Cl}_{2} \cdot{ }^{[7]}$

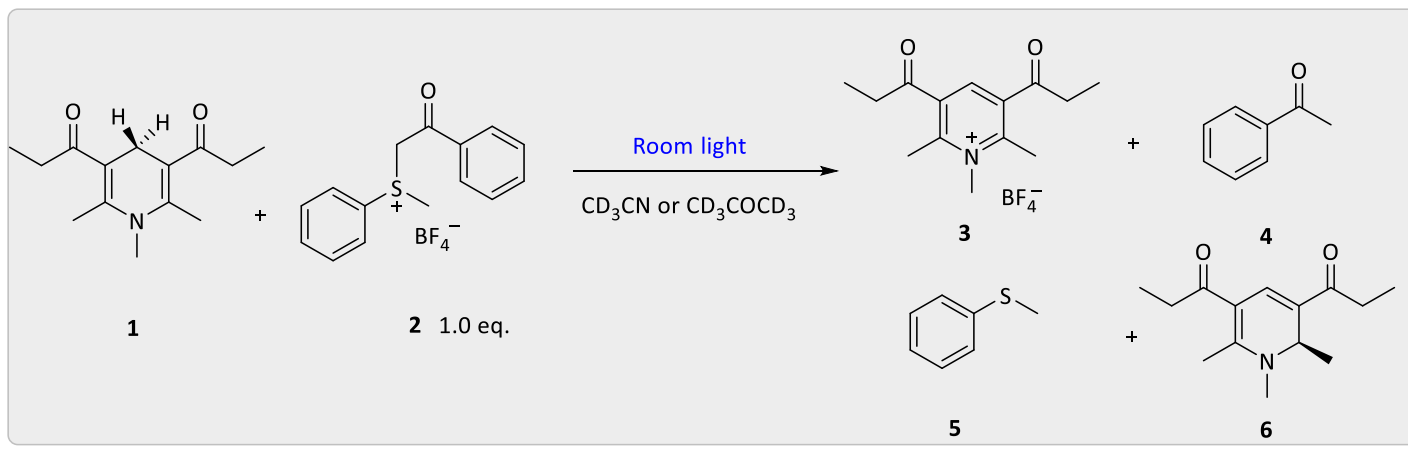

\begin{tabular}{lllllll}
\hline & Conditions & $\mathrm{t}_{1 / 2}(\mathrm{~h})$ & \multicolumn{5}{c}{ Yields (\%) } \\
\cline { 3 - 7 } & & $\mathbf{3}$ & $\mathbf{4}$ & $\mathbf{5}$ & $\mathbf{6}$ \\
\hline A & $60^{\circ} \mathrm{C}$, dark & 10 & 3 & 38 & 37 & 52 \\
B & $25^{\circ} \mathrm{C}$, dark & 72 & 0 & 0 & 0 & 0 \\
C & $25^{\circ} \mathrm{C}$, room light & 48 & 100 & 100 & 100 & 0 \\
D & $25^{\circ} \mathrm{C}$, room light, TPP & 3 & 100 & 100 & 100 & 0 \\
E & $25^{\circ} \mathrm{C}$, room light, eosin & 1 & 100 & 100 & 100 & 0 \\
F & $25^{\circ} \mathrm{C}$, room light, [Ru(bpy) $\left.)_{3}\right] \mathrm{Cl}_{2}$ & 0.3 & 100 & 100 & 100 & 0
\end{tabular}

Table 1.1 Light-induced reduction of sulfonium ions with different photocatalysts.

Heterogeneous catalysis involves complementary advantages including the ease of separation from the reaction mixture, higher chemical and photostability due to the restricted rotations and solid-state effects, the ability to create immobilized catalytic 
beds and above all the "reusability". ${ }^{[8]}$ Therefore, organic photocatalysis was also introduced into the heterogeneous realm which has been widely used in different fields, such as in water splitting, environmental remediation, $\mathrm{CO}_{2}$ reduction, disinfection etc. ${ }^{[9]}$ In the last decade, it has also been expanded towards the selective organic transformations to improve recyclability and stability of the photocatalyst. ${ }^{[10]}$ Detailed illustration is given in chapter 1.4.

\subsection{Visible-Light-Mediated Homogeneous Photocatalysis}

\subsubsection{General Mechanism of Homogeneous Photocatalysis}

In general, readily accessible transition metal complexes or organic dyes can facilitate the conversion of visible light into chemical energy under mild reaction conditions. ${ }^{[11]}$ Both of them exhibit strong and broad absorbance in the visible range that results in the production of long-lived excited states. In the photoredox (electron transfer) cycle, incoming visible light excites the photocatalyst by promoting an electron from the HOMO level to the LUMO level to form the triplet-excited state of the photocatalyst, where it has the remarkable property of being both more oxidizing and more reducing than the ground state species. Afterwards, the excited state of the photocatalyst can engage in a single-electron transfer (SET) event with organic (and organometallic) substrates via a reductive quenching cycle (pathway A) or via an oxidative quenching cycle (pathway B), thereby establishing access to a reaction environment that is unique in organic chemistry (Scheme 1.2). ${ }^{[12]}$

For instance, the half-reaction $\mathrm{Ru}(\mathrm{bpy}) 3^{3+}+\mathrm{e}^{-} \rightarrow{ }^{*} \mathrm{Ru}(\mathrm{bpy}) 3^{2+}$ has the reduction potential $E_{1 / 2}{ }^{I I I / * \|}=-0.81 \mathrm{~V}$ vs the saturated calomel electrode (SCE). This potential shows that the excited-state $\mathrm{Ru}(\mathrm{bpy})_{3}{ }^{2+}$ is a much more stronger electron donor than the ground-state of $\mathrm{Ru}(\mathrm{bpy}) 3_{3}{ }^{2+}\left(\mathrm{E}_{1 / 2}{ }^{\mathrm{III} / \mathrm{II}}=+1.29 \mathrm{~V}\right.$ vs SCE$)$. Meanwhile, the reduction potential of the excited state $\left(E_{1 / 2}{ }^{*} 1 / 1=+0.77 \mathrm{~V}\right.$ vs SCE $)$ indicates that this species is a much stronger oxidant than its ground state $\left(E_{1 / 2}{ }^{11 / I}=-1.33 \mathrm{~V}\right.$ vs SCE $) .{ }^{[4]}$ 


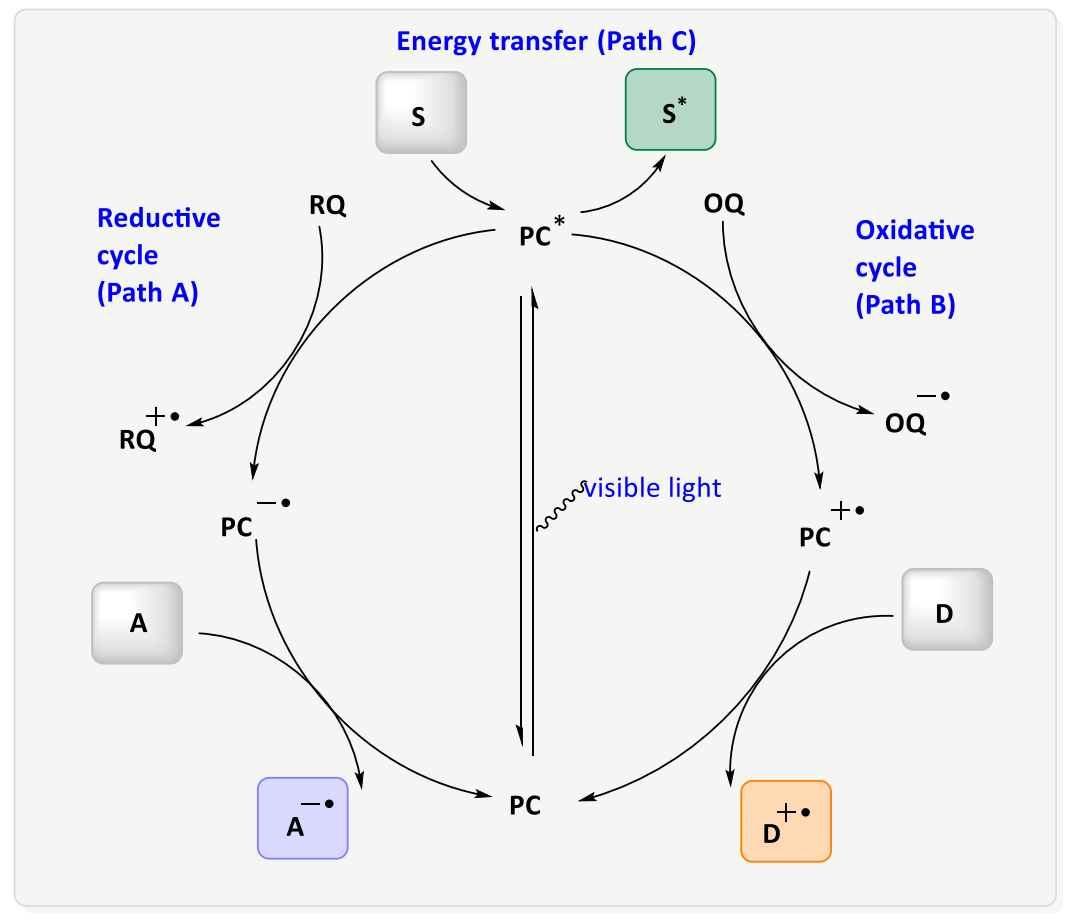

Scheme 1.2 General pathway of photocatalysis. A: acceptor; D: donor; PC: photocatalyst; S: starting material; RQ: reductive quencher; OQ: oxidative quencher.

Energy transfer catalysis (pathway C) has recently emerged to give access to the excited (triplet) state of organic compounds. ${ }^{[13]}$ Generally, electron and energy transfer processes need to be clearly distinguished within the field of visible lightmediated photocatalysis. Photoredox (electron transfer) catalysis relies on the higher redox activity of excited state photocatalyst to induce a single electron transfer (SET), which already has become a great synthetic process in the last decade. ${ }^{[14]}$ Nevertheless, energy transfer (EnT) catalysis has remained relatively underdeveloped since most of the photoredox catalysts are also powerful photosensitizers, which makes it difficult to illustrate their true mode of action. It should be noted that there is a possibility to undergo redox neutral reactions where the substrate participates in both the reductive and oxidative steps of the photocatalytic cycle, with no change to the overall oxidation state from the starting materials to the products. ${ }^{[15]}$

\section{Examples of Reductive Quenching Cycle (A)}

In 1981, Sakurai and coworkers described the $\mathrm{Ru}(\mathrm{bpy})_{3}{ }^{2+}$-mediated photocatalytic reduction of olefins with 1-benzyl-1,4-dihydronicotinamide (BNAH) as the 
reductant. ${ }^{[16]}$ In this case, the mechanistic studies clearly demonstrated that the reaction pathway underwent the reductive quenching cycle (Scheme 1.3). The redox potential of the $\mathrm{Ru}(\mathrm{bpy}) 3^{2+/+}$ was determined by cyclic voltammetry to be $-1.85 \mathrm{~V}$ vs. $\mathrm{Ag} / \mathrm{Ag}^{+}$in acetonitrile, at the same time the reduction potential of the olefin was also investigated from irreversible cyclic voltammograms. ${ }^{[17]}$ However, the group of Sakurai pointed out that the redox potential of the $\mathrm{Ru}(\mathrm{bpy}) 3^{2+/+}$ couple is close to the reduction potential of $9 \mathrm{a}(-2.20 \mathrm{~V})$, perhaps this is the lowest limit for the occurrence of electron transfer from $\mathrm{Ru}(\mathrm{bpy})^{3+}$ to olefins or these reactions occurred via energy transfer. It should be noted that preliminary experiments showed that the ground state reduction of olefins by BNAH was limited to such olefins that had reduction potentials ranging from $>-1.6$ to $-1.7 \mathrm{~V}$. Hence, this work extended the reduction of olefins a lot with the help of $\mathrm{Ru}(\mathrm{bpy}){ }_{3} \mathrm{Cl}_{2}$.

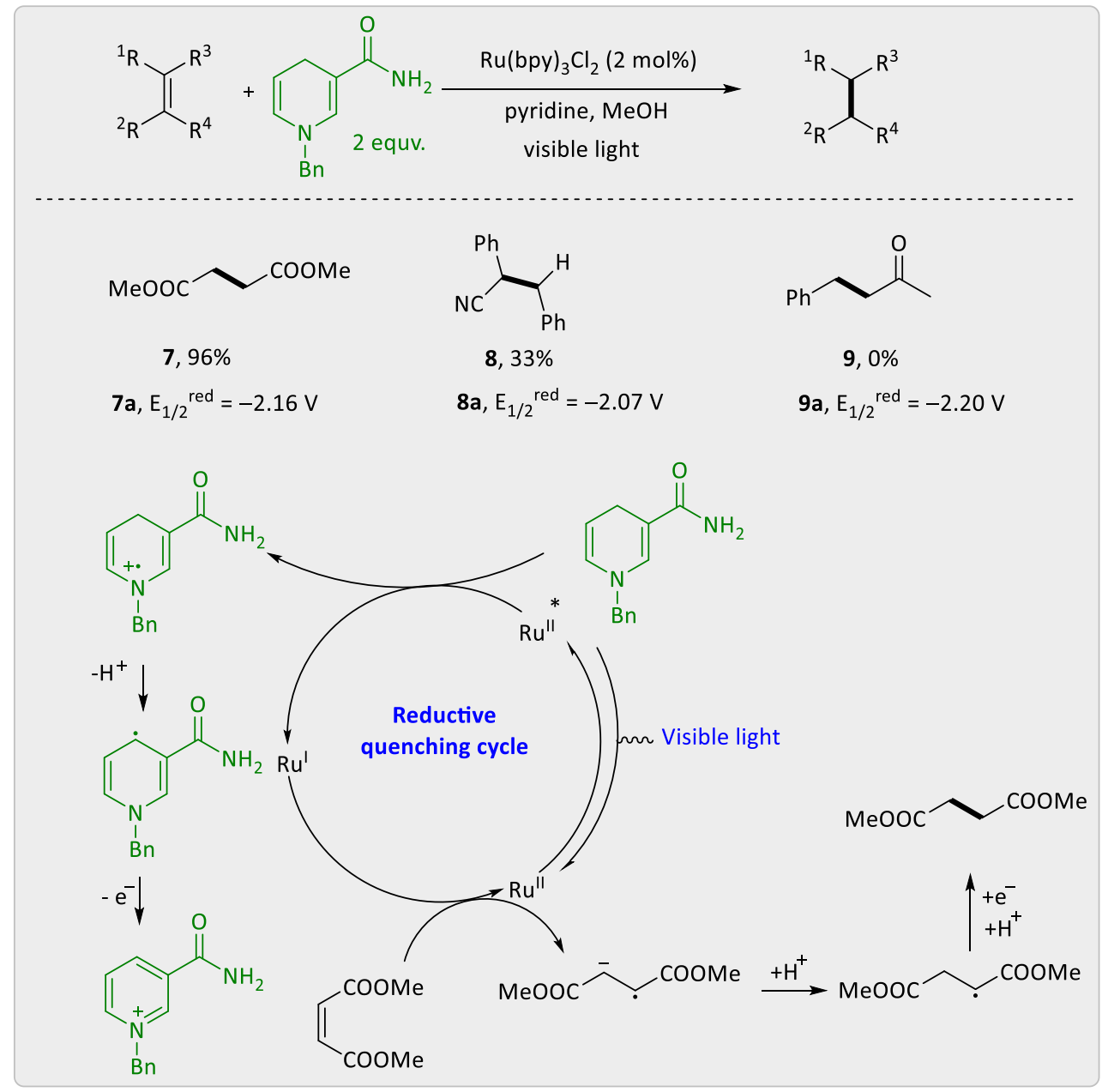

Scheme 1.3 Mechanism of photocatalytic reduction of electron-poor alkenes. 
In 2009, the Stephenson group described a mild strategy for the reductive dehalogenation using $\mathrm{Ru}(\mathrm{bpy}){ }_{3} \mathrm{Cl}_{2}$ as the photoredox catalyst and DIPEA derivatives as additives (Scheme 1.4). ${ }^{[18]}$ A wide scope of substrates containing a halogen atom attached to a carbonyl or tertiary benzyl group were dehalogenated in excellent to medium yields with good selectivity and tolerated functional groups. Notably, vinyl and aryl halides remained intact under the chosen reaction conditions. Through mechanistic investigations, a reductive quenching cycle pathway was also proposed where the photoredox catalyst $\mathrm{Ru}(\mathrm{bpy}){ }_{3} \mathrm{Cl}_{2}$ was subjected to irradiation to afford the excited state of $\mathrm{Ru}(\mathrm{bpy})_{3}{ }^{2+}$, which could be quenched by a sacrificial reductant such as DIPEA to form $\mathrm{Ru}(\mathrm{bpy})_{3}{ }^{+}$. Afterwards, another step of SET was involved from the reductant $\mathrm{Ru}(\mathrm{bpy})_{3}{ }^{+}$to the $\mathrm{C}-\mathrm{Br}$ bond, which then generated the corresponding radical.

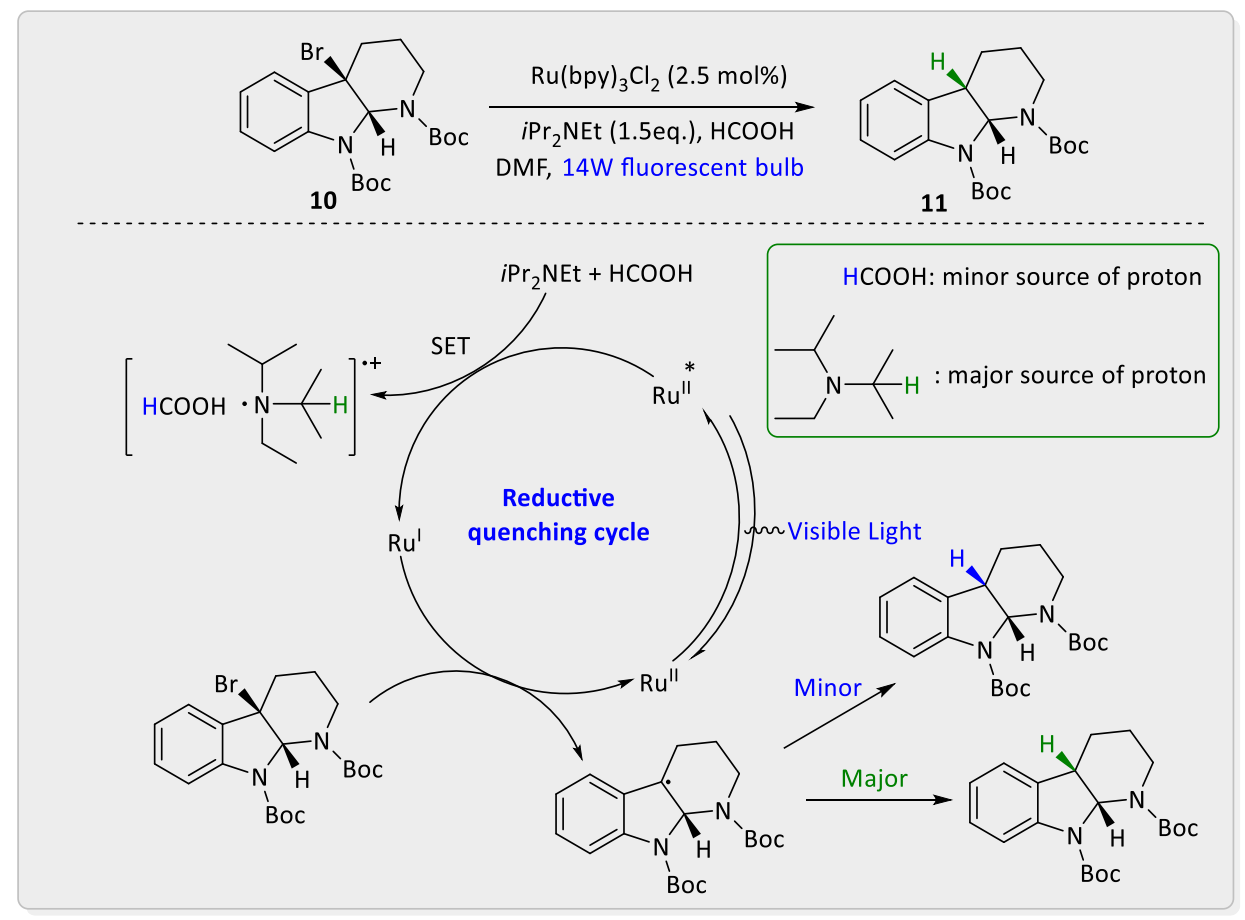

Scheme 1.4 Mechanism of visible-light-mediated reductive dehalogenation.

This mechanistic cycle was also confirmed by subsequent mechanistic studies. For example, a cyclopropane trapping experiment confirmed the radical nature of this reaction. Further investigation by isotope labelling suggested that the major hydrogen source in this reaction was DIPEA rather than formic acid. However, a basic limitation of this reaction was that only an activated halide was reactive enough to undergo this 
transformation. Essentially, this work offered an alternative strategy to form alkyl radicals which may be applied to find broad applications in organic synthesis.

Tertiary amines are desired electron donors, which can readily form the aminium radical cations by single-electron transfer. ${ }^{[19]}$ The latter could undergo $\alpha$ deprotonation to form an $\alpha$-amino radical by the addition of excessive amount of base. Thereby the $\alpha$-amino radical can either react with Michael acceptors to form new carbon-carbon bonds or react with reactive oxygen species to form amides. ${ }^{[20]}$ Based on this, Nishibayashi and Reiser simultaneously reported an efficient methodology for the visible-light-mediated addition of $\alpha$-aminoalkyl radicals, which are difficult to be generated directly from amines under thermal reaction conditions. The $\alpha$-aminoalkyl radicals then react with the Michael acceptors (electron-deficient alkenes) to construct the desired products via a reductive quenching cycle pathway (Scheme 1.5). ${ }^{[21]}$

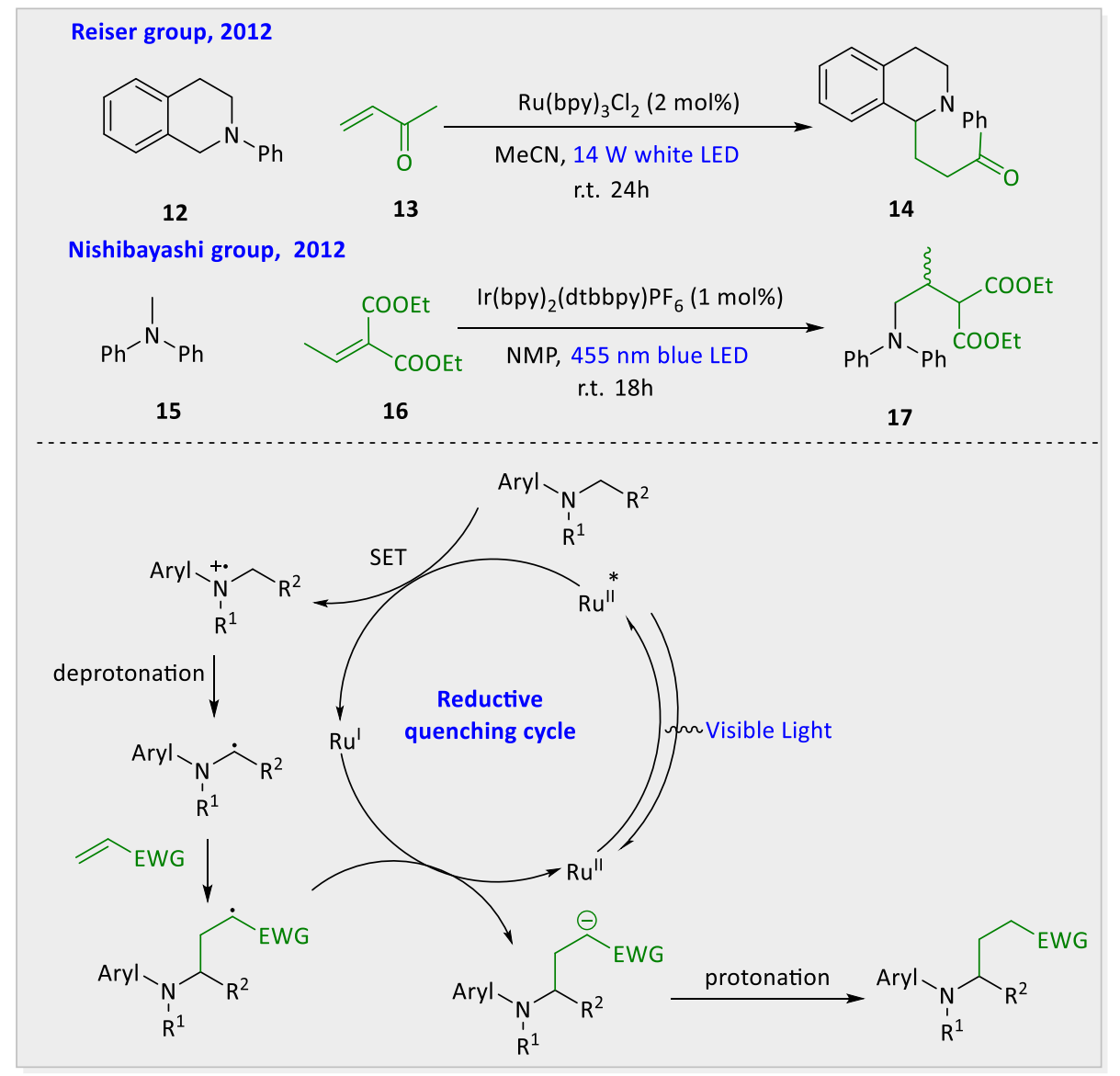

Scheme 1.5 Visible-light-mediated intermolecular addition of $\alpha$-aminoalkyl radicals to electron-deficient olefins. 


\section{Examples of Oxidative Quenching Cycle (B)}

The oxidative quenching cycle provided an alternative strategy, involving a fairly strong oxidant, which could complement the chemistry of the reductive quenching pathway, thereby expanding the utility of photoredox catalysis in organic synthesis. In terms of oxidative reactions, stoichiometric external oxidants such as oxygen or $\mathrm{CBr}_{4}$, and some bases are required to accept one electron from the excited-state photoredox catalyst. Then the substrate could be oxidized to the corresponding radical cation. In some cases, the substrate can play a role as an oxidant to accept one electron, thus it forms the substrate radical anion. ${ }^{[22]}$

For example, photoredox catalysis via an oxidative quenching cycle has been used for the synthesis of benzothiazoles and indoles with oxygen as a terminal oxidant. In 2013, $\mathrm{Li}$ and co-workers discovered a visible light-mediated photoredox approach to synthesize 2-substituted benzothiazoles through radical cyclization of thioanilides. In the plausible mechanism, the excited-state $\mathrm{Ru}(\mathrm{bpy})_{3}{ }^{2+}$ was oxidized to $\mathrm{Ru}(\mathrm{bpy})_{3^{3+}}$ by oxygen. Then the sulfur radical originated from the oxidation of thioamidate, could attack the benzene ring to form a radical intermediate and further generated the product by giving away a hydrogen to oxygen radical anion $\left(\mathrm{O}_{2}{ }^{--}\right)$(Scheme 1.6). ${ }^{[23]}$ To investigate the reaction kinetics, KIE experiments $\left(K_{H} / K_{D}=5\right)$ were performed and strongly suggested that the $\mathrm{C}-\mathrm{H}$ bond cleavage is the rate-determining step. 


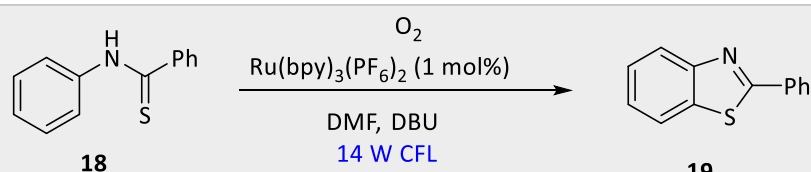

18

14 W CFL

19

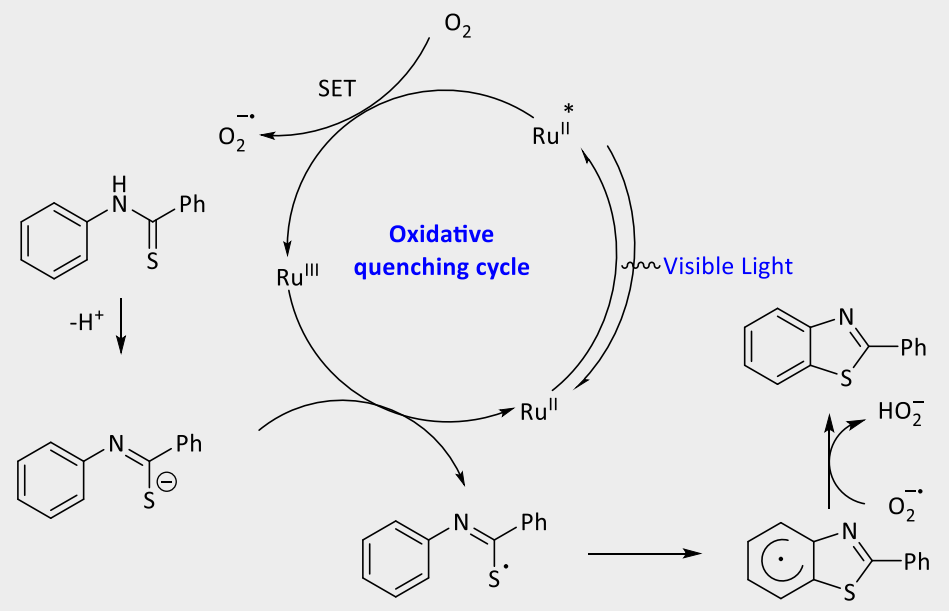

Scheme 1.6 Visible-light-mediated radical cyclization of thioanilides.

König and co-workers also reported Eosin $\mathrm{Y}$ as a photocatalyst for the direct $\mathrm{C}-\mathrm{H}$ bond arylation of heteroarenes with aryl diazonium salts (Scheme 1.7). ${ }^{[24]}$ This mild and facile procedure provides a metal-free alternative for the formation of aryl-heteroaryl bonds. More importantly, this methodology strongly proved that the application of an organic dye as a photocatalyst in organic chemistry is an efficient alternative compared with known transition-metal-catalysed ( $\mathrm{Pd}, \mathrm{Ru}, \mathrm{Ir}, \mathrm{Rh}$, and $\mathrm{Ti})$ and $t \mathrm{BuOK}-$ promoted strategies for $\mathrm{C}-\mathrm{H}$ arylation. ${ }^{[25]}$ The suggested mechanism also illustrated that the possible pathway is an oxidative quenching cycle. 


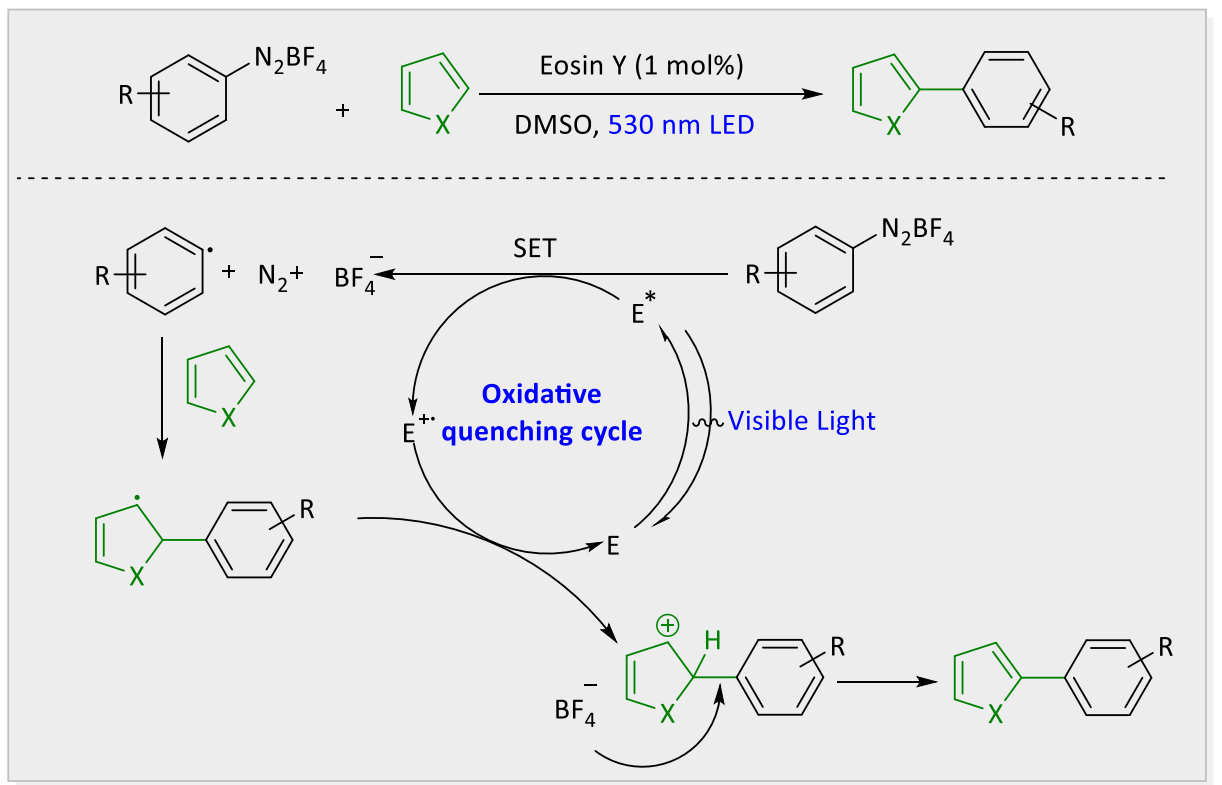

Scheme 1.7 Suggested mechanism of direct $\mathrm{C}-\mathrm{H}$ bond arylation of heteroarenes.

\section{Examples of Energy Transfer (C)}

Energy transfer in photocatalysis is defined typically as "the photophysical process in which an excited state of one molecular entity (the donor) is deactivated to a lowerlying state by transferring energy to a second molecular entity (the acceptor), which is thereby raised to a higher energy state". ${ }^{[13][26]}$ Regarding photocatalysis, absorption of a photon of the photosensitizer results in the excitation from the $S_{0}$ state to an excited singlet state $S_{1}$. The short-lived singlet state rapidly decays back to the ground state or undergoes intersystem crossing to its triplet state $\left(T_{1}\right)^{\left[{ }^{[13]}\right.}$ The photocatalyst resembles the donor, which is excited by the absorption of visible light directly. The excited state of the photocatalyst can further transfer its excited state energy to the starting material (acceptor), which is regarded as an indirectly excited or sensitized pathway.

Visible-light-mediated energy transfer catalysis is already applied to several realms of organic synthesis including cyclization reactions, photoisomerizations, bond dissociations and the sensitization of metal complexes. ${ }^{[27]}$ Yoon and coworkers made huge contributions in visible-light photocatalysis of cycloadditions. They have been able to exploit both photoreduction and photooxidation reactions of $\mathrm{Ru}(\mathrm{bpy})_{3}{ }^{2+}$ and related ruthenium(II) chromophores to design [2+2], [3+2] and [4+2] 
cycloadditions. ${ }^{[28]}$ The diversity of products is quite broad using their strategies. However, the nature of the photoinduced electron transfer processes that generate the radical ion intermediates necessarily limits the scope of these reactions.

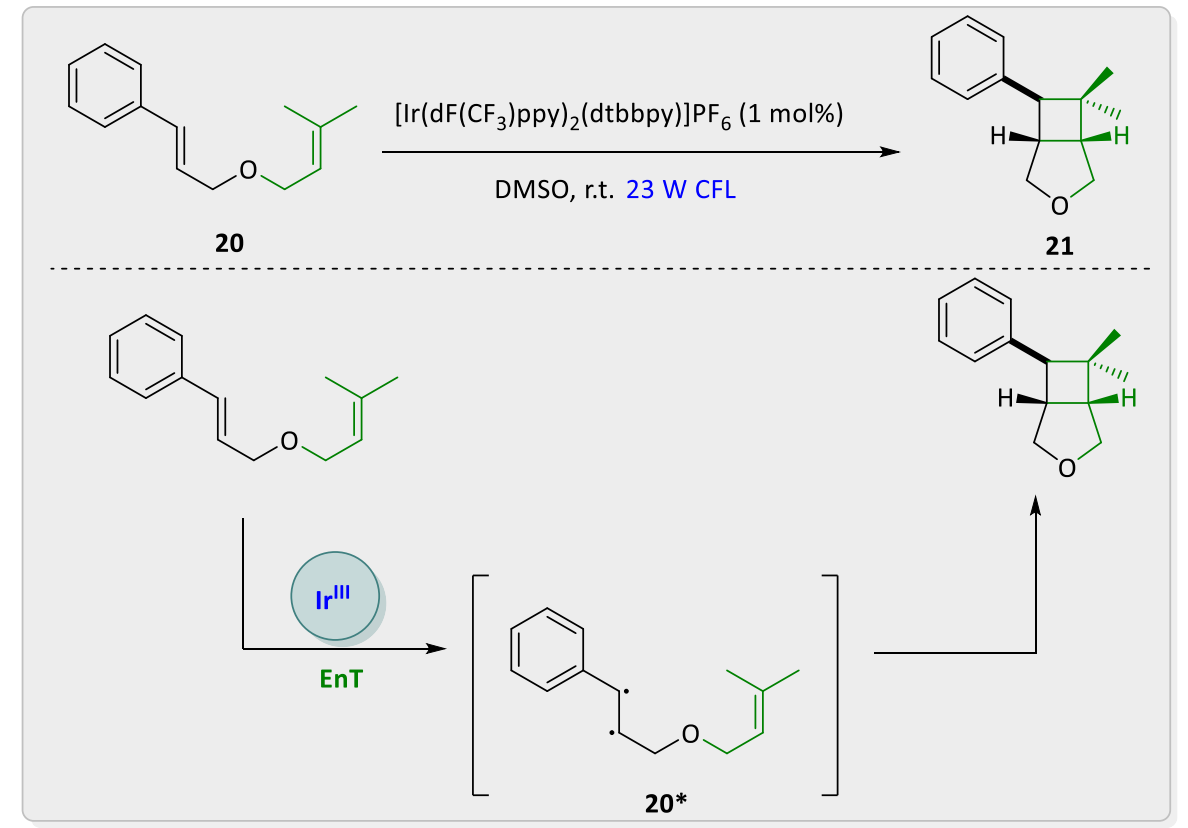

Scheme 1.8 EnT catalysis in the [2+2] photocycloadditions of styrenes.

For example, a reductive quenching pathway is an alternative in photocatalytic [2+2] cycloadditions. However, only sufficiently electron-deficient olefins can react with single-electron reduction, largely restricting the application via this pathway. ${ }^{[29]}$ Considering the oxidative quenching pathway, Yoon and co-workers further achieved the [2+2] cycloadditions of electron-rich olefins which could be oxidized to the corresponding radical cation by $\mathrm{Ru}(\mathrm{bpy}) 3^{3+}(1.29 \mathrm{~V}$ vs. SCE) generated from the oxidation of ${ }^{*} \mathrm{Ru}(\mathrm{bpy})_{3}{ }^{2+}$ by an external oxidant. Nevertheless, there are also some limitations in the substrates scope: at least one styrene must have an electrondonating substituent at the para or ortho position; meta-substituted and unsubstituted styrenes are not sufficiently electron-rich enough toward one-electron oxidation to generate the key radical cation intermediate; aliphatic olefins are not enough activated under this condition. ${ }^{[30]}$

To overcome the limitations of photoredox catalysis in cycloadditions, they described the intramolecular [2+2] photocycloadditions of alkene-tethered styrenes (Scheme 1.8). ${ }^{[31]}$ Firstly, the substrate has precluded it to participate in radical cation 
cycloadditions because of its oxidation potential $(+1.42 \mathrm{~V}$ vs SCE$) .\left[\operatorname{Ir}\left(\mathrm{dF}\left(\mathrm{CF}_{3}\right) \mathrm{ppy}\right)_{2^{-}}\right.$ (dtbbpy)]PF 6 , first reported by Malliaras and Bernhard ${ }^{[32]}$ and subsequently identified by Stephenson, ${ }^{[33]}$ did not provide a sufficient excited state oxidation potential $(+0.89$ V) to generate the radical cation of 20 . However, $E n T\left(E_{T}=61.8 \mathrm{~kJ} \mathrm{~mol}^{-1}\right)$ from this photoexcited Ir-based catalyst was used to access the triplet styrenes $20 *\left(E_{T}=55-60\right.$ $\mathrm{kJ} \mathrm{mol}^{-1}$ ), which readily reacted in an intramolecular fashion to construct the desired cyclobutane products in excellent yields. ${ }^{[34]}$

\subsubsection{Merger of Homogeneous Photoredox Catalysis with Other Catalytic Systems}

\section{Examples of Photoredox/Metal Dual Catalysis}

Photoredox catalysis can also be incorporated into a "dual catalysis" system, making the photocatalytic cycle coupled to different modes of catalysis. Indeed, these dual catalytic platforms have attracted tremendous attention recently. This can enable organic transformations that would not happen by applying the photocatalyst independently. In this regard, photoredox catalysis in combination with nickel catalysis has been used to discover novel reaction pathways. The nickel/photoredox dual catalysis was firstly reported for the direct decarboxylative $\mathrm{sp}^{3}-\mathrm{sp}^{2}$ cross-coupling of $\alpha$-amino carboxylic acids with aryl, alkenyl, or alkyl halides. According to the proposed mechanism, two crossed catalytic cycles are involved to simultaneously generate an organometallic nickel(II) species via the oxidative addition of a $\mathrm{Ni}(0)$ catalyst to a halide coupling partner and a carbon-centered radical generated through a photomediated oxidation event (Scheme 1.9). ${ }^{[35]}$

Besides nickel/photoredox catalysis, photoredox catalysis combined with other metal catalysis using palladium, copper or gold was also reported, whereby reactions can proceed under mild conditions along with high novelty. ${ }^{[36]}$ For example, $\mathrm{Pd} /$ photoredox dual catalysis was described for the $\mathrm{C}-\mathrm{H}$ arylation of arenes using aryldiazonium salts by Sanford group. ${ }^{[37]}$ This reaction can also be catalysed by palladium without photoredox catalyst, but harsh reaction conditions such as high temperature were required. Consequently, incorporation of a photocatalytic cycle allowed this reaction to proceed under mild conditions. 


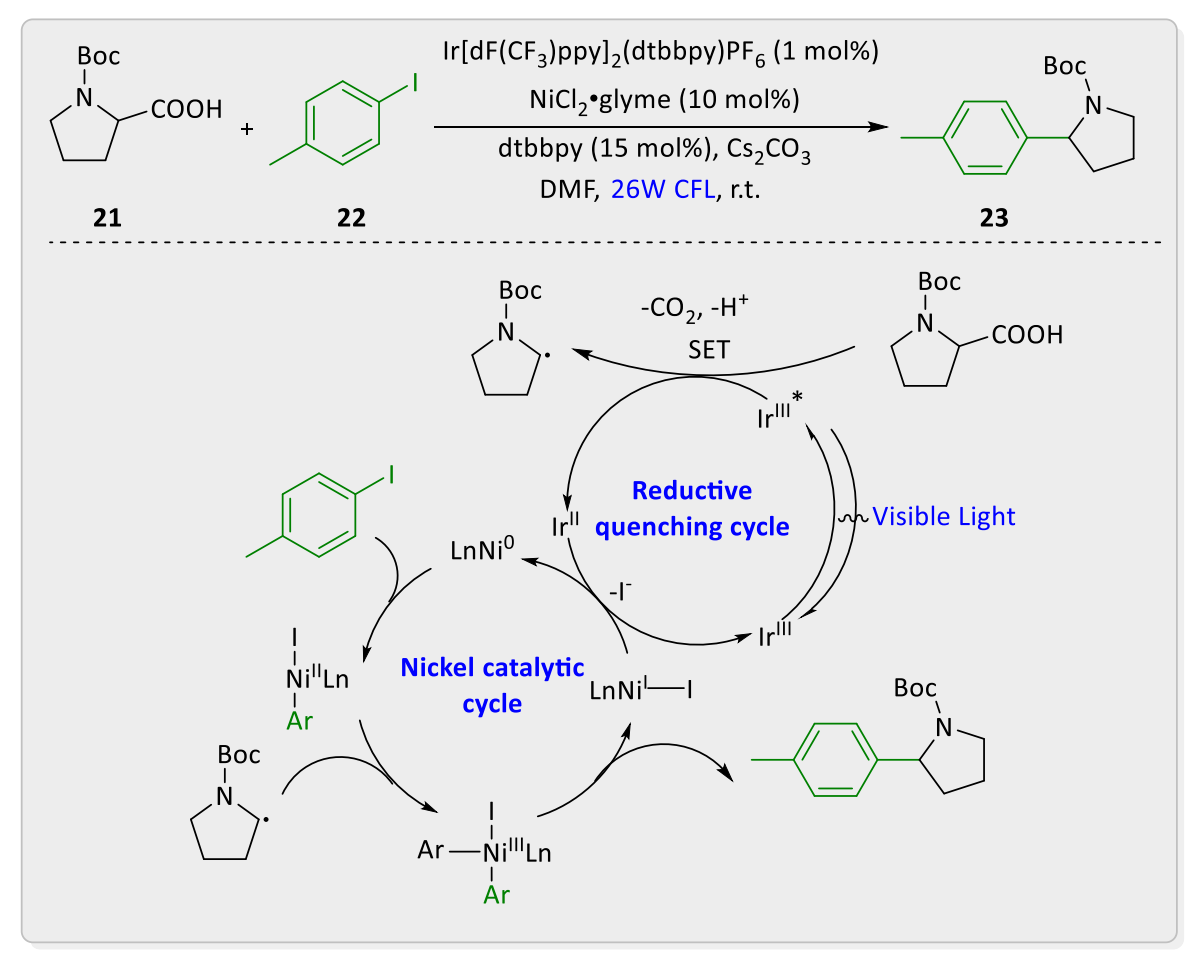

Scheme 1.9 Proposed mechanistic pathway of photoredox/nickel-catalysed decarboxylative arylation.

\section{Examples of Photoredox/HAT Dual Catalysis}

The variety of radical species generated via photoredox catalysis can be expanded by incorporation of hydrogen atom transfer (HAT). ${ }^{[38]}$ The HAT catalyst (generally thiols or $\mathrm{N}$-hydroxyphthalimide derivatives) is oxidized by a photocatalyst, and the resulting radical intermediate abstracts a hydrogen atom from the substrate, thereby generating the desired radical of the substrate. In 2015, photoredox/HAT dual catalysis has been employed for the alkylation of heteroarenes, with commercially available and abundant alcohols used as latent alkylating agents. Upon this dual catalytic system, some drug molecules can also be methylated or alkylated by methanol or other alcohols. In their proposed dual catalytic mechanism, the methanol was presumed to form the hydroxymethyl radical via hydrogen atom transfer with the thiyl radical as the key step (Scheme 1.10). 


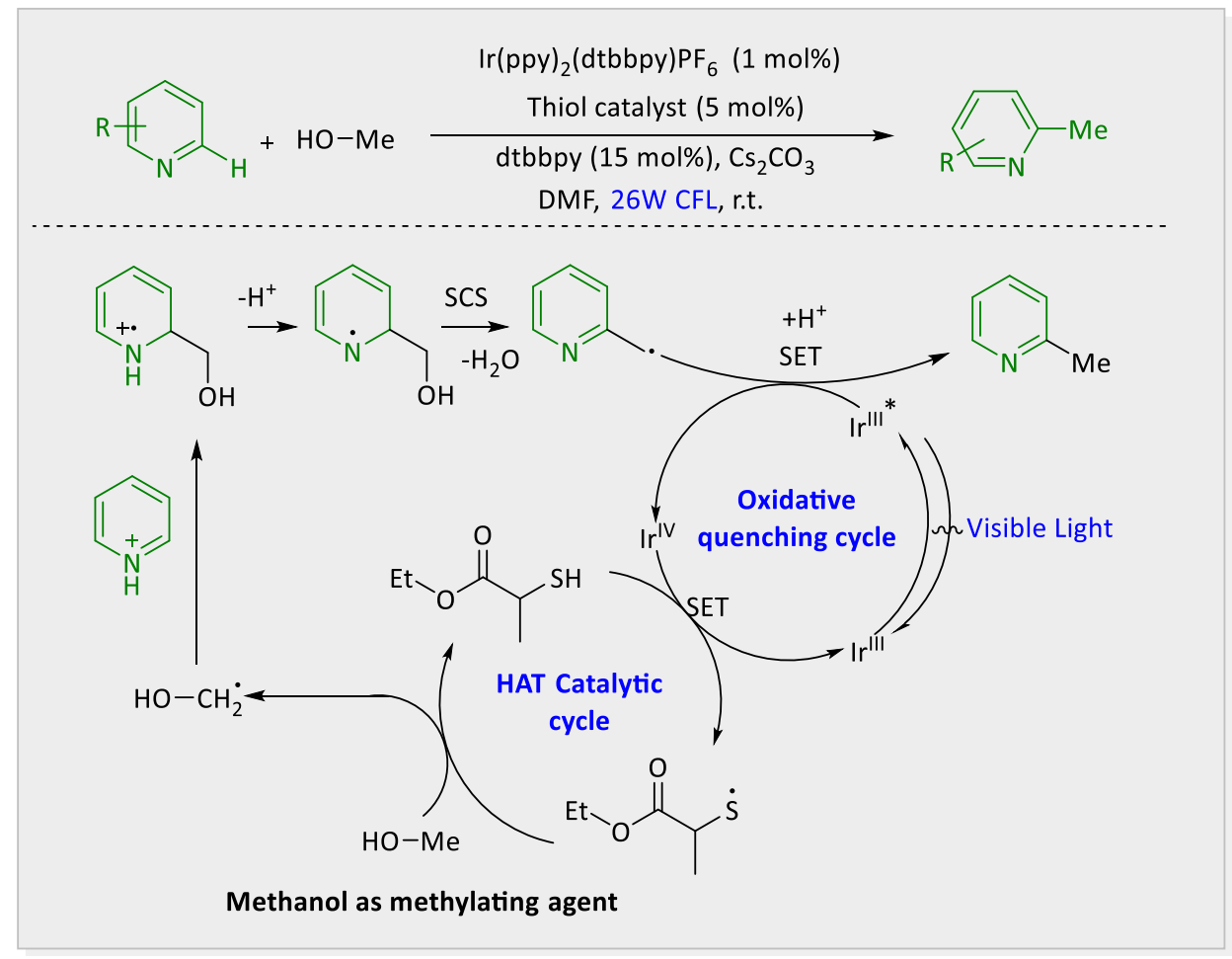

Scheme 1.10 Proposed mechanism for the direct alkylation of heteroaromatic $\mathrm{C}-\mathrm{H}$ bonds.

\section{Examples of Photoredox/NHC Dual Catalysis}

Another dual catalysis is the involvement of $\mathrm{N}$-heterocyclic carbene (NHC) along with the photocatalyst. ${ }^{[39]}$ In 2012, Rovis and coworkers reported an oxidative coupling of tetrahydroquinolines and aldehydes facilitated by a dual catalysis mode containing NHC and photoredox catalysis. In this process, two chemically distinct activation events emerged in the formation of the desired $\mathrm{C}-\mathrm{C}$ bond with the release of $\mathrm{H}_{2}$ as a byproduct. The reaction started initially with $\mathrm{Ru}(\mathrm{bpy})_{3}{ }^{2+}$ under the irradiation of blue LED, thereby generating the excited-state $R u(b p y) 3_{3}{ }^{2+}$. Then the powerful oxidant $\mathrm{Ru}(\mathrm{bpy}) 3^{3+}(1.29 \mathrm{~V}$ vs SCE$)$ was further generated in the presence of a suitable oxidant. Afterwards, oxidation of a tertiary amine followed by the hydrogen atom abstraction forming an iminium ion, and returned to the original state of $\mathrm{Ru}(\mathrm{bpy}) 3_{3}{ }^{2+}$ in the catalytic cycle. Interaction of an NHC with an aldehyde supposedly generates the nucleophilic Breslow intermediate, which could then intercept an iminium ion, forming the new $\mathrm{C}-\mathrm{C}$ bond. At last, elimination of the NHC provided the $\alpha$-amino ketone and allowed the NHC to return into the catalytic cycle (Scheme 1.11). 


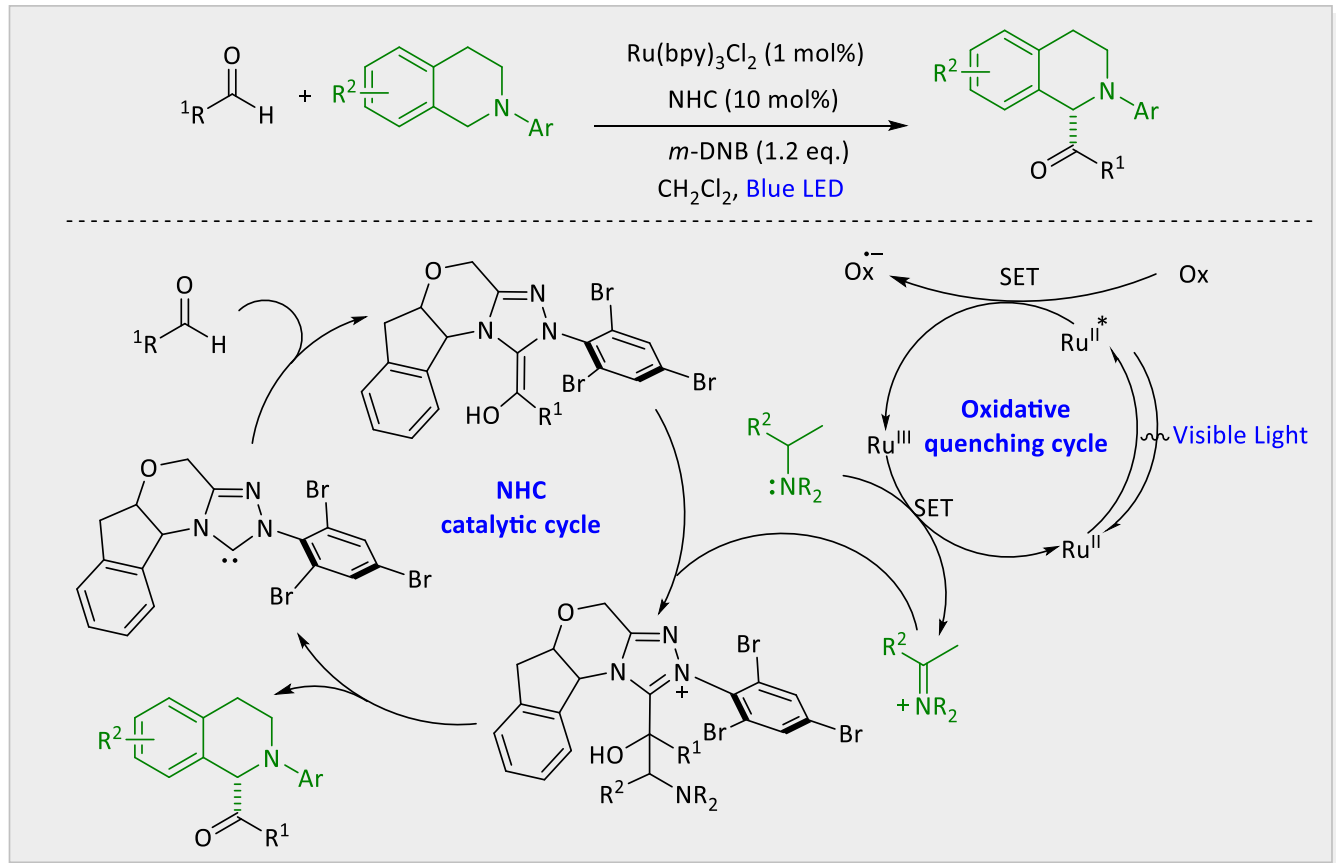

Scheme 1.11 Proposed dual catalytic cycles.

MacMillan and co-workers have also invented an organocatalytic/photoredox dual catalysis system. ${ }^{[40]}$ For example, aldehydes and ketones can be coupled with alkyl halides via utilizing a chiral secondary amine catalyst combined with a photocatalyst. This strategy was able to form the desired products with high enantioselectivity. The catalytic cycle contained the reduction of the alkyl halide to generate a radical species, then added to an enamine intermediate formed from the amine catalyst and the aldehyde substrate. The $\alpha$-amino radical was further oxidized to an iminium ion, which was finally hydrolyzed to provide the alkylated product. ${ }^{[41]}$

\subsection{Visible-Light-Mediated Heterogeneous Photocatalysis}

\subsubsection{General Mechanism of Visible-Light-Mediated Photocatalysis Using Polymeric Carbon Nitride}

Besides homogeneous visible-light-mediated photocatalysis using metal complexes or organocatalysts, heterogeneous photocatalysis has become an emerging tool for the development of new and valuable transformations in organic synthesis. It is highly promising and of great interest from the industrial point of view because of the inherent advantage of easy catalyst separation and recyclability. ${ }^{[42]}$ Generally, it can be divided into three parts according to the catalyst types. Typical metal oxides have 
large band gap such as $\mathrm{TiO}_{2}, \mathrm{Nb}_{2} \mathrm{O}_{5}$ and $\mathrm{ZnO}$, which are highly efficient photocatalysts under UV irradiation. However, the photocatalytic activity of these metal oxides usually forms radicals in a non-selective manner. Hence, they are widely used for the photodegradation of organic pollutants. ${ }^{[43]}$ Plasmonic photocatalysts are also wellknown as heterogeneous photocatalysts. The absorbance spectra surface plasmon bands of $\mathrm{Au}, \mathrm{Ag}$, and $\mathrm{Cu}$ spherical nanoparticles display maxima at $\sim 530, \sim 400$, and $\sim 580 \mathrm{~nm}$ respectively, which can be easily used within the range of visible light. ${ }^{[10 a][44]}$ For example, $\mathrm{Au} / \mathrm{TiO}_{2}(<5 \mathrm{~nm}$ diameter) could act as an efficient photocatalyst when exposed visible-light irradiation $(\lambda>450 \mathrm{~nm})$. The third type is about metal-free semiconductors, which draw considerable attention in catalysis as they show interesting photochemical properties with good stability.

Among metal-free semiconductors, the polymeric carbon nitride (PCN) is one of the most popular choices since it has a band gap of ca. $2.7 \mathrm{eV}\left(\lambda_{\mathrm{Ex}} \sim 460 \mathrm{~nm}\right)$, allowing it to be used as visible-light photoredox catalyst bypassing the use of UV light as energy input. Moreover, the band gap of PCN is narrower in comparison to the metal-based catalysts like $\mathrm{TiO}_{2}$, which even satisfies the condition of oxygen reduction reaction under solar light energy. ${ }^{[8]}$ It can even be comparable with well-known commercially available Ru or Ir-based photocatalysts or organic dyes as it has a suitable valence band gap. PCN has also excellent chemical and thermal stability up to $600^{\circ} \mathrm{C}$. Notably, the expense of synthesis of the catalyst is relatively low and in the few Euro/kg range, as both starting materials (e.g., urea or melamine) are quite cheap and the synthetic processes are simple. ${ }^{[45]}$

Additionally, PCN can be modified with different-functional starting material, which can adjust the band gap and the absorbance of wavelength. It is also expected to bind with the substrate or stabilize the reactive intermediates via changing the functional groups of the starting material. ${ }^{[8]}$ For example, the Wang and Xu groups reported a nitrogen deficient graphitic carbon nitride (UCNF) which has a slightly higher band gap $(2.8 \mathrm{eV})$ compared to the traditional $\mathrm{g}-\mathrm{C}_{3} \mathrm{~N}_{4}$. However, the nitrogen deficiency can optimize the electronic structure and probably serves as the active site for reactive species. ${ }^{[46]}$ 
Scheme 1.12 shows a general visible-light-mediated photocatalytic cycle using polymeric carbon nitride. In the first step, the catalyst is activated by the irradiation of visible light, hereby, producing oxidative valence band and reductive conduction band. Then, the valence band hole (1.4 V vs. NHE at $\mathrm{pH} 7)$ can oxidize the substrate to the corresponding radical cation. Oxidants such as molecular oxygen $\left(\mathrm{O}_{2}\right)$ can be activated through single electron transfer (SET) from the reductive conduction band of PCN and thereby form superoxide radical anion $\left(\mathrm{O}_{2}{ }^{--}\right)$, which accepts the photoinduced conduction band electrons and transports them to the cationic adduct intermediates to neutralize the charge. ${ }^{[8]}$
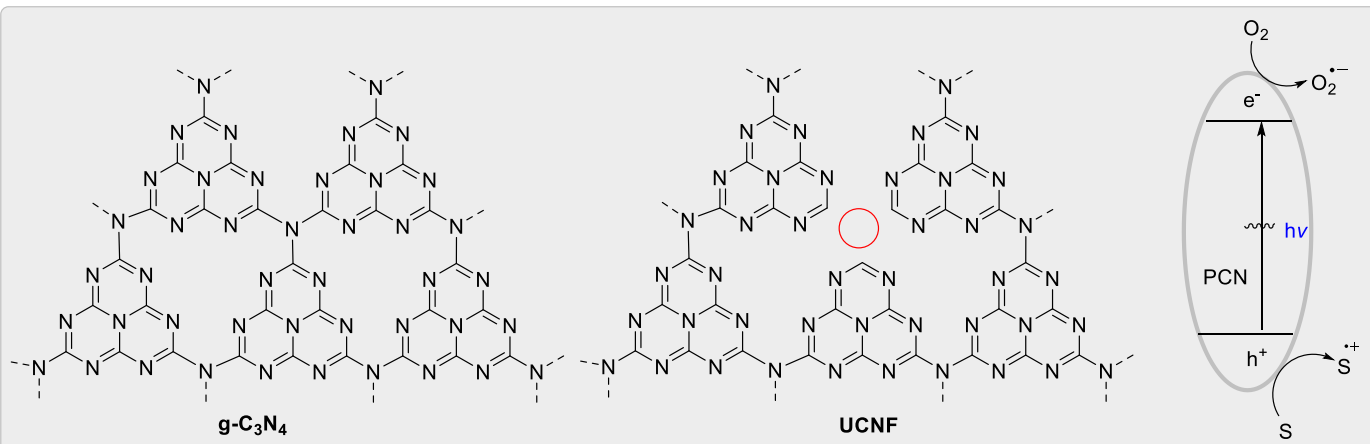

Scheme 1.12 Examples of different carbon nitrides and general mechanism; S: substrate.

\subsubsection{Recent Advances of Visible-Light-Mediated Photocatalysis Using Polymeric}

\section{Carbon Nitride}

In 2006, Antonietti et al. presented the PCN as a common available and simple photocatalyst that is able to perform Friedel-Crafts catalysis, serving as a pioneering work and unlocking the prosperity of photocatalysis using a PCN. ${ }^{[4]}$ Heterogeneous photocatalytic allylic and benzylic oxygenation ${ }^{[48]}$ and cycloadditions ${ }^{[49]}$ were already demonstrated using the PCN as a photocatalyst. In addition, it was able to drive many other reactions such as oxidation, hydrogenation, alkylations etc. Hence, different applications and recent advances of the PCN will be explained and discussed in this section. 


\section{Photocatalytic Oxidation of Amines by Polymeric Carbon Nitride}

Imines are regarded as important electrophilic intermediates in organic synthesis. Selective oxidation of secondary amines into imines can be achieved by employing stoichiometric amounts of an oxidant such as 2-iodoxybenzoic acid or oxygen/air as the terminal oxidant utilizing various transition metal catalysts and organic dyes. ${ }^{[50]}$ In 2010, the research group led by Blechert and Wang reported the aerobic oxidation of amines into imines using polymeric graphite carbon nitride. In addition, this oxidative coupling approach was applied to the one-pot synthesis of benzoxazoles, benzimidazoles and benzothiazoles. ${ }^{[51]}$

\section{a-Aminoalkyl Radical Additions, Alkylations, and Heteroarylations}

So far, applications of visible-light-mediated heterogeneous photocatalysis are mainly in photoredox oxidations and oxidative couplings under aerobic conditions. Inspired by the possibility to reductively activate $\mathrm{O}_{2}$ for the generation of highly reactive superoxide radical anions $\left(\mathrm{O}_{2}{ }^{\circ-}\right)$ through a one-electron photoreduction, the application of PCN as heterogeneous photocatalysts toward reactive $\alpha$-aminoalkyl radicals has been reported by the group of Rueping. In this case, the PCN was employed to generate the $\alpha$-tertiary and $\alpha$-secondary-aminoalkyl radicals from $\alpha$ silylamines and $\alpha$-amino acids under visible light irradiation, respectively. With combination of different acceptors, several photoredox catalysed transformations were realized including desilylative and decarboxylative additions to $\alpha, \beta$-unsaturated compounds, desilylative and decarboxylative allylation, and desilylative heteroarylations (Scheme 1.13). ${ }^{[52]}$ 


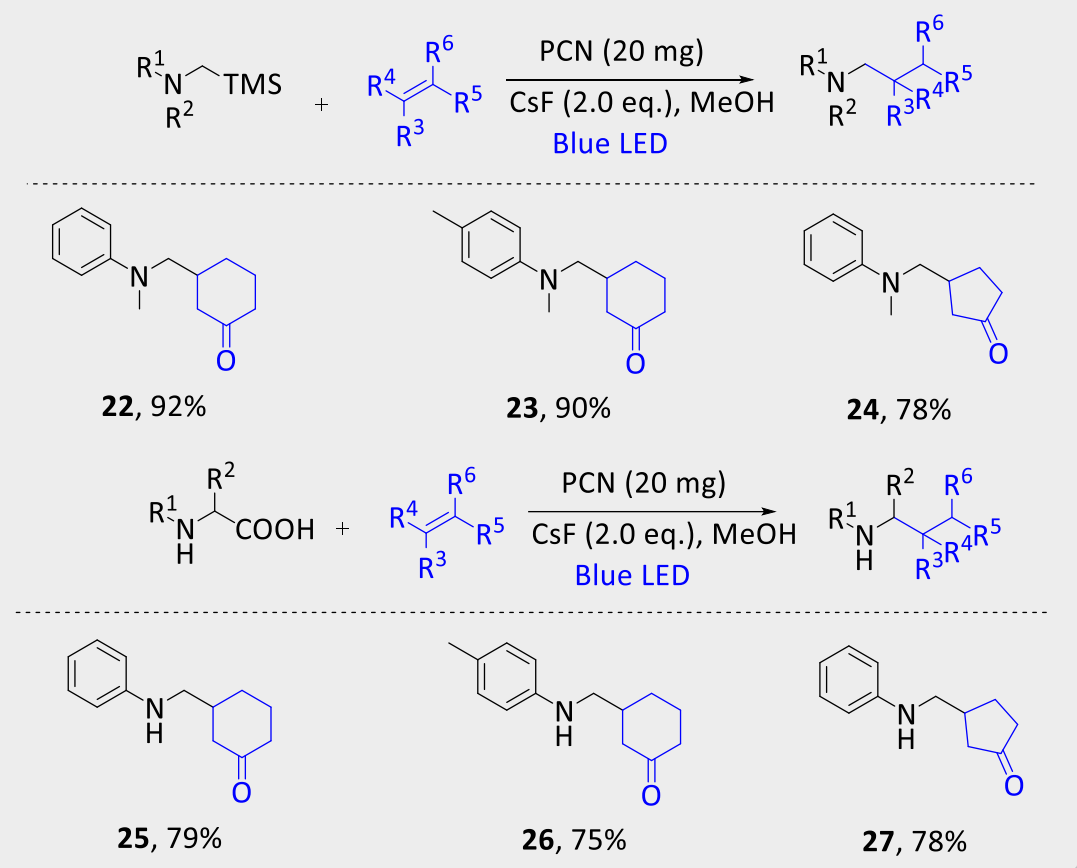

Scheme 1.13 Desilylative addition of $\alpha$-silylamines and decarboxylative addition of $\alpha$ amino acids.

\section{Bifunctionalization of Arenes and Heteroarenes using mpg-CN}

In 2019, the König group and the Antonietti group reported jointly the application of the mesoporous graphitic carbon nitride ( $\mathrm{mpg}-\mathrm{CN}$ ) as a heterogeneous photocatalyst for synthetic functionalizations of arenes and heteroarenes. ${ }^{[53]}$ In the process of arenes $\mathrm{C}-\mathrm{H}$ functionalization, the photogenerated hole and electron on the catalyst surface orchestrated oxidative and reductive redox steps to yield arene products functionalized at two distinct $\mathrm{C}-\mathrm{H}$ sites from either two or three starting materials. The synthetic examples of semiconductor photocatalytic arenes $C\left(s p^{2}\right)-C\left(s p^{3}\right) / C\left(s p^{2}\right)-$ heteroatom bifunctionalizations at two distinct $\mathrm{C}-\mathrm{H}$ sites are shown by using alkyl bromides as the source of two different functional groups (Scheme 1.14). 


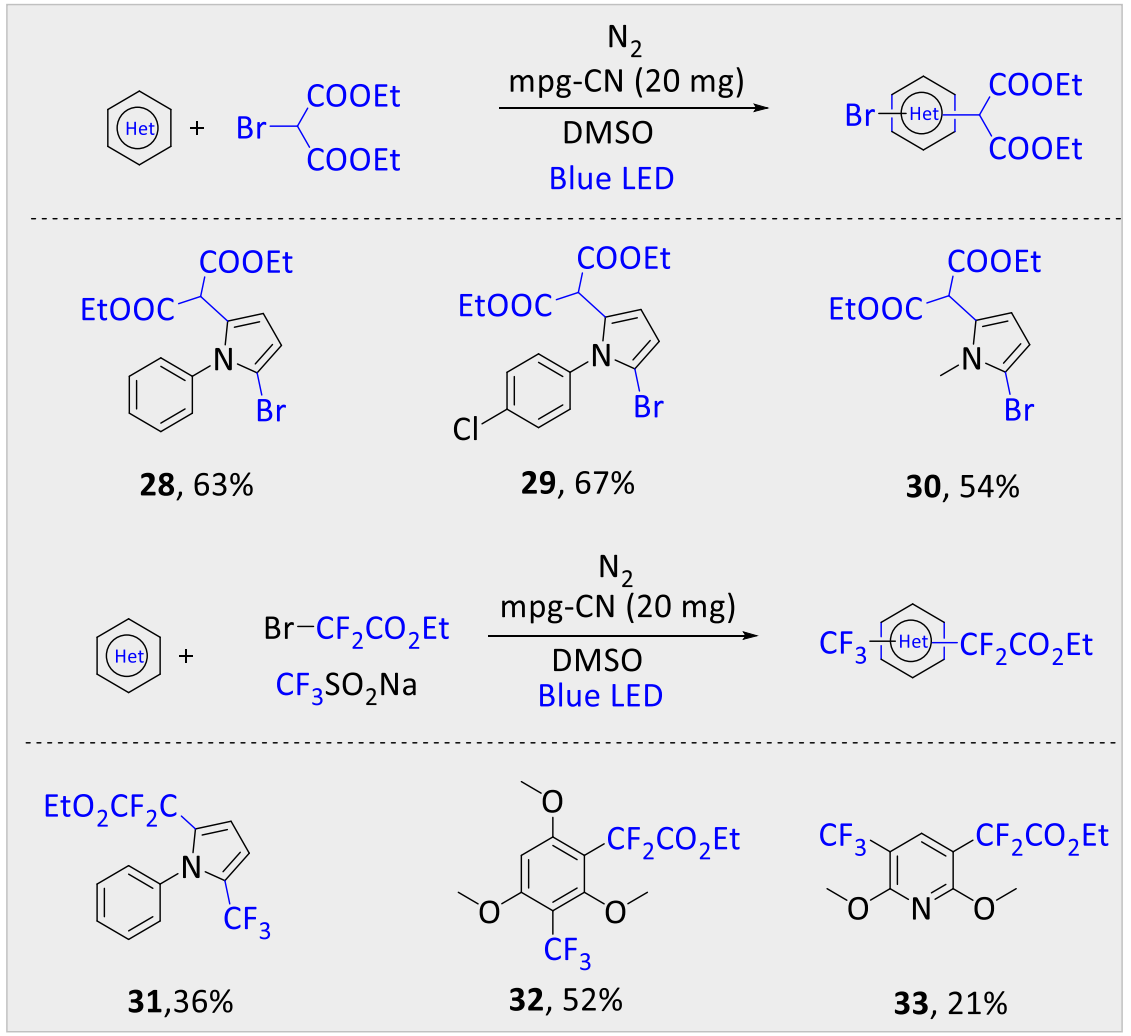

Scheme $1.14 \mathrm{C}-\mathrm{H}$ bifunctionalization of arenes using mpg-CN.

Upon single-electron reduction, the $\mathrm{C}\left(\mathrm{sp}^{3}\right)$-bromine bond in alkyl bromides breaks spontaneously, generating the relevant alkyl radical and a bromide anion for bifunctionalizations. In the presence of the $\mathrm{mpg}-\mathrm{CN}$, under the irradiation of blue light, the desired bifunctionalized arenes were formed. For direct $\mathrm{C}-\mathrm{H}$ bond monofunctionalizations of arenes and heteroarenes by the $\mathrm{mpg}-\mathrm{CN}, \mathrm{C}\left(\mathrm{sp}^{3}\right)$ - and $\mathrm{C}\left(\mathrm{sp}^{2}\right)$-centered radicals were generated. Particularly, the ${ }^{\circ} \mathrm{CF}_{3},{ }^{\circ} \mathrm{CH}_{2} \mathrm{CF}_{3},{ }^{\circ} \mathrm{CF} 2 \mathrm{H}$, or pentafluoro aryl ${ }^{\circ} \mathrm{C}_{6} \mathrm{~F}_{5}$ radical sources have been recently used extensively for latestage functionalization of pharmaceutically relevant molecules. Irradiation of a reaction mixture containing arenes, the $\mathrm{mpg}-\mathrm{CN}$, and the commercially available trifluoromethanesulfonic acid sodium salt under air led to the formation of the corresponding trifluoromethylated products with excellent isolated yields, providing a mild methodology to form trifluoromethylated products compared to the available methods which need difficult-to-handle trifluoro reagents or harsh conditions with the help of transition-metal catalysts (Scheme 1.15). 


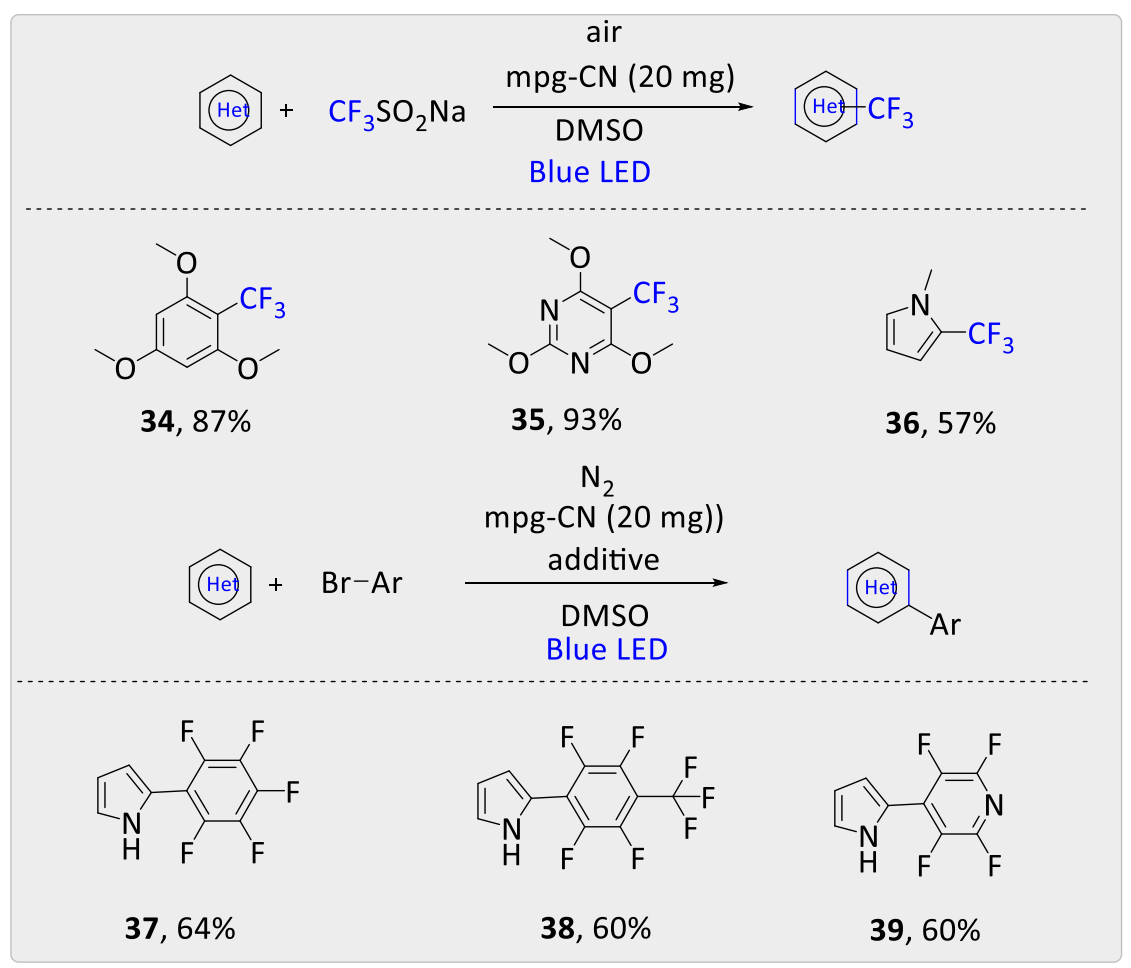

Scheme 1.15 Direct $\mathrm{C}-\mathrm{H}$ bond trifluoromethylations, difluoromethylations, perfluoroarylations of arenes.

This work also exhibited another pathway to facilitate the $\mathrm{C}-\mathrm{H}$ bond functionalization in arenes upon their reaction with nucleophiles. Inorganic or organic alkali metal salts (such as $\mathrm{KBr}, \mathrm{KCN}$ ) served as precursors for the direct $\mathrm{C}-\mathrm{H}$ bond functionalization of arenes. These experiments only involved mixing of the substrate, the respective alkali metal salts, and catalyst, followed by the addition of solvent under the irradiation of blue light. However, the reactions were quite slow and took more than four days with oxygen (in air) as the oxidant which could be improved in future. In addition, dual photoredox/nickel catalysis by combining heterogeneous mpg-CN photocatalyst and $\mathrm{NiBr}_{2} \bullet$ glyme was introduced, which afforded the corresponding $\mathrm{C}-\mathrm{N}$ functionalized products in excellent yields.

In conclusion, a great interest in applying the PCN as a photocatalyst for selective oxidation reactions, hydrogenation reactions and functionalization of $\mathrm{C}-\mathrm{H}$ bonds was observed. However, convincing reaction mechanism for carbon nitride and versatile synthetic strategies are still needed. Modifications of PCN catalyst to access suitable band gap and combining with other type of catalysts such as HAT or metal catalysts (dual catalysis) should provide broad applications of these catalysts and provide 
guidance for the catalyst design. ${ }^{[54]}$

\subsection{Metal-free Photocatalytic Benzylic $\mathrm{C}\left(\mathrm{sp}^{3}\right)-\mathrm{H}$ Oxygenation Using Oxygen as an}

\section{Oxidant}

\subsubsection{Advantages of Photocatalytic Oxygenation Using Oxygen}

Oxygenation is one of the popular methods in chemical industry. ${ }^{[55]}$ Compounds containing oxygen atoms play a crucial role in the biochemistry of living systems. ${ }^{[56]}$ Moreover, functional groups with oxygen are often designed part in the pharmacophores of diverse therapeutic agents. Numerous oxygen-containing drugs (>200) have been marketed worldwide, including widely used antibiotics, anticoagulants, and cholesterol-lowering statins (Scheme 1.16). ${ }^{[57]}$ Nevertheless, current synthetic methods employ stoichiometric and expensive terminal oxidants such as iodosobenzene, $\mathrm{PhCO} 3 \mathrm{tBu}$ and $\mathrm{RuO}_{2} / \mathrm{NalO}_{4}$, which generate equal or excessive amount of byproducts with high E-factors (Environmental factor, mass of waste per unit mass of product). ${ }^{[58]}$ Hence, cleaner and greener oxidants to minimize the amount and toxicity of the released wastes are in urgent demand to protect our everworsening environment and limited resources. In nature, oxygenases can directly incorporate an oxygen atom from molecular oxygen into the target molecule during biological syntheses. ${ }^{[59]}$ Those enzymatic processes have inspired organic chemists to develop synthetic analogues, as oxygen (or air) is cheaper and generates mostly water as byproduct. ${ }^{[60]}$

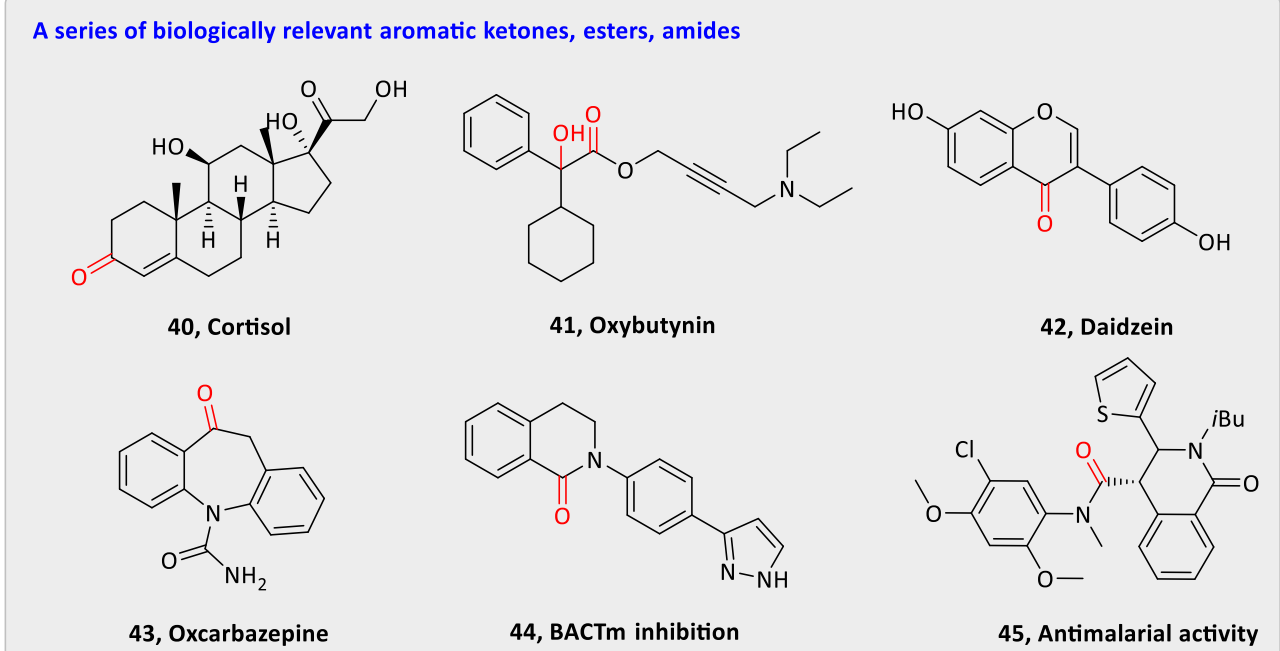

Scheme 1.16 Selective oxygenated products containing bioactive molecules and 
natural products.

The oxygenation reactions can also be employed through the functionalization of $\mathrm{C}-\mathrm{H}$ bonds which is indeed regarded as one of the emerging strategies. ${ }^{[61]}$ Principally, a relatively inert $\mathrm{C}-\mathrm{H}$ bond is activated and the hydrogen atom acts essentially as a leaving group. Particularly, a strategy involving regioselective $\mathrm{C}-\mathrm{H}$ bond functionalization shows high potential and offers promising new directions when $\mathrm{O}_{2}$ is used as the green oxidant. Indeed, there has been an explosive growth in the field of converting $\mathrm{C}-\mathrm{H}$ bonds to $\mathrm{C}-\mathrm{O}$ bonds and their applications towards the synthesis of natural products and pharmaceuticals over the past decades.

So far, transition-metal catalysts have been well explored for the formation of C-O bonds through the functionalization of $\mathrm{C}-\mathrm{H}$ bonds using molecular oxygen as oxidant. ${ }^{[62]}$ Moreover, other cheaper metals have also been applied to the $\mathrm{C}-\mathrm{H}$ bond oxygenation reactions. ${ }^{[63]}$ Alternative strategies for oxygenations, especially metalfree conditions, are highly attractive because of nontoxic, cheap, and easily separable organic compounds acting as catalysts. ${ }^{[64]}$ In general, organic molecules can activate oxygen and convert it to highly reactive compounds known as reactive oxygen species (ROS) under visible-light irradiation, which are the key to oxygenation reactions. ${ }^{[65]}$ So far, four major ROS have been recognized: superoxide radical anion $\left({ }^{\circ} \mathrm{O}_{2}^{-}\right)$, hydrogen peroxide $\left(\mathrm{H}_{2} \mathrm{O}_{2}\right)$, singlet oxygen $\left({ }^{1} \mathrm{O}_{2}\right)$, and hydroxyl radical $\left({ }^{\circ} \mathrm{OH}\right)$, and these are the key oxidants in many of the known oxygenation reactions via the functionalization of $\mathrm{C}-\mathrm{H}$ bonds. ${ }^{[66]}$ Upon excitation, oxygen molecules undergo single-electron transfer (SET) or energy transfer with excited-state catalysts to provide facile access to reactive species. Later, the reactive species from the substrate combine with the ROS and are thus transformed into the desired products with the release of water or hydrogen peroxide, which is obviously lower in toxicity and less polluting compared to the byproducts forming from other oxidants. There are a few comprehensive reviews about organic photocatalysis and $\mathrm{C}-\mathrm{H}$ bond functionalization. ${ }^{[67 a]}$ Moreover, several minireviews focusing on visible-light photoredox catalysis or visible-light-mediated oxygenation reactions are available and provide an overview of recent advances in this field. ${ }^{[67]}$ 


\subsubsection{Homogeneous Photocatalytic Benzylic C(sp $\left.{ }^{3}\right)-\mathrm{H}$ Oxygenation Using Oxygen}

\section{Visible-Light-Mediated Metal-Free Oxygenation of Amines}

In 2010, König and coworkers for the first time reported the photocatalytic oxygenation of amines to amides using riboflavin tetraacetate as the photocatalyst under blue-emitting LEDs (440 $\mathrm{nm}$ ) (Scheme 1.17). In this case, oxygen was used as the terminal oxidant and hydrogen peroxide appeared as the only byproduct in most cases. Besides the oxygenation of amines to amides, this catalytic system also exhibited reactivity towards the cleavage of trans-stilbene and oxidation of methylbenzenes. The mechanism of using flavin as an organocatalyst was also proposed. When an electron donor was present, the excited triplet form of flavin underwent two electron reduction and protonation subsequently to form dihydroflavin, which was further oxidized back to flavin by oxygen as the terminal oxidant. ${ }^{[68]}$

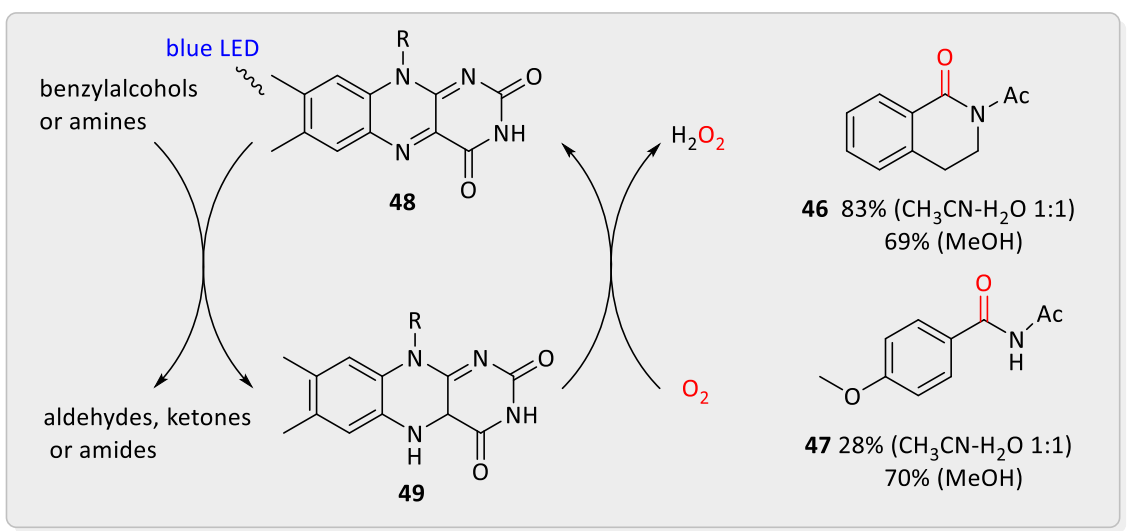

Scheme 1.17 Oxygenation of acylated benzylamines and proposed mechanism.

Later, Fu and coworkers reported an efficient aerobic oxygenation of $\mathrm{N}$ alkylpyridinium salts using Eosin $\mathrm{Y}$ as a metal-free photocatalyst. This method provided a novel and useful strategy for the synthesis of corresponding $\mathrm{N}$-methyl pyridones, quinolones, and isoquinolones derivatives. More importantly, a gram-scale experiment of 2-methyl-5-nitroisoquinolin-2-iumiodide was performed successfully under their mild reaction conditions. Clearly, it is promising to apply this metal-free protocol for the practical use (Scheme 1.18). ${ }^{[69]}$ 

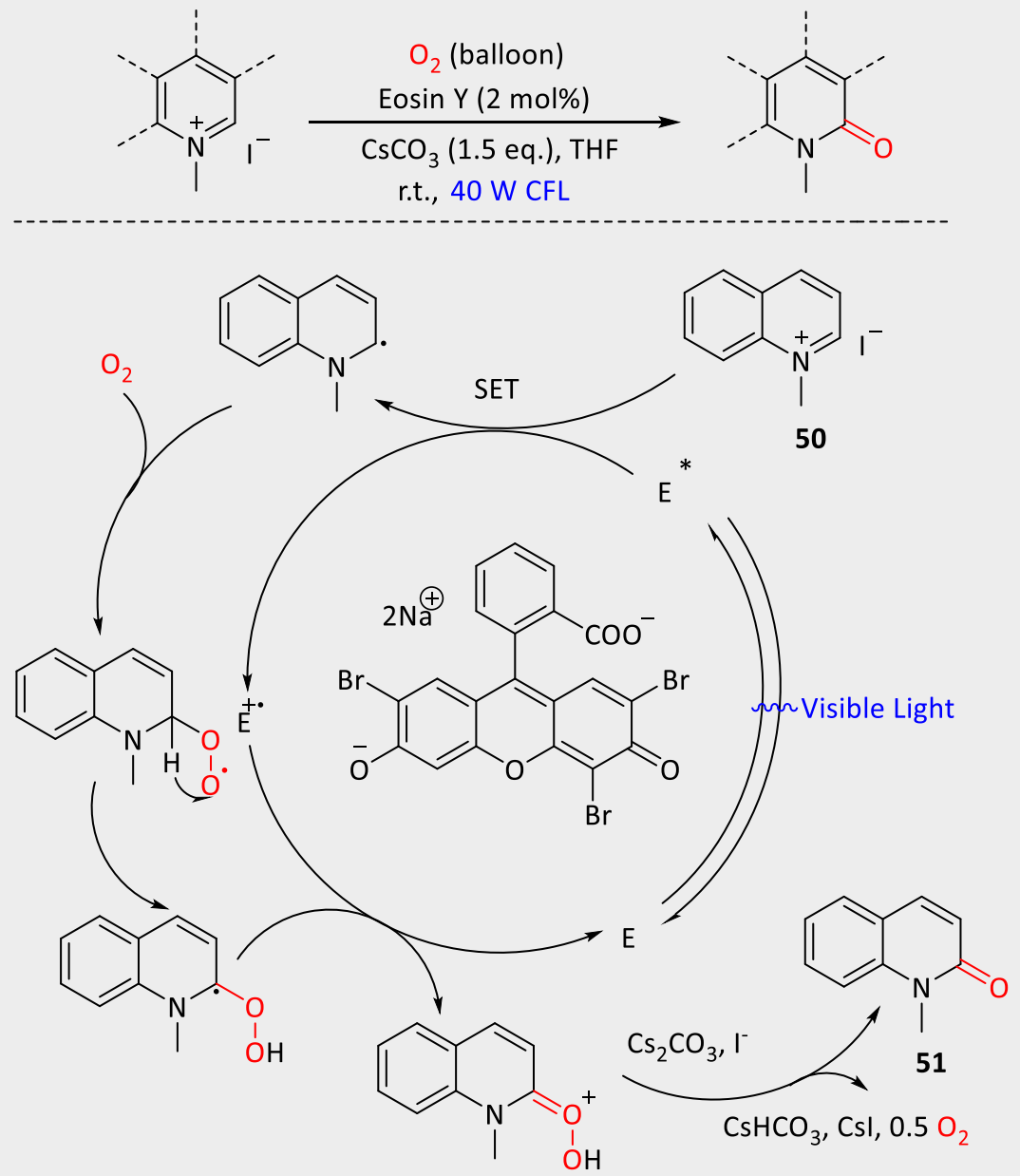

Scheme 1.18 Mechanism of oxygenation of $N$-alkylpyridinium salts.

Later, the group of Lee continued to work in this area and found that eosin $Y$ can also catalyse oxygenation of $\mathrm{N}$-substituted tetrahydroisoquinolines to dihydroisoquinolones. Notably, this methodology did not require any transition metal catalyst or reagent which is a promising way to synthesize diverse bioactive compounds containing the dihydroisoquinolone moiety (Scheme 1.19). ${ }^{[70]}$ However, the substrate scope was limited considering that only $\mathrm{N}$-substituted tetrahydroisoquinolines reacted under these conditions. In 2019, the Cai group also reported the $\mathrm{g}-\mathrm{C}_{3} \mathrm{~N}_{4}$ as a heterogeneous photocatalyst for the visible-light-mediated oxygenation of amines to amides and phthalides, the major improving aspect being the replacement of the homogeneous catalyst by a heterogeneous one. ${ }^{[48]}$ 


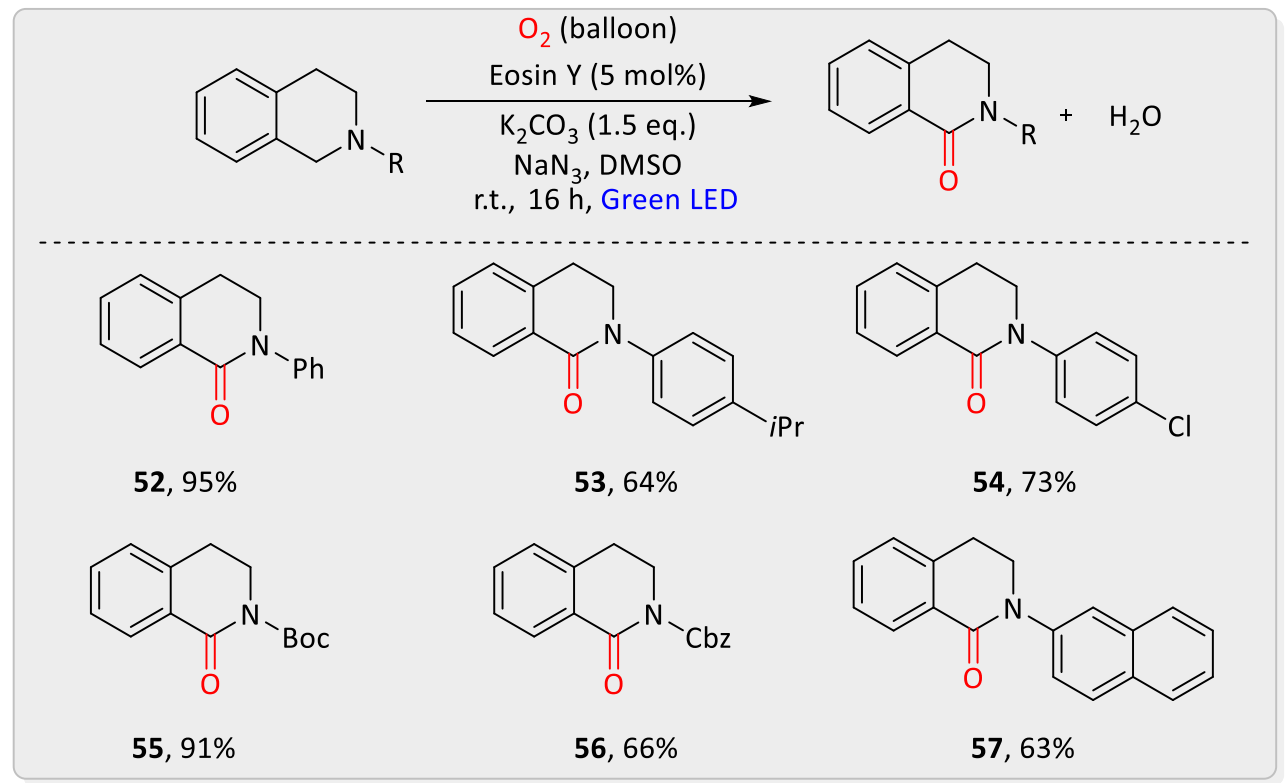

Scheme 1.19 Oxygenation of $\mathrm{N}$-substituted tetrahydroisoquinolines.

In summary, the oxygenation of amines or $\mathrm{N}$-alkylpyridinium salts still has some limitations and challenges in photocatalysis. For example, the reactions are limited to tertiary amines, it is also hard to active aliphatic amines, presumably due to the higher $\mathrm{BDE}$ (Bond-dissociation energy) of aliphatic $\mathrm{sp}^{3} \mathrm{C}-\mathrm{H}$ bonds than benzylic and allylic $\mathrm{sp}^{3}$ $\mathrm{C}-\mathrm{H}$ bonds. Moreover, primary or secondary amines mostly led to dehydrogenation, which resulted from the inefficient excited-state quenching or unproductive photoelectron transfer during the reaction. ${ }^{[71]}$

Photocatalytic Oxygenation of Benzylic C(sp $\left.{ }^{3}\right)-\mathrm{H}$ Bonds to Generate Aldehydes or Ketone Derivatives

For the first time, Fukuzumi et al. reported metal-free photocatalytic reactions to generate aromatic aldehydes from toluene using 10-methyl-9-phenylacridinium ion as a metal-free photocatalyst. In this process, the oxygenation reactions were initiated by the photoinduced electron transfer from the substituted toluenes to the singlet excited state of the photocatalyst. The high selectivity in this photocatalytic oxygenation reaction was mainly due to the photoinduced electron transfer mechanism that did not involve the autoxidation process (radical chain reactions). The selectivity in this case was very high but the reaction exhibited poor substrates scope and was only limited to xylene derivatives. ${ }^{[72]}$ 


$$
\widehat{P h} \widehat{R}_{\substack{\mathrm{CH}_{3} \mathrm{CN}(2.0 \mathrm{~mL}), 12 \mathrm{~h} \\ 3 \mathrm{~W} \text { blue LED }}}^{\stackrel{\mathrm{O}_{2}(1 \mathrm{~atm})}{\mathrm{Acr}^{+} \mathrm{MesClO}_{4}^{-}(1 \mathrm{~mol} \%)}}
$$

(a) Selective successful substrates<smiles>Cc1ccc(C(=O)c2ccccc2)cc1</smiles>

$58,64 \%$<smiles>O=C(c1ccccc1)c1ccc(Cl)cc1</smiles>

$59,75 \%$

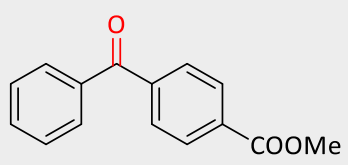

$60,58 \%$<smiles>O=C(c1ccccc1)c1ccc([N+](=O)[O-])cc1</smiles>

$61,28 \%$<smiles>Cc1cccc(C(=O)c2ccc(Cl)cc2)c1</smiles>

$62,51 \%$<smiles>O=C1CCCc2ccccc21</smiles>

$63,64 \%$

(b) Proposed mechanism

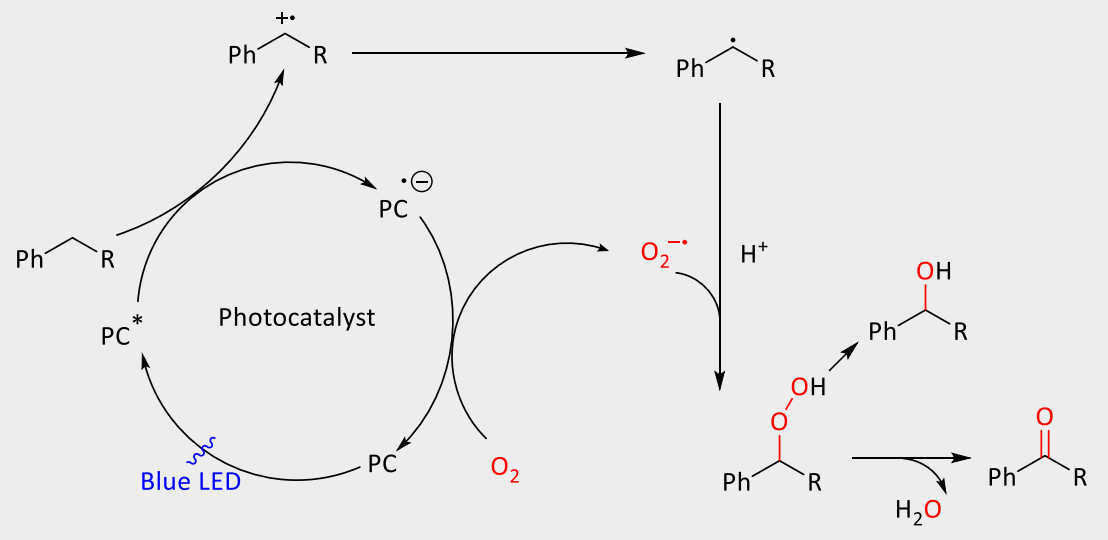

Scheme 1.20 Substrate scope and proposed mechanism for the oxygenation of benzylic $\mathrm{C}\left(\mathrm{sp}^{3}\right)-\mathrm{H}$ bonds to obtain keto derivatives. This figure and part of mechanism have been adapted from reference 70 .

In 2015, the Lei group described an aerobic oxygenation of benzylic C-H bonds using 9-mesityl-10-methylacridinium ion as a photocatalyst. ${ }^{[73]}$ With this mild reaction condition in hand, they were able to convert various benzylic $\mathrm{C}-\mathrm{H}$ bonds to the corresponding keto products. After that, mechanistic studies were performed and control experiments showed that the photocatalyst, visible light as well as the oxygen were all essential for the reaction. Radical-trapping reactions with TEMPO indicated a radical pathway, while ${ }^{18} \mathrm{O}_{2}$ and $\mathrm{H}_{2}{ }^{18} \mathrm{O}$ labeling showed that $\mathrm{O}_{2}$ was the only ketoxygen atom source in the final product. Based on previous reports and their experimental results, they proposed that the single electron transfer (SET) process occurred with the benzylic compound to generate the corresponding radical cation. Thereafter, the radical anion of the catalyst was oxidized by $\mathrm{O}_{2}$, meanwhile, the photocatalyst went 
back to the original state. Furthermore, the substrate radical cation lost one proton to afford the corresponding benzylic radical, which further reacted with the superoxide radical anion $\left(\mathrm{O}_{2}{ }^{\bullet-}\right)$ and a proton to achieve the hydroperoxide intermediate. With the release of water, the hydroperoxide intermediate transformed into the final desired product (Scheme 1.20).

\subsubsection{Heterogeneous Photocatalytic $\mathrm{C}\left(\mathrm{sp}^{3}\right)-\mathrm{H}$ Oxygenation Using Oxygen}

\section{Direct Oxygenation of Benzylic $\mathrm{C}\left(\mathrm{sp}^{3}\right)-\mathrm{H}$ Bonds to Generate Esters and Phthalides}

There is also a metal-free heterogeneous photocatalytic system for highly efficient oxygenations of benzylic $\mathrm{C}-\mathrm{H}$ bonds using oxygen as an oxidant (Scheme 1.21). Except for the successful oxygenation of amines to amides as introduced above, this mild strategy was also applied to the aerobic oxygenation of isochromans and phthalans, to obtain esters (lactams) and phthalides in excellent yields. Moreover, this reaction can be driven in water as sole solvent which is also quite promising for sustainable applications. Meanwhile, the Cai group further applied this condition to the synthesis of valuable bioactive and medicinal compounds such as corydaline, indoprofen and indobufen. A recycling procedure was also established to illustrate the practicability of the heterogeneous photocatalysis protocols. ${ }^{[48]}$

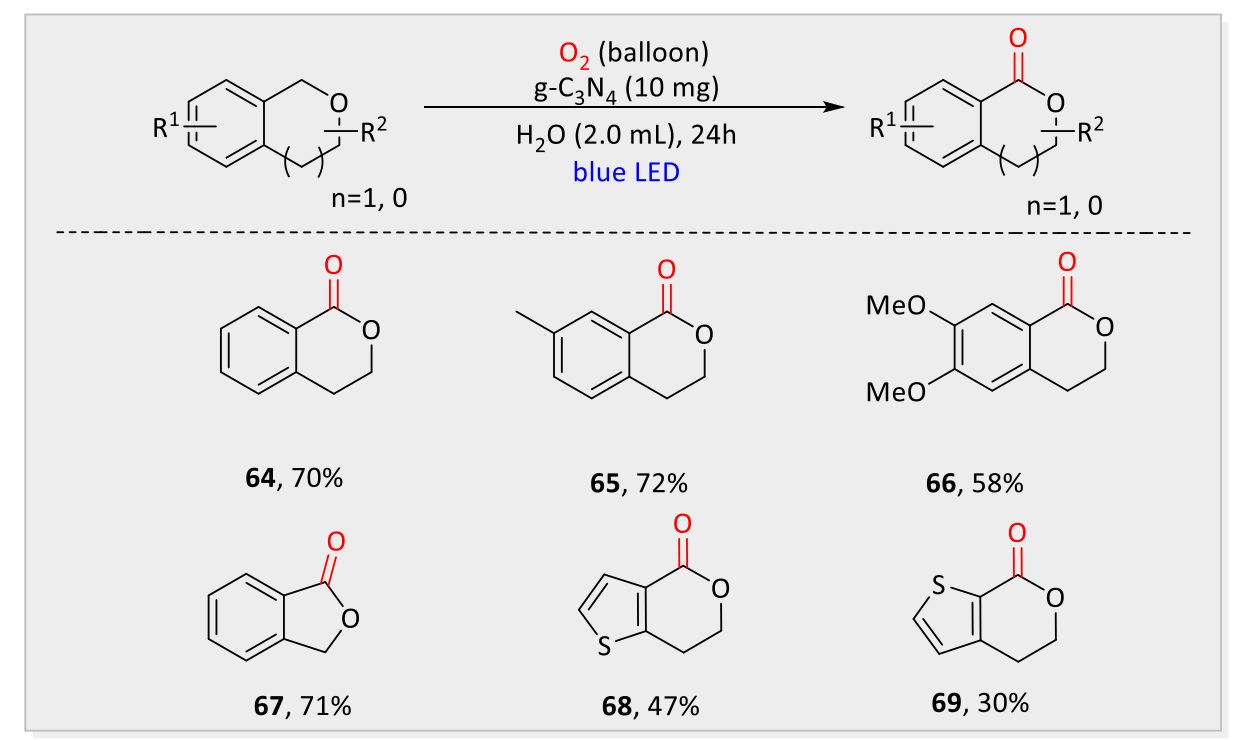

Scheme $1.21 \mathrm{~g}-\mathrm{C}_{3} \mathrm{~N}_{4}$ photocatalytic aerobic oxidation of isochromans and phthalans. 


\subsection{Metal-free Photocatalytic Oxidative C=C Bonds Cleavage}

\subsubsection{Earlier Examples of Oxidative $\mathrm{C}=\mathrm{C}$ Bonds Cleavage}

The oxidative scission of bulk chemical (olefins) into corresponding carbonyls is a highly significant organic transformation in the chemical industry. Since it introduces oxygen atoms into the substrate molecule, it provides numerous possibilities for synthetic chemistry, such as selective functionalization of olefins, degradation of complex molecules and the late-stage modification of drug molecules. ${ }^{[74]}$

However, the most popular protocols for the cleavage of $C=C$ bonds are still the ozonolysis and Lemieux-Johnson oxidation, ${ }^{[75]}$ which require an ozone generator and toxic $\mathrm{O}_{3}$ or utilize toxic and precious osmium tetroxide. Even though tremendous endeavors were made for developing the cleavage of olefins via cheaper and safer

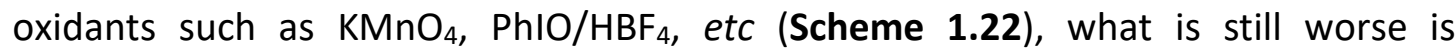
generating over amount of by-product and higher energy consumption which is far away from the goals of atom economy and environmental protection. ${ }^{[76]}$

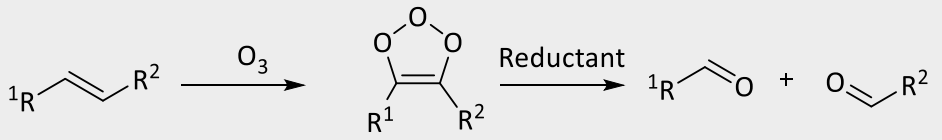

$$
\begin{aligned}
& { }_{1} \curvearrowright R^{2} \stackrel{\mathrm{KMnO}_{4}, \mathrm{PhIO} / \mathrm{HBF}_{4}}{\longrightarrow}{ }_{{ }_{R}} \overbrace{\mathrm{O}}+\mathrm{O}_{\Downarrow} \mathrm{R}^{2}
\end{aligned}
$$

Scheme 1.22 Earlier examples of $\mathrm{C}=\mathrm{C}$ bonds cleavage.

\subsubsection{Recent Advances of Oxidative $C=C$ Bonds Cleavage}

Based on the earlier investigation of oxidative cleavage of $C=C$ bonds, activation of oxygen as a clean and cheap oxidant will be ideal. For example, Xiao and coworkers firstly reported the selective cleavage of olefins using iron catalyst under $\mathrm{O}_{2}$ atmosphere, which exhibited good substrates scope including multi-substituted styrenes. ${ }^{[77]}$ Both $\alpha$ - and $\beta$-substituted styrenes were cleaved smoothly to afford the carbonyl compounds at 1 atm of $\mathrm{O}_{2}$ with high efficiency as well as chemoselectivity. In the case of $\alpha$-halo styrenes, migration of the halogen occurred, affording $\alpha$-halo acetophenones (Scheme 1.23). However, this reaction required a higher reaction temperature and an expensive ligand. Later, a commercially available iron catalyst for 
this reaction was described by Feng and coworkers. ${ }^{[78]}$ Furthermore, a copper complex was employed in $\mathrm{C}=\mathrm{C}$ bond cleavage by the group of Wang which can drive the reactions to generate carbonyl compounds from terminal alkenes. ${ }^{[79]}$

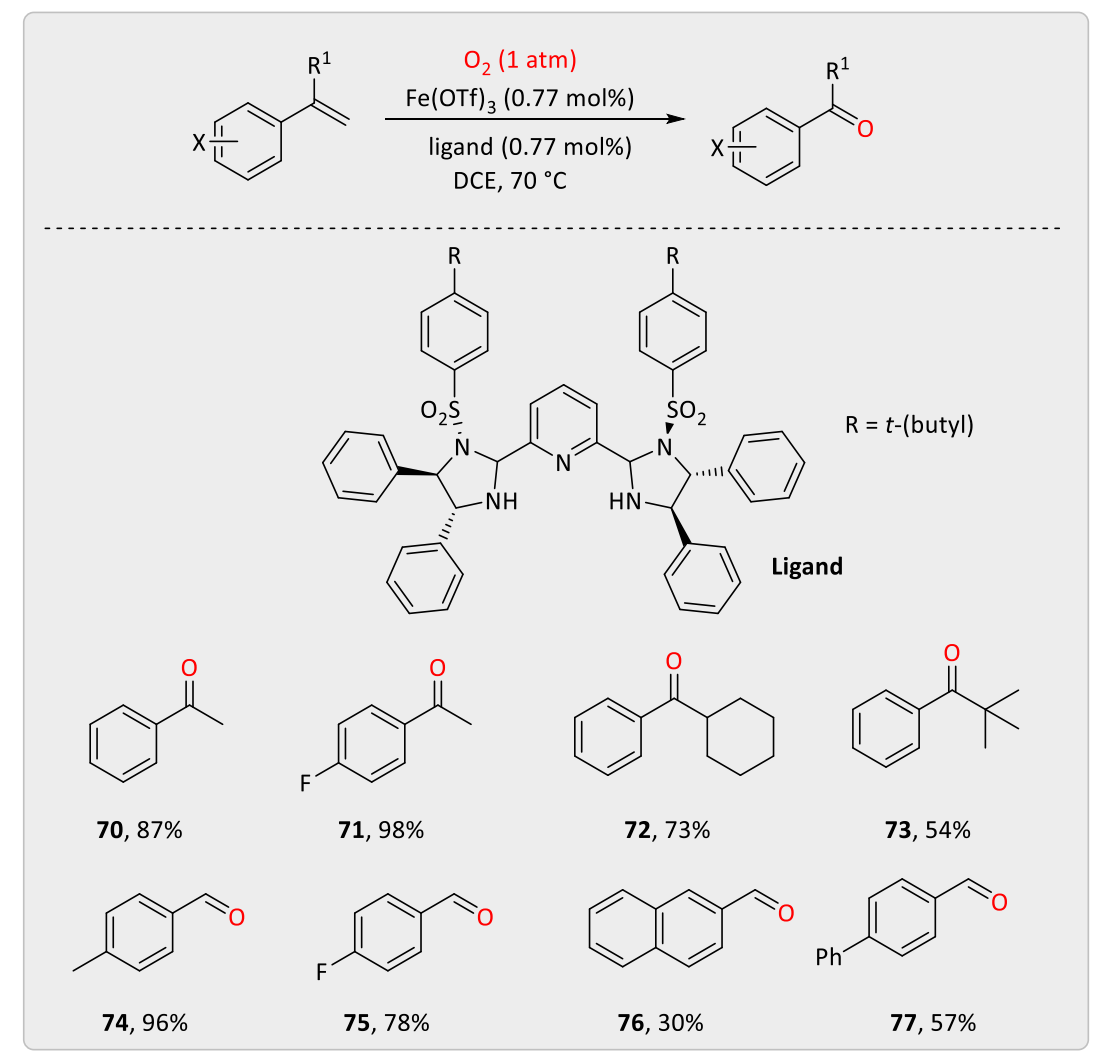

Scheme 1.23 Aerobic cleavage of $\mathrm{C}=\mathrm{C}$ bond in styrenes.

In the way of establishing a photocatalytic method under visible light, the Wang group reported the oxidative cleavage of $\mathrm{C}=\mathrm{C}$ bonds in the presence of disulfide [bis(4methox-yphenyl) disulfide] as a metal-free photocatalyst. In this case, terminal styrenes, $\alpha$ or $\beta$-substituted styrenes could be converted into corresponding aldehydes and ketones. Noteworthy, it underwent the oxidation-addition sequence rather than the single electron transfer (SET) oxidation pathway (Scheme 1.24). ${ }^{[80]}$ 


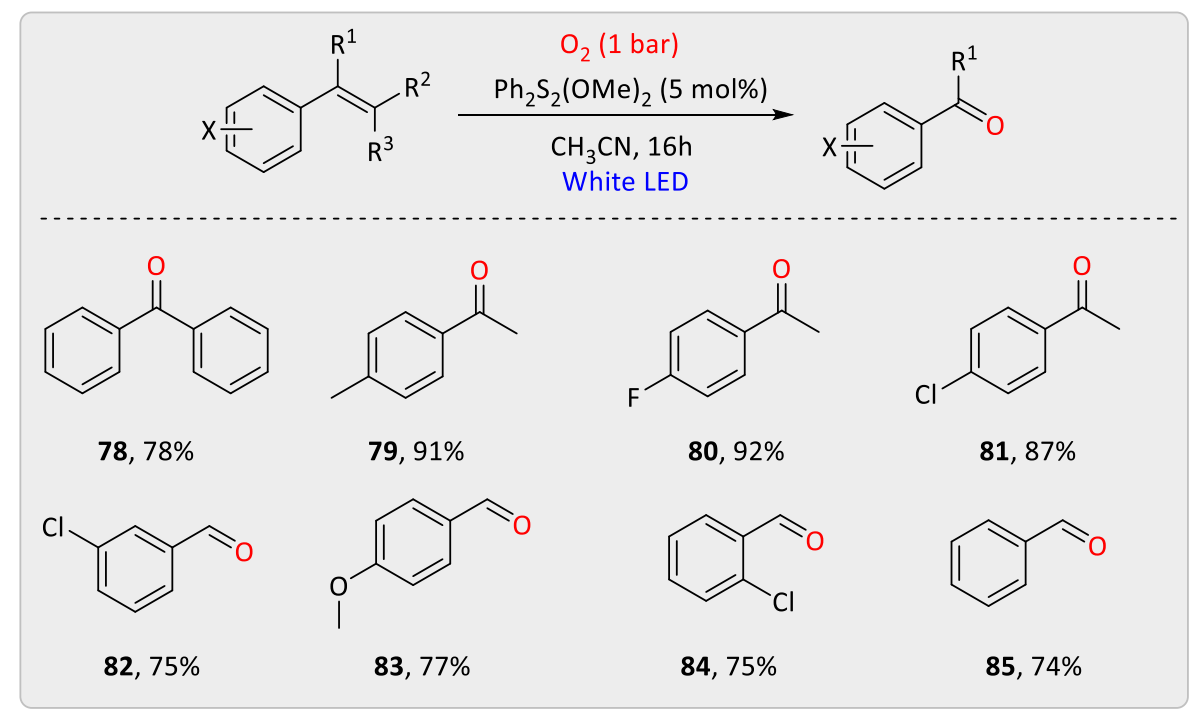

Scheme 1.24 Disulfide-catalysed photo-oxidation of aromatic olefins.

Recently, electrosynthesis has gained considerable momentum owing to its inherent sustainability and tunability which achieves redox transformations with traceless electricity instead of oxidants or reductants and thereby obviating the stoichiometric waste formation. ${ }^{[81]}$ Importantly, electrochemical methods offer a variety by means of exploring new modes of reactivity for a particular substrate (such as electrode, supporting electrolyte and current density). Hence, electrode material selective transformations of styrenes with $\mathrm{O}_{2}$ into carbonyls were demonstrated by the Chiba group. Interestingly, after changing the electrode from platinum to carbon, the structural moiety of 2,5-diaryltetrahydrofuran was found. The stereoselectivity was medium but most of the isomers could be separated via column chromatography (Scheme 1.25). ${ }^{[82]}$ 


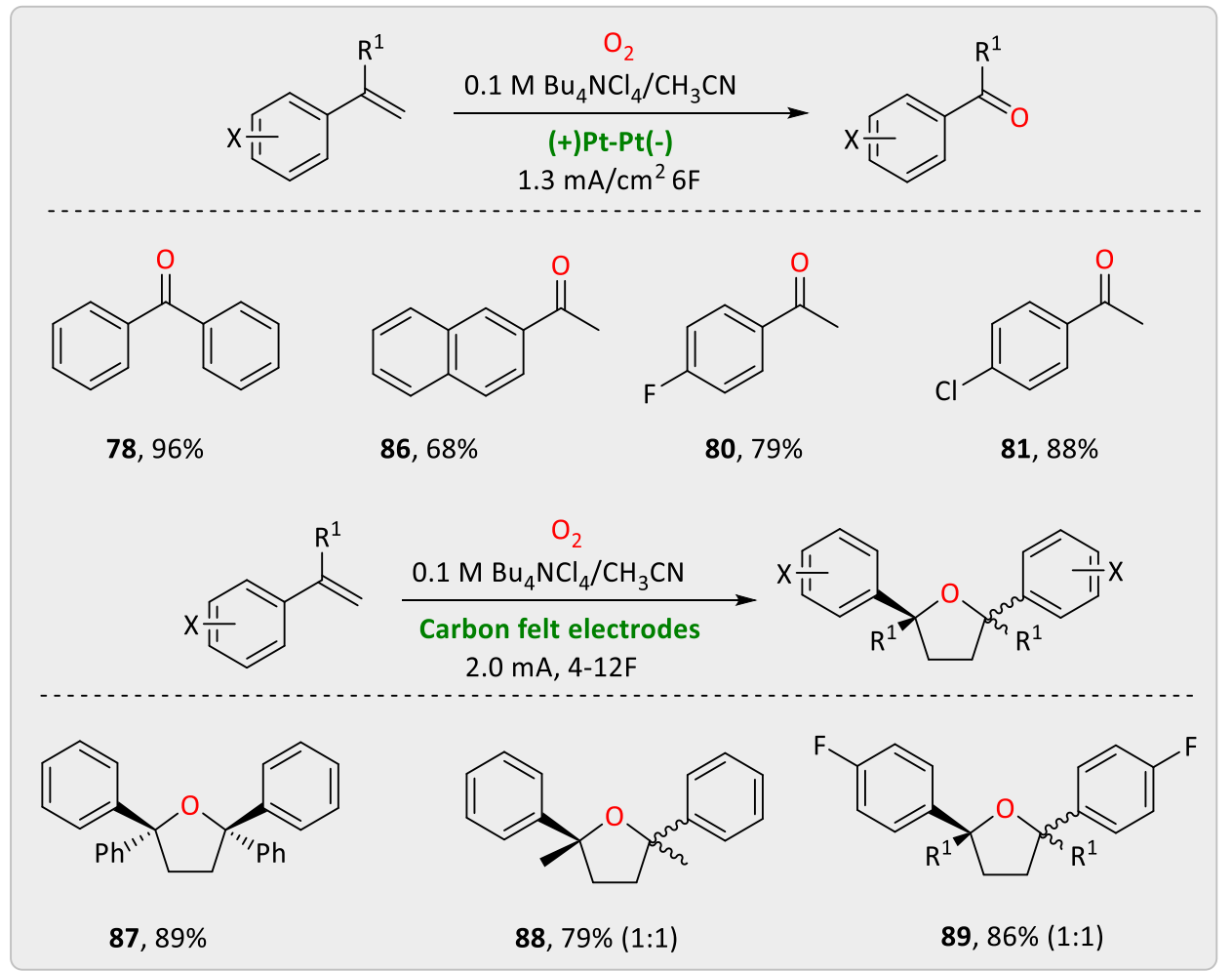

Scheme 1.25 Scope of oxidative cleavage of olefins and THF derivative synthesis.

\subsection{Metal-free Photocatalytic Cycloadditions}

Cycloadditions are powerful and popular transformations, which serve as fundamental strategies to assemble many structurally versatile carbocycles and heterocycles with the formation of up to four new stereogenic centers (e.g., steroid synthesis) with excellent atom economy, ${ }^{[83]}$ especially in the utility of organic synthesis, pharmaceutical industry and biochemistry (Scheme 1.26). ${ }^{[84]}$ Among them, two notable examples in this class of reactions are the Diels-Alder reactions and the 1,3-dipolar cycloaddition reactions. ${ }^{[85]}$ Recently, continuous efforts have been made by organic chemists to uncover new ways to promote various cycloaddition reactions with the development of photocatalysis which significantly expanded the scope of the cycloadditions. ${ }^{[27][28]}$ As outlined above (see Section 1.1.1), the excited-state photocatalyst can serve as an one-electron acceptor via a reductive quenching cycle (pathway A), as an one-electron donor via an oxidative quenching cycle (pathway B), or as a photosensitizer via energy transfer (pathway C). The photocatalyst's versatile mode of activation subsequently allows various cycloadditions to be explored. 


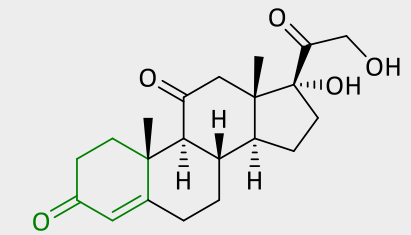

90, Cortisone

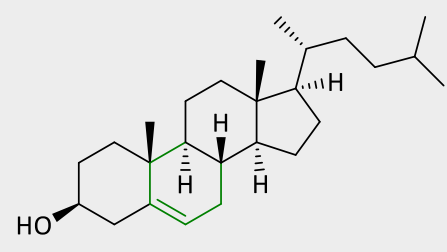

91, Cholesterol

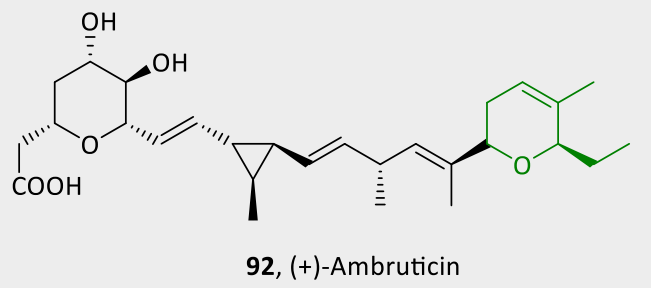

Scheme 1.26 Examples of synthesized products by cycloadditions.

\subsubsection{Examples of Photocatalytic [2+2] Cycloadditions}

A brief discussion about cycloadditions was already given in the general introduction of energy transfer mechanism. Cyclobutanes, the constructed products from [2+2] cycloadditions, are highly important as they are basic structural elements in numerous drug molecules and bioactive compounds, and they also produce strain-releasing fragmentations, which are useful in synthesizing medium-sized rings. ${ }^{[86]}$ Moreover, building up four new stereocenters and two new $\sigma$ bonds in a high-energy cyclobutane ring is a challenging task. Therefore, [2+2] cycloadditions are arguably regarded as the most straightforward ways to make such structures. As early as 1912, Ciamician and coworkers already realized that visible light had the potential to serve as an inexpensive and abundant reagent for [2+2] cycloadditions. Within his report, camphor carvone was obtained from carvone after 1-year exposure to the sunlight. ${ }^{[87]}$ Yoon and others have also made most important contributions to the development of visible-light-mediated [2+2] cycloadditions in the last decade. ${ }^{[27][28]}$

\section{[2+2] Cycloadditions via Reductive Quenching Cycle}

Krische and coworkers have reported the first metal-catalysed [2+2] cycloadditions of bis(enones) with cobalt and copper catalysts, respectively, which were initiated by one electron reduction of the enone. ${ }^{[88]}$ As the radical anion intermediate was authenticated by the formation of identical [2+2] cycloadducts using homogeneous one-electron reduction with well-studied photoredox catalyst of Ru(bipy) $3_{3}{ }^{2+}$, Yoon and 
coworkers discovered in 2008 that visible light-mediated photoredox catalysis could be used to generate the same radical anion intermediate (Scheme 1.27). In this case, various aromatic enones efficiently underwent the desired transformations with high diastereoselectivity upon irradiation of $275 \mathrm{~W}$ floodlight. However, at least one aromatic enone was essential for the transformation as only aromatic enones were able to be reduced under the chosen photoredox conditions. ${ }^{[27 e]}$

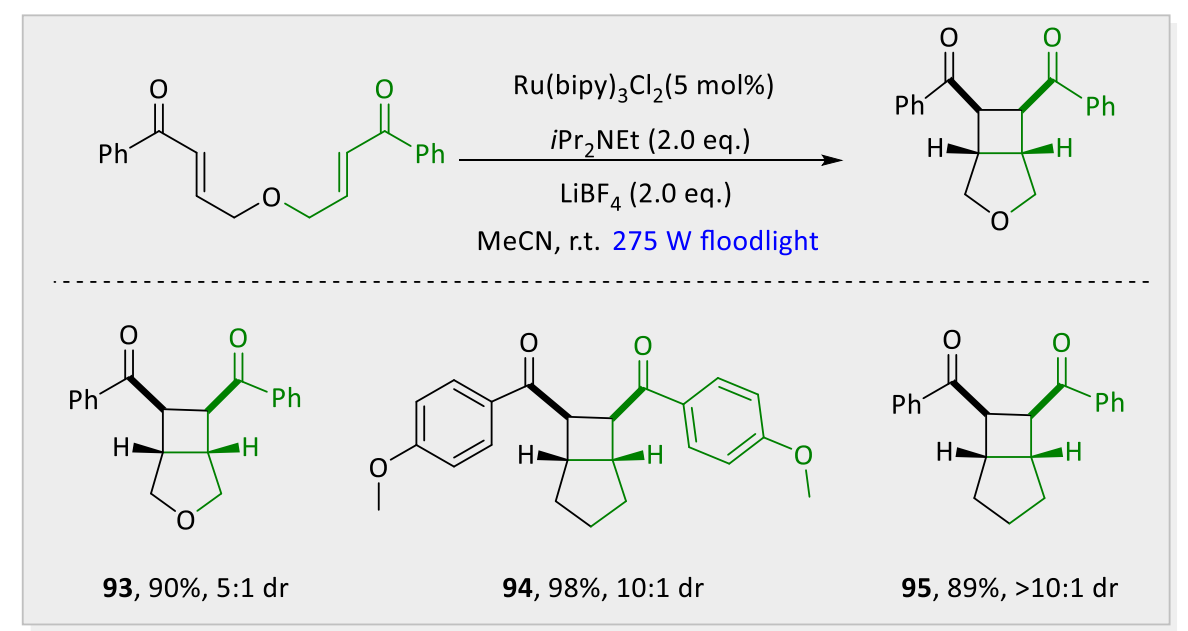

Scheme 1.27 Substrate scope for the visible-light-mediated cycloadditions of enones.

One year later, the Yoon group presented an efficient strategy for the intermolecular [2+2] cycloaddition reaction with excellent chemo- and stereoselectivity. In this case, they predicted that crossed intermolecular [2+2] heterodimerizations could be possible using two dissimilar enone substrates considering generation of the requisite radical anion intermediate easily, but the reacting partner needs to be suitable Michael acceptor. ${ }^{[89]}$ Later, the same group reported that $\alpha, \beta$-unsaturated 2 imidazolyl ketones underwent [2+2] cycloaddition with various Michael acceptors under the irradiation of visible light. ${ }^{[90]}$ Nevertheless, the formation of a radical anion intermediate still only worked for aromatic enones and required at least one alkene moiety, which is sufficiently electron-deficient.

\section{[2+2] Cycloadditions via Oxidative Quenching Cycle}

The [2+2] cycloaddition based on the reductive quenching cycle required at least one electron-deficient alkene moiety. To overcome this limitation in the substrate to reach electron-rich alkenes utilization, Yoon and coworkers developed a pioneering method 
relying on an oxidative quenching cycle.

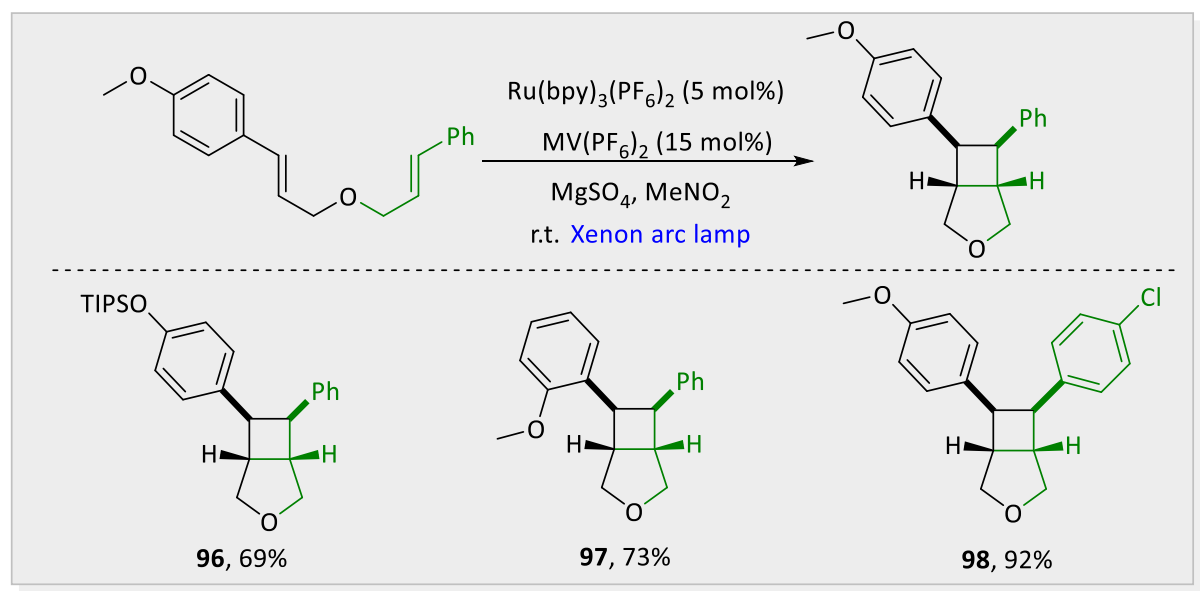

Scheme 1.28 Selected cycloadducts for the intramolecular [2+2] cycloaddition.

Indeed, electron-rich alkenes are known to undergo the [2+2] cycloaddition reactions via one-electron oxidation by chemical oxidants and photoinduced electron transfer by the organic sensitizers. ${ }^{[91]} \mathrm{Ru}(\mathrm{bpy}) 3^{3+}$, produced by oxidatively quenching of the excited-state $\mathrm{Ru}(\mathrm{bpy}) 3^{2+}$ with an electron acceptor (e.g., methyl viologen, $\mathrm{MV}^{2+}$ ), was able to oxidize electron-rich olefins to their corresponding radical cations. Given by this rational conjecture, they reported the first [2+2] cycloaddition by oxidative visible light photocatalysis. ${ }^{[30 a]}$ Notably, the reaction was modestly sensitive to water, so that the addition of magnesium sulfate could increase the yields and improve the reproducibility. In this case, it was also found that at least one electron-donating substituent must be present either in the ortho or para position of at least one styrene to achieve the desired transformation. However, the other alkene moieties, such as aliphatic olefins, did not react under this condition (Scheme 1.29).

In the proposed mechanism, the pattern for an oxidative quenching cycle was described. Here, the excited-state $\mathrm{Ru}(\mathrm{bpy})_{3}{ }^{2+}$ was formed under the irradiation of visible light, which can be further oxidized by $\mathrm{MV}^{2+}$, affording the highly oxidizing $\mathrm{Ru}(\mathrm{bpy}) 3^{3+}$. The ruthenium species then oxidized the electron-rich styrene to generate the radical cation, meanwhile returning to the ground state catalyst. The radical cation then underwent cyclization to afford the desired product (Scheme 1.29). 


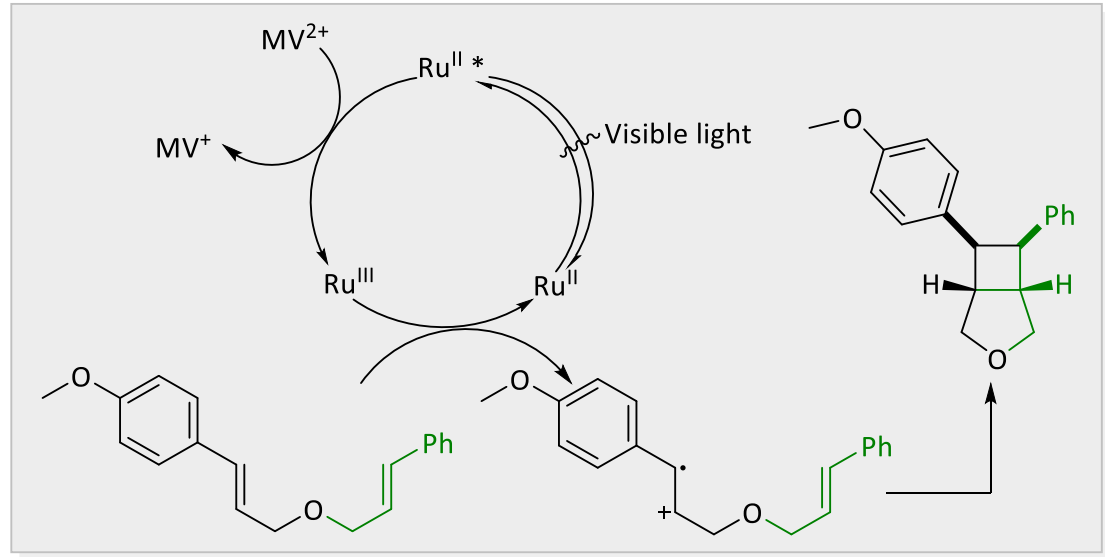

Scheme 1.29 Proposed mechanism of the intramolecular [2+2] cycloadditions of bis(styrenes).

The Yoon group then reported a complementary approach to cross [2+2] heterodimerization reaction of electron-rich olefins to construct asymmetric cyclobutanes. ${ }^{[30 \mathrm{~b}]}$ In their model reaction, initial studies showed that the conditions used in the intramolecular cases were ineffective for the intermolecular homodimerization. However, they could obtain the homodimerization product in a modest yield ( $57 \%$ after $2 \mathrm{~h}$ ) using more oxidizing conditions with $\mathrm{Ru}(\mathrm{bpz})_{3}{ }^{2+}$ and air. But full conversion was not achieved because the product of the cycloaddition was prone to oxidize and subsequent cycloreversion. ${ }^{[92]}$ This phenomenon was also proved since the oxidation potential of cycloadduct 100 was $+1.27 \mathrm{~V}$ vs. SCE, presumably indicating its susceptibility to oxidation by $\mathrm{Ru}(\mathrm{bpz})_{3}{ }^{2+*}$ (reported oxidation potential is $+1.45 \mathrm{~V})$. After adjusting the photoredox catalyst to $\mathrm{Ru}(\mathrm{bmp}) 3^{2+}$, which has the oxidation potential of $\mathrm{Ru}(\mathrm{bpm})_{3}{ }^{2+*}+1.20 \mathrm{~V}$ vs. $\mathrm{SCE},{ }^{[93]}$ it should be sufficient to promote the one-electron oxidation of 99 (+1.1 V) (Scheme 1.30).

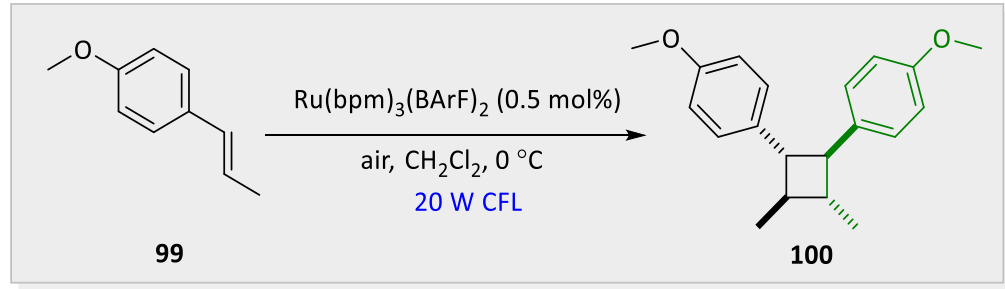

Scheme $1.30[2+2]$ dimerization of electron-rich styrenes.

Regarding the substrate scope of the heterodimerization, Yoon and coworkers made 
several strategic considerations. For example, the coupling partner must be less electron-rich than the electron-rich styrene so that only the styrene is oxidized to the key radical cation. Furthermore, the coupling partner needs to be a terminal alkene, so that steric hindrance may help to promote heterodimerization rather than forming the undesired homodimerization product. They also noticed an obvious increase in rate and yield when the reaction temperature was lowered to $-15{ }^{\circ} \mathrm{C}$. At last, if the homodimerization of the electron-rich styrenes became a major problem, a syringe pump could deliver the styrenes to the reaction mixture (Scheme 1.31).

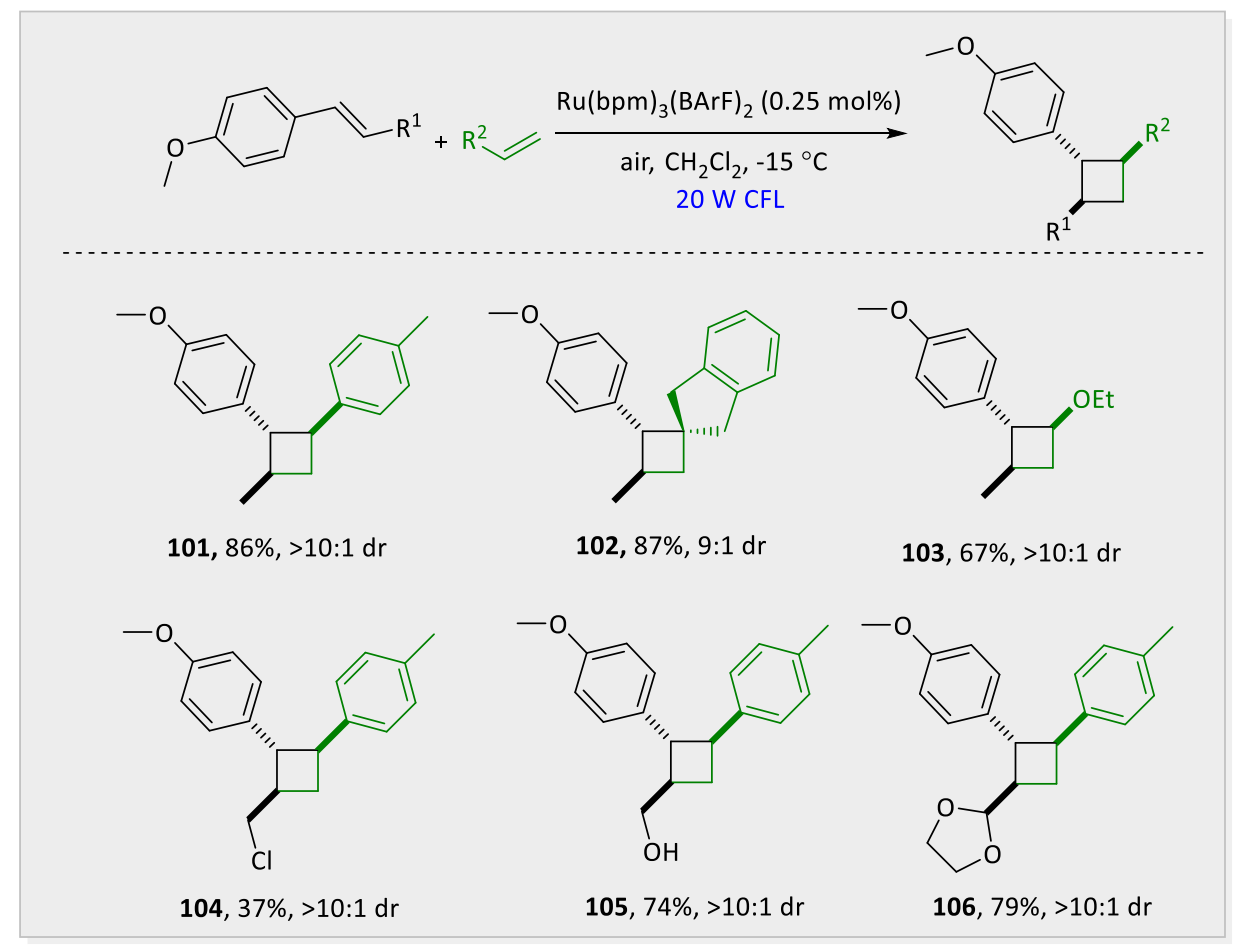

Scheme $1.31[2+2]$ heterodimerization of electron-rich styrenes.

\section{[2+2] Cycloadditions via Energy Transfer}

The [2+2] cycloadditions via photoredox catalysis rely on efficient electronic transfer between the catalyst and substrates, which depends on their redox properties. In the case of the reductive quenching cycle, the reduction potential of alkene substrates needs to be less negative than the reduction potential of the excited-state photocatalyst, while in the case of the oxidative quenching cycle, the oxidation potential of alkene substrates has to be less positive than the reduction potential of the excited-state photocatalyst. This constraint on the redox properties of substrates 
has fundamentally limited the substrate scope of cycloadditions. The first report via energy transfer was already illustrated in the first section which involves triplet sensitization of the alkene substrate by the photoexcited catalyst to promote the $[2+2]$ cycloadditions (Scheme 1.8). ${ }^{[31]}$

\subsubsection{Examples of Photocatalytic [4+2] Cycloadditions}

Typical Diels-Alder reactions only work well between electron-rich dienes and electron-poor dienophiles in accordance with frontier molecular orbital (FMO) theory. The electronically mismatched coupling between two electron-rich components has long been challenging. ${ }^{[94]}$ To overcome this restriction, mismatched systems can be forced to react at high temperatures or under Lewis-acid activation/catalysis, which limits their utility in synthesis. Alternatively, radical cations of electron-rich alkenes (dienophiles) undergo facile Diels-Alder cycloadditions with electron-rich dienes. In general, the radicals are produced using one-electron chemical oxidants with higher loadings, or using the organic photosensitizer usually requiring xenon lamps with higher energy consumption. ${ }^{[95]}$ Hence, using visible light to form the radical ion in Diels-Alder cycloadditions is much more promising and environmentally friendly.

\section{[4+2] Cycloadditions Using Radical Anions}

In 2011, Yoon and coworkers investigated hetero-Diels-Alder cycloadditions of tethered bis(enones) using radical anions based on their previous studies of intramolecular [2+2] cycloaddition. These reactions did not proceed well if the tether contains one extra methylene group. After optimization of the original reaction conditions by the addition of 10 equiv. of water and decreasing the reaction time from $9.5 \mathrm{~h}$ to $1 \mathrm{~h}$, they obtained the desired [4+2] cycloadduct products with excellent yields and good diastereoselectivity. ${ }^{[28]}$ In this case, symmetric aryl bis(enones) including both electron-rich and electron-deficient aryl enones reacted smoothly under the chosen reaction conditions. Similarly, unsymmetrical bis(enones) containing only one aryl group reacted favorably while other $\alpha$-alkoxy enones required further optimization by changing the Lewis acid and solvent system (Scheme 1.32). 


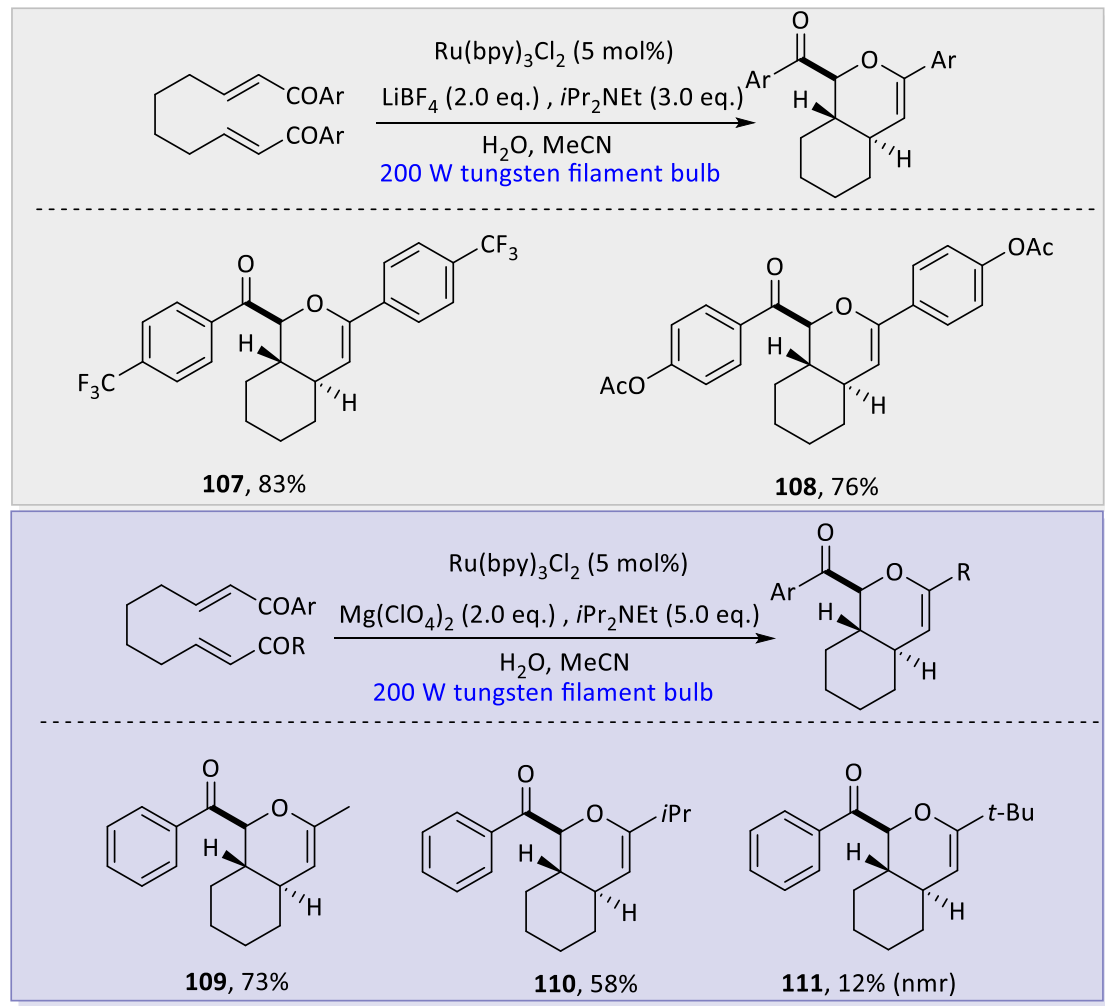

Scheme 1.32 Substrates scope of the [4+2] cycloaddition.

Mechanistically, the photoexcited $\mathrm{Ru}(\mathrm{bpy})_{3}{ }^{2+}$ underwent the reductive quenching by $i-\operatorname{Pr}_{2} \mathrm{NEt}$. The reduced $\mathrm{Ru}(\mathrm{bpy})_{3}{ }^{+}$then reduced the Lewis acid-activated enone complex, generating the activated radical anion while returning back the catalyst. The radical anion reacted efficiently via $\beta, \beta$-coupling, producing a monocyclized distonic radical anion intermediate, which formed the carbon-oxygen bond via path $\mathrm{A}$ or $\mathrm{B}$. They also expected that the aryl ketyl radical was more stable than its regioisomer, thereby forming the sole isomer. A one-electron loss of radical anion to either the amine radical cation or the Lewis acid enone complex resulted in the neutral Diels-Alder cycloadduct (Scheme 1.33). In 2015, the Shores and Ferreira groups continued to report a photocatalytic strategy using chromium catalysts for promoting radical cation cycloadditions. However, it had to be performed under the irradiation of UV light. ${ }^{[96]}$ 


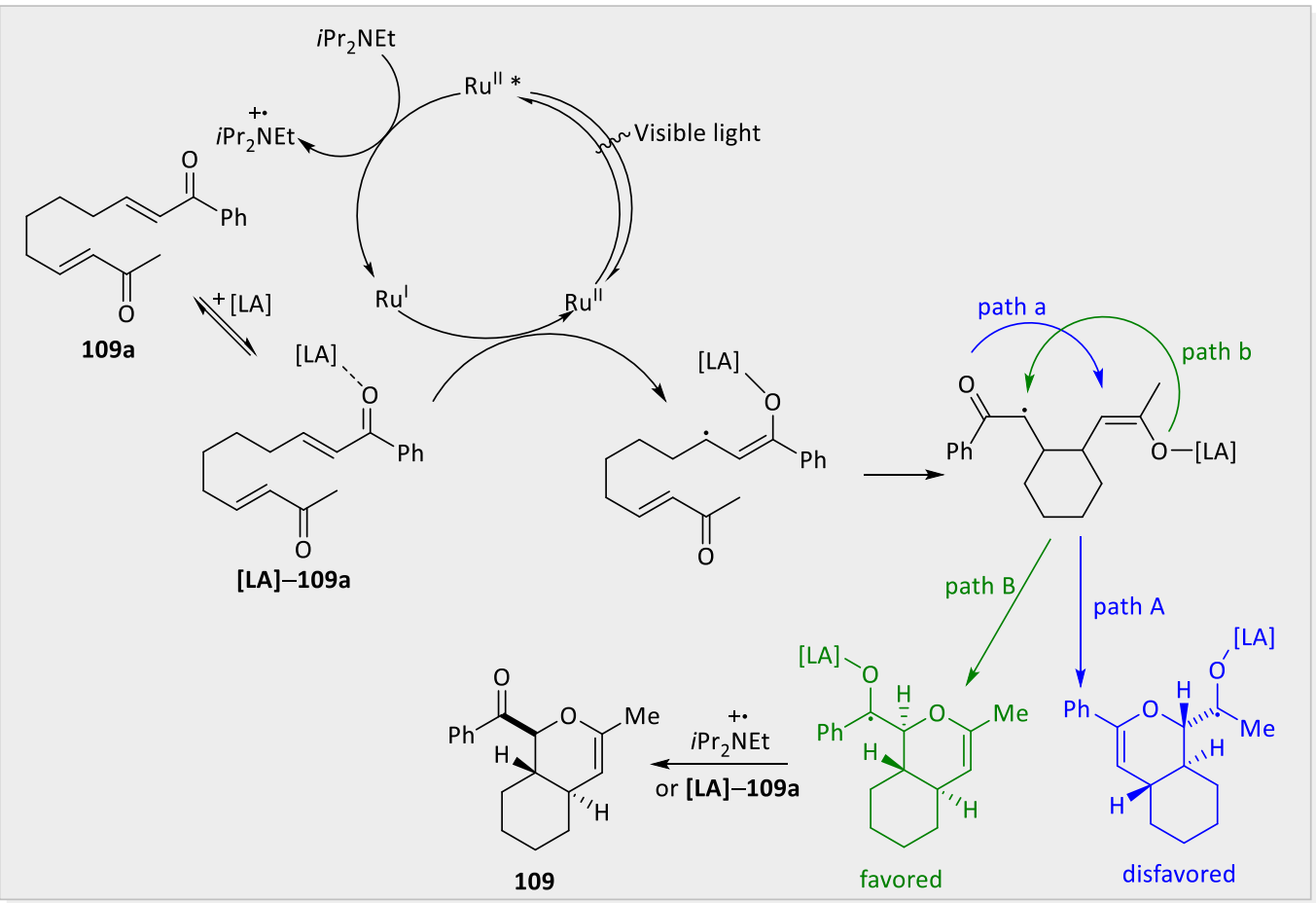

Scheme 1.33 Proposed mechanism of the regioselective hetero Diels-Alder cycloaddition of unsymmetrical bis(enones).

\section{[4+2] Cycloadditions Using Radical Cations}

Following the discovery of the intramolecular radical anion hetero-Diels-Alder cycloaddition, Yoon and coworkers continued to describe a facile intermolecular Diels-Alder cycloaddition using a photogenerated radical cation of an electron-rich olefin and an electron-rich diene (Scheme 1.34). ${ }^{[26]}$ Indeed, the cycloadduct was formed from electronically mismatched anethole and isoprene within $1 \mathrm{~h}$. Besides that, the reaction also showed a broad substrate scope. Various dienes including bulky, aryl substituted, and cyclic cases were reactive under this condition. Moreover, various substituted dienophiles including allylic substitutions, cyclic styrenes, and enamines were well tolerated as well. Notably, some dienes and dienophiles, such as the electron-rich Danishefsky dienes and electron-poor diene esters, did not work under this condition. A final limitation was that an electron-donating group in either ortho or para position of the styrene was necessary. 


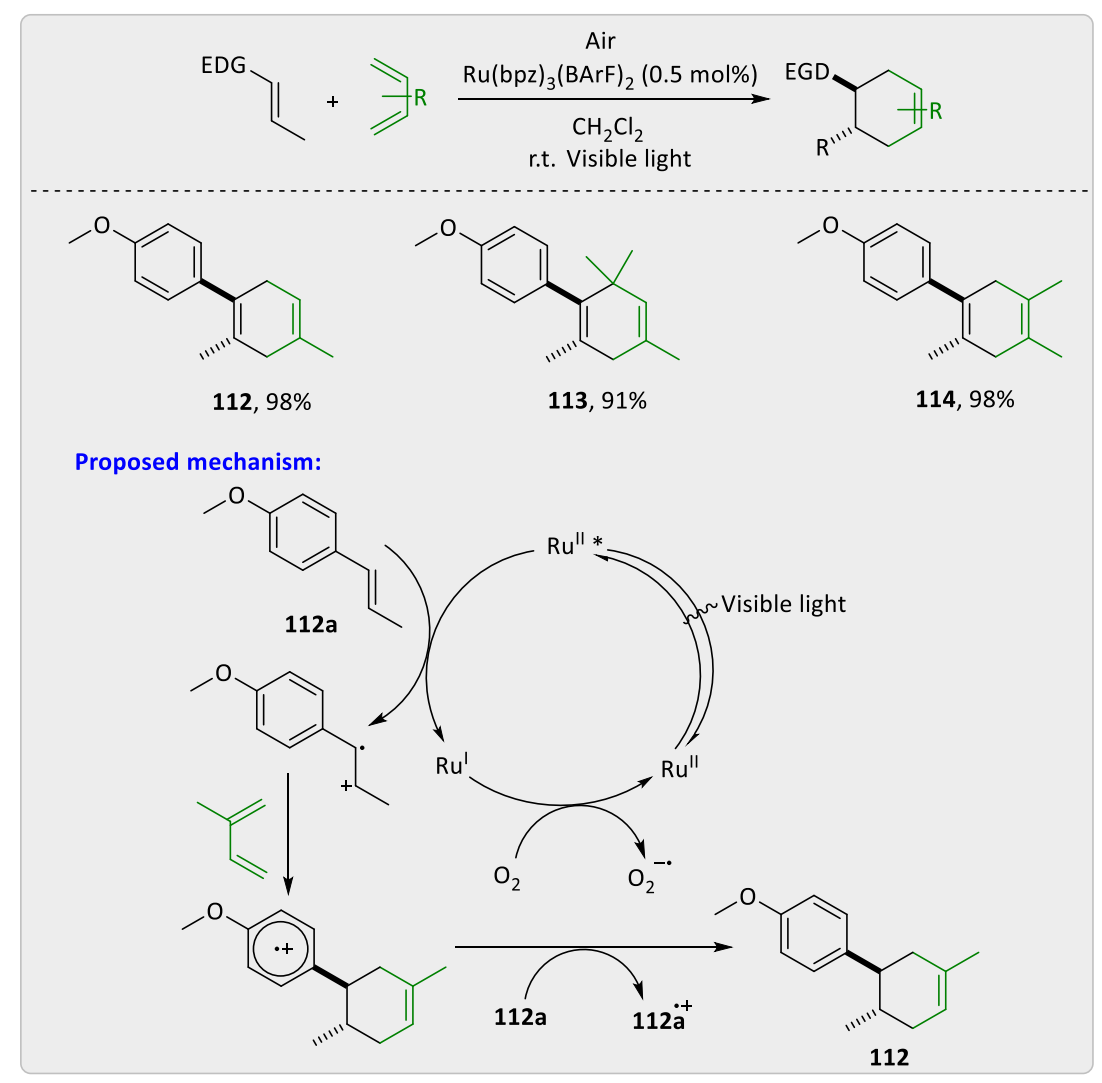

Scheme 1.34 Scope of the radical cation the [4+2] cycloadditions.

A proposed mechanism was also described for the whole process. In the beginning, the excited-state $\mathrm{Ru}(\mathrm{bpz})_{3}{ }^{2+}$ formed after the irradiation was considered to directly oxidize the anethole, then generating its radical cation and $\mathrm{Ru}(\mathrm{bpz})_{3}{ }^{+}$. The catalyst was turned over by aerobic oxygen, while the radical cation reacted with the electron-rich isoprene. The formed aromatic radical cation was then expected to generate the desired cycloadduct via one electron reduction by anethole. A chain propagation step was determined from the control experiments where the authors found that in degassed solvents in the absence of air, the cycloadduct was formed but not to completion. Later, the Yoon group discovered an intramolecular hetero Diels-Alder cycloaddition between electron-rich olefins and electron-rich dienes. ${ }^{[27 g]}$ Interestingly, these radical cation cycloadditions could even be conducted in gram scale using sunlight.

\section{Heterogeneous Photocatalytic [4+2] Cycloadditions}

g- $\mathrm{C}_{3} \mathrm{~N}_{4}$ was reported to be a promising photocatalyst to achieve the goal of radical cation Diels-Alder reactions. ${ }^{[49]}$ In this case, it exhibited a high apparent quantum yield 
(AQY) and tolerated a wide substrate scope as efficiently as the homogeneous photoredox catalysts. In the mechanistic part, the Zhao and Antonietti groups investigated the role of each substance. For example, only $5 \%$ yield was achieved without oxygen and the solvent was also critical since the yield will be dramatically lower for some less polar solvents. Then light on-off experiments were performed, proving that the light was the temporal control of the reaction. Additionally, ESR (electron spin resonance) spectra showed the characteristic peaks of the DMPO-O adduct (DMPO, 5,5-dimethyl-1-pyrroline $\mathrm{N}$-oxide), demonstrating the formation of a superoxide radical under the irradiation of the visible-light. The AQY was measured to prove the efficiency of this photocatalytic reaction system. The AQY of the reactions after 5 min irradiation of the visible light was $47.6 \pm 3.0 \%$, demonstrating the remarkable photoconversion efficiency of this photocatalytic system. The combination of all the above experimental facts provided the proposed mechanism as depicted in Scheme 1.35. Recently, the Wang group also reported a heterogeneous photoredox catalysis for the facile [4+2] cycloadditions in a continuous flow reactor with visible light. ${ }^{[49]}$

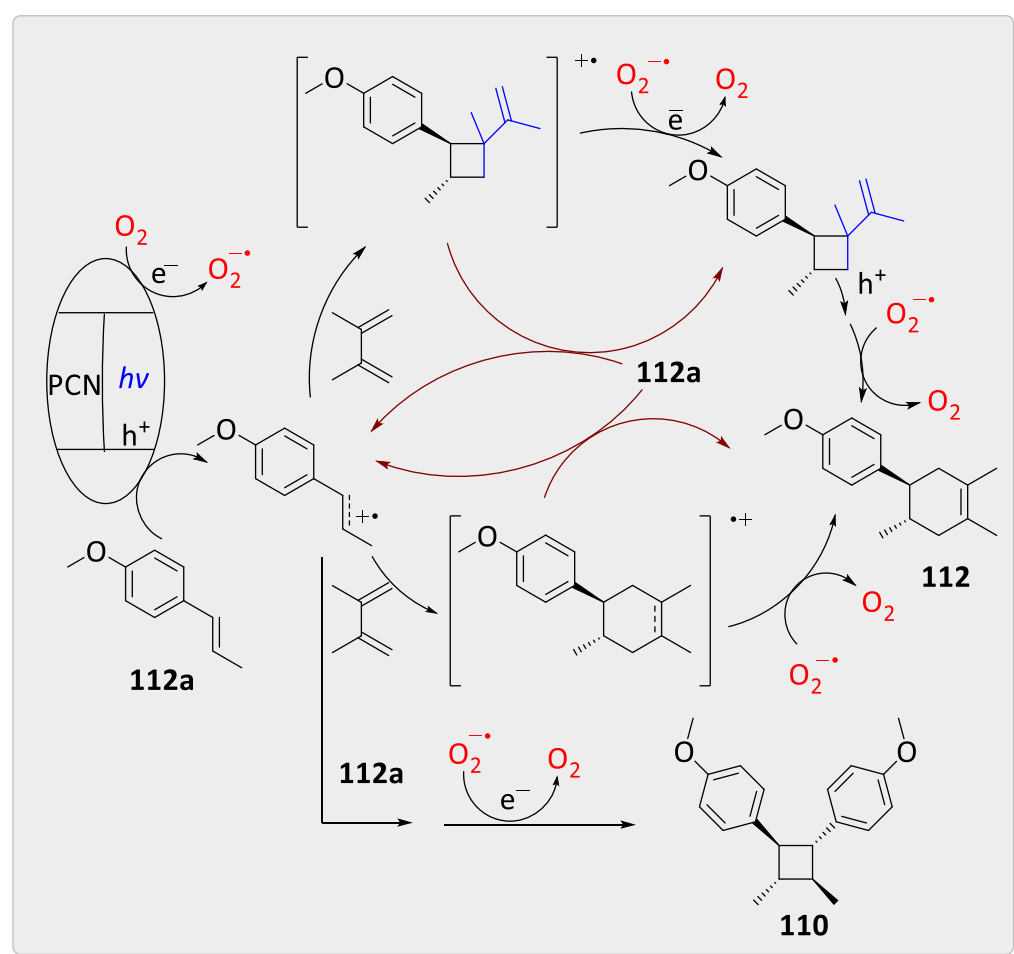

Scheme 1.35 Proposed mechanism of the heterogeneous photocatalytic [4+2] cycloadditions. 


\section{Objectives}

With the current ongoing developments in visible-light photocatalysis, we aimed to develop novel and green photocatalytic systems especially to avoid the expensive transition metal-based catalysts. Inspired by the known photocatalytic reactions for the functionalization of $\mathrm{C}-\mathrm{H}$ bonds in tertiary amines (Scheme 2.1), ${ }^{[21][67]}$ we aimed to merge those concepts and develop metal-free oxygenation reactions of amines to generate the corresponding amides.

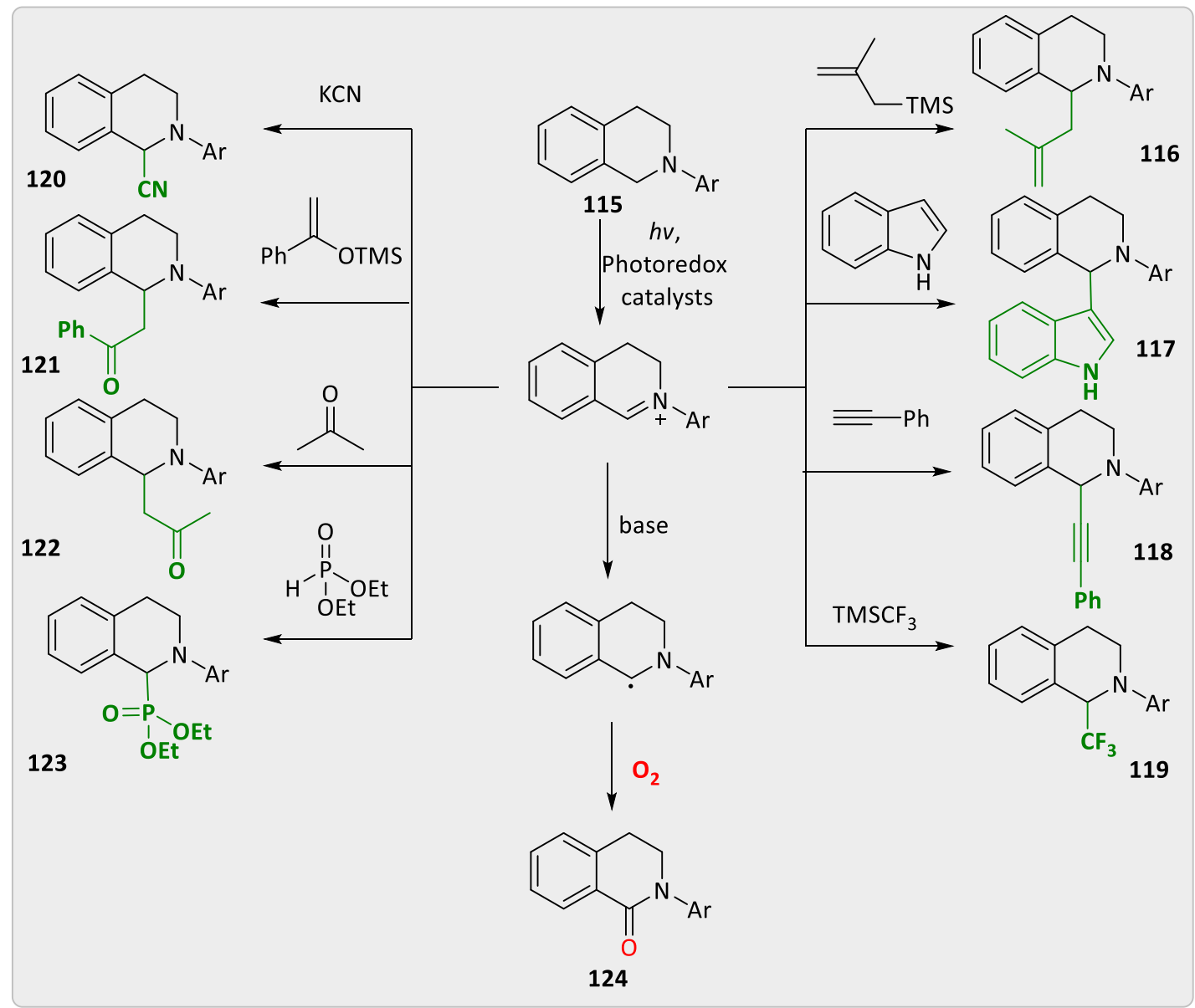

Scheme 2.1 Tetrahydroisoquinoline scaffolds trapped with a broad range of nucleophiles.

Moreover, there is no report of benzylic $\mathrm{C}\left(s p^{3}\right)-\mathrm{H}$ hydroxylation reactions utilizing metal-free photocatalyst. Hence, we aimed to develop a metal-free catalytic system which could generate versatile highly valuable hydroxylated products. The late-stage 
modification of natural products or drugs is an efficient alternative to generate various derivatives in the way of drug discovery and chemical biology. So we aimed to develop reaction conditions which can be applied to diversification of complex natural products. It would be promising if we could cooperate with other research groups of pharmacology to test the potential bioactivities of these synthesized natural products derivatives.

We were also interested in utilizing the metal-free heterogeneous catalyst to perform the oxidative cleavage of $\mathrm{C}=\mathrm{C}$ bonds considering that the cleavage of unreactive bonds such as $\mathrm{C}-\mathrm{C}, \mathrm{C}-\mathrm{H}$ and $\mathrm{C}-\mathrm{F}$ bond can lead to the development of efficient and selective processes for the utilization of hydrocarbons. So developing a metal-free heterogeneous photocatalytic system to achieve highly valuable desired carbonyl products is also one of our aims. Moreover, harvesting direct solar energy to replace the artificial blue LED would be also applied. At last, not only small molecules but the complex molecules were also employed into the reaction conditions under the presence of sunlight in gram scale.

Despite the research on light-initiated cycloadditions in recent years, most of them are based on transition-metal photoredox catalysts and graphitic carbon nitride. Against this background, the development of an optimized sustainable protocol for visible-light-mediated cycloadditions using an organic dye as a metal-free photocatalyst under mild conditions is of great interest. We aimed to find a photocatalytic system using cheaper organocatalyst to provide Diels-Alder and azaDiels-Alder reactions. 


\section{Results and Discussion}

\subsection{Visible-Light-Mediated Efficient Metal-Free $\alpha$-Oxygenation of Tertiary Amines to} Amides $^{1}$

\subsubsection{Optimization for the Synthesis of 1-Benzoylpiperidine}

In the beginning, we carried out several experiments to find out the best reaction conditions including changing the catalysts, catalyst loading, solvent and amount of solvent. For this purpose, we chose the oxygenation of 1-benzylpiperidine (125a) to 1benzoyl piperidine (125b) as model reaction under oxygen atmosphere (balloon). As shown in Table 3.1.1, different metal-free catalysts such as rose bengal, 9-fluorenone and riboflavin were screened with DMSO as solvent and DBN as base under the irradiation of $12 \mathrm{~W}$ blue LED for $16 \mathrm{~h}$ (entries 1-3). To our delight, rose bengal provided $78 \%$ yield of the corresponding amide (125b). Subsequently, other bases including $\mathrm{DBU}, \mathrm{Na}_{2} \mathrm{CO}_{3}, \mathrm{Et}_{3} \mathrm{~N}$, and DMAP were also applied but did not improve the yield of the reaction which means DBN still was the suitable base (entries 4-7). However, changing to other polar solvents such as DMF and DMA could improve the yields up to $95 \%$. At last, using higher concentration of the solution via less solvent loading provided the highest yield of $99 \%$ in $16 \mathrm{~h}$ (entries $\mathbf{8 - 1 3}$ ). It should be noted that the reaction also worked under air but only $59 \%$ of the product was observed (entry 14).

Table 3.1.1 Optimization for the visible-light-induced $\alpha$-oxygenation of 1benzylpiperidine (125a) to 1-benzoylpiperidine (125b). ${ }^{\text {[a-e] }}$

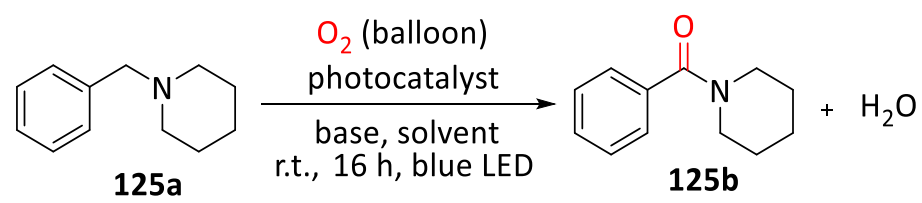

${ }^{1}$ The following chapter is based on the published paper. ${ }^{[97]}$ 


\begin{tabular}{|c|c|c|c|c|}
\hline Entry & Catalysts & Base & Solvent & Yield [\%] \\
\hline 1 & rose bengal & DBN & DMSO & 78 \\
\hline 2 & riboflavin & DBN & DMSO & 27 \\
\hline 3 & 9-fluorenone & DBN & DMSO & 10 \\
\hline 4 & Rose bengal & DBU & DMSO & 64 \\
\hline 5 & rose bengal & $\mathrm{Na}_{2} \mathrm{CO}_{3}$ & DMSO & 49 \\
\hline 6 & rose bengal & $\mathrm{NEt}_{3}$ & DMSO & 34 \\
\hline 7 & rose bengal & DMAP & DMSO & 28 \\
\hline 8 & rose bengal & DBN & DMF & 95 \\
\hline 9 & rose bengal & DBN & DMA & 88 \\
\hline 10 & rose bengal & DBN & $\mathrm{ACN}$ & 51 \\
\hline 11 & rose bengal & DBN & DMF & $95^{c}$ \\
\hline 12 & rose bengal & DBN & DMF & $96^{d}$ \\
\hline 13 & rose bengal & DBN & DMF & $99^{e}$ \\
\hline 14 & rose bengal & DBN & DMF & $59^{f}$ \\
\hline
\end{tabular}

[a] General reaction conditions: $\mathrm{O}_{2}$ (balloon), $12 \mathrm{~W}$ blue LED, $0.3 \mathrm{mmol} 125 \mathrm{a}$, photocatalyst ( $3 \mathrm{~mol} \%$ ), $0.45 \mathrm{mmol} \mathrm{DBN}, 2.5 \mathrm{~mL}$ solvent, room temperature, $16 \mathrm{~h}$. [b] Yields were determined by GC analysis using $n$-dodecane as an internal standard. [c] $2.0 \mathrm{~mL}$ DMF. [d] $1.5 \mathrm{~mL}$ DMF. [e] $1.0 \mathrm{~mL}$ DMF. [f] Under air. 


\subsubsection{Scope of $\alpha$-Oxygenation of Tertiary Amines to Amides}

\section{Visible-Light-Mediated Oxygenation of Tertiary Amines}

With these optimized reaction conditions in hand, we further applied this metal-free catalysed system to various tertiary amines (Scheme 3.1.1; entries 126b-144b). As shown in Scheme 3.1.1, this mild strategy was compatible with various substituted tertiary amines, including $N$-substituted piperidines, $N$-substituted $N, N$ dimethylbenzylamine, $\quad \mathrm{N}$-substituted tetrahydroquinoline and tetrahydroisoquinoline which afforded the corresponding products in moderate to excellent yields (Scheme 3.1.1; entries 126b-135b). It should be noted that $\mathbf{1 3 5 b}$ could further be dehydrogenated to form $\mathrm{N}$-substituted 1,2-dihydroquinolin-2-one with prolonging the reaction time, which limits the higher yield. For the $\mathrm{N}$-substituted anilines, most of them worked but exhibited limited reactivity (Figure 3.1.6). Among them, 140a could form the corresponding amides in medium isolated yield. Moreover, an $\mathrm{N}$-containing heterocyclic substrate, 1-benzylpyrrolidine, was converted to the corresponding amide as well (entry 136b) with satisfactory results. Inspired by the good results of benzylic amines, we sought to replace these with furan and thiophene moieties which also exhibited high reactivity (entries 137b-138b).

Different other substrates including heteroaromatic compounds were explored under our reaction conditions such as $\mathrm{N}$-substituted morpholine, and 4phenylpiperidine (entries 139b-143b) and afforded good yields. After the successful investigation of the aromatic and heteroaromatic amines, we became interested to find out the potential of this metal-free catalysed system for the conversion of aliphatic amines. To our delight, 1-methyl piperidine (entry 144a) showed moderate reactivity under our optimized reaction conditions with molecular sieves. 


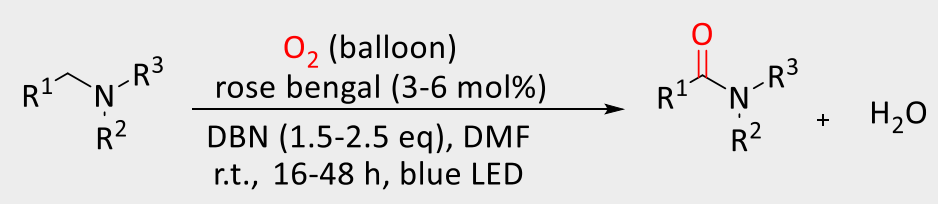<smiles>CN(C)C(=O)c1ccccc1</smiles>

126b, $70 \%$<smiles>Cc1ccc(C(=O)N2CCCCC2)cc1</smiles>

130b, $78 \%$<smiles>O=C(c1ccc(F)cc1)N1CCCCC1</smiles>

127b, $74 \%$<smiles>O=C1c2ccccc2CCN1c1ccccc1</smiles>

131b, $95 \%$<smiles>CCCCN1C(=O)CCc2ccccc21</smiles>
135b, $60 \%$<smiles>O=C(c1ccccc1)N1CCCC1</smiles>

139b, $76 \%$<smiles>O=C(c1ccc([N+](=O)[O-])cc1)N1CCCCC1</smiles>

128b, $88 \%$<smiles>CCN1CCc2ccccc2C1=O</smiles>

132b, $91 \%$<smiles>O=C1c2ccccc2CCN1CC1CC1</smiles>

133b, $85 \%$<smiles>O=C(c1ccncc1)N1CCCCC1</smiles>

136b, $72 \%$<smiles>O=C1CCCCN1c1ccccc1</smiles>

140b, $65 \%$<smiles>Cc1ccccc1C(=O)N1CCCCC1</smiles>

129b, 64\%<smiles>O=C(c1ccco1)N1CCCCC1</smiles>

137b, $80 \%$<smiles>O=C(/C=C/c1ccccc1)N1CCCCC1</smiles>

141b, $65 \%$

138b, $82 \%$<smiles>CCOC(=O)c1ccc(C(=O)N2CCOCC2)cc1</smiles>

142b, $71 \%$

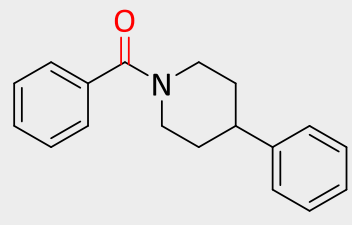

143b, $74 \%$

O

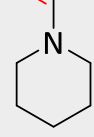

144b, 51\%

Scheme 3.1.1. Substrate scope for the visible-light-mediated oxygenation of tertiary amines. [a] Reaction conditions: $\mathrm{O}_{2}$ (balloon), $12 \mathrm{~W}$ blue LED, $0.3 \mathrm{mmol}$ starting material, photocatalyst (3-6 mol\%), $0.45-0.60 \mathrm{mmol} \mathrm{DBN}, 1.0 \mathrm{~mL} \mathrm{DMF}$, room temperature, $16-48 \mathrm{~h}$. All are isolated yields.

Based on the direct transformation of tertiary amines to amides, we found a one-pot synthesis of tertiary amides from secondary amines under metal-free conditions. We rationalized that tertiary amines can be directly synthesized from secondary amines and alkyl halides, and further oxygenation of tertiary amines under the optimized 
reaction conditions, thereby generating the corresponding tertiary amides in situ. Indeed, different benzylic and aliphatic bromides were reacted with piperidine and showed high formation of the desired amides (Scheme 3.1.2; entries 145b-149b). We hope this strategy can become highly valuable for the one-pot synthesis of pharmaceutical drugs and natural products.

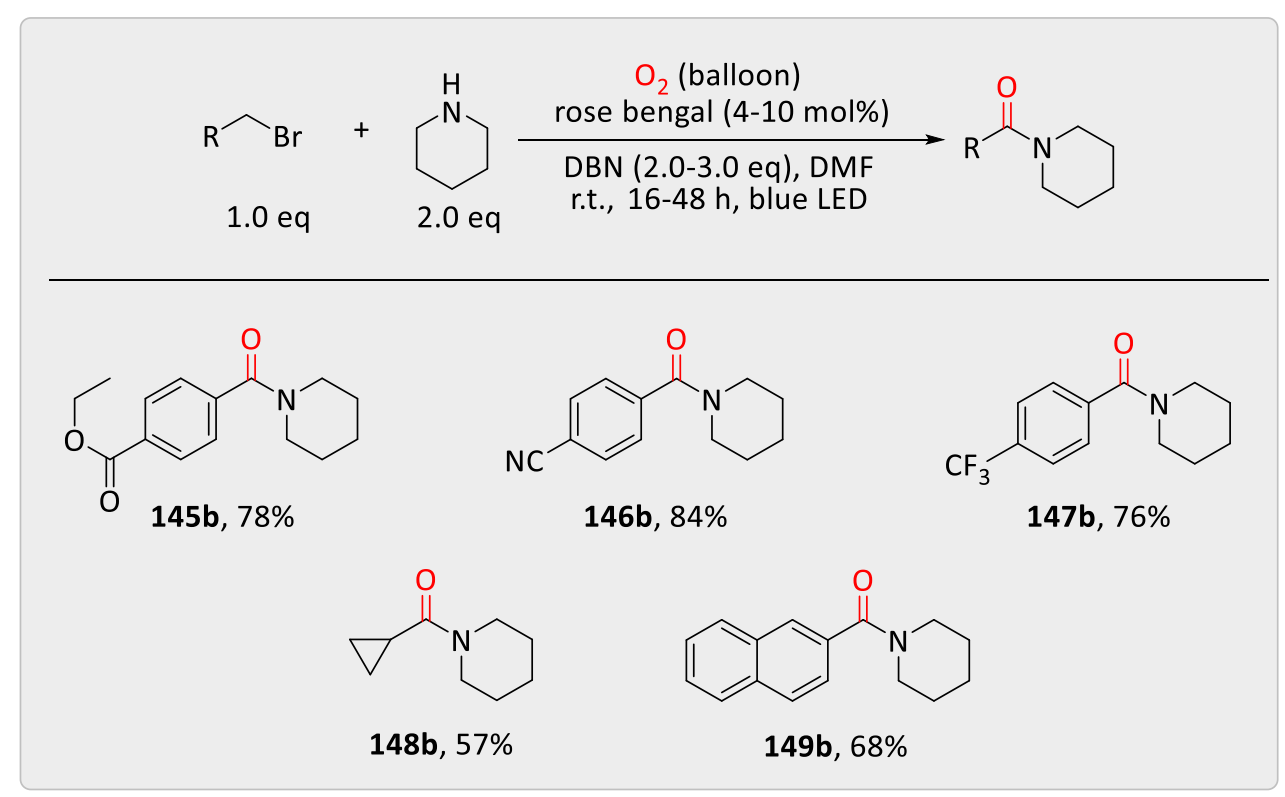

Scheme 3.1.2. One-pot syntheses of tertiary amides from secondary amines. [a] Reaction conditions: $\mathrm{O}_{2}$ (balloon), $12 \mathrm{~W}$ blue LED, $0.3 \mathrm{mmol}$ substrate, $4 \mathrm{~mol} \%$ rose bengal, $1 \mathrm{~mL}$ DMF, $0.6 \mathrm{mmol} \mathrm{DBN}, 16-24 \mathrm{~h}$. All are isolated yields. [c] 246b was performed with 3.0 eq DBN and 10 mol\% rose bengal with molecular sieves ( $3 \AA$ ).

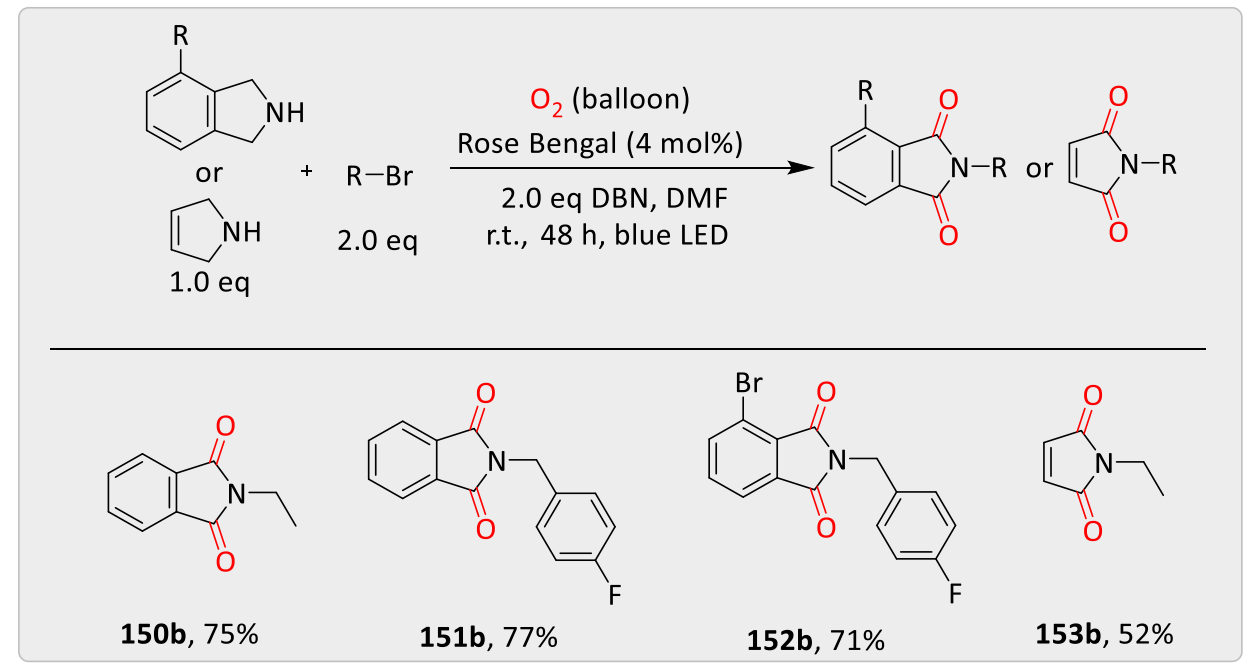

Scheme 3.1.3 One-pot synthesis of phthalimides and maleimide derivatives. [a] 
Reaction conditions: $\mathrm{O}_{2}$ (balloon), $12 \mathrm{~W}$ blue LED, $0.3 \mathrm{mmol}$ substrate, 4-6 mol\% rose bengal, $1 \mathrm{~mL}$ DMF, $0.45-0.75 \mathrm{mmol} \mathrm{DBN}, 16 \mathrm{~h}-48 \mathrm{~h}$. All are isolated yields.

In addition to these, we became interested in the one-pot syntheses of phthalimide derivatives as they are highly important as drug intermediates such as thalidomide, amphotalide, taltrimide, talmetoprim etc. ${ }^{[98]}$ Notably, it is challenging to afford $N$ substituted phthalimide directly because of the strong electron-withdrawing effect of phthalimide. Hence, we applied isoindoline derivatives and corresponding bromides to proceed in a one-pot oxidation reaction under optimized reaction conditions. To our delight, all of these substrates showed good yields of the desired phthalimides (Scheme 3.1.3; entries 150b-152b). Additionally, $N$-ethylmaleimide was also synthesized from 2,5-dihydropyrrole and ethyl bromide under the reaction conditions with medium yield (Scheme 3.1.3; entry 153b).

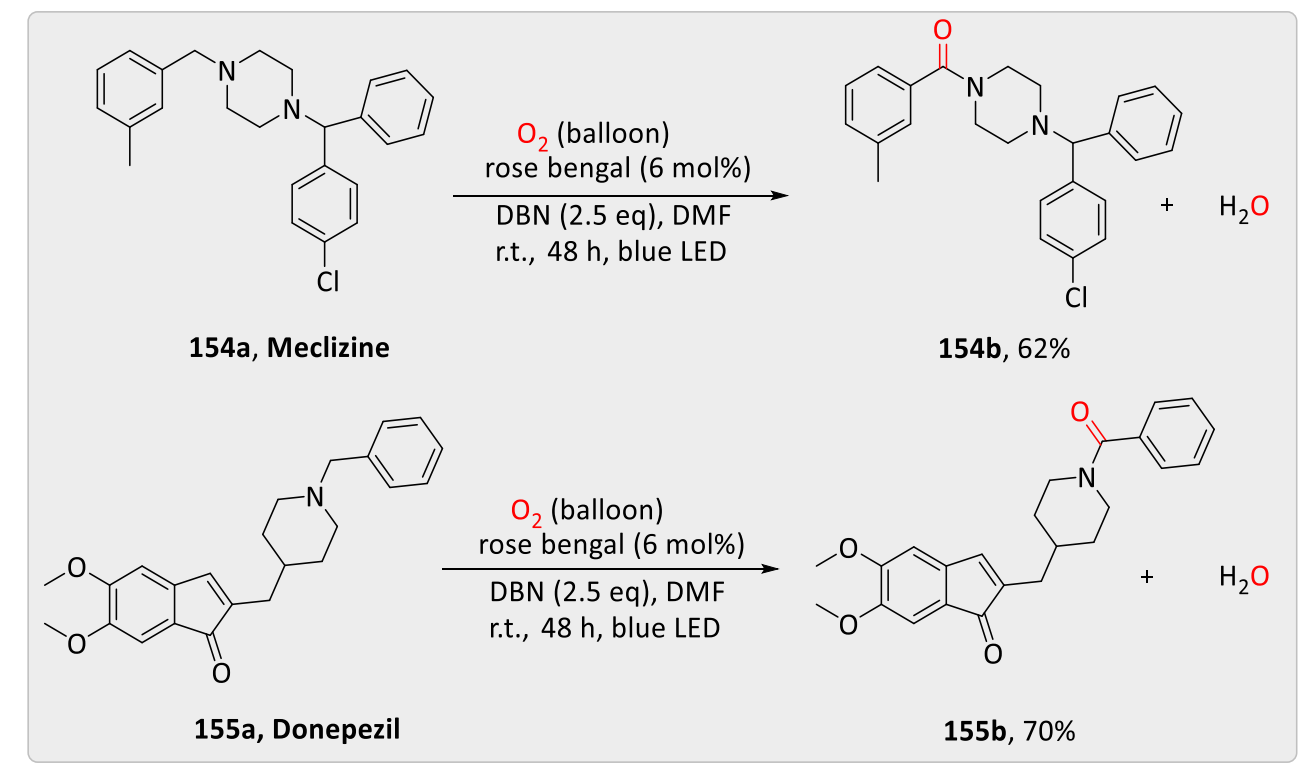

Scheme 3.1.4 Selective oxygenation of drug molecules.

Considering the synthetic practicability of the present metal-free $\alpha$-oxygenation protocol, oxygenation of drug molecules was performed. As shown in Scheme 3.1.4, oxygenation of meclizine (154a) (an antihistamine of the diphenylmethyl piperazine group considered to be an antiemetic) ${ }^{[99]}$ and donepezil (155a) (a medication used in the palliative treatment of Alzheimer's disease) was achieved in our system with good yields of the corresponding amides (Scheme 3.1.4; entries 154b-155b). ${ }^{[100]}$ Finally, 
we also investigated the application of the chosen conditions to the direct syntheses of natural products including (S)-(-)-8-oxoxylopinine and 17-oxosparteine (Scheme 3.1.5; entries $156 b-157 b) .{ }^{[100]}$ In fact, both of these natural products were synthesized directly in a single step using this metal-free pathway. To the best of our knowledge, currently there are no metal-free catalysts known for the oxygenation of sparteine (157a).

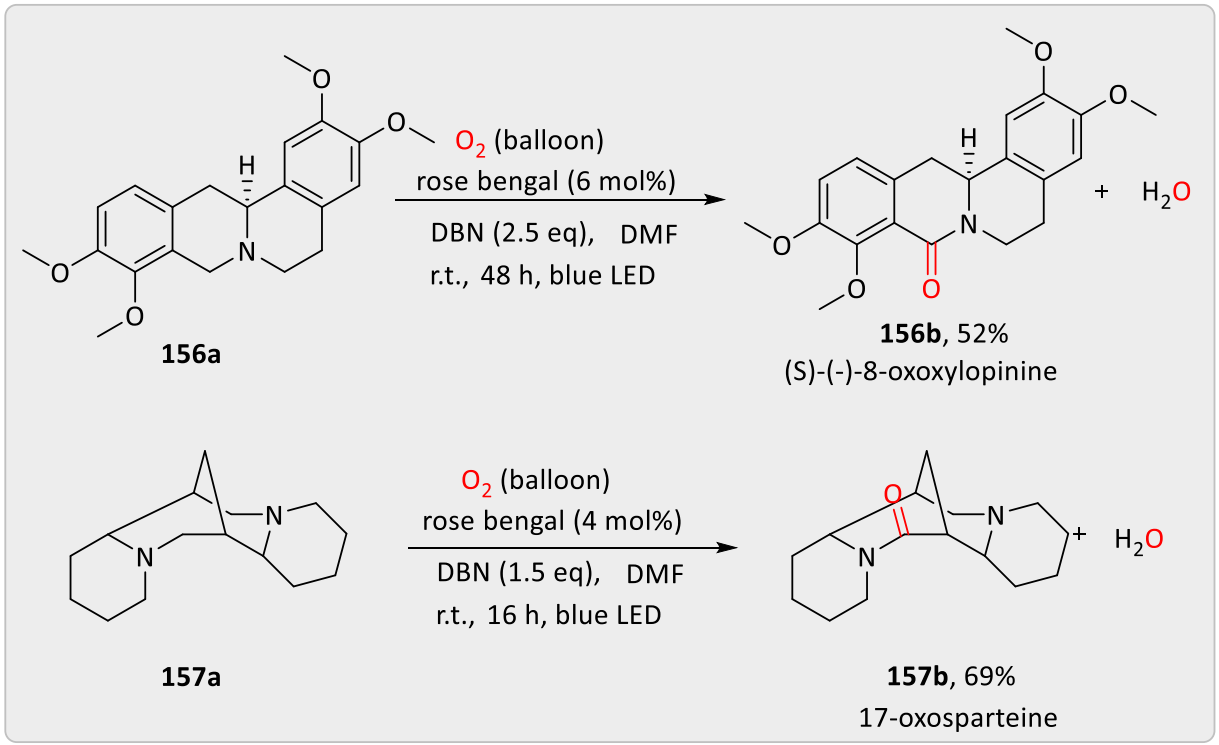

Scheme 3.1.5 One step syntheses of natural products.

\subsubsection{Mechanistic Studies}

\section{Control and Quenching Experiments}

We sought to collect more mechanistic information to figure out the role of the catalyst, base and light source in our reaction. Firstly, control experiments showed no product formation in the absence of light, photocatalyst or base (Table 3.1.2). Additionally, the yield also dropped to $0 \%$ without oxygen (under the nitrogen atmosphere) which clearly proved the significance of $\mathrm{O}_{2}$ in this reaction protocol. 
Table 3.1.2 Control experiments for the oxygenation of 1-benzylpiperidine. ${ }^{[a]}$

\begin{tabular}{lll}
\hline Entry & Controlled parameter & Yield [\%] \\
\hline 1 & Standard conditions & 99 \\
2 & N $_{2}$ atmosphere & 0 \\
3 & No light & 0 \\
4 & No catalyst & 2 \\
5 & No base & 3
\end{tabular}

[a] Reaction conditions: $\mathrm{O}_{2}$ (balloon), $12 \mathrm{~W}$ blue LED, $0.3 \mathrm{mmol} 125 \mathrm{a}, 3 \mathrm{~mol} \%$ rose bengal, $0.45 \mathrm{mmol} \mathrm{DBN}, 1.0 \mathrm{~mL}$ solvent, $18 \mathrm{~h}$. Yields were determined by GC analysis using $n$-dodecane as an internal standard.

In addition, the effect of different quenchers was investigated to identify the reactive oxygen species and possible intermediates (Table 3.1.3). When 2,6-di-tert-butyl-4methylphenol (BHT) or 2,2,6,6-tetramethyl-1-piperidinyloxyl (TEMPO) was added to the reaction mixture, the yield was decreased dramatically which proved a radical process to be involved. However, addition of tert-butanol did not decrease the yields which proved that the hydroxide radical was not involved. Furthermore, addition of $\mathrm{CuCl}_{2}$ and catalase to the reaction mixture showed lower yields which clearly showed the involvement of single electron processes and the presence of peroxide species in this photocatalytic system. Application of sodium azide and benzoquinone as quenchers revealed the presence of reactive oxygen species including singlet oxygen and superoxide radical anion, respectively, in the reaction system. 
Table 3.1.3 Quenching experiments for the oxygenation of 1-benzylpiperidine. ${ }^{\text {[a] }}$

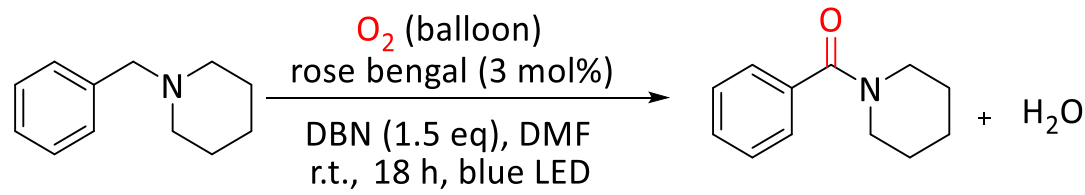

\begin{tabular}{cccc}
\hline Quenchers & Equivalents & Yield [\%] & Scavenger for \\
\hline BHT & 0.5 & 23 & radical \\
BHT & 1.0 & 0 & radical \\
TEMPO & 0.5 & 30 & radical \\
TEMPO & 1.0 & 2 & radical \\
tert-butanol & 1.0 & 75 & hydroxide radical \\
CuCl 2 & 1.0 & 2 & single electron \\
Catalase & $100 \mathrm{mg}$ & 6 & peroxide radical \\
Sodium azide & 1.0 & 1 & singlet oxygen \\
Benzoquinone & 1.0 & 2 & superoxide radical
\end{tabular}

[a] Reaction conditions: $\mathrm{O}_{2}$ (balloon), $12 \mathrm{~W}$ blue LED, $0.3 \mathrm{mmol} 125 \mathrm{a}, 3 \mathrm{~mol} \%$ rose bengal, $0.45 \mathrm{mmol} \mathrm{DBN}, 1.0 \mathrm{~mL} \mathrm{DMF}, 18 \mathrm{~h}$. Yields were determined by GC analysis using $n$-dodecane as an internal standard.

\section{Stern-Volmer Quenching Experiments}

To determine the reactive species in the photocatalytic reaction, absorptionemission spectra for a Stern-Volmer plot were acquired. Firstly, a 3D spectrum for excitation and emission of rose bengal was recorded in order to detect the maxima of absorption and emission. The resulting spectrum is depicted in (Figure 3.1.1). The excitation maximum was measured at $562 \mathrm{~nm}$ and the emission maximum at $574 \mathrm{~nm}$. These wavelengths were used for the further measurements. 


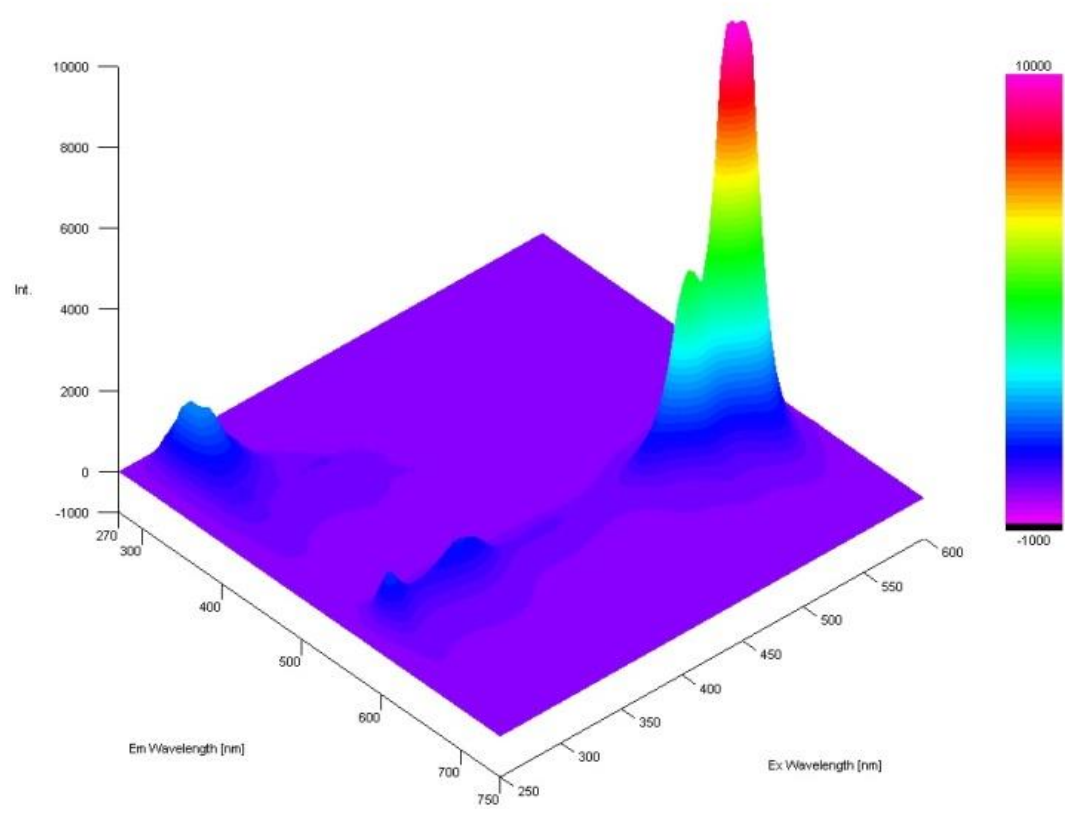

Figure 3.1.1 3D absorption-emission spectrum of rose bengal in DMF. The spectrum was measured by Daniel Riemer.

Afterwards, a blank sample was probed under $\mathrm{N}_{2}$ atmosphere without substrate and the received intensity was set as $I_{0}$. The effect of varied amounts of substrate 125a was investigated, as well as the saturation of the solution with air and oxygen. Figure 3.1.2 shows a summary of all three investigations. Depending on the concentration of substrate 125a, the emission decreases significantly. Saturation with air and oxygen shows only marginal effect in the magnitude of the uncertainty of the spectrometer, which means the excited state of the photocatalyst was quenched by 1-benzylpiperidine (125a). ${ }^{[102]}$ In conclusion, oxygen molecules did not react with the excited state of the photocatalyst, but rather the starting material does. 


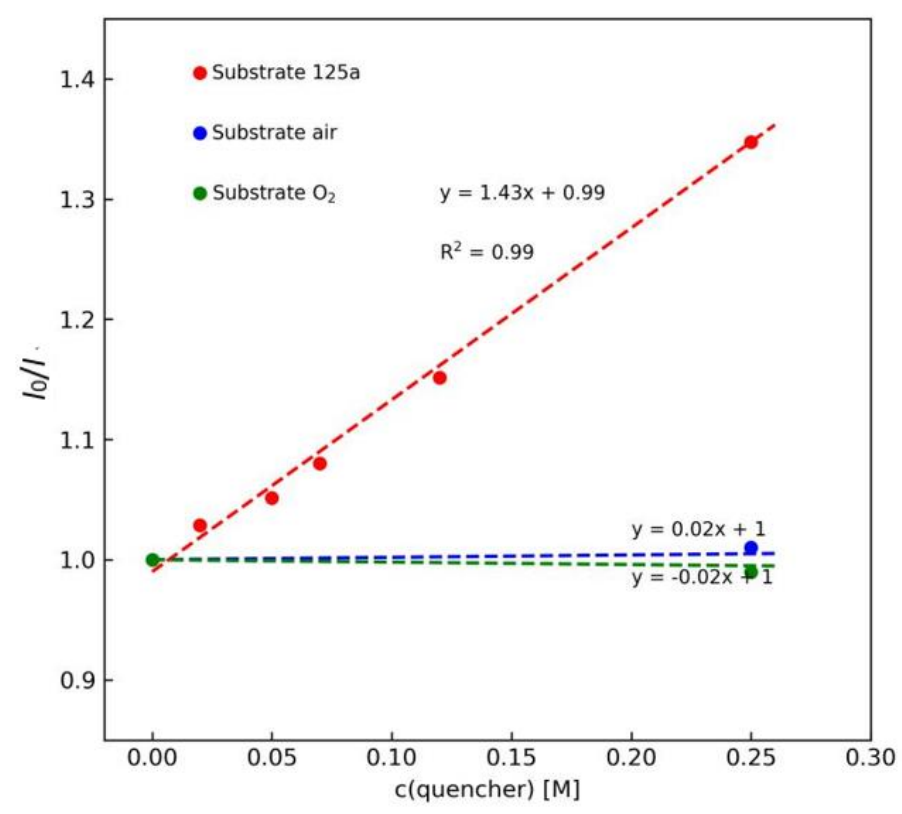

Figure 3.1.2 Stern-Volmer plot for the oxygenation of 1-benzylpiperidine.

\subsubsection{Proposed Mechanism}

Combining all the mechanistic results, a plausible mechanism for the visible-lightmediated metal-free catalytic oxygenation reaction is proposed in Scheme 3.1.4. We rationalized that, at first, the photocatalyst was excited by the irradiation of visible light and underwent a single electron transfer (SET) with 1-benzylpiperidine (125a). The role of the base in our system was to deprotonate radical I to form the morestable radical II. The photocatalyst radical anion then generated the actual oxidants superoxide radical anion and peroxide anion from $\mathrm{O}_{2}$ (reported value for the reduction potential of excited state of rose bengal residing at $-1.33 \mathrm{~V}$ vs SCE), which is sufficient for the reduction of molecular oxygen to its superoxide radical anion with the reduction potential residing at $-0.56 \mathrm{~V}$ vs SCE). ${ }^{[66][103]}$ The radical II reacted with peroxide radical from superoxide radical anion to form intermediate III. At last, a proton abstraction by a base would generate the desired product $\mathbf{1 2 5 b}$. At the same time, water was released as the only byproduct. 


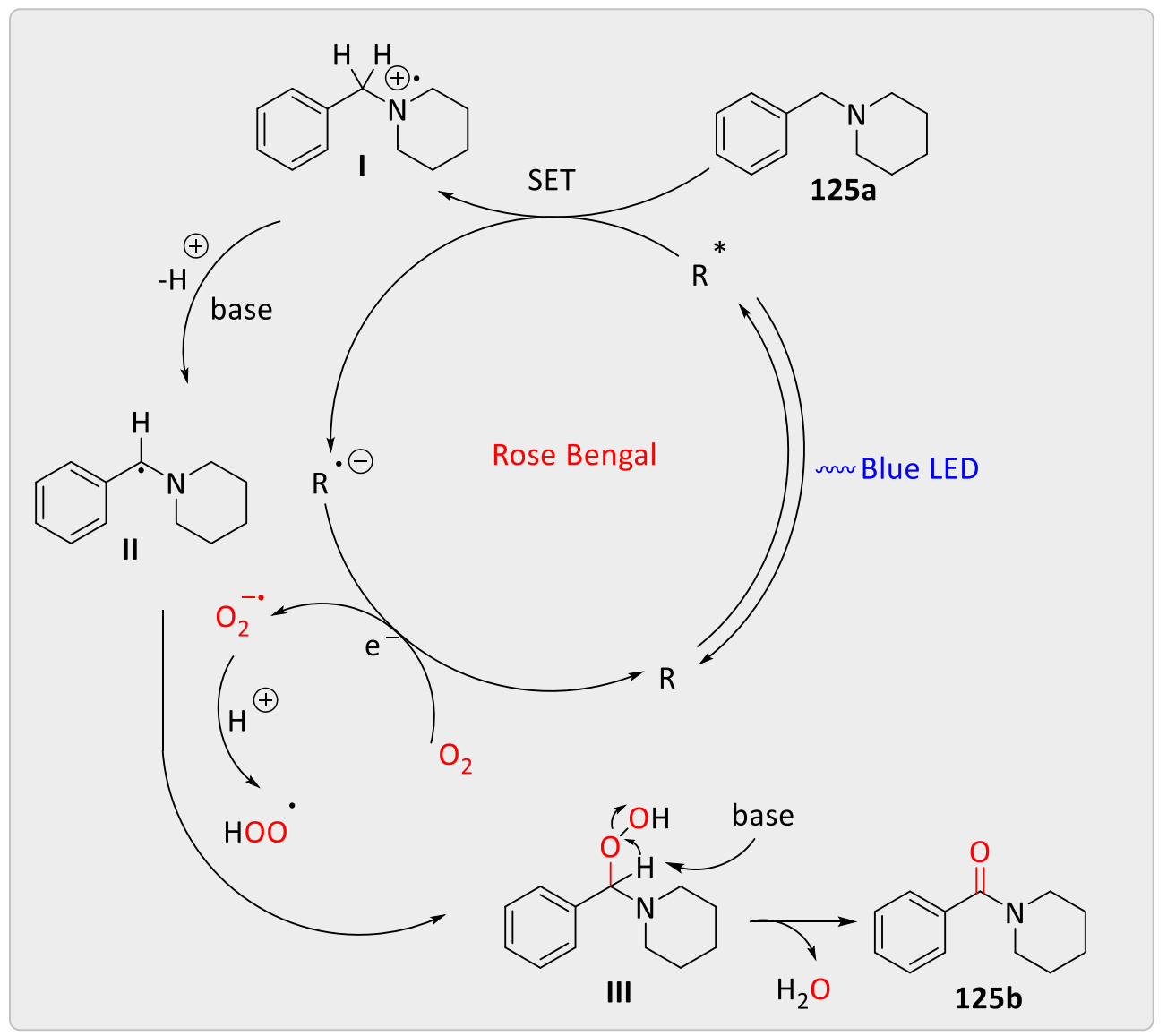

Scheme 3.1.4 Proposed mechanism of tertiary amines to amides.

\subsubsection{Unsuccessful Substrates Scope}

Besides the reported substrates, there were some tertiary amines which did not work in our system (Scheme 3.1.5). For example, it seems that electron-poor amines block the reactivity completely (Scheme 3.1.5, F1, F5, F9, F11, F12, F17). Moreover, the substrates with primary and secondary amines (such as unprotected piperazines) showed no reactivity in our system either (Scheme 3.1.5, F23-F26). Especially, we observed that secondary benzylic amines will form the imines instead of amides. We also tried to apply our system to aliphatic tertiary amines, but almost all of them did not work (F6-F8, F10, F16). There were some substrates which exhibited limited reactivity, but higher yields could not be achieved under applied conditions (Scheme 3.1.5). 
<smiles>O=S1(=O)CCN(Cc2ccccc2)CC1</smiles>

F1, 48h, n. ob.<smiles>CCN(C)c1ccc(C#N)cc1</smiles>

F5, 24h, n. ob.<smiles>c1cc(N2CCCC2)ccn1</smiles>

F9, 24h, n. ob.<smiles>C1CN2CCN1CC2</smiles>

F13, 24h, n. ob<smiles>CCN(Cc1ccccc1)c1ccccc1</smiles>

F2, 48h, n. ob.<smiles>C=CCN1CCCCC1</smiles>

F6, 24h, n. ob.<smiles>C1CCC(N2CCCCC2)CC1</smiles>

F10, 24h, n. ob.<smiles>c1ccc(N2CCOCC2)cc1</smiles>

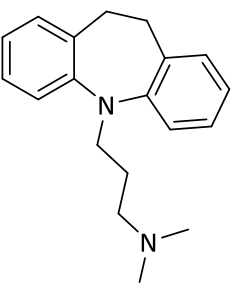

F19, 24h, n. ob.

F18, 48h, n. ob.<smiles>C1=CNCC(CN2CCSc3ccccc32)C1</smiles>

F4, 48h, n. ob.<smiles>CN1CCCC1</smiles>

F8, 24h, n. ob.<smiles>c1ccc(CN(Cc2ccccc2)Cc2ccccc2)cc1</smiles>

F11, 24h, n. ob.

F15, 48h, t. a.<smiles>O=C1CCCN1c1ccccc1</smiles><smiles>O=C1CN(Cc2ccccc2)C(=O)N1</smiles>

F12, 48h, n. ob<smiles>CCN1CCCCC1</smiles>

F16, 24h, n. ob.<smiles>COc1cc2cc3[n+](cc2cc1OC)CCc1cc2c(cc1-3)OCO2</smiles>

F21, 48h, n. ob.

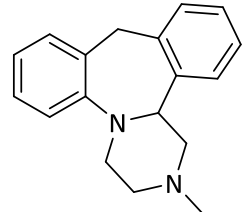

F22, 48h, t. a<smiles>c1ccc(CCN2CCCCC2)cc1</smiles>

F20, 48h, n. ob.<smiles>c1cc2c(cc1CN1CCNCC1)OCO2</smiles><smiles>COc1cc(CN2CCNCC2)cc(OC)c1OC</smiles>

F25, 48h, n. ob.

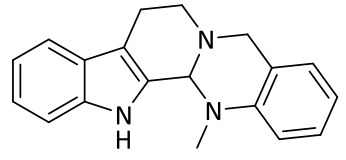

F23, 48h, n. ob.

F24, 48h, n. ob.

Scheme 3.1.5 Substrates which did not afford products in this system. The reaction was monitored via GC-MS and TLC. Part of the reactions were performed with the assistance of Jiri Kollmann and Daniel Riemer. t.a.: trace amount; n. ob.: not observed. 


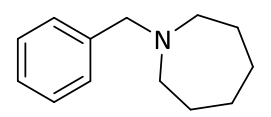

F28, 24h, 10\%<smiles>c1cc2c3c(c1)CCCN3CCC2</smiles>

F31, 48h, <5\%<smiles></smiles>

F36, 24h, 17\%<smiles>CCCCCCN(c1ccccc1)c1ccccc1</smiles>

F29, 48h, 34\%<smiles>CCCCN1CCc2cccnc21</smiles>

F32, $48 h,<5 \%$<smiles>OC1CCN(Cc2ccccc2)CC1</smiles>

F37, 24h, 31\%<smiles>CC(C)(C)CN(c1ccccc1)c1ccccc1</smiles>

F30, 24h, 20\%<smiles>CCN1CCc2ccccc21</smiles>

F33, 24h, <10\%

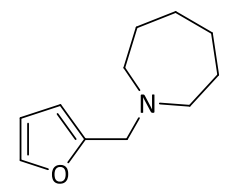

F38, 24h, 23\%

Scheme 3.1.6 Substrates with limited reactivity. The reaction was monitored via GCMS and TLC. The yields were determined via NMR using iodoform as an internal standard. Part of the reactions were performed by Jiri Kollmann and Daniel Riemer. 


\subsection{Heterogeneous Photocatalytic Benzylic C(sp $\left.{ }^{3}\right)-H$ Hydroxylation}

\subsubsection{Optimization for Synthesis of 2-Phenyl-2-propanol}

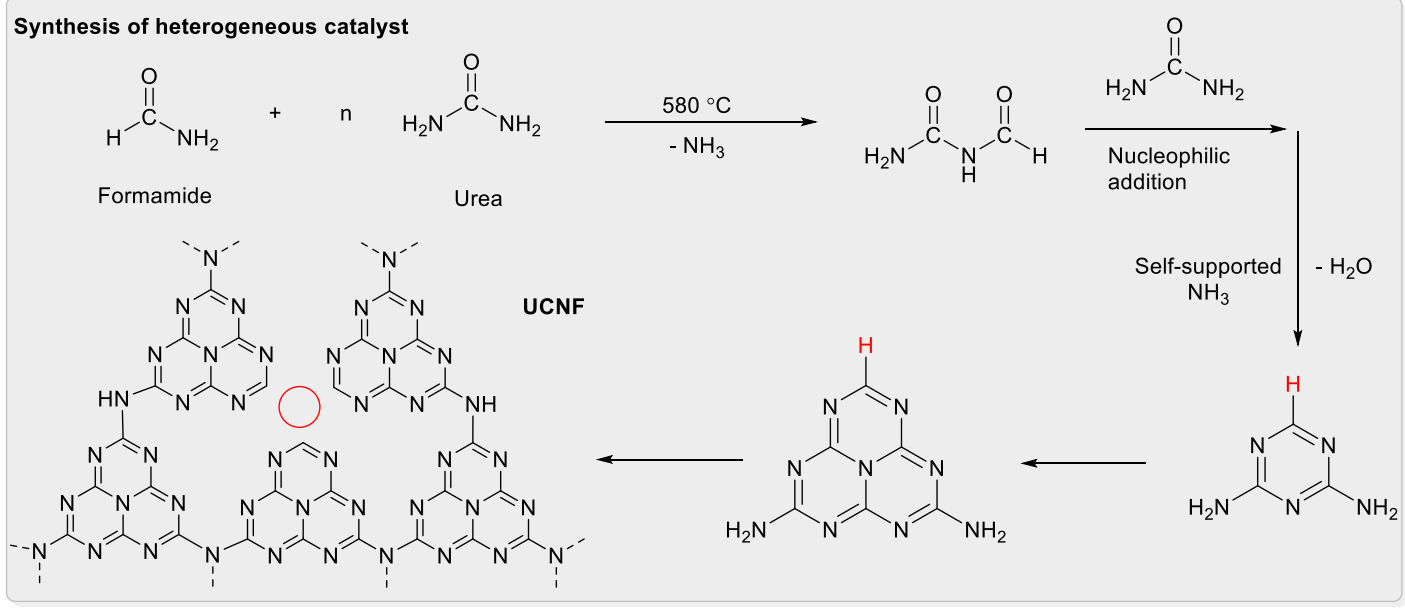

Figure 3.2.1 Synthesis of novel nitrogen-deficient polymeric carbon nitride. The catalyst was synthesized by Waldemar Schilling.

In the beginning, we have synthesized a novel nitrogen-deficient polymeric carbon nitride (UCNF) (Figure 3.2.2) according to a literature report. ${ }^{[46]}$ It is further used for the development of hydroxylation reactions of benzylic $\mathrm{C}\left(\mathrm{sp}^{3}\right)-\mathrm{H}$ bonds. With this catalyst in hand, we started to optimize for the best reaction conditions using cumene (159a) as the model substrate. The results of the screening and optimizations are shown in Table 3.2.1. The first screened parameter was the heterogeneous catalyst without any HAT reagents (Entry 1), which showed that the heterogeneous catalyst only generated a limited yield of the product. Afterwards, a HAT reagent $\left(\mathrm{Cl}_{4} \mathrm{NHPI}, \mathrm{N}\right.$ Hydroxy tetrachlorophthalimide) was added into reaction conditions. To our delight, the yield was improved to $65 \%$ yield. In this case, $\mathrm{Cl}_{4} \mathrm{NHPI}$ was proposed to give an oxyl radical which can abstract of a hydrogen atom from the substrate. Thereby, a benzylic carbon radical was generated mainly via HAT. However, adding higher amount of the catalyst could not further improve the yields (Entries 2-4). Besides that, different solvents were screened but could not further improve the yields of reaction (Entries 5-7). Notably, only the HAT reagent itself without the presence of the catalyst also achieved the product but with limited yield (Entry 8). It is supposed that the initiation of $\mathrm{Cl}_{4} \mathrm{PINO}$ (PINO = phthalimide $\mathrm{N}$-oxyl) radicals is the key step, without co-catalyst for 
$\mathrm{Cl}_{4} \mathrm{NHPI}$ activation, $\mathrm{Cl}_{4} \mathrm{PINO}$ radicals can only partially be activated under the irradiation of blue-LED. Cooperation with the catalyst accelerated this step and generated more $\mathrm{Cl}_{4} \mathrm{PINO}$ radicals. The further control experiments clearly proved the indispensable role of the light and oxygen in this reaction (Entries 9-10).

Table 3.2.1 Optimization studies for the synthesis of 2-phenyl-2-propanol. ${ }^{[a-b]}$

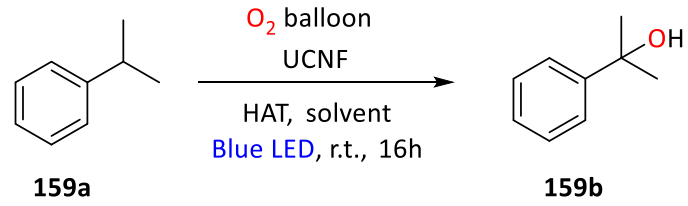

\begin{tabular}{|c|c|c|c|c|c|c|c|}
\hline Entry & UCNF & $\mathrm{Cl}_{4} \mathrm{NHPI}$ & Solvents & $T(h)$ & Light & $\begin{array}{l}\text { Atmos- } \\
\text { phere }\end{array}$ & Yield (\%) \\
\hline 1 & $10 \mathrm{mg}$ & 0 eq. & $\mathrm{MeCN}$ & 16 & on & $\mathrm{O}_{2}$ & 23 \\
\hline 2 & $10 \mathrm{mg}$ & 0.3 eq. & $\mathrm{MeCN}$ & 16 & on & $\mathrm{O}_{2}$ & 65 \\
\hline 3 & $12 \mathrm{mg}$ & 0.3 eq. & $\mathrm{MeCN}$ & 16 & on & $\mathrm{O}_{2}$ & 65 \\
\hline 4 & $15 \mathrm{mg}$ & 0.3 eq. & $\mathrm{MeCN}$ & 16 & on & $\mathrm{O}_{2}$ & 63 \\
\hline 5 & $10 \mathrm{mg}$ & 0.3 eq. & THF & 16 & on & $\mathrm{O}_{2}$ & 15 \\
\hline 6 & $10 \mathrm{mg}$ & 0.3 eq. & DMSO & 16 & on & $\mathrm{O}_{2}$ & 9 \\
\hline 7 & $10 \mathrm{mg}$ & 0.3 eq. & DMF & 16 & on & $\mathrm{O}_{2}$ & 24 \\
\hline 8 & $0 \mathrm{mg}$ & 0.3 eq. & $\mathrm{MeCN}$ & 16 & on & $\mathrm{O}_{2}$ & 16 \\
\hline 9 & $10 \mathrm{mg}$ & 0.3 eq. & $\mathrm{MeCN}$ & 16 & off & $\mathrm{O}_{2}$ & 0 \\
\hline 10 & $10 \mathrm{mg}$ & 0.3 eq. & $\mathrm{MeCN}$ & 16 & on & $\mathrm{N}_{2}$ & 1 \\
\hline
\end{tabular}

[a] General reaction conditions: oxygen, $12 \mathrm{~W}$ blue LED, $0.3 \mathrm{mmol}$ 159a (1.0 eq.), 0.3 eq. $\mathrm{Cl}_{4} \mathrm{NHPI}, 10 \mathrm{mg}$ photocatalyst, $1 \mathrm{~mL}$ solvent, room temperature, $16 \mathrm{~h}$. [b] Yields were determined by GC using dodecane as an internal standard.

\subsubsection{Substrate Scope for the Hydroxylation under Blue LED}

The optimized reaction conditions were obtained from Table 3.2.1. Various substrates were employed to afford the corresponding oxygenated products. As shown in Figure 3.2.2, the scope was firstly extended to various substituted isopropyl benzenes such 

(160b-166b, 172b-174b). To our delight, all of them exhibited good reactivity under the standard conditions.

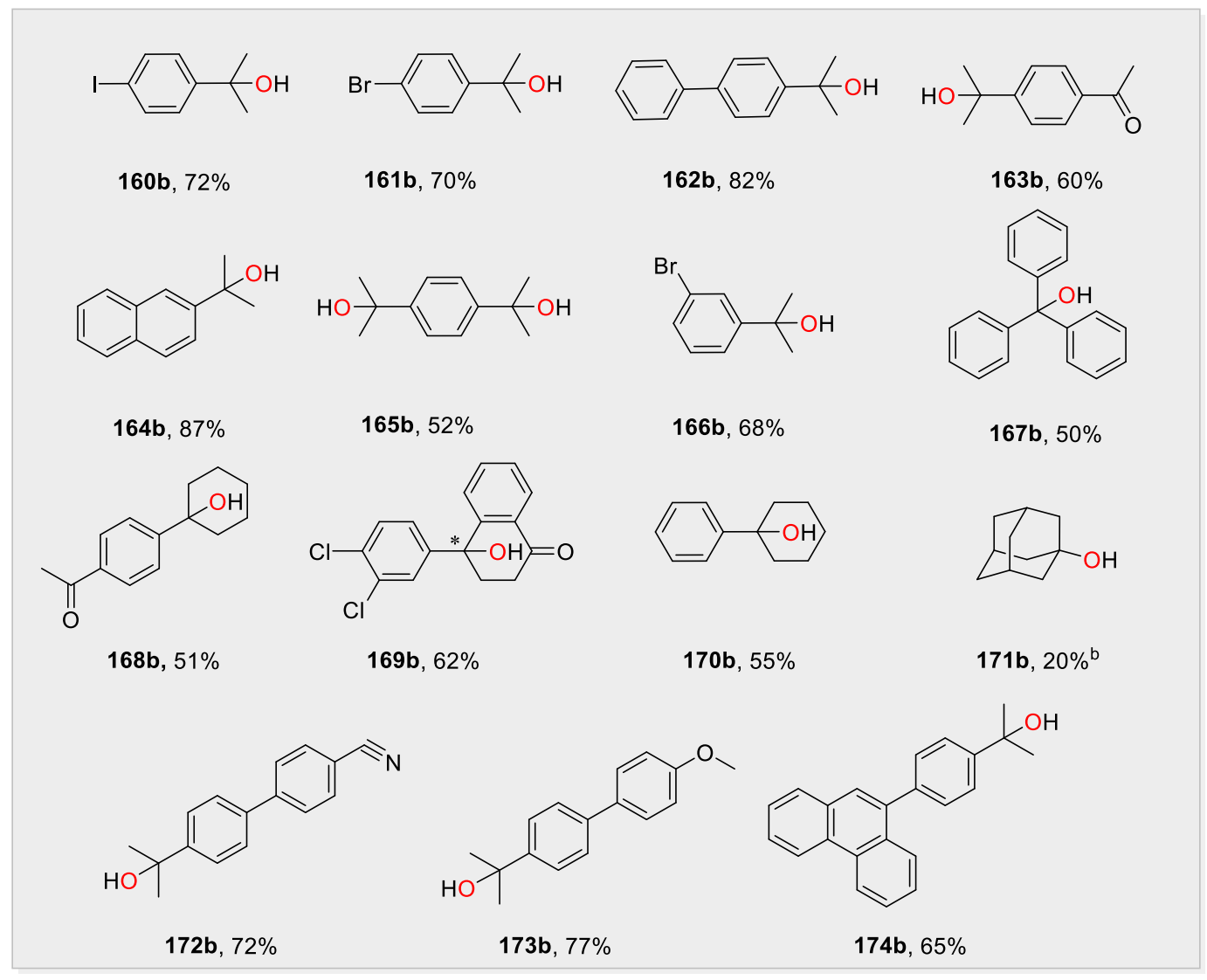

Figure 3.2.2 Substrates scope of photocatalytic hydroxylation. [a] Reaction conditions: $\mathrm{O}_{2}$ (balloon), $12 \mathrm{~W}$ blue LED, $0.3 \mathrm{mmol}$ substrate, $10 \mathrm{mg}$ catalyst, 0.3 eq. $\mathrm{Cl}_{4} \mathrm{NHPI}, 1 \mathrm{~mL} \mathrm{ACN}, 16 \mathrm{~h}-48 \mathrm{~h}$. [b] The yield was determined by GC with dodecane as an internal standard.

Furthermore, we also applied these mild reaction conditions to triphenylmethane which also formed the corresponding hydroxylated product with medium isolated yield probably due to the steric hindrance of the triphenyl structure (167b). It should be also mentioned that $161 b$ (115 $€ / g$, CAS No. 2077-19-2, TCI Europe), 163b (770 \$/g, CAS No. 54549-72-3, Enamine), 164b (571 \$/g, CAS No. 20351-54-6, Enamine) are highly expensive chemcials, which proves that this photocatalytic method could play an important role for the synthesis of benzylic tertiary alcohols. The scope was further extended to other substrates having benzylic $\mathrm{C}\left(\mathrm{sp}^{3}\right)-\mathrm{H}$ bonds including 
phenylcyclohexane and 4'-cyclohexylacetophenone (168b-170b) under this condition with medium yields. Adamantane (171a) was also employed in our reaction conditions, which showed limited reactivity under our system.

After achieving desirable products using blue LED as the light source, we aimed to replace the bule LED by harvesting direct sunlight and even for the gram scale reactions. To our delight, 2 -isopropyl naphthalene was highly compatible with an excellent isolated yield of the desired product (Figure 3.2.3). Considering the high value of tertiary alcohol products in chemical industry, this metal-free heterogeneous catalytic system will provide a novel and valuable methodology to construct tertiary alcohols.

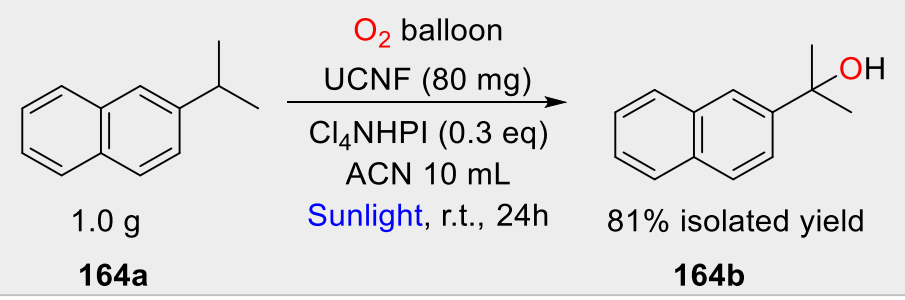

Figure 3.2.3 Hydroxylation under sunlight. Reaction setup under sunlight irradiation on the rooftop of the institute and temperature data on a sunny day for the reactions.

\subsubsection{Recycling Experiments and ${ }^{18} \mathrm{O}$-Labelling Experiments}

To ensure the stability of the catalyst, experiments with a recycled catalyst were performed 5 times under the same reaction conditions. This catalyst can be recycled with stable reactivity (Figure 3.2.4). The detailed procedure of recycling the catalyst is given in the experimental section. The ${ }^{18} \mathrm{O}$-labeling experiment was performed with ${ }^{18} \mathrm{O}_{2}$ (Sigma Aldrich, ${ }^{18} \mathrm{O}$ atom 99.7\%), and analyzed with ESI-HRMS and NMR showing the ${ }^{18} \mathrm{O}$-labeled product with an isolated yield of $51 \%$. The result showed that the origin of the oxygen atom in the desired product was only from oxygen gas since no ${ }^{16} \mathrm{O}$-labeled product was found (Figure 3.2.5). 

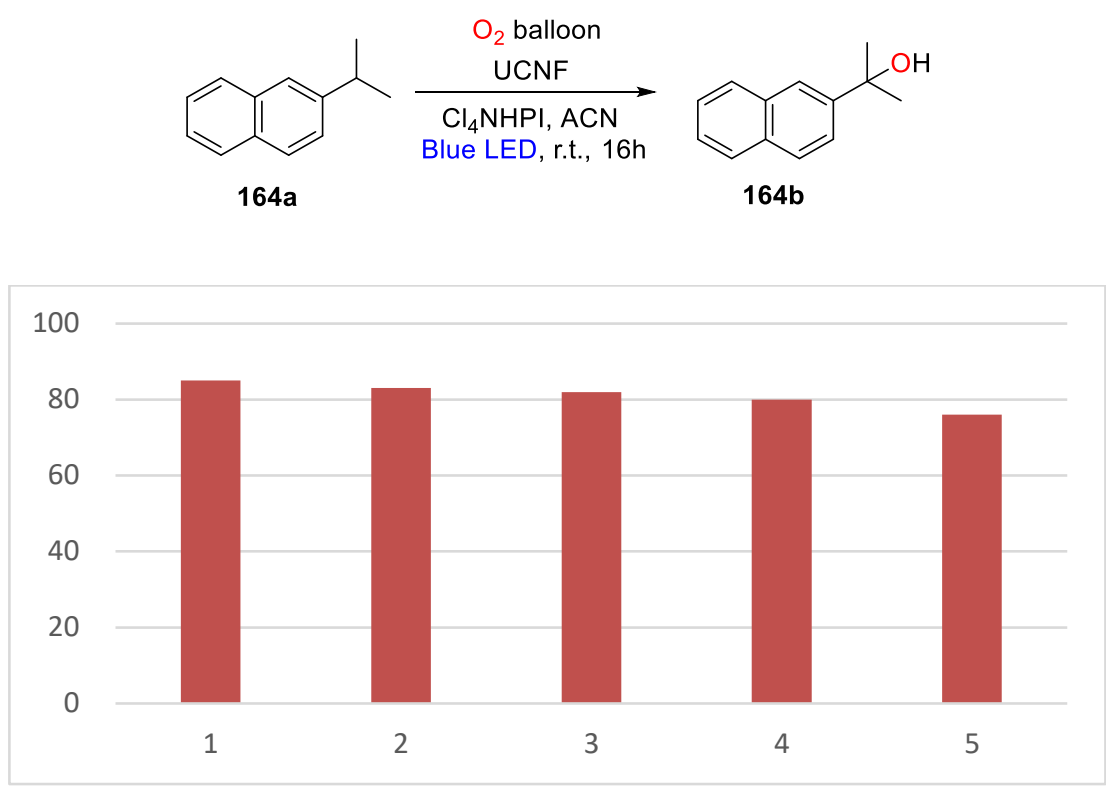

Figure 3.2.4 Reusability of UCNF for hydroxylation of 2-isopropylnaphthalene.

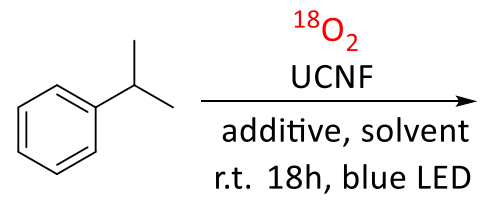<smiles>CC(O)(O)c1ccccc1</smiles>

$51 \%$ isolated yield

Figure 3.2.5 Hydroxylation of cumene in presence of ${ }^{18} \mathrm{O}$-labeled oxygen.

\subsubsection{Suggested Mechanism}

Based on our previous investigations of photocatalytic reactions and literature reports of the heterogeneous photocatalysis and HAT, ${ }^{[48][104]}$ we suggested a mechanism in Figure 3.2.6. In the first step, the catalyst (UCNF) was activated under the irradiation of blue LED and positive holes $\left(\mathrm{h}^{+}\right)$and electrons $\left(\mathrm{e}^{-}\right)$were generated on the surface of the catalyst. Then, the $\mathrm{O}_{2}$ was activated through SET by the conduction band of the catalyst and formed the superoxide radical anion $\left(\mathrm{O}_{2}{ }^{--}\right)$. The $\mathrm{Cl}_{4} \mathrm{PINO}$ radical was generated through HAT process with the $\mathrm{O}_{2}{ }^{\bullet-}$, meanwhile the $\mathrm{O}_{2}{ }^{\bullet-}$ was reduced to the $\mathrm{HOO}^{-}$. Afterwards, the $\mathrm{HOO}^{-}$reacted with a hydrogen atom from HAT to form hydrogen peroxide and one electron, the electron could transfer into the valence band (VB) of the catalyst to neutralize the charge. Meanwhile, another $\mathrm{Cl}_{4} \mathrm{PINO}$ radical was activated through this process. 
In the second step, the substrate was activated by the $\mathrm{Cl}_{4} \mathrm{PINO}$ radical through HAT process and formed a radical at the benzylic position of 159. At the same time, $\mathrm{Cl}_{4} \mathrm{NHPI}$ was regenerated. This intermediate reacted with the oxygen and formed the intermediate $159 \mathrm{~d}$, then the $\mathrm{H}$ atom from $\mathrm{Cl}_{4} \mathrm{NHPI}$ was abstracted by $159 \mathrm{~d}$ to form 159e. Finally, the product $159 \mathrm{~b}$ was generated by eliminating the oxygen.

The KIE experiment for the $\mathrm{Cl}_{4} \mathrm{NHPI}$-based catalytic system (oxidation reactions of a range of primary, secondary, andcyclic alcohols) has been investigated by Buckingham et $a l^{[105]}$ and turned out that the $\mathrm{C}-\mathrm{H}$ hydrogen abstraction process is the rate determining step for the reaction.

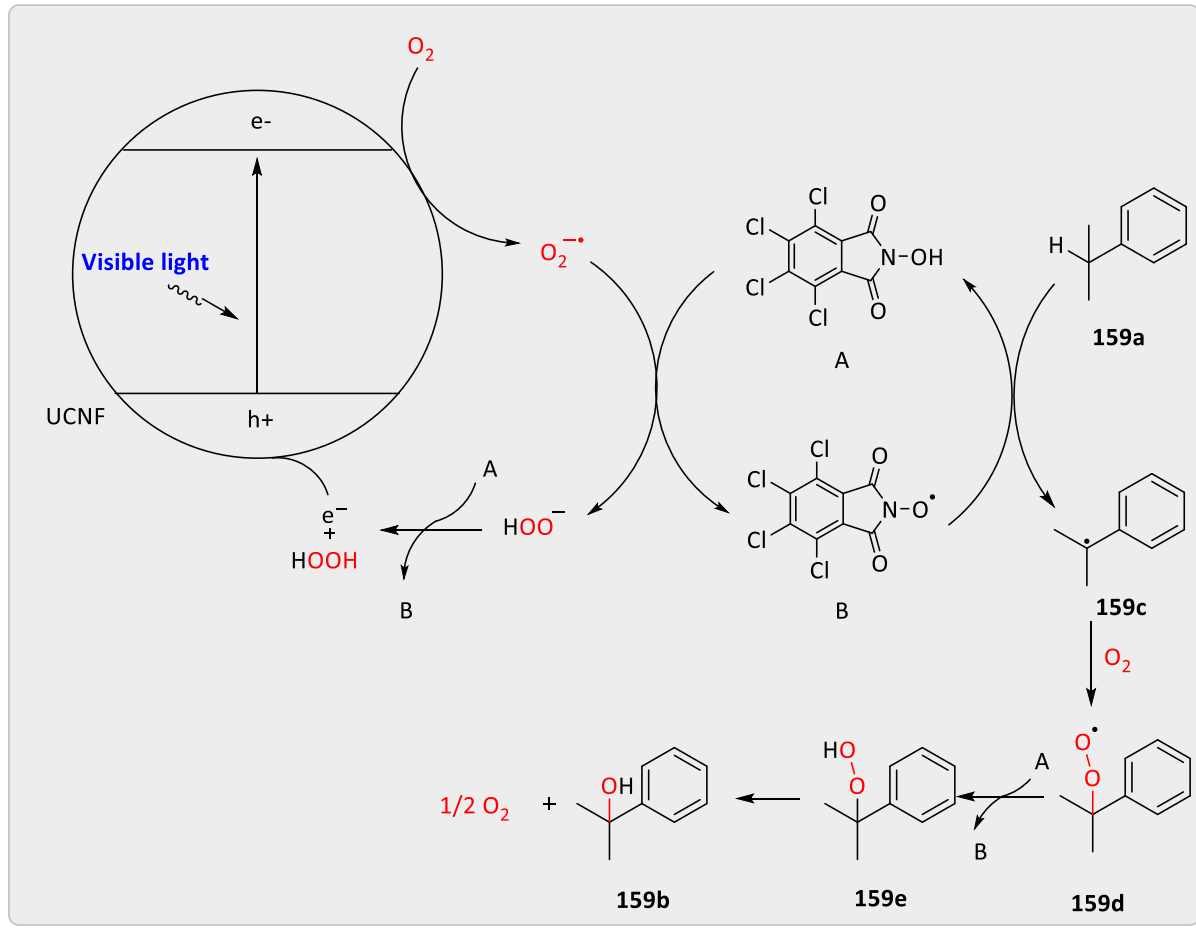

Figure 3.2.6 Suggested mechanism of photocatalytic hydroxylation.

\subsubsection{Unsuccessful Substrates}

However, during the extension of the scope of the photocatalytic benzylic $\mathrm{C}\left(\mathrm{sp}^{3}\right)-\mathrm{H}$ hydroxylation, there were some challenges and limitations. Especially, the selectivity was not good when the reaction conditions were applied to sec-butylbenzene (Figure 3.2.7, F42). Only a side product, dehydrogenated alkene, was generated and furthermore, corresponding ketone via $\mathrm{C}=\mathrm{C}$ bonds cleavage was also obtained. The same side reactions occurred in the case of 1,1-diphenylpropane (Figure 3.2.7, F4950) and its derivatives such as 1,1-diphenylpentane. In these cases, the 
dehydrogenated alkenes and the following ketones were observed under our reaction conditions. Some substrates such as 2-bromocumene and 2,6-diisopropylnaphthalene (Figure 3.2.7, F39-40) did not exhibit good reactivities.

Even though phenyl cyclohexane exhibited moderate activity, the 5-phenyl-1,3cyclohexanedione and 4-phenylpiperidine did not show the formation of products (Figure 3.2.7, F47-48). Furthermore, 4-phenylpiperidine and benzoyl protected 4phenylpiperidine were also employed into our system, but both of them did not furnish the products (Figure 3.2.7, F51, F53). Another pharmaceuticals derivative (F52) was also subjected to our reaction conditions, but in this case no product was observed.

In addition, other derivatives especially containing EWG groups did not work under our reaction conditions (Figure 3.2.7, F41, F45-F46, F51-F54). After the hydroxylation of triphenylmethane, several similar complex molecules including leuco malachite green and leuco crystal violet (Figure 3.2.7, F55-F56) were employed under our conditions. But the desired hydroxylated products were not observed in any of the cases. 
<smiles>CC(C)c1ccccc1Br</smiles>

F39, 48h, $15 \%$<smiles>CC(C)c1ccc2cc(C(C)C)ccc2c1</smiles>

F40, 48h, 30\%<smiles>N#CCC(c1ccccc1)c1ccccc1</smiles>

F41, 48h, n. ob.<smiles>COC(=O)C(c1ccccc1)c1ccccc1</smiles>

F45, 48h, n. ob.<smiles>CCC(C)c1ccccc1</smiles>

F42, 48h, n. ob.<smiles>CC(C)C(=O)c1ccccc1</smiles>

F43, 48h, n. ob.<smiles>CC(C)c1ccc(N)cc1</smiles>

F44, 48h, n. ob<smiles>CCC(c1ccccc1)c1ccccc1</smiles>

F49, 48 h, n. ob<smiles>O=C1c2ccccc2C(=O)C1C1C=CC=CC1</smiles>

F46, 48h, n. ob.

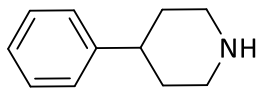

F47, 48h, n. ob.

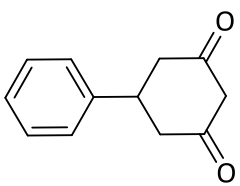

F48, 48h, n. ob.<smiles>CCCCCC(c1ccccc1)c1ccccc1</smiles>

F50, 48h, n. ob.<smiles>Oc1ccc(C2CCCCC2)cc1</smiles>

F51, 48h, n. ob.<smiles>CN(C)C1CCC(c2ccc(Cl)c(Cl)c2)c2ccccc21</smiles>

F52, 48h, n. ob.

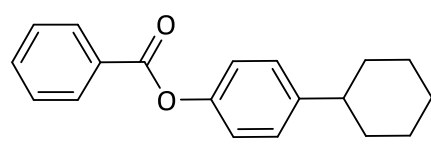

F53, 48h, n. ob.<smiles>O=C(c1ccccc1)N1CCC(c2ccccc2)CC1</smiles>

F54, 48h, n. ob.<smiles>CN(C)c1ccc(C(c2ccccc2)c2ccc(N(C)C)cc2)cc1</smiles>

F55, 48h, n. ob.<smiles>CN(C)c1ccc(C(c2ccc(N(C)C)cc2)c2ccc(N(C)C)cc2)cc1</smiles>

F56, 48h, n. ob.

Figure 3.2.7 Unsuccessful substrates in photocatalytic hydroxylation. The reaction was monitored via GC-MS and TLC. Part of reactions were performed under the assistance of Shaowei Qin.

\subsubsection{Applications to Other Benzylic $\mathrm{C}\left(\mathrm{sp}^{3}\right)-\mathrm{H}$ Oxygenations}

Besides that, the same reaction conditions could be employed to other benzylic $C\left(\mathrm{sp}^{3}\right)$ $\mathrm{H}$ oxygenations such as oxygenation of $\mathrm{C}\left(\mathrm{sp}^{3}\right)-\mathrm{H}$ bonds to form ketones, esters and amides (with base) in medium to excellent isolated yields (Figure 3.2.8). However, 
similar concepts and results were published already by the group of Cai before this project was finished. ${ }^{[48 b]}$

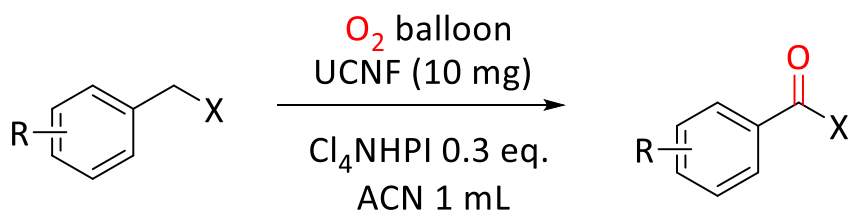

Blue LED, r.t., $16 \mathrm{~h}$<smiles>O=C1OCCc2ccccc21</smiles>

F57, $83 \%$<smiles>O=C1CCCc2ccccc21</smiles>

F61, 71\%<smiles>CC(C)CCOC(=O)c1ccccc1</smiles>

F58, $54 \%$<smiles>O=C1Cc2cccc3cccc1c23</smiles>

F62, 88\%<smiles>O=C1OCc2ccccc21</smiles>

$\mathbf{F 5 9 , 7 3 \%}$<smiles>CC(=O)c1cc(C(C)(C)C)cc2c1C(=O)CC2(C)C</smiles>

F63, $41 \%$<smiles>O=C1CCc2ccccc21</smiles>

$\mathbf{F} 60,67 \%$<smiles>CCN1CCc2ccccc2C1=O</smiles>

F64, $68 \%^{\mathrm{b}}$

Figure 3.2.8 Substrate scope of visible-light-mediated photocatalytic oxygenations using UCNF. [a] Reaction conditions: $\mathrm{O}_{2}$ (balloon), $12 \mathrm{~W}$ blue LED, $0.3 \mathrm{mmol}$ substrate, $10 \mathrm{mg}$ catalyst, 0.3 eq. $\mathrm{Cl}_{4} \mathrm{NHPI}, 1 \mathrm{~mL} \mathrm{ACN}, 16 \mathrm{~h}$. [b] additional 1.5 eq DBN as base. 


\subsection{A Metal-Free Heterogeneous Photocatalyst for the Selective Oxidative Cleavage} of $\mathrm{C}=\mathrm{C}$ Bonds via Harvesting Direct Solar Energy ${ }^{2}$

\subsubsection{Optimization of the $\mathrm{C}=\mathrm{C}$ Bonds Cleavage}

We aimed to develop a metal-free heterogeneous photocatalytic system which is currently an interesting avenue in organic synthesis. Hence, we focused on utilizing polymeric carbon nitride (PCN) which is easy to synthesize and even commercially available. At the outset of our reaction, 4-fluoro- $\alpha$-methylstyrene (175a) was chosen as a model substrate to optimize the reaction conditions (Table 3.3.1). In the beginning, different amounts of PCN were loaded using acetonitrile as the solvent under the irradiation of $12 \mathrm{~W}$ blue LED for $24 \mathrm{~h}$. Gratifyingly, $43 \%$ of 4 fluoroacetophenone (175b) was achieved using $8 \mathrm{mg}$ of PCN (entries 1-4). However, increasing amounts of catalyst did not improve the yields of reactions. Afterwards, different additives (10 mol\%) such as $N$-hydroxysuccinimide (NHS), ascorbic acid (AA) and 3-mercaptopropionic acid (MPA) were investigated (entries 5-7). Among them, NHS increased the formation of the desired product to $61 \%$. Finally, the addition of 20 mol\% of NHS, increased the yield to $80 \%$ of $1 \mathrm{~b}$. However, further loading of NHS did not perform any further improvement (entries 8-9). At last, investigation of different solvents also did not increase yields of reactions since acetonitrile has higher oxygen solubility than others (entries 10-12).

${ }^{2}$ The following chapter is based on the published paper. ${ }^{[106]}$ 
Table 3.3.1 Optimization of the $\mathrm{C}=\mathrm{C}$ bonds cleavage using a heterogeneous photocatalyst. ${ }^{\text {a-e }}$

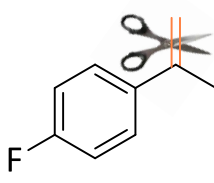

$175 a$

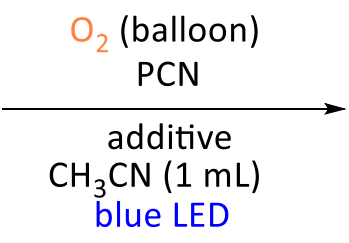

blue LED

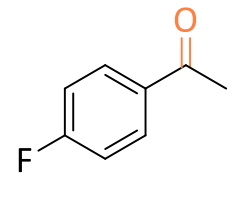

$175 b$

\begin{tabular}{lllll}
\hline Entry & Cat. Amount & additive & Solvent & Yield [\%] \\
\hline 1 & $3 \mathrm{mg}$ & $\mathrm{No}$ & $\mathrm{CH}_{3} \mathrm{CN}$ & 18 \\
2 & $5 \mathrm{mg}$ & $\mathrm{No}$ & $\mathrm{CH}_{3} \mathrm{CN}$ & 31 \\
3 & $8 \mathrm{mg}$ & $\mathrm{No}$ & $\mathrm{CH}_{3} \mathrm{CN}$ & 40 \\
4 & $12 \mathrm{mg}$ & $\mathrm{No}$ & $\mathrm{CH}_{3} \mathrm{CN}$ & 43 \\
5 & $8 \mathrm{mg}$ & $\mathrm{AA}$ & $\mathrm{CH}_{3} \mathrm{CN}$ & 0 \\
6 & $8 \mathrm{mg}$ & $\mathrm{MPA}$ & $\mathrm{CH}_{3} \mathrm{CN}$ & 6 \\
7 & $8 \mathrm{mg}$ & $\mathrm{NHS}^{\mathrm{c}}$ & $\mathrm{CH}_{3} \mathrm{CN}$ & 61 \\
8 & $8 \mathrm{mg}$ & $\mathrm{NHS}^{\mathrm{d}}$ & $\mathrm{CH}_{3} \mathrm{CN}$ & 80 \\
9 & $8 \mathrm{mg}$ & $\mathrm{NHS}^{\mathrm{e}}$ & $\mathrm{CH}_{3} \mathrm{CN}$ & 79 \\
10 & $8 \mathrm{mg}$ & $\mathrm{NHS}^{\mathrm{d}}$ & $\mathrm{DMSO}$ & 46 \\
11 & $8 \mathrm{mg}$ & $\mathrm{NHS}^{\mathrm{d}}$ & $\mathrm{DMF}$ & 30 \\
12 & $8 \mathrm{mg}$ & $\mathrm{NHS}^{\mathrm{d}}$ & $\mathrm{DMA}$ & 48
\end{tabular}

[a] General reaction conditions: $\mathrm{O}_{2}$ balloon, $12 \mathrm{~W}$ blue LED, $0.25 \mathrm{mmol}$ of $175 \mathrm{a}, \mathrm{PCN}$, $1 \mathrm{~mL}$ of acetonitrile, additive, room temperature, $24 \mathrm{~h}$. [b] Yields were determined by GC calibration using dodecane as internal standard. [c] $N$-hydroxysuccinimide (10 mol\%). [d] $N$-hydroxysuccinimide (20 mol\%). [e] $N$-hydroxysuccinimide (30 mol\%).

\subsubsection{Scope of the $C=C$ Bonds Cleavage}

With optimized reaction conditions in hand, the scope of substrates was extended to other $\alpha$-substituted styrenes (Scheme 3.3.1). Firstly, other substituted $\alpha$ methylstyrenes such as 4-chloro- $\alpha$-methylstyrene, 2-methoxy- $\alpha$-methylstyrene and 4-methyl- $\alpha$-methylstyrene afforded the corresponding carbonyl compounds in 
excellent yields (70b, 79b, 81b, 176b, 177b). Furthermore, 1,1'-diphenylethylenes and other substituted 1,1'-diphenylethylenes also worked well to form the benzophenone derivatives (78b, 178b, 179b). Other $\alpha$-substituted styrene derivatives such as $\alpha$ - $n$-propylstyrene, $\alpha-n$-butylstyrene and $\alpha$-iso-butylstyrene etc. generated the corresponding ketones in moderate to high yields (entries 180b184b). Further investigations towards the hetero-aromatic ring substituted styrenes were also carried out. To our delight, these substrates could form the 4benzoylpyridine, 2-benzoylpyridine and 2-(Prop-1-en-2-yl)thiophene with good isolated yields (entries $\mathbf{1 8 5 b - 1 8 7 b ) . ~}$ 


$$
\begin{aligned}
& \mathrm{O}_{2} \text { (balloon) } \\
& \text { PCN (8 mg) }
\end{aligned}
$$

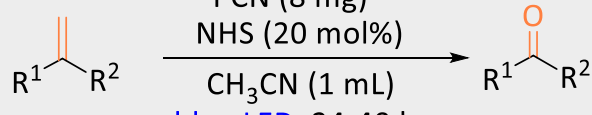

$$
\begin{aligned}
& \text { blue LED, 24-40 h }
\end{aligned}
$$

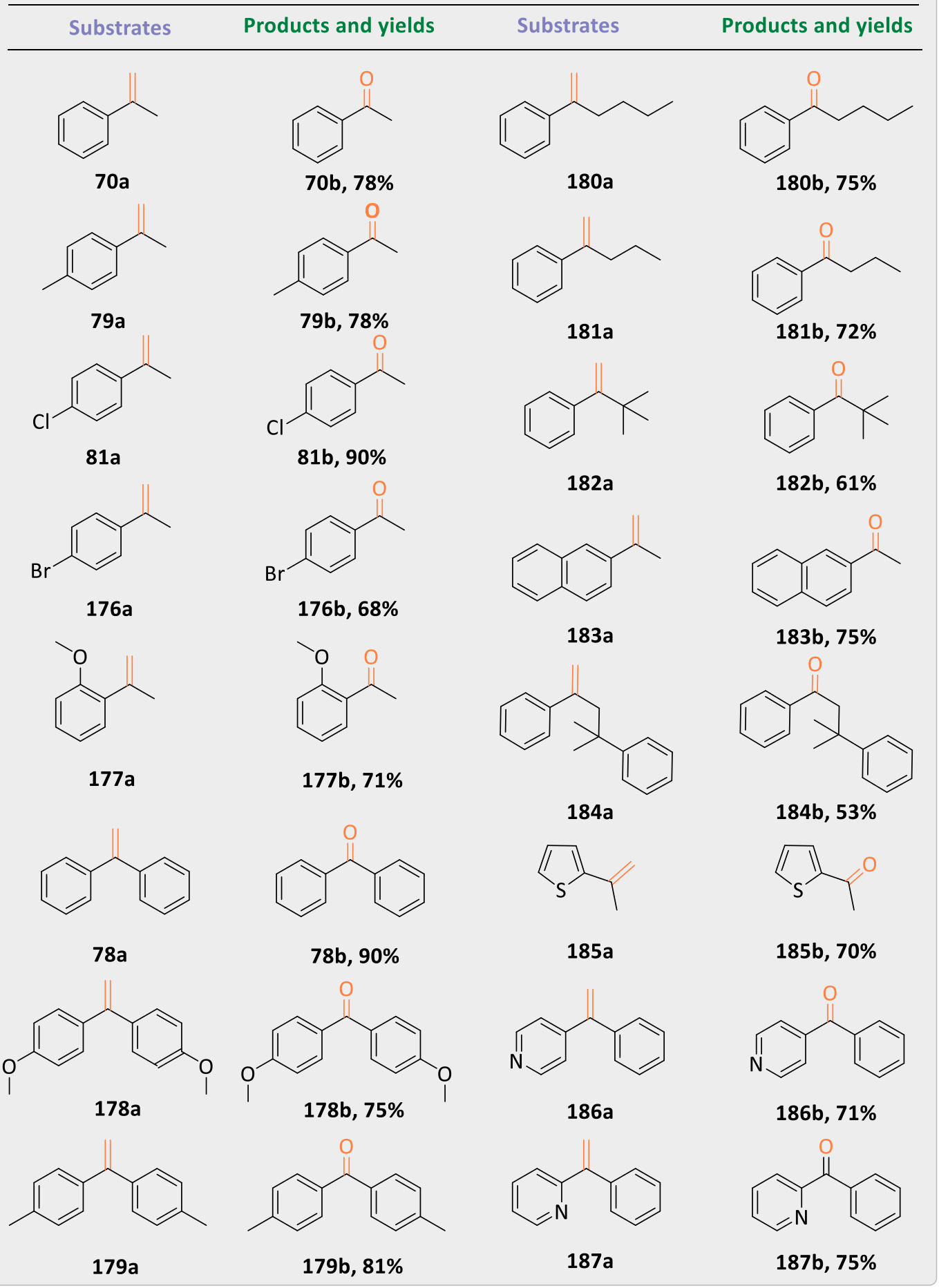

Scheme 3.3.1 Scope of the $\mathrm{C}=\mathrm{C}$ bonds cleavage of $\alpha$-substituted styrenes. [a] $\mathrm{O}_{2}$ balloon, $12 \mathrm{~W}$ blue LED, $0.25 \mathrm{mmol}$ of starting material, $8 \mathrm{mg}$ of PCN, $1 \mathrm{~mL}$ of acetonitrile, 20 mol\% of NHS, room temperature. [b] isolated yields. 
Next, we aimed to apply this photocatalytic system to mono-substituted olefins since they could generate high-valued aldehydes (Scheme 3.3.2). For example, veratraldehyde (189b) is a key intermediate for the synthesis of various pharmaceuticals such as verazide, hoqquizil, amiquinsin, etc. ${ }^{[107]}$ To our delight, this heterogeneous photocatalyst exhibited medium reactivity for the mono-substituted styrenes such as 3,4-dimethylstyrene, 3,4-dimethoxystyrene, 2,4,5trimethoxystyrene and 4-vinylbiphenyl to generate the corresponding aldehydes (entries 188b-191b).

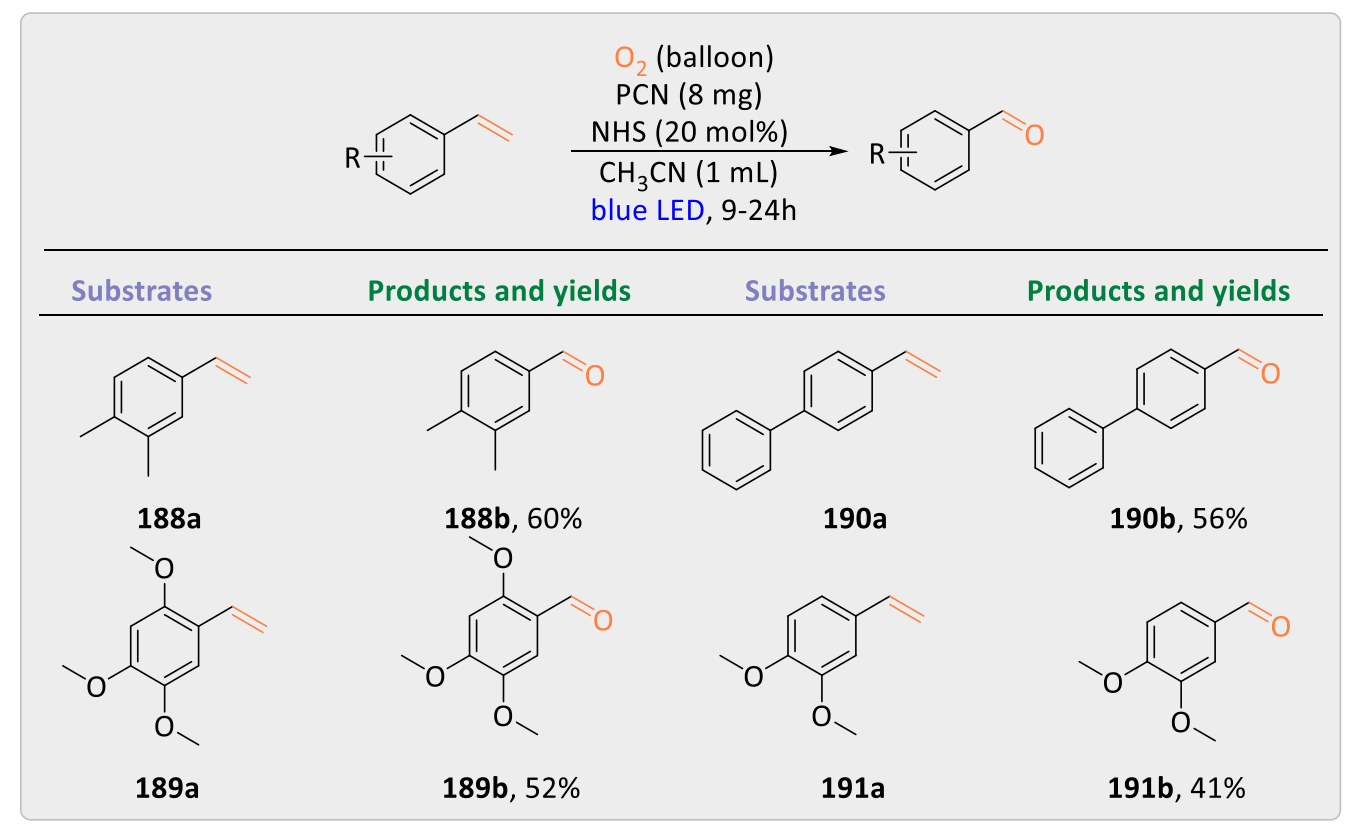

Scheme 3.3.2 Scope of the $C=C$ bonds cleavage of terminal styrenes. ${ }^{a-b}[a] \mathrm{O}_{2}$ balloon, $12 \mathrm{~W}$ blue LED, $0.25 \mathrm{mmol}$ of starting material, $8 \mathrm{mg}$ of PCN, $1 \mathrm{~mL}$ of acetonitrile, 20 mol\% of NHS, room temperature. [b] isolated yields.

Inspired by the excellent reactivity, we aimed to expand the substrates scope only using the heterogeneous photocatalyst (Scheme 3.3.3). Besides the model substrate (271a) (see Table 3.3.1, entry 3), anethole, trans- $\beta$-methylstyrene, etc. also showed moderate reactivity even under air (entries 192b-196b). Interestingly, $\alpha$ bromostyrene derivatives underwent the migration of the bromide to the $\beta$-carbon and afforded the phenacyl bromide derivatives (197b-199b). Notably, some of the complex molecules such as triacetylresveratrol (Scheme 3.3.4; 200a), ${ }^{[108]}$ transchlorprothixene (201a), ${ }^{[109]}$ are highly challenging molecules to cleave under the 
normal circumstances. But selective cleavage of these complex molecules generated the corresponding oxygenated products in high yields with this robust applied method.

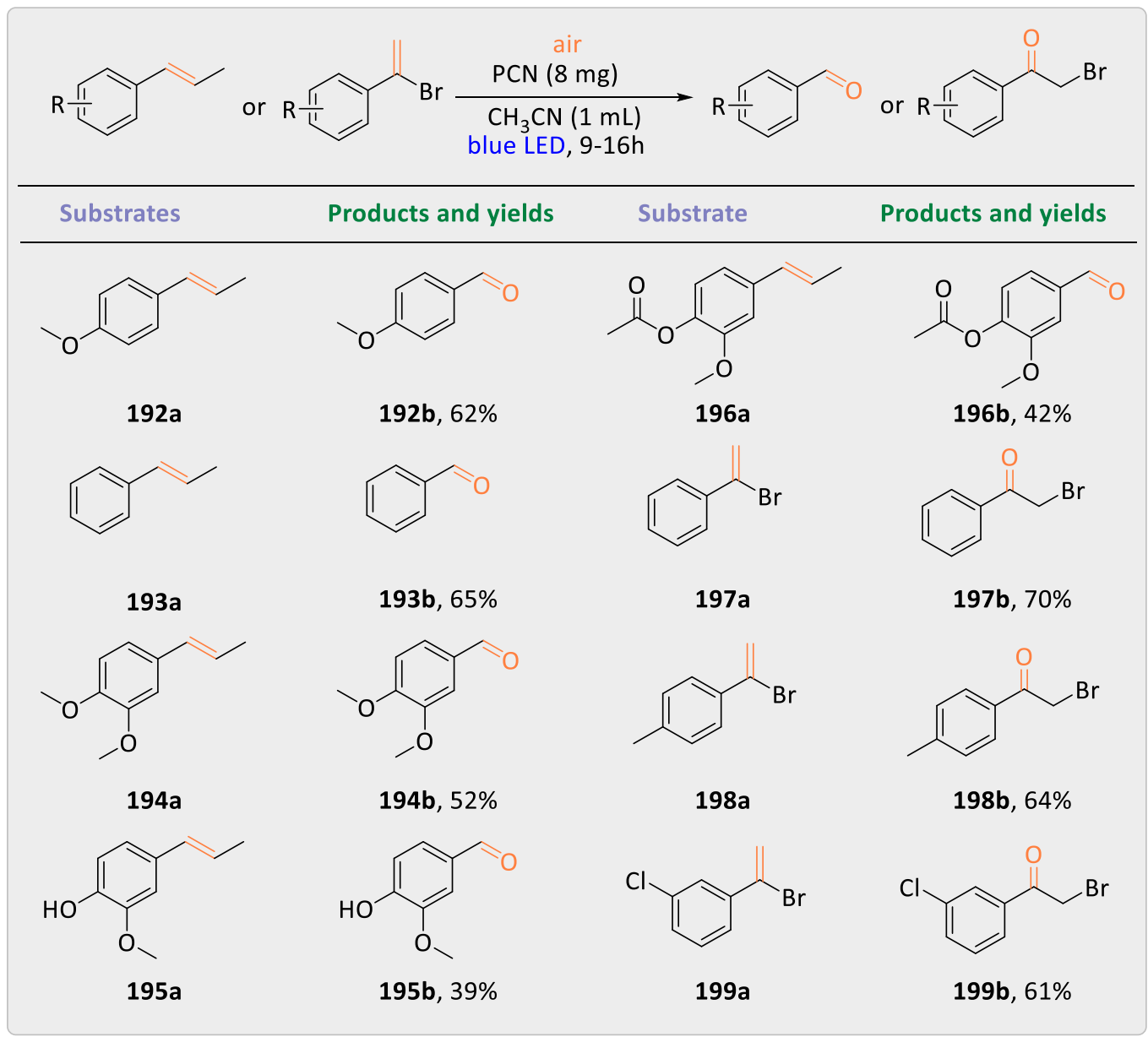

Scheme 3.3.3 Scope of the $C=C$ bond cleavage of $\beta$-substituted styrenes and $\alpha$-bromo styrenes. 


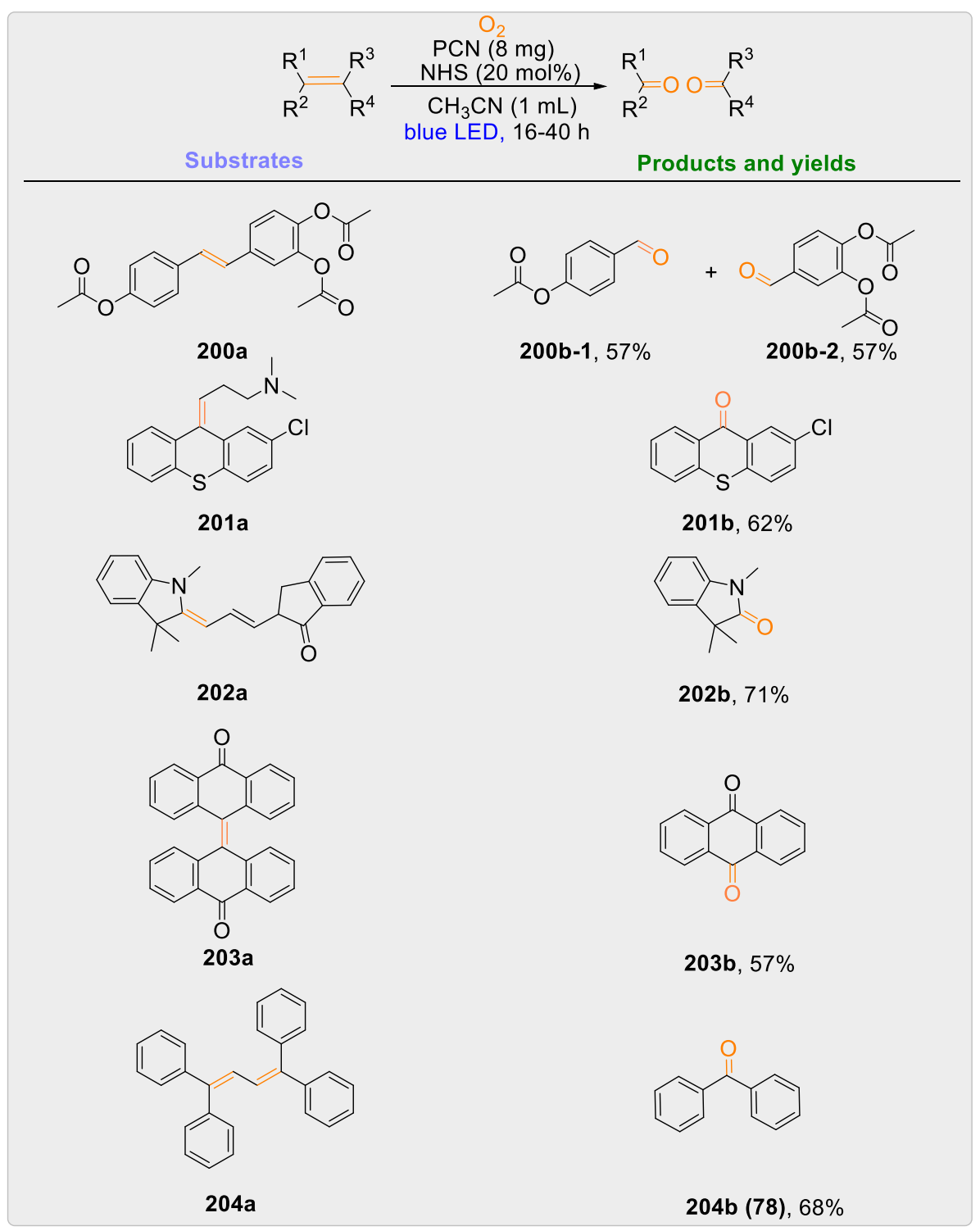

Scheme 3.3.4 Scope of the $\mathrm{C}=\mathrm{C}$ bond cleavage of complex molecules.

Natural sunlight is an inexpensive and widely available energy source as we mentioned in the introduction. Therefore, we aimed to harvest solar energy directly for the selective cleavage of $C=C$ bonds. Not only the small molecule (178a) was cleaved into the corresponding ketone, but the complex molecule such as 200 a, was also cleaved selectively under the irradiation of sunlight. These reactions were easily scaled up to g-scale. Additionally, fenofibrate, a pharmaceutical drug used for the treatment of abnormal lipid levels, was synthesized in g-scale as well from the corresponding olefin (Scheme 3.3.5; entry 205b). ${ }^{[110]}$ 


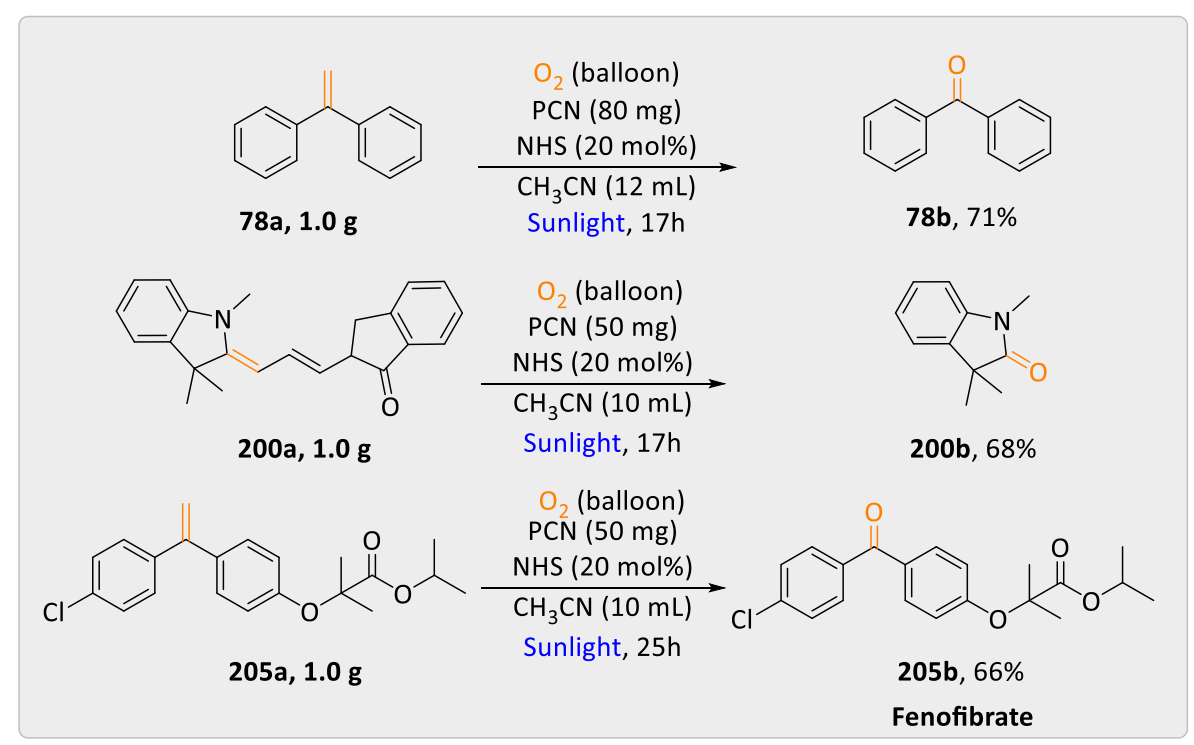

Scheme 3.3.5 Direct solar energy mediated $\mathrm{C}=\mathrm{C}$ bond cleavage in g-scale. Reaction setup under sunlight irradiation on the rooftop of the institute and temperature data on a sunny day for the reactions under sunlight.

\subsubsection{Recycling Experiments and Spectroscopic Analyses}

To ensure the stability and reusability of the photocatalyst, the recycled catalysts were employed multiple times under the optimized reaction conditions. To our delight, the activities of recycled catalysts keep well in 5 times. In fact, catalyst was recycled 5 times and can be reused further (Figure 3.3.1). 


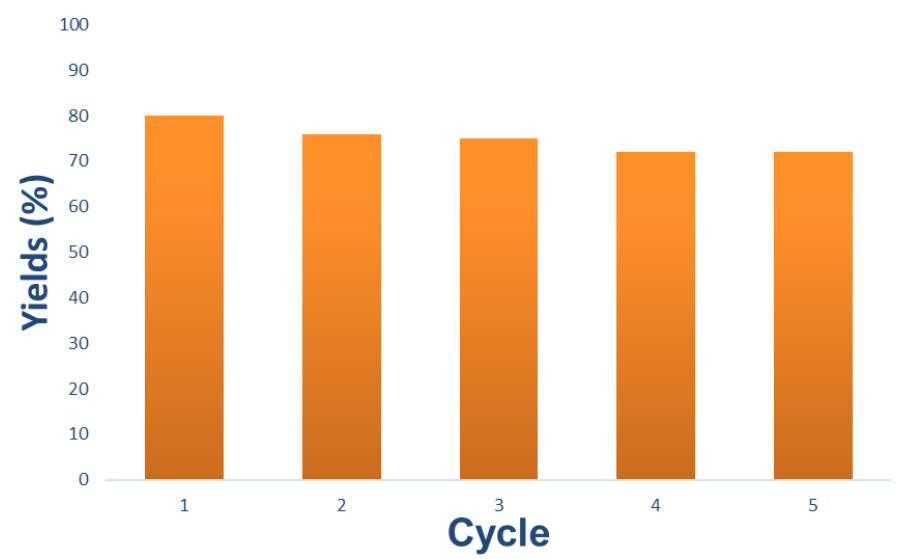

Figure 3.3.1 Reusability of PCN for the $\mathrm{C}=\mathrm{C}$ bond cleavage of 4-fluoro- $\alpha$ methylstyrene.

To further investigate the stability of the catalyst, elemental analysis (Table 3.3.2), Xray powder diffraction (XRD), fourier-transform infrared spectroscopy (FTIR), UV/Vis, scanning electron microscopy (SEM), and high resolution transmission electron microscopy (HRTEM) were measured and compared the difference between the freshly prepared catalyst and the recycled catalyst. Analysis of the catalyst before and after a reaction using XRD showed the same characteristic peaks at $27.3^{\circ}$ and $13.2^{\circ}$ which also matches a literature report (Figure 3.3.2). ${ }^{[111]}$

Table 3.3.2 The constituents of fresh and reused catalyst.

\begin{tabular}{cccc}
\hline Name & $\mathrm{C} \mathrm{( \% )}$ & $\mathrm{H}(\%)$ & $\mathrm{N}(\%)$ \\
\hline New catalyst & 35.18 & 2.00 & 62.00 \\
Reused catalyst & 35.26 & 2.01 & 61.36 \\
\hline
\end{tabular}




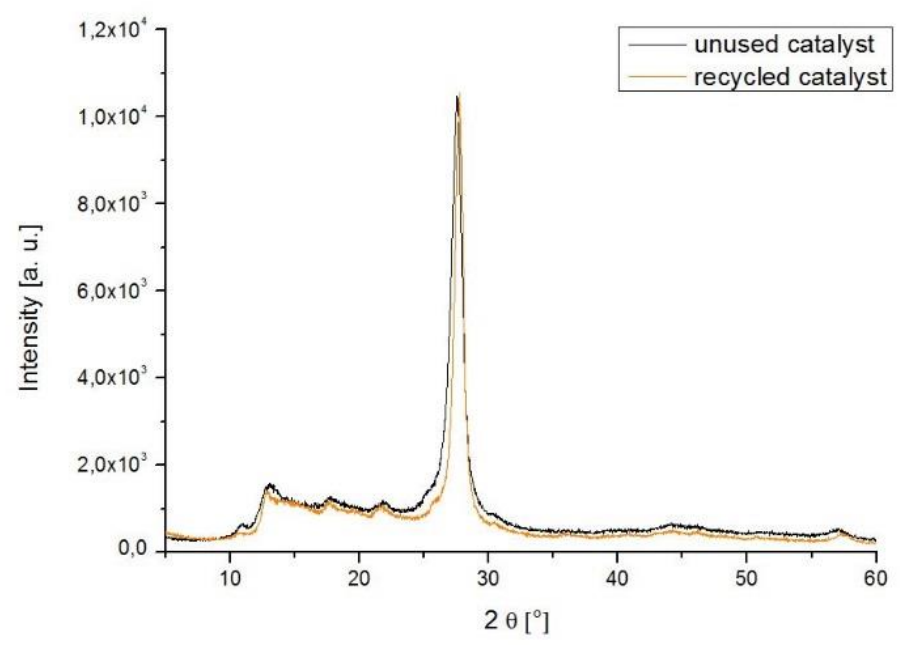

Figure 3.3.2 Analysis of new and recovered PCN by powder XRD.
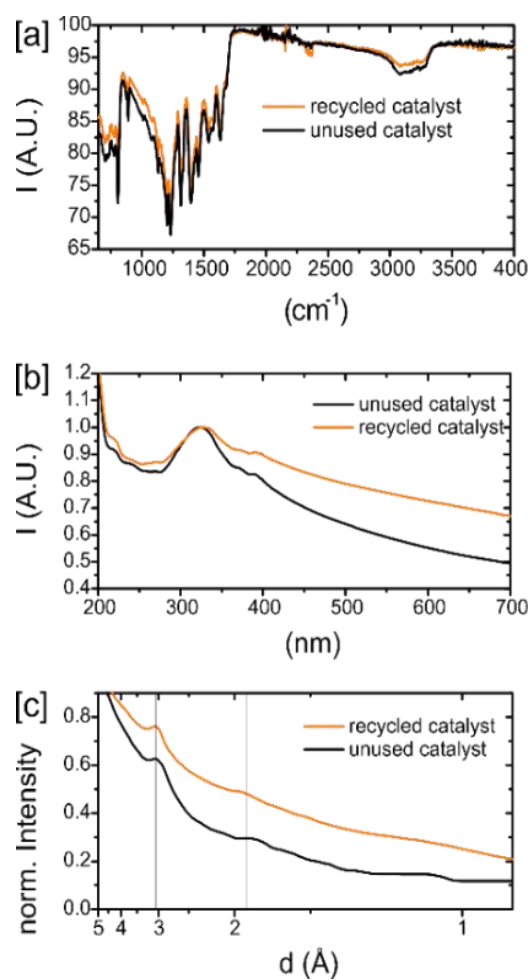
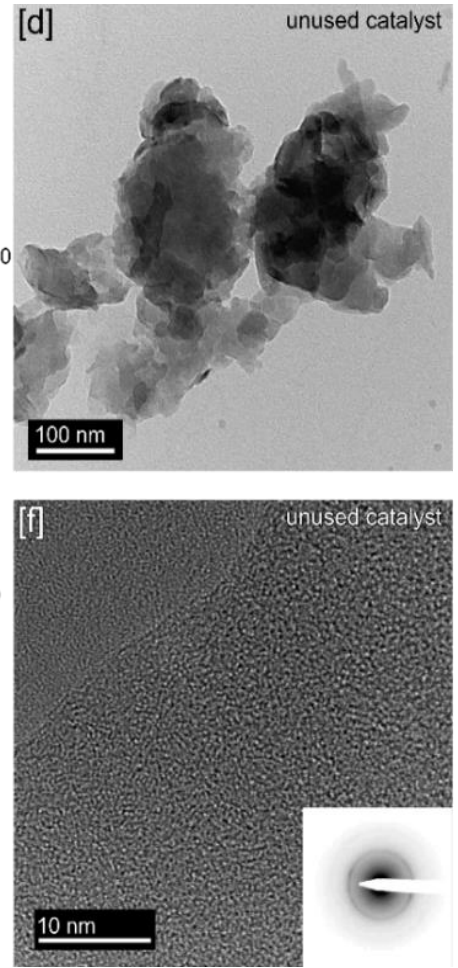
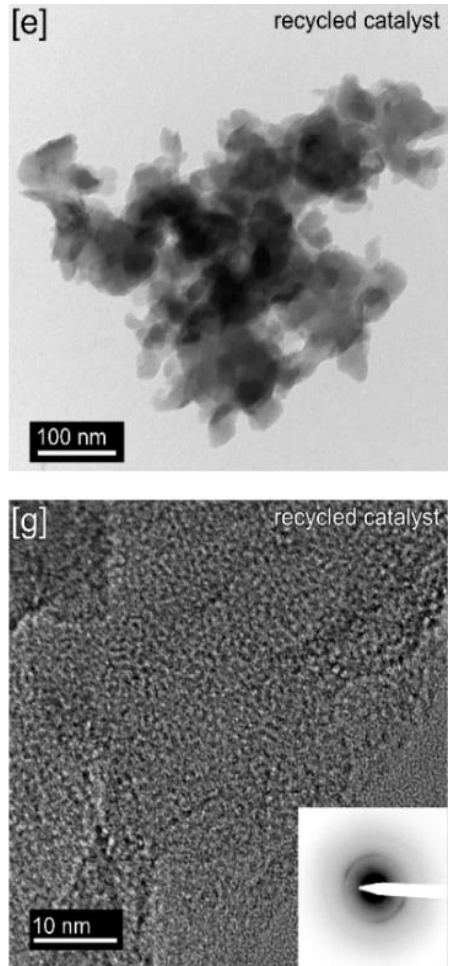

Figure 3.3.3 Analysis of new and recovered PCN by FTIR [a]: UV/Vis [b]: intensity profile of the selected area electron diffraction pattern (SAED) [c]: brightfield TEM [de]: as well as HRTEM with SAED as an inset [f-g].

From the UV-Vis and FTIR, no difference in the absorbance was observed. TEM and SEM revealed the morphology of the PCN particles before and after the recycling. They formed rounded plates with an average diameter of $(30 \pm 10) \mathrm{nm}$ and a thickness 
of the order of a few angstroms. Due to the agglomeration, bigger particles were composed by stacks of the plates. No significant changes in the morphology were observed with TEM (Figure 3.3.3 [d-e]) and SEM (Figure 3.3.4) after recovering the catalyst. To get more insight into the structure, selected area electron diffraction measurements were carried out. The diffuse rings did not exhibit any sign of crystallinity. A more detailed analysis of the intensity profile did not provide any evidence of a change during catalysis. In addition, the HRTEM images demonstrated clearly the amorphous nature of the catalyst.
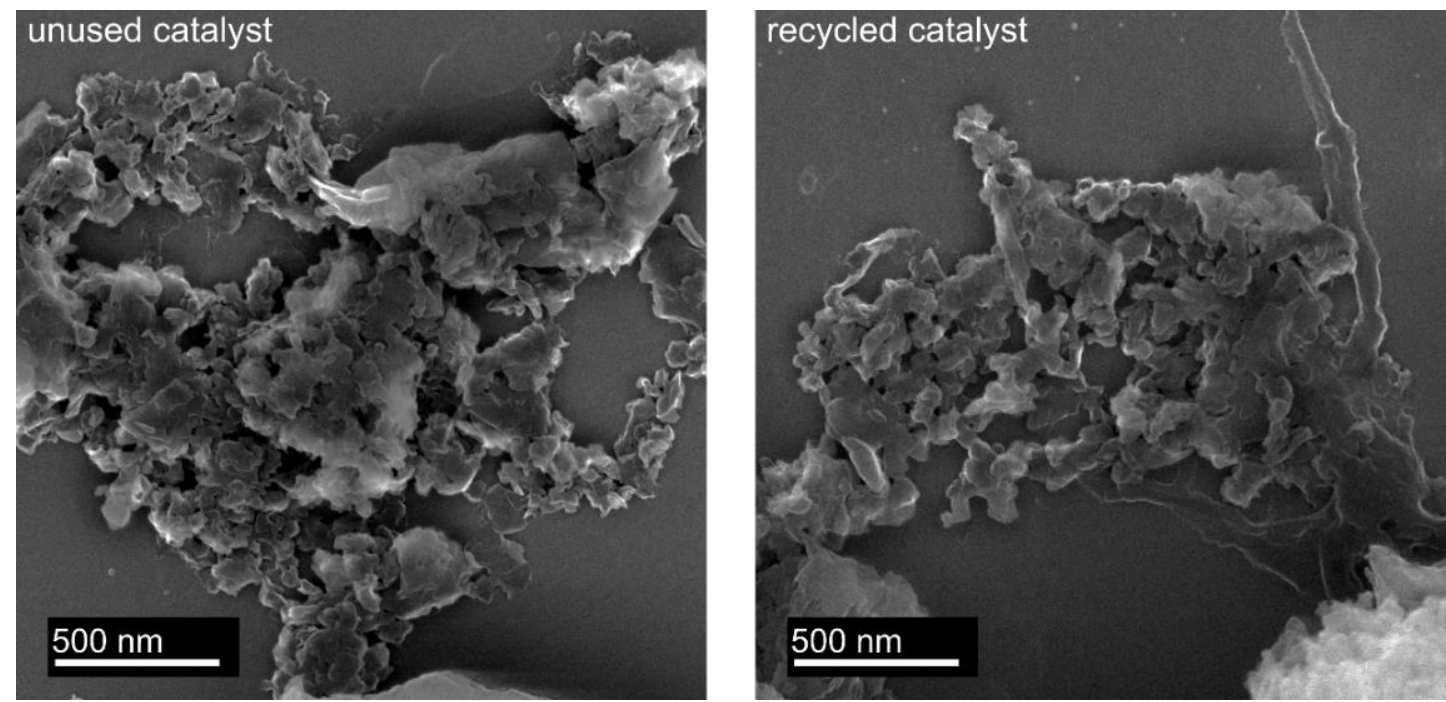

Figure 3.3.4 SEM of synthesized of PCN. (left) unused catalyst; (right) recycled catalyst.

\subsubsection{Mechanistic Studies}

Afterwards, we sought to gather mechanistic information about the roles of the light, oxygen, catalyst and additive in our reactions. According to the control experiments, no product was observed in the absence of light, photocatalyst or oxygen. Only a trace amount of the product (5\%) was observed in the presence of just the additive which clearly demonstrated the necessity of the photocatalyst and oxygen in the reaction (Table 3.3.3). Further, on/off light experiments were also carried out to determine that the yield of the product was completely suppressed in the absence of light (Figure 3.3.5). 
Table 3.3.3 Control experiments for the $\mathrm{C}=\mathrm{C}$ bond cleavage of 4-fluoro$\alpha-$ methylstyrene.

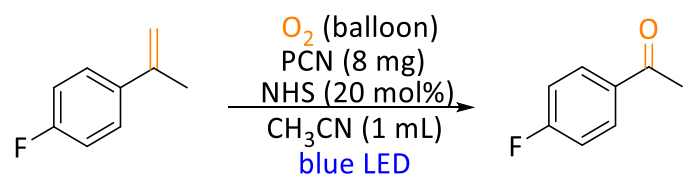

$\begin{array}{ccccc}\mathrm{O}_{2} & \mathrm{NHS} & \mathrm{g}-\mathrm{C}_{3} \mathrm{~N}_{4} & \text { Light } & \text { Yield (\%) } \\ - & + & + & + & 0 \\ + & - & + & + & 43 \\ + & + & - & + & 5 \\ + & + & + & - & 0 \\ + & + & + & + & 80\end{array}$

Furthermore, the effect of different quenchers was investigated to figure out the reactive oxygen species and possible intermediates in our reactions (Table 3.3.4). When BHT or TEMPO were added, the yields were decreased to $0 \%$ which proved that the reaction was undergoing a radical pathway. Further addition of benzoquinone in the reaction decreased the yield dramatically, pointing the presence of superoxide radical anion. Furthermore, addition of $\mathrm{CuCl}_{2}$ and catalase to the reaction mixture showed lower yields which proved that there could be involvement of single electron processes and the presence of peroxide species in this reaction. In contrast, singlet oxygen did not play a main role as proved by adding the sodium azide.

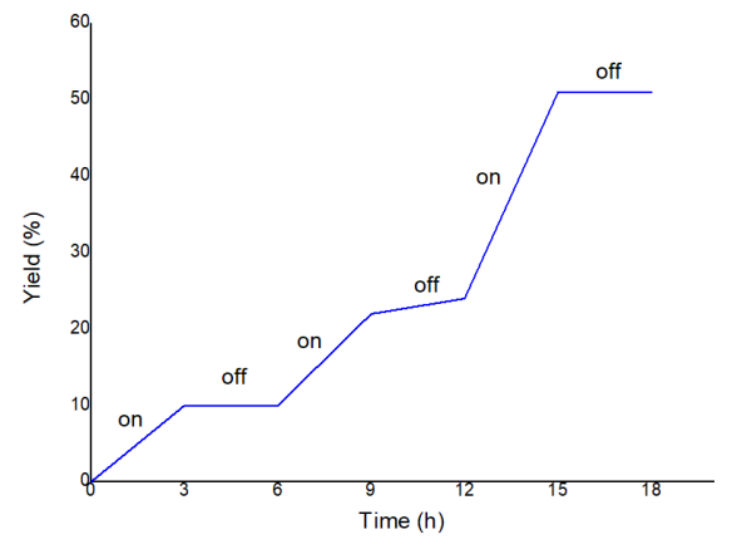

Figure 3.3.5 On/off light experiments of 4-(1-propenyl)-1,2-dimethoxybenzene. Yields were determined by NMR using iodoform as an internal standard. 
Table 3.3.4 Quenching experiments for the $\mathrm{C}=\mathrm{C}$ bond cleavage. ${ }^{\mathrm{a}-\mathrm{b}}$

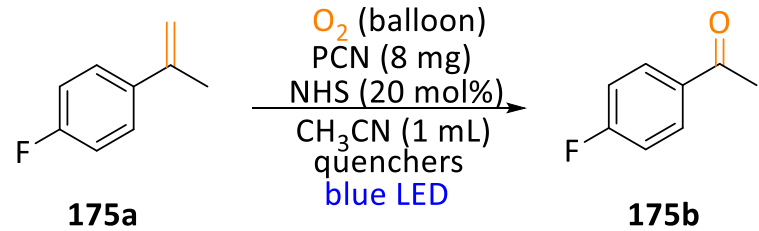

\begin{tabular}{cccc}
\hline Quencher & Equivalent & Yield [\%] & Scavenger for \\
\hline BHT & 0.5 & 0 & radical \\
TEMPO & 0.5 & 0 & radical \\
tert-butanol & 1.0 & 42 & hydroxide radical \\
$\mathrm{CuCl}_{2}$ & 1.0 & 46 & single electron \\
Catalase & $100 \mathrm{mg}$ & 9 & peroxide radical \\
Benzoquinone & 1.0 & 2 & superoxide radical \\
Sodium azide & 2.0 & 43 & singlet oxygen \\
\hline
\end{tabular}

[a] $\mathrm{O}_{2}$ balloon, $12 \mathrm{~W}$ blue LED, $0.25 \mathrm{mmol}$ of starting material, $8 \mathrm{mg}$ of PCN, $1 \mathrm{~mL}$ of acetonitrile, 20 mol\% of $\mathrm{N}$-hydroxysuccinimide, room temperature, different quenchers. [b] Yields were determined by GC analysis using $n$-dodecane as an internal standard.

To get further information about the reaction mechanism, Stern-Volmer quenching experiments were carried out (Figure 3.3.6). To find the suitable excitation and emission, several wavelengths were tested and the excitation maximum at $300 \mathrm{~nm}$ with corresponding emission maximum at $600 \mathrm{~nm}$ gave the best signal. A blank sample was recorded without the substrate and the received intensity was set as $I_{0}$. The effect of varied amounts of 4-fluoro- $\alpha$-methylstyrene was investigated. Depending on the concentration of 4-fluoro- $\alpha$-methylstyrene, emission decreases significantly. The concentration of the additive and oxygen atmosphere had no measurable effect on the emission of the photocatalyst, which shows that the excited state of the photocatalyst was quenched by the olefin and not by the oxygen or additive. 


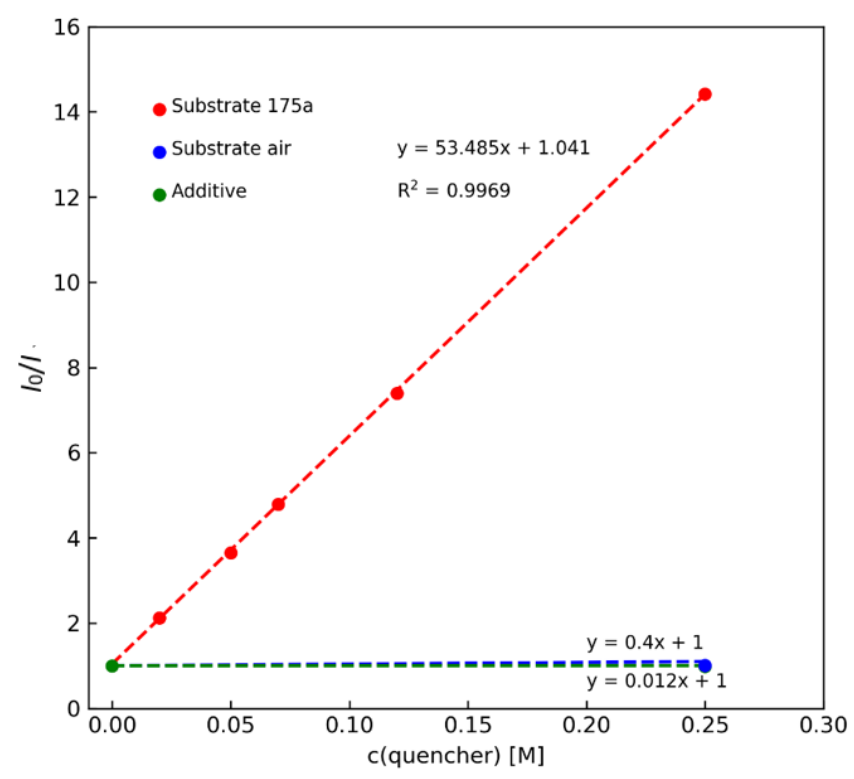

Figure 3.3.6 Stern-Volmer plot for the different concentrations of olefins, $\mathrm{O}_{2}$ and additive.

To show the actual source of oxygen in the product, the reaction was investigated under ${ }^{18} \mathrm{O}$-labelled oxygen atmosphere. To our delight, only ${ }^{18} \mathrm{O}$-labelled product was observed via GC-MS and further isolated from the reaction mixture. Interestingly, we also detected the ${ }^{18} \mathrm{O}$-labelled intermediate in this reaction by HR-MS, and in this case both of the oxygen atoms in dioxetane came from the oxygen atmosphere (Figure 3.3.7).<smiles>C=CC</smiles><smiles>CC1(c2ccc(F)cc2)CO[Ge]1</smiles>

detected

$(\mathrm{M}+\mathrm{H})^{+}=173.0744$

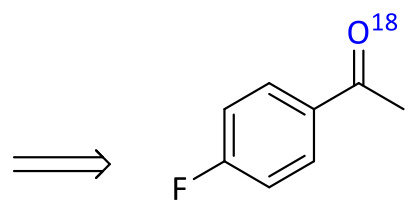

isolated

$(\mathrm{M}+\mathrm{H})^{+}=141.0596$

Figure 3.3.7 Oxidation of 4-fluoro- $\alpha$-methylstyrene in presence of ${ }^{18} \mathrm{O}$-labeled oxygen.

\subsubsection{Proposed Mechanism}

Combing all the mechanistic information, the mechanism of this reaction was proposed (Figure 3.3.8). At first, visible-light irradiation created charge separation in PCN, produced oxidative valence band and reductive conduction-band. ${ }^{[112]}$ Then the conduction-band electron reduced the oxygen to produce the superoxide 
radical anion. The valence band hole oxidized $175 \mathrm{a}$ to the corresponding radical cation 175 c. This radical cation $175 c$ combined with the superoxide radical anion and $\mathbf{1 7 5 f}$ was formed via cyclization. However, there is a possible other pathway (pathway B) which involved the additive $N$-hydroxysuccinimide (NHS). Firstly, 175h was generated through NHS via hydrogen atom transfer (HAT). Meanwhile, the hydrogenperoxide ( $\mathrm{HOO}$ ) anion was generated. Afterwards, $175 \mathrm{~h}$ attacked $\mathbf{1 7 5 a}$ to form the cation $\mathbf{1 7 5} \mathrm{d}$ which further reacted with the hydrogenperoxide anion to afford $\mathbf{1 7 5 e}$ which later formed the intermediate $\mathbf{1 7 5 f}$ by releasing the NHS. ${ }^{[113]}$ At the end, the cleavage of dioxetane generated the final product (175b).

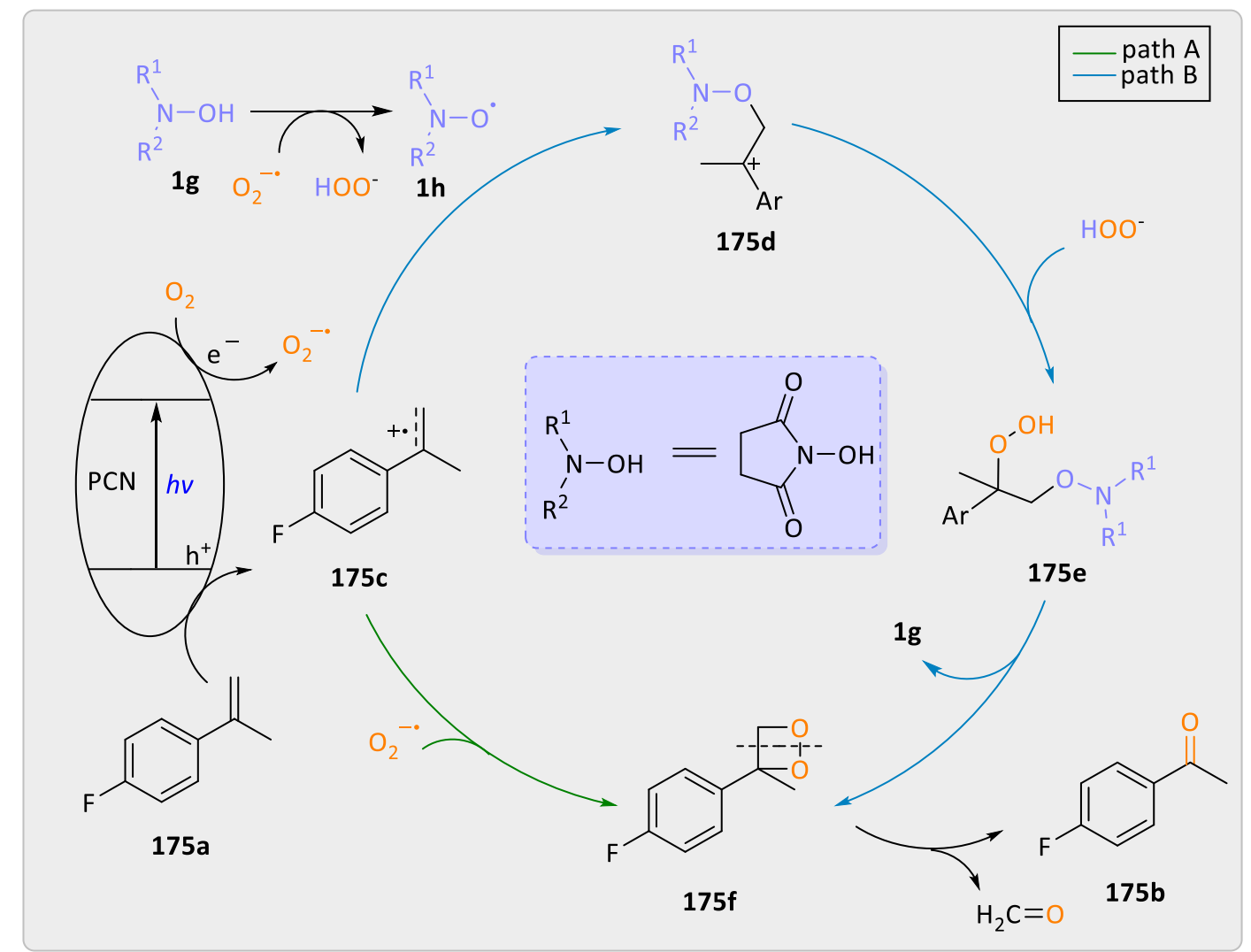

Figure 3.3.8 Plausible mechanism of this reaction. 


\subsubsection{Unsuccessful Substrates}<smiles>C=Cc1c(C)cc(C)cc1C</smiles>

F65, 71h, t. a.<smiles>O=C1CCCCC1C1=CCCCC1</smiles>

F69, 24h, n. ob<smiles>CC(=O)CCC=C(C)C</smiles>

F73, 24h, n. ob.<smiles>C=Cc1ccc(F)cc1</smiles>

F77, 71h, t. a.<smiles>C=Cc1ccccn1</smiles>

F66, 71h, t. a.<smiles>CC1=CCC(C(C)C)CC1O</smiles>

F70, 24h, n. ob<smiles>CCCCC=CCO</smiles>

F74, 24h, n. ob.<smiles>C=Cc1ccc(O)cc1</smiles>

F78, 71h, t. a.<smiles>N#CC(C#N)=Cc1ccccc1</smiles>

F67, 71h, n. ob.<smiles>O=C(O)/C=C/c1ccccc1</smiles>

F71, 24h, n. ob.<smiles>CC(=O)/C=C/C1=CCCCC1(C)C</smiles>

F75, 24h, n. ob.<smiles>O=C1/C(=C2\Nc3ccccc3C2=O)Nc2ccccc21</smiles>

F79, 24h, n. ob.<smiles>C=CCCCCCBr</smiles>

F68, 71h, n. ob.<smiles>C=C(C)[C@@H]1CCC2=CC(=O)C[C@H](C)[C@]2(C)C1</smiles>

F72, 24h, n. ob.<smiles>c1ccc(C(=C2c3ccccc3-c3ccccc32)c2ccccc2)cc1</smiles>

F76, 24h, n. ob.<smiles>ClC(Cl)=C(c1ccccc1)c1ccccc1</smiles>

F80, 24h, n. ob.<smiles>C1=CC(=C(c2ccccc2)c2ccccc2)C=C1</smiles>

F81, $24 h$, n. ob.<smiles>CC=C(/C(=C/C)c1ccc(OC(C)=O)cc1)c1ccc(OC(C)=O)cc1</smiles>

F82, 24h, n. ob.<smiles>C(=C\c1ccccc1)\c1ccccc1</smiles>

F83, 24h, n. ob.

Figure 3.3.9 Substrates with trace of amounts of products or no observation of product formation. t.a.: Trace of amounts of products detected by GC-MS. Part of the reactions were performed with the assistance of Nareh Hatami and Niklas Simon Lange.

During the investigation of the substrate scope, there were some substrates which exhibited limited reactivity. The PCN has the band gap of $2.7 \mathrm{eV}$ (enough to harvest solar energy), with the valence band (VB) residing at $+1.6 \mathrm{~V}$ and conduction band (CB) at $-1.10 \mathrm{~V} \cdot{ }^{[49]}$ So if the substrates have oxidation potential lower than $+1.6 \mathrm{~V}$, it will work properly under applied conditions. However, if the substrates have much higher 
oxidation potentials such as aliphatic olefins (Figure 3.3.9, F68-70, F73-F74), it will not work under our reaction conditions. To the best of our knowledge, such type of cleavage of aliphatic olefins has been rarely reported. But if the starting material has conjugated double bonds, the decreasing oxidation potential will promote the reactions to proceed towards the products formation. For example, calciferol (Figure 3.3.10, F101), worked under our photocatalytic system to form the corresponding ketone, unfortunately it could not be isolated.<smiles>C=C(C)c1cccc(C(=C)C)c1</smiles>

F84, 48h, 31\%<smiles>C=Cc1ccc(Br)cc1</smiles>

F88, 69h, 35\%<smiles>C=Cc1ccc(C(C)(C)C)cc1</smiles>

F92, 42h, 36\%<smiles>C=Cc1ccc([N+](=O)[O-])cc1</smiles>

F96, $69 \mathrm{~h}, 21 \%$<smiles>C(=C/c1ccccc1)\c1ccccc1</smiles>

F85, $48 \mathrm{~h}, 35 \%$<smiles>C=Cc1ccc(Cl)cc1</smiles>

F89, $71 h, 35 \%$

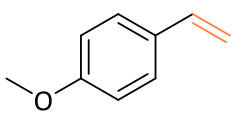

F93, $42 \mathrm{~h}, 5 \%$<smiles>C=Cc1cccc(Br)c1</smiles>

F97, 71h, 30\%<smiles>OC/C=C/c1ccccc1</smiles>

F86, $26 h, 14 \%$<smiles>C=Cc1ccc(C#N)cc1</smiles>

F90, 71h, $12 \%$<smiles>C=Cc1ccc(C)cc1</smiles>

F94, $42 \mathrm{~h}, 19 \%$<smiles>C=Cc1c(F)c(F)c(F)c(F)c1F</smiles>

F98, $71 \mathrm{~h}, 17 \%$<smiles>O=C/C=C/c1ccccc1</smiles>

F87, 26h, $26 \%$<smiles>O=C/C=C/c1ccoc1</smiles>

F91, $71 \mathrm{~h}, 5 \%$<smiles>C=Cc1ccccc1C</smiles>

F95, 42h, 7\%<smiles>C=C(C)c1cccnc1</smiles>

F99, 71h, 10\%<smiles>Cc1ccc(N(c2ccc(C)cc2)c2ccc(C=C(c3ccccc3)c3ccccc3)cc2)cc1</smiles>

F100, 48h, 35\%

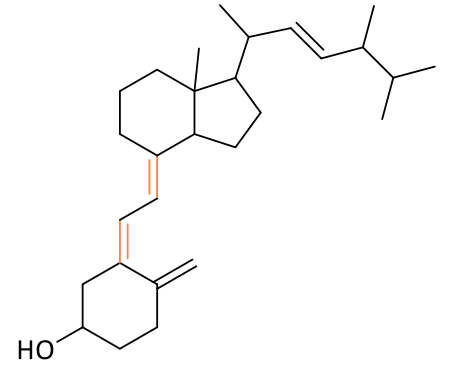

F101, 16h, failed isolation

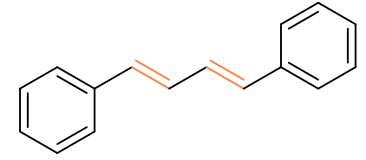

F102, 48h, 32\%

Figure 3.3.10 Substrates with limited reactivity. Yield was determined via NMR with iodoform as an internal standard. Part of the reactions were carried out with the assistance of Nareh Hatami and Niklas Simon Lange. 
Another main limitation of this photocatalytic system was the applications towards terminal styrenes (Figure 3.3.8). Even though all of them did work under our conditions, the yields were lower because of the formation of the corresponding acids. Therefore, it was hard to achieve satisfactory yields of the corresponding aldehydes even though the reactions had complete conversion. It should be noted that some large and more complex molecules were also applied in our reaction conditions. However, the reactions rate was limited (Figure 3.3.8, F100, F102). 


\subsection{A Simple Ketone as An Efficient Metal-free Catalyst for Visible-Light-Mediated} Cycloadditions $^{3}$

\subsubsection{Optimization of Diels-Alder Reactions.}

At the outset of the reaction, we started the optimization using trans-anethole and 2,3-dimethyl-1,3-butadiene as model substrates under air, respectively (Table 3.4.1). At first, eight different organic dyes such as 9-fluorenone, methylene blue, and rose bengal were screened using nitromethane $\left(\mathrm{MeNO}_{2}\right)$ as solvent and $\mathrm{MgSO}_{4}$ as desiccant under the irradiation of a $12 \mathrm{~W}$ blue LED for $4 \mathrm{~h}$ (Table 3.4.1, entries 1-8). Among them, 9-fluorenone $(0.56 € / g)$ as a photocatalyst afforded $81 \%$ yield of the corresponding Diels-Alder product. Other catalysts showed no or lower reactivity. Afterwards, the increase of amounts of catalysts showed a trend towards a slight increase in the yields (Table 3.4.1, entries 9-10). Since the turnover efficiency was the highest at 3 mol\% and the yields increased only slightly with a significant increase in catalyst loading, the optimized catalyst loading was $3 \mathrm{~mol} \%$ for this reaction. The yields decreased with rising amount of the desiccant magnesium sulfate. The increased turbidity of the suspension was likely to interfere with the photoexcitation of the catalyst (Table 3.4.1, entries 11-14). Later, other aprotic polar solvents such as THF, DMF, DMSO and ACN were applied but did not improve the yields (Table 3.4.1, entries 15-17). Consequently, nitromethane was the best solvent in this reaction as aprotic polar solvents with higher dipole moment at comparable polarities can solvate the formed radical cation intermediate which enhances the product formation.

Table 3.4.1 Optimization for Diels-Alder Reactions. ${ }^{[a-f]}$

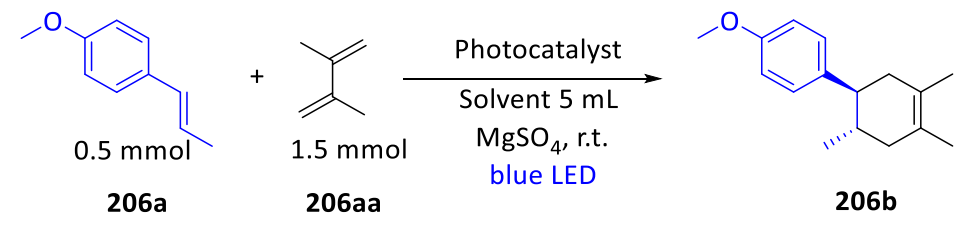

${ }^{3}$ The following chapter is based on the published paper. ${ }^{[114]}$ 


\begin{tabular}{|c|c|c|c|c|}
\hline Entry & Catalysts & Catalyst amount & Solvent & Yield $[\%]^{[b]}$ \\
\hline 1 & 9-fluorenone & $3 \mathrm{~mol} \%$ & $\mathrm{MeNO}_{2}$ & 81 \\
\hline 2 & methylene blue & $3 \mathrm{~mol} \%$ & $\mathrm{MeNO}_{2}$ & 0 \\
\hline 3 & rose bengal & 3 mol\% & $\mathrm{MeNO}_{2}$ & 0 \\
\hline 4 & eosin $Y$ & 3 mol\% & $\mathrm{MeNO}_{2}$ & 0 \\
\hline 5 & michler's ketone & $3 \mathrm{~mol} \%$ & $\mathrm{MeNO}_{2}$ & 0 \\
\hline 6 & xanthone & 3 mol\% & $\mathrm{MeNO}_{2}$ & 6 \\
\hline 7 & thioxanthen-9-one & 3 mol\% & $\mathrm{MeNO}_{2}$ & 8 \\
\hline 8 & acriflavine & 3 mol\% & $\mathrm{MeNO}_{2}$ & 0 \\
\hline 9 & 9-fluorenone & $5 \mathrm{~mol} \%$ & $\mathrm{MeNO}_{2}$ & 83 \\
\hline 10 & 9-fluorenone & $10 \mathrm{~mol} \%$ & $\mathrm{MeNO}_{2}$ & 88 \\
\hline 11 & 9-fluorenone & 3 mol\% & $\mathrm{MeNO}_{2}$ & $81^{[c]}$ \\
\hline 12 & 9-fluorenone & $3 \mathrm{~mol} \%$ & $\mathrm{MeNO}_{2}$ & $79^{[d]}$ \\
\hline 13 & 9-fluorenone & 3 mol\% & $\mathrm{MeNO}_{2}$ & $73^{[\mathrm{e}]}$ \\
\hline 14 & 9-fluorenone & 3 mol\% & $\mathrm{MeNO}_{2}$ & $74^{[\mathrm{f}]}$ \\
\hline 15 & 9-fluorenone & $3 \mathrm{~mol} \%$ & THF & 0 \\
\hline 16 & 9-fluorenone & $3 \mathrm{~mol} \%$ & DMF & 0 \\
\hline 17 & 9-fluorenone & $3 \mathrm{~mol} \%$ & DMSO & 0 \\
\hline
\end{tabular}

[a] General reaction conditions: air, $12 \mathrm{~W}$ blue LED, $0.5 \mathrm{mmol} 206 \mathrm{a}$ (1 eq.), $1.5 \mathrm{mmol}$ 1 b (3 eq.), photocatalyst ( $3 \mathrm{~mol} \%$ ), $5 \mathrm{~mL}$ solvent, $30 \mathrm{mg} \mathrm{MgSO}_{4}$, room temperature, 4 h. [b] Yields were determined by NMR yield using iodoform as an internal standard. [c] no $\mathrm{MgSO}_{4}$. [d] $10 \mathrm{mg} \mathrm{MgSO}$. [e] $60 \mathrm{mg} \mathrm{MgSO}$. [f] $100 \mathrm{mg} \mathrm{MgSO}$.

\subsubsection{Scope of the Diels-Alder Reactions under the Irradiation of Visible-Light.}

With these optimized reaction conditions in hand, we aimed to extend the scope of this metal-free system to other electron-rich dienophiles and dienes (Scheme 3.2.2; 206b-216c). The formation of [4+2] cycloadducts can be employed smoothly using 2,3-dimethyl-1,3-butadiene as diene and other electron-rich dienophiles including 
myrcene, isoprene etc. (entries 206b-209b).

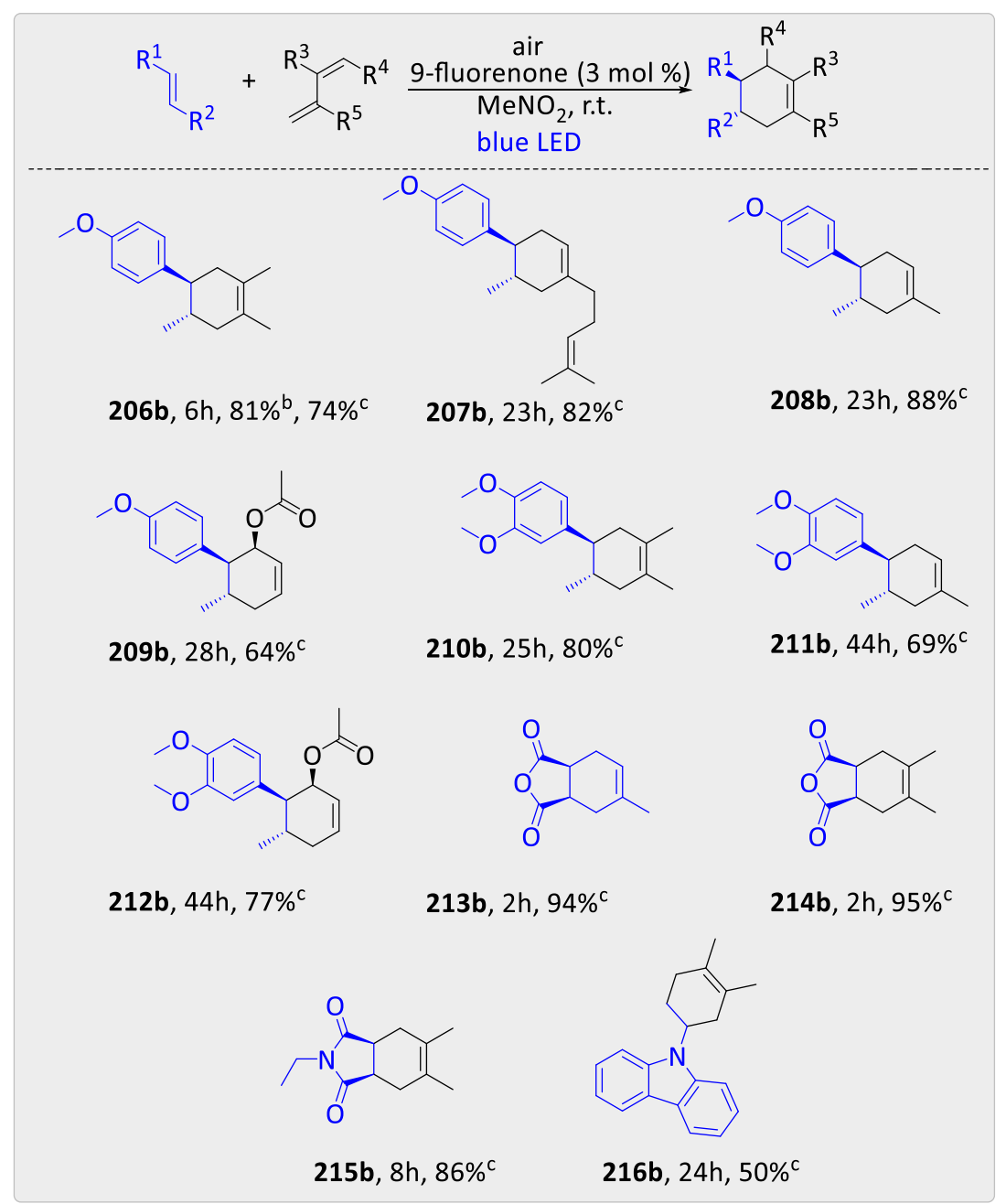

Scheme 3.4.2 Scope of the 9-fluorenone-catalysed Diels-Alder Reactions under the irradiation of visible-light. Reaction conditions: air, 12 W blue LED, dienophiles (0.5 mmol, 1 eq.), dienes (3 eq.), photocatalyst (3 mol\%), $5 \mathrm{~mL}$ solvent, room temperature. [b] Yield was determined by NMR yield using iodoform as an internal standard. [c] Isolated yields.

Another dienophile, 1,2-dimethoxy-4-propenylbnzene, reacted also effectively with a series of dienes (entries 210b-212b). Additionally, 2,5-furandione was also employed in our system with excellent yield of the corresponding cyclohexene products in $2 \mathrm{~h}$ using two different electron-rich dienes (entries 213b-214b). It should be noted that for 2,5-furandione, in previous studies, expensive metal-based catalysts were required to obtain the desired product. ${ }^{[115]}$ With $\mathrm{N}$-ethylmaleimide as 
the dienophile, [4+2] cycloaddition reaction with isoprene provided $\mathbf{2 1 5 b}$ with $86 \%$ yield. The 9-vinylcarbazole was also capable to react with trans-anethole resulting 216b using applied method in medium isolated yield.

\subsubsection{Optimization of aza-Diels-Alder Reactions}

Inspired by the good reactivity in [4+2] cycloadditions, we aimed to utilize this mild strategy into aza-Diels-Alder reactions and synthesize important $N$-heterocycles in a straightforward way. For this purpose, we synthesized various substituted 2,3dihydropyridin- $4(1 H)$-one derivatives as these have potential applications for the treatment of emergent infectious diseases. ${ }^{[116]}$ Importantly, these compounds have been utilized for the syntheses of piperidine-containing natural products and bioactive molecule synthesis. ${ }^{[117]}$ For example, bremazocine, a k-opioid receptor agonist related to pentazocine, could be afforded by 6 steps from a 2,3dihydropyridin-4(1H)-one derivative. ${ }^{[117 b]}$

It should be noted that previous syntheses of 2,3-dihydropyridin-4(1H)-one derivatives had required either expensive metal based catalysts or longer reaction times. ${ }^{[118]}$ To overcome these challenges, we were interested in developing a more efficient photocatalytic system for aza-Diels-Alder reactions using (E)-1-(4methoxyphenyl)- $N$-phenylmethanimine as a dienophile and Danishefsky's diene to synthesize 2-(4-methoxyphenyl)-1-phenyl-2,3-dihydropyridin-4(1H)-one (217b) as the product (Table 3.4.3). Gratifyingly, after screening a series of photocatalysts with the nitromethane $\left(\mathrm{MeNO}_{2}\right)$ as solvent under the irradiation of $12 \mathrm{~W}$ blue LED for $2 \mathrm{~h}$, 9-fluorenone performed better compared to other metal-free photocatalysts with excellent yields. The yields decreased with lower amounts of solvent (Table 3.4.3; entries 1-3). In order to save the diene, lower ratios between imines and dienes were explored. To our delight, a ratio of 1:2 also gave a good yield of 96\% (Table 3.4.2; entries 4-5). Afterwards, various solvents were screened but they did not increase the yields (Table 3.4.3; entries 6-9). 
Table 3.4.2 Optimization of aza-Diels-Alder reactions. ${ }^{[a-d]}$

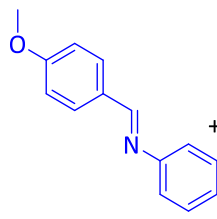

217a

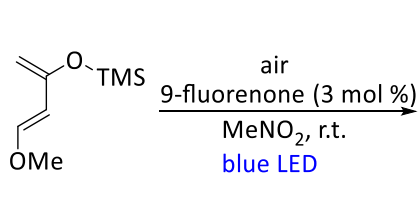

217aa

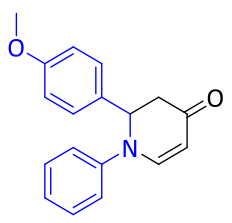

217b

\begin{tabular}{lllll}
\hline Entry & Catalyst & Solvent & Volume $(\mathrm{mL})$ & Yield [\%] \\
\hline 1 & 9-fluorenone & $\mathrm{MeNO}_{2}$ & 5 & 99 \\
2 & 9-fluorenone & $\mathrm{MeNO}_{2}$ & 1 & 23 \\
3 & 9-fluorenone & $\mathrm{MeNO}_{2}$ & 3 & 58 \\
4 & 9-fluorenone & $\mathrm{MeNO}_{2}$ & 5 & $55^{\text {[c] }}$ \\
5 & 9-fluorenone & $\mathrm{MeNO}_{2}$ & 5 & $96^{[\mathrm{d}]}$ \\
6 & 9-fluorenone & $\mathrm{DCM}^{\mathrm{b}}$ & 5 & 0 \\
7 & 9-fluorenone & $\mathrm{CHCl}_{3}$ & 5 & 0 \\
8 & 9-fluorenone & $\mathrm{DMSO}^{2}$ & 5 & 25 \\
9 & 9-fluorenone & $\mathrm{DMF}^{2}$ & 5 & 0
\end{tabular}

[a] General reaction conditions: air, $12 \mathrm{~W}$ blue LED, $0.5 \mathrm{mmol}$ 217a (1 eq.), $1.5 \mathrm{mmol}$ 217aa (3 eq.), photocatalyst ( $3 \mathrm{~mol} \%$ ), $5 \mathrm{~mL}$ solvent, room temperature, $2 \mathrm{~h}$. [b] Yields were determined by NMR yield using iodoform as an internal standard. [c] $0.5 \mathrm{mmol} 217 \mathrm{a}$ (1 eq.), $0.5 \mathrm{mmol} 217 \mathrm{aa}$ (1 eq.). [d] $0.5 \mathrm{mmol} 217 \mathrm{a}$ (1 eq.), $1.0 \mathrm{mmol}$ 217aa (2 eq.).

\subsubsection{Scope of aza-Diels-Alder Reactions under the Irradiation of Visible-light.}

With these optimized reaction conditions in hand, we applied the 9-fluorenone catalyst to other imines to obtain diverse 2,3 -dihydropyridin-4(1H)-one derivatives (Scheme 3.4.3). Various electron-rich imines showed high reactivity with the formation of desired products in $2 \mathrm{~h}$ with up to $96 \%$ yield. The catalyst showed high tolerance towards alkyl, aryl and heteroaryl substituents (entries 217-228b). Additionally, six new 2,3-dihydropyridin-4(1H)-one derivatives were synthesized 
whose biological activities should be further investigated considering the promising lead compounds against human infectious diseases. ${ }^{[118]}$ Expediently, with this 9fluorenone catalyst, we have been able to synthesize previously reported bioactive derivatives which were evaluated in vitro against a wide spectrum of viruses (entries 218b, 227b-228b).

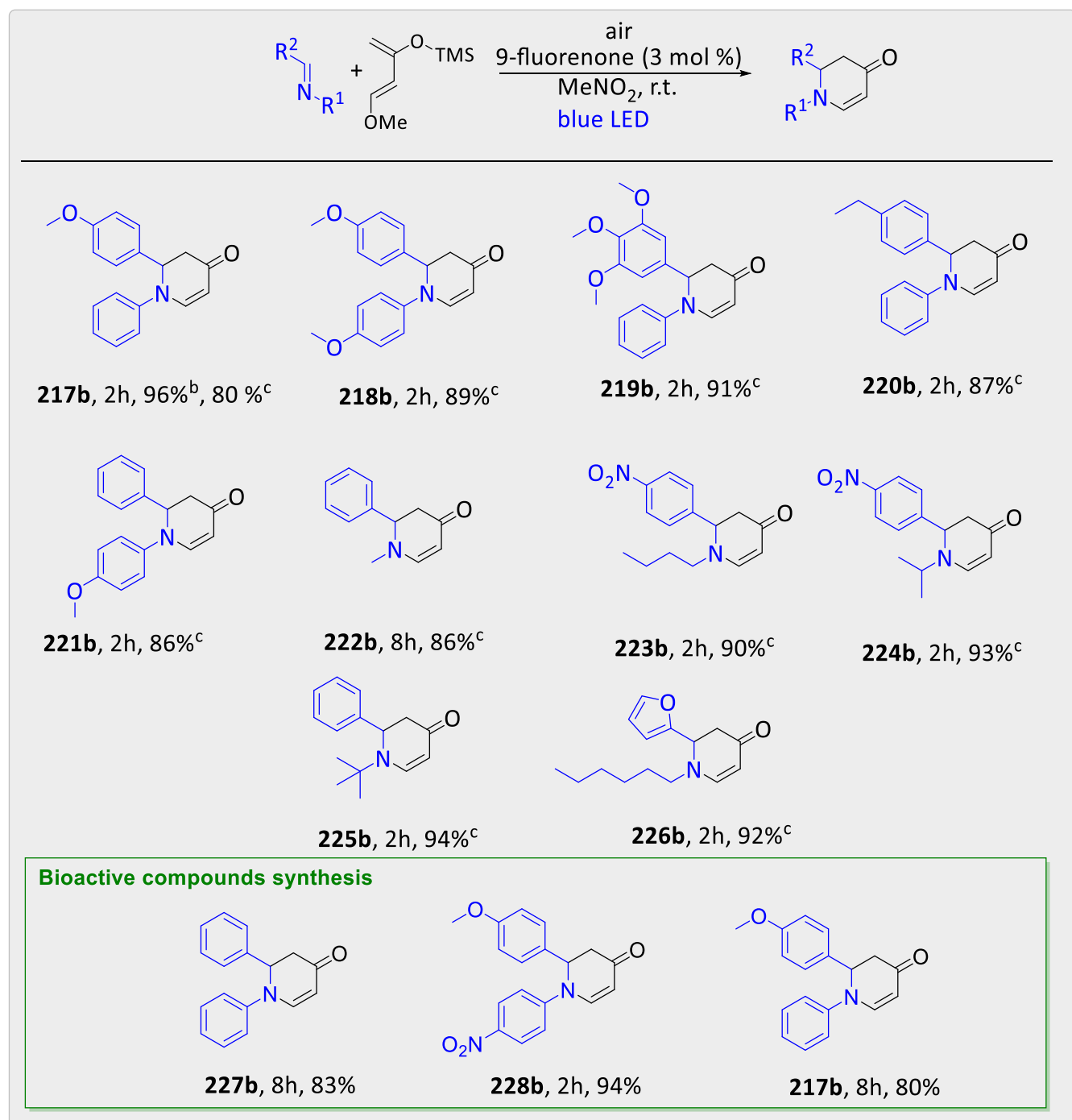

Scheme 3.4.3 Scope of the 9-fluorenone-catalysed aza-Diels-Alder reactions under visible light irradiation. [a] Reaction conditions: air, $12 \mathrm{~W}$ blue LED, imines (0.10 mmol, 1 eq.), diene ( 2 eq.), photocatalyst (3 mol\%), $5 \mathrm{~mL}$ solvent, room temperature. [b] Yield was determined by NMR spectroscopy using iodoform as an internal standard. [c] Isolated yields. 
The scope of the optimized protocol for blue LED-mediated (aza)-Diels-Alder reactions was not limited to the synthesis of 2,3-dihydropyridin-4(1H)-ones as shown in Scheme 3.4.4. In addition to the reaction of imines with Danishefsky's diene, the model imine was converted with 1-methoxy-1,3-butadiene to a 1,2,3,6tetrahydropyridine (entry 229b). In addition to this, 2,3-dimethyl-1,3-butadiene reacted with 1,4-benzoquinone to generate 3,4-dimethyl-1-oxaspiro[5.5] undeca3,7,10-trien-9-one (entry 230b).

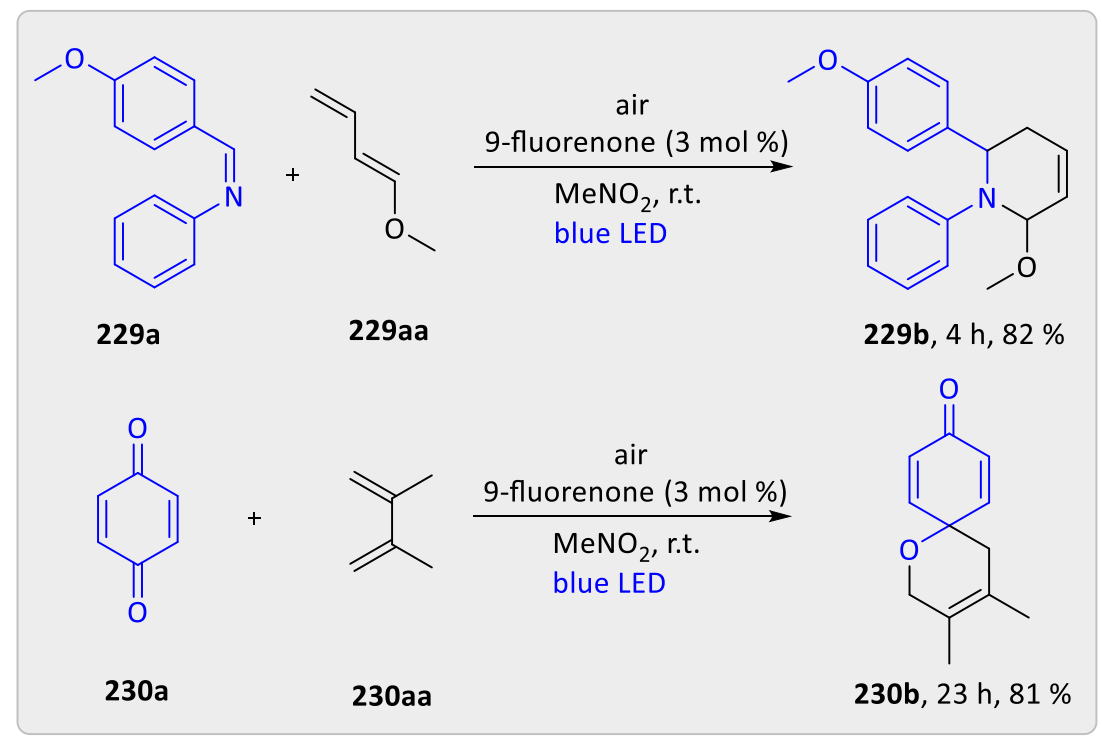

Scheme 3.4.4 9-fluorenone-catalysed hetero-Diels-Alder reactions under visible light irradiation.

\subsubsection{Scope of [2+2] cycloadditions}

The reactions also proceeded via [2+2] cycloadditions in the absence of any diene. In fact, cyclobutane derivatives have wide applications in natural product syntheses and many of them already have become promising candidates as anticancer, antiviral and antifungal drugs. Additionally, lignan and neolignan type compounds also contain a cyclobutane motif (Scheme 3.4.5). ${ }^{[119]}$ Based on the efficient [4+2] cycloaddition in our system and the indispensable role of the cyclobutane motif in the pharmaceutical industry, we conjectured that 9-fluorenone could promote [2+2] cycloaddition reactions to furnish cyclobutane derivatives in the absence of dienes (Scheme 3.4.5; entries 233b-234b). To our delight, the catalyst not only showcased the activity for homodimerization of the alkenes (233b) but also showed an excellent reactivity 
towards the heterodimerization of alkene (234b). Interestingly, this reaction was favorable to proceed via [2+2] rather than $[2+4]$ cycloadditions. We presumed this reaction was prone to undergo the energy transfer (triplet-state reactions) rather than single electron oxidations according to literature reports. ${ }^{[120]}$

(a) Examples of bioactive cyclobutane lignan natural products

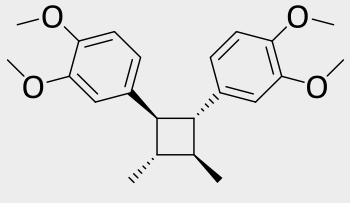

231, di-O-methylendiandrin A

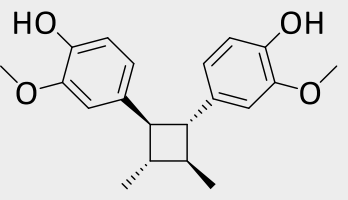

232, endiandrin A

(b) Our work on [2+2] cycloadditions

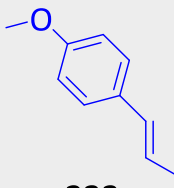

233a

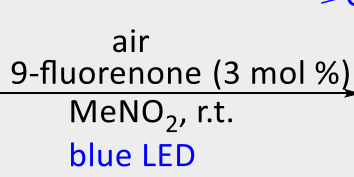

blue LED

234a

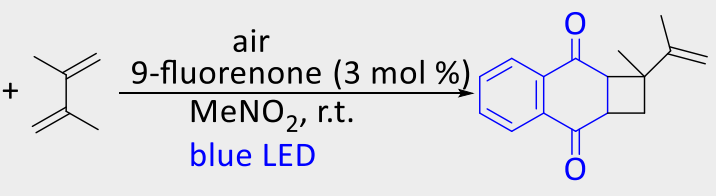

blue LED

234b, 23 h, 72\%

Scheme 3.4.5 (a) Examples of bioactive cyclobutane lignan natural products. (b) Our work on [2+2] cycloadditions.

\subsubsection{Mechanistic Studies and Proposed Mechanism}

Considering the broad scope of these mild reaction conditions, we sought to elucidate the role of the catalyst, air, and light source in this reaction. Control experiments clearly showed no product formation in the absence of light or photocatalyst (Table 3.4.3). It should be noted that $60 \%$ yield was achieved under nitrogen, which clearly showed that air did not play any important role in the reaction process. This finding indicated that a chain propagation mechanism exists in this reaction which is consistant matched with the discovery by Yoon et al. ${ }^{[27 f]}$ Furthermore, the effect of different quenchers was investigated to identify the reactive oxygen species from air and possible intermediates (Table 3.2.3). When 2,6-di-tert-butyl-4-methylphenol 
(BHT) or 2,2,6,6-tetramethyl-1-piperidinyloxyl (TEMPO) were added to the reaction mixture, the reaction was completely inhibited, which proved a radical pathway. Furthermore, addition of $\mathrm{CuCl}_{2}$ to the reaction mixture showed comparable yields, which showed the possible involvement of single electron processes in this photocatalytic system. The addition of sodium azide did not decreased yield obviously, which showed no involvement of singlet oxygen, or other activated oxygen species. But application of benzoquinone as quencher revealed the presence of the superoxide radical anion.

Table 3.4.3 Quenching experiments for 9-fluorenone catalysed cycloadditions.

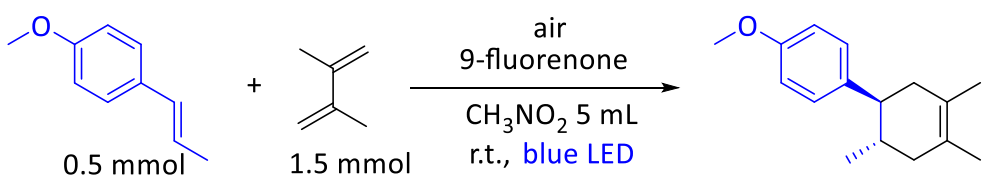

\begin{tabular}{ccccc}
\hline Condition & Quencher & Equivalent & Yield [\%] & Scavenger for \\
\hline Std. & --- & --- & 81 & --- \\
No Oxygen & --- & --- & 60 & --- \\
No catalyst & --- & --- & 2 & --- \\
No light & --- & --- & 0 & --- \\
Std. & BHT & 1.0 & 0 & radical \\
Std. & TEMPO & 1.0 & 0 & radical \\
Std. & Benzoquinone & 1.0 & 0 & superoxide radical \\
Std. & Catalase & $100 \mathrm{mg}$ & 0 & peroxide radical \\
Std. & $\mathrm{CuCl}_{2}$ & 1.0 & 73 & single electron \\
Std. & $\mathrm{NaN}_{3}$ & 1.0 & 63 & singlet oxygen \\
\hline
\end{tabular}

To acquire further information about the reaction mechanism, Stern-Volmer quenching experiments were carried out. To determine the reactive species in the beginning of the photocatalytic reaction, absorption-emission spectra for a SternVolmer plot were acquired. Firstly, a 3D spectrum for excitation and emission of 9fluorenone was recorded in order to detect the maxima of absorption and emission. 
The resulting spectrum is depicted in Figure 3.4.1. The excitation maximum was determined at $402 \mathrm{~nm}$ and the emission maximum at $519 \mathrm{~nm}$. These wavelengths were used for further measurements.
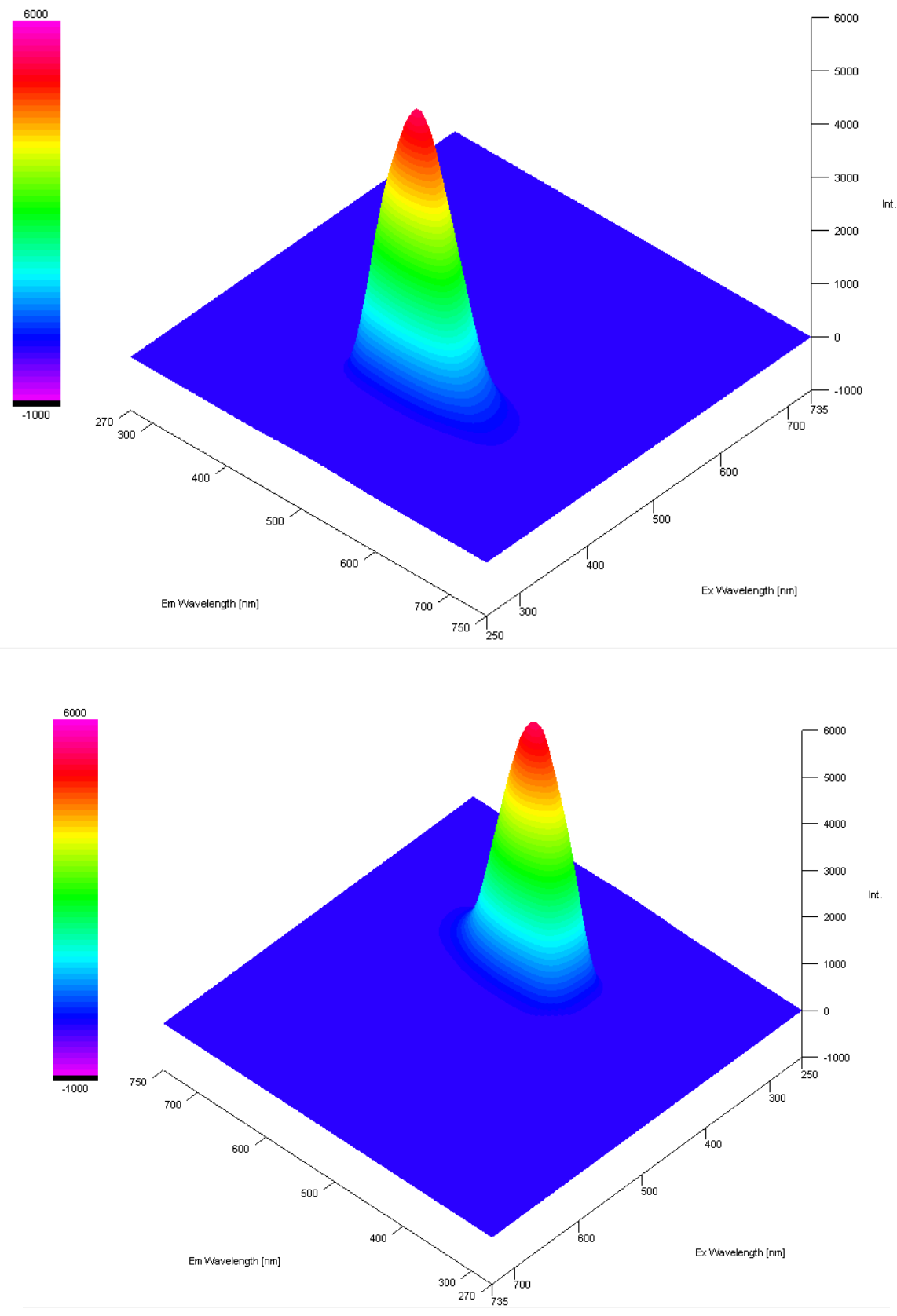

Figure 3.4.1 3D Absorption-emission spectra of 9-fluorenone in nitromethane. The spectra were measured by Waldemar Schilling.

A blank sample was recorded without substrate and the received intensity was set as I0. The effect of varied amounts of trans-anethole and 2,3-dimethyl-1,3-butadiene 
was investigated. Figure 3.4.2 shows a summary of the obtained results. Depending on the concentration of trans-anethole, the emission decreased significantly. The concentration of 2,3-dimethyl-1,3-butadiene had no measurable effect on the emission of 9-fluorenone.

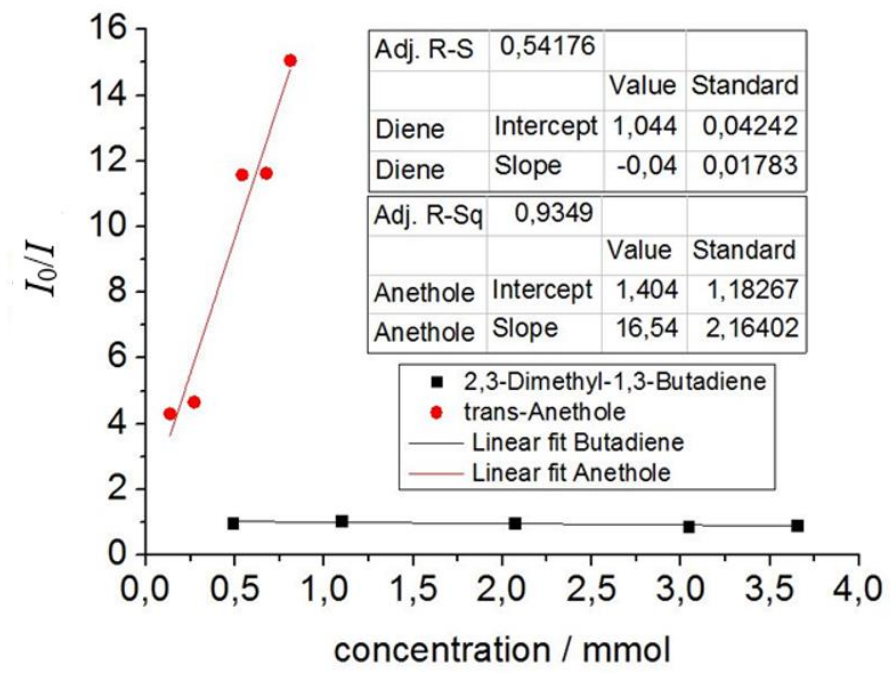

Figure 3.4.2 Stern-Volmer plot for different concentrations of trans-anethole (red) and 2,3-dimethyl-1,3-butadiene (black). The experiments were carried out and calculated by Waldemar Schilling.

Combining all the mechanistic results, a plausible mechanism has been outlined for the visible-light-mediated Diels-Alder reactions in Figure 3.4.3. At first, we proposed that the photocatalyst was converted into the photo-excited state under the irradiation of visible light and underwent a single electron transfer (SET) with transanethole. The resulting radical cation of 206a further reacted with the diene via the [4+2] cycloaddition to afford the radical cation of the product. Afterwards, this radical cation of the product accepted one electron mainly from another equivalent of 206a with a chain propagation step to generate the final product (206b). Meanwhile superoxide radical anion was also involved partly as electron donor which was not dominant role. Here the role of air/oxygen was to turn over the reduced photocatalyst to the original state. [2+2] cycloaddition is also proposed to occur via SET pathway to form the product $\mathbf{2 3 3 b}$ in the absence of the diene. 


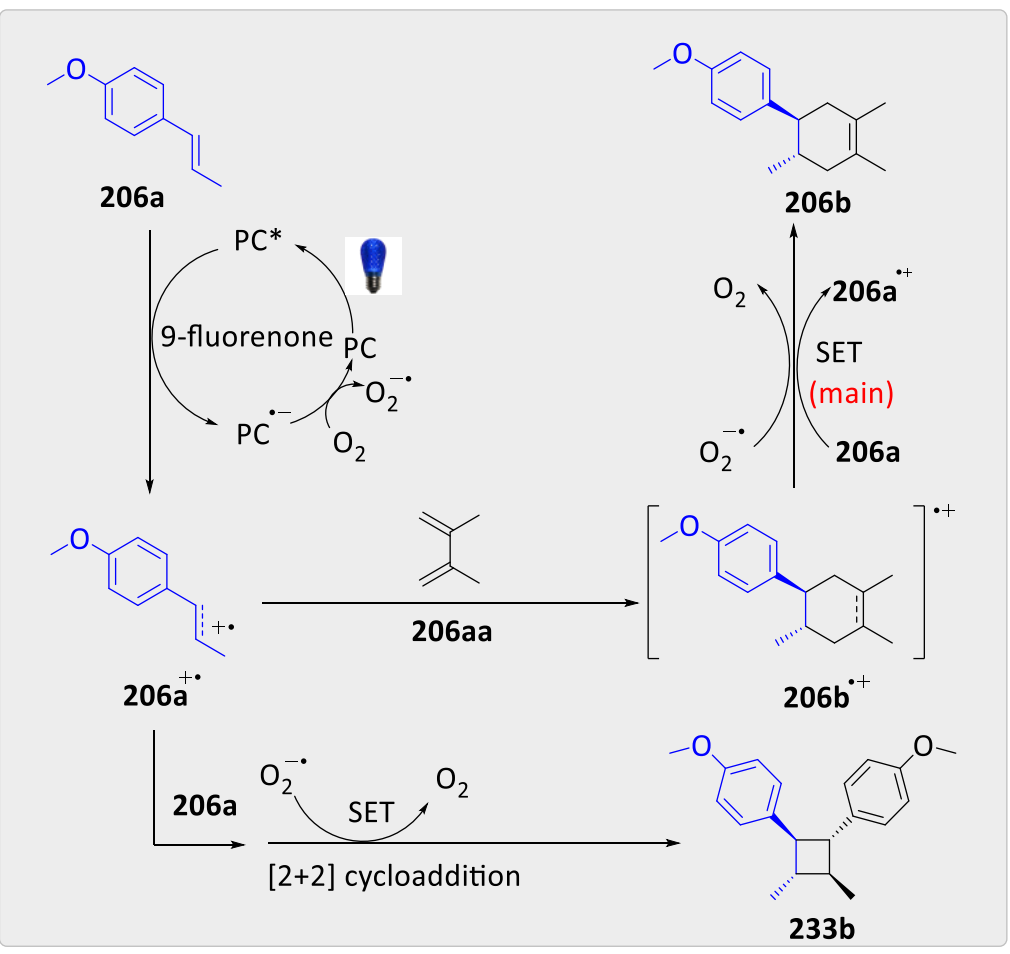

Figure 3.4.3 Proposed reaction mechanism for the 9-fluorenone catalysed Diels-Alder reactions.

\subsubsection{Unsuccessful Substrates}

During the extension of substrate scope, there were some unreactive substrates. For example, 9-vinylcarbazole was tested with different dienes such as (e)-buta-1,3-dienyl benzene, 1-acetoxy-1,3-butadiene and 2,4-dimethyl-1,3-pentadiene. However, the desired products were not observed under our reaction conditions (Scheme 3.4.6, F103-F105). Afterwards, these dienes were also applied to Diels-Alder reactions with $\beta$-methylstyrene as dienophile, but none of the desired products were formed. (Scheme 3.4.6, F106-F109). Interestingly, 3,4-dihydroisoquinoline could act as a dienophile in aza-Diels-Alder reactions. However, the isolation was problematic and the pure NMR data was failed to collect. The rest of unreactive substrates in this project was reported in the master thesis of Jiri Kollmann. ${ }^{[121]}$ 


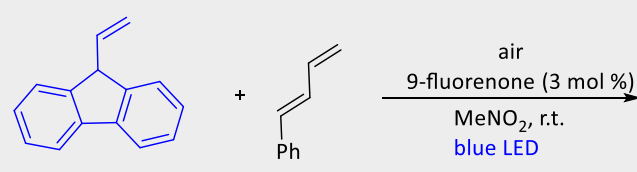<smiles></smiles>

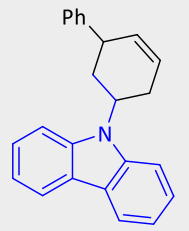

F103, 24h, n. ob.<smiles>C=C/C=C/OC(C)=O</smiles>

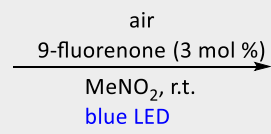<smiles>CC(=O)OC1C=CCCC1n1c2ccccc2c2ccccc21</smiles><smiles>CC(=O)OC1C=CCC(n2c3ccccc3c3ccccc32)C1</smiles>

F104, 24h, n. ob.<smiles>C=CC1c2ccccc2-c2ccccc21</smiles><smiles>C=C(C)C=C(C)C</smiles>
blue LED

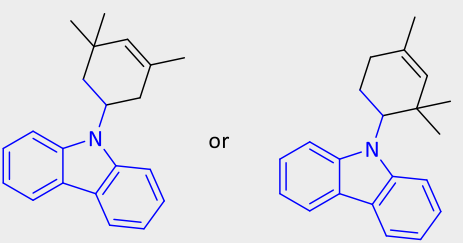

F105, 24h, n. ob.
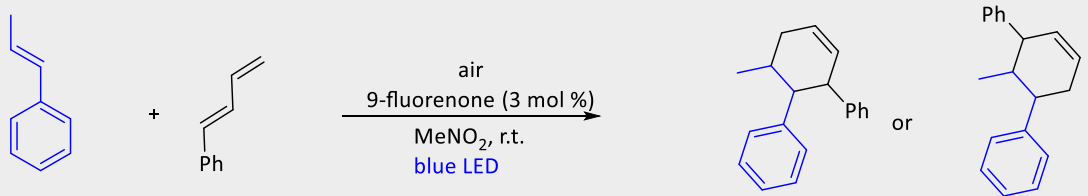

$106,24 h, n . o b$
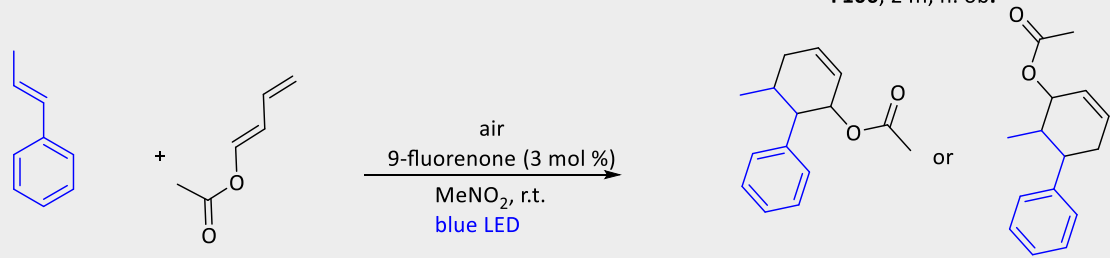

F107, 24h, n. ob
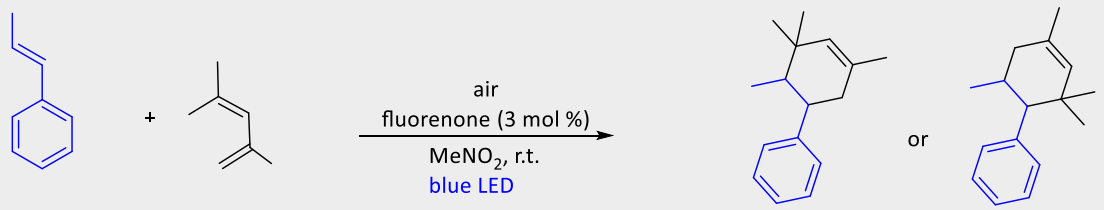

F108, 24h, n. ob.
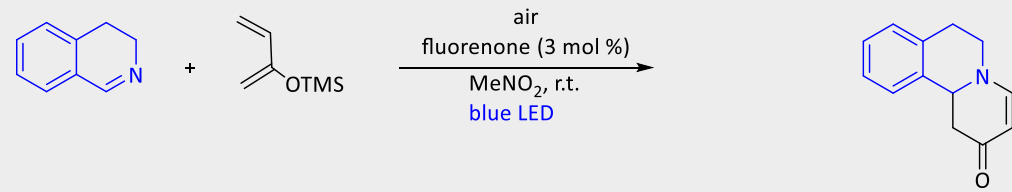

F109, 24h, failed isolation

Scheme 3.4.6 Unreactive substrates in (aza)-Diels-Alder reactions.

As di-O-methylendiandrin A (F110) and endiandrin A (F111) are potential bioactive natural products, we aimed to utilize our system to synthesize these molecules. However, both of them were not formed. Instead, the cleavage of $\mathrm{C}=\mathrm{C}$ bonds of the starting materials was observed. 


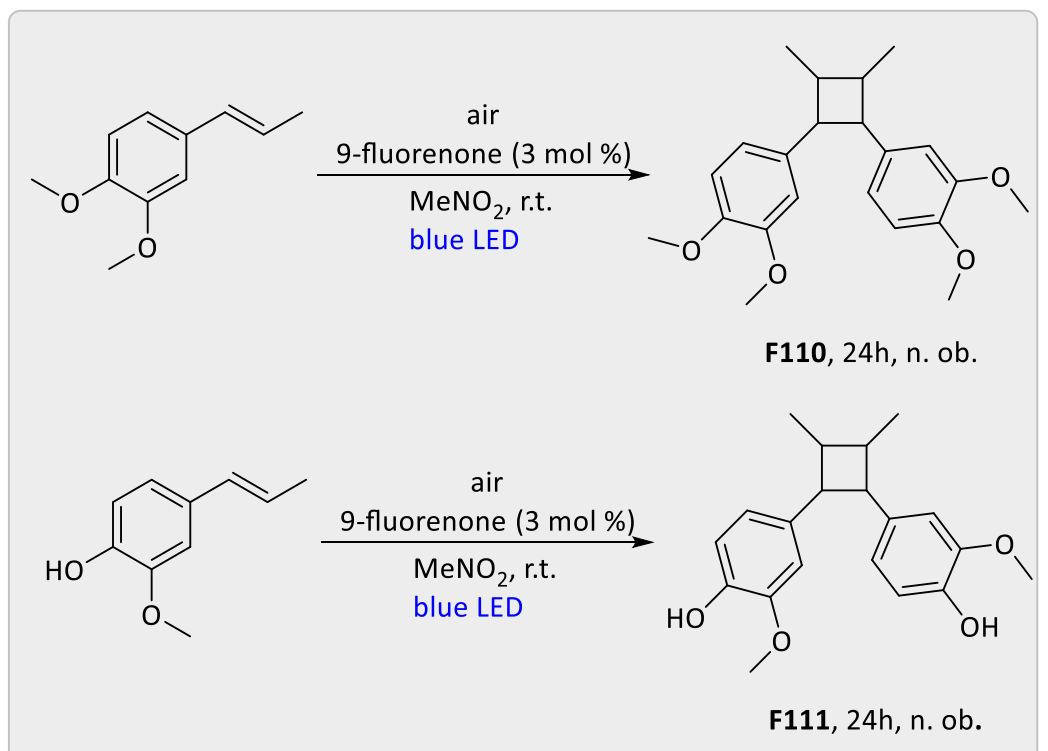

Scheme 3.4.7 Unreactive substrates in [2+2] cycloadditions. 


\section{Summary and Outlook}

In the first project, visible-light-mediated oxygenation of tertiary amines has been achieved using rose bengal as a cheap and commercially available metal-free catalyst and produced water as the sole by-product (Scheme 4.1). In fact, the catalyst exhibited an excellent substrate scope for $\mathrm{N}$-substituted piperidines, $\mathrm{N}, \mathrm{N}$ dimethylbenzylamine, $\mathrm{N}$-substituted tetrahydroquinoline, $\mathrm{N}$-substituted tetrahydroisoquinoline, nitrogen-containing heterocyclic substrates, $\mathrm{N}$-substituted morpholine, and 4-phenylpiperidine etc. Furthermore, one-pot syntheses of tertiary amides from secondary amines and one-pot syntheses of phthalimides and maleimide also worked very well under these catalytic conditions. Finally, we applied this photocatalyst to the late-stage modifications of existing natural products and pharmaceuticals. We believe this protocol can be applied to the oxygenation of drug molecules and towards the syntheses of natural products, which will be a promising method for the synthesis and modifications of pharmaceuticals. Additionally, detailed mechanistic studies revealed the role of the photocatalyst, base and oxygen and led to a mechanistic proposal of this reaction. ${ }^{[97][122][123]}$

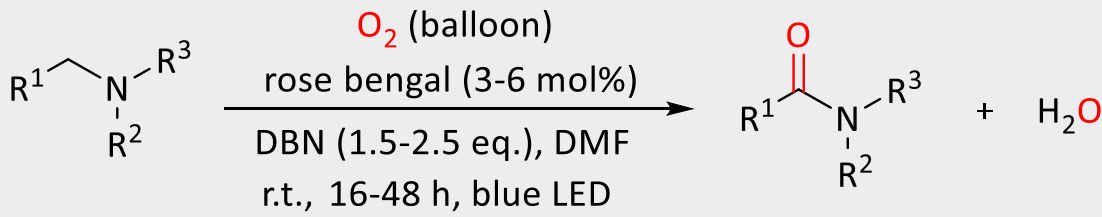

$$
\begin{aligned}
& \text { Metal-free catalyst } \quad \mathrm{O}_{2} \text { as oxidant } \\
& 33 \text { substrate } \quad \text { Natural products syntheses }
\end{aligned}
$$

Scheme 4.1 Metal-free photocatalytic oxygenation of tertiary amines.

In the second project, a clean and "green" heterogeneous UCNF/Cl${ }_{4} N H P I$ catalytic system with molecular oxygen as oxidant was designed and employed under mild conditions (room temperature, $12 \mathrm{~W}$ blue LED mediation). Versatile benzylic oxygenation/hydroxylation reactions afforded moderate to excellent isolated yields under this heterogenous photocatalytic system (Scheme 4.2). More importantly, the reaction can process with even cleaner and greener energy, by using sunlight- 
irradiation with good isolated yields. Furthermore, the reaction system could be applied as late stage modification for relevant drug molecules. Although the late stage modifications for some drug molecules have already been carried out in this work, most of them were accompanied with side products and therefore were hard to isolate.

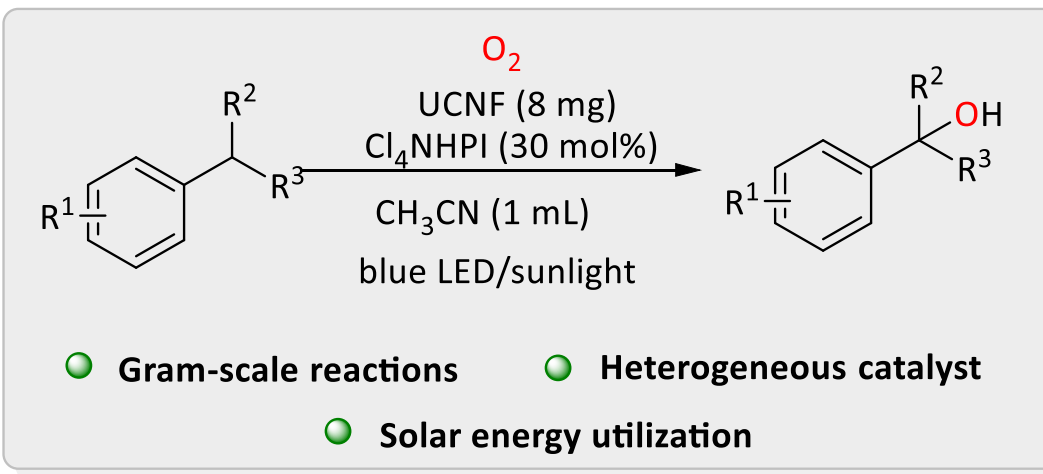

Scheme 4.2 Metal-free heterogeneous photocatalysis for the hydroxylation of benzylic $\mathrm{Cs} p^{3}-\mathrm{H}$ bond.

In the third project, polymeric carbon nitrides (PCN) as a photocatalyst have been developed for the selective cleavage of $C=C$ bonds in aryl olefins (Scheme 4.3). This method was selective, scalable under the presence of sunlight and the photocatalyst was easily recyclable. Excellent substrates scope with high selectivity is the main advantage of this recyclable catalyst. In addition, we have been able to perform the late-stage $\mathrm{C}=\mathrm{C}$ bond cleavage in aryl olefins and degradation of complex molecules to achieve highly valuable products and pharmaceuticals. The stability of PCN has been well investigated by different spectroscopic analyses. We believe that this protocol can be applied and extended for the cleavage of olefins in bulk chemistry, for the syntheses of pharmaceuticals and natural products. ${ }^{[106]}$ 


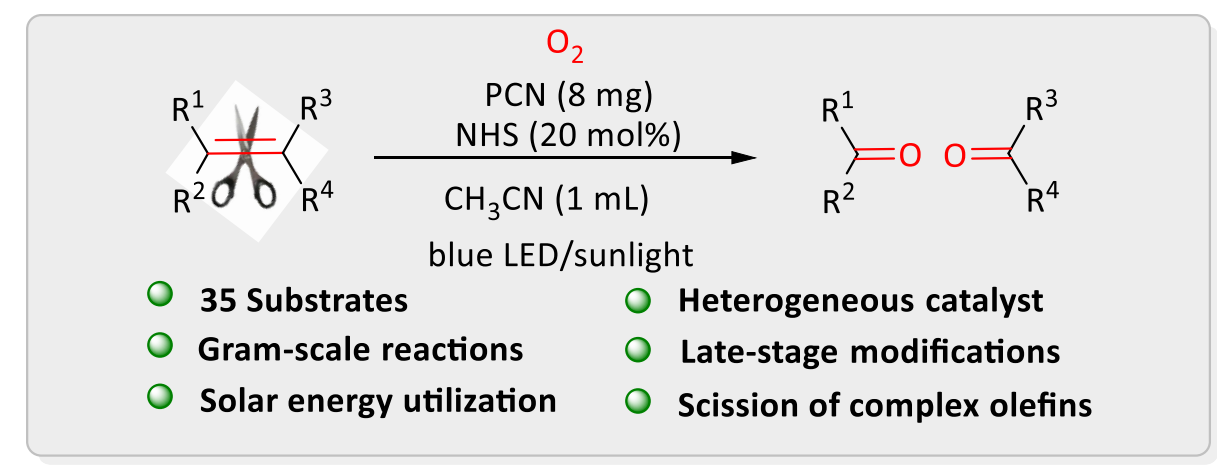

Scheme 4.3 Metal-free heterogeneous photocatalyst for the selective oxidative cleavage of $\mathrm{C}=\mathrm{C}$ bonds.

In the last project, we have used 9-fluorenone as a metal-free photocatalyst for the visible-light-mediated cycloaddition reactions which has proven to be an efficient, sustainable and metal-free possibility to synthesize cyclohexene derivatives (Scheme 4.4). This catalyst is very cheap and commercially available and has shown excellent substrate scopes for [4+2] and [2+2] cycloaddition reactions with electron-rich dienophiles. In addition, these mild conditions can be employed to synthesize bioactive 2,3-dihydropyridin-4(1)-one derivatives via aza-Diels-Alder reactions. We believe that this protocol can be further applied and extended for the syntheses of pharmaceuticals and natural products. ${ }^{[123][124]}$

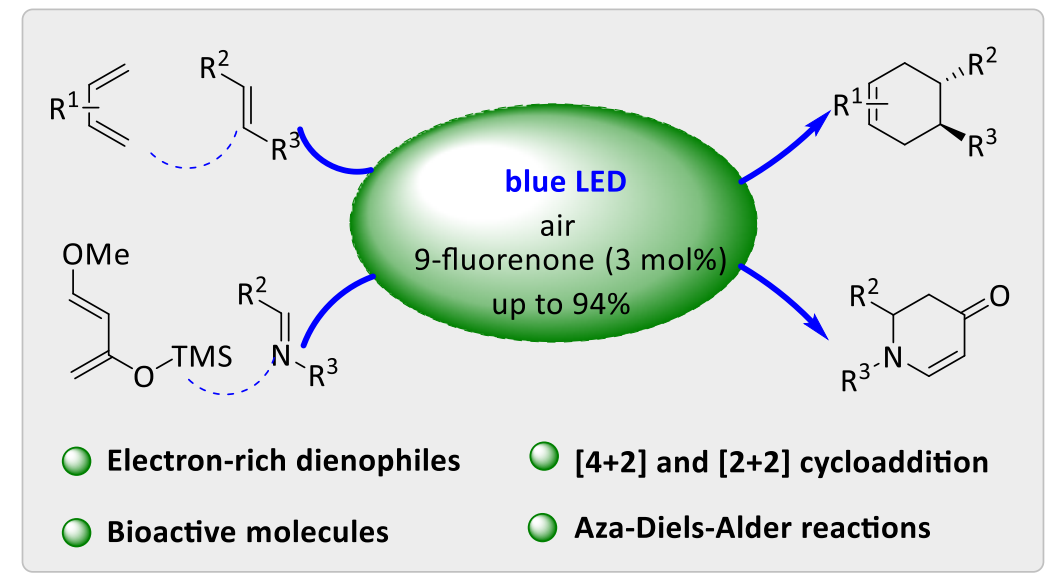

Scheme 4.4 Efficient metal-free photocatalytic (aza)-Diels-Alder reactions. 


\section{Experimental Section}

\subsection{General Remarks}

In general, photocatalytic oxygenation reactions of tertiary amines, hydroxylation, and $\mathrm{C}=\mathrm{C}$ double bond cleavage were performed under $\mathrm{O}_{2}-$ balloon (Dräger, 2.0L 2165694; Cawila Football bladder 1.6 L). However, several reactions including the $C=C$ bond cleavage and hydroxylation were carried out under sunlight. (Aza)-Diels-Alder reactions were performed under air. If not otherwise noted, yields refer to the isolated compounds, estimated to be $>95 \%$ pure as determined by ${ }^{1} \mathrm{H}-\mathrm{NMR}$.

\section{Vacuum}

The following pressure was measured on the used vacuum pump and was not corrected: oil pump vacuum (OPV): 0.1 mbar.

\section{Chromatography}

Analytical thin layer chromatography (TLC) was performed on $0.25 \mathrm{~mm}$ silica gel 60Fplates (MACHEREY-NAGEL) with $254 \mathrm{~nm}$ fluorescent indicator from MERCK. Plates were visualized under UV-light or developed by the treatment with a $\mathrm{KMnO}_{4}$ solution followed by careful applying a heat gun. Chromatographic purification of products was accomplished by flash column chromatography on MERCK silica gel, grade 60 (0.040-0.063 $\mathrm{mm}$ and $0.063-0.200 \mathrm{~mm})$

\section{Gas Chromatography}

To monitor the progress of the reaction and to determine its yield, after stopping the reaction, the reaction flask was placed on the balance and four drops of $n$-dodecane (normally the weight was $20-30 \mathrm{mg}$ ) were added to the flask. The whole sample was then transferred into a $20-\mathrm{mL}$ snap cap vial using EtOAc as solvent and the contents of the flask were washed for three times. The resulting mixture was subjected to an aqueous workup (adding $9 \mathrm{~mL}$ of deionized water and $8 \mathrm{~mL}$ of ethyl acetate) and the organic layer was transferred into a gas chromatography (GC) vial and proceeded with a GC analysis using a chromatograph 7890A GC System (Supelcowax 10 Fused 
Silica Capillary Column; $30 \mathrm{~m} \times 0.32 \mathrm{~mm} \times 0.25 \mu \mathrm{m}$ ). The reaction yield was determined using the added $n$-dodecane as the internal standard based on a GC calibration curve. Please note that GC analysis was performed using $\mathrm{N}_{2}$ as carrier gas with the following parameters: $50{ }^{\circ} \mathrm{C}$ at $1 \mathrm{~min}$, then heating to $250^{\circ} \mathrm{C}$ at a rate of $10^{\circ} \mathrm{C} / \mathrm{min}$, and holding $1 \min$ at $250^{\circ} \mathrm{C}$.

The typical order of elution was as follows: $n$-dodecane, starting material (if any was left), and the product. The mentioned elution order has been proven using authentic samples of each substance by myself. ${ }^{[122]}$

\section{Gel Permeation Chromatography (GPC)}

GPC purifications were performed on a JAI system (JAI-LC-9260 II NEXT) equipped with two sequential columns (JAIGEL-2HR, gradient rate: 5.000; JAIGEL-2.5HR, gradient rate: 20.000 ; internal diameter $=20 \mathrm{~mm}$; length $=600 \mathrm{~mm}$; flush rate $=10.0$ $\mathrm{mL} / \mathrm{min}$ and chloroform (HPLC-quality with $0.6 \%$ ethanol as stabilizer) was used as the eluent.

\section{Gas Chromatography-Mass Spectrometry (GC-MS)}

To monitor the progress of the reaction (in projects of oxygenation of tertiary amines, hydroxylation, and oxidative $\mathrm{C}=\mathrm{C}$ bond cleavage), $0.05 \mathrm{~mL}$ of reaction mixture was collected from the reaction flask using a syringe, which had been purged three times with the reaction gas atmosphere through the septum, and transfer it to a $20-\mathrm{mL}$ snap cap vial. In the case of reactions performed using atmospheric air (cycloadditions), the reaction mixture sample was collected using a Pasteur pipette. EtOAc $(8 \mathrm{~mL})$ and water $(9 \mathrm{~mL})$ were then added into the collected reaction mixture. Then transfer the organic phase into a GC vial after filtering and proceed with performing gas chromatography-mass spectrometry (GC-MS) analysis using a Thermo Finnigan spectrometers TRACE and DSQ together. Upon this method, the reaction process can be monitored or confirm whether the products were formed. 
The general parameters: Use $\mathrm{He}$ as the carrier gas, $50{ }^{\circ} \mathrm{C}$ for $1 \mathrm{~min}$, then heating to $300{ }^{\circ} \mathrm{C}$ at a rate of $15^{\circ} \mathrm{C} / \mathrm{min}$, and holding 1 minute at $300{ }^{\circ} \mathrm{C}$. If solvents with a high boiling point are present in the sample after workup (e.g. DMSO, DMA, or DMF) set the solvent delay to $4 \mathrm{~min}$. On the other hand, if only solvents with a low boiling points, set the solvent delay 2 min. ${ }^{[120]}$

\section{Mass Spectrometry}

Electron ionization (EI) and El high resolution mass spectra (HR-MS) were measured on a time-of-flight mass spectrometer AccuTOF from JOEL. Electrospray ionization (ESI) mass spectra were recorded on an ion-trap mass spectrometer LCQ from FINNIGAN, a quadrupole time-of-flight maXis from BRUKER DALTONIC or on a timeof-flight mass spectrometer microTOF from BRUKER DALTONIC. ESI-HRMS spectra were recorded on a BRUKER Apex IV, fourier transform ion cyclotron resonance (FTICR) mass spectrometer. The ratios of mass to charge $(\mathrm{m} / \mathrm{z})$ are indicated and intensities relative to the base peak $(I=100)$ are written in parentheses.

\section{Nuclear Magnetic Resonance Spectroscopy (NMR)}

Nuclear magnetic resonance (NMR) spectra were recorded on VARIAN Inova 500, 600, VARIAN Mercury 300, VX 300, VARIAN Avance 300, VARIAN VNMRS 300 and BRUKER Avance III 300, 400 and HD 500 spectrometers. All chemical shifts are given as $\delta$-values in ppm relative to the residual proton peak of the deuterated solvent or its carbon atom, respectively. ${ }^{1} \mathrm{H}$ and ${ }^{13} \mathrm{C}$ NMR spectra were referenced using the residual proton or solvent carbon peak (see table), respectively. ${ }^{13} \mathrm{C}$ and ${ }^{19} \mathrm{~F} N M R$ were measured as proton-decoupled spectra.

\begin{tabular}{lll}
\hline & ${ }^{1} \mathrm{H}-\mathrm{NMR}$ & ${ }^{13} \mathrm{C}-\mathrm{NMR}$ \\
\hline $\mathrm{CDCl}_{3}$ & 7.26 & 77.16 \\
{$[\mathrm{D}]_{6}$-DMSO } & 2.50 & 39.52 \\
\hline
\end{tabular}


The observed resonance-multiplicities were described by the following abbreviations: s (singlet), $d$ (doublet), t (triplet), q (quartet), hept (heptet), $m$ (multiplet) or analogous representations. The coupling constants $J$ are reported in Hertz $(\mathrm{Hz})$. Analysis of the recorded spectra was carried out with MestReNova 10 software.

\section{Solvents}

Solvents such as $N, N$-dimethylformamide (DMF), tetrahydrofuran (THF), acetonitrile (MeCN) in these projects (oxygenation of tertiary amines, hydroxylation, and oxidative $\mathrm{C}=\mathrm{C}$ bond cleavage) were purchased from Acros (extra dry over molecular sieve). For taking the dry solvents used in these three projects, firstly the bottle was purged with nitrogen gas, then dry solvents were transferred with a nitrogen-flushed (3 times) syringe through a rubber septum into the reaction flask. The solvents used in cycloadditions were of analytical standard. These solvents could be added directly with a syringe under air.

\section{Reagents}

Chemicals obtained from commercial sources with purity above 95\% were used without further purification. Some of the substrates in $\mathrm{C}=\mathrm{C}$ double bond cleavage project were synthesized according to previously described methods.

\section{Preparative Thin-Layer Chromatography}

Preparative TLC plates $(20 \mathrm{~cm} \times 20 \mathrm{~cm})$ with a $0.20 \mathrm{~mm}$ silica gel 60 layer on alumina containing a fluorescent indicator F254 from Macherey Nagel by Merck were used to isolate compounds of smaller amount $(<0.5 \mathrm{mmol})$. The TLC plates were analyzed by near $(366 \mathrm{~nm})$ and middle $(254 \mathrm{~nm})$ UV light. If necessary, iodine, potassium permanganate solution or phosphomolybdic acid (PMA) solution were used to stain the TLC plates. 


\section{Flash Column Chromatography}

Flash column chromatography was used to purify crude products of larger amount (>0.5 mmol) using silica gel with a pore size of $60 \AA$ and a particle size of $40-63 \mu \mathrm{m}$ (Merck) and the respective eluent.

\subsection{Reaction Procedures of $\alpha$-Oxygenation of Tertiary Amines}

\section{General Procedure for Photocatalytic Reactions}

The reaction setup consisted of a self-constructed light source configuration, made up of a crystallizing dish with a diameter of $140 \mathrm{~mm}$. Inside of the crystallizing dish, a commercially available $5 \mathrm{~m}$ LED-Strip was glued with separable LED elements. In the designed setup, $3 \mathrm{~m}$ LED strip was used in a crystallizing dish, with a total power of $24 \mathrm{~W}$. Light intensity of the light source could be adjusted by a self-constructed dimmer. Construction of the reaction setup and the dimmer was performed by the electronic services of the faculty for chemistry of the Georg-August-Universität Göttingen. Cooling of the setup was performed by a commercially available $120 \mathrm{~mm}$ computer fan. To ensure a constant room temperature, the dimmer setting was used at $50 \%(12 \mathrm{~W})$. During the first experiment the temperature was monitored inside the crystallizing dish and did not exceed the room temperature $\left(25-30^{\circ} \mathrm{C}\right)$. Magnetic stirring was performed with $250 \mathrm{rpm}$. It should be noted that the reaction temperature will increase if the higher power (more than $12 \mathrm{~W}$ ) was employed. 

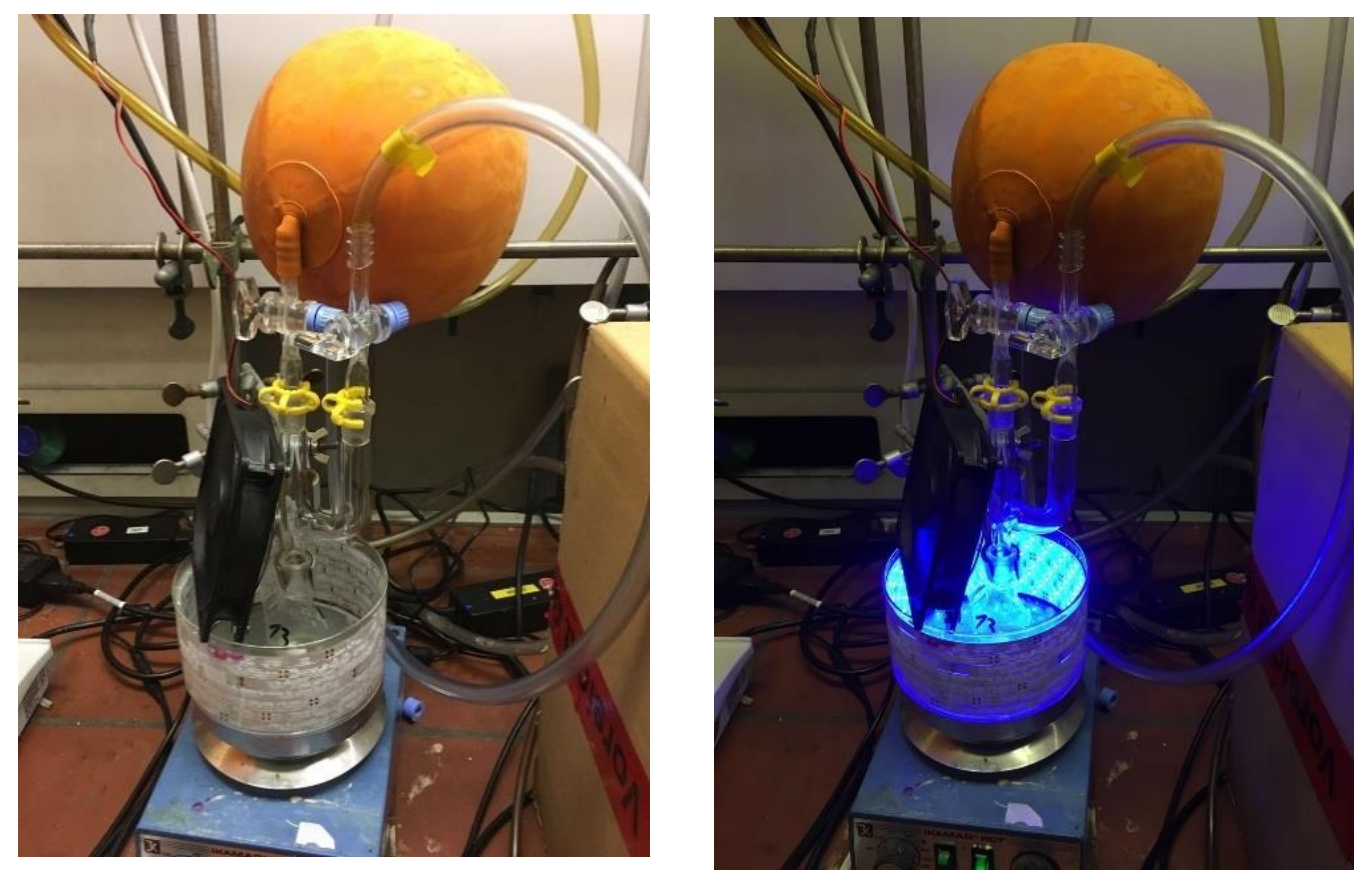

Figure 5.2.1 LED reaction setup for photocatalytic reactions.

\section{Measurement of Wavelength about Blue LED}

The emission spectra of the light setup were measured with a UV-Vis probe from Ocean optics (P200-5-UV-Vis). The emission spectra showed a clear wavelength band between 404 and $553 \mathrm{~nm}$ with a maximum at $456 \mathrm{~nm}$ (Figure 5.2.2).[122]

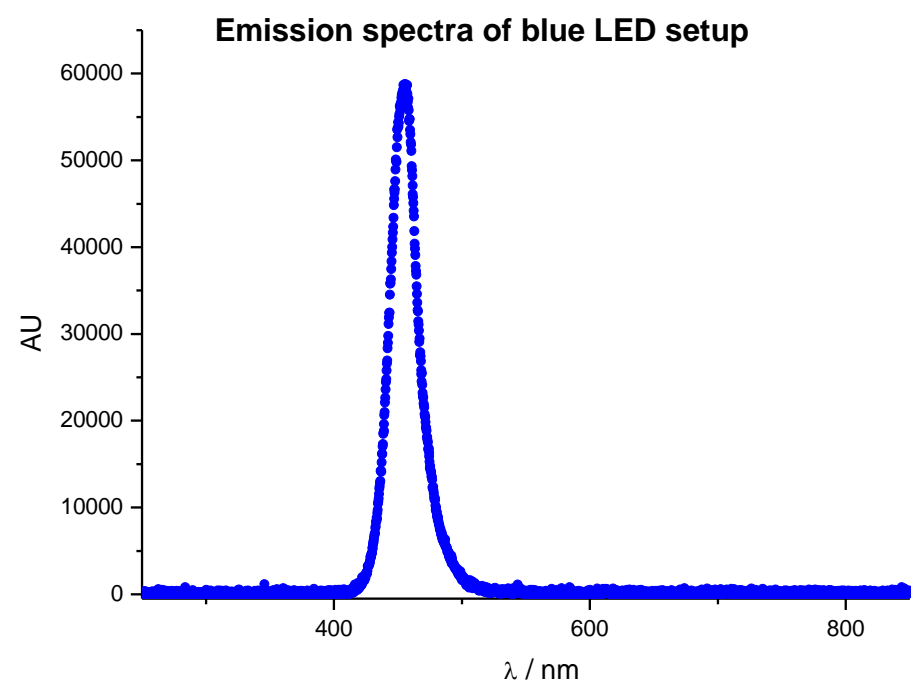

Figure 5.2.2 Wavelength of blue LED. 


\section{General Procedure for The Oxygenation of Tertiary Amines}

A dry $10 \mathrm{~mL}$ two-necked flask containing a stirring bar was charged with $0.30 \mathrm{mmol}$ of substrate and $0.009 \mathrm{mmol}$ of rose bengal. The flask was degassed three times ( 5 min each) under nitrogen in the dark, oxygen atmosphere was incorporated through an $\mathrm{O}_{2}$ balloon subsequently. Finally, dry DMF $(1.0 \mathrm{~mL})$ and a dry DBN solution $(1 \mathrm{~mol} / \mathrm{L}$ in DMF) were added. The resulting mixture was stirred for $16-48 \mathrm{~h}$ under $12 \mathrm{~W}$ blue LED irradiation (the progress was monitored via GC-MS or TLC).
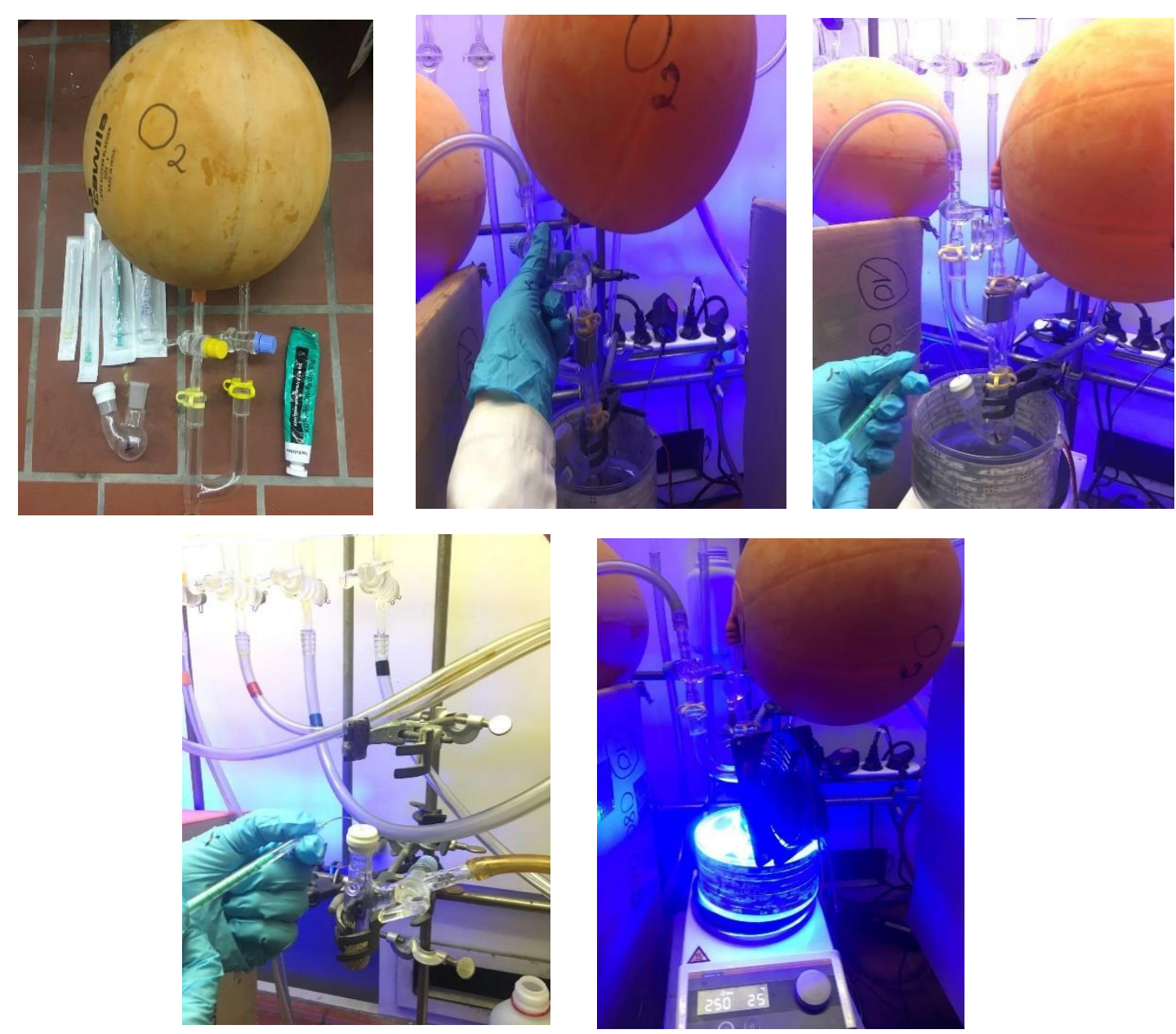

Figure 5.2.3 A: preparation of the reaction; B: reaction setup under vacuum; C: transferring DBN from Schlenk tube with syringe under nitrogen; D: Injecting DBN into the flask through the septum; E: Starting the magnetic stirring, cooling fan, and LED light. ${ }^{[122]}$

Then, the resulting mixture was subjected to an aqueous workup (using distilled 
water; or brine in case of slurry phase separation) and was extracted three times with ethyl acetate. The combined organic layers were dried over anhydrous $\mathrm{Na}_{2} \mathrm{SO}_{4}$, filtered and concentrated in vacuo. Products were purified via silica gel chromatography or GPC (see details of products) with ethyl acetate and $n$-hexane as solvents (Figure 5.2.3).

\section{Synthesis of $\boldsymbol{N}$-Phenyl-tetrahydroisoquinoline}

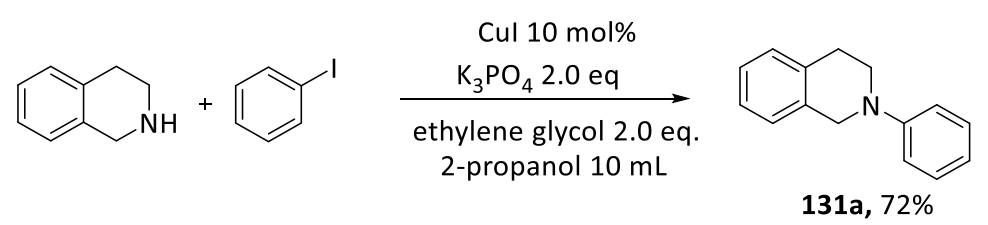

A typical procedure is described as following for the synthesis of $\mathrm{N}$-phenyltetrahydroisoquinoline: Copper $(\mathrm{I})$ iodide $(1.0 \mathrm{mmol}, 10 \mathrm{~mol} \%)$, and potassium phosphate ( $20 \mathrm{mmol}, 2.0$ eq.) were added to a Schlenk tube. The tube was evacuated and back filled with nitrogen. 2-Propanol (10 mL), ethylene glycol (20 mmol, 2.0 eq.), 1,2,3,4-tetrahydroisoquinoline ( $2.0 \mathrm{~g}, 15 \mathrm{mmol}, 1.5 \mathrm{eq}$.) and iodobenzene (10 mmol, 1.0 eq.) were added successively at room temperature. The reaction mixture was heated at $90{ }^{\circ} \mathrm{C}$ and kept for $24 \mathrm{~h}$ and then allowed to cool to room temperature. Diethyl ether $(20 \mathrm{~mL})$ and water $(20 \mathrm{~mL})$ were then added. The aqueous layer was extracted with diethyl ether $(2 \times 20 \mathrm{~mL})$. The combined organic phases were washed with brine and dried over magnesium sulfate. The solvent was removed via rotary evaporation, and the remaining residue was purified via flash column chromatography to give the desired product with $72 \%$ yield. The products were obtained and the analytical data were consistent with those given in literature. ${ }^{[125]}$

Synthesis of $\mathbf{N}$-Ethyl-tetrahydroisoquinoline and Derivatives

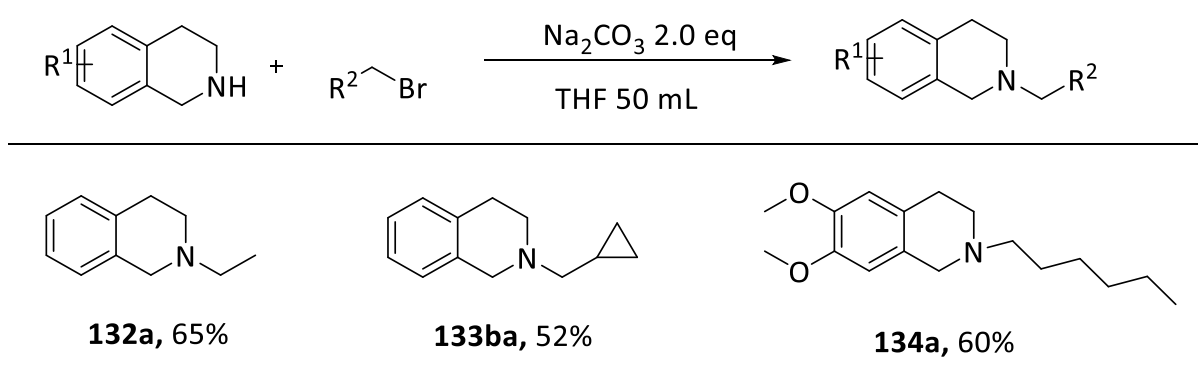


A typical procedure is described as following for the synthesis of $\mathrm{N}$-phenyltetrahydroisoquinoline: tetrahydroisoquinolines (10 mmol, 1.0 eq.), bromides (12 mmol, 1.2 eq.), and sodium carbonate ( $20 \mathrm{mmol}, 2.0$ eq.) were added into THF (50 $\mathrm{mL})$. Afterwards, diethyl ether $(20 \mathrm{~mL})$ and water $(20 \mathrm{~mL})$ were added. The aqueous layer was extracted with diethyl ether $(2 \times 20 \mathrm{~mL})$. The combined organic phases were washed with brine and dried over magnesium sulfate. The solvent was removed via rotary evaporation, and the remaining residue was purified via flash column chromatography to give the desired product with medium yields. The products were obtained and the analytical data were consistent with those given in literature. ${ }^{[126]}$

\section{Synthesis of $\mathbf{N}$-Benzyl Piperidine}

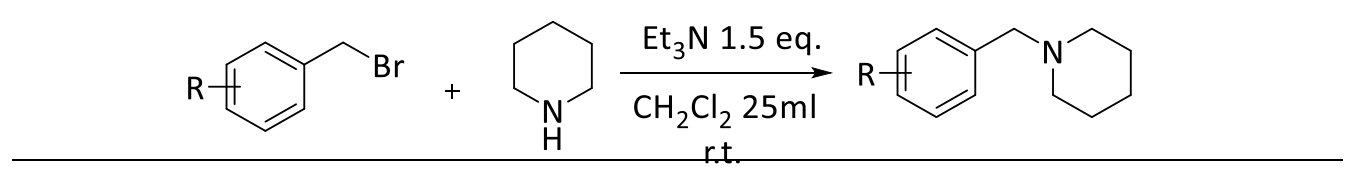

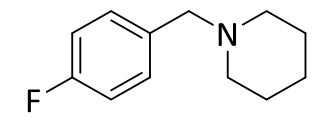

$127 a, 92 \%$

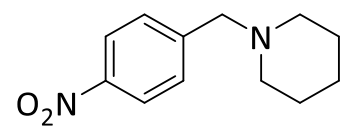

$128 a, 95 \%$

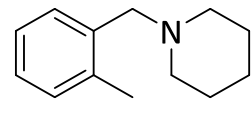

$129 a, 89 \%$

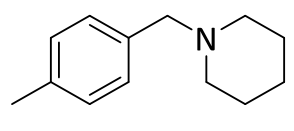

$130 a, 91 \%$

Adapting a known procedure, ${ }^{[127]}$ piperidine $(12.5 \mathrm{mmol}, 2.5$ equiv.) was added at 0 ${ }^{\circ} \mathrm{C}$ to a solution of benzyl bromide ( $5 \mathrm{mmol}, 1.0$ equiv.) in $\mathrm{CH}_{2} \mathrm{Cl}_{2}(25 \mathrm{~mL})$. Afterwards, $\mathrm{Et}_{3} \mathrm{~N}$ (7.5 mmol, 1.5 equiv.) was add into the solution slowly. The reaction mixture was stirred overnight at room temperature, then concentrated in vacuo and aqueous $\mathrm{HCl}$ $(20 \mathrm{~mL}, 2 \mathrm{M})$ was added. The mixture was extracted with $n$-hexane $(3 \times 40 \mathrm{~mL})$. The aqueous layer was made strongly basic by adding solid sodium hydroxide and the resulting solution was extracted with diethyl ether $(3 \times 40 \mathrm{~mL})$. The combined organic phase was dried over $\mathrm{MgSO}_{4}$, filtered and solvents were removed in vacuo. The products were isolated by flash column chromatography ( $n$-hexane/EtOAc 80:20) to give the desired product with $88 \%$ yield. The products were obtained and the analytical data were consistent with those given in literature. ${ }^{[127]}$ 
Synthesis of 1-(Furan-2-ylmethyl)piperidine and 1-(Thiophen-2-ylmethyl)piperidine

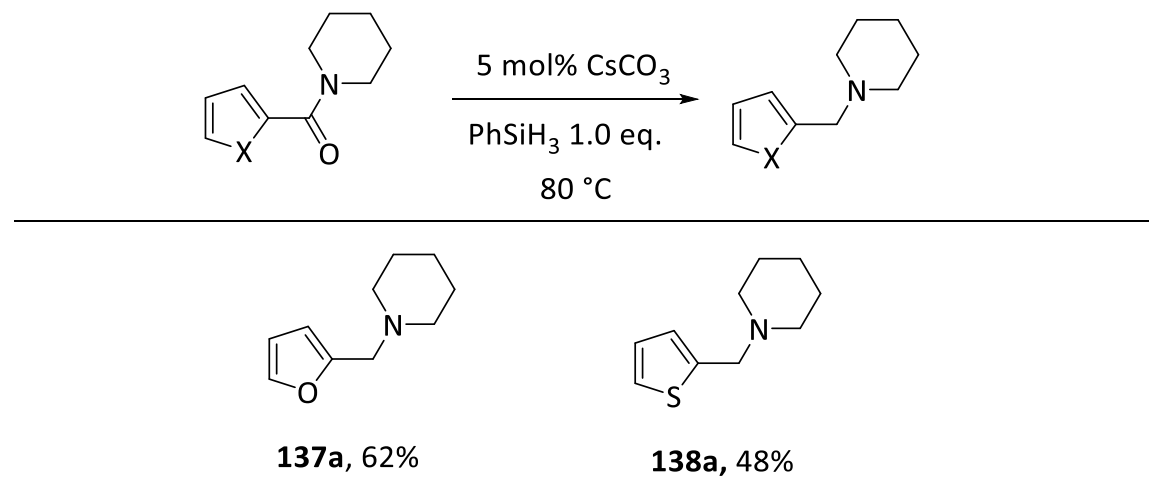

Adapting a known procedure, ${ }^{[128]}$ a $25 \mathrm{~mL}$ Schlenk tube containing a stir bar was charged with $5.0 \mathrm{~mol} \% \mathrm{Cs}_{2} \mathrm{CO}_{3}(0.15 \mathrm{mmol}$ ). Subsequently, amides ( $3.0 \mathrm{mmol}, 1.0$ eq.) and $\mathrm{PhSiH}_{3}(3.0 \mathrm{mmol}, 1.0$ eq.) were added. The mixture was stirred at room temperature for $24 \mathrm{~h}$. Dichloromethane $(0.5 \mathrm{~mL})$ was added to the mixture. The mixture was purified on a short silica gel column to give the products. The products were obtained and the analytical data were consistent with those given in literature. [128]

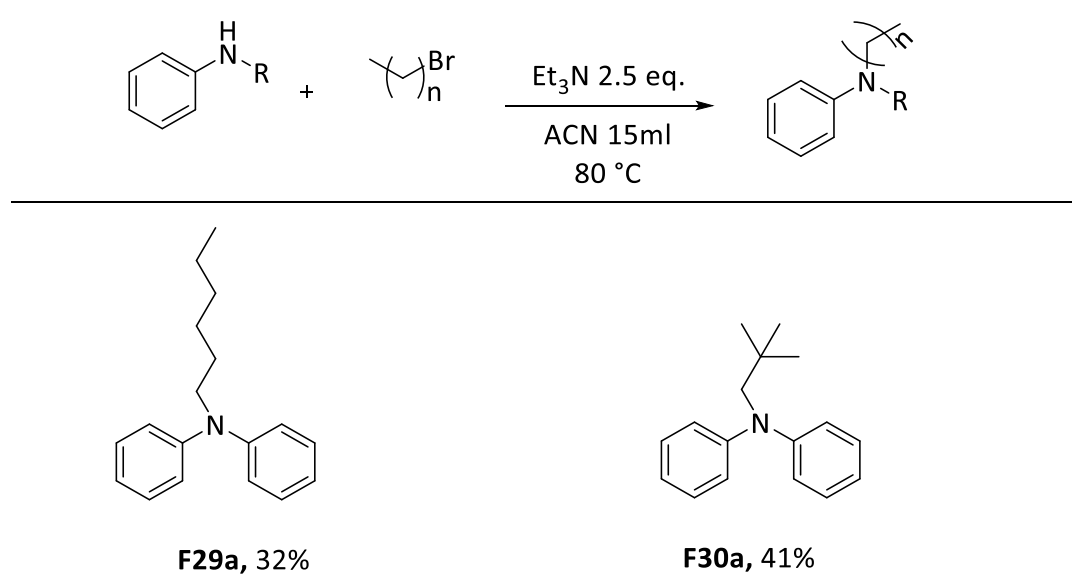

Those tertiary amines were synthesized in accordance with a known procedure. ${ }^{[126]}$ To a 50-mL round-bottom flask fitted with a reflux condenser were added the reactants including the secondary amines $(5.0 \mathrm{mmol}, 1.0$ equiv.), acetonitrile $(15 \mathrm{~mL})$, aliphatic bromides ( $8.5 \mathrm{mmol}, 1.7$ equiv.), and triethylamine (12.5 mmol, 2.5 equiv.), respectively. The reaction was heated to reflux overnight. After cooling to room temperature, the mixture was partitioned between water $(20 \mathrm{~mL})$ and $\mathrm{DCM}(20 \mathrm{~mL})$. The organic layer was washed with an additional portion of water $(20 \mathrm{~mL})$, dried over 
$\mathrm{MgSO}_{4}$, filtered, and concentrated under reduced pressure. Then the remaining residue was purified via flash column chromatography to give the desired products with medium yields. The products were obtained and the analytical data were consistent with those given in literature. ${ }^{[126]}$

\section{${ }^{18} \mathrm{O}-$-labelling and KIE Experiments}

Oxidation of 1-Benzylpiperidine in Presence of ${ }^{18} \mathrm{O}$-labeled Oxygen.

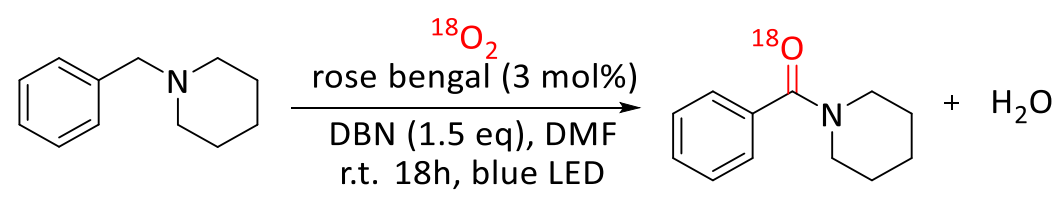

The ${ }^{18} \mathrm{O}$-labeling experiment was performed with ${ }^{18} \mathrm{O}_{2}$ (Sigma Aldrich, ${ }^{18} \mathrm{O}$ atom 99.7\%), and analyzed with ESI-HRMS and GC showing the ${ }^{18} \mathrm{O}$-labeled product with a yield of $91 \%$ ( $88 \%$ of isolated yield). The result showed that the origin of the oxygen atom in the desired product $222 \mathrm{c}$ was only from oxygen gas since no ${ }^{16} \mathrm{O}$-labeled product was found.

ESI-HRMS: $m / z$ calcd. for $\mathrm{C}_{12} \mathrm{H}_{15} \mathrm{~N}^{18} \mathrm{O}[\mathrm{M}+\mathrm{H}]^{+}:$192.1269, found 192.1274; calcd. for $\mathrm{C}_{12} \mathrm{H}_{15} \mathrm{~N}^{18} \mathrm{O}[\mathrm{M}+\mathrm{Na}]^{+}:$214.1088, found 214.1092.

Synthesis of Deuterium-labeled 1-Benzylpiperidine.<smiles>c1ccc(CN2CCCCC2)cc1</smiles>

$$
\underset{\text { DMSO- } d_{6}, 80^{\circ} \mathrm{C}}{\stackrel{\mathrm{KO}}{\mathrm{t}} \mathrm{Bu}(0.5 \text { eq. })}
$$<smiles>[2H]C([2H])(c1ccccc1)N1CCCCC1</smiles>

The synthesis was performed according to the literature. ${ }^{[129]} \mathrm{KO}^{t} \mathrm{Bu}(280.5 \mathrm{mg}$, $2.5 \mathrm{mmol}, 0.5 \mathrm{eq})$ and 1-benzylpiperidine $(0.88 \mathrm{~mL}, 5 \mathrm{mmol})$ were added into a $25 \mathrm{~mL}$ two-necked flask. After purging the flask three times with vacuum $/ \mathrm{N}_{2}, 7.5 \mathrm{~mL}$ of dry DMSO- $d_{6}$ was added and the reaction mixture was stirred at $80^{\circ} \mathrm{C}$ for $2 \mathrm{~h}$. The reaction was quenched with water and the product was extracted with ethyl acetate. The solution was dried over $\mathrm{Na}_{2} \mathrm{SO}_{4}$ and the solvent was removed under reduced pressure providing the product in > 95 atom $\%$ D and $99 \%$ yield which was used without further purification. 
KIE: Oxidation of Deuterium-labeled 1-Benzylpiperidine.

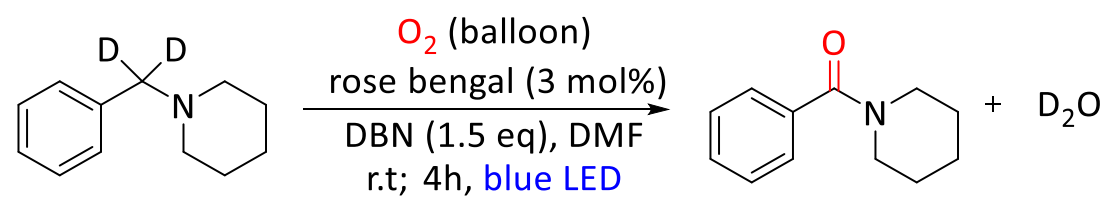

Deuterium-labeled 1-benzylpiperidine was oxidized by the general procedure. under $\mathrm{O}_{2}$ atmosphere. After $4 \mathrm{~h}$, the yield was determined by GC using $n$-dodecane as internal standard and compared with a non-deuterated sample under the same conditions. The calculated KIE is a result of the average (the average of three runs with a standard deviation of 1.5) of three independent runs, which strongly suggested the $\mathrm{C}-\mathrm{H}$ bond cleavage in the rate-determining step. The signal of $\mathrm{D}_{2} \mathrm{O}$ was also detected by NMR.

$$
\frac{k_{\mathrm{H}}}{k_{\mathrm{D}}} \sim \frac{n\left(P_{\mathrm{H}}\right)}{n\left(P_{\mathrm{D}}\right)}=\frac{0.0922}{0.0283}=3.2
$$

\subsection{Reaction Procedures of Blue LED-Mediated Hydroxylation}

\section{General Procedure for Blue LED-Mediated Hydroxylation}

Hydroxylation reactions were also carried out under water-free condition and oxygen atmosphere. For this purpose, the reaction flask and stirring bar were dried in an oven over four hours to remove moisture. Afterwards, to the dried two necked flask (with stirring bar) a mixture of $0.3 \mathrm{mmol}$ (1.0 eq.) substrates, $10 \mathrm{mg}$ nitrogen deficient g- $\mathrm{C}_{3} \mathrm{~N}_{4}$, and $27 \mathrm{mg} \mathrm{Cl} \mathrm{Cl}_{4} \mathrm{NHPI}$ (0.3 eq.) was added. Afterwards, the flask was evacuated and flushed with nitrogen $(2 \mathrm{x})$ and oxygen $(1 \mathrm{x})$ via Schlenk techniques. Dry MeCN solvent $(1.0 \mathrm{~mL})$ was transferred with a nitrogen-flushed $(3 \mathrm{x})$ syringe through a rubber septum into the reaction flask. The reaction mixture was stirred at room temperature under the irradiation of blue LED ( $16 \mathrm{~W})$ for $16-48 \mathrm{~h}$. The reactions were monitored by TLC and GC-MS. The reactions were quenched with $10 \mathrm{~mL}$ of distilled water and $10 \mathrm{~mL}$ of ethyl acetate. The combined organic layers were extracted with ethyl acetate and concentrated in vacuo then purified via flash column chromatography. 


\section{General Procedure for the Hydroxylation using Sunlight}

A 50-mL two-necked flask containing a stirring bar was charged with $1.0 \mathrm{~g}$ of substrate, $80 \mathrm{mg}$ of UCNF and $30 \mathrm{~mol} \%$ of $\mathrm{Cl}_{4} \mathrm{NHPI}$. After evacuating the flask three times and purging it two times with nitrogen, oxygen atmosphere was incorporated through an $\mathrm{O}_{2}$ balloon. Finally, dry acetonitrile $(10.0 \mathrm{~mL})$ was added. The resulting mixture was stirred under sunlight (the setup was put on the roof of building, seeing Figure 5.3.1) and the progress was monitored via GC-MS). Then, the resulting mixture was subjected to an aqueous workup (using distilled water; or brine in case of slurry phase separation) and was extracted three times with ethyl acetate. The combined organic layers were dried over anhydrous $\mathrm{Na}_{2} \mathrm{SO}_{4}$, filtered and concentrated in vacuo. Products were purified via silica gel chromatography with ethyl acetate and $n$-hexane as solvents.

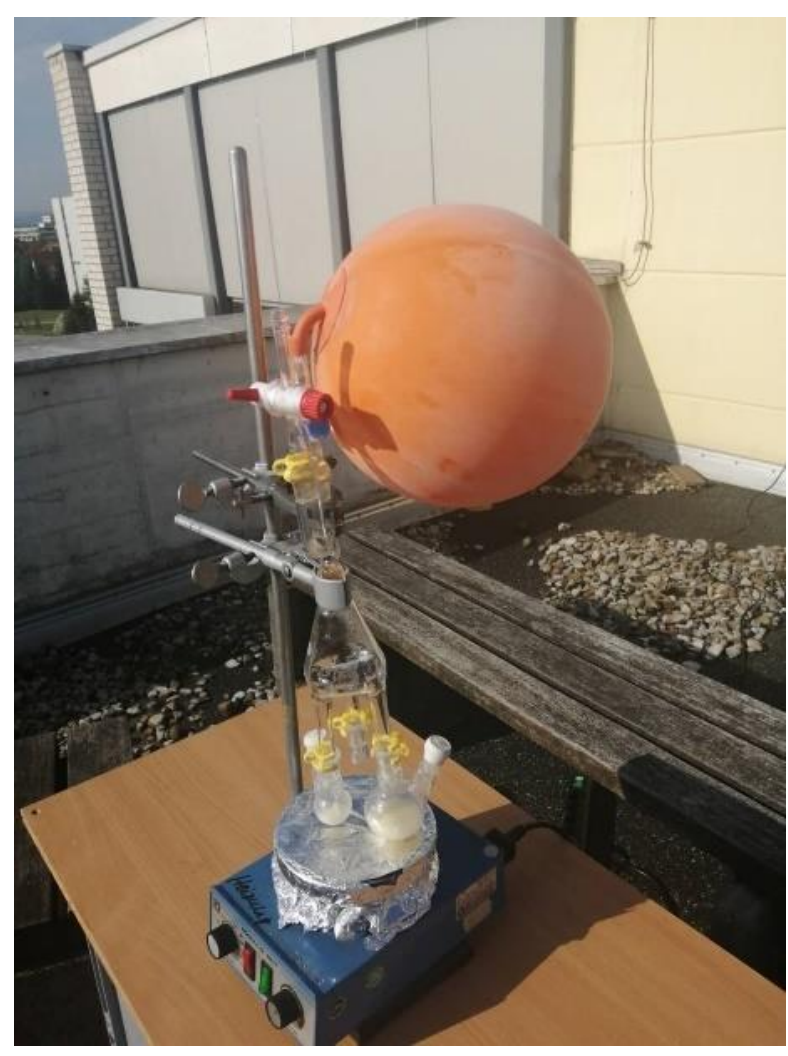

Figure 5.3.1 Hydroxylation and cleavage of $\mathrm{C}=\mathrm{C}$ bonds under sunlight.

\section{Synthesis of Substrates for Hydroxylation via Suzuki-Miyaura Coupling}




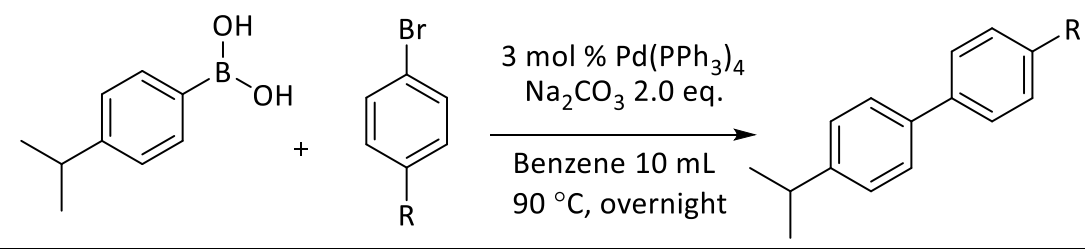

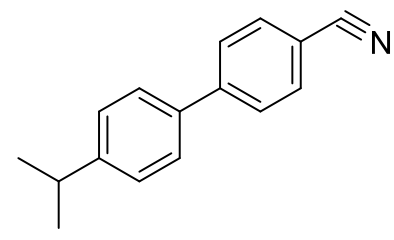

$172 a, 42 \%$<smiles>COc1ccc(-c2ccc(C(C)C)cc2)cc1</smiles>

$173 a, 57 \%$<smiles>CC(C)c1ccc(-c2cc3ccccc3c3ccccc23)cc1</smiles>

$174 a, 55 \%$

Several substrates were synthesized according to the Suzuki Coupling, ${ }^{[130]}$ Firstly, a 50$\mathrm{mL}$ two-necked flask containing a stirring bar was charged with $3.0 \mathrm{mmol}$ of an aryl halide, 3 mol\% catalyst, 2.0 equiv. of Base and 1.1 equiv. of boronic acid. After evacuating the flask three times and filling it two times with nitrogen, nitrogen atmosphere was incorporated. Finally, dry benzene $(10.0 \mathrm{~mL})$ was added. The resulting mixture was stirred overnight under $90^{\circ} \mathrm{C}$. Then, the resulting mixture was subjected to an aqueous workup (using distilled water; or brine in case of slurry phase separation) and was extracted three times with ethyl acetate. The combined organic layers were dried over anhydrous $\mathrm{Na}_{2} \mathrm{SO}_{4}$, filtered and concentrated in vacuo. Products were purified via silica gel chromatography ethyl acetate and $n$-hexane as solvents. The products were obtained and the analytical data were consistent with those given in literature. ${ }^{[130]}$

\section{${ }^{18} \mathrm{O}$-labelling Experiments}

The ${ }^{18} \mathrm{O}$-labeling experiment was performed with ${ }^{18} \mathrm{O}_{2}$ (Sigma Aldrich, ${ }^{18} \mathrm{O}$ atom 99.7\%), and analyzed with ESI-HRMS and NMR showing the ${ }^{18} \mathrm{O}$-labeled product with $a$ isolated yield of $51 \%$. The result showed that the origin of the oxygen atom in the desired product was only from oxygen gas since no ${ }^{16} \mathrm{O}$-labeled product was found.

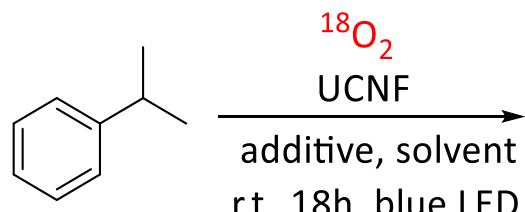<smiles>CC(C)(O)c1ccccc1</smiles>

$51 \%$ isolated yield 
Figure 5.3.2 Hydroxylation of cumene in presence of ${ }^{18} \mathrm{O}$-labeled oxygen.

ESI-HRMS: $m / z$ calcd. for $\mathrm{C}_{9} \mathrm{H}_{12}{ }^{18} \mathrm{O}[\mathrm{M}+\mathrm{H}]^{+}:$139.0966, found 139.1015 .

\section{Procedure for synthesis of nitrogen deficient polymeric carbon nitride}

Nitrogen deficient polymeric carbon nitride was synthesized according to a literature report (Figure 3.2.1). ${ }^{[46]}$ This photocatalyst was synthesized by waldermar Schilling. For its preparation a self-constructed reaction chamber made of VA steel, containing urea $(50 \mathrm{~g})$ and formamide $(0.5 \mathrm{~g})$, fitted in a VA steel tube, was directly heated to $580^{\circ} \mathrm{C}$ in a Gero carbolite oven (type: F70-200, power: $1.5 \mathrm{~kW}$ ) for 244 minutes. The temperature inside the chamber was monitored via a thermoelement and held for $2 \mathrm{~h}$, finally the chamber was cooled to the room temperature.

\subsection{Reaction Procedures for $\mathrm{C}=\mathrm{C}$ Bond Cleavage Reactions}

\section{General Procedure for The Cleavage of Olefins Using Blue LED}

A $10-\mathrm{mL}$ two-necked flask containing a stirring bar was charged with $0.25 \mathrm{mmol}$ of substrate, $8 \mathrm{mg}$ of $\mathrm{g}-\mathrm{C}_{3} \mathrm{~N}_{4}$ and 20 mol\% of $\mathrm{N}$-hydroxysuccinimide. After purging the flask three times with vacuum and two times with nitrogen, oxygen atmosphere was incorporated through an $\mathrm{O}_{2}$ balloon. Finally, dry acetonitrile $(1.0 \mathrm{~mL})$ was added. The resulting mixture was stirred for 9-40 h under $12 \mathrm{~W}$ blue LED irradiation (the progress monitored via GC-MS or TLC). Then, the resulting mixture was subjected to an aqueous workup (using distilled water; or brine in case of slurry phase separation) and was extracted three times with ethyl acetate. The combined organic layers were dried over anhydrous $\mathrm{Na}_{2} \mathrm{SO}_{4}$, filtered and concentrated in vacuo. Products were purified via silica gel chromatography or GPC (see details of products) with ethyl acetate and $n$-hexane as solvents (Figure 5.2.3).

\section{General Procedure for the Cleavage of Olefins Using Sunlight}

A 50-mL two-necked flask containing a stirring bar was charged with $1.0 \mathrm{~g}$ of substrate, $80 \mathrm{mg}$ or $50 \mathrm{mg}$ of g- $\mathrm{C}_{3} \mathrm{~N}_{4}$ and 20 mol\% of $\mathrm{N}$-hydroxysuccinimide depending on the amount of starting material. After purging the flask three times with vacuum 
and two times with nitrogen, oxygen atmosphere was incorporated through an $\mathrm{O}_{2}$ balloon. Finally, dry acetonitrile $(12.0 \mathrm{~mL}$ or $10.0 \mathrm{~mL})$ was added. The resulting mixture was stirred under sunlight (the setup was put on the roof of building, seeing the (Figure 5.2.5). And the progress was monitored via GC-MS). Then, the resulting mixture underwent an aqueous workup (using distilled water; or brine in case of slurry phase separation) and was extracted three times with ethyl acetate. The combined organic layers were dried over anhydrous $\mathrm{Na}_{2} \mathrm{SO}_{4}$, filtered and concentrated in vacuo. Products were purified via silica gel chromatography with ethyl acetate and $n$-hexane as solvents.

\section{${ }^{18} \mathrm{O}$-Labelling Experiments}

The ${ }^{18} \mathrm{O}$-labeling experiment was performed with ${ }^{18} \mathrm{O}_{2}$ (Sigma Aldrich, ${ }^{18} \mathrm{O}$ atom 99.7\%), and analyzed with ESI-HRMS and NMR showing the ${ }^{18} \mathrm{O}-$ labeled product with an isolated yield of $72 \%$. The result showed that the origin of the oxygen atom in the desired product was only from oxygen gas since no ${ }^{16} \mathrm{O}$-labeled product was found.<smiles>C=C(C)c1ccc(F)cc1</smiles>

$175 a$

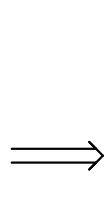

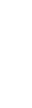

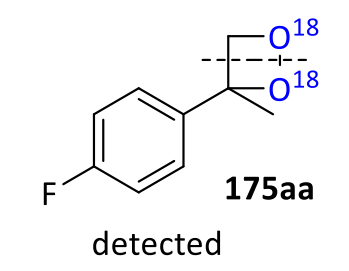

$(\mathrm{M}+\mathrm{H})^{+}=173.0744$

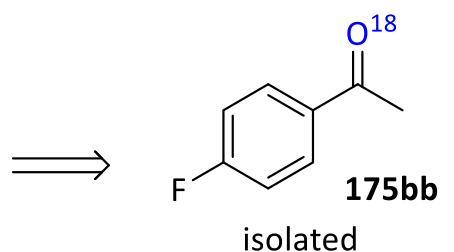

$(\mathrm{M}+\mathrm{H})^{+}=141.0596$

Figure 3.3.7 Oxidation of 4-fluoro- $\alpha$-methylstyrene in presence of ${ }^{18} \mathrm{O}$-labeled oxygen.

ESI-HRMS: $m / z$ calcd. for $\mathrm{C}_{8} \mathrm{H}_{7} \mathrm{~F}^{18} \mathrm{O}[\mathrm{M}+\mathrm{H}]^{+}:$: 141.0559, found 141.0596 .

The intermediate was also detected with ESI-HRMS: $m / z$ calcd. for $\mathrm{C}_{9} \mathrm{H}_{9} \mathrm{~F}^{18} \mathrm{O}_{2}[\mathrm{M}+\mathrm{H}]^{+}$: 173.0665, found 173.0744 .

\section{General Procedure for Synthesis and Characterization of Catalyst}

Melamine (15 g), was loaded into a stainless-steel chamber with a semi-closed lid. Prepared chamber inserted into a stainless-steel heating chamber, which was heated 
to $600{ }^{\circ} \mathrm{C}$ (inside temperature) by setting the temperature of the GERO carbolite oven to $635^{\circ} \mathrm{C}$ (outside temperature at the heating coil) (type F70-200, power: $1.5 \mathrm{~kW}$ ). The heating ramp was set to achieve the target temperature in 30 minutes. The temperature was maintained for about two hours, followed by cooling the chamber to room temperature in 6 hours (Figure 5.2.6).

An aqueous hydrochloric acid $(\mathrm{HCl})$ solution (18\%) was used to neutralize the formed ammonia gas leaving the reaction chamber. ${ }^{[131]}$ Afterwards, the synthesized PCN was characterized by Infrared Spectroscopy (IR) and Scanning Electron Microscope (SEM) (Figure 5.4.1).

IR (FTIR): $v\left(\mathrm{~cm}^{-1}\right)=1635,1397,1316,1234,808$. Compared to the reference, ${ }^{[132]}$ the morphology of PCN is similar but our particles have relatively more branches.<smiles>Nc1nc(N)nc(N)n1</smiles>

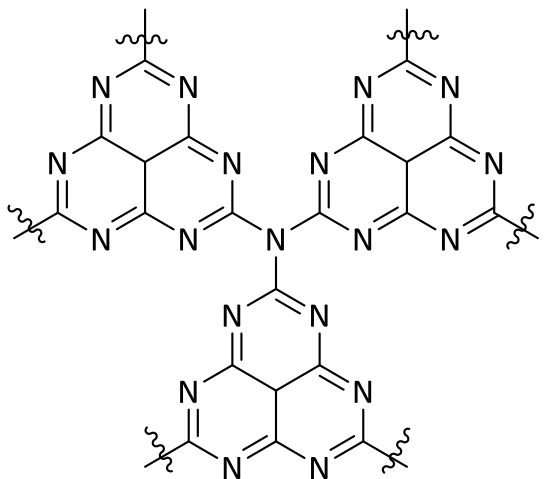

polymeric carbon nitride

Figure 5.4.1 Synthesis of the polymeric carbon nitride (PCN).

For the TEM and SEM analysis, $5 \mathrm{mg}$ of the catalyst was mixed with $1 \mathrm{ml}$ of THF and treated with ultrasonic sound. One drop of this mixture was applied to a TEM Grid. The TEM analysis including electron diffraction was carried out with a Phillips CM12 instrument at $120 \mathrm{kV}$. For the HRTEM analysis an aberration corrected FEI Titan electron microscope with $300 \mathrm{keV}$ electrons was deployed. The resolution limit in high vacuum is about $0.08 \mathrm{~nm}$ for this microscope.

SEM analysis was carried out at the same Grids which were used for the TEM analysis. Here a Nova NanoSem 650 in-situ SEM from FEI was employed. At an acceleration 
voltage of $15 \mathrm{kV}$ a "through the lens" (TTL) detector was used to take images (Figure 5.2.7).
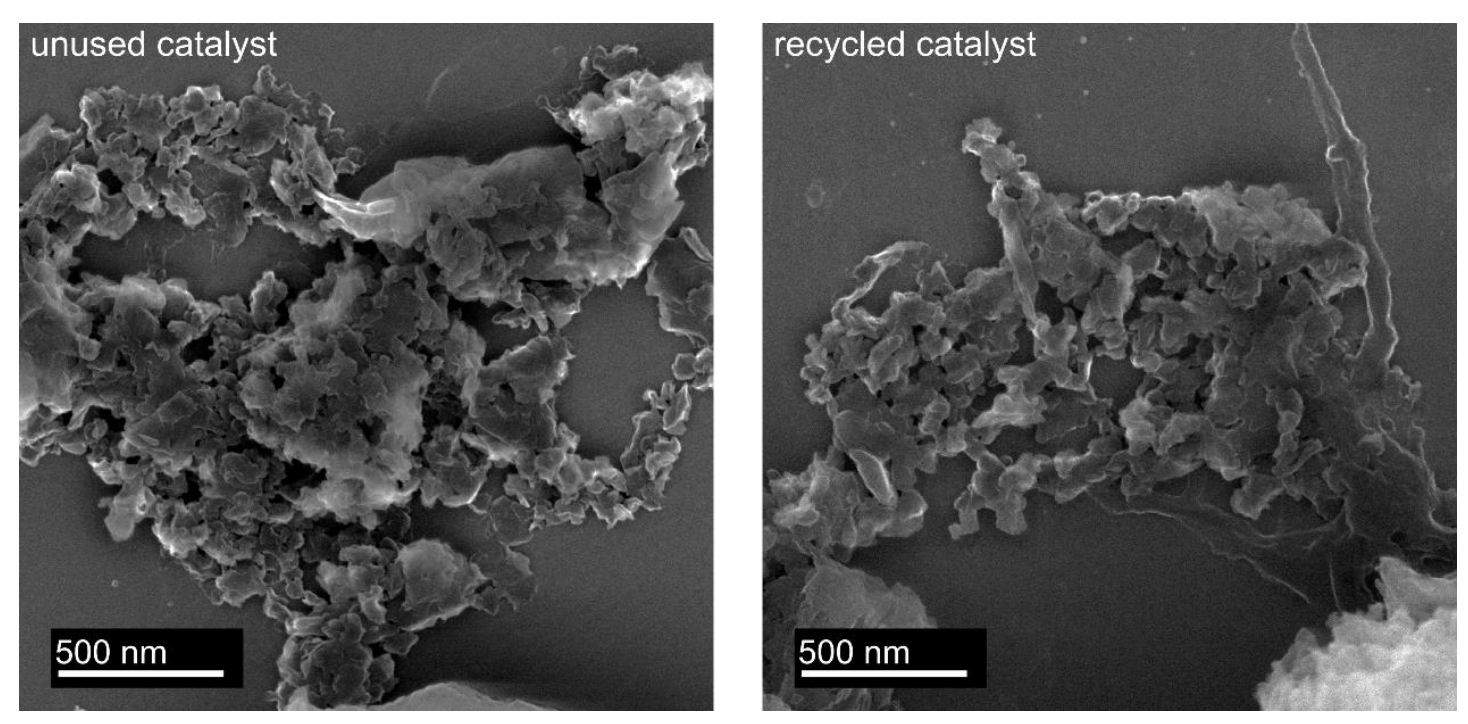

Figure 5.2.7 SEM of synthesized of PCN. (left) unused catalyst; (right) recycled catalyst.

\section{Recycling of The Catalysts}

After normal reactions according to the general procedure for the cleavage of olefins using Blue LED, the reaction mixture was transferred into centrifuge tube with addition of $20 \mathrm{ml}$ acetone. Meanwhile, the same weight of centrifuge tube was prepared filling with water. Then both centrifuge tubes were put into the centrifuge. The deposit of catalyst was formed after 10 min running of centrifuge with speed of $9000 \mathrm{r} / \mathrm{s}$. Afterwards, the catalyst in the centrifuge tube was washed with acetone and water three times, respectively. At last, the catalyst was dried in the oven overnight with $60^{\circ} \mathrm{C}$ and reused for the next reaction.

\subsection{Reaction Procedures of Blue LED-Mediated (aza)-Diels-Alder Reactions}

\section{General Procedure for Blue LED-Mediated Diels-Alder Reactions}

A $10-\mathrm{mL}$ one-necked flask containing a stirring bar was charged with the dienophile (1.0 equiv., $0.50 \mathrm{mmol})$, fluorenone $(3 \mathrm{~mol} \%, 15 \mu \mathrm{mol})$ and nitromethane $(5.0 \mathrm{~mL})$. The diene (3.0 equiv., $1.5 \mathrm{mmol}$ ) was added to the stirred solution (for $12 \mathrm{c}$ no diene was added). The resulting mixture was stirred for $2-44 \mathrm{~h}$ at room temperature under $12 \mathrm{~W}$ blue LED irradiation (the progress monitored via GC-MS or TLC). The resulting 
mixture is subjected to an aqueous workup using brine $(3.0 \mathrm{~mL})$ and was extracted three times with ethyl acetate $(3 \times 3.0 \mathrm{~mL})$. The combined organic layers were dried over anhydrous $\mathrm{Na}_{2} \mathrm{SO}_{4}$, filtered and concentrated in vacuo. The crude product was purified by silica gel preparative TLC using a mixture of $n$-hexane and ethyl acetate as eluent.

\section{General Procedure for Blue LED-Mediated aza-Diels-Alder Reactions}

A $10-\mathrm{mL}$ one-necked flask containing a stirring bar was charged with the imine (1.0 equiv., $0.1 \mathrm{mmol}$ ) or ketone, 9-fluorenone (3 mol\%, $3.0 \mu \mathrm{mol})$ and nitromethane (5.0 mL). Danishefsky's diene (3.0 equiv., $0.3 \mathrm{mmol}$ ) was added to the stirred solution (other dienes were used in some cases). The resulting mixture was stirred for 2-8 $\mathrm{h}$ at room temperature under $12 \mathrm{~W}$ blue LED irradiation (the progress can be monitored via GC-MS or TLC). The resulting mixture is subjected to an aqueous workup using brine $(3.0 \mathrm{~mL})$ and was extracted three times with ethyl acetate $(3 \times 3.0 \mathrm{~mL})$. The combined organic layers were dried over anhydrous $\mathrm{Na}_{2} \mathrm{SO}_{4}$, filtered and concentrated in vacuo. The crude product was purified by silica gel preparative TLC using a mixture of $n$-hexane and ethyl acetate as eluent (Figure 5.2.4).
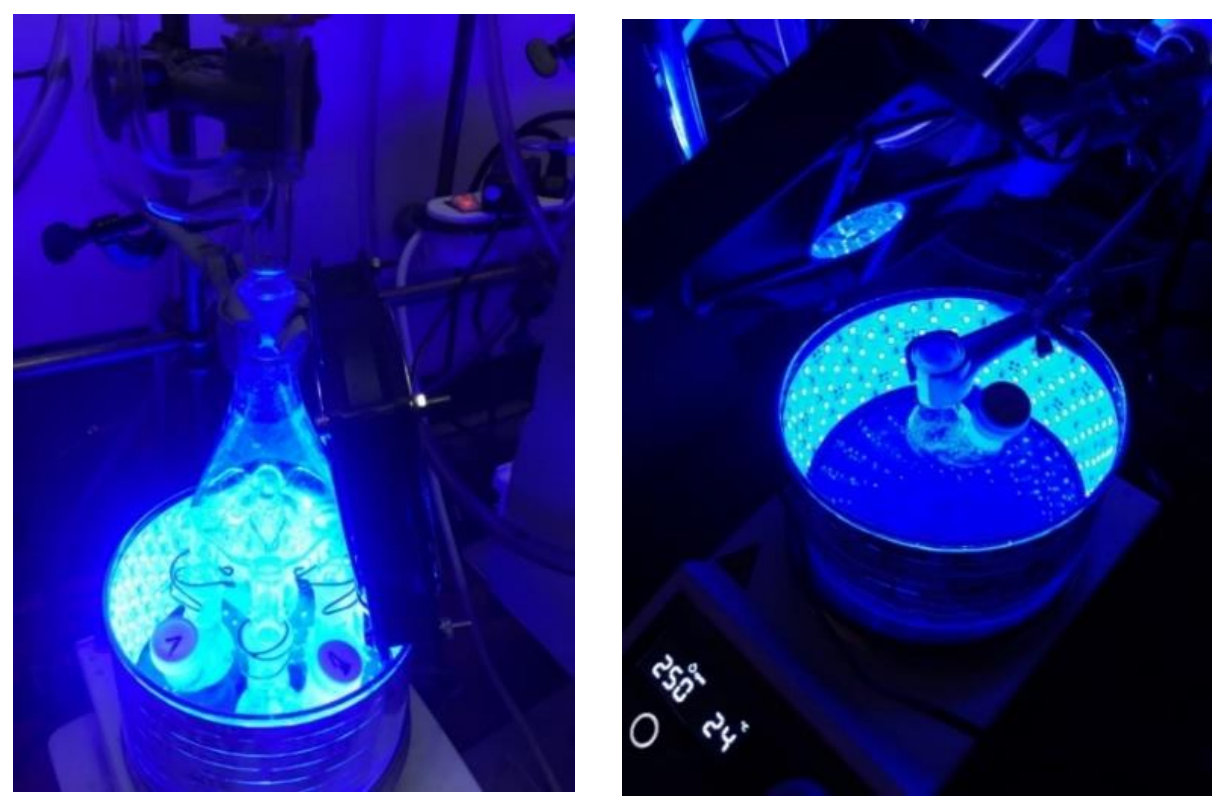

Figure 5.2.4 Set-up for blue LED-mediated (aza)-Diels-Alder reactions. 


\section{General Procedures of Synthesizing the Imines}

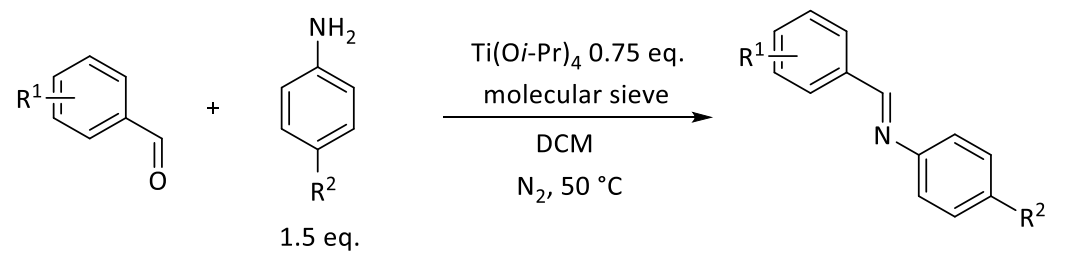

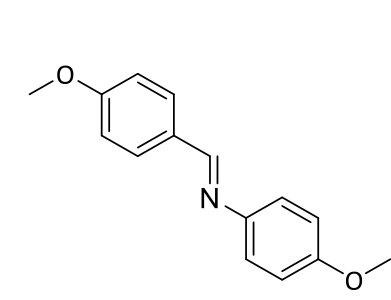

218a, 21\%, 93h

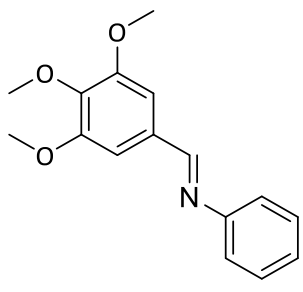

219a,79\%, 88h

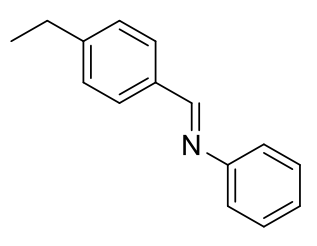

220 a, $48 \%, 88 h$

Molecular sieve $3 \AA \AA$, the aldehyde (1.0 equiv., $20 \mathrm{mmol}$ ) and 4-nitroaniline (1.0 equiv., $20 \mathrm{mmol}, 2.8 \mathrm{~g}$ ) were added to a Schlenk tube. The tube was evacuated and back filled with nitrogen and the $\mathrm{DCM}(40 \mathrm{~mL})$ was added at room temperature. Then the titanium isopropoxide ( 0.75 equiv., $15 \mathrm{mmol}, 4.4 \mathrm{~mL}$ ) was added slowly into the reaction solution. The reaction mixture was stirred for $88 \mathrm{~h}-93 \mathrm{~h}$ at $50{ }^{\circ} \mathrm{C}$ under nitrogen atmosphere. After cooling to ambient temperature, the suspension was filtered and concentrated in vacuo. The crude product was purified by silica gel column chromatography using a mixture of $n$-pentane and EtOAc $(9: 1)$ as eluent with an addition of 5 vol\% triethylamine. Imines were obtained and the analytical data were consistent with those given in literature. ${ }^{[132]}$

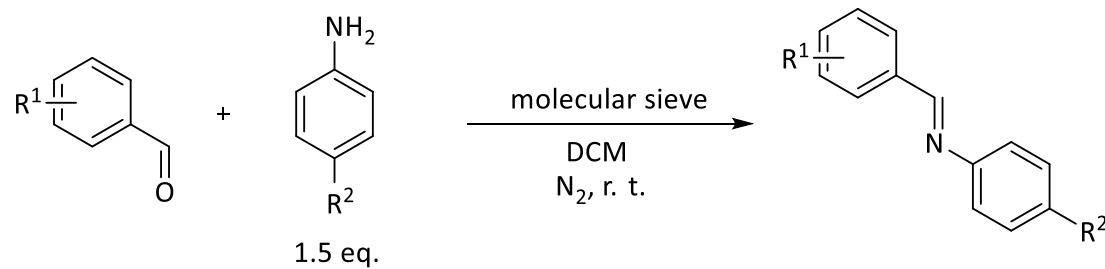

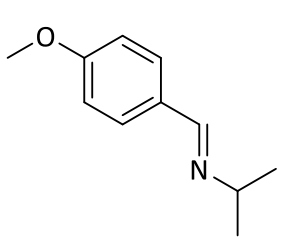

223a, $66 \%, 19 h$

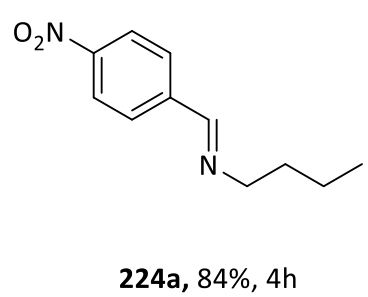

224a, $84 \%, 4 h$

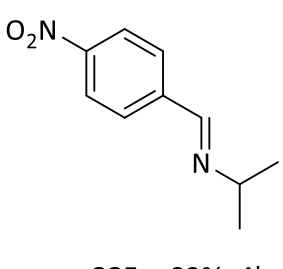

225a, $83 \%, 4 h$

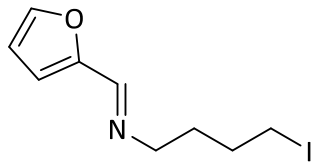

226a, $87 \%, 5 \mathrm{~h}, 50^{\circ} \mathrm{C}$

Molecular sieve $3 \AA$, the aldehyde (1.0 equiv., $20 \mathrm{mmol}$ ) were added to the Schlenk 
tube. The tube was evacuated and back filled with nitrogen and the DCM ( $40 \mathrm{~mL})$ were added at room temperature. Then the aliphatic primary amine (1.0 equiv., $10 \mathrm{mmol}$ ) was added. The reaction mixture was stirred for $2 \mathrm{~h}-19 \mathrm{~h}$ at room temperature under nitrogen atmosphere. The suspension was filtered and concentrated in vacuo. The crude product was purified by silica gel column chromatography using a mixture of $n$ pentane and EtOAc (19:1) as eluent with an addition of 5 vol\% triethylamine. The imines products were obtained and the analytical data were consistent with those given in literature. ${ }^{[133]}$ 
5.6 Analytical Data for the Products

Data for Visible-Light-Mediated Efficient Metal-Free Catalyst for $\alpha$-Oxygenation of Tertiary Amines to Amides<smiles>CN(C)C(=O)c1ccccc1</smiles>

3 mol\% rose bengal, 1.5 eq DBN, 24 h; $\boldsymbol{N}, \boldsymbol{N}$-Diethylbenzamide (126b): ${ }^{1} \mathrm{H}$ NMR ( $\mathrm{CDCl}_{3}$, $300 \mathrm{MHz}): \delta 7.39(\mathrm{~m}, 5 \mathrm{H}), 3.13(\mathrm{~s}, 3 \mathrm{H}), 2.99(\mathrm{~s}, 3 \mathrm{H}) ;{ }^{13} \mathrm{C} \mathrm{NMR}\left(\mathrm{CDCl}_{3}, 75 \mathrm{MHz}\right): \delta$ 171.6, 136.4, 129.5, 128.3, 127.0, 39.6, 35.3; MS (GC-MS): $m / z 149\left(\mathrm{M}^{+}\right) ;{ }^{[134]}$ Yield: $70 \%$.<smiles>O=C(c1ccc(F)cc1)N1CCCCC1</smiles>

4 mol\% rose bengal, 2.0 eq DBN, 40 h; 1-(4-Fluorobenzoyl)piperidine (127b): ${ }^{1} \mathrm{H}$ NMR $\left(\mathrm{CDCl}_{3}, 300 \mathrm{MHz}\right): \delta 7.43-7.39(\mathrm{~m}, 2 \mathrm{H}), 7.12-7.06(\mathrm{~m}, 2 \mathrm{H}), 3.66(\mathrm{~s}, 2 \mathrm{H}), 3.36(\mathrm{br} \mathrm{s}$,

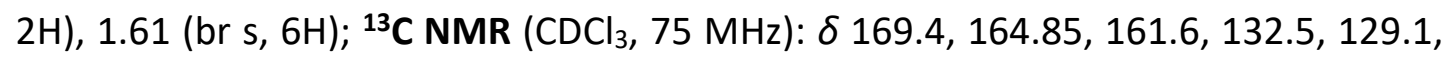
115.6, 115.3, 48.9, 43.3, 26.4, 25.8, 24.6; MS (GC-MS): m/z 207 (M+); ;135] Yield: 74\%.<smiles>O=C(c1ccc([N+](=O)[O-])cc1)N1CCCCC1</smiles>

4 mol\% rose bengal, 2.0 eq DBN, 24 h; 1-(4-Nitrobenzoyl)piperidine (128b): ${ }^{1} \mathbf{H}$ NMR $\left(\mathrm{CDCl}_{3}, 300 \mathrm{MHz}\right): \delta 8.28(\mathrm{~d}, J=8.0 \mathrm{~Hz}, 2 \mathrm{H}), 7.57(\mathrm{~d}, J=8.0 \mathrm{~Hz}, 2 \mathrm{H}), 3.74(\mathrm{br} \mathrm{s}, 2 \mathrm{H})$, 3.29 (br s, 2H), 1.71 (br s, 4H), 1.54 (br s, 2H). $\left.{ }^{13} \mathrm{C} \mathrm{NMR} \mathrm{(CDCl}, 75 \mathrm{MHz}\right): \delta$ 168.0, 148.3, 142.8, 127.9, 124.0, 48.8, 43.3, 26.6, 25.6, 24.5; MS (GC-MS): $m / z 233\left(\mathrm{M}^{+}\right)$; ${ }^{[136]}$ Yield: $83 \%$. 
<smiles>Cc1ccccc1C(=O)N1CCCCC1</smiles>

4 mol\% rose bengal, 2.0 eq DBN, 24 h; 1-(2-Methylbenzoyl)piperidine (129b): ${ }^{1} \mathrm{H}$ NMR $\left(\mathrm{CDCl}_{3}, 300 \mathrm{MHz}\right): \delta 7.28-7.13(\mathrm{~m}, 4 \mathrm{H}), 3.80$ (br s, 1H), 3.71 (br s, 1H), 3.19 (br s, 2H), 2.31 (s, 3H), 1.66 (br s, 4H), 1.46 (br s, 2H); $\left.{ }^{13} \mathrm{C} \mathrm{NMR} \mathrm{(CDCl} 3,75 \mathrm{MHz}\right): \delta 169.8$, $136.8,134.0,130.3,128.5,125.8,125.6,47.8,42.4,26.6,25.7,24.6,19.0$; MS (GCMS): $m / z 203\left(\mathrm{M}^{+}\right) ;{ }^{[136]}$ Yield: $64 \%$.<smiles>Cc1ccc(C(=O)N2CCCCC2)cc1</smiles>

4 mol\% rose bengal, 2.0 eq DBN, 24 h; 1-(2-Methylbenzoyl)piperidine (130b): ${ }^{1} \mathbf{H}$ NMR $\left(\mathrm{CDCl}_{3}, 300 \mathrm{MHz}\right): \delta 7.24(\mathrm{~d}, J=8.0 \mathrm{~Hz}, 2 \mathrm{H}), 7.12(\mathrm{~d}, J=8.0 \mathrm{~Hz}, 2 \mathrm{H}), 3.63$ (br s,

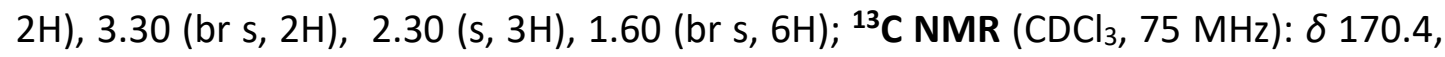
139.3, 133.5, 128.9, 126.8, 48.7, 43.1, 26.5, 25.7, 24.6, 21.3; MS (GC-MS): m/z 203 $\left(\mathrm{M}^{+}\right) ;{ }^{[136]}$ Yield: $78 \%$.<smiles>O=C1c2ccccc2CCN1c1ccccc1</smiles>

3 mol\% rose bengal, 1.5 eq DBN, 16 h; 2-Phenyl-3,4-dihydroisoquinolin-1(2H)-one (131b): ${ }^{1} \mathrm{H}$ NMR $\left(\mathrm{CDCl}_{3}, 300 \mathrm{MHz}\right): \delta 8.19$ (dd, $\left.J=7.7,1.5 \mathrm{~Hz}, 1 \mathrm{H}\right), 7.43(\mathrm{~m}, 6 \mathrm{H}), 7.28$ $(\mathrm{m}, 2 \mathrm{H}), 4.01(\mathrm{t}, J=6.0 \mathrm{~Hz}, 2 \mathrm{H}), 3.16(\mathrm{t}, J=6.4 \mathrm{~Hz}, 2 \mathrm{H}) ;{ }^{13} \mathrm{C} \mathrm{NMR}\left(\mathrm{CDCl}_{3}, 75 \mathrm{MHz}\right): \delta$ 164.2, 143.1, 138.3, 132.0, 129.7, 128.9, 128.7, 127.2, 127.0, 126.3, 125.3, 49.4, 28.7; MS (GC-MS): $m / z 223\left(\mathrm{M}^{+}\right) ;{ }^{[137]}$ Yield: 95\%. 
<smiles>CCN1CCc2ccccc2C1=O</smiles>

3 mol\% rose bengal, 1.5 eq DBN, 16 h; 2-Ethyl-3,4-dihydroisoquinolin-1(2H)-one (132b): ${ }^{1} \mathrm{H}$ NMR $\left(\mathrm{CDCl}_{3}, 300 \mathrm{MHz}\right): \delta 8.08(\mathrm{dd}, J=7.5,1.6 \mathrm{~Hz}, 1 \mathrm{H}), 7.29(\mathrm{~m}, 3 \mathrm{H})$, 3.63-3.55 (m, 4H), $2.99(\mathrm{t}, J=6.6 \mathrm{~Hz}, 2 \mathrm{H}), 1.22(\mathrm{t}, J=7.2 \mathrm{~Hz}, 3 \mathrm{H}) ;{ }^{13} \mathrm{C} \mathrm{NMR}\left(\mathrm{CDCl}_{3}, 75\right.$ $\mathrm{MHz}$ ): $\delta$ 164.0, 137.9, 131.4, 129.7, 128.2, 127.0, 126.8, 45.5, 42.2, 28.2, 12.8; MS (GC-MS): $m / z 175\left(\mathrm{M}^{+}\right) ;{ }^{[138]}$ Yield: $91 \%$.<smiles>O=C1c2ccccc2CCN1CC1CC1</smiles>

3 mol\% rose bengal, 1.5 eq DBN, 16 h; 2-(Cyclopropylmethyl)-3,4dihydroisoquinolin-1(2H)-one (133b): ${ }^{1} \mathrm{H}$ NMR (300 MHz, $\left.\mathrm{CDCl}_{3}\right): \delta 8.11$ (dd, J= 7.6, $1.5 \mathrm{~Hz}, 1 \mathrm{H}), 7.45-7.43(\mathrm{~m}, 2 \mathrm{H}), 7.19(\mathrm{~d}, J=8.1 \mathrm{~Hz}, 1 \mathrm{H}), 3.73-3.62(\mathrm{~m}, 2 \mathrm{H}), 3.50$ $(\mathrm{d}, J=6.9 \mathrm{~Hz}, 2 \mathrm{H}), 3.03(\mathrm{t}, J=6.6 \mathrm{~Hz}, 2 \mathrm{H}), 1.10(\mathrm{~s}, 1 \mathrm{H}), 0.63-0.23(\mathrm{~m}, 4 \mathrm{H}) ;{ }^{13} \mathrm{C}$ NMR $\left(75 \mathrm{MHz} \mathrm{CDCl}_{3}\right) \delta 164.3,138.0,131.4,129.7,128.3,127.0,126.8,51.4,46.1,28.2$, 9.6, 3.5; ESI-HRMS: $m / z$ calcd. for $\mathrm{C}_{13} \mathrm{H}_{15} \mathrm{NO}[\mathrm{M}+\mathrm{H}]^{+}:$202.1232, found 202.1227; Yield: $85 \%$.<smiles>CCCCCCN1CCc2cc(OC)c(OC)cc2C1=O</smiles>

3 mol\% rose bengal, 1.5 eq DBN, 16 h; 2-Hexyl-6,7-dimethoxy-3,4-

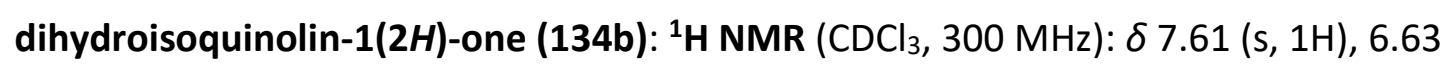
$(\mathrm{s}, 1 \mathrm{H}), 3.93(\mathrm{~s}, 3 \mathrm{H}), 3.92(\mathrm{~s}, 3 \mathrm{H}), 3.53(\mathrm{~m}, 4 \mathrm{H}), 2.91(\mathrm{t}, J=6.8 \mathrm{~Hz}, 2 \mathrm{H}), 1.62(\mathrm{~m}, 2 \mathrm{H})$, $1.33(\mathrm{~m}, 6 \mathrm{H}), 0.89(\mathrm{~m}, 3 \mathrm{H}) ;{ }^{13} \mathrm{C} \mathrm{NMR}\left(\mathrm{CDCl}_{3}, 75 \mathrm{MHz}\right): \delta 164.2,151.6,147.9,131.5$, $122.3,110.6,109.2$, 56.0, 56.0, 47.5, 46.3, 31.6, 27.82, 27.8, 26.6, 22.6, 14.0; ESIHRMS: $m / z$ calcd. for $\mathrm{C}_{17} \mathrm{H}_{25} \mathrm{NO}_{3}[\mathrm{M}+\mathrm{Na}]^{+}: 314.1732$, found 314.1727; Yield: $78 \%$. 


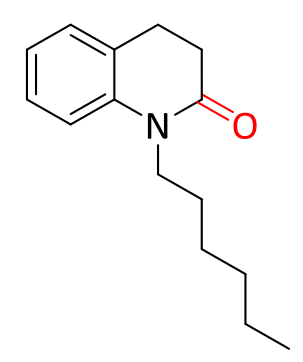

6 mol\% rose bengal, 2.0 eq DBN, 40 h; 1-Hexyl-3,4-dihydroquinolin-2(1H)-one (135b): ${ }^{1} \mathrm{H}$ NMR (300 MHz, $\left.\mathrm{CDCl}_{3}\right): \delta 7.24-7.15(\mathrm{~m}, 2 \mathrm{H}), 7.02-6.97(\mathrm{~m}, 2 \mathrm{H}), 3.92(\mathrm{~m}$, $2 \mathrm{H}), 2.88(\mathrm{~m}, 2 \mathrm{H}), 2.63(\mathrm{~m}, 2 \mathrm{H}), 1.64(\mathrm{~m}, 2 \mathrm{H}), 1.30(\mathrm{~m}, 6 \mathrm{H}), 0.88(\mathrm{~m}, 3 \mathrm{H}) ;{ }^{13} \mathrm{C}$ NMR $(75$ $\left.\mathrm{MHz}, \mathrm{CDCl}_{3}\right): \delta 170.1,139.7,128.0,127.4,126.6,122.6,114.8,42.2,32.0,31.5,27.2$, 26.6, 25.6, 22.6, 14.0; ESI-HRMS: $m / z$ calcd. for $\mathrm{C}_{15} \mathrm{H}_{21} \mathrm{NO}[\mathrm{M}+\mathrm{H}]^{+}: 232.1701$, found 232.1695; Yield: 60\%; Purification with GPC.<smiles>O=C(c1ccncc1)N1CCCCC1</smiles>

4 mol\% rose bengal, 1.5 eq DBN, 24 h; 1-(4-Pyridinecarbonyl)piperidine (136b): ${ }^{1} \mathrm{H}$ NMR $\left(\mathrm{CDCl}_{3}, 300 \mathrm{MHz}\right): \delta 8.70(\mathrm{~s}, 4 \mathrm{H}), 3.75(\mathrm{~m}, 2 \mathrm{H}), 3.32(\mathrm{t}, J=5.0 \mathrm{~Hz}, 2 \mathrm{H}), 1.71$ (br s,

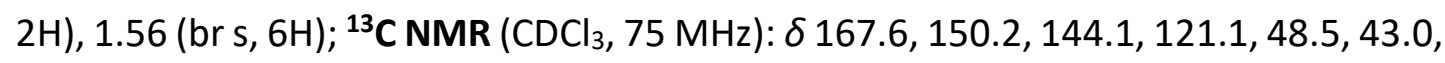
26.5, 25.5, 24.4; MS (GC-MS): $m / z 190\left(\mathrm{M}^{+}\right) ;{ }^{[136]}$ Yield: $72 \%$.<smiles>O=C(c1ccco1)N1CCCCC1</smiles>

3 mol\% rose Bengal, 1.5 eq DBN, 20 h; Furan-2-yl-piperidin-1-yl-methanone (137b): ${ }^{1} \mathrm{H}$ NMR $\left(300 \mathrm{MHz} \mathrm{CDCl}_{3}\right.$ ): $\delta 7.44$ (dd, $J=0.9 \mathrm{~Hz}, 1.9 \mathrm{~Hz}, 1 \mathrm{H}$ ), 6.88 (dd, $J=0.9,3.5 \mathrm{~Hz}$, 1H), 6.37 (dd, $J=1.8,3.5 \mathrm{~Hz}, 1 \mathrm{H}$ ) ), 3.64 (br s, 4H), 1.63 (br s, 6H); ${ }^{13} \mathrm{C}$ NMR (75 MHz, $\mathrm{CDCl}_{3}$ ): $\delta$ 159.2, 148.2, 143.3, 115.3, 111.0, 47.4, 44.4, 26.2, 24.7; MS (GC-MS): $\mathrm{m} / \mathrm{z}$ $179\left(\mathrm{M}^{+}\right) ;{ }^{[139]}$ Yield: $80 \%$. 
<smiles>O=C(c1cccs1)N1CCCCC1</smiles>

3 mol\% rose Bengal, 1.5 eq DBN, 20 h; Piperidin-1-yl-thiophen-2-yl-methanone (138b): ${ }^{1} \mathbf{H}$ NMR (300 MHz, CDCl 3 ): $\delta 7.42-7.40$ (dd, $\left.J=1.1 \mathrm{~Hz}, J=5.5 \mathrm{~Hz}, 1 \mathrm{H}\right), 7.28-$ $7.26(\mathrm{dd}, J=1.2,3.7 \mathrm{~Hz}, 1 \mathrm{H}), 7.05-7.02(\mathrm{dd}, 1 \mathrm{H}, J=3.6,4.8 \mathrm{~Hz}, 1 \mathrm{H}), 3.66(\mathrm{t}, J=5.6 \mathrm{~Hz}$, $4 \mathrm{H}), 1.72-1.63(\mathrm{~m}, 6 \mathrm{H}) ;{ }^{13} \mathrm{C} \mathrm{NMR}\left(75 \mathrm{MHz}, \mathrm{CDCl}_{3}\right): \delta 163.5,137.6,128.2,128.0,126.5$, 46.6, 26.1, 24.6; MS (GC-MS): $m / z 195\left(\mathbf{M}^{+}\right)$; ${ }^{[139]}$ Yield: 82\%.<smiles>O=C(c1ccccc1)N1CCCC1</smiles>

4 mol\% rose bengal, 1.5 eq DBN, $24 \mathrm{~h}$; Phenyl(pyrrolidin-1-yl)methanone (139b): ${ }^{1} \mathrm{H}$ NMR $\left(\mathrm{CDCl}_{3}, 300 \mathrm{MHz}\right): \delta 7.45(\mathrm{~m}, 5 \mathrm{H}), 3.58(\mathrm{t}, J=6.9 \mathrm{~Hz}, 2 \mathrm{H}), 3.42(\mathrm{t}, J=6.5 \mathrm{~Hz}, 2$

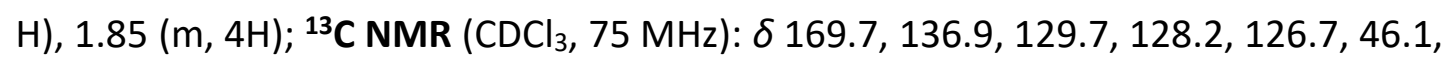
45.0, 26.2, 24.2; MS (GC-MS): $m / z 175\left(\mathrm{M}^{+}\right)$; ${ }^{[140]}$ Yield: 76\%.<smiles>O=C1CCCCN1c1ccccc1</smiles>

3 mol\% rose bengal, 1.5 eq DBN, 24 h; Phenylpiperidin-2-one (140b): ${ }^{1} \mathrm{H}$ NMR (300 $\left.\mathrm{MHz}, \mathrm{CDCl}_{3}\right): \delta 7.46-7.38(\mathrm{~m}, 2 \mathrm{H}), 7.29-7.25(\mathrm{~m}, 3 \mathrm{H}), 3.69(\mathrm{t}, J=5.9 \mathrm{~Hz}, 2 \mathrm{H}), 2.60$ $(\mathrm{t}, J=5.8 \mathrm{~Hz}, 2 \mathrm{H}), 1.95(\mathrm{~m}, 4 \mathrm{H}) ;{ }^{13} \mathrm{C} \mathrm{NMR}\left(75 \mathrm{MHz} \mathrm{CDCl}_{3}\right): \delta 170.0,129.2,129.2,126.7$, 126.2, 51.7, 32.9, 23.6, 21.5; MS (GC-MS): $m / z 175\left(\mathrm{M}^{+}\right)$; ${ }^{[141]}$ Yield: 65\%. 
<smiles>O=C(/C=C/c1ccccc1)N1CCCCC1</smiles>

3 mol\% rose bengal, 2.0 eq DBN, $40 \mathrm{~h}$; 1-Cinnamoylpiperidine (141b): ${ }^{1} \mathrm{H}$ NMR $\left(\mathrm{CDCl}_{3}\right.$, $300 \mathrm{MHz}): \delta 7.66(\mathrm{~d}, J=15.5 \mathrm{~Hz}, 1 \mathrm{H}), 7.52(\mathrm{~m}, 2 \mathrm{H}), 7.39-7.34(\mathrm{~m}, 3 \mathrm{H}), 6.91(\mathrm{~d}, J=$ $15.5 \mathrm{~Hz}, 1 \mathrm{H}), 3.65$ (br s, 4H), 1.63 (br s, 6H); ${ }^{13} \mathrm{C} \mathrm{NMR}\left(\mathrm{CDCl}_{3}, 75 \mathrm{MHz}\right.$ ): $\delta 165.2,14201$, 135.4, 129.3, 128.6, 127.7, 117.7, 47.0, 43.4, 26.8, 25.7, 24.7; MS (GC-MS): m/z 215 $\left(\mathrm{M}^{+}\right) ;[136]$ Yield: 65\%. Purification with GPC.<smiles>CCOC(=O)c1ccc(C(=O)N2CCOCC2)cc1</smiles>

3 mol\% rose bengal, 1.5 eq DBN, 40 h; Ethyl 4-(morpholine-4-carbonyl)benzoate (142b): ${ }^{1} \mathrm{H}$ NMR (300 MHz, $\mathrm{CDCl}_{3}$ ): $\delta 8.11$ (d, J=8.5 Hz, 2H), $7.48(\mathrm{~d}, J=8.5 \mathrm{~Hz}, 2 \mathrm{H}$ ), $4.41(\mathrm{q}, J=7.1 \mathrm{~Hz}, 2 \mathrm{H}), 3.80-3.40(\mathrm{~m}, 8 \mathrm{H}), 1.43(\mathrm{t}, J=7.1 \mathrm{~Hz}, 3 \mathrm{H}) ;{ }^{13} \mathrm{C}$ NMR $(100$ $\left.\mathrm{MHz}, \mathrm{CDCl}_{3}\right): \delta 169.4,165.8,139.5,131.7,129.8,127.0,66.8,61.3,14.3$; MS (GC-MS): $m / z 263\left(\mathrm{M}^{+}\right) ;{ }^{[142]}$ Yield: 71\%.<smiles>O=C(c1ccccc1)N1CCC(c2ccccc2)CC1</smiles>

3 mol\% rose bengal, 1.5 eq DBN, 40 h; 1-Benzoyl-4-phenylpiperidine (143b): ${ }^{1} \mathbf{H}$ NMR (300 MHz, $\left.\mathrm{CDCl}_{3}\right): \delta 7.45-7.22(\mathrm{~m}, 10 \mathrm{H}), 4.86$ (br s, 1H), 3.89 (br s, 1H), 3.14 (br s, 1H), $2.86-2.78(\mathrm{~m}, 2 \mathrm{H}), 1.94-1.59$ (br s, 4H); ${ }^{13} \mathrm{C} \mathrm{NMR}\left(\mathrm{CDCl}_{3}, 75 \mathrm{MHz}\right): \delta$ 170.4, 145.1, 136.3, 129.5, 128.7, 128.6, 126.9, 126.7, 126.5, 53.4, 42.8, 33.7, 33.0; MS (GC-MS): m/z 265 (M+); $^{+136]}$ Yield: 74\%. 
${ }^{N}$

6 mol\% rose bengal, 2.5 eq DBN, 14 h; 1-Formylpiperidine (144b): ${ }^{1} \mathbf{H}$ NMR (300 $\left.\mathrm{MHz}, \mathrm{CDCl}_{3}\right): \delta 7.87(\mathrm{~s}, 1 \mathrm{H}), 3.34(\mathrm{~m}, 2 \mathrm{H}), 3.18(\mathrm{~m}, 2 \mathrm{H}), 1.64-1.48(\mathrm{~m}, 2 \mathrm{H}), 1.44$ (m, 4H); ${ }^{13} \mathrm{C} \mathrm{NMR}\left(\mathrm{CDCl}_{3}, 75 \mathrm{MHz}\right): \delta 160.64,46.68,40.45,26.47,24.98,24.58$; MS (GC-MS): $m / z 113\left(\mathrm{M}^{+}\right) ;{ }^{[143]}$ GC-MS Yield: $51 \%$.<smiles>CCOC(=O)c1ccc(C(=O)N2CCCCC2)cc1</smiles>

4 mol\% rose bengal, 2.0 eq DBN, 40 h; Ethyl 4-(piperidine-1-carbonyl)benzoate (145b): ${ }^{1} \mathrm{H}$ NMR (300 MHz, $\mathrm{CDCl}_{3}$ ): $\delta 8.10-8.07(\mathrm{~m}, 2 \mathrm{H}), 7.47-7.44(\mathrm{~m}, 2 \mathrm{H}), 4.40$ (q, J = $7.1 \mathrm{~Hz}, 2 \mathrm{H}), 3.72$ (br s, $2 \mathrm{H}), 3.29$ (br s, $2 \mathrm{H}), 1.69(\mathrm{~s}, 4 \mathrm{H}), 1.52(\mathrm{~m}, 2 \mathrm{H}), 1.43$ $(\mathrm{t}, J=7.1 \mathrm{~Hz}, 3 \mathrm{H}) ;{ }^{13} \mathrm{C}$ NMR $\left(75 \mathrm{MHz}, \mathrm{CDCl}_{3}\right): \delta 169.2,165.9,140.7,131.2,129.7$, 126.7, 61.2 48.6, 43.1, 26.5, 25.6, 24.5, 14.3; MS (GC-MS): m/z $260\left(\mathrm{M}^{+}\right)$; ${ }^{[144]}$ Yield: $78 \%$.<smiles>N#Cc1ccc(C(=O)N2CCCCC2)cc1</smiles>

4 mol\% rose bengal, 2.0 eq DBN, 40 h; 1 -(4-Cyanobenzoyl)piperidine (146b): ${ }^{1} \mathrm{H}$ NMR $\left(\mathrm{CDCl}_{3}, 300 \mathrm{MHz}\right): \delta 7.72(\mathrm{~d}, J=8.5 \mathrm{~Hz}, 2 \mathrm{H}), 7.51(\mathrm{~d}, J=8.5 \mathrm{~Hz}, 2 \mathrm{H}), 3.75(\mathrm{br}$ s, 2H), 3.34 (br s, 2H), 1.71 (br s, 4H), 1.54 (br s, 2H); ${ }^{13} \mathrm{C} \mathrm{NMR}\left(\mathrm{CDCl}_{3}, 75 \mathrm{MHz}\right): \delta$ 168.2, 140.9, 132.4, 127.5, 118.2, 113.2, 48.6, 43.2, 26.6, 25.5, 24.4; MS (GC-MS): $m / z 214\left(\mathrm{M}^{+}\right) ;{ }^{[145]}$ Yield: 84\%. 
<smiles>O=C(c1ccc(C(F)(F)F)cc1)N1CCCCC1</smiles>

4 mol\% rose bengal, 2.0 eq DBN, 40 h; 1-(4-Trifluoromethylbenzoyl)piperidine (147b): ${ }^{1} \mathbf{H}$ NMR $\left(\mathrm{CDCl}_{3}, 300 \mathrm{MHz}\right): \delta 7.69(\mathrm{~d}, J=8.0 \mathrm{~Hz}, 2 \mathrm{H}), 7.52(\mathrm{~d}, J=8.0 \mathrm{~Hz}$, 2H), 3.74 (br s, 2H), 3.32 (br s, 2H), $1.70-1.65$ (br s, 4H), 1.54 (br s, 2H); ${ }^{13} \mathrm{C}$ NMR $\left(\mathrm{CDCl}_{3}, 75 \mathrm{MHz}\right): \delta 168.8,140.1,131.6,131.1,127.1,125.6,122.0,48.7,43.2,26.5$, 25.6, 24.5; MS (GC-MS): m/z $257\left(\mathbf{M}^{+}\right) ;{ }^{[136]}$ Yield: 76\%.<smiles>O=C(C1CC1)N1CCCCC1</smiles>

10 mol\% rose bengal, 3.0 eq DBN, 20 h; Cyclopropyl-1-piperidinylmethanone (148b): ${ }^{1}$ H NMR $\left(\mathrm{CDCl}_{3}, 300 \mathrm{MHz}\right): \delta 3.60(\mathrm{br}, 4 \mathrm{H}), 1.76(\mathrm{~m}, 1 \mathrm{H}), 1.69-1.49(\mathrm{~m}$, $6 \mathrm{H}), 0.99-0.93(\mathrm{~m}, 2 \mathrm{H}), 0.76-0.69(\mathrm{~m}, 2 \mathrm{H}) ;{ }^{13} \mathrm{C} \mathrm{NMR}\left(\mathrm{CDCl}_{3}, 75 \mathrm{MHz}\right): \delta 171.6$, 46.6, 43.3, 26.2, 24.7, 11.0, 7.1; MS (GC-MS): $m / z 153\left(\mathrm{M}^{+}\right) ;{ }^{[146]}$ Yield: 57\%.<smiles>O=C(c1ccc2ccccc2c1)N1CCCCC1</smiles>

4 mol\% rose bengal, 2.0 eq DBN, 40 h; 1-(2-Naphthalenecarbonyl)piperidine (149b): ${ }^{1} \mathbf{H}$ NMR $\left(300 \mathrm{MHz}, \mathrm{CDCl}_{3}\right): \delta 7.91-7.86(\mathrm{~m}, 4 \mathrm{H}), 7.56-7.48(\mathrm{~m}, 3 \mathrm{H}), 3.71$ (br s, 4H), $1.70-1.53$ (br s, 6H); ${ }^{13} \mathrm{C}$ NMR (75 MHz, $\left.\mathrm{CDCl}_{3}\right): \delta 170.5,133.9,133.6$, 132.8, 128.4, 128.2, 127.8, 126.9, 126.6, 126.5, 124.3, 49.0, 46.3, 26.5, 26.2, 24.6; MS (GC-MS): $m / z 239\left(\mathrm{M}^{+}\right) ;{ }^{[136]}$ Yield: 68\%. 
<smiles>CCN1C(=O)c2ccccc2C1=O</smiles>

4 mol\% rose Bengal, 2.0 eq DBN, 48 h; 2-Ethyl isoindole-1,3-dione (150b): ${ }^{1} \mathrm{H}$ NMR $\left(\mathrm{CDCl}_{3}, 300 \mathrm{MHz}\right): \delta 7.86(\mathrm{~m}, 2 \mathrm{H}), 7.72(\mathrm{~m}, 2 \mathrm{H}), 3.77(\mathrm{q}, \mathrm{J}=7.2 \mathrm{~Hz}, 2 \mathrm{H}), 1.30(\mathrm{t}, J=$ $7.5 \mathrm{~Hz}, 3 \mathrm{H}) ;{ }^{13} \mathrm{C}$ NMR $\left(\mathrm{CDCl}_{3}, 75 \mathrm{MHz}\right): \delta 168.2,133.8,132.2,123.1,32.9,13.9 ; \mathrm{MS}$ (GC-MS): $m / z 175\left(\mathrm{M}^{+}\right) ;{ }^{[147]}$ Yield: 75\%.

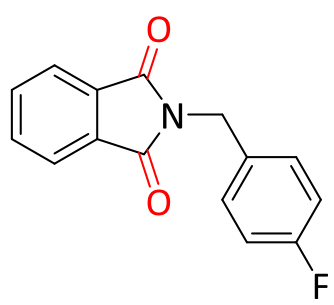

4 mol\% rose bengal, 2.0 eq DBN, 48 h; 2-(3-Fluorobenzyl)isoindoline-1,3-dione (151b): $\left.{ }^{1} \mathrm{H} \mathrm{NMR} \mathrm{(CDCl} 3,300 \mathrm{MHz}\right): \delta 7.87$ (dd, $\left.J=5.5,3.0 \mathrm{~Hz}, 2 \mathrm{H}\right), 7.73$ (dd, $J=5.4$, $3.1 \mathrm{~Hz}, 2 \mathrm{H}), 7.44(\mathrm{~m}, 2 \mathrm{H}), 7.01(\mathrm{~m}, 2 \mathrm{H}), 4.83(\mathrm{~m}, 2 \mathrm{H}) ;{ }^{13} \mathrm{C} \mathrm{NMR}\left(\mathrm{CDCl}_{3}, 75 \mathrm{MHz}\right): \delta$ $168.0,164.0,134.1,132.1,130.6,130.5,123.4,115.7,115.4,40.9 ;$ MS (GC-MS): $m / z 255\left(\mathrm{M}^{+}\right) ;^{[147]}$ Yield: 77\%.<smiles>O=C1c2cccc(Br)c2C(=O)N1Cc1ccc(F)cc1</smiles>

4 mol\% rose bengal, 2.0 eq DBN, 48 h; 4-Bromo-2-(3-fluorobenzyl)isoindoline1,3-dione (152b): ${ }^{1} \mathrm{H}$ NMR $\left(\mathrm{CDCl}_{3}, 300 \mathrm{MHz}\right): \delta 7.85$ (dd, J = 4.0, $\left.0.9 \mathrm{~Hz}, 1 \mathrm{H}\right), 7.82$ (dd, $J=4.0,0.9 \mathrm{~Hz}, 1 \mathrm{H}$ ), $7.57(\mathrm{~m}, 1 \mathrm{H}), 7.46(\mathrm{dd}, J=8.0,7.4 \mathrm{~Hz}, 2 \mathrm{H}), 7.33(\mathrm{t}, J=8.7$ $\mathrm{Hz}, 2 \mathrm{H}), 4.83(\mathrm{~m}, 2 \mathrm{H}) ;{ }^{13} \mathrm{C} \mathrm{NMR}\left(\mathrm{CDCl}_{3}, 75 \mathrm{MHz}\right): \delta 164.1,160.9,138.9,135.0$, 134.3, 131.9, 130.9, 130.7, 122.5, 118.7, 115.8, 115.5, 41.2; ESI-HRMS: $m / z$ calcd. 
for $\mathrm{C}_{15} \mathrm{H}_{9} \mathrm{BrFNO}_{2}[\mathrm{M}+\mathrm{H}]^{+}: 333.9879 \mathrm{~m} / \mathrm{z}$, found $333.9861 \mathrm{~m} / \mathrm{z}$; Yield: $71 \%$.<smiles>CCN1C(=O)C=CC1=O</smiles>

4 mol\% rose bengal, 2.0 eq DBN, 48 h; $\boldsymbol{N}$-Ethylmaleimide (153b): ${ }^{1} \mathrm{H}$ NMR (300 $\left.\mathrm{MHz}, \mathrm{CDCl}_{3}\right): \delta 6.67(\mathrm{~s}, 2 \mathrm{H}), 3.54(\mathrm{q}, J=7.2 \mathrm{~Hz}, 2 \mathrm{H}), 1.16(\mathrm{t}, J=7.2 \mathrm{~Hz}, 3 \mathrm{H}) ;{ }^{13} \mathrm{C} \mathrm{NMR}$ (75 MHz, $\mathrm{CDCl}_{3}$ ): $\delta 170.63,134.09,32.72,13.87$; MS (GC-MS): $m / z 160\left(\mathrm{M}^{+}\right) ;{ }^{[148]}$ $52 \%$ yield.<smiles>Cc1cccc(C(=O)N2CCN(C(c3ccccc3)c3ccc(Cl)cc3)CC2)c1</smiles>

6 mol\% rose bengal, 2.5 eq DBN, 48 h; (4-((4Chlorophenyl)(phenyl)methyl)piperazin-1-yl)(m-tolyl)methanone (154b): ${ }^{1} \mathrm{H}$ NMR (300 MHz, $\left.\mathrm{CDCl}_{3}\right): \delta 7.39-7.14$ (br s, 13H), 4.18 (s, 1H), 3.83 (br s, 4H), 2.48 - 2.37 (m, 7H); ${ }^{13} \mathrm{C}$ NMR $\left(\mathrm{CDCl}_{3}, 75 \mathrm{MHz}\right): \delta 170.5,141.7,140.9,138.9,138.4$, $135.9,133.0,130.4,129.2,128.9,128.9,128.3,127.9,127.8,127.5,124.1,75.4$, 60.5, 51.8, 48.2, 42.4, 21.5; ESI-HRMS: $m / z$ calcd. for $\mathrm{C}_{25} \mathrm{H}_{25} \mathrm{ClN}_{2} \mathrm{O}[\mathrm{M}+\mathrm{H}]^{+}$: 405.1734, found 405.1716; Yield: 61\%. 


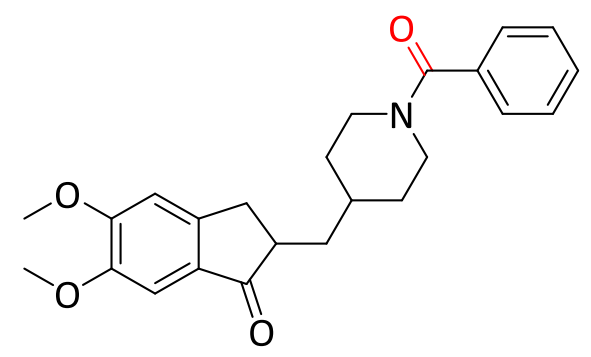

6 mol\% rose bengal, 2.5 eq DBN, 48 h; 2-((1-Benzoylpiperidin-4-yl)methyl)-5,6dimethoxy-2,3-dihydro-1 $\boldsymbol{H}$-inden-1-one (155b): ${ }^{1} \mathrm{H} \mathbf{N M R}\left(300 \mathrm{MHz}, \mathrm{CDCl}_{3}\right): \delta 7.41$ (br s, 5H), 7.18 (br s, 1H), 6.87 (br s, 1H), 4.76 (br s, 1H), 3.97 (s, 3H), 3.92 (s, 3H), $3.78(\mathrm{br} \mathrm{s}, 1 \mathrm{H}), 3.33-3.24(\mathrm{~m}, 1 \mathrm{H}), 2.93-2.68(\mathrm{~m}, 4 \mathrm{H}), 2.00-1.68(\mathrm{~m}, 4 \mathrm{H}), 1.44$ - $1.24(\mathrm{~m}, 3 \mathrm{H}) ;{ }^{13} \mathrm{C}$ NMR $\left(\mathrm{CDCl}_{3}, 75 \mathrm{MHz}\right): \delta 207.3,170.3,155.6,149.5,148.56$, 136.4, 129.4, 129.2, 128.4, 126.8, 107.3, 104.4, 56.2, 56.1, 48.0, 45.1, 38.7, 34.7, 33.4, 26.9; ESI-HRMS: $m / z$ calcd. for $\mathrm{C}_{24} \mathrm{H}_{27} \mathrm{NO}_{4}[\mathrm{M}+\mathrm{H}]^{+}$: 416.1838, found 416.1824; Yield: 70\%.<smiles>COc1cc2c(cc1OC)[C@H]1Cc3ccc(OC)c(OC)c3C(=O)N1CC2</smiles>

6 mol\% rose bengal, 2.0 eq DBN, 48 h; 8-Oxotetrahydropalmatine (156b): ${ }^{1} \mathrm{H}$ NMR $\left(300 \mathrm{MHz}, \mathrm{CDCl}_{3}\right): \delta 7.50-7.57(\mathrm{~m}, 1 \mathrm{H}), 7.47-7.58(\mathrm{~m}, 1 \mathrm{H}), 6.95-7.10(\mathrm{~s}, 1 \mathrm{H})$, $6.70(\mathrm{~s}, 1 \mathrm{H}), 4.93-5.03(\mathrm{~m}, 2 \mathrm{H}), 3.82-4.10(\mathrm{~s}, 12 \mathrm{H}), 3.34(\mathrm{~m}, 1 \mathrm{H}), 2.26-3.19(\mathrm{~m}$, $4 \mathrm{H}) ;{ }^{13} \mathrm{C}$ NMR $\left(\mathrm{CDCl}_{3}, 75 \mathrm{MHz}\right): \delta 165.9,157.5,152.2,147.2,147.0,134.8,124.6$, $121.9,120.6,119.1,115.7,114.0,68.7,62.5,56.6,55.9,38.9,33.6,26.2$; ESIHRMS: $m / z$ calcd. for $\mathrm{C}_{21} \mathrm{H}_{23} \mathrm{NO}_{5}[\mathrm{M}+\mathrm{H}]^{+}: 392.1474 \mathrm{~m} / \mathrm{z}$, found $392.1459 \mathrm{~m} / \mathrm{z}$; Yield: 54\%. 


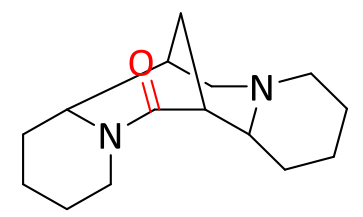

4 mol\% rose bengal, 1.5 eq DBN, 16 h; (+)-17-Oxosparteine (157b): ${ }^{1} \mathbf{H}$ NMR (DMSO- $\left.d_{6}, 300 \mathrm{MHz}\right): \delta 4.58(\mathrm{~m}, 1 \mathrm{H}), 3.22(\mathrm{~m}, 1 \mathrm{H}), 2.75(\mathrm{~m}, 3 \mathrm{H}), 2.32(\mathrm{~m}, 1 \mathrm{H}), 2.11$ (m, 1H), $1.96(\mathrm{~m}, 1 \mathrm{H}), 1.81-1,70(\mathrm{~m}, 3 \mathrm{H}), 1.03(\mathrm{~m}, 13 \mathrm{H}) ;{ }^{13} \mathrm{C}$ NMR (DMSO-d 6,75 $\mathrm{MHz}): \delta 168.6,64.8,62.9,61.2,56.4,44.0,42.1,34.5,33.2,30.3,27.1,25.7,25.5$, 25.2, 24.7; MS (GC-MS): $m / z 248\left(\mathrm{M}^{+}\right) ;{ }^{[149]}$ Yield: 69\%.

Data for Visible-Light-Mediated Heterogeneous Photocatalytic Hydroxylation<smiles>CC(C)(O)c1ccc(I)cc1</smiles>

40h, 2-(4-iodophenyl)propan-2-ol (160b), ${ }^{1} \mathrm{H}$ NMR (300 MHz, $\mathrm{CDCl}_{3}$ ): $\delta 7.69$ (dd, $J=8.8,2.4 \mathrm{~Hz}, 2 \mathrm{H}$ ), 7.27 (dd, $J=8.8,2.4 \mathrm{~Hz}, 2 \mathrm{H}$ ), 1.83 (bs, $1 \mathrm{H}), 1.57(\mathrm{~s}, 6 \mathrm{H}) ;{ }^{13} \mathrm{C}$ NMR (75 MHz, $\mathrm{CDCl}_{3}$ ): $\delta$ 148.9, 137.2, 126.6, 92.1, 72.3, 31.7; MS (GC-MS): m/z $262\left(\mathrm{M}^{+}\right) ;{ }^{[150]}$ Yield: $72 \%$.

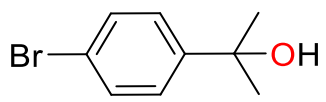

40h, 2-(4-bromophenyl)propan-2-ol (161b), ${ }^{1} \mathbf{H}$ NMR (300 MHz, $\left.\mathrm{CDCl}_{3}\right): \delta 7.48$ (d, $J=8.6 \mathrm{~Hz}, 2 \mathrm{H}), 7.38(\mathrm{~d}, J=8.5 \mathrm{~Hz}, 2 \mathrm{H}), 2.05$ (bs, $1 \mathrm{H}), 1.57(\mathrm{~s}, 6 \mathrm{H}) ;{ }^{13} \mathrm{C}$ NMR $(75$ $\left.\mathrm{MHz}, \mathrm{CDCl}_{3}\right): \delta$ 148.2, 131.2, 126.4, 120.6, 72.3, 31.7. MS (GC-MS): m/z 214 $\left(\mathrm{M}^{+}\right) ;{ }^{[151]}$ Yield: $70 \%$.

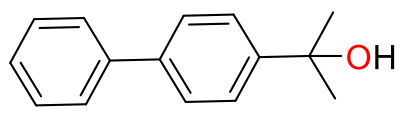

24h, 2-([1,1'-biphenyl]-4-yl)propan-2-ol (162b), ${ }^{1} \mathrm{H}$ NMR (300 MHz, CDCl 3 ): $\delta 7.66$ - 7.26 (m, 9H), 1.93 (bs, 1H), 1.67 (s, 6H); $\left.{ }^{13} \mathrm{C} \mathrm{NMR} \mathrm{(75} \mathrm{MHz,} \mathrm{CDCl}_{3}\right): \delta$ 48.2, 140.9, 139.6, 128.8, 127.2, 127.1, 127.0, 124.9, 72.5, 31.8. MS (GC-MS): $m / z 212\left(\mathrm{M}^{+}\right)$; ${ }^{[152]}$ Yield: 82\%. 


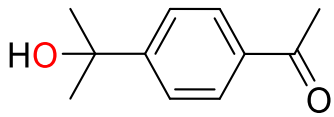

48h, 1-(4-(2-hydroxypropan-2-yl)phenyl)ethan-1-one (163b), ${ }^{1} \mathrm{H}$ NMR (300 MHz, $\left.\mathrm{CDCl}_{3}\right): \delta(\mathrm{ppm})=7.97(\mathrm{dt}, J=8.7,2.0 \mathrm{~Hz}, 2 \mathrm{H}), 7.54(\mathrm{dt}, J=8.7,2.0 \mathrm{~Hz}, 2 \mathrm{H}), 2.63$ (s, 3H), 1.59 (s, 6H); ${ }^{13} \mathrm{C}$ NMR $\left(75 \mathrm{MHz}, \mathrm{CDCl}_{3}\right): \delta 197.9,151.5,135.8,128.1,125.8$, 81.8, 26.8, 26.6. MS (GC-MS): $m / z 178\left(\mathrm{M}^{+}\right) ;{ }^{[153]}$ Yield: 60\%.<smiles>CC(C)(O)c1ccc2ccccc2c1</smiles>

24h, 2-(naphthalen-2-yl)propan-2-ol (164b), ${ }^{1} \mathbf{H}$ NMR $\left(300 \mathrm{MHz}, \mathrm{CDCl}_{3}\right): \delta 7.97$ (m, 1H), $7.87(\mathrm{~m}, 3 \mathrm{H}), 7.66(\mathrm{dd}, 1 \mathrm{H}), 7.52(\mathrm{~m}, 2 \mathrm{H}), 2.05$ (bs, $1 \mathrm{H}), 1.71(\mathrm{~s}, 6 \mathrm{H}) ;{ }^{13} \mathrm{C}$ NMR (75 MHz, $\left.\mathrm{CDCl}_{3}\right): \delta 146.5,133.2,132.3,128.2,128.0,127.5,126.1,125.7$, 123.6, 122.4, 72.7, 31.7. MS (GC-MS): $m / z 186\left(\mathrm{M}^{+}\right) ;{ }^{[152]}$ Yield: 87\%.

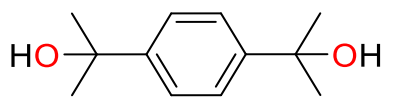

48h, 2,2'-(1,4-phenylene)bis(propan-2-ol) (165b), ${ }^{1} \mathrm{H}$ NMR (300 MHz, $\left.\mathrm{CDCl}_{3}\right): \delta$ 7.48 (br-s, 4H), 1.61 (s, $12 \mathrm{H}) ;{ }^{13} \mathrm{C}$ NMR (75 MHz, $\left.\mathrm{CDCl}_{3}\right): \delta 147.5,124.3,72.4,31.7$. MS (GC-MS): $m / z 194\left(\mathrm{M}^{+}\right) ;{ }^{[154]}$ Yield: 42\%.

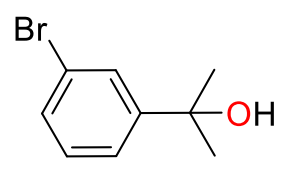

16h, 2-(3-bromophenyl)propan-2-ol (166b), ${ }^{1} \mathbf{H}$ NMR $\left(300 \mathrm{MHz}, \mathrm{CDCl}_{3}\right): \delta 7.68$ (t, $J=1.8 \mathrm{~Hz}, 1 \mathrm{H}), 7.41(\mathrm{tdd}, J=2.9,2.3,1.0 \mathrm{~Hz}, 2 \mathrm{H}), 7.34-7.16(\mathrm{~m}, 1 \mathrm{H}), 1.89(\mathrm{~s}, 1 \mathrm{H})$, $1.59(\mathrm{~s}, 1 \mathrm{H}) ;{ }^{13} \mathrm{C}$ NMR $\left(75 \mathrm{MHz}, \mathrm{CDCl}_{3}\right): \delta 151.4,129.8,129.8,127.8,123.1,122.51$, 72.6, 31.7; MS (GC-MS): $m / z 214\left(\mathrm{M}^{+}\right),{ }^{[151]}$ Yield: 68\%. 
<smiles>OC(c1ccccc1)(c1ccccc1)c1ccccc1</smiles>

40h, Triphenylmethanol (167b), ${ }^{1} \mathbf{H}$ NMR (300 MHz, $\left.\mathrm{CDCl}_{3}\right): \delta 7.38-7.30(\mathrm{~m}, 15 \mathrm{H})$, $2.83(\mathrm{~s}, 1 \mathrm{H}) ;{ }^{13} \mathrm{C}$ NMR $\left(75 \mathrm{MHz}, \mathrm{CDCl}_{3}\right): \delta$ 146.9, 127.9, 127.3, 82.0. MS (GC-MS): $m / z 260\left(\mathrm{M}^{+}\right) ;{ }^{[155]}$ Yield: 50\%.<smiles>CC(=O)c1ccc(C2(O)CCCCC2)cc1</smiles>

40h, 1-(4-(1-hydroxycyclohexyl)phenyl)ethan-1-one (168b), ${ }^{1} \mathrm{H}$ NMR (300 MHz, $\left.\mathrm{CDCl}_{3}\right): \delta 7.97(\mathrm{~d}, J=8.5 \mathrm{~Hz}, 1 \mathrm{H}), 7.63(\mathrm{~d}, J=8.5 \mathrm{~Hz}, 1 \mathrm{H}), 1.81-1.76(\mathrm{~m}, 4 \mathrm{H}), 1.70$ -1.58 (m, 6H); ${ }^{13} \mathrm{C} \mathrm{NMR}\left(75 \mathrm{MHz}, \mathrm{CDCl}_{3}\right): \delta 197.9,154.8,135.6,128.5,128.4,124.9$, 73.4, 38.7, 26.6, 25.4, 22.1. MS (GC-MS): $\mathrm{m} / \mathrm{z} 218\left(\mathrm{M}^{+}\right)$; Yield: 46\%.

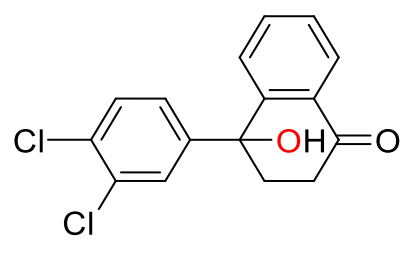

40h, 4-(3,4-dichlorophenyl)-4-hydroxy-3,4-dihydronaphthalen-1(2H)-one, (169b), ${ }^{1} \mathrm{H}$ NMR (300 MHz, $\left.\mathrm{CDCl}_{3}\right): \delta 8.05-7.08$ (m, 7H), $2.94(2 \mathrm{H}), 2.52(2 \mathrm{H})$. The products were mixture of enantiomers. Even though it was analysed by TLC or GCMS, it was one spot. We could further purify them via chiral columns in future. SIHRMS: $m / z$ calcd. for $\mathrm{C}_{15} \mathrm{H}_{21} \mathrm{NO}[\mathrm{M}+\mathrm{H}]^{+}: 291.0337 \mathrm{~m} / \mathrm{z}$; found $291.0343 \mathrm{~m} / \mathrm{z}$; Yield: $62 \%$. 


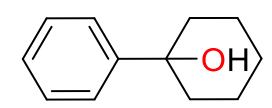

42h, Phenylcyclohexan-1-ol (170b), ${ }^{1} \mathrm{H}$ NMR (300 MHz, $\left.\mathrm{CDCl}_{3}\right): \delta 7.57$ - 7.52 (m, $2 \mathrm{H}), 7.42-7.34(\mathrm{~m}, 2 \mathrm{H}), 7.31-7.24(\mathrm{~m}, 1 \mathrm{H}), 1.90-1.63(\mathrm{~m}, 10 \mathrm{H}), 1.42-1.26(\mathrm{~m}$, 1H); ${ }^{13} \mathrm{C} \mathrm{NMR}\left(75 \mathrm{MHz} \mathrm{CDCl}_{3}\right): \delta 149.4,128.3,128.2,126.7,124.6,73.2,38.9,25.5$, 22.2. MS (GC-MS): $m / z 176\left(\mathrm{M}^{+}\right) ;{ }^{[156]}$ Yield: 55\%.<smiles>CC(C)(O)c1ccc(-c2ccc(C#N)cc2)cc1</smiles>

16h, 4'-(1 -Hydroxy-1-methyl-ethyl)-biphenyl-4-carbonitrile (172b), ${ }^{1}$ H NMR (300 $\left.\mathrm{MHz}, \mathrm{CDCl}_{3}\right): \delta 7.69(\mathrm{q}, J=8.6 \mathrm{~Hz}, 4 \mathrm{H}), 7.59(\mathrm{dd}, J=8.6 \mathrm{~Hz}, 4 \mathrm{H}), 1.63(\mathrm{~s}, 6 \mathrm{H}) ;{ }^{13} \mathrm{C}$ NMR $\left(75 \mathrm{MHz}_{1} \mathrm{CDCl}_{3}\right): \delta 149.8,145.3,137.5,132.8,132.6,127.9,127.6,127.0$, 125.2, 118.9, 110.8, 72.4, 31.8. MS (GC-MS): $m / z 237\left(\mathrm{M}^{+}\right) ;{ }^{[150]}$ Yield: $72 \%$<smiles>COc1ccc(-c2ccc(C(C)(C)O)cc2)cc1</smiles>

16h, 2-(4'-methoxy-[1,1'-biphenyl]-4-yl)propan-2-ol (173b), ${ }^{1} \mathbf{H}$ NMR (300 MHz, $\left.\mathrm{CDCl}_{3}\right): \delta 7.53-7.50(\mathrm{~m}, 6 \mathrm{H}), 6.98-6.95(\mathrm{~m}, 2 \mathrm{H}), 3.85(\mathrm{~s}, 3 \mathrm{H}), 1.61(\mathrm{~m}, 6 \mathrm{H}) ;{ }^{13} \mathrm{C}$ NMR $\left(75 \mathrm{MHz}_{2} \mathrm{CDCl}_{3}\right): \delta 159.1,147.5,139.2,133.4,128.1,126.5,124.9,114.2$, 72.4, 55.3, 31.8. MS (GC-MS): $m / z 242\left(\mathrm{M}^{+}\right) ;{ }^{[157]}$ Yield: 77\%. 
<smiles>CC(C)(O)c1ccc(-c2cc3ccccc3c3ccccc23)cc1</smiles>

16h, 2-(4-(phenanthren-9-yl)phenyl)propan-2-ol (174b), ${ }^{1} \mathbf{H}$ NMR (300 MHz, $\left.\mathrm{CDCl}_{3}\right): \delta 8.74(\mathrm{dd}, J=22.1,8.2 \mathrm{~Hz}, 2 \mathrm{H}), 7.91(\mathrm{dd}, J=24.9,8.3 \mathrm{~Hz}, 2 \mathrm{H}), 7.67-7.51$ (m, 9H), 1.69 (s, 6H); ${ }^{13} \mathrm{C} \mathrm{NMR}\left(75 \mathrm{MHz}, \mathrm{CDCl}_{3}\right): \delta 148.2,139.2,138.5,131.6,131.2$, 130.7, 130.0, 129.9, 128.6, 127.5, 126.9, 126.8, 126.6, 126.5, 126.4, 124.4, 122.9, 122.5, 72.6, 31.9. ESI-HRMS: $m / z$ calcd. for $\mathrm{C}_{15} \mathrm{H}_{21} \mathrm{NO}[\mathrm{M}+\mathrm{H}]^{+}: 313.1590 \mathrm{~m} / z$, found $312.1592 \mathrm{~m} / \mathrm{z}$; Yield: $65 \%$.<smiles>O=C1OCCc2ccccc21</smiles>

16h, Isochroman-1-one (F57) ${ }^{1} \mathbf{H}$ NMR (300 MHz, CDCl 3 ): $\delta 8.09$ (dd, $J=7.7,1.3 \mathrm{~Hz}$, $1 \mathrm{H}), 7.56(\mathrm{td}, J=7.5,1.4 \mathrm{~Hz}, 1 \mathrm{H}), 7.42(\mathrm{td}, J=7.6,1.2 \mathrm{~Hz}, 1 \mathrm{H}), 7.29(\mathrm{~d}, J=7.5 \mathrm{~Hz}, 1 \mathrm{H})$, $4.56(\mathrm{~m}, 2 \mathrm{H}), 3.06(\mathrm{t}, J=6.0 \mathrm{~Hz}, 2 \mathrm{H}) ;{ }^{13} \mathrm{C}$ NMR $\left(75 \mathrm{MHz}, \mathrm{CDCl}_{3}\right): 165.0,139.9,133.7$, $130.5,127.7,127.2,125.3,67.3,27.8 . \mathrm{MS}$ (GC-MS): $\mathrm{m} / \mathrm{z} 148\left(\mathrm{M}^{+}\right) ;{ }^{[48 b]}$ isolated yields: $83 \%$.<smiles>CC(C)CCOC(=O)c1ccccc1</smiles>

24h, Isopentyl benzoate (F58), ${ }^{1} \mathrm{H}$ NMR $\left(300 \mathrm{MHz}, \mathrm{CDCl}_{3}\right): \delta 8.06(\mathrm{~m}, 2 \mathrm{H}), 7.55$ (dd, $J=$ 8.5, 6.3 Hz, 1H), $7.44(\mathrm{t}, J=7.6 \mathrm{~Hz}, 2 \mathrm{H}), 4.38(\mathrm{t}, J=6.7 \mathrm{~Hz}, 2 \mathrm{H}), 1.88(\mathrm{~m}, 2 \mathrm{H}), 1.65(\mathrm{~m}$, $1 \mathrm{H}), 1.01(\mathrm{~d}, J=6.6 \mathrm{~Hz}, 6 \mathrm{H}) ;{ }^{13} \mathrm{C}$ NMR $\left(75 \mathrm{MHz}, \mathrm{CDCl}_{3}\right): \delta 166.6,132.8,130.5,129.5$, 128.3, 63.6, 37.4, 25.2, 22.5. MS (GC-MS): m/z $192\left(\mathrm{M}^{+}\right) ;{ }^{[158]}$ isolated yields: 54\%. 
<smiles>O=C1OCc2ccccc21</smiles>

16h, Isobenzofuran-1(3H)-one (F59), ${ }^{1} \mathbf{H}$ NMR (300 MHz, CDCl 3 ): $\delta 7.92$ (d, J = $7.6 \mathrm{~Hz}$, $1 \mathrm{H}), 7.69(\mathrm{t}, J=7.4 \mathrm{~Hz}, 1 \mathrm{H}), 7.53-7.48(\mathrm{~m}, 2 \mathrm{H}), 5.32(\mathrm{~s}, 2 \mathrm{H}) ;{ }^{13} \mathrm{C} \mathrm{NMR}\left(75 \mathrm{MHz}, \mathrm{CDCl}_{3}\right)$ : $\delta 166.33,141.77,129.25,124.28,121.02,121.00,117.34,72.60,72.49,72.28,71.97$, 64.90. MS (GC-MS): $\mathrm{m} / \mathrm{z} 134\left(\mathrm{M}^{+}\right) ;{ }^{[159]}$ isolated yields: $71 \%$.<smiles>O=C1CCc2ccccc21</smiles>

24h, 1-indanone (F60), ${ }^{1} \mathrm{H}$ NMR $\left(300 \mathrm{MHz}, \mathrm{CDCl}_{3}\right): \delta 7.78(\mathrm{~d}, J=7.6 \mathrm{~Hz}, 1 \mathrm{H}), 7.60$ (td, $J=7.5,1.3 \mathrm{~Hz}, 1 \mathrm{H}), 7.50(\mathrm{br} \mathrm{d}, J=7.8 \mathrm{~Hz}, 1 \mathrm{H}), 7.39(\mathrm{brt}, J=7.5 \mathrm{~Hz}, 1 \mathrm{H}), 3.17(\mathrm{~m}, 2 \mathrm{H})$, $2.70(\mathrm{~m}, 2 \mathrm{H}) ;{ }^{13} \mathrm{C}$ NMR $\left(75 \mathrm{MHz}, \mathrm{CDCl}_{3}\right): \delta$ 198.4, 144.5, 133.4, 132.6, 128.8, 127.1, 126.6, 39.2, 29.7, 23.4, 23.3. MS (GC-MS): $\mathrm{m} / \mathrm{z} 132\left(\mathrm{M}^{+}\right) ;{ }^{[160]}$ isolated yields: $67 \%$.<smiles>O=C1CCCc2ccccc21</smiles>

16h, 1-Tetralone (F61) ${ }^{1} \mathrm{H}$ NMR $\left(300 \mathrm{MHz}, \mathrm{CDCl}_{3}\right): \delta 8.03(\mathrm{~d}, J=6.9 \mathrm{~Hz}, 1 \mathrm{H}), 7.47$ (td, $J$ = 7.5, $1.5 \mathrm{~Hz}, 1 \mathrm{H}), 7.49-7.25(\mathrm{~m}, 1 \mathrm{H}), 2.97(\mathrm{td}, J=6.9 \mathrm{~Hz}, 2 \mathrm{H}), 2.66(\mathrm{dd}, J=7.3,5.8 \mathrm{~Hz}$, 2H), 1.96-1.91 (m, 2H). ${ }^{13} \mathrm{C}$ NMR (75 MHz, $\left.\mathrm{CDCl}_{3}\right): \delta 198.4,144.5,133.4,132.6,128.8$, 127.1, 126.6, 39.2, 29.7, 23.4, 23.3. MS (GC-MS): $\mathrm{m} / \mathrm{z} 146\left(\mathrm{M}^{+}\right) ; ;^{[161]}$ isolated yields: $71 \%$. 


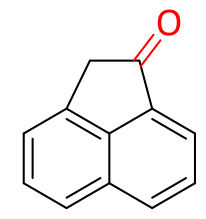

16h, acenaphthylen-1(2H)-one (F62): ${ }^{1} \mathbf{H}$ NMR (300 MHz, $\mathrm{CDCl}_{3}$ ): $\delta 8.10$ (dd, $J=8.1$, $0.8 \mathrm{~Hz}, 1 \mathrm{H}$ ), 7.97 (dd, $J=7.0,0.7 \mathrm{~Hz}, 1 \mathrm{H}$ ), 7.83 (dd, $J=8.4,0.8 \mathrm{~Hz}, 1 \mathrm{H}$ ), 7.72 (dd, $J=8.1$, $7.0 \mathrm{~Hz}, 1 \mathrm{H}), 7.61(\mathrm{dd}, J=8.4,6.9 \mathrm{~Hz}, 1 \mathrm{H}), 7.52-7.44(\mathrm{~m}, 1 \mathrm{H}), 3.83(\mathrm{t}, J=0.9 \mathrm{~Hz}, 2 \mathrm{H})$; ${ }^{13} \mathrm{C}$ NMR $\left(75 \mathrm{MHz}, \mathrm{CDCl}_{3}\right): \delta 203.08,141.59,135.17,134.86,131.62,131.11,128.52$, 128.15, 124.10, 121.59, 121.18, 42.16; MS (GC-MS): $m / z 168\left(\mathrm{M}^{+}\right) ;{ }^{[162]}$ isolated yields: $88 \%$.<smiles>CC(=O)c1cc(C(C)(C)C)cc2c1C(=O)CC2(C)C</smiles>

24h, 7-acetyl-5-(tert-butyl)-3,3-dimethyl-2,3-dihydro-1H-inden-1-one (F63), ${ }^{1} \mathrm{H}-\mathrm{NMR}$ $\left(300 \mathrm{MHz}, \mathrm{CDCl}_{3}\right): \delta(\mathrm{ppm})=7.56(\mathrm{~s}, 1 \mathrm{H}), 7.35(\mathrm{~s}, 1 \mathrm{H}), 2.66(\mathrm{~s}, 3 \mathrm{H}), 2.63(\mathrm{~s}, 2 \mathrm{H}), 1.45$ (s, $6 \mathrm{H}), 1.38(\mathrm{~s}, 9 \mathrm{H}) .{ }^{13} \mathrm{C}-\mathrm{NMR}\left(75 \mathrm{MHz}, \mathrm{CDCl}_{3}\right): \delta(\mathrm{ppm})=204.7,203.7,164.6,159.4,123.3$, 121.8, 100.0, 53.3, 31.1, 31.0, 30.0; MS (GC-MS): $\mathrm{m} / \mathrm{z} 253\left(\mathrm{M}^{+}\right) ;{ }^{[163]}$ isolated yields: 49\%.

Data for Solar Energy Mediated Scalable and Selective Oxidative Cleavage of $\mathrm{C}=\mathrm{C}$ Bonds in Aryl Olefins

The following starting materials were synthesized according to the literature. ${ }^{[164]}$<smiles>C=C(C)c1ccccc1OC</smiles>

2-(2'-Methoxy-phenyl)-propene (177a): NMR (300 MHz, $\left.\mathrm{CDCl}_{3}\right): \delta 7.26$ - $7.17(\mathrm{~m}$, 2H), $6.94-6.86(\mathrm{~m}, 2 \mathrm{H}), 5.19-5.08(\mathrm{~m}, 1 \mathrm{H}), 5.09-5.02(\mathrm{~m}, 1 \mathrm{H}), 3.83(\mathrm{~s}, 3 \mathrm{H}), 2.11$ (s, 3H); ${ }^{13} \mathrm{C} \mathrm{NMR}\left(75 \mathrm{MHz}, \mathrm{CDCl}_{3}\right): \delta 156.5,144.3,132.8,129.4,128.3,120.5,115.1$, 110.6, 55.4, 23.1; MS (GC-MS): $m / z 148\left(\mathrm{M}^{+}\right) ;{ }^{[164]}$ Yield: 50\%. 


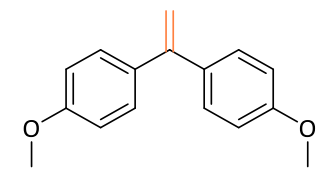

4,4'-(ethene-1,1-diyl)bis(methoxybenzene) (178a): NMR (300 MHz, $\left.\mathrm{CDCl}_{3}\right): \delta 7.31$ - $7.25(\mathrm{~m}, 4 \mathrm{H}), 6.89-6.86(\mathrm{~m}, 4 \mathrm{H}), 5.31(\mathrm{~s}, 2 \mathrm{H}), 3.84(\mathrm{~s}, 6 \mathrm{H}) .{ }^{13} \mathrm{C}$ NMR $(75 \mathrm{MHz}$, $\left.\mathrm{CDCl}_{3}\right): \delta 159.3,148.9,134.2,129.4,113.4,111.6,55.3$. MS (GC-MS): $\mathrm{m} / z 240$ $\left(\mathrm{M}^{+}\right) ;{ }^{[164]}$ Yield: $55 \%$.

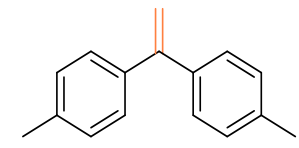

4,4'-(Ethene-1,1-diyl)bis(methylbenzene) (179a) : NMR (300 MHz, $\mathrm{CDCl}_{3}$ ): $\delta 7.25$ $(\mathrm{d}, J=8.0 \mathrm{~Hz}, 4 \mathrm{H}), 7.15(\mathrm{~d}, J=8.0 \mathrm{~Hz}, 4 \mathrm{H}), 5.39(\mathrm{~s}, 2 \mathrm{H}), 2.38(\mathrm{~s}, 6 \mathrm{H}) ;{ }^{13} \mathrm{C}$ NMR $(75$ $\left.\mathrm{MHz}, \mathrm{CDCl}_{3}\right): \delta 149.5,138.6,137.2,128.7,128.1,113.0,21.2 . \mathrm{MS}$ (GC-MS): $\mathrm{m} / \mathrm{z} 208$ $\left(\mathrm{M}^{+}\right) ;{ }^{[165]}$ Yield: $58 \%$.

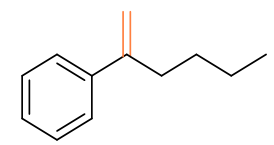

hex-1-en-2-ylbenzene (180a): NMR (300 MHz, $\mathrm{CDCl}_{3}$ ): $\delta 7.3(\mathrm{~d}, J=6.9 \mathrm{~Hz}, 2 \mathrm{H}), 7.2$ $-7.0(\mathrm{~m}, 2 \mathrm{H}), 7.1(\mathrm{~d}, J=6.9 \mathrm{~Hz}, 1 \mathrm{H}), 5.1(\mathrm{~d}, J=1.2 \mathrm{~Hz}, 1 \mathrm{H}), 4.9(\mathrm{~d}, J=1.2 \mathrm{~Hz}, 1 \mathrm{H})$, $2.4(\mathrm{t}, J=7.1 \mathrm{~Hz}, 2 \mathrm{H}), 1.4-1.2(\mathrm{~m}, 4 \mathrm{H}), 0.8(\mathrm{t}, J=7.3 \mathrm{~Hz}, 3 \mathrm{H}) .{ }^{13} \mathrm{C}$ NMR $(75 \mathrm{MHz}$, $\mathrm{CDCl}_{3}$ ): $\delta 148.6,141.4,128.2,127.2,126.2,112.0,35.0,30.4,22.4,13.9$. MS (GCMS): $m / z 160\left(\mathrm{M}^{+}\right) ;{ }^{[166]}$ Yield: 48\%. 


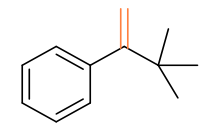

(3, 3-Dimethylbut-1-en-2-yl)benzene (182a): NMR (300 MHz, $\mathrm{CDCl}_{3}$ ): $\delta 7.25$ - 7.15 (m, 3H), 7.08 (dd, J = 7.6, 1.5 Hz, 2H), $5.11(\mathrm{~d}, J=1.7 \mathrm{~Hz}, 1 \mathrm{H}), 4.70(\mathrm{~d}, J=1.7 \mathrm{~Hz}, 1 \mathrm{H})$, 1.08 (s, 9H). ${ }^{13} \mathrm{C}$ NMR $\left(75 \mathrm{MHz} \mathrm{CDCl}_{3}\right): \delta 159.8,143.4,129.0,127.2,126.2,111.5$, 29.6. MS (GC-MS): $m / z$ 160; ${ }^{[167]}$ Yield: $55 \%$.

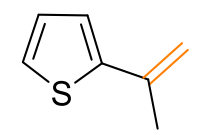

2-(Prop-1-en-2-yl)thiophene (185a) NMR (300 MHz, $\left.\mathrm{CDCl}_{3}\right): \delta 7.17$ (dd, J = 5.1, 1.2 $\mathrm{Hz}, 1 \mathrm{H}), 7.03$ (dd, J = 3.6, 1.2 Hz, 1H), 6.98 (dd, J = 5.1, 3.6 Hz, 1H), $5.38(\mathrm{~s}, 1 \mathrm{H}), 4.95$ $(\mathrm{t}, J=1.4 \mathrm{~Hz}, 1 \mathrm{H}), 2.15$ (dd, $J=1.5,0.8 \mathrm{~Hz}, 3 \mathrm{H}) .{ }^{13} \mathrm{C} \mathrm{NMR}\left(75 \mathrm{MHz}, \mathrm{CDCl}_{3}\right): \delta 145.8$, 137.2, 127.3, 124.2, 123.5, 111.2, 21.8. MS (GC-MS): m/z 124; ;164] Yield: 60\%.

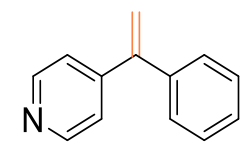

4-(1-phenylvinyl)pyridine (176a): NMR (300 MHz, $\left.\mathrm{CDCl}_{3}\right): \delta 8.56(\mathrm{~d}, J=4.0 \mathrm{~Hz}, 2 \mathrm{H})$, $7.35-7.33(\mathrm{~m}, 3 \mathrm{H}), 7.28-7.24(\mathrm{~m}, 2 \mathrm{H}), 7.22-7.21(\mathrm{~m}, 2 \mathrm{H}), 5.60(\mathrm{~s}, 1 \mathrm{H}), 5.59$ (s, 1H). ${ }^{13} \mathrm{C}$ NMR (75 MHz, $\left.\mathrm{CDCl}_{3}\right): \delta 149.9,148.9,147.9,139.8,128.5,128.3,128.2$, 122.8, 117.0. MS (GC-MS): $m / z$ 181; ${ }^{[168]}$ Yield: $45 \%$.

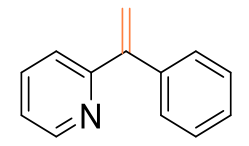

2-(1-phenylvinyl)pyridine (186a): NMR (300 MHz, $\mathrm{CDCl}_{3}$ ): $\delta 8.66$ (s, 1H), 7.63 (t, J = $7.7 \mathrm{~Hz}$ ), $7.39-7.37(\mathrm{~m}, 5 \mathrm{H}), 7.27(\mathrm{~d}, J=8.0 \mathrm{~Hz}, 1 \mathrm{H}), 7.20(\mathrm{t}, J=6.3 \mathrm{~Hz}), 6.02(\mathrm{~s}, 1 \mathrm{H})$, $5.63(\mathrm{~s}, 1 \mathrm{H}) .{ }^{13} \mathrm{C}$ NMR $\left(75 \mathrm{MHz}, \mathrm{CDCl}_{3}\right): \delta 158.5,149.4,149.1,140.4,136.4,128.5$, 128.3, 127.9, 122.9, 122.5, 117.9. MS (GC-MS): $m / z$ 181; ${ }^{[169]}$ Yield: 50\%. 
事

1-(1-bromovinyl)-3-chlorobenzene (205a): NMR (300 MHz, $\mathrm{CDCl}_{3}$ ): $\delta 7.29-7.22$ $(\mathrm{m}, 4 \mathrm{H}), 7.17(\mathrm{~d}, J=8.8 \mathrm{~Hz}, 2 \mathrm{H}), 6.77(\mathrm{~d}, J=8.8 \mathrm{~Hz}, 2 \mathrm{H}), 5.38(\mathrm{~s}, 1 \mathrm{H}), 5.32(\mathrm{~s}, 1 \mathrm{H})$, 5.13-5.03 (m, 1H), $1.60(\mathrm{~s}, 6 \mathrm{H}), 1.21(\mathrm{~d}, J=6.0 \mathrm{~Hz}, 6 \mathrm{H}), .{ }^{13} \mathrm{C} \mathrm{NMR}\left(75 \mathrm{MHz}, \mathrm{CDCl}_{3}\right): \delta$ 173.6, 155.4, 148.3, 140.0, 134.3, 133.4, 129.6, 128.8, 128.1, 118.2, 113.6, 79.0, 69.0, 25.3, 21.5. MS (GC-MS): $m / z$ 358; ${ }^{[168]}$ Yield: 59\%.

$\alpha$-Bromostyrene derivatives were synthesized according to the literature. ${ }^{[170]}$<smiles>C=C(Br)c1ccc(C)cc1</smiles>

1-(1-Bromovinyl)-4-methylbenzene (28a): NMR (300 MHz, $\mathrm{CDCl}_{3}$ ): $\delta 7.50$ (d, J = 7.0 $\mathrm{Hz}, 2 \mathrm{H}$ ), 7.12 (d, J=7.0 Hz, 2H), 6.11 (d, J=1.8 Hz, 1H), 6.78 (d, J=1.8 Hz, 1H), 2.40 (s, 3H). ${ }^{13} \mathrm{C}$ NMR $\left(75 \mathrm{MHz} \mathrm{CDCl}_{3}\right): \delta 139.1,135.7,131.0,128.9,125.9,116.8,21.1$. MS (GC-MS): $m / z$ 196; ${ }^{[171]}$ Yield: $65 \%$.<smiles>CC(Br)c1cccc(Cl)c1</smiles>

1-(1-Bromovinyl)-3-chlorobenzene (29a): NMR (300 MHz, $\left.\mathrm{CDCl}_{3}\right): \delta 7.59-7.57(\mathrm{~m}$, 1H), $7.48-7.46(m, 1 H), 7.31-7.26(m, 2 H), 6.13(d, J=2.3 \mathrm{~Hz}, 1 \mathrm{H}), 5.81(\mathrm{~d}, J=2.3$ $\mathrm{Hz}, 1 \mathrm{H}) .{ }^{13} \mathrm{C}$ NMR $\left(75 \mathrm{MHz}, \mathrm{CDCl}_{3}\right): \delta 140.2,134.3,129.5,129.2,129.1,127.4,125.4$, 118.8. MS (GC-MS): $m / z$ 196; ${ }^{[171]}$ Yield: $61 \%$. 
Data for products of photocatalytic $\mathrm{C}=\mathrm{C}$ bonds cleavage<smiles>CC(=O)c1ccc(F)cc1</smiles>

24h, 4-Fluroacetophenon (175b). ${ }^{1} \mathrm{H}$ NMR (300 MHz, $\left.\mathrm{CDCl}_{3}\right): \delta 7.84$ (dd, $J=8.9$, $5.4 \mathrm{~Hz}, 2 \mathrm{H}), 7.14-7.08(\mathrm{~m}, 2 \mathrm{H}), 2.57(\mathrm{~s}, 3 \mathrm{H}) ;{ }^{13} \mathrm{C}$ NMR $\left(75 \mathrm{MHz}, \mathrm{CDCl}_{3}\right): \delta 196.4$, 167.4, 133.6, 130.9 (d, $J=9.3 \mathrm{~Hz}$ ), 115.6 (d, $J=21.9 \mathrm{~Hz}$ ), 26.5; MS (GC-MS): $m / z$ $138\left(\mathrm{M}^{+}\right) ;{ }^{[77]}$ Yield: $80 \%$.<smiles>CC(=O)c1ccccc1</smiles>

24h, Acetophenone (70b). ${ }^{1} \mathrm{H}$ NMR (300 MHz, $\left.\mathrm{CDCl}_{3}\right): \delta 7.98-7.95(\mathrm{~m}, 2 \mathrm{H}), 7.60-$ $7.55(\mathrm{~m}, 1 \mathrm{H}), 7.50-7.44(\mathrm{~m}, 2 \mathrm{H}), 2.61(\mathrm{~s}, 3 \mathrm{H}) ;{ }^{13} \mathrm{C} \mathrm{NMR}\left(75 \mathrm{MHz}, \mathrm{CDCl}_{3}\right): \delta 198.1$, 137.1, 133.1, 128.6, 128.3, 26.6; MS (GC-MS): $m / z 120\left(\mathrm{M}^{+}\right) ;$;77] $^{[\mathrm{T}}$ Yield: $78 \%$.<smiles>CC(=O)c1ccc(Cl)cc1</smiles>

40h, 4-Chloroacetophenone (81b). ${ }^{1} \mathrm{H}$ NMR (300 MHz, $\left.\mathrm{CDCl}_{3}\right): \delta 7.91-7.88(\mathrm{~m}$, 2H), $7.45-7.42(\mathrm{~m}, 2 \mathrm{H}), 2.59(\mathrm{~s}, 3 \mathrm{H}) ;{ }^{13} \mathrm{C}$ NMR (75 MHz, $\left.\mathrm{CDCl}_{3}\right): \delta 196.8,139.5$, 135.4, 129.7, 128.9, 26.5; MS (GC-MS): $m / z 154\left(\mathrm{M}^{+}\right) ;{ }^{[77]}$ Yield: $90 \%$.<smiles>CC(=O)c1ccc(Br)cc1</smiles>

24h, 4-Bromacetophenon (176b). ${ }^{1} \mathbf{H}$ NMR (300 MHz, $\mathrm{CDCl}_{3}$ ): $\delta 7.84$ (dd, $J=8.2,1.0$ $\mathrm{Hz}, 2 \mathrm{H}), 7.64(\mathrm{~d}, J=0.9 \mathrm{~Hz}, 2 \mathrm{H}), 2.59(\mathrm{~s}, 3 \mathrm{H}) ;{ }^{13} \mathrm{C} \mathrm{NMR}\left(75 \mathrm{MHz}, \mathrm{CDCl}_{3}\right): \delta$ 197.0, 
135.8, 131.9, 129.8, 128.3, 26.5; MS (GC-MS): m/z $197\left(\mathrm{M}^{+}\right) ;{ }^{[77]}$ Yield: 68\%.<smiles>COc1ccccc1C(C)=O</smiles>

40h, 2-Methoxyacetophenone (177b). ${ }^{1} \mathrm{H}$ NMR (300 MHz, $\left.\mathrm{CDCl}_{3}\right): \delta 7.76-7.73(\mathrm{~m}$, 1H), $7.50-7.44(\mathrm{~m}, 1 \mathrm{H}), 7.03-6.96(\mathrm{~m}, 1 \mathrm{H}), 3.90(\mathrm{~s}, 3 \mathrm{H}), 2.62(\mathrm{~s}, 3 \mathrm{H}) ;{ }^{13} \mathrm{C}$ NMR $(75$ $\mathrm{MHz}, \mathrm{CDCl}_{3}$ ): $\delta 199.9,158.9,133.6,130.3,128.3,120.5,111.6,55.5,26.5 ;$ MS (GCMS): $m / z 150\left(\mathrm{M}^{+}\right) ;{ }^{[172]}$ Yield: $71 \%$.<smiles>CC(=O)c1ccc(C)cc1</smiles>

24h, 4-Methylacetophenone (79b) ${ }^{1} \mathrm{H}-\mathrm{NMR}\left(300 \mathrm{MHz}, \mathrm{CDCl}_{3}\right): \delta 7.88$ (d, J= $7.4 \mathrm{~Hz}$, $2 \mathrm{H}), 7.27(\mathrm{~d}, J=7.4 \mathrm{~Hz}, 2 \mathrm{H}), 2.58(\mathrm{~s}, 3 \mathrm{H}), 2.42(\mathrm{~s}, 3 \mathrm{H}) ;{ }^{13} \mathrm{C} \mathrm{NMR}\left(\mathrm{CDCl}_{3}, 75 \mathrm{MHz}\right): \delta$ 197.8, 143.9, 134.7, 129.2, 128.4, 26.5, 21.6; MS (GC-MS): $m / z 134\left(\mathrm{M}^{+}\right) ;{ }^{[77]}$ Yield: $78 \%$.<smiles>O=C(c1ccccc1)c1ccccc1</smiles>

24h, Benzophenone (78b). ${ }^{1} \mathrm{H}$ NMR (300 MHz, $\left.\mathrm{CDCl}_{3}\right): \delta 7.85-7.81(\mathrm{~m}, 4 \mathrm{H}), 7.64-$ $7.59(\mathrm{~m}, 2 \mathrm{H}), 7.54-7.48(\mathrm{~m}, 4 \mathrm{H}) ;{ }^{13} \mathrm{C}$ NMR $\left(75 \mathrm{MHz}, \mathrm{CDCl}_{3}\right): \delta$ 196.8, 137.6, 132.4, 130.1, 128.3; MS (GC-MS): m/z $182\left(\mathbf{M}^{+}\right) ;{ }^{[164]} 90 \%$ yield for $\mathbf{1 b}, 68 \%$ yield for $\mathbf{3 4 b .}$<smiles>COc1ccc(C(=O)c2ccc(OC)cc2)cc1</smiles>

24h, 4,4'-Dimethoxybenzophenone (178b): ${ }^{1} \mathrm{H}$ NMR (300 MHz, $\left.\mathrm{CDCl}_{3}\right): \delta 7.83-$ $7.74(\mathrm{~m}, 4 \mathrm{H}), 6.99-6.91(\mathrm{~m}, 4 \mathrm{H}), 3.89(\mathrm{~s}, 6 \mathrm{H}) ;{ }^{13} \mathrm{C} \mathrm{NMR}\left(75 \mathrm{MHz}, \mathrm{CDCl}_{3}\right): \delta 194.42$, 
162.83, 132.20, 130.78, 113.45, 55.45; MS (GC-MS): $m / z 242\left(\mathrm{M}^{+}\right) ;{ }^{[173]} 75 \%$ yield.<smiles>Cc1ccc(C(=O)c2ccc(C)cc2)cc1</smiles>

24h, 4,4'-Dimethylbenzophenone (179b). ${ }^{1} \mathrm{H}$ NMR (300 MHz, $\left.\mathrm{CDCl}_{3}\right): \delta 7.74-7.71$ (m, 4H), 7.31 - $7.28(\mathrm{~m}, 2 \mathrm{H}), 2.46(\mathrm{~s}, 6 \mathrm{H}) ;{ }^{13} \mathrm{C} \mathrm{NMR}\left(75 \mathrm{MHz}, \mathrm{CDCl}_{3}\right): \delta 196.3,142.9$, 135.2, 130.2, 128.9, 21.6; MS (GC-MS): $m / z 210\left(\mathbf{M}^{+}\right) ;{ }^{[173]}$ Yield: 81\%.<smiles>CCCCC(=O)c1ccccc1</smiles>

40h, 1-Phenylpentan-1-one (180b) ${ }^{1} \mathrm{H}-\mathrm{NMR}\left(300 \mathrm{MHz}, \mathrm{CDCl}_{3}\right): \delta 7.99-7.96(\mathrm{~m}, 2 \mathrm{H})$, 7.59 - $7.55(\mathrm{~m}, 1 \mathrm{H}), 7.50-7.45(\mathrm{~m}, 2 \mathrm{H}), 3.00(\mathrm{t}, J=7.4 \mathrm{~Hz}, 2 \mathrm{H}), 1.76(\mathrm{~m}, 2 \mathrm{H}), 1.44$ (m, 2H), 1.03 (t, $J=7.3 \mathrm{~Hz}, 3 \mathrm{H}) ;{ }^{13} \mathrm{C}$ NMR $\left(\mathrm{CDCl}_{3}, 75 \mathrm{MHz}\right): \delta 200.6,137.1,132.9$, 128.5, 128.1, 38.3, 26.5, 22.5, 14.0; MS (GC-MS): m/z $162\left(\mathrm{M}^{+}\right) ;{ }^{[174]}$ Yield: 75\%.<smiles>CCCC(=O)c1ccccc1</smiles>

40h, 1-Phenylbutan-1-one (181b) ${ }^{1} \mathrm{H}-\mathrm{NMR}\left(300 \mathrm{MHz}, \mathrm{CDCl}_{3}\right): \delta 7.99-7.96(\mathrm{~m}, 2 \mathrm{H})$, $7.60-7.54(\mathrm{~m}, 1 \mathrm{H}), 7.50-7.45(\mathrm{~m}, 2 \mathrm{H}), 2.97(\mathrm{t}, J=7.3 \mathrm{~Hz}, 2 \mathrm{H}), 1.80$ (q, J = $7.4 \mathrm{~Hz}$, 2H), $1.03(\mathrm{t}, J=7.4 \mathrm{~Hz}, 3 \mathrm{H}) ;{ }^{13} \mathrm{C} \mathrm{NMR}\left(\mathrm{CDCl}_{3}, 75 \mathrm{MHz}\right): \delta 200.4,137.1,132.9,128.5$, 128.0, 40.5, 17.8, 13.9; MS (GC-MS): $m / z 148\left(\mathrm{M}^{+}\right) ; ;^{[175]}$ Yield: $72 \%$. 


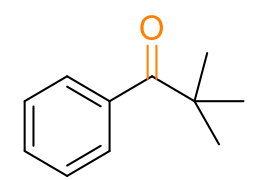

40h, 2,2-dimethyl-1-phenylpropan-1-one (182b) ${ }^{1} \mathrm{H}-\mathrm{NMR}\left(300 \mathrm{MHz}, \mathrm{CDCl}_{3}\right): \delta 7.71$ - $7.64(\mathrm{~m}, 2 \mathrm{H}), 7.51$ - $7.39(\mathrm{~m}, 3 \mathrm{H}), 1.37$ (s, 9H); ${ }^{13} \mathrm{C} \mathrm{NMR}\left(\mathrm{CDCl}_{3}, 75 \mathrm{MHz}\right): \delta 209.3$, 138.6, 130.8, 128.0, 127.8, 44.2, 28.0; MS (GC-MS): $m / z 162\left(\mathrm{M}^{+}\right) ;{ }^{[171]}$ Yield: $61 \%$.<smiles>CC(=O)c1ccc2ccccc2c1</smiles>

24h, 2'-Acetonaphthone (183b). ${ }^{1} \mathbf{H}$ NMR (300 MHz, $\mathrm{CDCl}_{3}$ ): $\delta 8.50$ (br-s, 1H), 8.09 - $7.98(\mathrm{~m}, 2 \mathrm{H}), 7.94$ - $7.89(\mathrm{~m}, 2 \mathrm{H}), 7.67-7.56(\mathrm{~m}, 2 \mathrm{H}), 2.76(\mathrm{~s}, 3 \mathrm{H}) ;{ }^{13} \mathrm{C}$ NMR (75 $\left.\mathrm{MHz}, \mathrm{CDCl}_{3}\right): \delta 198.1,135.6,134.5,132.5,130.2,129.6,128.5,128.4,127.8,126.8$, 123.9, 26.7; MS (GC-MS): m/z $170\left(\mathbf{M}^{+}\right)$; ${ }^{[82]}$ Yield: 75\%.<smiles>CC(C)(CC(=O)c1ccccc1)c1ccccc1</smiles>

40h, 3-methyl-1,3-diphenylbutan-1-one (184b). ${ }^{1} \mathbf{H}$ NMR (300 MHz, $\mathrm{CDCl}_{3}$ ): $\delta 7.85$ - $7.82(\mathrm{~m}, 2 \mathrm{H}), 7.54-7.49(\mathrm{~m}, 1 \mathrm{H}), 7.42$ - $7.37(\mathrm{~m}, 4 \mathrm{H}), 7.32$ - $7.30(\mathrm{~m}, 2 \mathrm{H}), 7.20$ $7.15(\mathrm{~m}, 1 \mathrm{H}), 3.33(\mathrm{~s}, 2 \mathrm{H}), 2.76(\mathrm{~s}, 6 \mathrm{H}) ;{ }^{13} \mathrm{C} \mathrm{NMR}\left(75 \mathrm{MHz}, \mathrm{CDCl}_{3}\right): \delta$ 199.0, 148.9, 138.2, 132.7, 128.4, 128.2, 128.1, 125.8, 125.4, 50.9, 37.5, 29.1, 15.3; ESI-HRMS: $\mathrm{m} / \mathrm{z}$ calcd. for $\mathrm{C}_{17} \mathrm{H}_{18} \mathrm{O}[\mathrm{M}+\mathrm{H}]^{+}: 239.1436 \mathrm{~m} / \mathrm{z}$, found $239.1428 \mathrm{~m} / \mathrm{z}$; Yield: $53 \%$. Purified with GPC. 
$\left.4\right|^{0}$

40h, 2-Acetylthiophene (185b). ${ }^{1} \mathbf{H}$ NMR (300 MHz, $\mathrm{CDCl}_{3}$ ): $\delta 7.69$ (dd, $J=3.8,1.1$ $\mathrm{Hz}, 1 \mathrm{H}), 7.63(\mathrm{dd}, J=5.0,1.1 \mathrm{~Hz}, 1 \mathrm{H}),, 7.12(\mathrm{td}, J=5.0,3.8 \mathrm{~Hz}, 1 \mathrm{H}), 2.55(\mathrm{~s}, 3 \mathrm{H}) ;{ }^{13} \mathrm{C}$ NMR (75 MHz, $\left.\mathrm{CDCl}_{3}\right): \delta 190.7,144.5,133.8,132.5,128.1,26.9$; MS (GC-MS): $\mathrm{m} / \mathrm{z}$ $126\left(\mathrm{M}^{+}\right) ;{ }^{[176]}$ Yield: $70 \%$.<smiles>O=C(c1ccccc1)c1ccncc1</smiles>

40h, Phenyl(pyridin-4-yl)methanone (186b) ${ }^{1} \mathrm{H}-\mathrm{NMR}\left(300 \mathrm{MHz}, \mathrm{CDCl}_{3}\right.$ ): $\delta 8.81$ (dd, $J=4.4,1.6 \mathrm{~Hz}, 2 \mathrm{H}), 7.89-7.77(\mathrm{~m}, 2 \mathrm{H}), 7.64(\mathrm{~s}, 1 \mathrm{H}), 7.58(\mathrm{dd}, J=4.4,1.6 \mathrm{~Hz}, 2 \mathrm{H}), 7.51$ $(\mathrm{t}, J=7.7 \mathrm{~Hz}, 2 \mathrm{H}) ;) ;{ }^{13} \mathrm{C}$ NMR $\left(\mathrm{CDCl}_{3}, 75 \mathrm{MHz}\right): \delta 195.1,150.4,144.4,135.9,133.50$, 130.1, 128.7, 122.8; MS (GC-MS): $m / z 183\left(\mathrm{M}^{+}\right) ;{ }^{[177]}$ Yield: $75 \%$.<smiles>O=C(c1ccccc1)c1ccccn1</smiles>

40h, Phenyl(pyridine-2-yl)methanone (187b): ${ }^{1} \mathrm{H}$ NMR (300 MHz, $\mathrm{CDCl}_{3}$ ): $\delta 8.74$ (ddd, $J=4.8,1.7,0.9 \mathrm{~Hz}, 1 \mathrm{H}), 8.09-8.02(\mathrm{~m}, 3 \mathrm{H}), 7.91$ (td, $J=7.7,1.7 \mathrm{~Hz}, 1 \mathrm{H}), 7.63$ - $7.56(\mathrm{~m}, 1 \mathrm{H}), 7.53-7.45(\mathrm{~m}, 3 \mathrm{H}) ;{ }^{13} \mathrm{C}$ NMR $\left(75 \mathrm{MHz}, \mathrm{CDCl}_{3}\right): \delta$ 194.0, 155.1, 148.6, 137.1, 136.3, 132.9, 131.0, 128.2, 126.2, 124.6; MS (GC-MS): $m / z 183\left(\mathrm{M}^{+}\right) ;{ }^{[177]}$ Yield: $71 \%$.

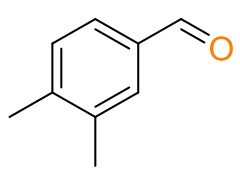

16h, 3, 4-Dimethylbenzaldehyde (188b). ${ }^{1} \mathrm{H}$ NMR (300 MHz, $\left.\mathrm{CDCl}_{3}\right): \delta 9.95(\mathrm{~s}, 1 \mathrm{H})$, $7.66-7.61(\mathrm{~m}, 2 \mathrm{H}), 7.32-7.29(\mathrm{~m}, 1 \mathrm{H}), 2.29(\mathrm{~s}, 6 \mathrm{H}) ;{ }^{13} \mathrm{C} \mathrm{NMR}\left(75 \mathrm{MHz}, \mathrm{CDCl}_{3}\right): \delta$ 
192.3, 144.3, 137.5, 134.6, 130.6, 130.2, 127.7, 20.3, 19.7.; MS (GC-MS): m/z 134 $\left(\mathrm{M}^{+}\right) ;[178]$ Yield: $60 \%$.<smiles>COc1cc(OC)c(OC)cc1C=O</smiles>

9h, 2, 4, 5-Trimethoxybenzaldehyde (189b). ${ }^{1} \mathbf{H} \mathbf{N M R}\left(300 \mathrm{MHz}, \mathrm{CDCl}_{3}\right.$ ): $\delta 10.3$ (s, 1H), $7.34(\mathrm{~s}, 1 \mathrm{H}), 6.51(\mathrm{~s}, 1 \mathrm{H}), 3.99(\mathrm{~s}, 3 \mathrm{H}), 3.94(\mathrm{~s}, 3 \mathrm{H}), 3.89(\mathrm{~s}, 3 \mathrm{H}) ;{ }^{13} \mathrm{C}$ NMR (75 $\left.\mathrm{MHz}, \mathrm{CDCl}_{3}\right): \delta 188.0,158.3,155.8,143.6,117.4,109.0,96.0,56.3,56.2,56.2 ; \mathrm{MS}$ (GC-MS): $m / z 196\left(\mathrm{M}^{+}\right) ;{ }^{[172]}$ Yield: 53\%.<smiles>O=Cc1ccc(-c2ccccc2)cc1</smiles>

24h, Biphenyl-4-carboxaldehyde (190b). ${ }^{1} \mathrm{H}$ NMR (300 MHz, $\left.\mathrm{CDCl}_{3}\right): \delta 10.09(\mathrm{~s}, 1 \mathrm{H})$, 8.00-7.97 (m, 2H), $7.80-7.77(\mathrm{~m}, 2 \mathrm{H}), 7.69-7.64$ (dd, $J=1.8,8.4 \mathrm{~Hz}, 2 \mathrm{H}), 7.54-$ $7.41(\mathrm{~m}, 3 \mathrm{H}) ;{ }^{13} \mathrm{C}$ NMR $\left(75 \mathrm{MHz}, \mathrm{CDCl}_{3}\right): \delta$ 191.9, 147.2, 139.7, 135.2, 130.3, 129.0, 128.5, 127.7, 127.4; MS (GC-MS): m/z $182\left(\mathrm{M}^{+}\right) ;{ }^{[171]}$ Yield: 56\%.<smiles>COc1ccc(C=O)cc1OC</smiles>

9h, 3,4-Dimethoxybenzaldehyde (191b): ${ }^{1} \mathrm{H}$ NMR (300 MHz, $\left.\mathrm{CDCl}_{3}\right): \delta 9.81$ (s, $\left.1 \mathrm{H}\right)$, 7.41 (dd, $J=8.2,1.9 \mathrm{~Hz}, 1 \mathrm{H}$ ), 7.36 (d, $J=1.9 \mathrm{~Hz}, 1 \mathrm{H}$ ), 6.94 (d, J=8.2 Hz, 1H), 3.92 (s, 3H), $3.90(\mathrm{~s}, 3 \mathrm{H}),{ }^{13} \mathrm{C} \mathrm{NMR}\left(75 \mathrm{MHz}, \mathrm{CDCl}_{3}\right): \delta 190.9,154.5,149.6,130.1,126.9,110.4$, 108.9, 56.18, 56.2; MS (GC-MS): $m / z 166\left(\mathrm{M}^{+}\right) ;{ }^{[172]} 41 \%$ yield for 21b, 52\% for 24b. 
皮

16h, 4-Methoxybenzaldehyde (192b). ${ }^{1} \mathrm{H}$ NMR (300 MHz, $\left.\mathrm{CDCl}_{3}\right): \delta 9.88(\mathrm{~s}, 1 \mathrm{H})$, $7.82(\mathrm{~m}, 2 \mathrm{H}), 6.99(\mathrm{~m}, 2 \mathrm{H}), 3.89(\mathrm{~s}, 3 \mathrm{H}) ;{ }^{13} \mathrm{C} \mathrm{NMR}\left(75 \mathrm{MHz}, \mathrm{CDCl}_{3}\right): \delta$ 190.8, 164.6, 132.0, 130.0, 114.3, 55.6; MS (GC-MS): $m / z 136\left(\mathrm{M}^{+}\right) ;{ }^{[171]}$ Yield: 62\%.<smiles>O=Cc1ccccc1</smiles>

16h, Benzaldehyde (193b): ${ }^{1} \mathrm{H}$ NMR (300 MHz, $\mathrm{CDCl}_{3}$ ): $\delta 10.00$ (s, 1H), $7.91-7.83$ (m, 2H), $7.67-7.59(\mathrm{~m}, 1 \mathrm{H}), 7.55-7.46(\mathrm{~m}, 2 \mathrm{H}) ;{ }^{13} \mathrm{C} \mathrm{NMR}\left(\mathrm{CDCl}_{3}, 75 \mathrm{MHz}\right): \delta 192.4$, 136.4, 134.5, 129.7, 129.0; MS (GC-MS): $m / z 106\left(\mathrm{M}^{+}\right) ;{ }^{[171]}$ Yield: 65\%.<smiles>COc1cc(C=O)ccc1O</smiles>

40h, Vanillin (195b): ${ }^{1} \mathrm{H}$ NMR $\left(300 \mathrm{MHz}, \mathrm{CDCl}_{3}\right): \delta 9.85(\mathrm{~s}, 1 \mathrm{H}), 7.47-7.44(\mathrm{~m}, 2 \mathrm{H})$, 7.07 (br-d, $J=8.8 \mathrm{~Hz}, 1 \mathrm{H}), 6.28$ (br-s, $1 \mathrm{H}), 3.99$ (s, 3H); ${ }^{13} \mathrm{C} \mathrm{NMR}\left(\mathrm{CDCl}_{3}, 75 \mathrm{MHz}\right): \delta$ 13C NMR (75 MHz, CDCl3) $\delta$ 190.89, 151.68, 147.14, 129.89, 127.53, 114.38, 108.76, 56.13; MS (GC-MS): $m / z 152\left(\mathrm{M}^{+}\right) ;[179]$ Yield: 65\%.<smiles>COc1cc(C=O)ccc1OC(C)=O</smiles>

16h, Methyl 4-formyl-2-methoxybenzoate (196b): ${ }^{1} \mathbf{H}$ NMR (300 MHz, $\left.\mathrm{CDCl}_{3}\right): \delta$ $9.97(\mathrm{~s}, 1 \mathrm{H}), 7.55-7.46(\mathrm{~m}, 2 \mathrm{H}), 7.24(\mathrm{~d}, J=8.2 \mathrm{~Hz}, 1 \mathrm{H}), 3.92(\mathrm{~s}, 3 \mathrm{H}), 2.37(\mathrm{~s}, 3 \mathrm{H})$; ${ }^{13} \mathrm{C} \mathrm{NMR}\left(\mathrm{CDCl}_{3}, 75 \mathrm{MHz}\right): \delta$ 191.0, 168.3, 152.0, 144.9, 135.2, 124.7, 123.4, 110.8, 56.1, 20.6. MS (GC-MS): $m / z 194\left(\mathrm{M}^{+}\right) ;{ }^{[180]}$ Yield: $42 \%$. 
<smiles>O=C(CBr)c1ccccc1</smiles>

16h, 2-Bromo-1-phenylethan-1-one (197b). ${ }^{1} \mathrm{H}$ NMR (300 MHz, $\left.\mathrm{CDCl}_{3}\right): \delta 8.03-$ $8.00(\mathrm{~m}, 2 \mathrm{H}), 7.67-7.61(\mathrm{~m}, 1 \mathrm{H}), 7.54-7.50(\mathrm{~m}, 2 \mathrm{H}), 4.49(\mathrm{~s}, 2 \mathrm{H}) ;{ }^{13} \mathrm{C} \mathrm{NMR}(75 \mathrm{MHz}$, $\left.\mathrm{CDCl}_{3}\right): \delta 191.3,134.0,133.9,130.0,128.9,30.9 ; \mathrm{MS}$ (GC-MS): $\mathrm{m} / z 197\left(\mathrm{M}^{+}\right) ;{ }^{[171]}$ Yield: 70\%.<smiles>Cc1ccc(C(=O)CBr)cc1</smiles>

16h, 2-Bromo-1-(p-tolyl)ethan-1-one (198b): ${ }^{1} \mathrm{H}$ NMR (300 MHz, $\mathrm{CDCl}_{3}$ ): $\delta 7.88$ (d, $J=8.3 \mathrm{~Hz}, 2 \mathrm{H}), 7.29-7.27(\mathrm{~m}, 2 \mathrm{H}), 4.41(\mathrm{~s}, 2 \mathrm{H}), 2.42(\mathrm{~s}, 3 \mathrm{H}) ;{ }^{13} \mathrm{C} \mathrm{NMR}\left(\mathrm{CDCl}_{3}\right.$, $75 \mathrm{MHz}): \delta$ 190.9, 145.0, 131.5, 31.2, 21.8; MS (GC-MS): $\mathrm{m} / \mathrm{z} 211\left(\mathrm{M}^{+}\right) ;{ }^{[171]}$ Yield: $64 \%$.<smiles>O=C(CBr)c1cccc(Cl)c1</smiles>

16h, 2-Bromo-1-(3-chlorophenyl)ethan-1-one (199b): ${ }^{1} \mathbf{H}$ NMR (300 MHz, $\mathrm{CDCl}_{3}$ ): $\delta$ $8.06-7.92(\mathrm{~m}, 1 \mathrm{H}), 7.88$ (ddd, $J=7.7,1.7,1.1 \mathrm{~Hz}, 1 \mathrm{H}), 7.61(\mathrm{ddd}, J=8.0,2.1,1.1 \mathrm{~Hz}$, 1H), 7.53 - 7.39 (m, 1H), 4,44 (s, 2H); ${ }^{13} \mathrm{C}$ NMR (CDCl, $\left.75 \mathrm{MHz}\right): \delta 190.1,135.5$, 135.3, 133.9, 130.2, 129.0, 127.0, 30.5; MS (GC-MS): m/z 231 (M+); ;181] Yield: 61\%. 
<smiles>CC(=O)Oc1ccc(C=O)cc1</smiles>

40h, 4-Acetoxybenzaldehyde (200b-1): ${ }^{1} \mathrm{H}$ NMR (300 MHz, CDCl 3 ): $\delta 10.02$ (s, $\left.1 \mathrm{H}\right)$, 7.97 - $7.92(\mathrm{~m}, 2 \mathrm{H}), 7.32-7.28(\mathrm{~m}, 2 \mathrm{H}), 2.36(\mathrm{~s}, 3 \mathrm{H}) ;{ }^{13} \mathrm{C} \mathrm{NMR}\left(\mathrm{CDCl}_{3}, 75 \mathrm{MHz}\right): \delta$ 190.9, 168.7, 155.3, 134.0, 131.2, 122.4, 21.2; MS (GC-MS): $m / z 164\left(\mathrm{M}^{+}\right) ;{ }^{[182]}$ Yield: $57 \%$.<smiles>CC(=O)Oc1ccc(C=O)cc1OC(C)=O</smiles>

40h, 3,4-Diacetoxybenzaldehyde (200b-2): ${ }^{1} \mathrm{H}$ NMR (300 MHz, $\mathrm{CDCl}_{3}$ ): $\delta 9.98(\mathrm{~s}, 1 \mathrm{H}$ ), $7.88(\mathrm{dd}, J=2.3,0.6 \mathrm{~Hz}, 2 \mathrm{H}), 7.23(\mathrm{~m}, 1 \mathrm{H}), 2.32(\mathrm{~s}, 6 \mathrm{H}) ;{ }^{13} \mathrm{C} \mathrm{NMR}\left(\mathrm{CDCl}_{3}, 75 \mathrm{MHz}\right): \delta$ 190.0, 168.6, 151.6, 138.1, 121.4, 119.9, 21.0; MS (GC-MS): $m / z 222\left(\mathrm{M}^{+}\right) ;{ }^{[182]}$ Yield: $57 \%$.<smiles>O=c1c2ccccc2sc2ccc(Cl)cc12</smiles>

40h, 2-Chlorothioxanthone (201b): ${ }^{1} \mathrm{H}-\mathrm{NMR}\left(300 \mathrm{MHz}, \mathrm{CDCl}_{3}\right): \delta 8.65-8.63(\mathrm{~m}$, 2H), $7.70-7.29(\mathrm{~m}, 5 \mathrm{H}) ;{ }^{13} \mathrm{C}$ NMR $\left(\mathrm{CDCl}_{3}, 75 \mathrm{MHz}\right): \delta 178.9,136.9,135.4,132.6$, 132.6, 132.5, 130.3, 130.0, 129.3, 128.8, 127.4, 126.6, 126.0; MS (GC-MS): m/z 208 $\left(\mathrm{M}^{+}\right) ;[183]$ Yield: $62 \%$. 


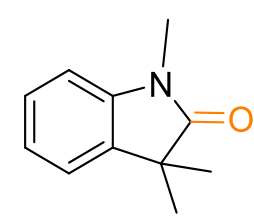

24h, 1,3,3-Trimethylindolin (202b): ${ }^{1} \mathrm{H}-\mathrm{NMR}\left(300 \mathrm{MHz}, \mathrm{CDCl}_{3}\right): \delta 7.26$ (dt, $J=7.6$, $1.2 \mathrm{~Hz}, 1 \mathrm{H}), 7.21(\mathrm{dd}, J=7.2,0.4 \mathrm{~Hz}, 1 \mathrm{H}), 7.06(\mathrm{dt}, J=7.6,0.8 \mathrm{~Hz}, 1 \mathrm{H}), 6.85(\mathrm{~d}, J=8.0$ $\mathrm{Hz}, 1 \mathrm{H}), 3.21(\mathrm{~s}, 3 \mathrm{H}), 1.37(\mathrm{~s}, 6 \mathrm{H}) ;{ }^{13} \mathrm{C}$ NMR $\left(\mathrm{CDCl}_{3}, 75 \mathrm{MHz}\right): \delta 181.3,142.5,135.7$, 127.6, 122.4, 122.2, 107.9, 44.1, 26.1, 24.3; MS (GC-MS): $m / z 175\left(\mathrm{M}^{+}\right)$; $^{[184]}$ Yield: $71 \%$.<smiles>O=C1c2ccccc2C(=O)c2ccccc21</smiles>

24h, Anthraquinone (203b): ${ }^{1} \mathrm{H}-\mathrm{NMR}\left(300 \mathrm{MHz}, \mathrm{CDCl}_{3}\right): \delta 7.7-7.82(\mathrm{~m}, 4 \mathrm{H}), 8.24$

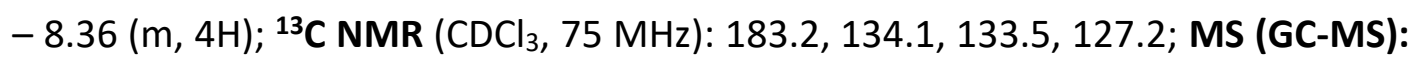
$m / z 208\left(\mathrm{M}^{+}\right) ;{ }^{[185]}$ Yield: 68\%.<smiles>CC(C)OC(=O)C(C)(C)Oc1ccc(C(=O)c2ccc(Cl)cc2)cc1</smiles>

18h, Fenofibrate (205b). ${ }^{1} \mathrm{H}$ NMR (300 MHz, $\left.\mathrm{CDCl}_{3}\right) \delta 7.74(\mathrm{~s}, 4 \mathrm{H}), 7.50-7.39(\mathrm{~m}, 2 \mathrm{H})$, $6.93-6.81(\mathrm{~m}, 2 \mathrm{H}), 5.11$ (hept, $J=6.2 \mathrm{~Hz}, 1 \mathrm{H}), 1.66(\mathrm{~s}, 6 \mathrm{H}), 1.20(\mathrm{~d}, J=6.5 \mathrm{~Hz}, 6 \mathrm{H}) ;{ }^{13} \mathrm{C}$ NMR $\left(\mathrm{CDCl}_{3}, 75 \mathrm{MHz}\right): \delta 194.2,173.1,159.7,138.3,136.4,131.9,131.1,130.2,128.5$, 117.2, 79.4, 69.3, 25.4, 21.5; MS (GC-MS): $m / z 360\left(\mathrm{M}^{+}\right) ;{ }^{[186]}$ Yield: 75\%. 
Data for A Simple Ketone as An Efficient Metal-free Catalyst for Visible-LightMediated Cycloaddition Reactions

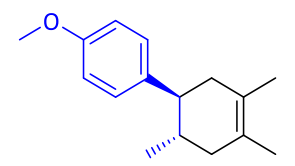

(1S,2S)-4'-methoxy-2,4,5-trimethyl-1,2,3,6-tetrahydro-1,1'-biphenyl

(206b):

${ }^{1} \mathbf{H}$ NMR $\left(\mathrm{CDCl}_{3}, 300 \mathrm{MHz}\right): \delta 7.12-7.07(\mathrm{~m}, 2 \mathrm{H}), 6.88-6.83(\mathrm{~m} .2 \mathrm{H}), 3.80(\mathrm{~s}, 3 \mathrm{H})$, $2.35(\mathrm{td}, J=10.2,6.2 \mathrm{~Hz}, 1 \mathrm{H}), 2.22-2.05(\mathrm{~m}, 3 \mathrm{H}), 1.94-1.78(\mathrm{~m}, 2 \mathrm{H}), 1.66(\mathrm{~s}, 3 \mathrm{H})$, $1.63(\mathrm{~s}, 3 \mathrm{H}), 0.71$ (d, J=6.1 Hz, 3H); ${ }^{13} \mathrm{C}$ NMR $\left(\mathrm{CDCl}_{3}, 75 \mathrm{MHz}\right): \delta 157.9,138.3$, 128.6, 125.6, 125.4, 113.8, 55.3, 48.0, 42.0, 41.8, 34.4, 20.2, 18.9, 18.8; MS (ESI): $\mathrm{m} / \mathrm{z} 231.1[\mathrm{M}+\mathrm{H}]^{+}, 253.1[\mathrm{M}+\mathrm{Na}]^{+} ; \boldsymbol{R}_{\mathbf{F}}=0.55\left(n-\mathrm{Hex}:\right.$ EtOAc 19:1); Yield: 74 \%. ${ }^{[27 f]}$<smiles>COc1ccc([C@H]2CC=C(CCC=C(C)C)C[C@H]2C)cc1</smiles>

(1S,2S)-4'-methoxy-2-methyl-4-(4-methylpent-3-en-1-yl)-1,2,3,6-tetrahydro-1,1'biphenyl (207b): ${ }^{1} \mathrm{H}$ NMR $\left(\mathrm{CDCl}_{3}, 300 \mathrm{MHz}\right): \delta 7.11-7.06(\mathrm{~m}, 2 \mathrm{H}), 6.86-6.81(\mathrm{~m}, 2 \mathrm{H})$, $5.45(\mathrm{~s}, 1 \mathrm{H}), 5.16-5.11(\mathrm{~m}, 1 \mathrm{H}), 3.79(\mathrm{~s}, 3 \mathrm{H}), 2.37-2.07(\mathrm{~m}, 6 \mathrm{H}), 2.01-1.76(\mathrm{~m}, 4 \mathrm{H})$, $1.70(\mathrm{~s}, 3 \mathrm{H}), 1.62(\mathrm{~s}, 3 \mathrm{H}), 0.71(\mathrm{~d}, J=6.2 \mathrm{~Hz}, 3 \mathrm{H}) ;{ }^{13} \mathrm{C} \mathrm{NMR}\left(\mathrm{CDCl}_{3}, 75 \mathrm{MHz}\right): \delta 157.9$, 138.4, 137.6, 131.6, 128.6, 124.5, 120.6, 113.8, 55.4, 47.2, 38.3, 37.7, 35.3, 34.1, 26.6, 25.9, 20.4, 17.9; ESI-HRMS: $m / z$ calcd. for $\left[\mathrm{C}_{20} \mathrm{H}_{28} \mathrm{O}+\mathrm{Na}\right]^{+}: 307.2032$, found 307.2029; Yield: $82 \%{ }^{[27 f]}$ 


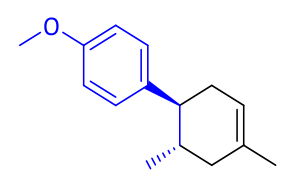

(1S,2S)-4'-methoxy-2,4-dimethyl-1,2,3,6-tetrahydro-1,1'-biphenyl

(208b):

${ }^{1} \mathrm{H}$ NMR $\left(\mathrm{CDCl}_{3}, 300 \mathrm{MHz}\right): \delta 7.11-7.06(\mathrm{~m}, 2 \mathrm{H}), 6.87-6.82(\mathrm{~m}, 2 \mathrm{H}), 5.45(\mathrm{~s}, 1 \mathrm{H})$, $3.80(\mathrm{~s}, 3 \mathrm{H}), 2.31(\mathrm{td}, J=10.4,5.3 \mathrm{~Hz}, 1 \mathrm{H}), 2.22-2.05(\mathrm{~m}, 3 \mathrm{H}), 1.97-1.75(\mathrm{~m}, 2 \mathrm{H})$, $1.69(\mathrm{~s}, 3 \mathrm{H}), 0.71$ (d, $J=6.2 \mathrm{~Hz}, 3 \mathrm{H}) ;{ }^{13} \mathrm{C} \mathrm{NMR}\left(\mathrm{CDCl}_{3}, 75 \mathrm{MHz}\right): \delta 157.9,138.3$, 134.0, 128.6, 121.0, 113.8, 55.4, 47.1, 40.0, 35.4, 34.1, 23.5, 20.4; MS (ESI): $\mathrm{m} / \mathrm{z}$ $217.1[\mathrm{M}+\mathrm{H}]^{+} ; \boldsymbol{R}_{\mathrm{F}}=0.35\left(n-\mathrm{Hex}:\right.$ EtOAc 19:1); Yield: $88 \%{ }^{[27 f]}$

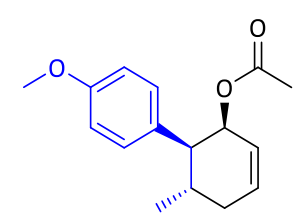

(1S,2S,6S)-4'-methoxy-6-methyl-1,2,5,6-tetrahydro-[1,1'-biphenyl]-2-yl acetate (209b): ${ }^{1} \mathrm{H}$ NMR $\left(\mathrm{CDCl}_{3}, 300 \mathrm{MHz}\right): \delta 7.15-7.11(\mathrm{~m}, 2 \mathrm{H}), 6.85-6.80(\mathrm{~m}, 2 \mathrm{H}), 6.03$ (ddd, J = 9.9, 4.8, 2.2 Hz, 1H), $5.90-5.84(\mathrm{~m}, 1 \mathrm{H}), 5.24-5.21(\mathrm{~m}, 1 \mathrm{H}), 3.79(\mathrm{~s}, 3 \mathrm{H})$, $2.62(\mathrm{dd}, J=11.8,3.8 \mathrm{~Hz}, 1 \mathrm{H}), 2.43-2.27(\mathrm{~m}, 2 \mathrm{H}), 1.91-1.78(\mathrm{~m}, 1 \mathrm{H}), 1.88(\mathrm{~s}, 3 \mathrm{H})$, $0.79(\mathrm{~d}, J=6.3 \mathrm{~Hz}, 3 \mathrm{H}) ;{ }^{13} \mathrm{C}$ NMR $\left(\mathrm{CDCl}_{3}, 75 \mathrm{MHz}\right): \delta 170.3,158.3,133.0,132.4$, 130.4, 125.1, 113.5, 70.1, 55.3, 50.8, 35.2, 27.2, 21.3, 20.0; ESI-HRMS: $\mathrm{m} / \mathrm{z}$ calcd. for $\left[\mathrm{C}_{16} \mathrm{H}_{20} \mathrm{O}_{3}+\mathrm{NH}_{4}\right]^{+}$: 278.1751, found 278.1753; calcd. for $\left[\mathrm{C}_{16} \mathrm{H}_{20} \mathrm{O}_{3}+\mathrm{Na}\right]^{+}$: 283.1305, found 283.1306; calcd. for $\left[\mathrm{C}_{16} \mathrm{H}_{20} \mathrm{O}_{3}+\mathrm{K}\right]^{+}$: 299.1044, found 299.1050; Yield: $64 \%{ }^{[49 a]}$

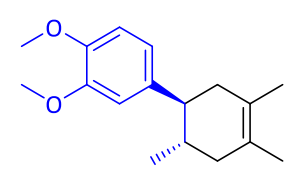

(1S,2S)-3',4'-dimethoxy-2,4,5-trimethyl-1,2,3,6-tetrahydro-1,1'-biphenyl (210b): ${ }^{1} \mathrm{H}$ NMR $\left(\mathrm{CDCl}_{3}, 300 \mathrm{MHz}\right): \delta 6.82-6.79(\mathrm{~m}, 1 \mathrm{H}), 6.73-6.70(\mathrm{~m}, 2 \mathrm{H}), 3.87(\mathrm{~s}, 3 \mathrm{H})$, $3.86(\mathrm{~s}, 3 \mathrm{H}), 2.33(\mathrm{td}, J=10.1,6.3 \mathrm{~Hz}, 1 \mathrm{H}), 2.19-2.02(\mathrm{~m}, 3 \mathrm{H}), 1.93-1.77(\mathrm{~m}, 2 \mathrm{H})$, 
$1.64(\mathrm{~s}, 3 \mathrm{H}), 1.62(\mathrm{~s}, 3 \mathrm{H}), 0.71(\mathrm{~d}, J=6.1 \mathrm{~Hz}, 3 \mathrm{H}) ;{ }^{13} \mathrm{C} \mathrm{NMR}\left(\mathrm{CDCl}_{3}, 75 \mathrm{MHz}\right): \delta$ 149.0, 147.3, 139.0, 125.5, 125.5, 119.7, 111.2, 110.8, 56.0, 56.0, 48.5, 41.9, 41.7, 34.4, 20.1, 18.9, 18.8; ESI-HRMS: $m / z$ calcd. for $\left[\mathrm{C}_{17} \mathrm{H}_{24} \mathrm{O}_{2}+\mathrm{H}\right]^{+}:$261.1849, found 261.1848; calcd. for [ $\left.\mathrm{C}_{17} \mathrm{H}_{24} \mathrm{O}_{2}+\mathrm{Na}\right]^{+}:$283.1669, found 283.1662; Yield: $80 \%{ }^{\text {[49a] }}$

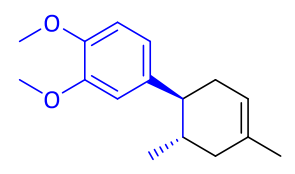

(1S,2S)-3',4'-dimethoxy-2,4-dimethyl-1,2,3,6-tetrahydro-1,1'-biphenyl (211b): ${ }^{1} \mathrm{H}$ NMR $\left(\mathrm{CDCl}_{3}, 300 \mathrm{MHz}\right): \delta 6.82-6.79(\mathrm{~m}, 1 \mathrm{H}), 6.73-6.70(\mathrm{~m}, 2 \mathrm{H}), 5.44(\mathrm{~s}, 1 \mathrm{H})$, $3.87(\mathrm{~s}, 3 \mathrm{H}), 3.86(\mathrm{~s}, 3 \mathrm{H}), 2.29(\mathrm{td}, J=10.3,4.9 \mathrm{~Hz}, 1 \mathrm{H}), 2.23-2.04(\mathrm{~m}, 3 \mathrm{H}), 1.96-$ $1.74(\mathrm{~m}, 2 \mathrm{H}), 1.69(\mathrm{~s}, 3 \mathrm{H}), 0.72(\mathrm{~d}, J=6.2 \mathrm{~Hz}, 3 \mathrm{H}) ;{ }^{13} \mathrm{C} \mathrm{NMR}\left(\mathrm{CDCl}_{3}, 75 \mathrm{MHz}\right): \delta$ 149.0, 147.3, 139.0, 134.0, 120.9, 119.8, 111.2, 110.9, 56.0, 56.0, 47.6, 40.0, 35.3, 34.2, 23.5, 20.4; ESI-HRMS: $\mathrm{m} / \mathrm{z}$ calcd. for $\left[\mathrm{C}_{16} \mathrm{H}_{22} \mathrm{O}_{2}+\mathrm{H}\right]^{+}:$:247.1693, found 247.1693; calcd. for [ $\left.\mathrm{C}_{16} \mathrm{H}_{22} \mathrm{O}_{2}+\mathrm{Na}\right]^{+}:$269.1512, found 269.1511; Yield: 69 \%. ${ }^{\text {[49a] }}$

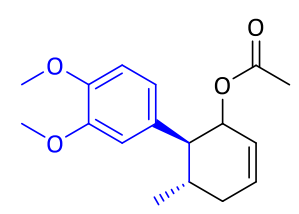

(1S,6S)-3',4'-dimethoxy-6-methyl-1,2,5,6-tetrahydro-[1,1'-biphenyl]-2-yl acetate (212b): ${ }^{1} \mathrm{H}$ NMR $\left(\mathrm{CDCl}_{3}, 300 \mathrm{MHz}\right): \delta 6.81-6.77(\mathrm{~m}, 3 \mathrm{H}), 6.03$ (ddd, J = 9.9, 4.8, 2.2 Hz, 1H), $5.92-5.86(\mathrm{~m}, 1 \mathrm{H}), 5.26-5.22(\mathrm{~m}, 1 \mathrm{H}), 3.87(\mathrm{~s}, 3 \mathrm{H}), 3.86(\mathrm{~s}, 3 \mathrm{H})$, $2.61(\mathrm{dd}, J=11.8,3.7 \mathrm{~Hz}, 1 \mathrm{H}), 2.50-2.17(\mathrm{~m}, 3 \mathrm{H}), 1.90(\mathrm{~s}, 3 \mathrm{H}), 0.81(\mathrm{~d}, J=6.3 \mathrm{~Hz}$, $3 \mathrm{H}) ;{ }^{13} \mathrm{C} \mathrm{NMR}\left(\mathrm{CDCl}_{3}, 75 \mathrm{MHz}\right): \delta 170.2,153.0,148.5,137.7,133.0,125.1,121.9$, 112.7, 110.9, 70.2, 56.0, 55.9, 51.1, 35.2, 27.3, 21.4, 20.0; ESI-HRMS: $\mathrm{m} / \mathrm{z}$ calcd. for $\left[\mathrm{C}_{17} \mathrm{H}_{22} \mathrm{O}_{4}+\mathrm{NH}_{4}\right]^{+}$: 308.1856, found 308.1860; calcd. for $\left[\mathrm{C}_{17} \mathrm{H}_{22} \mathrm{O}_{4}+\mathrm{Na}\right]^{+}$: 313.1410, found 313.1403; Yield: $77 \%$. 


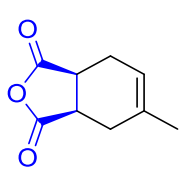

(3aR,7aS)-5-methyl-3a,4,7,7a-tetrahydroisobenzofuran-1,3-dione

(213b):

${ }^{1} \mathrm{H}$ NMR $\left(\mathrm{CDCl}_{3}, 300 \mathrm{MHz}\right): \delta 5.63-5.58(\mathrm{~m}, 1 \mathrm{H}), 3.43-3.29(\mathrm{~m}, 2 \mathrm{H}), 2.55$ (ddd, $J=16.3,6.8,2.6 \mathrm{~Hz}, 1 \mathrm{H}), 2.47(\mathrm{dd}, J=15.6,2.8 \mathrm{~Hz}, 1 \mathrm{H}), 2.29-2.18(\mathrm{~m}, 2 \mathrm{H}), 1.74$ $(\mathrm{s}, 3 \mathrm{H}) ;{ }^{13} \mathrm{C} \mathrm{NMR}\left(\mathrm{CDCl}_{3}, 75 \mathrm{MHz}\right): \delta 174.6,174.5,136.7,120.2,40.2,39.5,28.5$, 24.2, 23.6; ESI-HRMS: $\mathrm{m} / \mathrm{z}$ calcd. for $\left[\mathrm{C}_{9} \mathrm{H}_{10} \mathrm{O}_{3}+\mathrm{H}\right]^{+}$: 167.0703, found 167.0705; calcd. for $\left[\mathrm{C}_{9} \mathrm{H}_{10} \mathrm{O}_{3}+\mathrm{Na}\right]^{+}:$189.0522, found 189.0521; Yield: $94 \% .{ }^{[187]}$

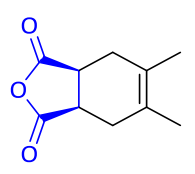

(3aR,7aS)-5,6-dimethyl-3a,4,7,7a-tetrahydroisobenzofuran-1,3-dione (214b): ${ }^{1} \mathrm{H}$ NMR $\left(\mathrm{CDCl}_{3}, 300 \mathrm{MHz}\right): \delta 3.34-3.31(\mathrm{~m}, 2 \mathrm{H}), 2.43(\mathrm{~d}, J=15.1 \mathrm{~Hz}, 2 \mathrm{H}), 2.25(\mathrm{~d}$, $J=13.7 \mathrm{~Hz}, 2 \mathrm{H}), 1.68(\mathrm{~d}, J=0.9 \mathrm{~Hz}, 6 \mathrm{H}) ;{ }^{13} \mathrm{C} \mathrm{NMR}\left(\mathrm{CDCl}_{3}, 75 \mathrm{MHz}\right): \delta 174.7,127.4$, 40.5, 30.5, 19.4; ESI-HRMS: $m / z$ calcd. for $\left[\mathrm{C}_{10} \mathrm{H}_{12} \mathrm{O}_{3}+\mathrm{H}\right]^{+}:$: 181.0859, found 181.0860; calcd. for $\left[\mathrm{C}_{10} \mathrm{H}_{12} \mathrm{O}_{3}+\mathrm{Na}\right]^{+}:$203.0679, found 203.0678; Yield: $95 \%$. $^{[188]}$

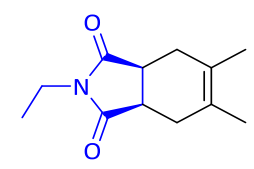

(3aR,7aS)-2-ethyl-5,6-dimethyl-3a,4,7,7a-tetrahydro-1H-isoindole-1,3(2H)dione (215b): ${ }^{1} \mathrm{H}$ NMR $\left(\mathrm{CDCl}_{3}, 300 \mathrm{MHz}\right): \delta 3.47$ (q, $\left.J=7.2 \mathrm{~Hz}, 2 \mathrm{H}\right), 3.00-2.97(\mathrm{~m}$, $2 \mathrm{H}$ ), 2.42 (d, J = 14.6 Hz, 2H), 2.20 (d, J = $14.0 \mathrm{~Hz}, 2 \mathrm{H}$ ), 1.64 (d, J = 1.0 Hz, 6H), 1.05 $(\mathrm{t}, J=7.2 \mathrm{~Hz}, 3 \mathrm{H}) ;{ }^{13} \mathrm{C} \mathrm{NMR}\left(\mathrm{CDCl}_{3}, 75 \mathrm{MHz}\right): \delta 180.2,127.0,40.0,33.8,30.9,19.3$, 13.2; ESI-HRMS: $m / z$ calcd. for $\left[\mathrm{C}_{12} \mathrm{H}_{17} \mathrm{O}_{2}+\mathrm{H}\right]^{+}:$208.1332, found 208.1328; calcd. for $\left[\mathrm{C}_{12} \mathrm{H}_{17} \mathrm{O}_{2}+\mathrm{Na}\right]^{+}:$230.1151, found 230.1145; Yield: $86 \%{ }^{[189]}$ 


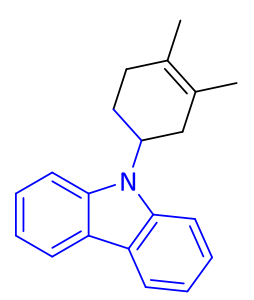

9-(3,4-dimethylcyclohex-3-en-1-yl)-9H-fluoren (216b): ${ }^{1} \mathrm{H}$ NMR $\quad\left(\mathrm{CDCl}_{3}\right.$, $500 \mathrm{MHz}): \delta 8.11-8.07(\mathrm{~m}, 2 \mathrm{H}), 7.52-7.49(\mathrm{~m}, 2 \mathrm{H}), 7.43-7.37(\mathrm{~m}, 2 \mathrm{H}), 7.22-$ $7.16(\mathrm{~m}, 2 \mathrm{H}), 4.82-4.71(\mathrm{~m}, 1 \mathrm{H}), 3.06-2.94(\mathrm{~m}, 1 \mathrm{H}), 2.72-2.58(\mathrm{~m}, 1 \mathrm{H}), 2.43-$ $2.31(\mathrm{~m}, 1 \mathrm{H}), 2.25-2.16(\mathrm{~m}, 2 \mathrm{H}), 2.04-1.99(\mathrm{~m}, 1 \mathrm{H}), 1.72(\mathrm{~s}, 3 \mathrm{H}), 1.66(\mathrm{~s}, 3 \mathrm{H})$; ${ }^{13} \mathrm{C} \mathrm{NMR}\left(\mathrm{CDCl}_{3}, 125 \mathrm{MHz}\right): \delta$ 139.7, 125.9, 125.3, 124.4, 123.3, 120.3, 118.5, 110.1, 52.4, 34.9, 32.6, 27.8, 19.1, 18.8; ESI-HRMS: $m / z$ calcd. for $\left[\mathrm{C}_{21} \mathrm{H}_{22}+\mathrm{H}\right]^{+}$: 276.1752, found 276.1759; Yield: 50\%. ${ }^{\text {[49a] }}$<smiles>COc1ccc(C2CC(=O)C3CCN2C3)cc1</smiles>

2-(4-methoxyphenyl)-1-phenyl-2,3-dihydropyridin-4(1H)-one (217b): ${ }^{1} \mathrm{H}$ NMR $\left(\mathrm{CDCl}_{3}, 300 \mathrm{MHz}\right): \delta 7.64(\mathrm{dd}, J=7.8,1.1 \mathrm{~Hz}, 1 \mathrm{H}), 7.33-7.27(\mathrm{~m}, 2 \mathrm{H}) 7.20-7.15$ $(\mathrm{m}, 2 \mathrm{H}), 7.10(\mathrm{tt}, J=6.9,1.0 \mathrm{~Hz}, 1 \mathrm{H}), 7.04-7.00(\mathrm{~m}, 2 \mathrm{H}), 6.86-6.81(\mathrm{~m}, 2 \mathrm{H}), 5.27$ $(\mathrm{dd}, J=7.8,1.1 \mathrm{~Hz}, 1 \mathrm{H}), 5.24(\mathrm{dd}, J=6.9,3.2 \mathrm{~Hz}, 1 \mathrm{H}), 3.77(\mathrm{~s}, 3 \mathrm{H}), 3.26(\mathrm{dd}, J=16.3$, $7.0 \mathrm{~Hz}, 1 \mathrm{H}), 2.76(\mathrm{ddd}, J=16.3,3.3,1.1 \mathrm{~Hz}, 1 \mathrm{H}) ;{ }^{13} \mathrm{C} \mathrm{NMR}\left(\mathrm{CDCl}_{3}, 75 \mathrm{MHz}\right): \delta 190.6$, 159.3, 148.4, 144.9, 129.9, 129.6, 127.5, 124.5, 118.8, 114.5, 102.9, 61.4, 55.4, 43.8; ESI-HRMS: $m / z$ calcd. for $\left[\mathrm{C}_{18} \mathrm{H}_{17} \mathrm{NO}_{2}+\mathrm{H}\right]^{+}:$: 280.1332, found 280.1330; calcd. for $\left[\mathrm{C}_{18} \mathrm{H}_{17} \mathrm{NO}_{2}+\mathrm{Na}\right]^{+}$: 302.1151, found 302.1144; calcd. for $\left[\mathrm{C}_{18} \mathrm{H}_{17} \mathrm{NO}_{2}+\mathrm{K}\right]^{+}$: 318.0891, found 318.0881; Yield: $80 \% .^{[190]}$ 


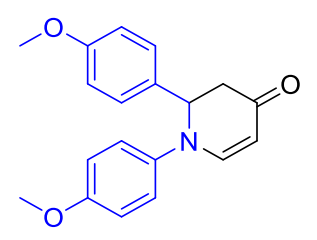

1,2-bis(4-methoxyphenyl)-2,3-dihydropyridin-4(1H)-one (218b): ${ }^{1} \mathrm{H}$ NMR ( $\mathrm{CDCl}_{3}$, $300 \mathrm{MHz}): \delta 7.19-7.14(\mathrm{~m}, 2 \mathrm{H}), 6.98-6.93(\mathrm{~m}, 2 \mathrm{H}), 6.85-6.77(\mathrm{~m}, 4 \mathrm{H}), 7.50(\mathrm{~d}$, $J=7.8 \mathrm{~Hz}, 1 \mathrm{H}), 5.21(\mathrm{~d}, J=7.7 \mathrm{~Hz}, 1 \mathrm{H}), 5.13(\mathrm{dd}, J=6.9,4.3 \mathrm{~Hz}, 1 \mathrm{H}), 3.77(\mathrm{~s}, 3 \mathrm{H})$, $3.76(\mathrm{~s}, 3 \mathrm{H}), 3.21(\mathrm{dd}, J=16.3,7.0 \mathrm{~Hz}, 1 \mathrm{H}), 2.74(\mathrm{dd}, J=16.3,4.2 \mathrm{~Hz}, 1 \mathrm{H}) ;{ }^{13} \mathrm{C}$ NMR $\left(\mathrm{CDCl}_{3}, 75 \mathrm{MHz}\right): \delta 190.5,159.3,157.1,149.8,138.5,130.4,127.8,121.5,114.7$, 114.4, 101.6, 62.2, 55.7, 55.4, 43.8; ESI-HRMS: $\mathrm{m} / \mathrm{z}$ calcd. for $\left[\mathrm{C}_{19} \mathrm{H}_{19} \mathrm{NO}_{3}+\mathrm{H}\right]^{+}$: 310.1438, found 310.1440; calcd. for $\left[\mathrm{C}_{19} \mathrm{H}_{19} \mathrm{NO}_{3}+\mathrm{Na}\right]^{+}$: 332.1257, found 332.1256; Yield: $89 \% .{ }^{[191]}$<smiles>COc1cc(C2CC(=O)C=CN2c2ccccc2)cc(OC)c1OC</smiles>

1-phenyl-2-(3,4,5-trimethoxyphenyl)-2,3-dihydropyridin-4(1H)-one (219b): ${ }^{1} \mathrm{H}$ NMR $\left(\mathrm{CDCl}_{3}, 300 \mathrm{MHz}\right): \delta 7.66(\mathrm{dd}, J=7.8,0.8 \mathrm{~Hz}, 1 \mathrm{H}), 7.34-7.28(\mathrm{~m}, 2 \mathrm{H}), 7.15$ - $7.10(\mathrm{~m}, 1 \mathrm{H}), 7.05-7.03(\mathrm{~m}, 2 \mathrm{H}), 6.46(\mathrm{~s}, 2 \mathrm{H}), 5.29(\mathrm{dd}, J=7.8,0.7 \mathrm{~Hz}, 1 \mathrm{H}), 5.18$ (dd, $J=7.1,2.9 \mathrm{~Hz}, 1 \mathrm{H}), 3.81(\mathrm{~s}, 3 \mathrm{H}), 3.77(\mathrm{~s}, 6 \mathrm{H}), 3.28(\mathrm{dd}, J=16.4,7.2 \mathrm{~Hz}, 1 \mathrm{H}$ ), 2.77 (ddd, $J=16.3,3.1,0.8 \mathrm{~Hz}, 1 \mathrm{H}) ;{ }^{13} \mathrm{C} \mathrm{NMR}\left(\mathrm{CDCl}_{3}, 75 \mathrm{MHz}\right): \delta 190.4,153.7$, 148.5, 144.9, 137.7, 133.9, 129.7, 124.8, 119.0, 103.3, 102.7, 62.1, 60.9, 56.3, 43.7; ESI-HRMS: $m / z$ calcd. for $\left[\mathrm{C}_{20} \mathrm{H}_{21} \mathrm{NO}_{4}+\mathrm{H}\right]^{+}:$340.1543, found 340.1536; calcd. for $\left[\mathrm{C}_{20} \mathrm{H}_{21} \mathrm{NO}_{4}+\mathrm{Na}\right]^{+}:$362.1363, found 362.1357; Yield: $91 \%$. 


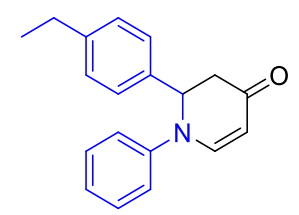

2-(4-ethylphenyl)-1-phenyl-2,3-dihydropyridin-4(1H)-one $\quad$ (220b): $\quad{ }^{1} \mathrm{H}$ NMR $\left(\mathrm{CDCl}_{3}, 300 \mathrm{MHz}\right): \delta 7.67(\mathrm{dd}, J=7.8,1.0 \mathrm{~Hz}, 1 \mathrm{H}), 7.32-7.27(\mathrm{~m}, 2 \mathrm{H}), 7.19-7.08$ $(\mathrm{m}, 5 \mathrm{H}), 7.08-7.02(\mathrm{~m}, 2 \mathrm{H}), 5.29-5.24(\mathrm{~m}, 2 \mathrm{H}), 3.29(\mathrm{dd}, J=16.3,7.1 \mathrm{~Hz}, 1 \mathrm{H})$, $2.78(\mathrm{ddd}, J=16.3,3.0,1.0 \mathrm{~Hz}, 1 \mathrm{H}), 2.62(\mathrm{q}, J=7.6 \mathrm{~Hz}, 2 \mathrm{H}), 1.21(\mathrm{t}, J=7.6 \mathrm{~Hz}, 3 \mathrm{H})$; ${ }^{13} \mathrm{C} \mathrm{NMR}\left(\mathrm{CDCl}_{3}, 75 \mathrm{MHz}\right): \delta$ 190.5, 148.2, 145.0, 144.0, 135.1, 129.7, 128.6, 126.2, 124.5, 118.7, 103.1, 61.7, 43.7, 28.6, 15.5; ESI-HRMS: $m / z$ calcd. for $\left[\mathrm{C}_{19} \mathrm{H}_{19} \mathrm{NO}+\mathrm{H}\right]^{+}:$278.1539, found 278.1541; calcd. for [ $\left.\mathrm{C}_{19} \mathrm{H}_{19} \mathrm{NO}+\mathrm{Na}\right]^{+}: 300.1359$, found 300.1358; Yield: $87 \%$.<smiles>COc1ccc(N2C=CC(=O)CC2c2ccccc2)cc1</smiles>

1-(4-methoxyphenyl)-2-phenyl-2,3-dihydropyridin-4(1H)-one (221b): ${ }^{1} \mathrm{H}$ NMR $\left(\mathrm{CDCl}_{3}, 300 \mathrm{MHz}\right): \delta 7.54(\mathrm{dd}, J=7.8,0.8 \mathrm{~Hz}, 1 \mathrm{H}), 7.34-7.22(\mathrm{~m}, 5 \mathrm{H}), 6.98-6.93$ $(\mathrm{m}, 2 \mathrm{H}), 6.83-6.77(\mathrm{~m}, 2 \mathrm{H}), 5.23(\mathrm{dd}, J=7.8,0.8 \mathrm{~Hz}, 1 \mathrm{H}), 5.18(\mathrm{dd}, J=7.1,3.9 \mathrm{~Hz}$, $1 \mathrm{H}), 3.75(\mathrm{~s}, 3 \mathrm{H}), 3.26(\mathrm{dd}, J=16.4,7.2 \mathrm{~Hz}, 1 \mathrm{H}$ ), 2.77 (ddd, $J=16.4,3.9,0.8 \mathrm{~Hz}, 1 \mathrm{H}$ ); ${ }^{13} \mathrm{C} \mathrm{NMR}\left(\mathrm{CDCl}_{3}, 75 \mathrm{MHz}\right): \delta$ 190.2, 157.0, 149.7, 138.5, 138.4, 129.1, 128.0, 126.5, 121.3, 114.8, 101.8, 62.6, 55.7, 43.6; ESI-HRMS: $m / z$ calcd. for $\left[\mathrm{C}_{18} \mathrm{H}_{17} \mathrm{NO}_{2}+\mathrm{H}\right]^{+}$: 280.1332, found 280.1331; calcd. for $\left[\mathrm{C}_{18} \mathrm{H}_{17} \mathrm{NO}_{2}+\mathrm{Na}\right]^{+}:$302.1151, found 302.1148; Yield: $86 \%{ }^{[30 \mathrm{~b}]}$<smiles>CN1C=CC(=O)CC1c1ccccc1</smiles>

1-methyl-2-phenyl-2,3-dihydropyridin-4(1H)-one (222b): ${ }^{1} \mathrm{H} \mathrm{NMR} \quad\left(\mathrm{CDCl}_{3}\right.$, $300 \mathrm{MHz}): \delta 7.41-7.29(\mathrm{~m}, 5 \mathrm{H}), 7.09(\mathrm{~d}, J=7.6 \mathrm{~Hz}, 1 \mathrm{H}), 5.03(\mathrm{~d}, J=7.6 \mathrm{~Hz}, 1 \mathrm{H})$, 
$4.47(\mathrm{dd}, J=9.6,6.7 \mathrm{~Hz}, 1 \mathrm{H}), 2.83(\mathrm{dd}, J=16.5,6.8 \mathrm{~Hz}, 1 \mathrm{H}), 2.83(\mathrm{~s}, 3 \mathrm{H}), 2.69$ (dd, $J=16.5,9.6 \mathrm{~Hz}, 1 \mathrm{H}) ;{ }^{13} \mathrm{C}$ NMR $\left(\mathrm{CDCl}_{3}, 75 \mathrm{MHz}\right): \delta 190.7,155.3,139.0,129.2,128.5$, 127.1, 98.7, 64.0, 44.1, 41.6; ESI-HRMS: $m / z$ calcd. for $\left[\mathrm{C}_{12} \mathrm{H}_{13} \mathrm{NO}+\mathrm{H}\right]^{+}:$188.1070, found 188.1072; calcd. for [ $\left.\mathrm{C}_{12} \mathrm{H}_{13} \mathrm{NO}+\mathrm{Na}\right]^{+}:$210.0889, found 210.0889; Yield: 86 $\%^{[192]}$

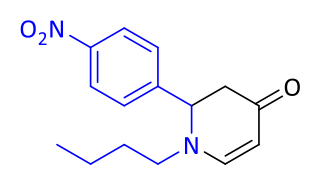

1-butyl-2-(4-nitrophenyl)-2,3-dihydropyridin-4(1H)-one (223b): ${ }^{1} \mathrm{H}$ NMR $\left(\mathrm{CDCl}_{3}\right.$, $300 \mathrm{MHz}): \delta 8.24-8.19(\mathrm{~m}, 2 \mathrm{H}), 7.49-7.45(\mathrm{~m}, 2 \mathrm{H}), 7.19(\mathrm{~d}, J=7.6 \mathrm{~Hz}, 1 \mathrm{H}), 5.04$ (d, $J=7.6 \mathrm{~Hz}, 1 \mathrm{H}), 4.73(\mathrm{dd}, J=7.4,5.9 \mathrm{~Hz}, 1 \mathrm{H}), 3.24-3.14(\mathrm{~m}, 1 \mathrm{H}), 3.10-2.96$ $(\mathrm{m}, 2 \mathrm{H}), 2.60(\mathrm{dd}, J=16.4,5.9 \mathrm{~Hz}, 1 \mathrm{H}), 1.54(\mathrm{q}, J=7.1 \mathrm{~Hz}, 2 \mathrm{H}), 1.39-1.25(\mathrm{~m}, 2 \mathrm{H})$, $0.91(\mathrm{t}, J=7.3 \mathrm{~Hz}, 3 \mathrm{H}) ;{ }^{13} \mathrm{C} \mathrm{NMR}\left(\mathrm{CDCl}_{3}, 75 \mathrm{MHz}\right): \delta 188.8,153.7,147.9,146.3$, 127.8, 124.5, 98.9, 60.3, 54.1, 43.2, 31.1, 19.9, 13.8; ESI-HRMS: $\mathrm{m} / \mathrm{z}$ calcd. for $\left[\mathrm{C}_{15} \mathrm{H}_{18} \mathrm{~N}_{2} \mathrm{O}_{3}+\mathrm{H}\right]^{+}:$275.1390, found 275.1391; calcd. for $\left[\mathrm{C}_{15} \mathrm{H}_{18} \mathrm{~N}_{2} \mathrm{O}_{3}+\mathrm{Na}\right]^{+}$: 297.1210, found 297.1206; Yield: $90 \%$.

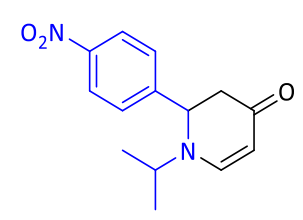

1-isopropyl-2-(4-nitrophenyl)-2,3-dihydropyridin-4(1H)-one (224b): ${ }^{1} \mathrm{H}$ NMR $\left(\mathrm{CDCl}_{3}, 300 \mathrm{MHz}\right): \delta 8.23-8.18(\mathrm{~m}, 2 \mathrm{H}), 7.51-7.46(\mathrm{~m}, 2 \mathrm{H}), 7.33(\mathrm{~d}, J=7.7 \mathrm{~Hz}$, $1 \mathrm{H}), 5.10(\mathrm{~d}, J=7.7 \mathrm{~Hz}, 1 \mathrm{H}), 4.77(\mathrm{dd}, J=7.3,5.7 \mathrm{~Hz}, 1 \mathrm{H}), 3.41(\mathrm{sept}, J=6.6 \mathrm{~Hz}, 1 \mathrm{H})$, 3.00 (dd, $J=16.3,7.4 \mathrm{~Hz}, 1 \mathrm{H}$ ), 2.57 (dd, $J=16.3,5.5 \mathrm{~Hz}, 1 \mathrm{H}$ ), 1.30 (d, $J=6.7 \mathrm{~Hz}$, $3 \mathrm{H}), 1.16(\mathrm{~d}, J=6.6 \mathrm{~Hz}, 3 \mathrm{H}) ;{ }^{13} \mathrm{C} \mathrm{NMR}\left(\mathrm{CDCl}_{3}, 75 \mathrm{MHz}\right): \delta 188.9,150.0,147.3$, 127.6, 124.5, 99.4, 59.6, 54.3, 43.4, 21.9; ESI-HRMS: $\mathrm{m} / \mathrm{z}$ calcd. for $\left[\mathrm{C}_{14} \mathrm{H}_{16} \mathrm{~N}_{2} \mathrm{O}_{3}+\mathrm{H}\right]^{+}$: 261.1234, found 261.1235; calcd. for $\left[\mathrm{C}_{14} \mathrm{H}_{16} \mathrm{~N}_{2} \mathrm{O}_{3}+\mathrm{Na}\right]^{+}$: 283.1053, found 283.1050; Yield: $93 \%$. 


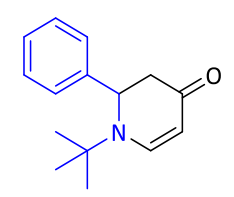

1-(tert-butyl)-2-phenyl-2,3-dihydropyridin-4(1H)-one (225b): ${ }^{1} \mathrm{H}$ NMR $\left(\mathrm{CDCl}_{3}\right.$, $300 \mathrm{MHz}$ ): $\delta 7.57$ (dd, $J=7.7,1.3 \mathrm{~Hz}, 1 \mathrm{H}), 7.30-7.18(\mathrm{~m}, 5 \mathrm{H}), 5.02$ (dd, $J=7.7$, $1.0 \mathrm{~Hz}, 1 \mathrm{H}$ ), 4.95 (d, $J=7.7 \mathrm{~Hz}, 1 \mathrm{H}$ ), 3.10 (dd, $J=16.2,7.7 \mathrm{~Hz}, 1 \mathrm{H}$ ), 2.50 (td, $J=16.2$, $1.4 \mathrm{~Hz}, 1 \mathrm{H}) 1.33(\mathrm{~s}, 9 \mathrm{H}) ;{ }^{13} \mathrm{C} \mathrm{NMR}\left(\mathrm{CDCl}_{3}, 75 \mathrm{MHz}\right): \delta 189.0,149.3,140.1,128.8$, 127.6, 126.2, 98.7, 58.7, 56.3, 43.5, 29.4; ESI-HRMS: $m / z$ calcd. for $\left[\mathrm{C}_{15} \mathrm{H}_{19} \mathrm{NO}+\mathrm{H}\right]^{+}$: 230.1539, found 230.1542; calcd. for [ $\left.\mathrm{C}_{15} \mathrm{H}_{19} \mathrm{NO}+\mathrm{Na}\right]^{+}: 252.1359$, found 252.1351; Yield: $94 \%$.

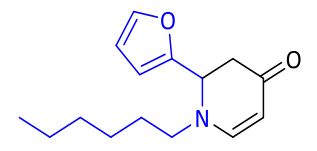

2-(furan-2-yl)-1-hexyl-2,3-dihydropyridin-4(1H)-one (226b): ${ }^{1} \mathrm{H} \mathrm{NMR}\left(\mathrm{CDCl}_{3}\right.$, $300 \mathrm{MHz}): \delta 7.38(\mathrm{dd}, J=1.8,0.8 \mathrm{~Hz}, 1 \mathrm{H}), 6.96(\mathrm{~d}, J=7.6 \mathrm{~Hz}, 1 \mathrm{H}), 6.32(\mathrm{dd}, J=3.3$, $1.8 \mathrm{~Hz}, 1 \mathrm{H}), 6.28(\mathrm{~d}, J=3.3 \mathrm{~Hz}, 1 \mathrm{H}), 4.96(\mathrm{~d}, J=7.6 \mathrm{~Hz}, 1 \mathrm{H}), 4.66(\mathrm{t}, J=6.4 \mathrm{~Hz}, 1 \mathrm{H})$, $3.31-3.09(\mathrm{~m}, 2 \mathrm{H}), 2.83(\mathrm{dd}, J=16.4,6.6 \mathrm{~Hz}, 1 \mathrm{H}), 2.75(\mathrm{dd}, J=16.6,6.2 \mathrm{~Hz}, 1 \mathrm{H})$, $1.62-1.52(\mathrm{~m}, 2 \mathrm{H}), 1.34-1.23(\mathrm{~m}, 6 \mathrm{H}), 0.91-0.86(\mathrm{~m}, 3 \mathrm{H}) ;{ }^{13} \mathrm{C} \mathrm{NMR}\left(\mathrm{CDCl}_{3}\right.$, $75 \mathrm{MHz}): \delta$ 190.3, 152.9, 151.4, 142.7, 110.5, 108.6, 97.7, 54.4, 54.2, 40.0, 31.5, 29.2, 26.3, 22.6, 14.1; ESI-HRMS: $m / z$ calcd. for $\left[\mathrm{C}_{15} \mathrm{H}_{21} \mathrm{NO}_{2}+\mathrm{H}\right]^{+}: 248.1645$, found 248.1647; calcd. for $\left[\mathrm{C}_{15} \mathrm{H}_{21} \mathrm{NO}_{2}+\mathrm{Na}\right]^{+}: 270.1465$, found 270.1465; calcd. for $\left[\mathrm{C}_{15} \mathrm{H}_{21} \mathrm{NO}_{2}+\mathrm{K}\right]^{+}:$286.1204, found 286.1206; Yield: $92 \%$.

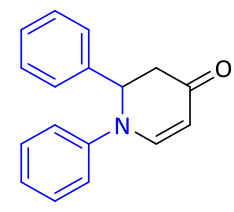

1,2-diphenyl-2,3-dihydropyridin-4(1H)-one (227b): ${ }^{1} \mathrm{H}$ NMR $\left(\mathrm{CDCl}_{3}, 300 \mathrm{MHz}\right): \delta 7.68$ (dd, $J=7.8,1.1 \mathrm{~Hz}, 1 \mathrm{H}), 7.36-7.24(\mathrm{~m}, 7 \mathrm{H}), 7.11(\mathrm{tt}, J=7.4,1.1 \mathrm{~Hz}, 1 \mathrm{H}), 7.04-7.00$ 
(m, 2H), 5.29 (dd, J = 7.8, 1.0 Hz, 1H), $5.29-5.27$ (m, 1H), 3.31 (dd, J = 16.4, 7.1 Hz, 1H), 2.80 (ddd, $J=16.4,3.2,1.1 \mathrm{~Hz}, 1 \mathrm{H}) ;{ }^{13} \mathrm{C}$ NMR $\left(\mathrm{CDCl}_{3}, 75 \mathrm{MHz}\right): \delta 190.4,148.4$, 144.9, 138.1, 129.7, 129.1, 128.0, 126.3, 124.6, 118.7, 103.2, 61.9, 43.6; ESI-HRMS: $\mathrm{m} / z$ calcd. for $\left[\mathrm{C}_{17} \mathrm{H}_{15} \mathrm{NO}+\mathrm{H}\right]^{+}: 250.1226$, found 250.1227 ; calcd. for $\left[\mathrm{C}_{17} \mathrm{H}_{15} \mathrm{NO}+\mathrm{Na}\right]^{+}$: 272.1046, found 272.1047; Yield: $83 \%$. ${ }^{[191]}$<smiles>COc1ccc(C2CC(=O)C=CN2c2ccc([N+](=O)[O-])cc2)cc1</smiles>

2-(4-methoxyphenyl)-1-(4-nitrophenyl)-2,3-dihydropyridin-4(1H)-one

(228b):

${ }^{1} \mathrm{H} \mathrm{NMR}\left(\mathrm{CDCl}_{3}, 300 \mathrm{MHz}\right): \delta 8.20-8.15(\mathrm{~m}, 2 \mathrm{H}), 7.72(\mathrm{dd}, J=8.1,1.2 \mathrm{~Hz}, 1 \mathrm{H}), 7.17-$ $7.07(\mathrm{~m}, 4 \mathrm{H}), 6.89-6.84(\mathrm{~m}, 2 \mathrm{H}), 5.44(\mathrm{dd}, J=8.0,1.0 \mathrm{~Hz}, 1 \mathrm{H}), 5.34(\mathrm{~d}, J=5.2 \mathrm{~Hz}, 1 \mathrm{H})$, $3.78(\mathrm{~s}, 3 \mathrm{H}), 3.31$ (dd, $J=16.4,6.9 \mathrm{~Hz}, 1 \mathrm{H}), 2.84$ (ddd, $J=16.4,2.6,1.2 \mathrm{~Hz}, 1 \mathrm{H}$ ); ${ }^{13} \mathrm{C} \mathrm{NMR}\left(\mathrm{CDCl}_{3}, 75 \mathrm{MHz}\right): \delta 190.4,159.6,149.4,145.4,143.2,128.4,127.2,125.8$, 117.1, 114.9, 106.6, 61.2, 55.5, 43.9; ESI-HRMS: $m / z$ calcd. for $\left[\mathrm{C}_{18} \mathrm{H}_{16} \mathrm{~N}_{2} \mathrm{O}_{4}+\mathrm{H}\right]^{+}$: 325.1183, found 325.1184; calcd. for $\left[\mathrm{C}_{18} \mathrm{H}_{16} \mathrm{~N}_{2} \mathrm{O}_{4}+\mathrm{Na}\right]^{+}$: 347.1002, found 347.1004; Yield: $94 \% .{ }^{[193]}$

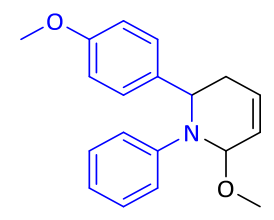

6-methoxy-2-(4-methoxyphenyl)-1-phenyl-1,2,3,6-tetrahydropyridine

(229b):

${ }^{1} \mathrm{H}$ NMR $\left(\mathrm{CDCl}_{3}, 300 \mathrm{MHz}\right): \delta 7.36-7.31(\mathrm{~m}, 2 \mathrm{H}), 7.18(\mathrm{~d}, J=7.6 \mathrm{~Hz}, 1 \mathrm{H}), 7.02(\mathrm{t}$, $J=7.6 \mathrm{~Hz}, 1 \mathrm{H}), 6.92-6.87(\mathrm{~m}, 2 \mathrm{H}), 6.68(\mathrm{td}, J=7.5,1.0 \mathrm{~Hz}, 1 \mathrm{H}), 6.53-6.49(\mathrm{~m}, 2 \mathrm{H})$, $4.71(\mathrm{dd}, J=12.6,9.6 \mathrm{~Hz}, 1 \mathrm{H}), 4.45(\mathrm{dd}, J=11.2,2.6 \mathrm{~Hz}, 1 \mathrm{H}), 3.99(\mathrm{~s}, 1 \mathrm{H}), 3.82(\mathrm{~s}, 3 \mathrm{H})$, $3.60-3.51(\mathrm{~m}, 1 \mathrm{H}), 3.56(\mathrm{~s}, 3 \mathrm{H}), 2.07$ (ddd, $J=13.0,5.5,2.8 \mathrm{~Hz}, 1 \mathrm{H}), 1.97-1.85(\mathrm{~m}$, 1H); ${ }^{13} \mathrm{C} \mathrm{NMR}\left(\mathrm{CDCl}_{3}, 75 \mathrm{MHz}\right): \delta 159.3,148.7,145.1,136.4,129.0,127.8,124.5$, 117.5, 114.1, 106.4, 100.1, 56.1, 55.5, 41.1, 37.5; ESI-HRMS: $\mathrm{m} / \mathrm{z}$ calcd. for $\left[\mathrm{C}_{19} \mathrm{H}_{21} \mathrm{NO}_{2}+\mathrm{H}\right]^{+}:$296.1645, found 296.1642; Yield: $82 \%{ }^{[194]}$ 


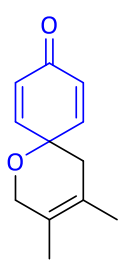

3,4-dimethyl-1-oxaspiro[5.5] undeca-3,7,10-trien-9-one (230b): ${ }^{1} \mathrm{H}$ NMR $\left(\mathrm{CDCl}_{3}\right.$, $300 \mathrm{MHz}): \delta 7.01-6.96(\mathrm{~m}, 2 \mathrm{H}), 6.24-6.18(\mathrm{~m}, 2 \mathrm{H}), 4.14-4.10(\mathrm{~m}, 2 \mathrm{H}), 2.12-2.11$ $(\mathrm{m}, 2 \mathrm{H}), 1.69-1.68(\mathrm{~m}, 3 \mathrm{H}), 1.63-1.62(\mathrm{~m}, 3 \mathrm{H}) ;{ }^{13} \mathrm{C} \mathrm{NMR}\left(\mathrm{CDCl}_{3}, 75 \mathrm{MHz}\right): \delta 185.7$, 148.4, 128.6, 123.6, 121.6, 69.0, 65.6, 38.6, 18.6, 14.0; ESI-HRMS: $\mathrm{m} / \mathrm{z}$ calcd. for $\left[\mathrm{C}_{12} \mathrm{H}_{14} \mathrm{O}_{2}+\mathrm{H}\right]^{+}:$191.1067, found 191.1065; calcd. for $\left[\mathrm{C}_{12} \mathrm{H}_{14} \mathrm{O}_{2}+\mathrm{Na}\right]^{+}: 213.0886$, found 213.0888; $\boldsymbol{R}_{\mathrm{F}}=0.61\left(n-\right.$ Hex:EtOAc 19:1); Yield: $81 \%{ }^{[195]}$

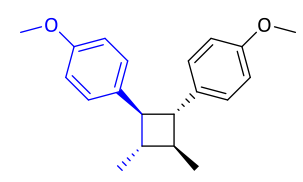

4,4'-((1R,2R,3S,4S)-3,4-dimethylcyclobutane-1,2-diyl)bis(methoxybenzene) (231b): ${ }^{1} \mathrm{H}$ NMR $\left(\mathrm{CDCl}_{3}, 300 \mathrm{MHz}\right): \delta 7.15-7.10(\mathrm{~m}, 4 \mathrm{H}), 6.85-6.80(\mathrm{~m}, 4 \mathrm{H}), 3.78(\mathrm{~s}, 6 \mathrm{H})$, $2.81-2.78(\mathrm{~m}, 2 \mathrm{H}), 1.87-1.78(\mathrm{~m}, 2 \mathrm{H}), 1.18(\mathrm{~d}, J=6.0 \mathrm{~Hz}, 6 \mathrm{H}) ;{ }^{13} \mathrm{C} \mathrm{NMR}\left(\mathrm{CDCl}_{3}\right.$, $75 \mathrm{MHz}$ ): $\delta$ 158.1, 136.1, 127.9, 113.8, 55.4, 52.6, 43.4, 19.0; ESI-HRMS: $\mathrm{m} / \mathrm{z}$ calcd. for $\left[\mathrm{C}_{20} \mathrm{H}_{24} \mathrm{O}_{2}+\mathrm{H}\right]^{+}:$:297.1849, found 297.1837; calcd. for $\left[\mathrm{C}_{20} \mathrm{H}_{24} \mathrm{O}_{2}+\mathrm{NH}_{4}\right]^{+}: 314.2115$, found 314.2110; calcd. for $\left[\mathrm{C}_{20} \mathrm{H}_{24} \mathrm{O}_{2}+\mathrm{Na}\right]^{+}: 319.1669$, found 319.1665; Yield: $76 \%{ }^{[196]}$

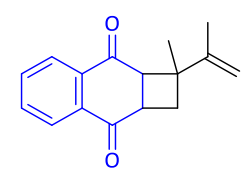

1-methyl-1-(prop-1-en-2-yl)-1,2,2a,8a-tetrahydrocyclobuta[b]naphthalene-3,8dione (232b): ${ }^{1} \mathrm{H}$ NMR $\left(\mathrm{CDCl}_{3}, 300 \mathrm{MHz}\right): \delta 8.16-8.09(\mathrm{~m}, 2 \mathrm{H}), 7.80-7.74(\mathrm{~m}, 2 \mathrm{H})$, $4.99(\mathrm{~s}, 1 \mathrm{H}$ ), 4.86 (quint, $J=1.4 \mathrm{~Hz}, 1 \mathrm{H}$ ), 3.75 (dd, $J=9.4,0.9 \mathrm{~Hz}, 1 \mathrm{H}$ ), 3.40 (ddd, $J=0.8$, 9.4, $3.9 \mathrm{~Hz}, 1 \mathrm{H}), 2.87-2.79(\mathrm{~m}, 1 \mathrm{H}), 2.27(\mathrm{ddd}, J=11.9,3.9,1.0 \mathrm{~Hz}, 1 \mathrm{H}), 1.81(\mathrm{dd}$, $J=1.3,0.6 \mathrm{~Hz}, 3 \mathrm{H}), 1.04(\mathrm{~s}, 3 \mathrm{H}) ;{ }^{13} \mathrm{C} \mathrm{NMR}\left(\mathrm{CDCl}_{3}, 75 \mathrm{MHz}\right): \delta 199.5,195.8,151.2$, 
136.9, 136.0, 134.4, 127.3, 109.6, 51.8, 49.8, 38.6, 37.1, 23.4, 18.3; ESI-HRMS: $m / z$ calcd. for $\left[\mathrm{C}_{16} \mathrm{H}_{16} \mathrm{O}_{2}+\mathrm{H}\right]^{+}:$241.1223, found 241.1220; calcd. for $\left[\mathrm{C}_{16} \mathrm{H}_{16} \mathrm{O}_{2}+\mathrm{Na}\right]^{+}$: 263.1043, found 263.1039; Yield: $72 \%{ }^{[194]}$ 


\subsection{NMR Spectra}

\section{6b}

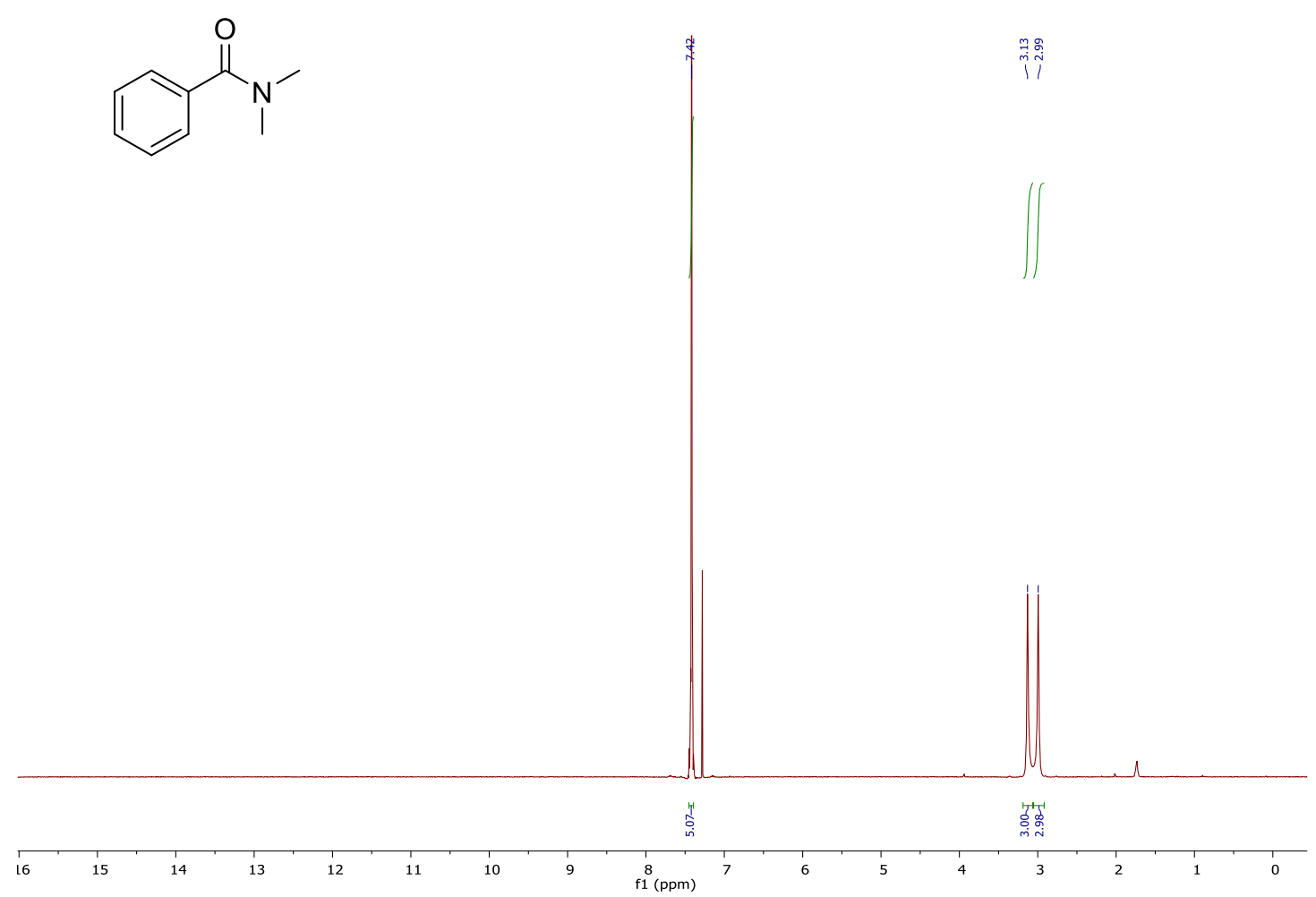

${ }^{1} \mathrm{H}$ NMR spectrum in $\mathrm{CDCl}_{3}$.
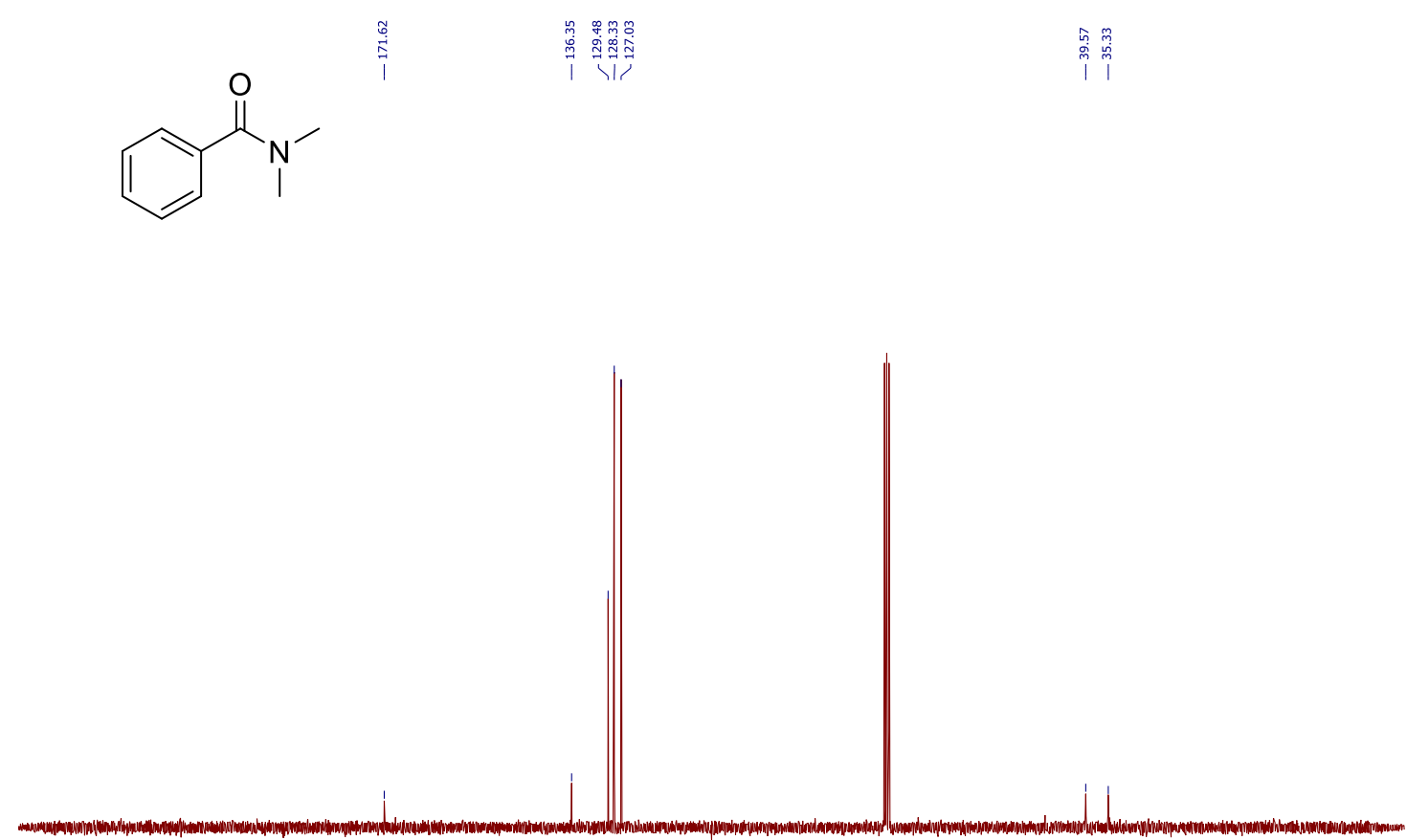

$\begin{array}{llllllllllllllllllllllllllllllll}1 & 230 & 220 & 210 & 200 & 190 & 180 & 170 & 160 & 150 & 140 & 130 & 120 & 110 & 100 & 90 & 80 & 70 & 60 & 50 & 40 & 30 & 20 & 10 & 0 & -10 & -2\end{array}$

${ }^{13} \mathrm{C}$ NMR spectrum in $\mathrm{CDCl}_{3}$. 
Experimental Section

127b

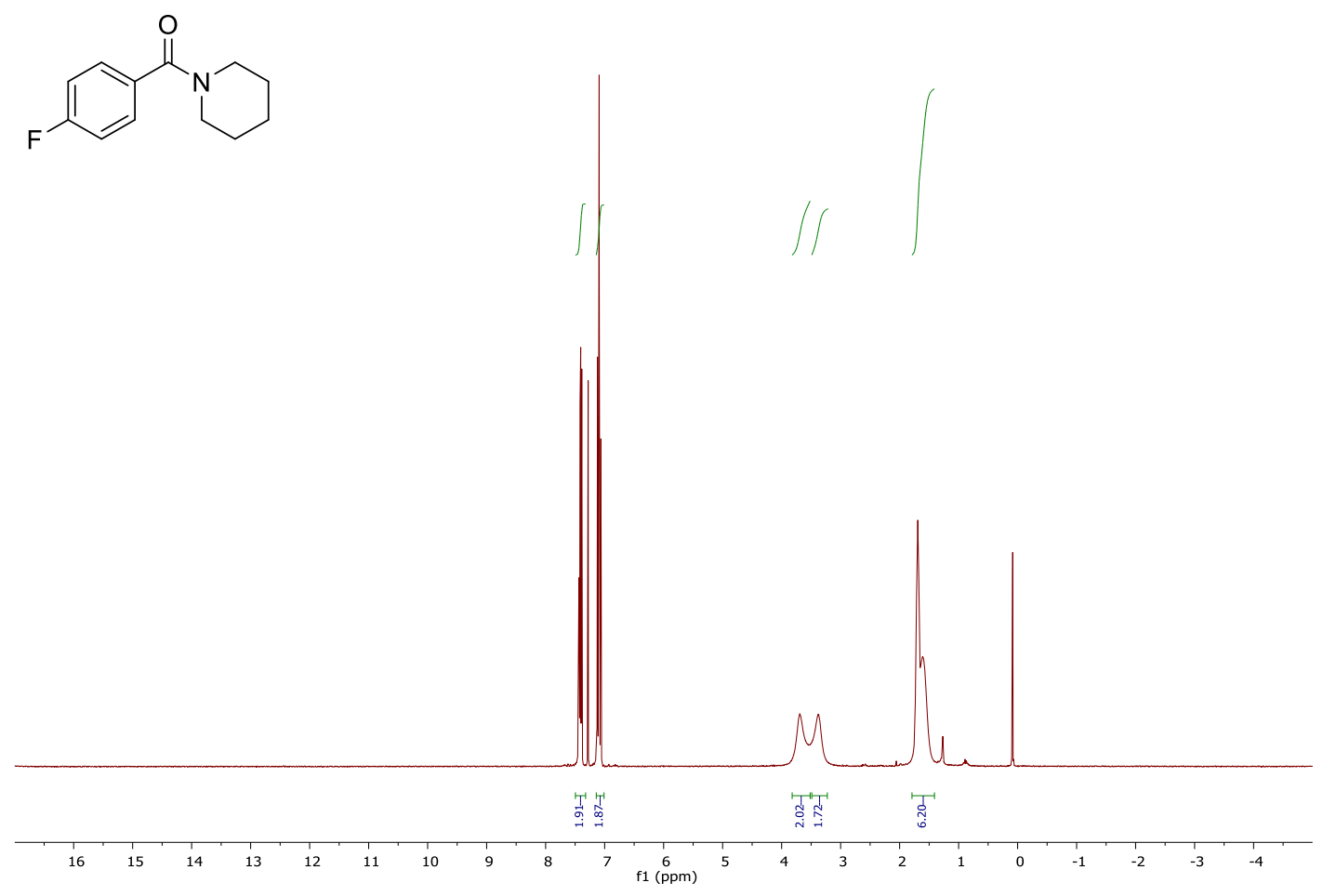

${ }^{1} \mathrm{H}$ NMR spectrum in $\mathrm{CDCl}_{3}$.

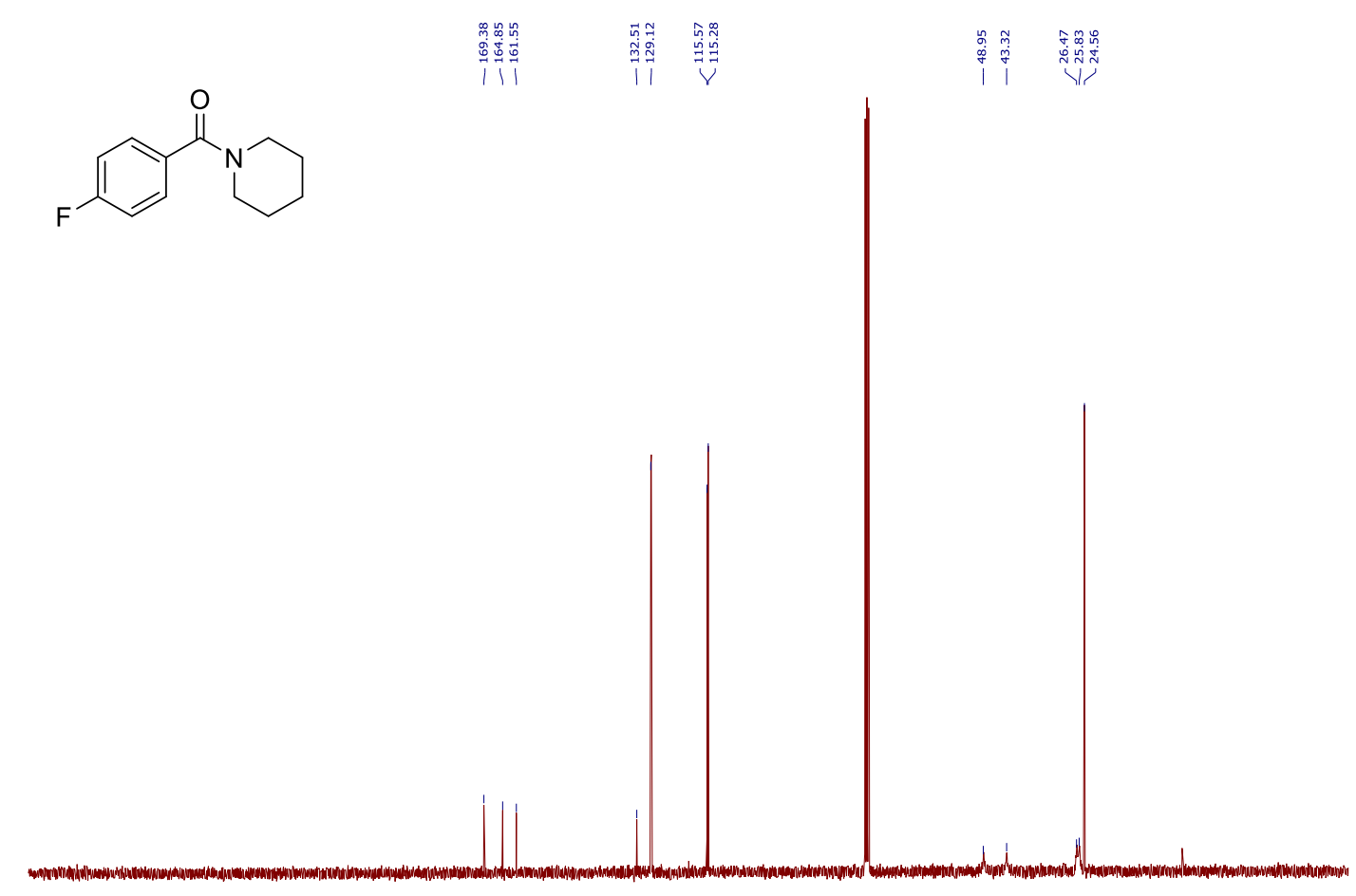

\begin{tabular}{rlllllllllllllllllllllllllllllllll}
\hline 270 & 260 & 250 & 240 & 230 & 220 & 210 & 200 & 190 & 180 & 170 & 160 & 150 & 140 & 130 & 120 & 110 & 100 & 90 & 80 & 70 & 60 & 50 & 40 & 30 & 20 & 10 & 0 & -10 & -20 & -30
\end{tabular}

${ }^{13} \mathrm{C}$ NMR spectrum in $\mathrm{CDCl}_{3}$.

169 
Experimental Section

$128 b$

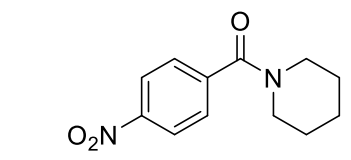

$\stackrel{\substack{0 \\ 0}}{1}$

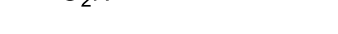

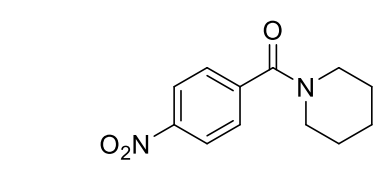
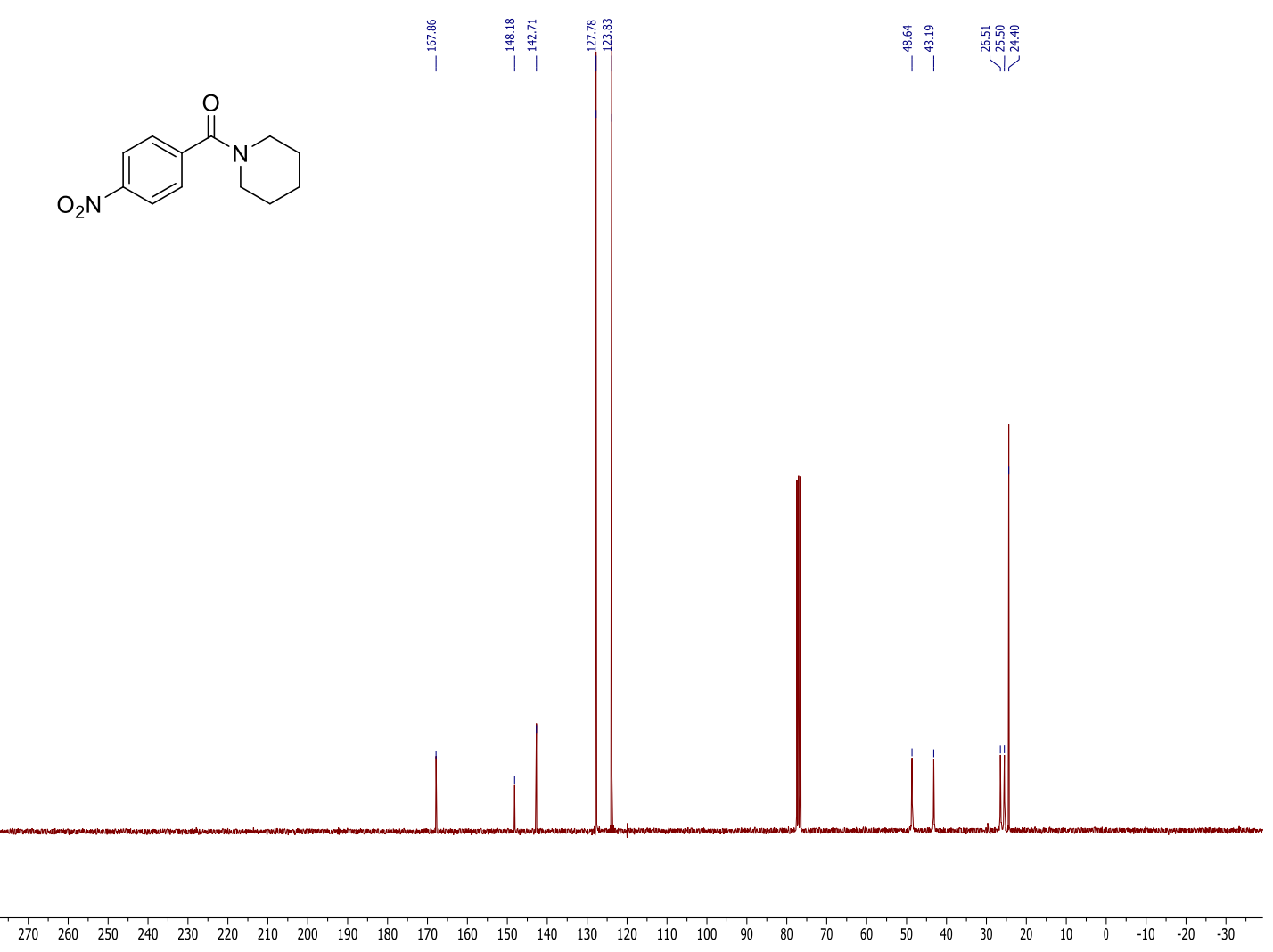

${ }^{13} \mathrm{C}$ NMR spectrum in $\mathrm{CDCl}_{3}$.

170 
Experimental Section

$129 b$
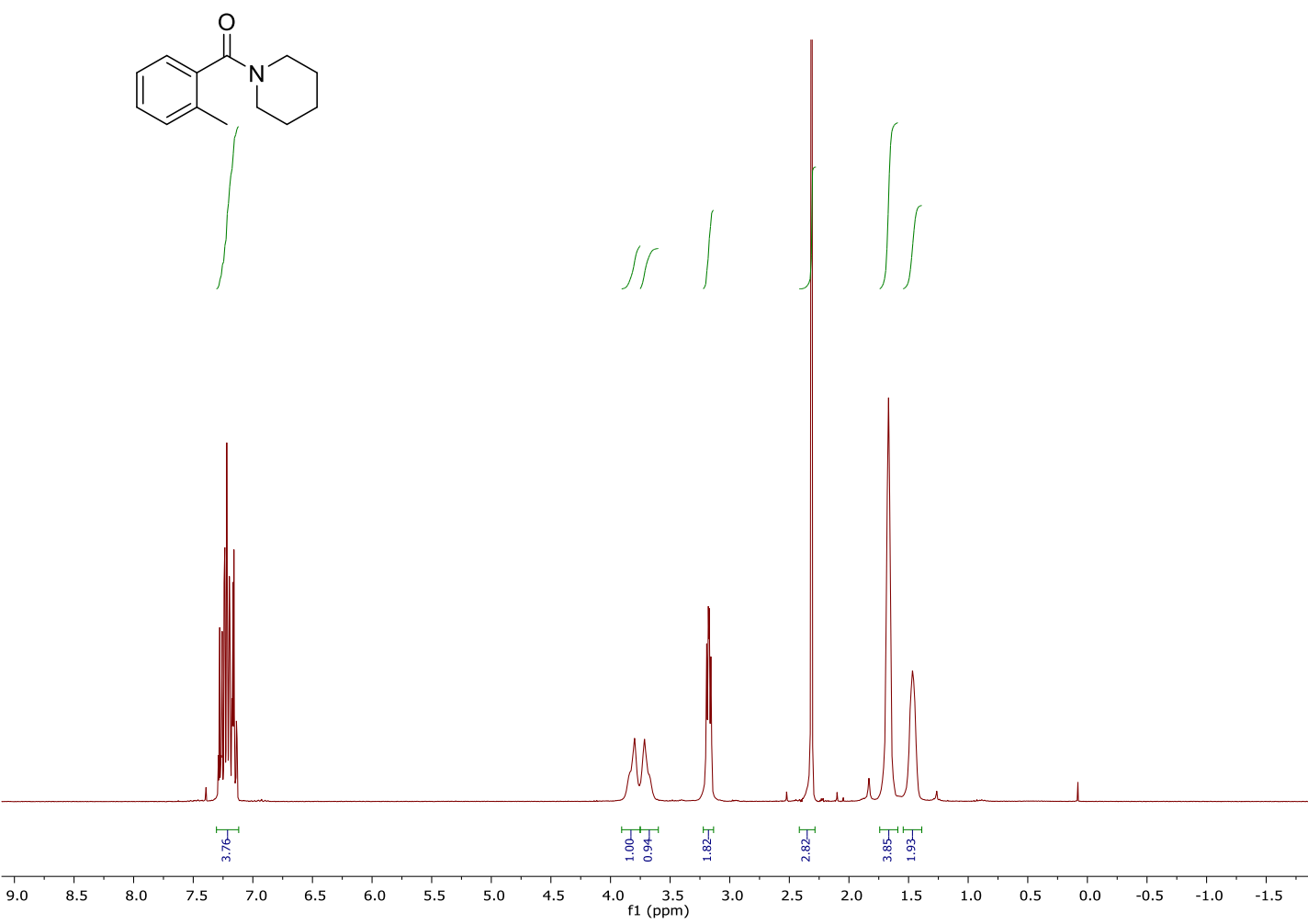

${ }^{1} \mathrm{H}$ NMR spectrum in $\mathrm{CDCl}_{3}$.

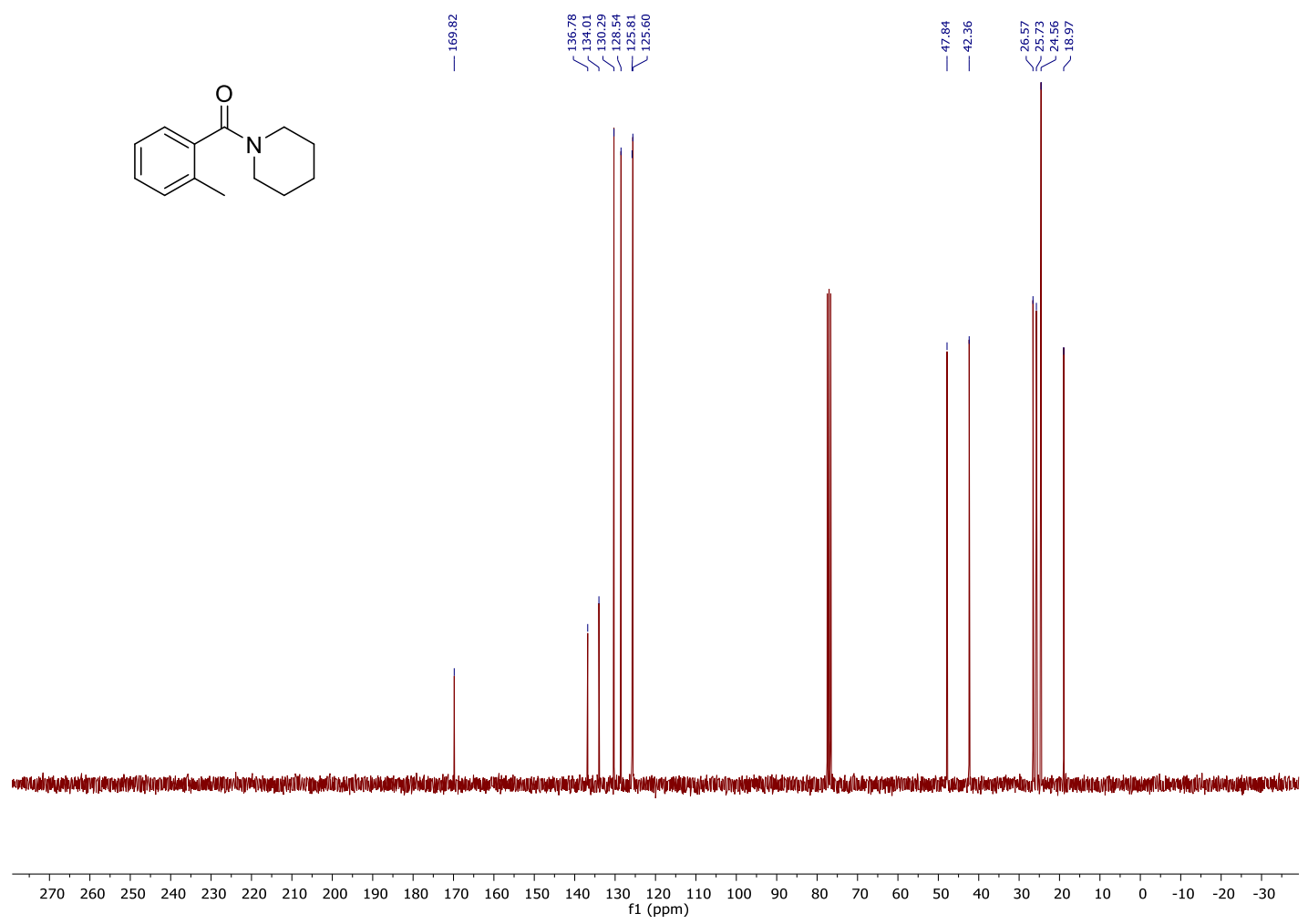

${ }^{13} \mathrm{C}$ NMR spectrum in $\mathrm{CDCl}_{3}$.

171 
130b

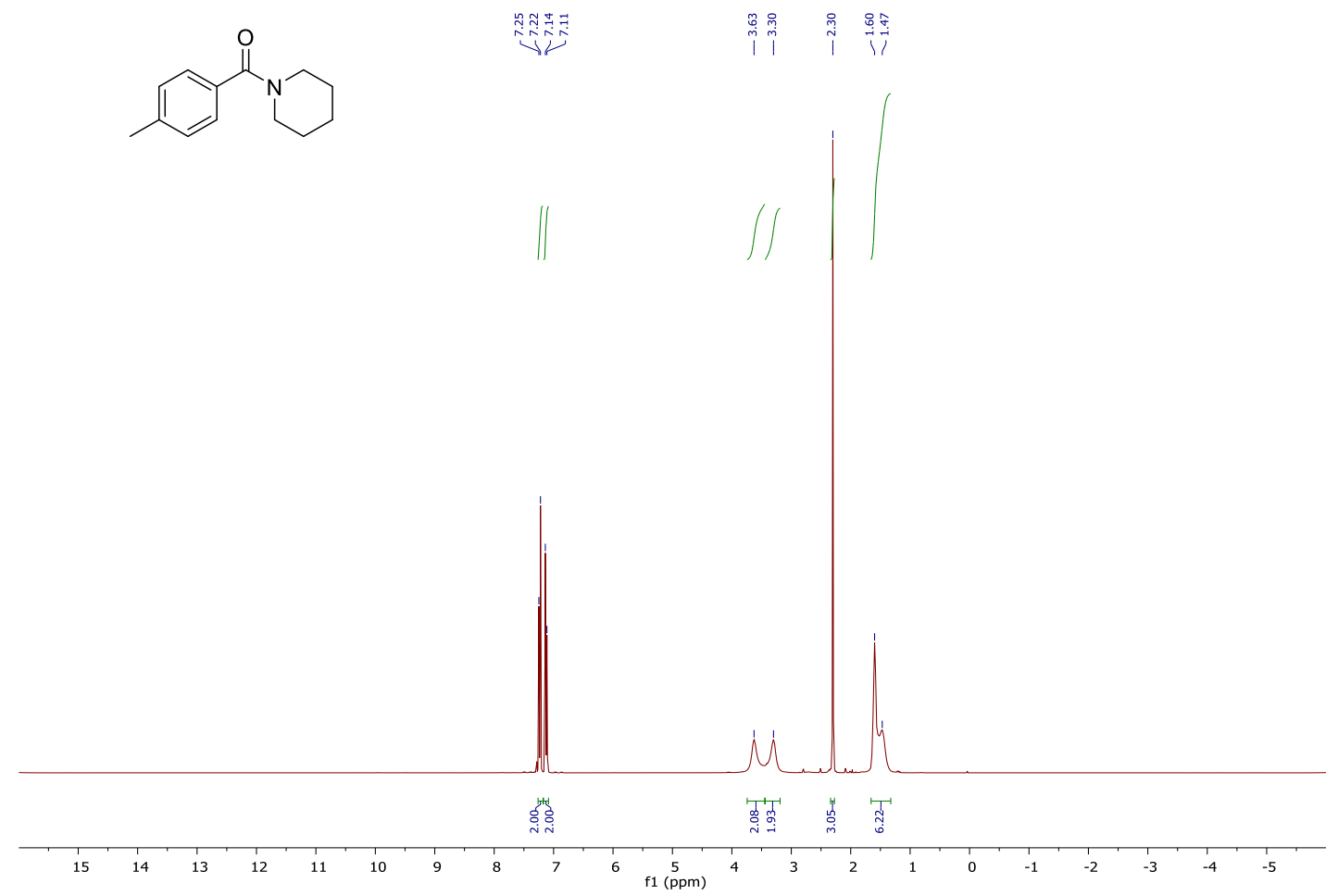

${ }^{1} \mathrm{H}$ NMR spectrum in $\mathrm{CDCl}_{3}$.
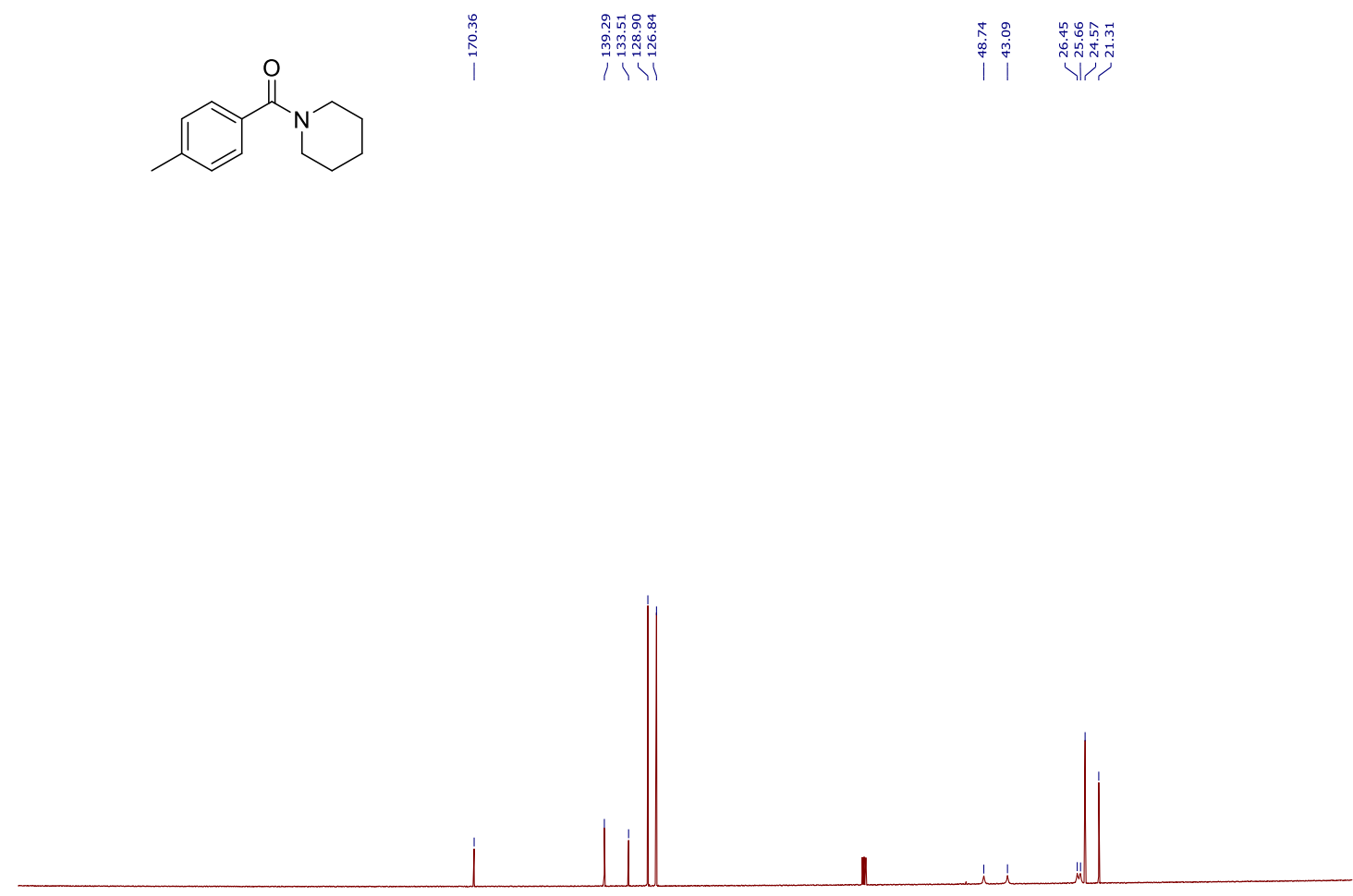

$\begin{array}{llllllllllllllllllllllllllllllllll}270 & 260 & 250 & 240 & 230 & 220 & 210 & 200 & 190 & 180 & 170 & 160 & 150 & 140 & 130 & 120 & 110 & 100 & 90 & 80 & 70 & 60 & 50 & 40 & 30 & 20 & 10 & 0 & -10 & -20 & -30\end{array}$ ${ }^{13} \mathrm{C}$ NMR spectrum in $\mathrm{CDCl}_{3}$. 
131b

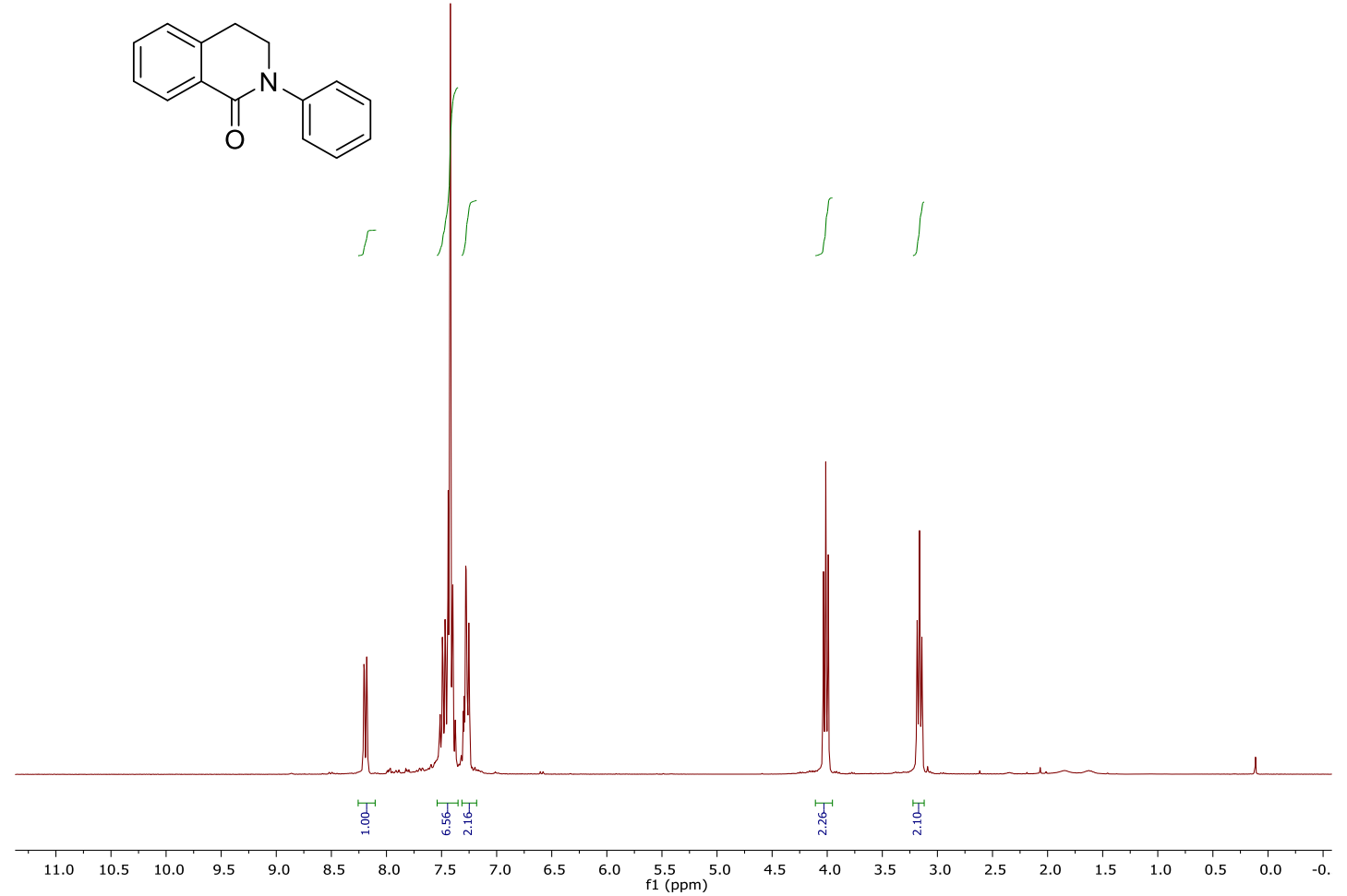

${ }^{1} \mathrm{H}$ NMR spectrum in $\mathrm{CDCl}_{3}$.<smiles>O=C1c2ccccc2CCN1c1ccccc1</smiles>

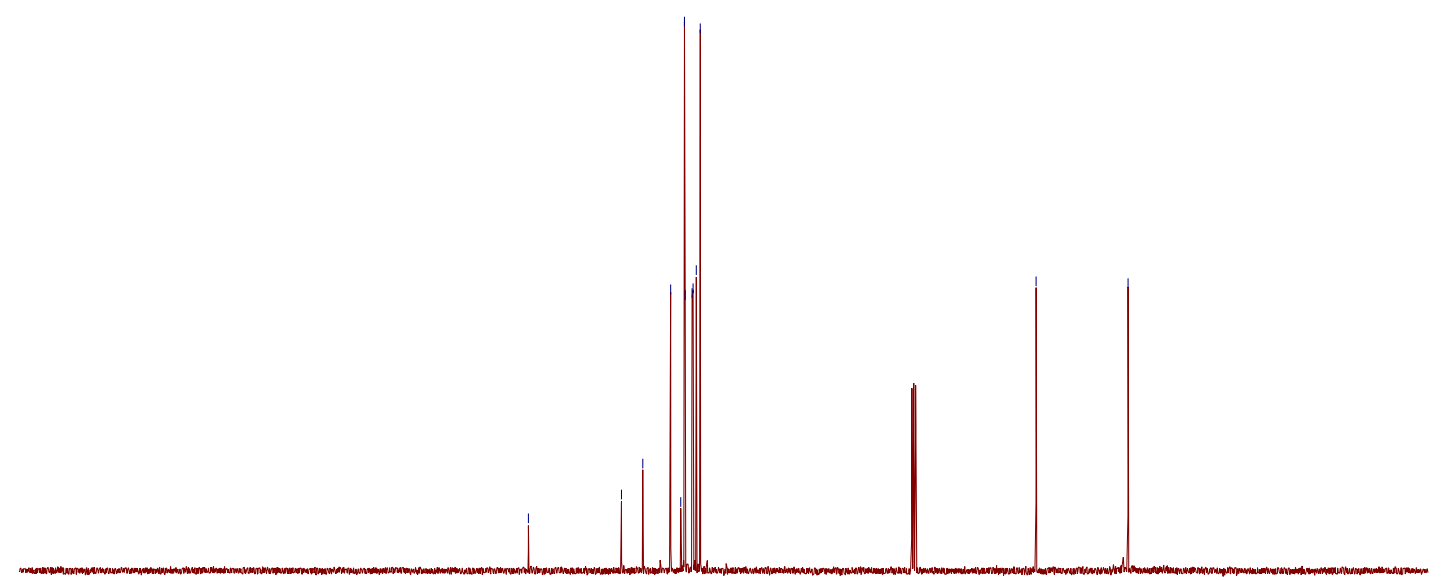

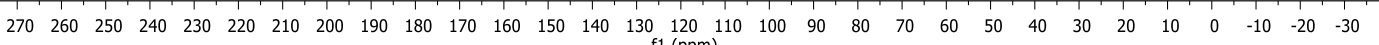

${ }^{13} \mathrm{C}$ NMR spectrum in $\mathrm{CDCl}_{3}$. 
$132 b$

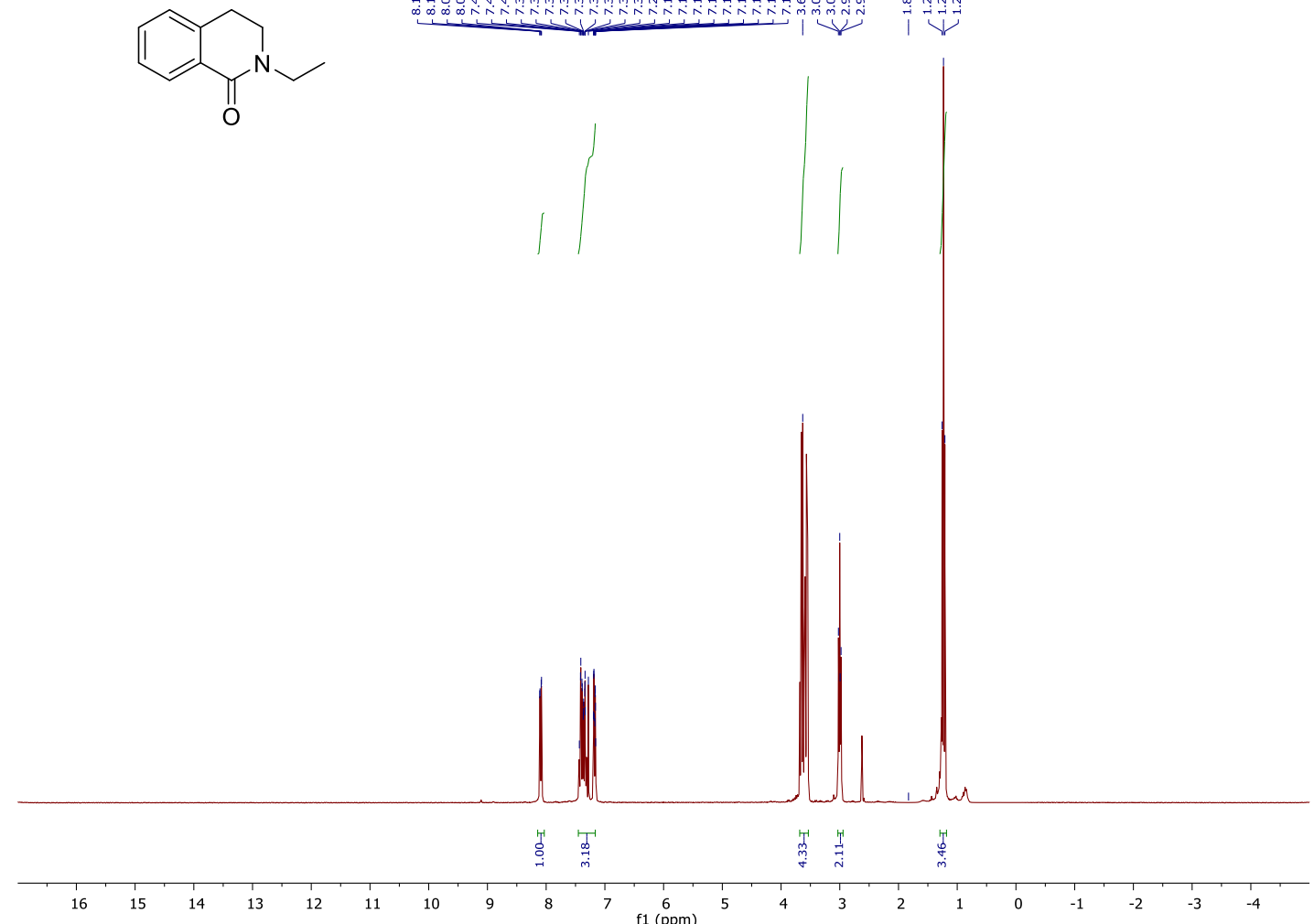

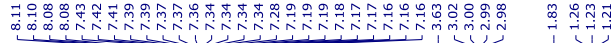

${ }^{1} \mathrm{H}$ NMR spectrum in $\mathrm{CDCl}_{3}$.<smiles>CCN1CCc2ccccc2C1=O</smiles>

\section{|}

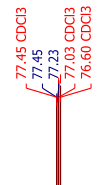

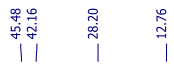

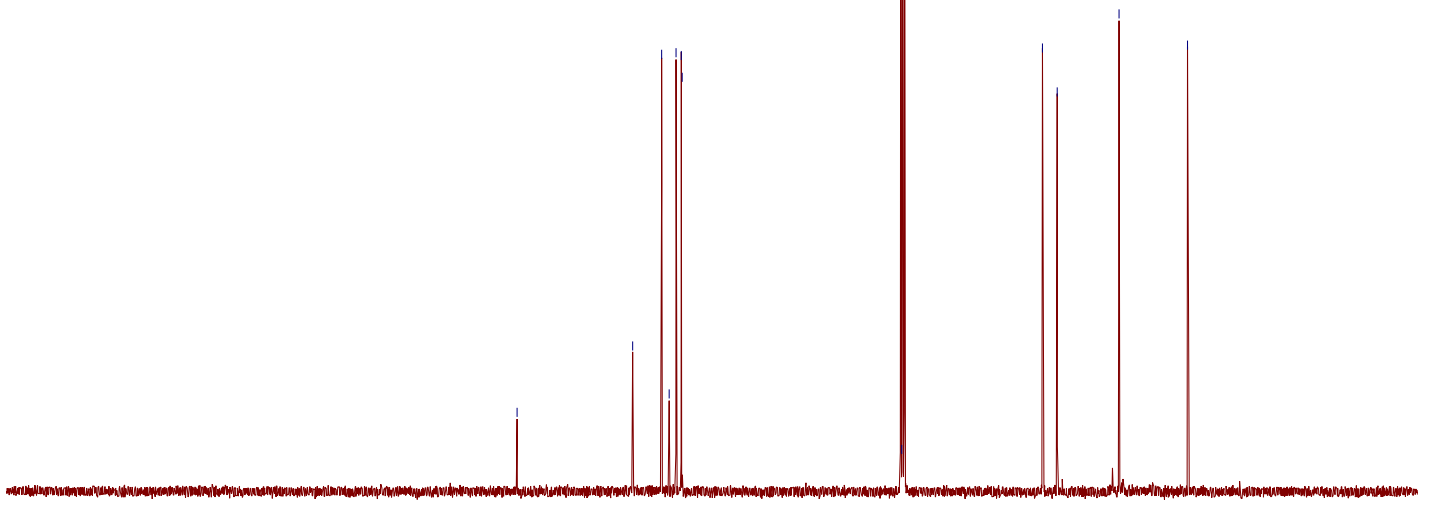

$\begin{array}{lllllllllllllllllllllllllllllllllll}270 & 260 & 250 & 240 & 230 & 220 & 210 & 200 & 190 & 180 & 170 & 160 & 150 & 140 & 130 & 120 & 110 & 100 & 90 & 80 & 70 & 60 & 50 & 40 & 30 & 20 & 10 & 0 & -10 & -20 & -30\end{array}$ ${ }^{13} \mathrm{C}$ NMR spectrum in $\mathrm{CDCl}_{3}$. 
133b

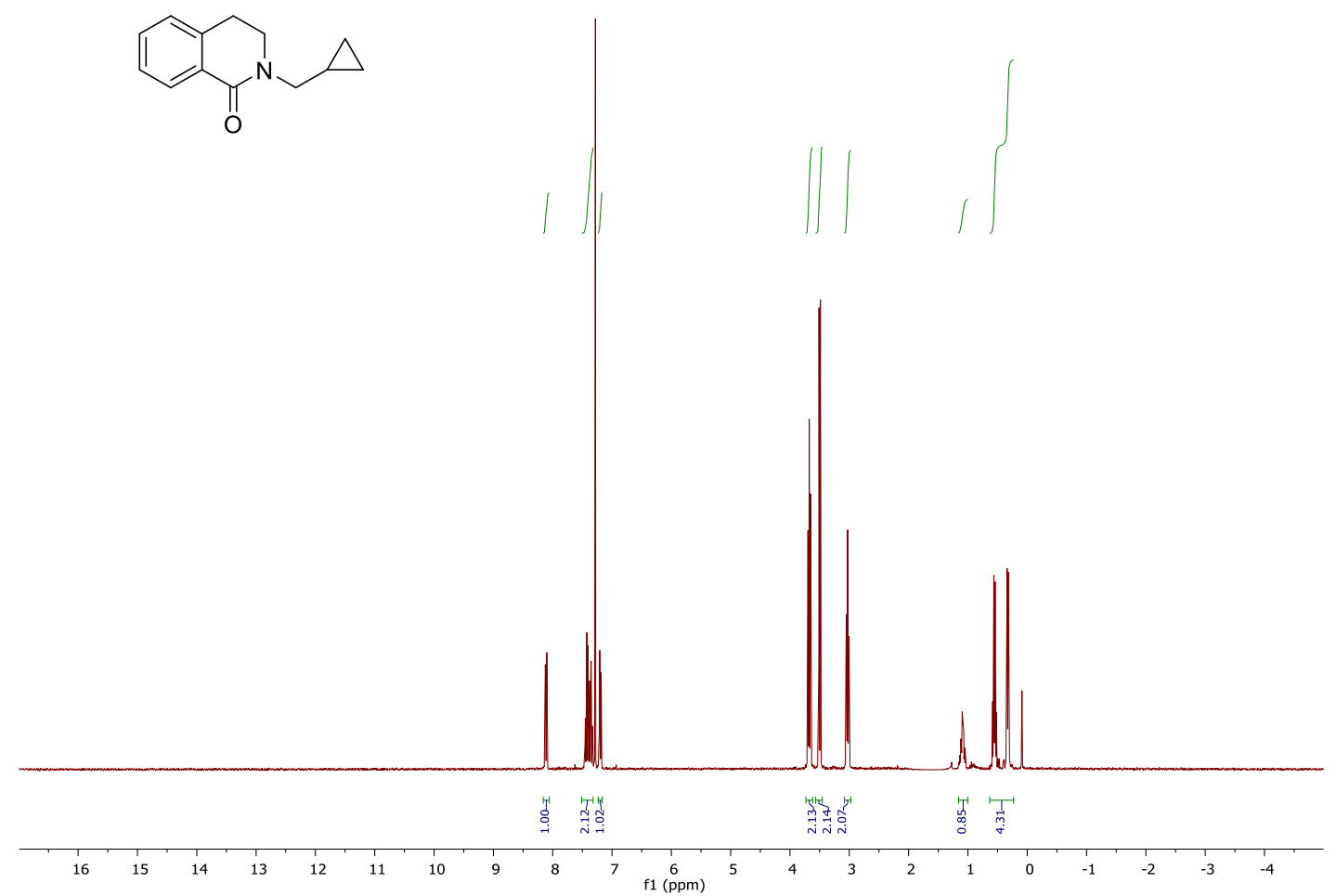

${ }^{1} \mathrm{H}$ NMR spectrum in $\mathrm{CDCl}_{3}$.<smiles>O=C1c2ccccc2CCN1CC1CC1</smiles>

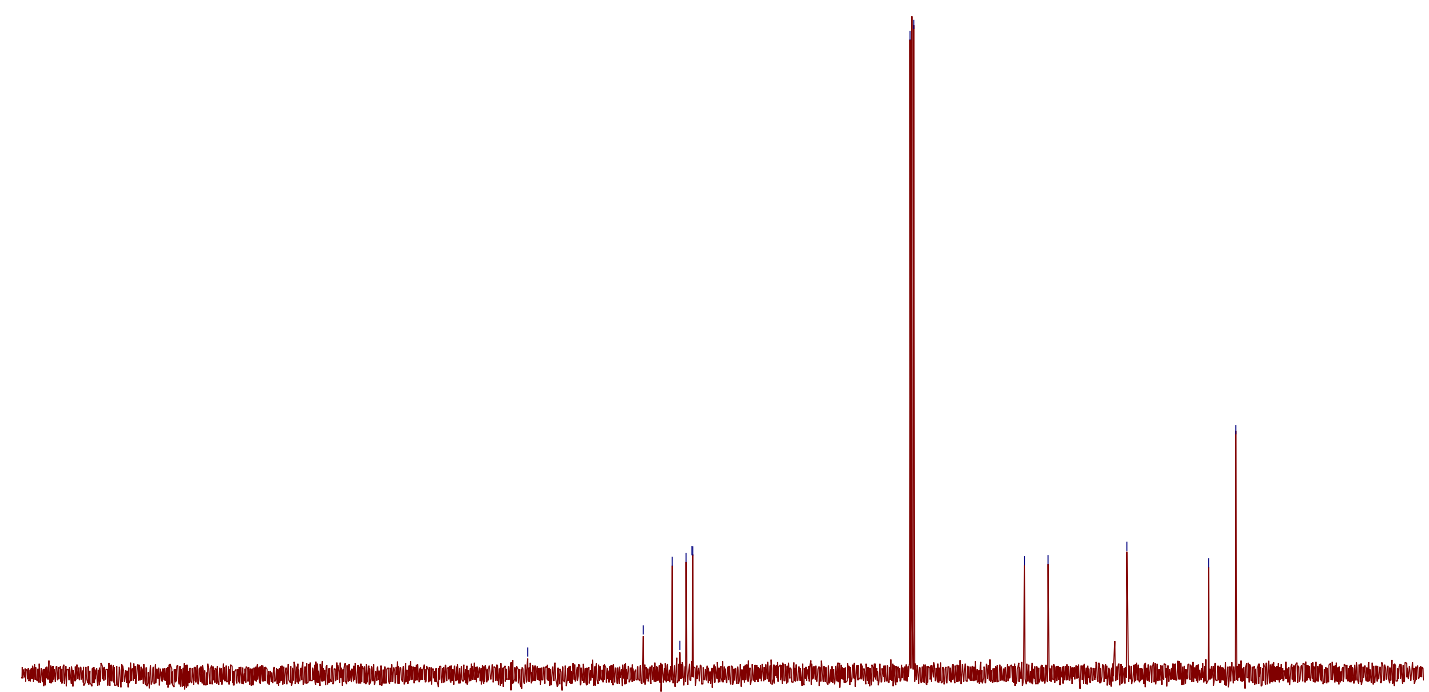

$\begin{array}{llllllllllllllllllllllllllllllllll}270 & 260 & 250 & 240 & 230 & 220 & 210 & 200 & 190 & 180 & 170 & 160 & 150 & 140 & 130 & 120 & 110 & 100 & 90 & 80 & 70 & 60 & 50 & 40 & 30 & 20 & 10 & 0 & -10 & -20 & -30\end{array}$ ${ }^{13} \mathrm{C}$ NMR spectrum in $\mathrm{CDCl}_{3}$. 
134b<smiles>CCCCCCN1CCc2cc(OC)c(OC)cc2C1=O</smiles>

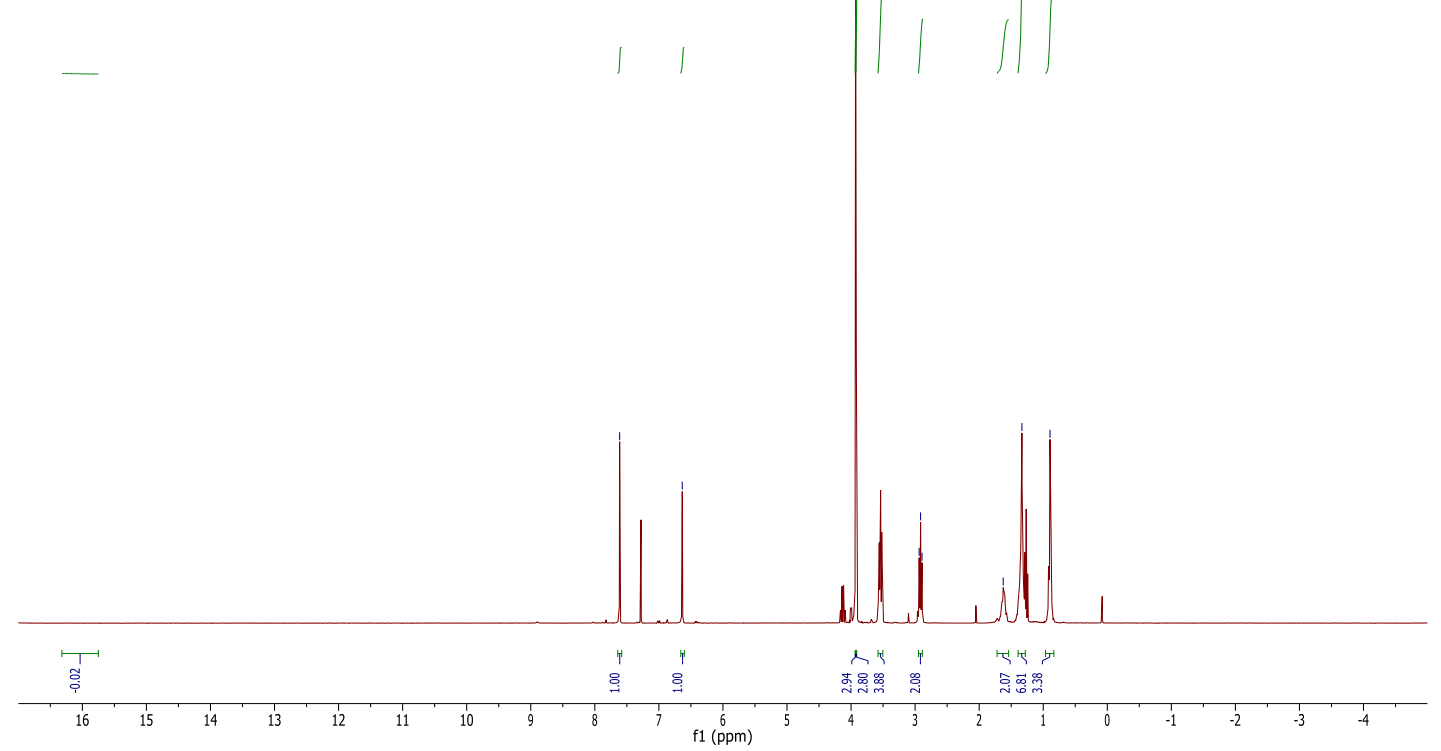

${ }^{1} \mathrm{H}$ NMR spectrum in $\mathrm{CDCl}_{3}$.

$$
\text { | }
$$

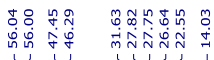<smiles>CCCCCCN1CCc2cc(OC)c(OC)cc2C1=O</smiles>

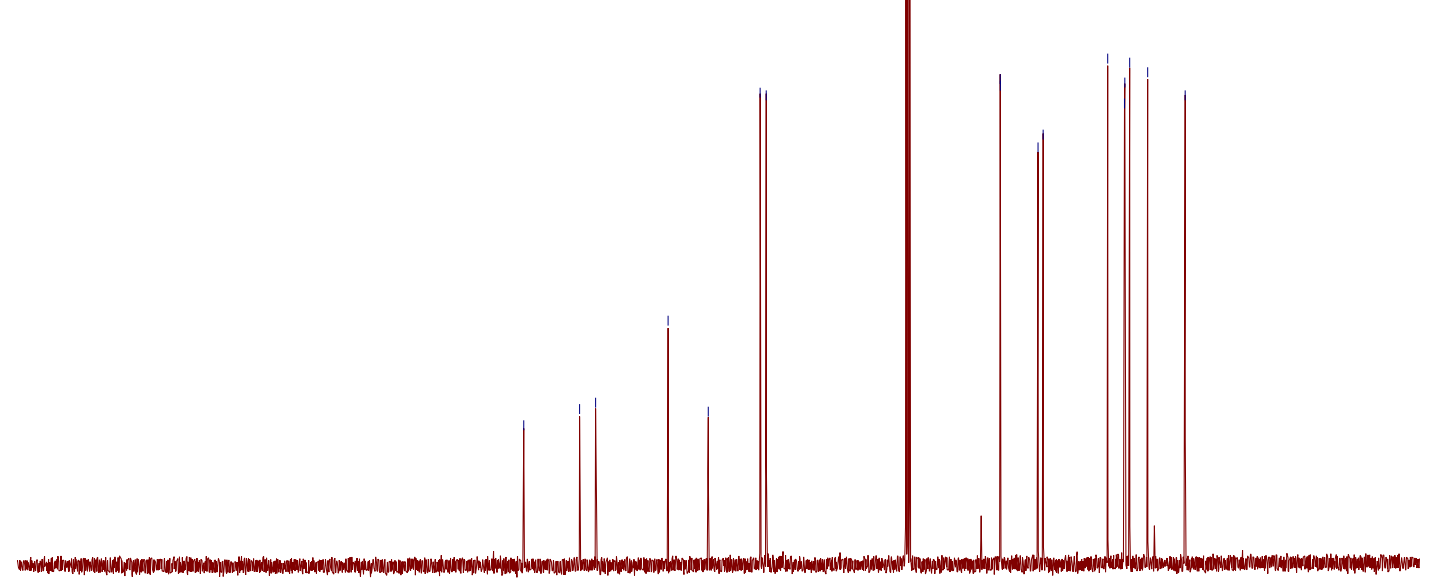

$\begin{array}{lllllllllllllllllllllllllllllllllllll}270 & 260 & 250 & 240 & 230 & 220 & 210 & 200 & 190 & 180 & 170 & 160 & 150 & 140 & 130 & 120 & 110 & 100 & 90 & 80 & 70 & 60 & 50 & 40 & 30 & 20 & 10 & 0 & -10 & -20 & -30\end{array}$ ${ }^{13} \mathrm{C}$ NMR spectrum in $\mathrm{CDCl}_{3}$. 
135b

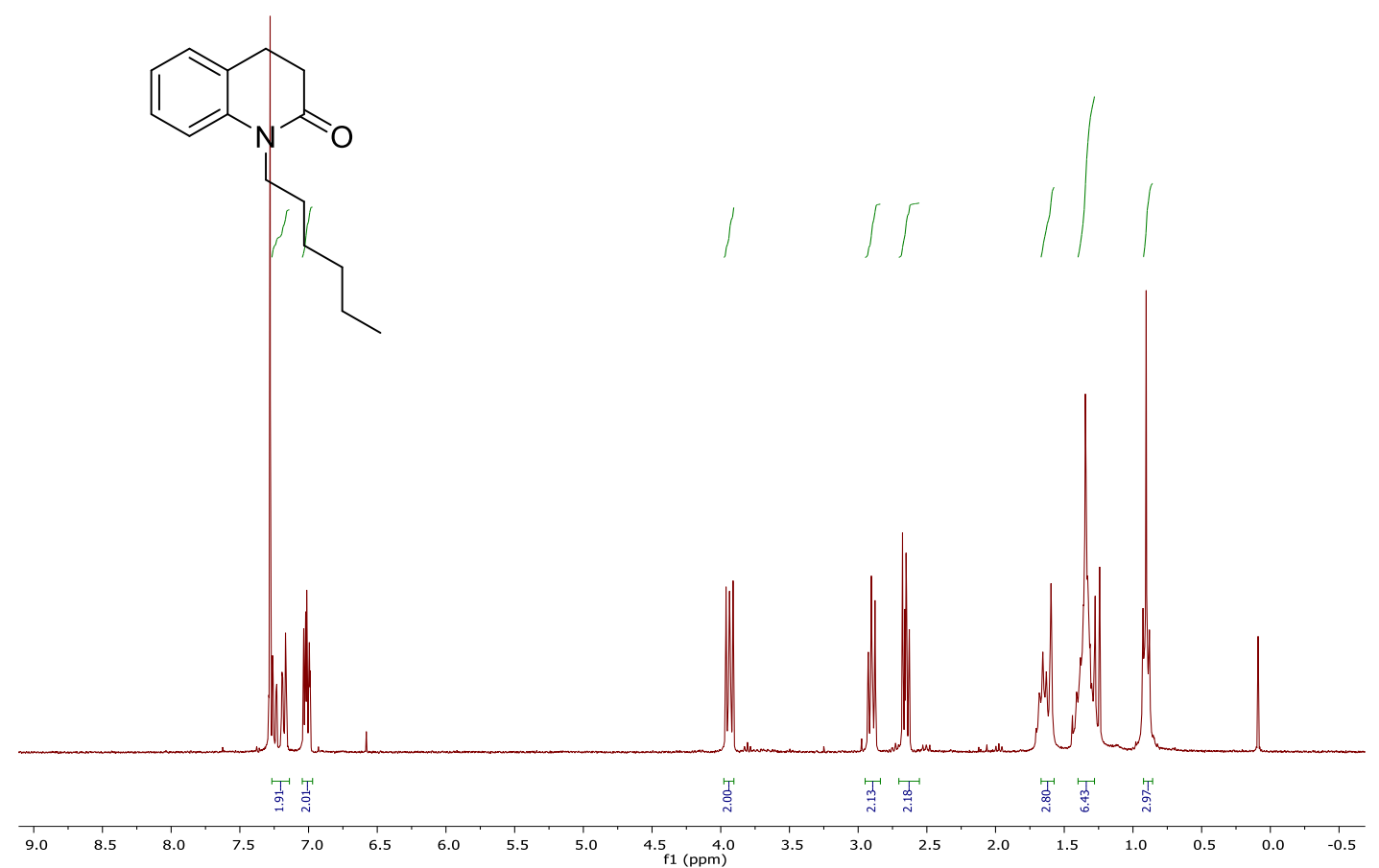

${ }^{1} \mathrm{H}$ NMR spectrum in $\mathrm{CDCl}_{3}$.

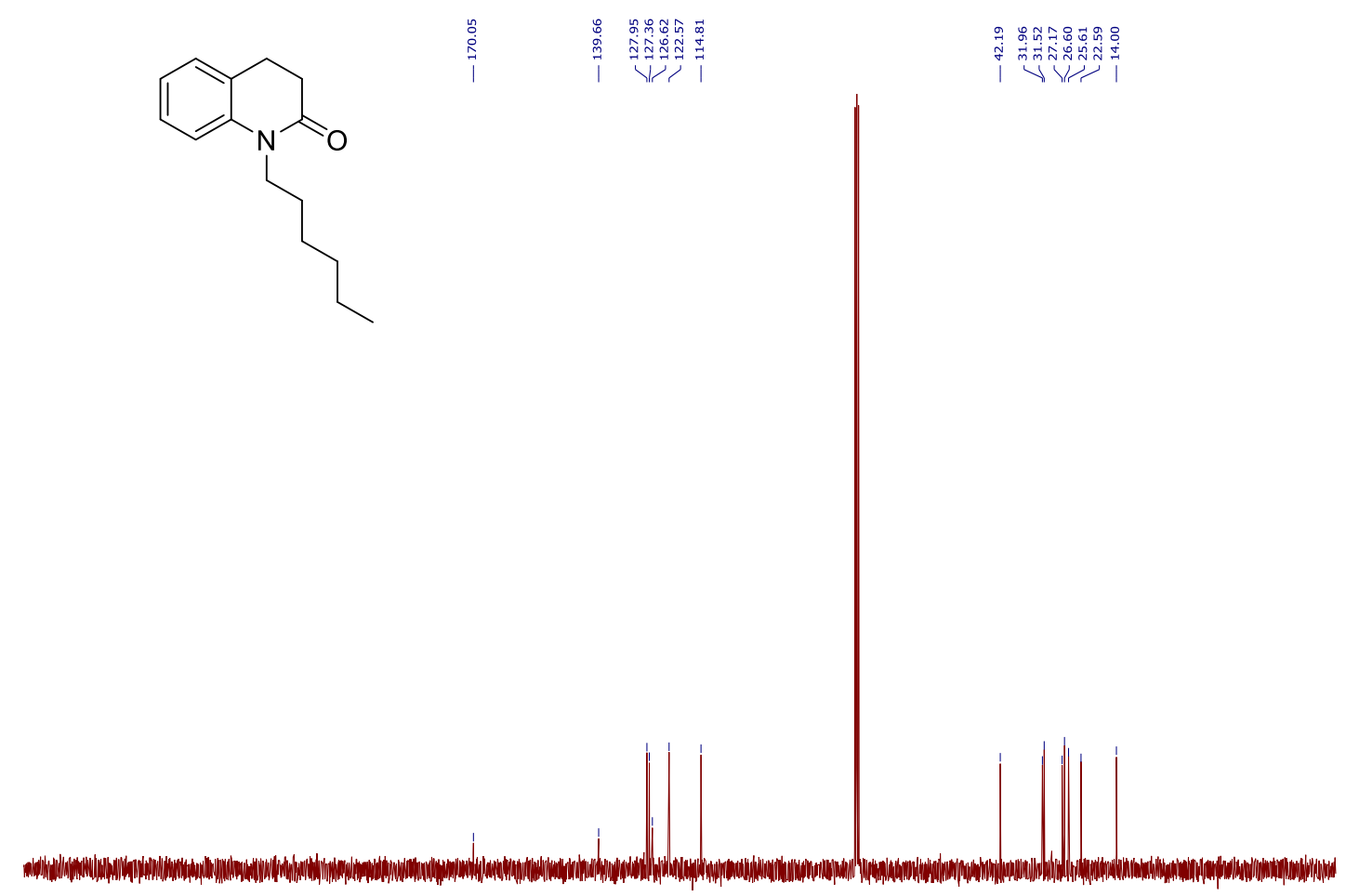

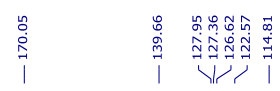

|

$\begin{array}{llllllllllllllllllllllllllllllllllllllllllllllll}270 & 260 & 250 & 240 & 230 & 220 & 210 & 200 & 190 & 180 & 170 & 160 & 150 & 140 & 130 & 120 & 110 & 100 & 90 & 80 & 70 & 60 & 50 & 40 & 30 & 20 & 10 & 0 & -10 & -20 & -30\end{array}$

${ }^{13} \mathrm{C}$ NMR spectrum in $\mathrm{CDCl}_{3}$. 
136b

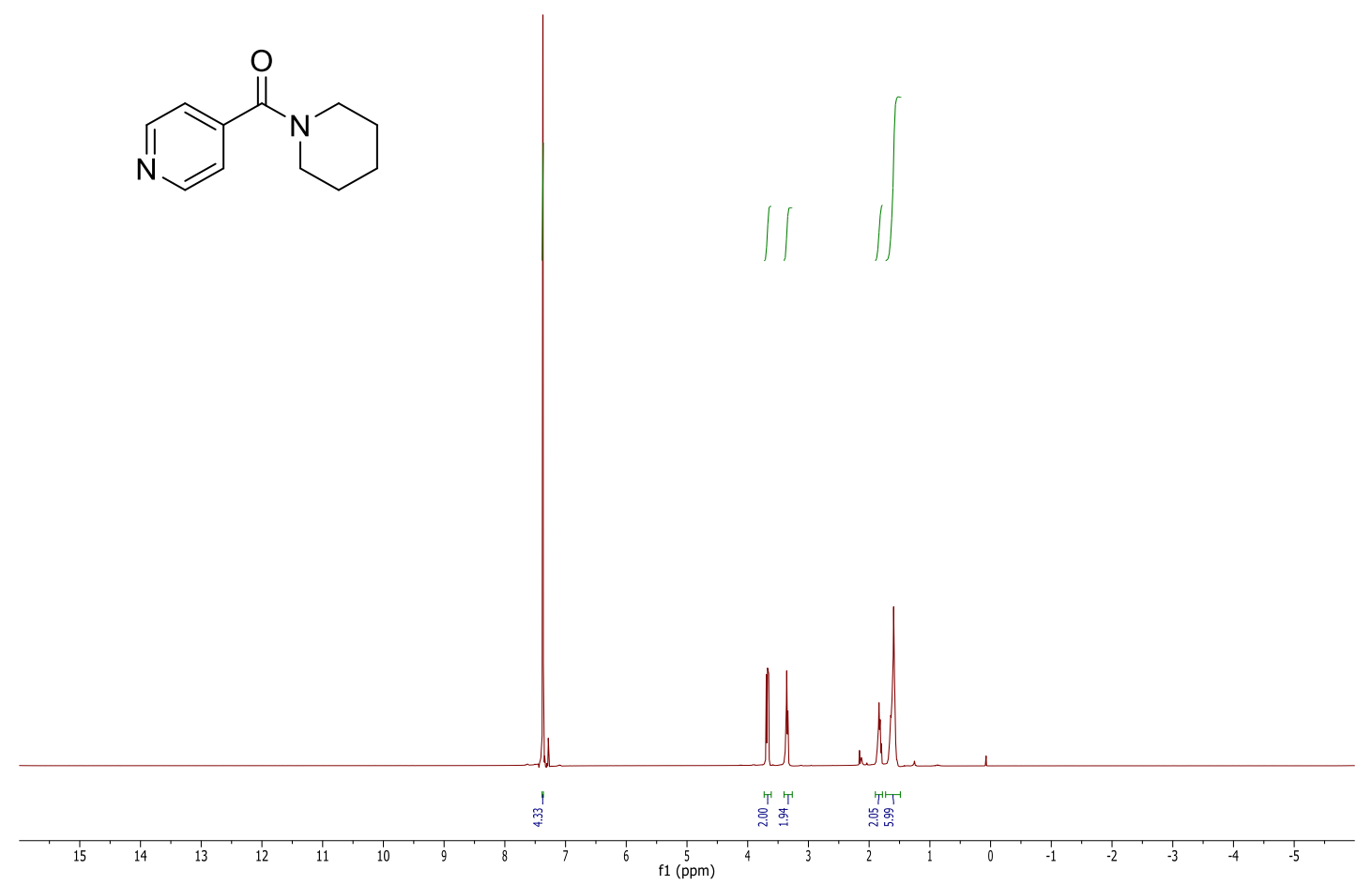

${ }^{1} \mathrm{H}$ NMR spectrum in $\mathrm{CDCl}_{3}$.

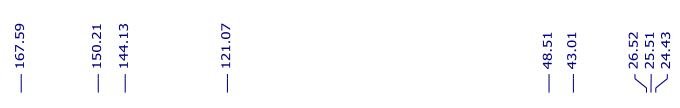<smiles>O=C(c1ccncc1)N1CCCCC1</smiles>

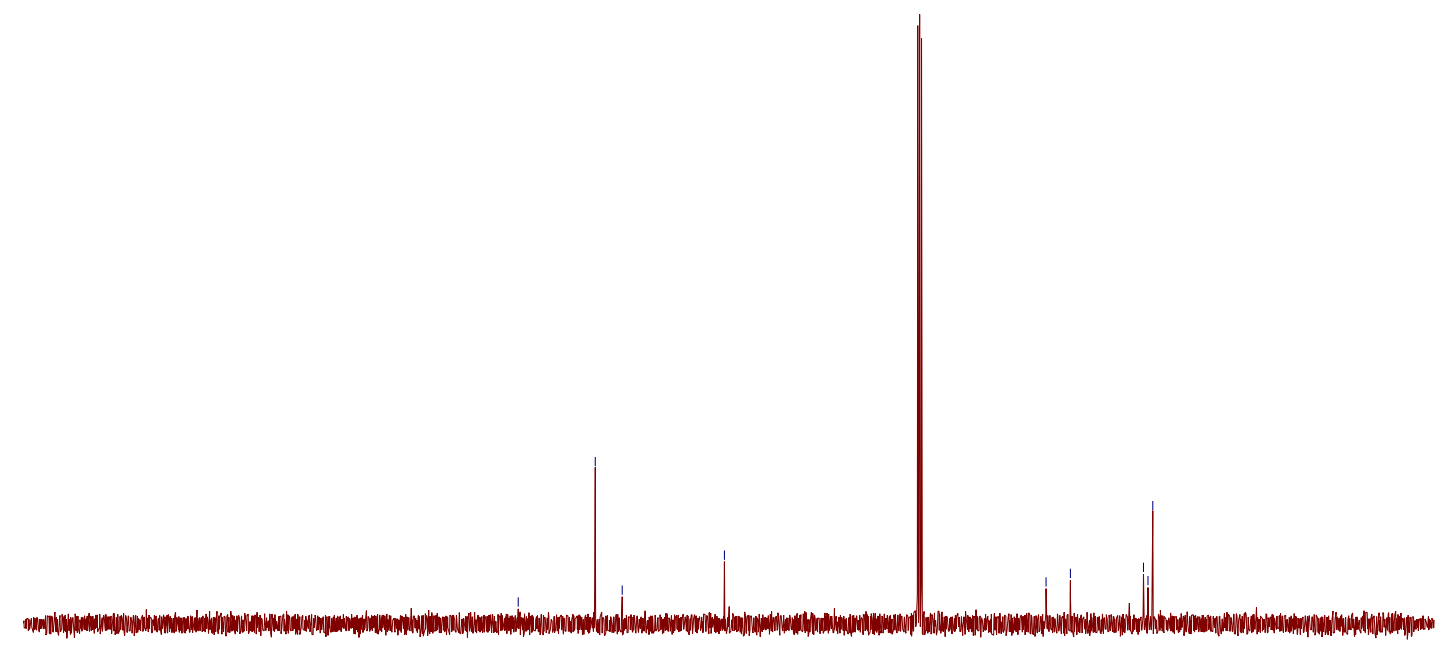

$\begin{array}{llllllllllllllllllllllllllllllllll}270 & 260 & 250 & 240 & 230 & 220 & 210 & 200 & 190 & 180 & 170 & 160 & 150 & 140 & 130 & 120 & 110 & 100 & 90 & 80 & 70 & 60 & 50 & 40 & 30 & 20 & 10 & 0 & -10 & -20 & -30\end{array}$

${ }^{1} \mathrm{H}$ NMR spectrum in $\mathrm{CDCl}_{3}$. 
Experimental Section

$137 b$

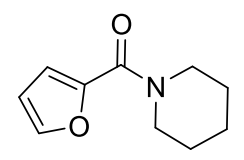

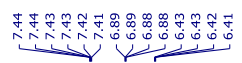

111

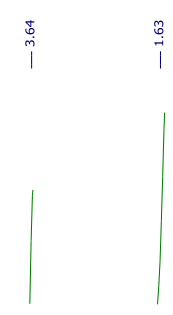

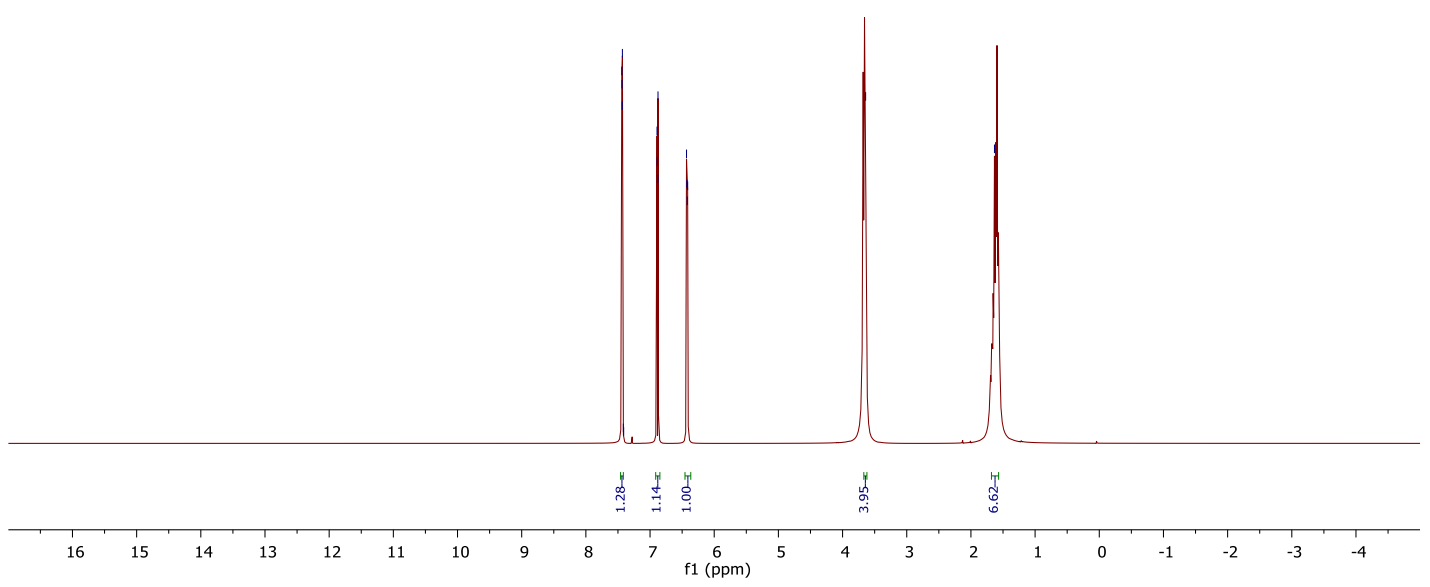

${ }^{1} \mathrm{H}$ NMR spectrum in $\mathrm{CDCl}_{3}$.
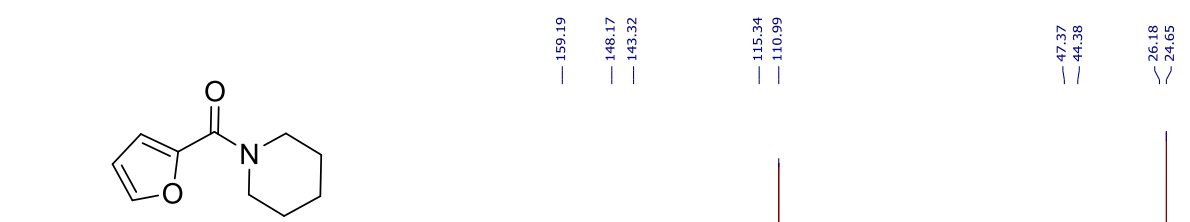

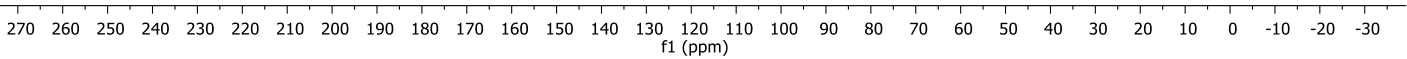
${ }^{13} \mathrm{C}$ NMR spectrum in $\mathrm{CDCl}_{3}$.

179 
Experimental Section

$138 b$

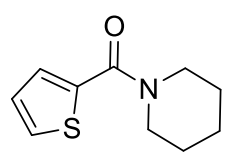

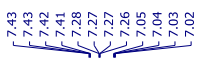
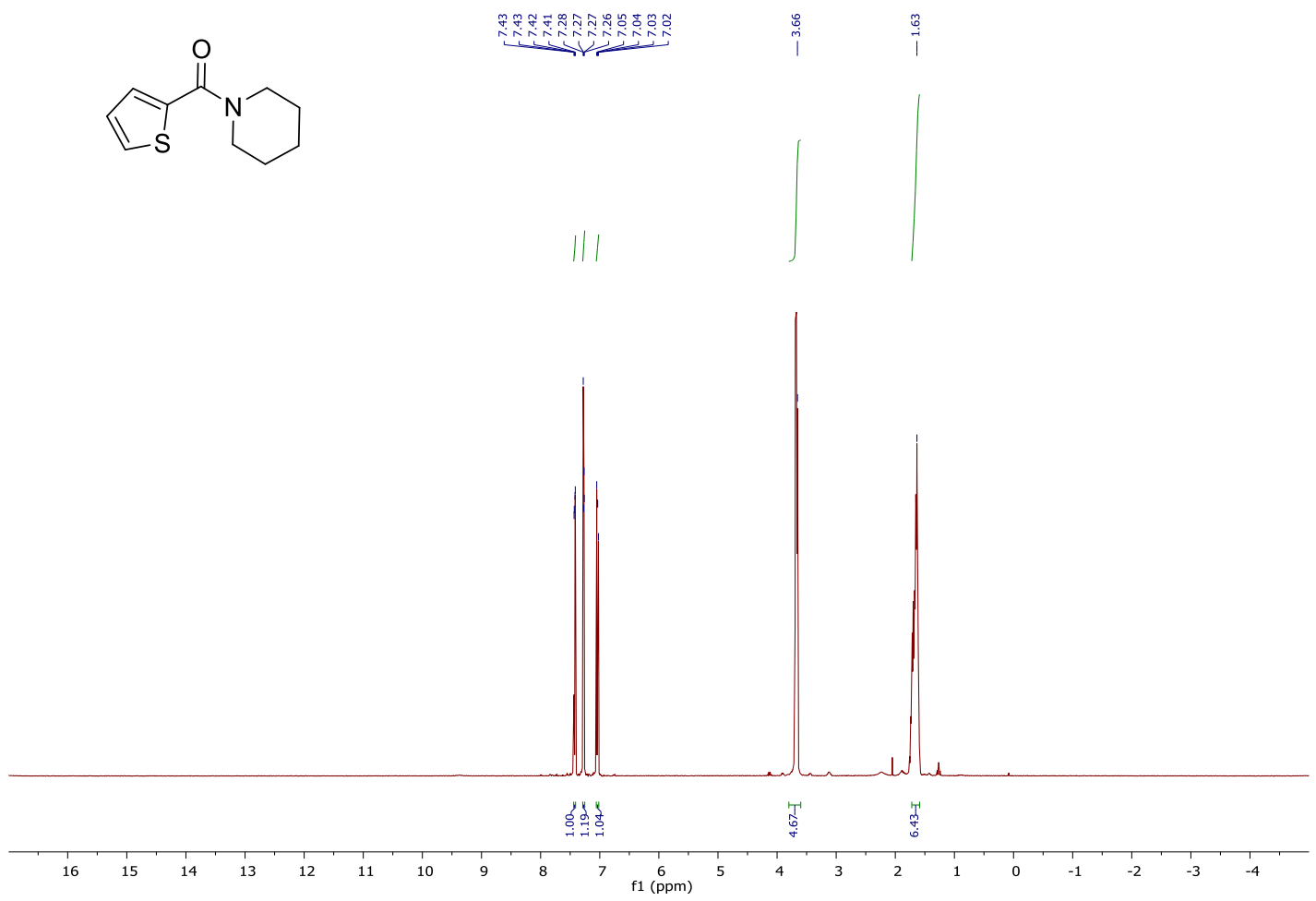

${ }^{1} \mathrm{H}$ NMR spectrum in $\mathrm{CDCl}_{3}$.
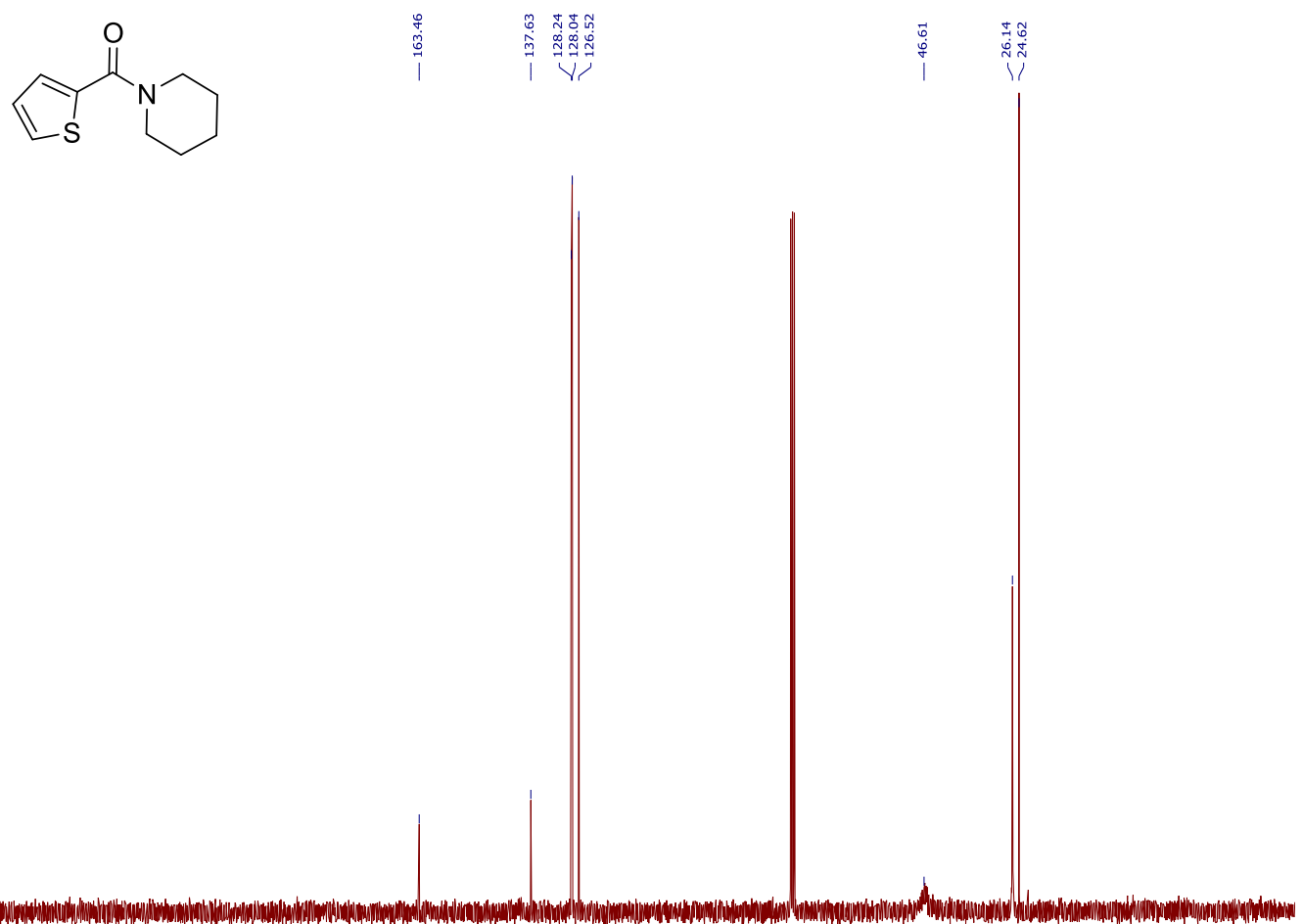

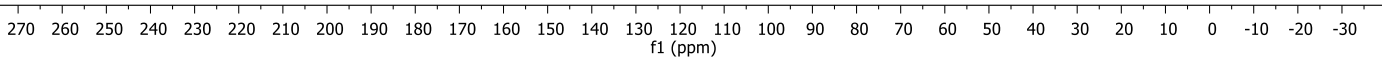

${ }^{13} \mathrm{C}$ NMR spectrum in $\mathrm{CDCl}_{3}$.

180 
139b

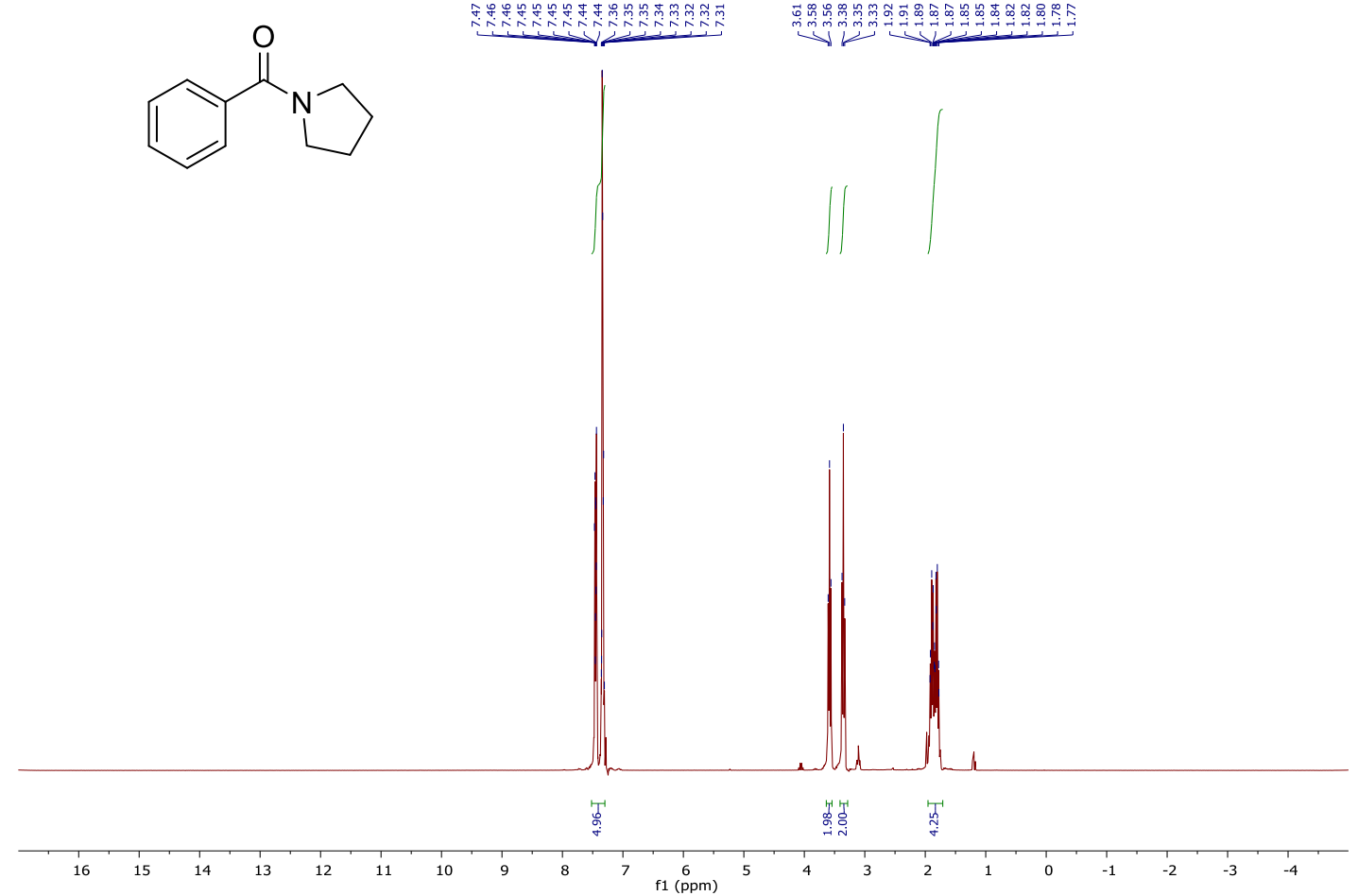

${ }^{1} \mathrm{H}$ NMR spectrum in $\mathrm{CDCl}_{3}$.<smiles>O=C(c1ccccc1)N1CCCC1</smiles>

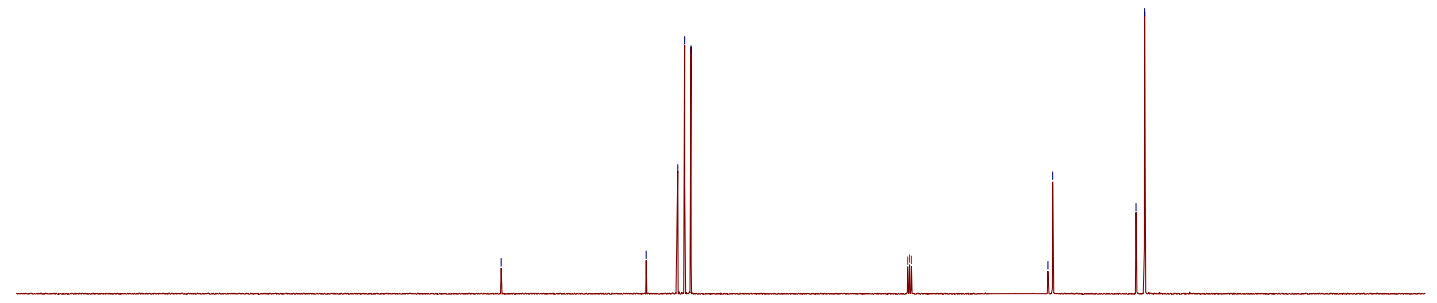

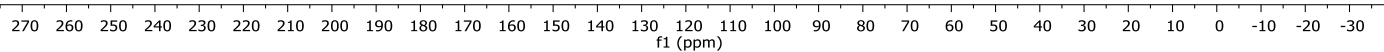
${ }^{13} \mathrm{C}$ NMR spectrum in $\mathrm{CDCl}_{3}$. 
Experimental Section

140b
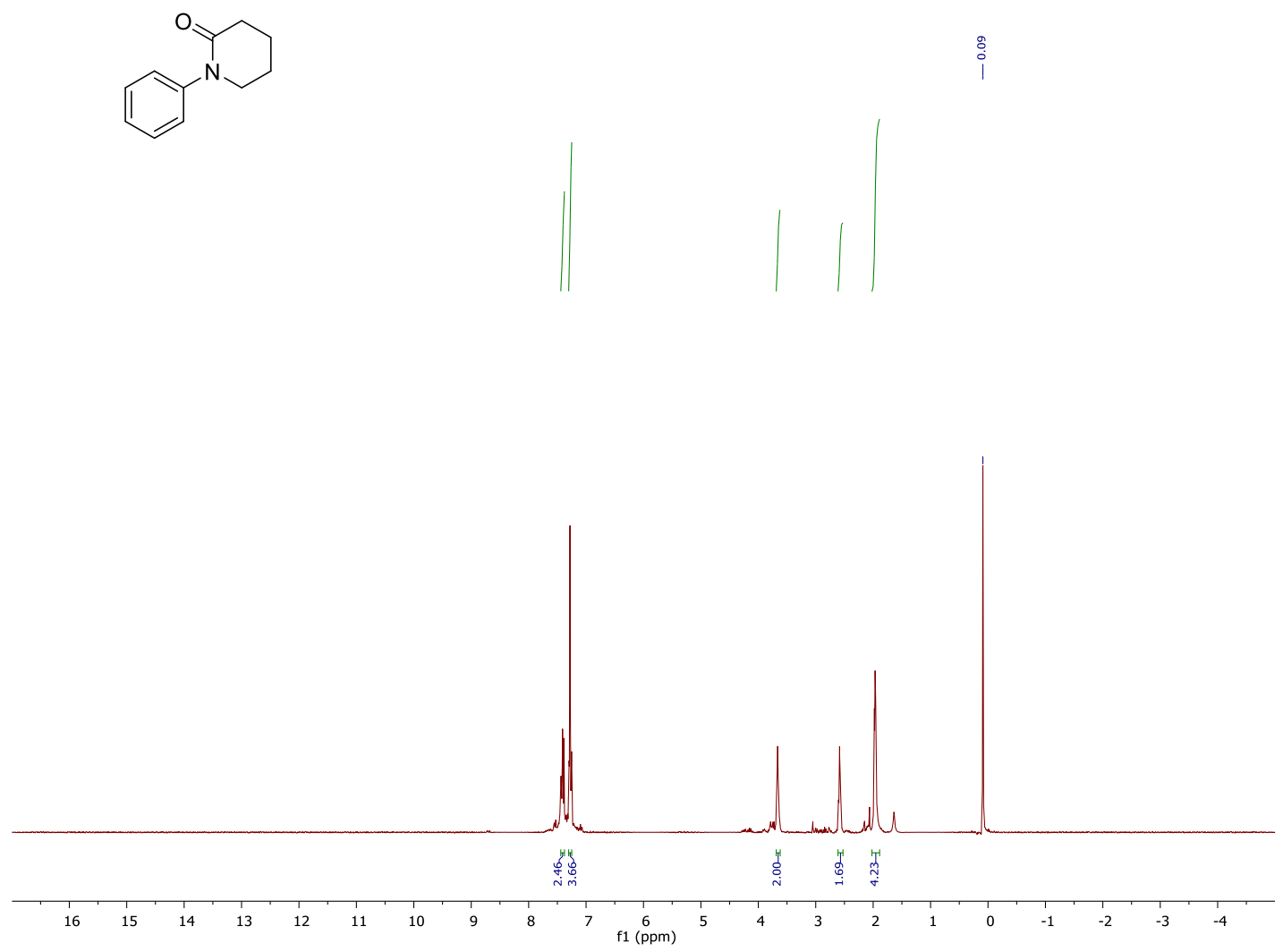

${ }^{1} \mathrm{H}$ NMR spectrum in $\mathrm{CDCl}_{3}$.
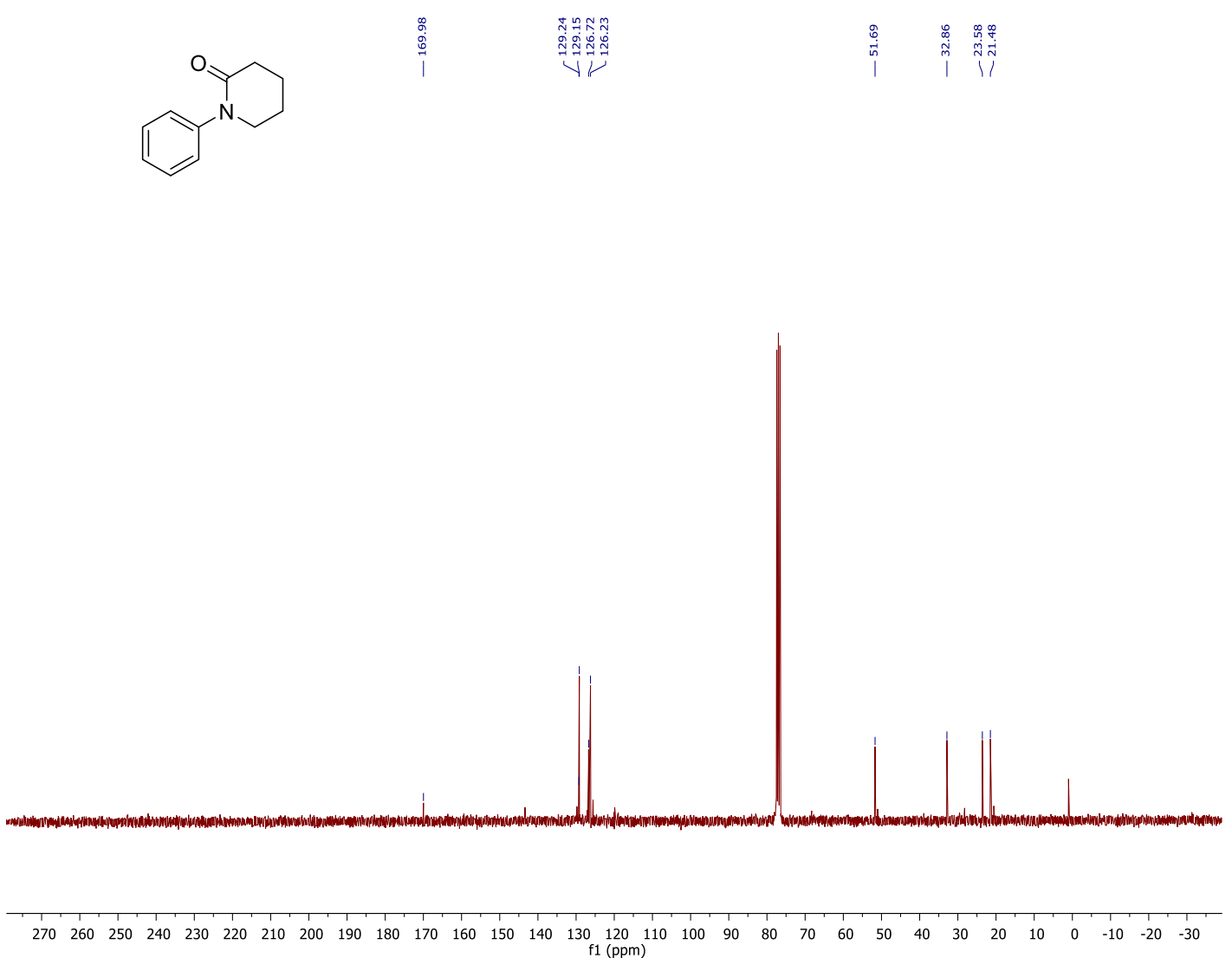

${ }^{13} \mathrm{C}$ NMR spectrum in $\mathrm{CDCl}_{3}$.

182 
141b

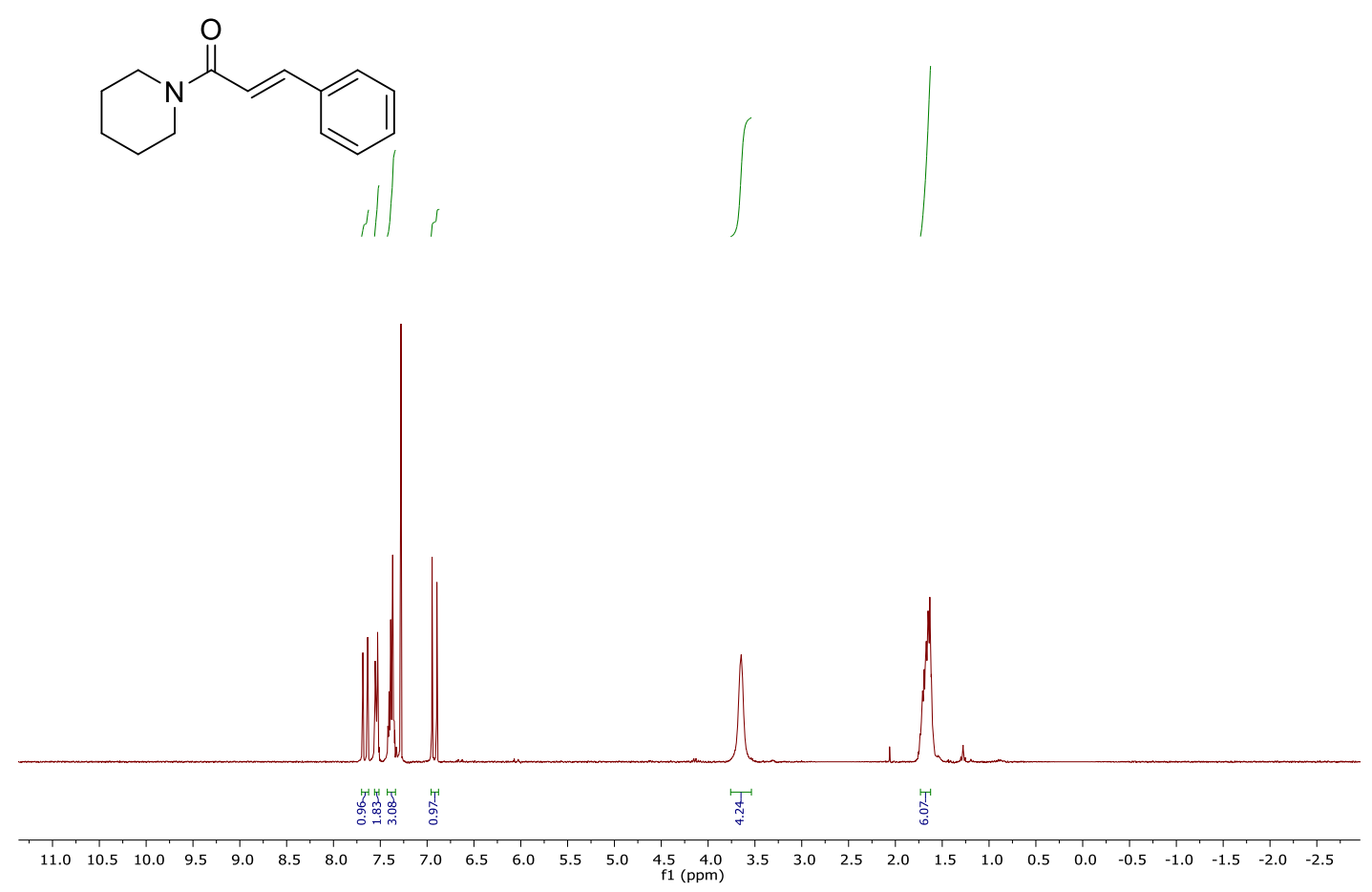

${ }^{1} \mathrm{H}$ NMR spectrum in $\mathrm{CDCl}_{3}$.
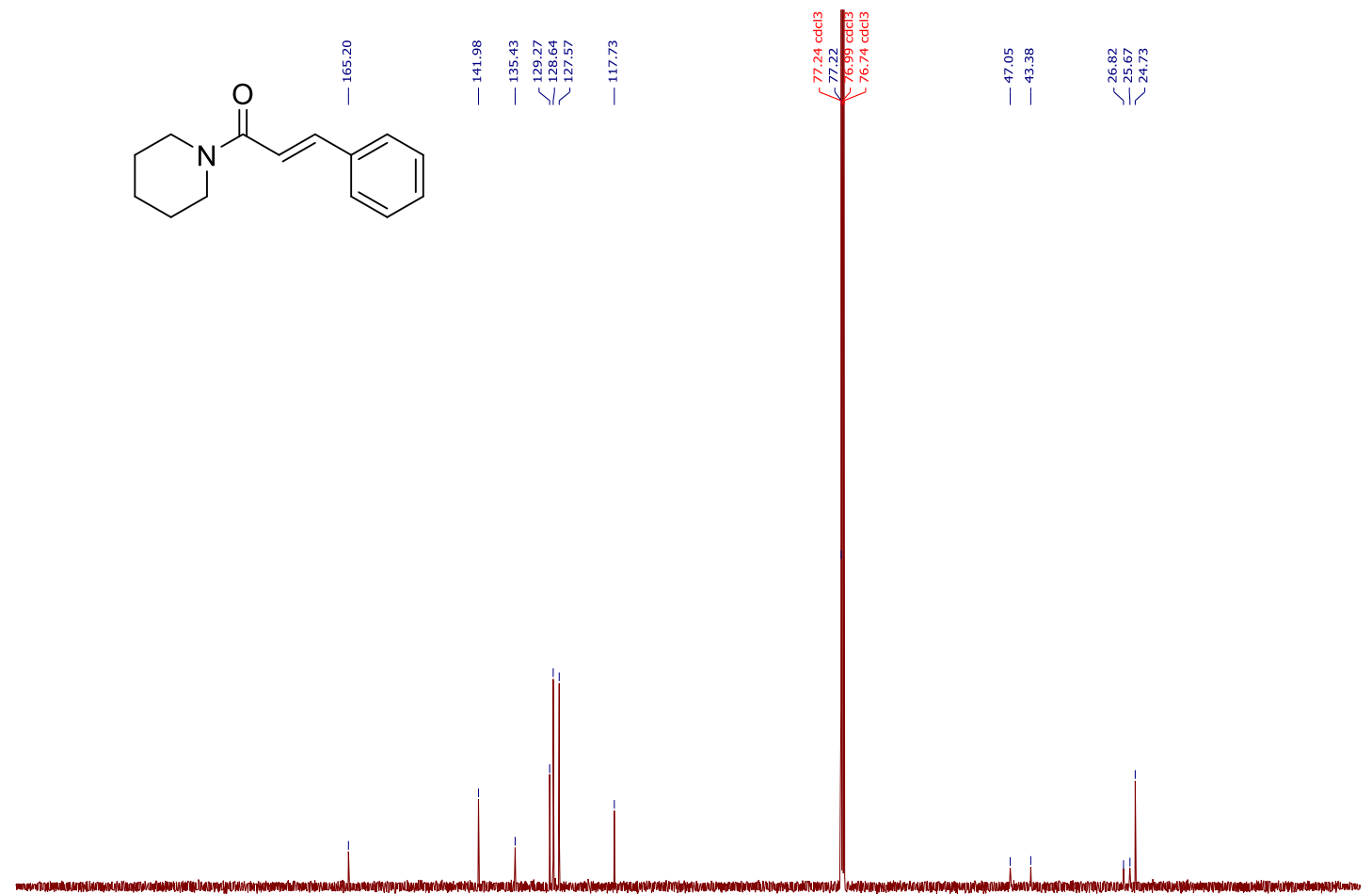

$\begin{array}{lllllllllllllllllllllllllllll}220 & 210 & 200 & 190 & 180 & 170 & 160 & 150 & 140 & 130 & 120 & 110 & 100 & 90 & 80 & 70 & 60 & 50 & 40 & 30 & 20 & 10 & 0 & -10\end{array}$

${ }^{13} \mathrm{C}$ NMR spectrum in $\mathrm{CDCl}_{3}$. 
$142 b$<smiles>CCOC(=O)c1ccc(C(=O)N2CCOCC2)cc1</smiles>

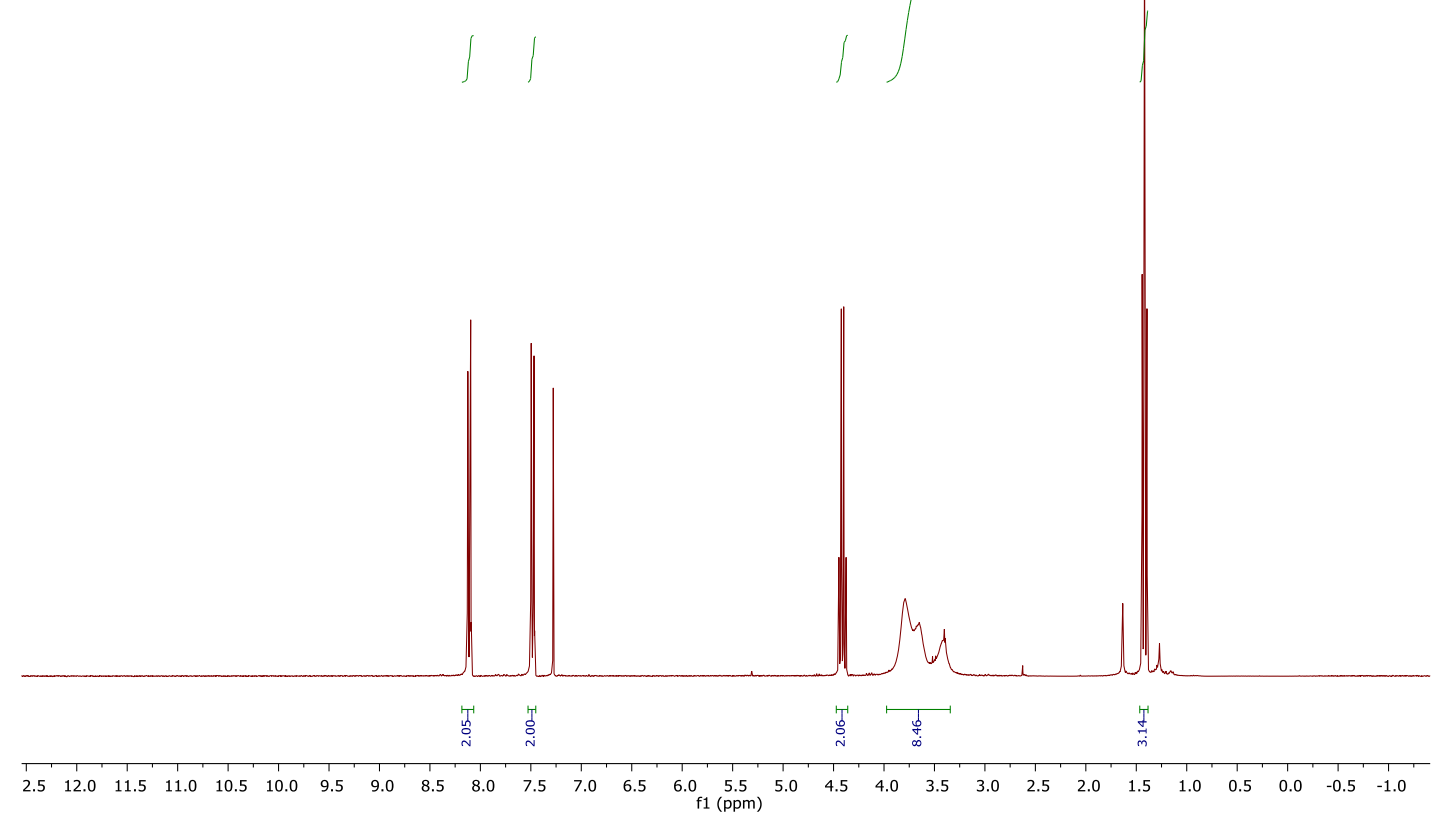

${ }^{1} \mathrm{H}$ NMR spectrum in $\mathrm{CDCl}_{3}$.<smiles>CCOC(=O)c1ccc(C(=O)N2CCOCC2)cc1</smiles>

${ }^{13} \mathrm{C}$ NMR spectrum in $\mathrm{CDCl}_{3}$. 
Experimental Section

$143 b$
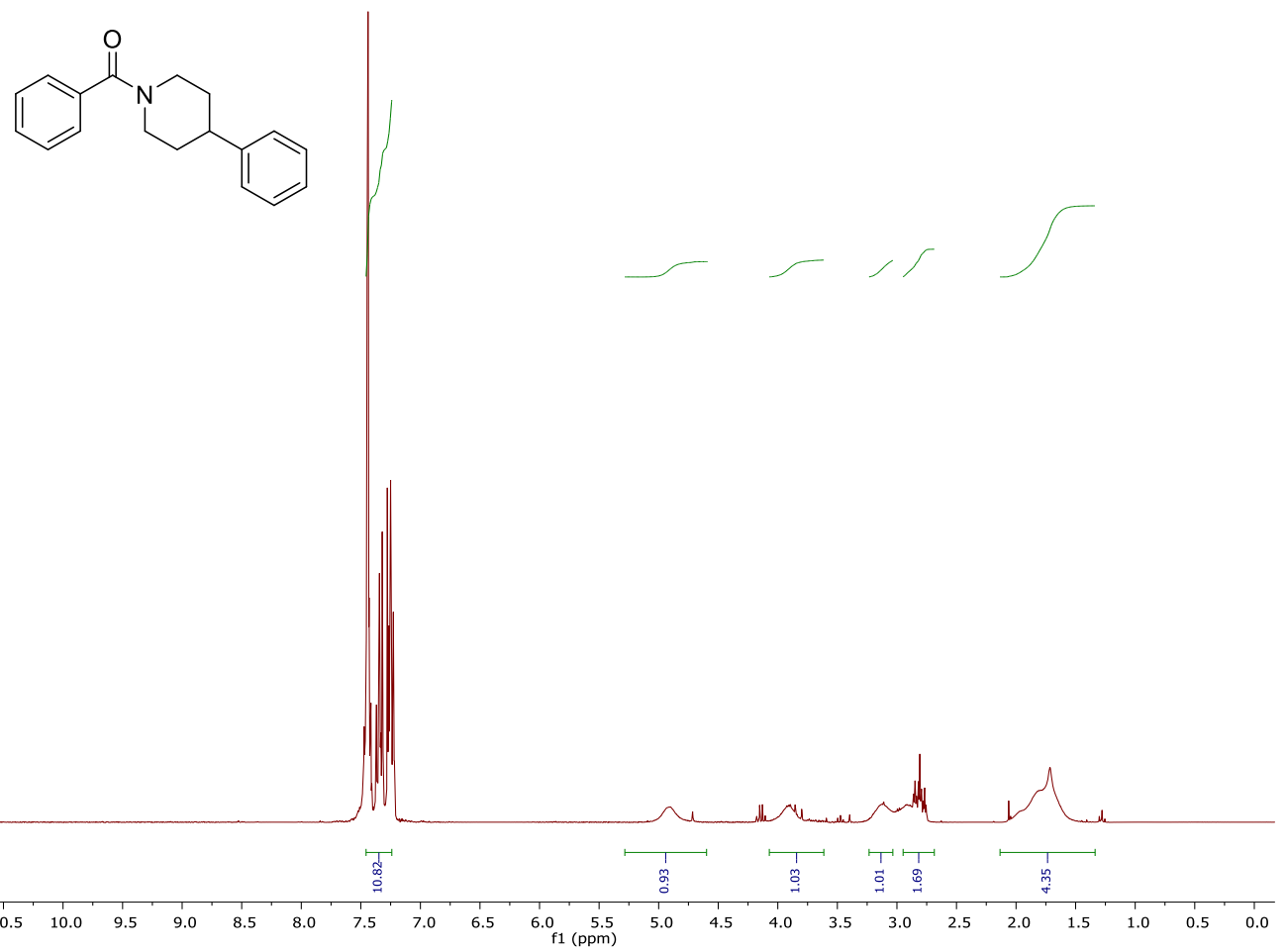

${ }^{1} \mathrm{H}$ NMR spectrum in $\mathrm{CDCl}_{3}$.
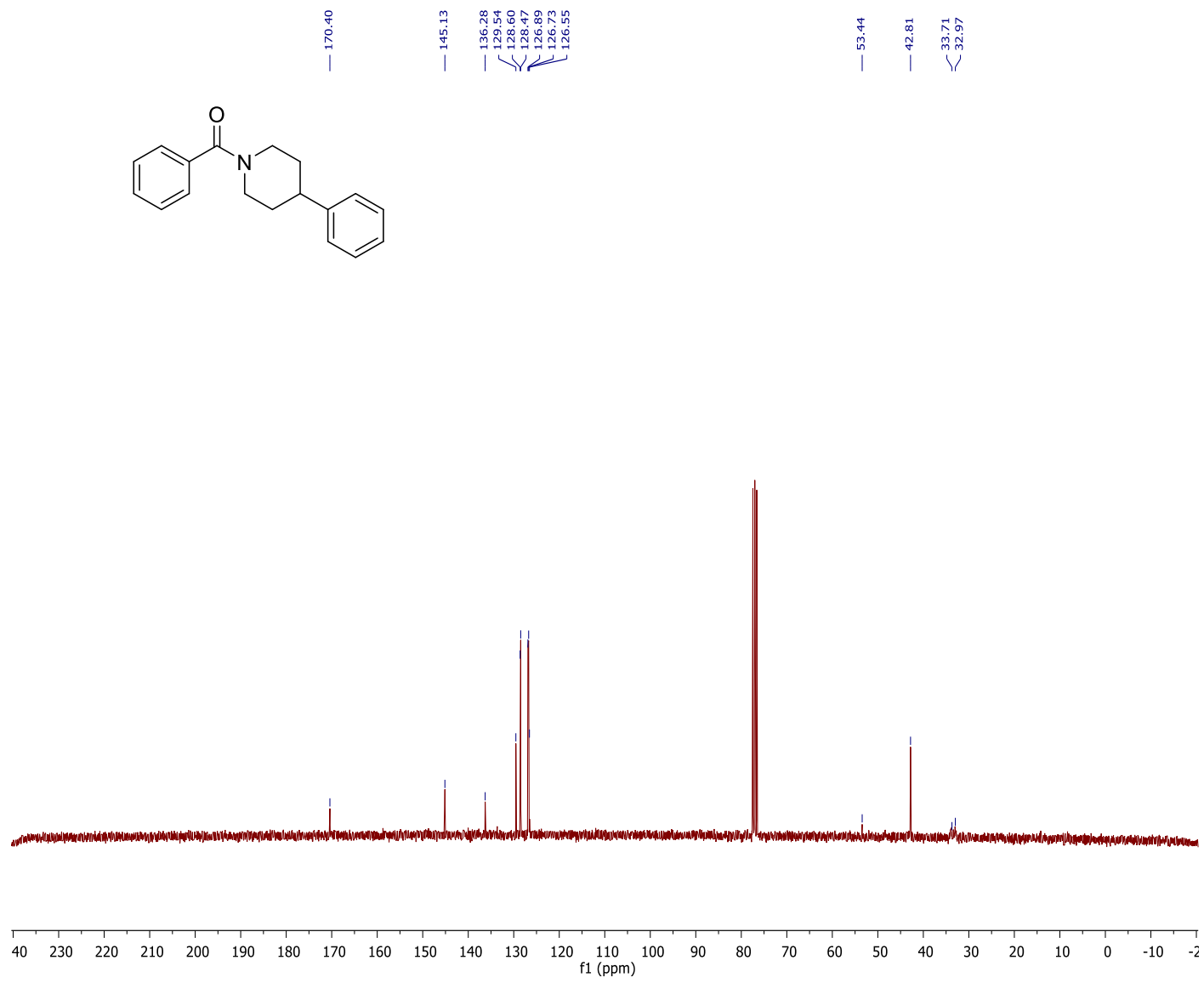

${ }^{13} \mathrm{C}$ NMR spectrum in $\mathrm{CDCl}_{3}$.

185 
144b<smiles>O=CN1CCCCC1</smiles>

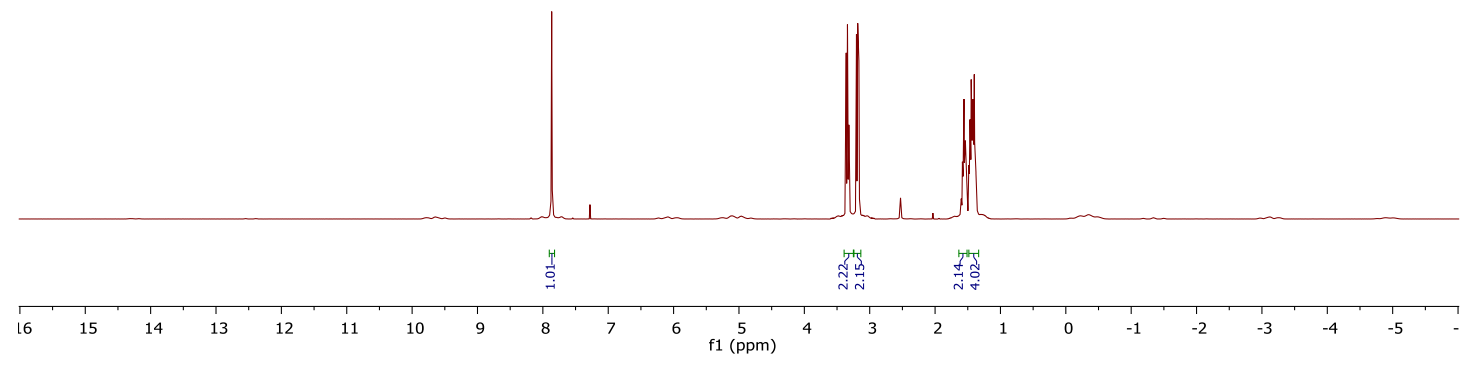

${ }^{1} \mathrm{H}$ NMR spectrum in $\mathrm{CDCl}_{3}$.
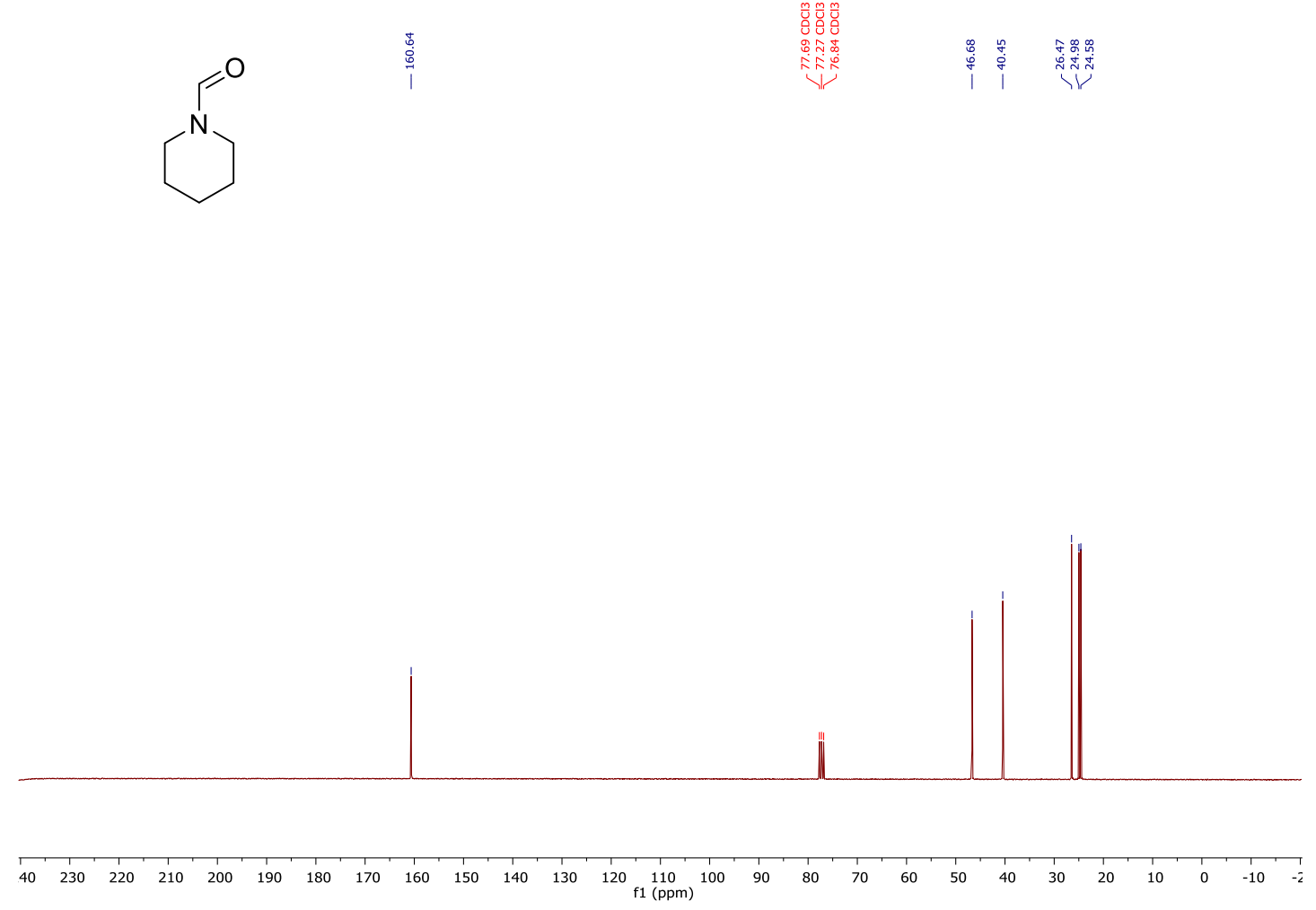

${ }^{13} \mathrm{C}$ NMR spectrum in $\mathrm{CDCl}_{3}$. 


\section{5b}

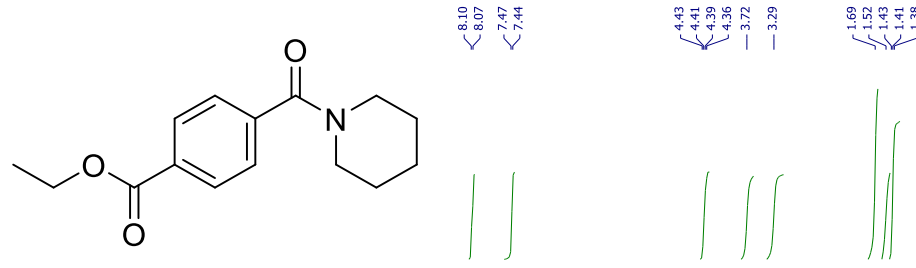

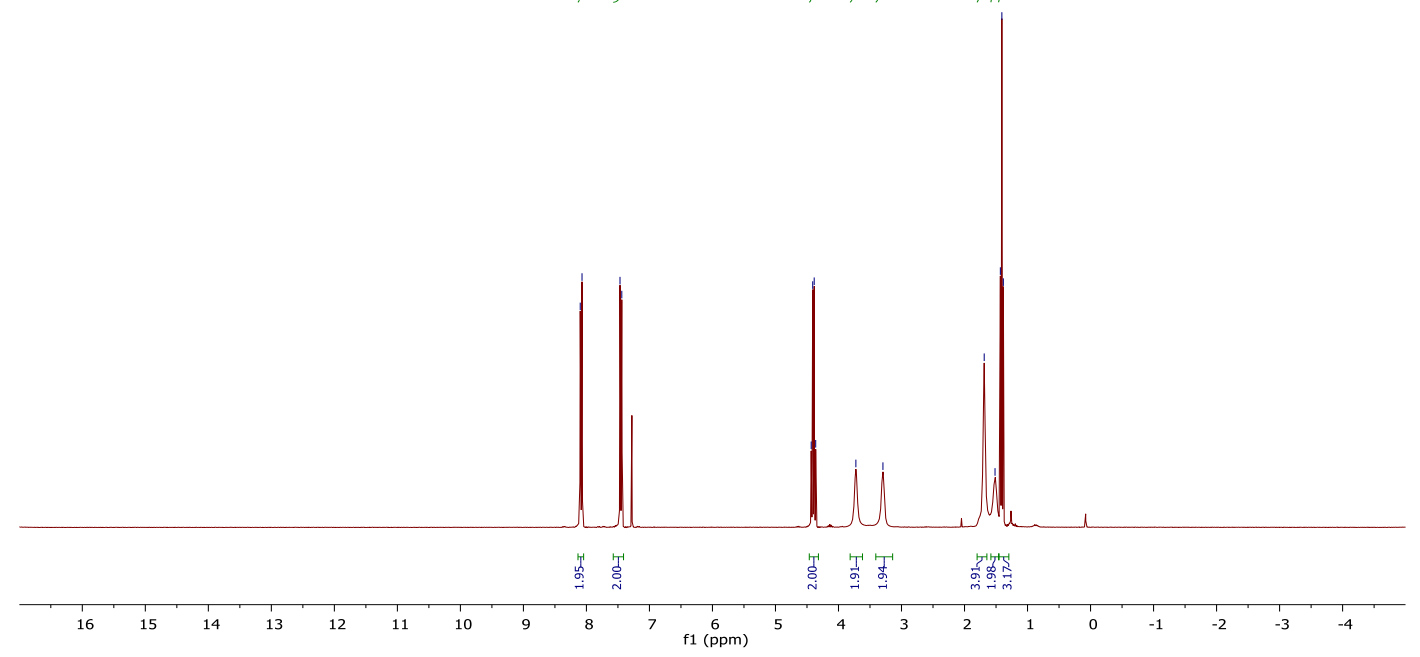

${ }^{1} \mathrm{H}$ NMR spectrum in $\mathrm{CDCl}_{3}$.

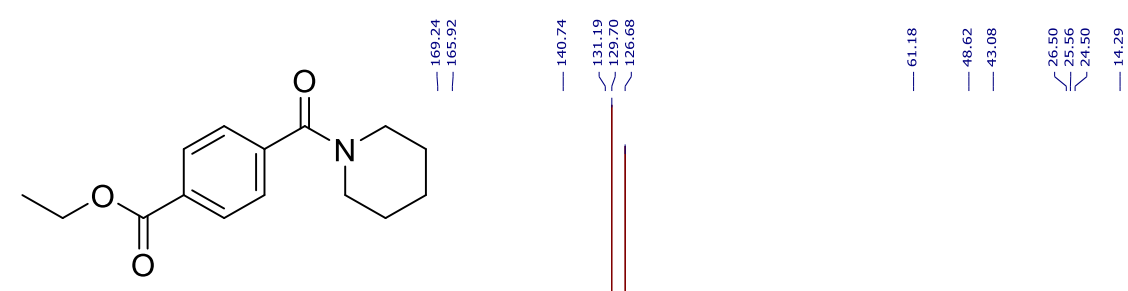

$\begin{array}{lllllllllllllllllllllllllllllll}270 & 260 & 250 & 240 & 230 & 220 & 210 & 200 & 190 & 180 & 170 & 160 & 150 & 140 & 130 & 120 & 110 & 100 & 90 & 80 & 70 & 60 & 50 & 40 & 30 & 20 & 10 & 0 & -10 & -20 & -30\end{array}$ ${ }^{13} \mathrm{C}$ NMR spectrum in $\mathrm{CDCl}_{3}$. 
Experimental Section

$146 b$

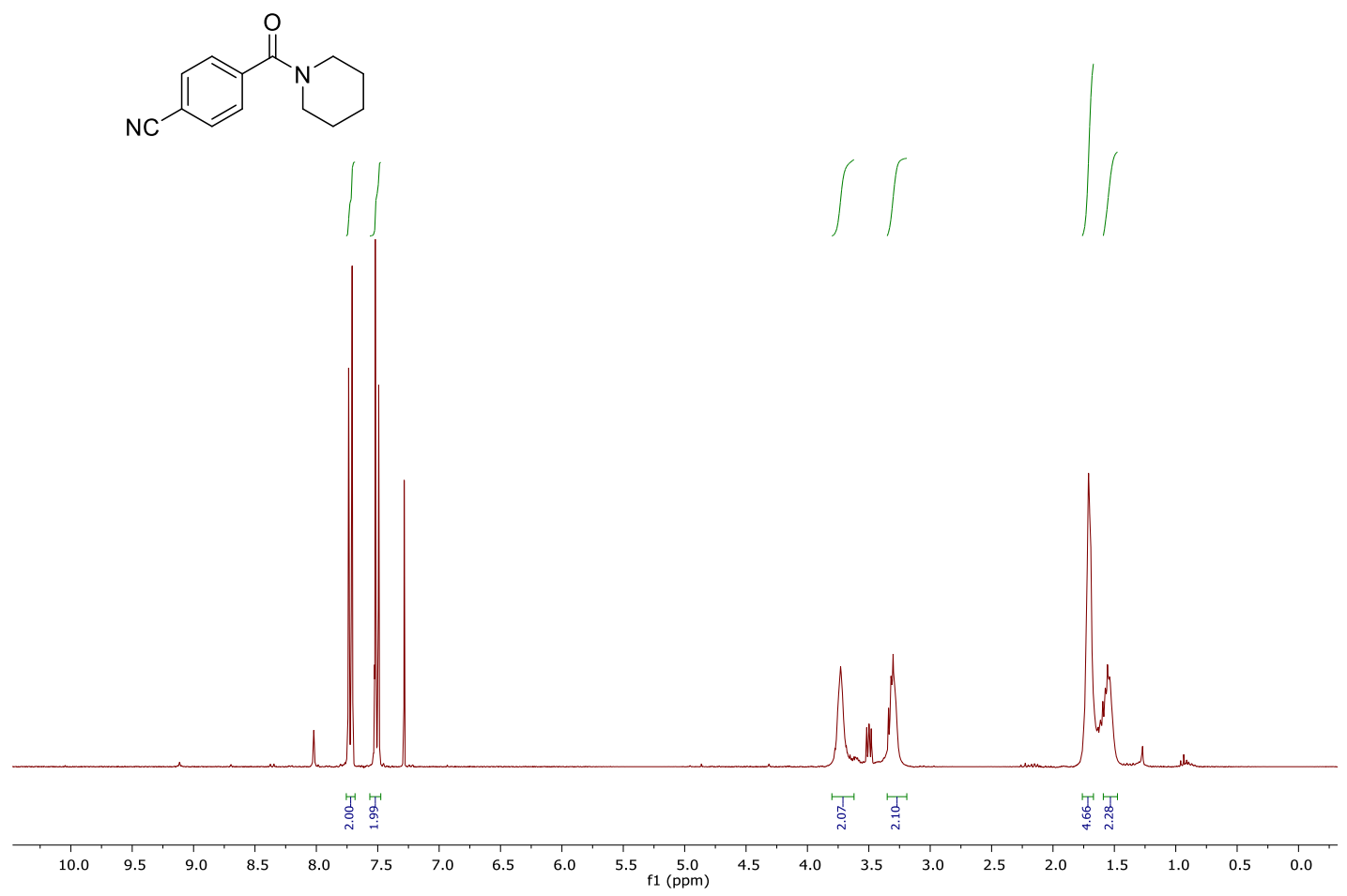

${ }^{1} \mathrm{H}$ NMR spectrum in $\mathrm{CDCl}_{3}$.

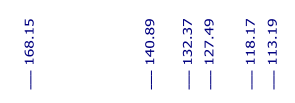

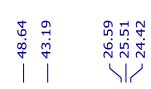
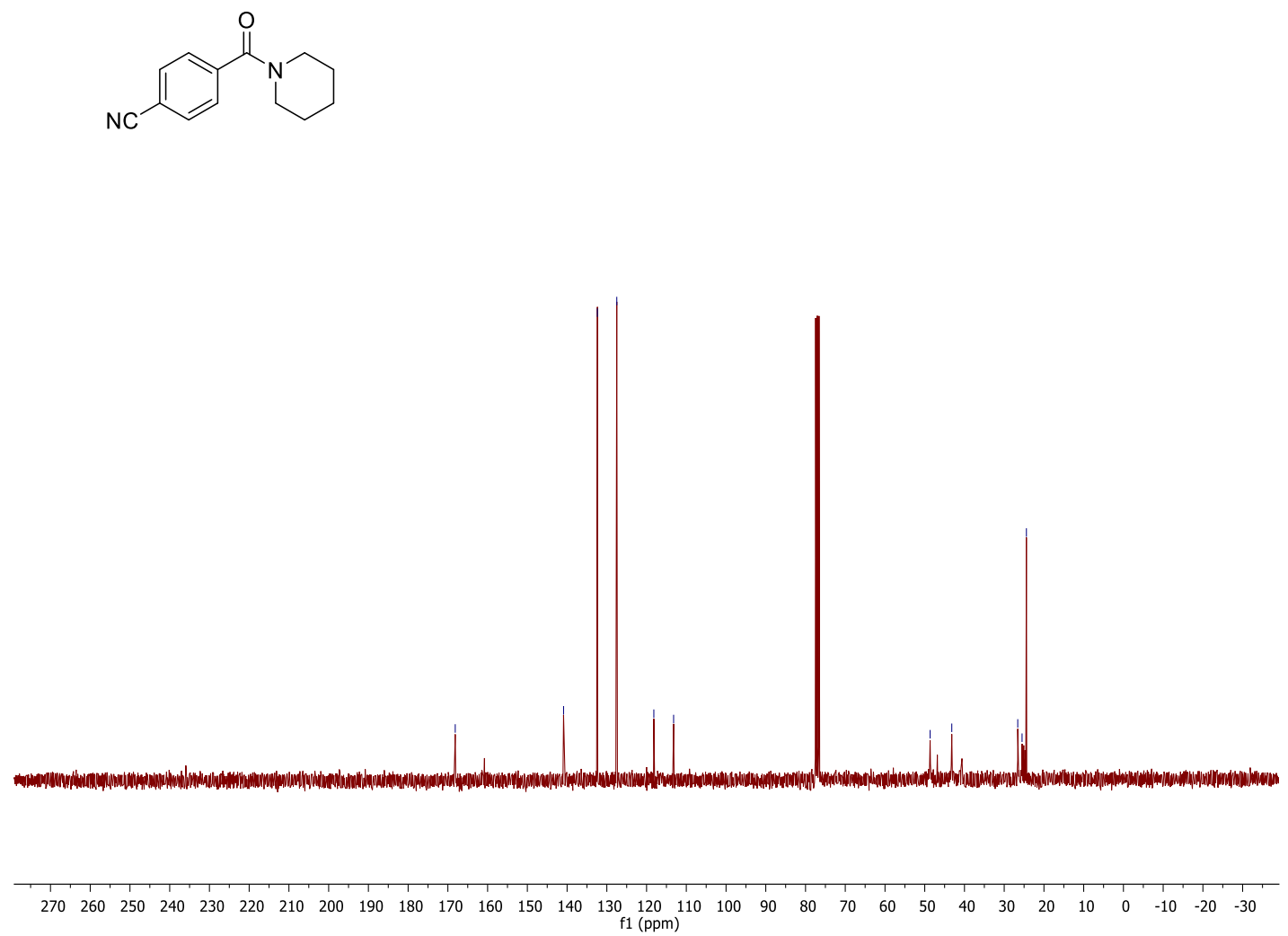

${ }^{13} \mathrm{C}$ NMR spectrum in $\mathrm{CDCl}_{3}$.

188 
Experimental Section

$147 b$
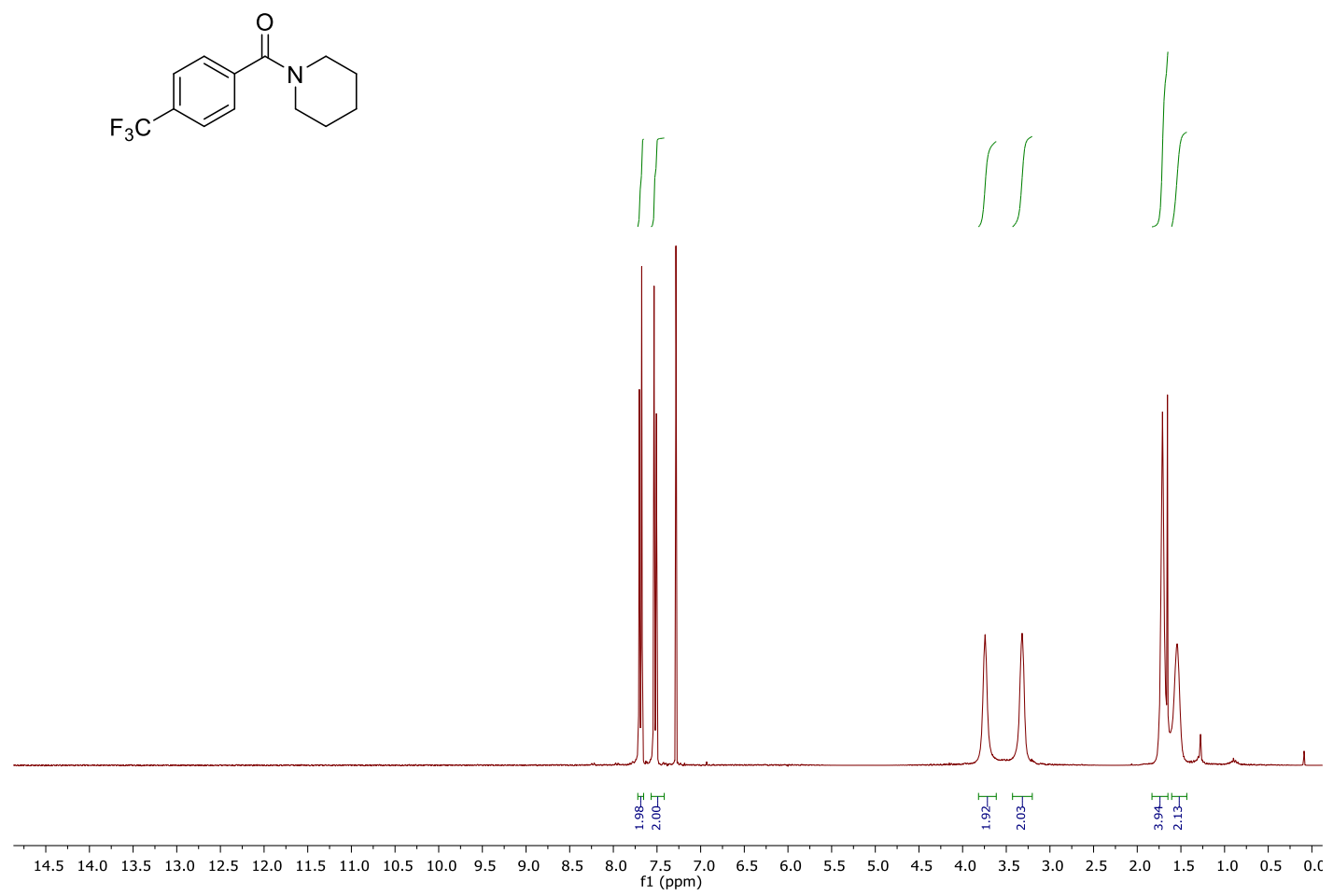

${ }^{1} \mathrm{H}$ NMR spectrum in $\mathrm{CDCl}_{3}$.
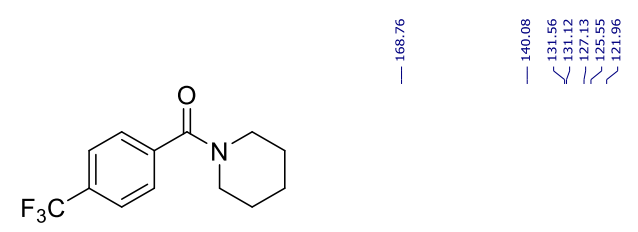

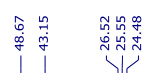

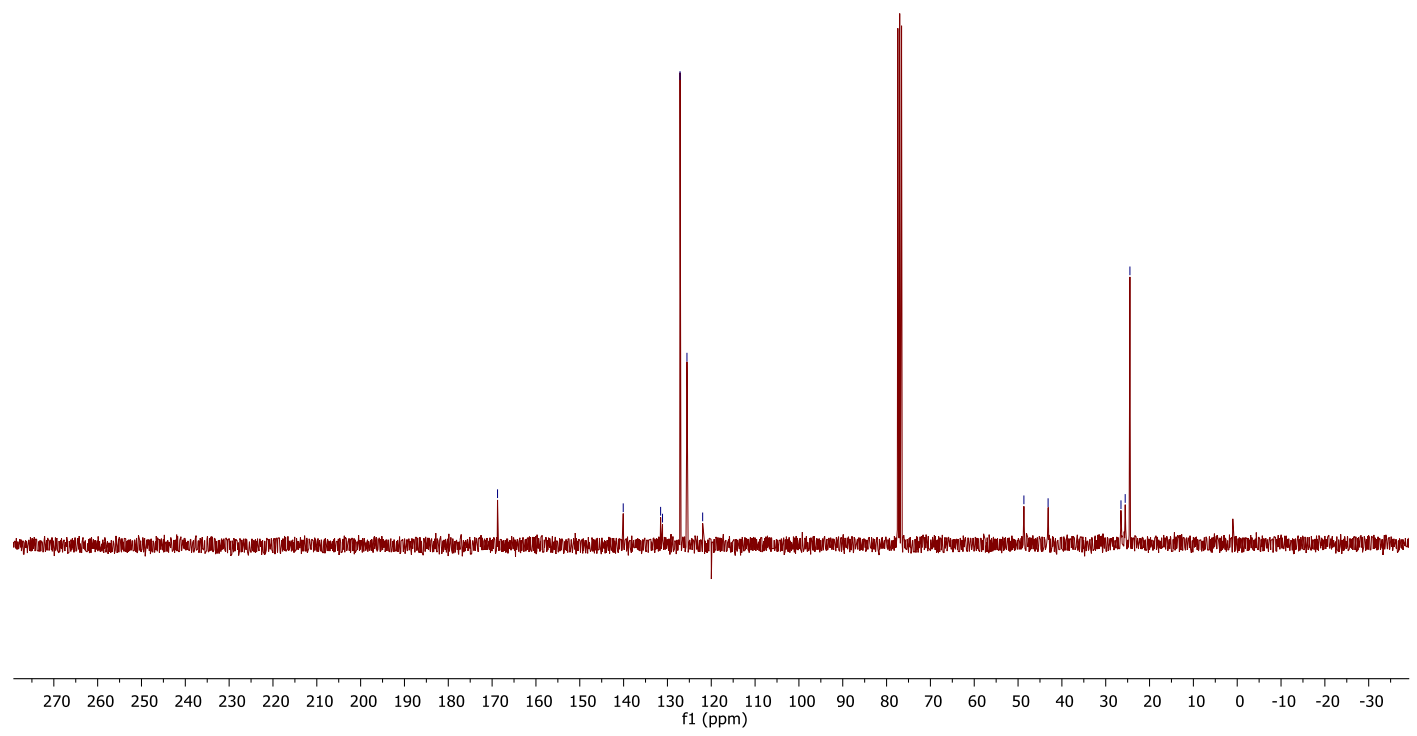

${ }^{13} \mathrm{C} \mathrm{NMR}$ spectrum in $\mathrm{CDCl}_{3}$.

189 
$148 b$

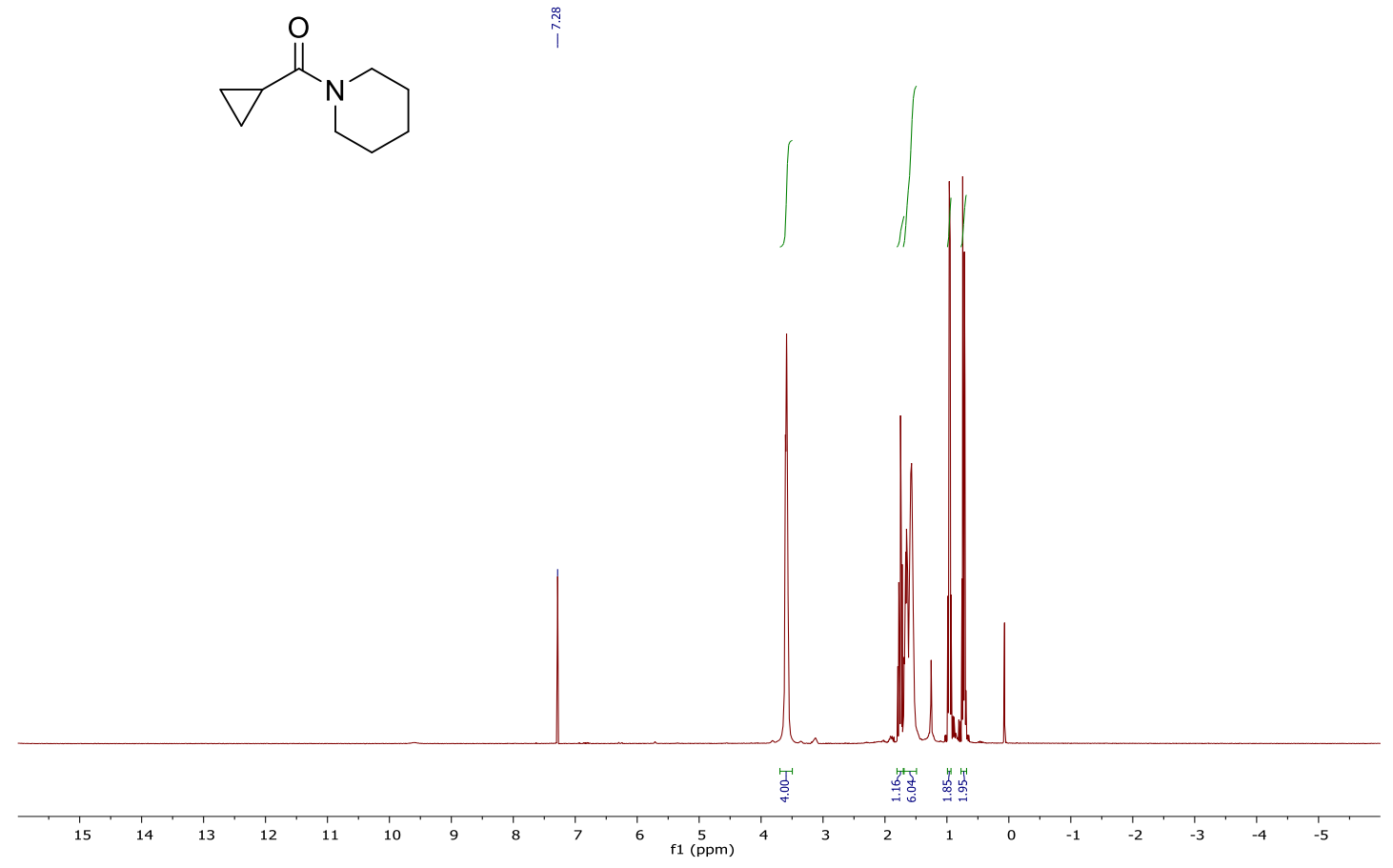

${ }^{1} \mathrm{H}$ NMR spectrum in $\mathrm{CDCl}_{3}$.<smiles>COC(=O)N1CCCCC1</smiles>

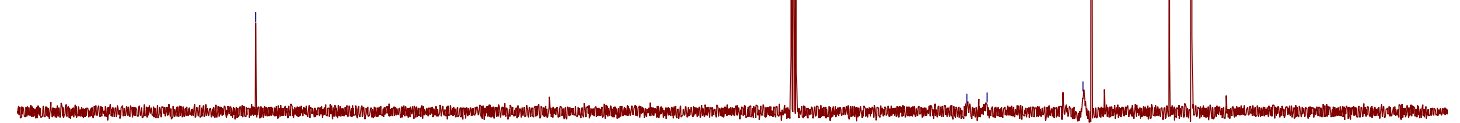
\begin{tabular}{lllllllllllllllllllllllllll}
\hline 210 & 200 & 190 & 180 & 170 & 160 & 150 & 140 & 130 & 120 & 110 & 100 & 90 & 80 & 70 & 60 & 50 & 40 & 30 & 20 & 10 & 0 & -10 & -20 & -30
\end{tabular} ${ }^{13} \mathrm{C}$ NMR spectrum in $\mathrm{CDCl}_{3}$. 
149b

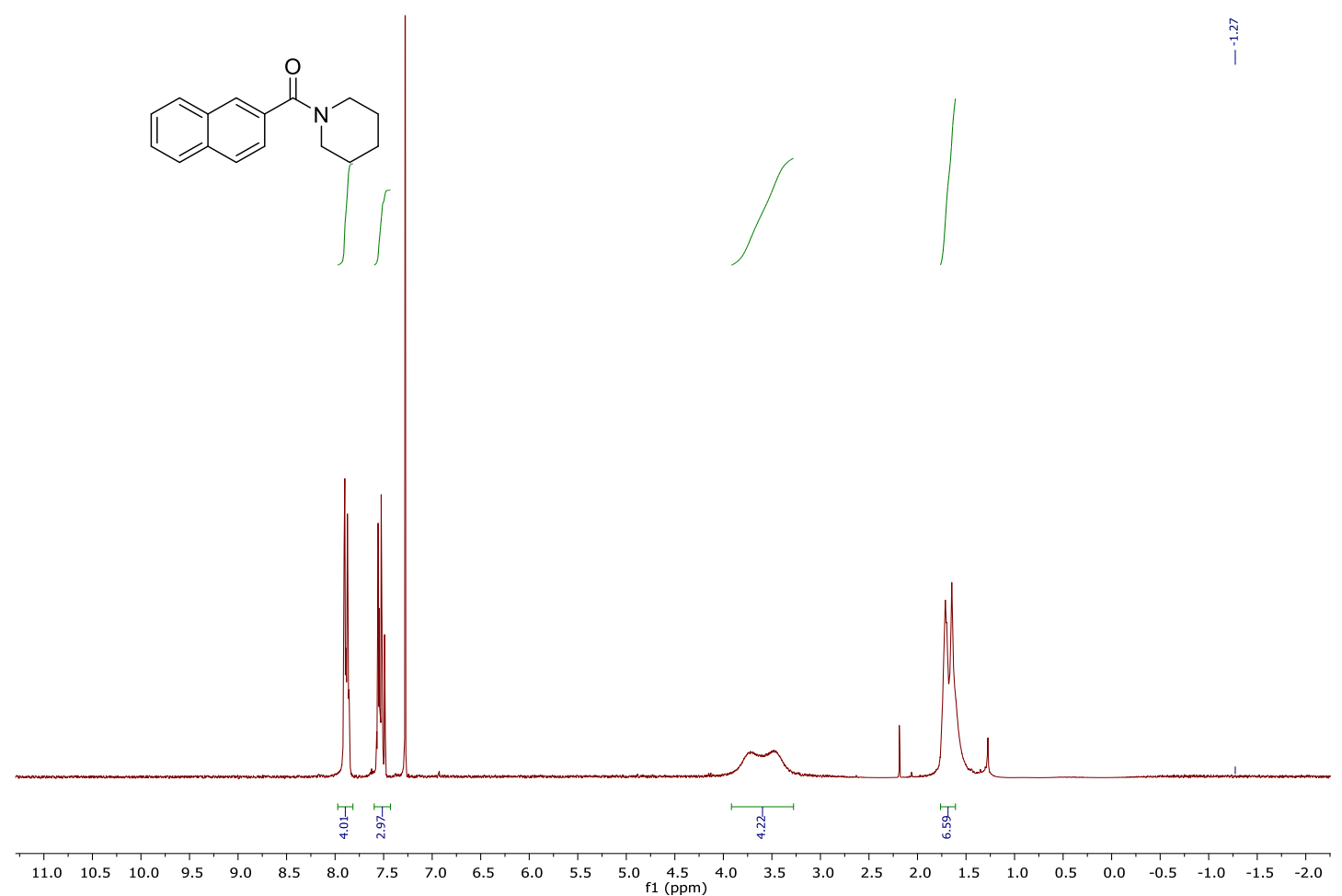

${ }^{1} \mathrm{H}$ NMR spectrum in $\mathrm{CDCl}_{3}$.
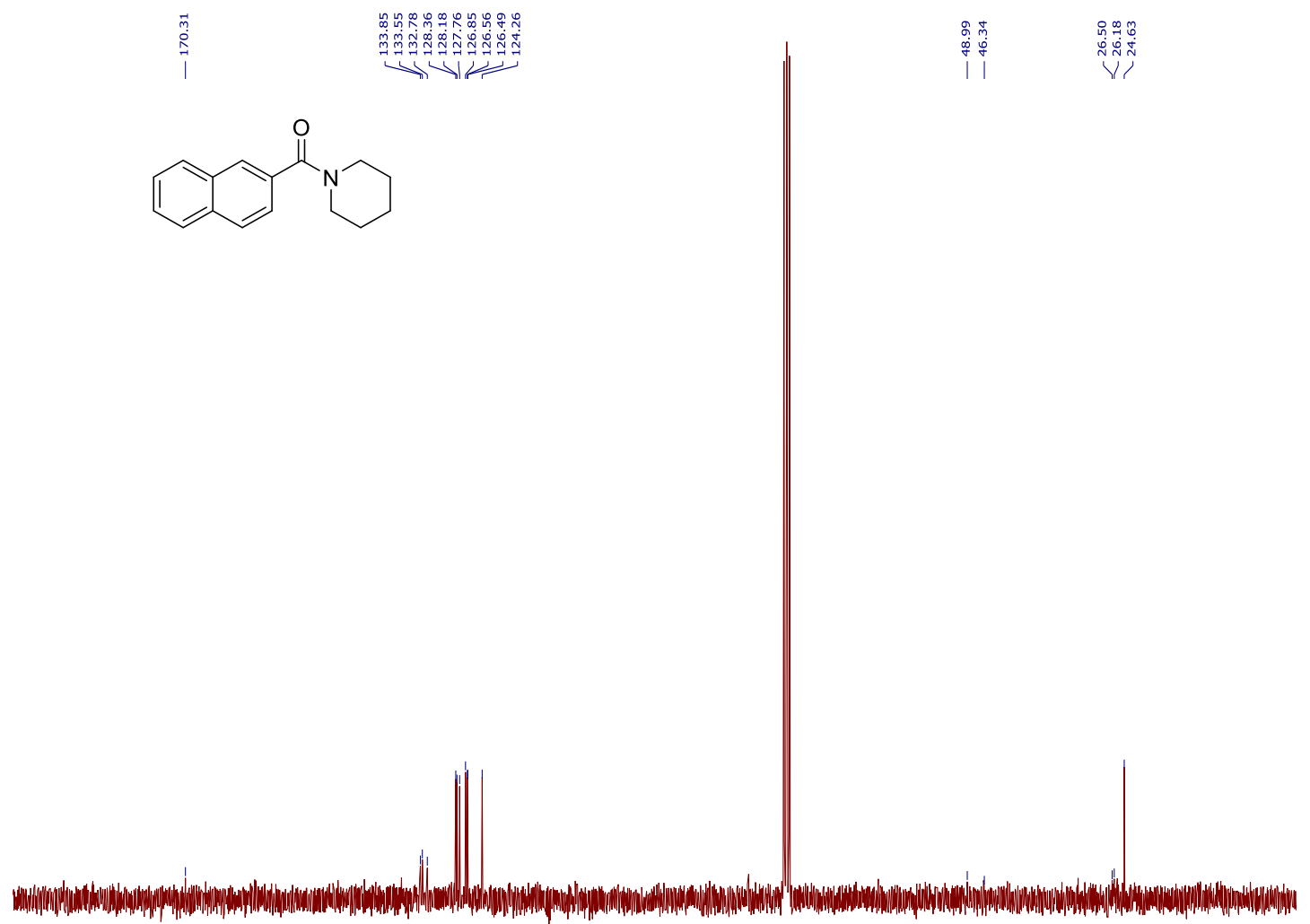

$\begin{array}{lllllllllllllllllllll}190 & 180 & 170 & 160 & 150 & 140 & 130 & 120 & 110 & \underset{f 1}{100}(\mathrm{pmm}) & 90 & 80 & 70 & 60 & 50 & 40 & 30 & 20 & 10 & 0\end{array}$

${ }^{13} \mathrm{C}$ NMR spectrum in $\mathrm{CDCl}_{3}$. 
150b

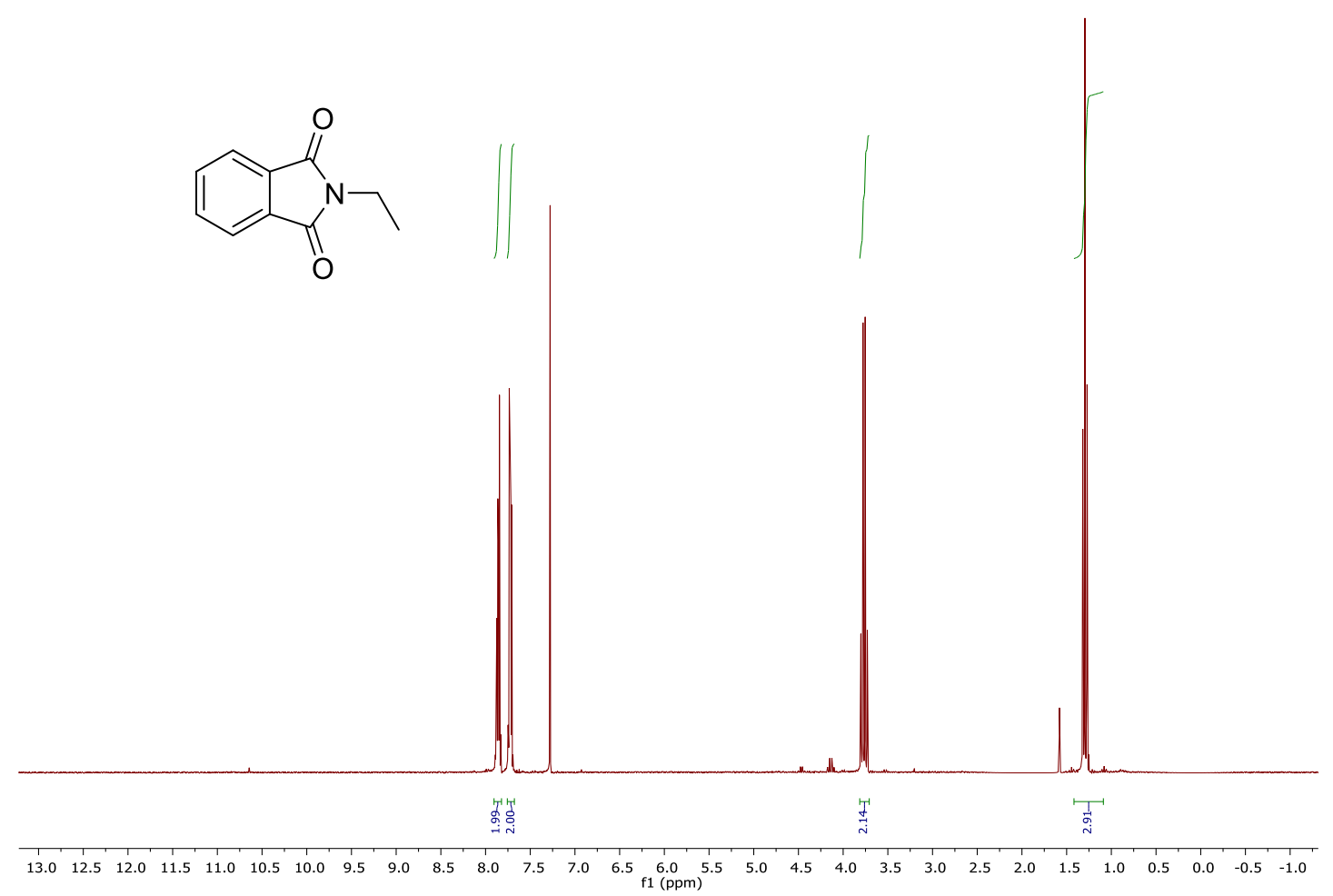

${ }^{1} \mathrm{H}$ NMR spectrum in $\mathrm{CDCl}_{3}$.
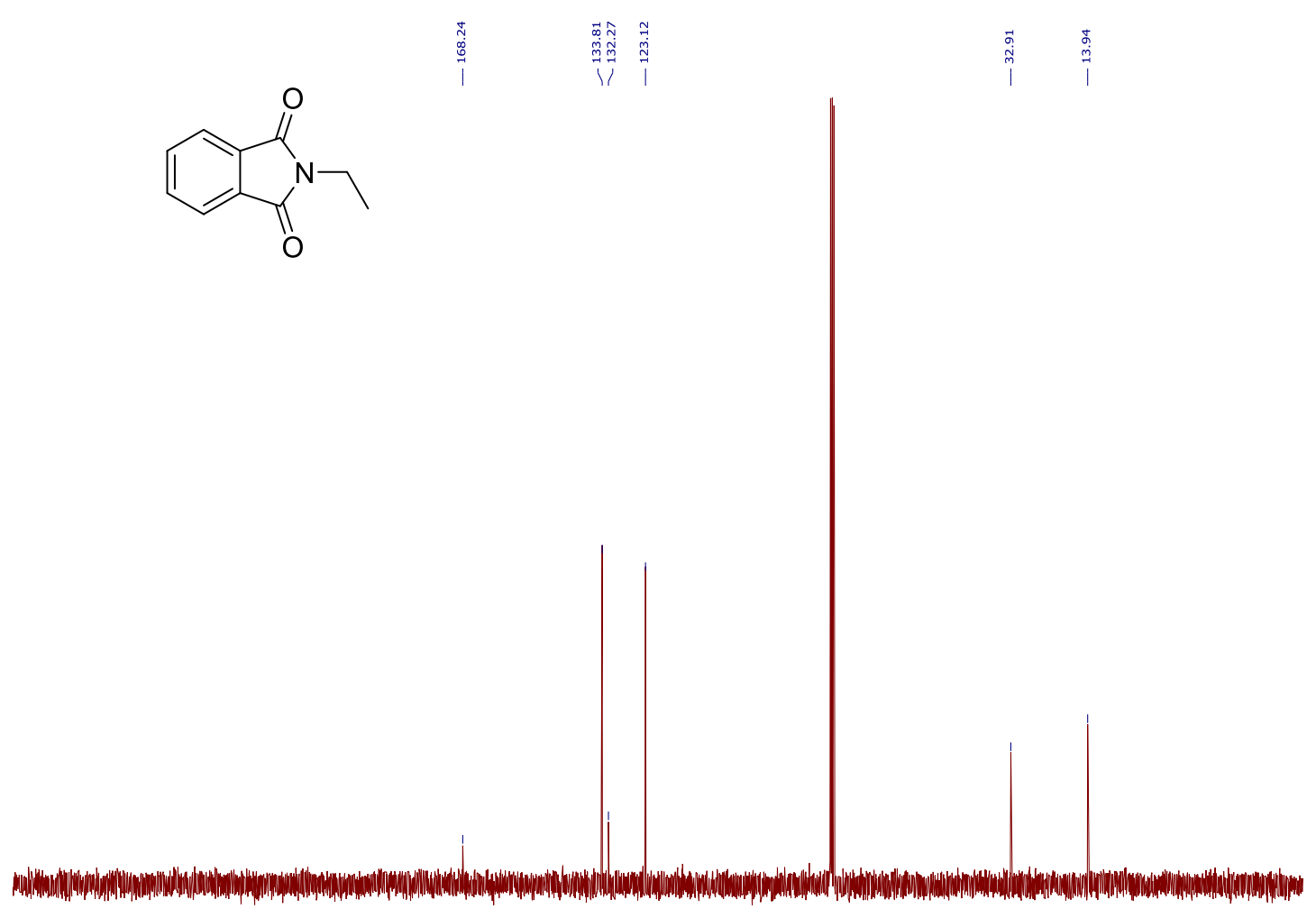

$\begin{array}{lllllllllllllllllllllllllllllllll}270 & 260 & 250 & 240 & 230 & 220 & 210 & 200 & 190 & 180 & 170 & 160 & 150 & 140 & 130 & 120 & 110 & 100 & 90 & 80 & 70 & 60 & 50 & 40 & 30 & 20 & 10 & 0 & -10 & -20 & -30\end{array}$ ${ }^{13} \mathrm{C}$ NMR spectrum in $\mathrm{CDCl}_{3}$. 
151b
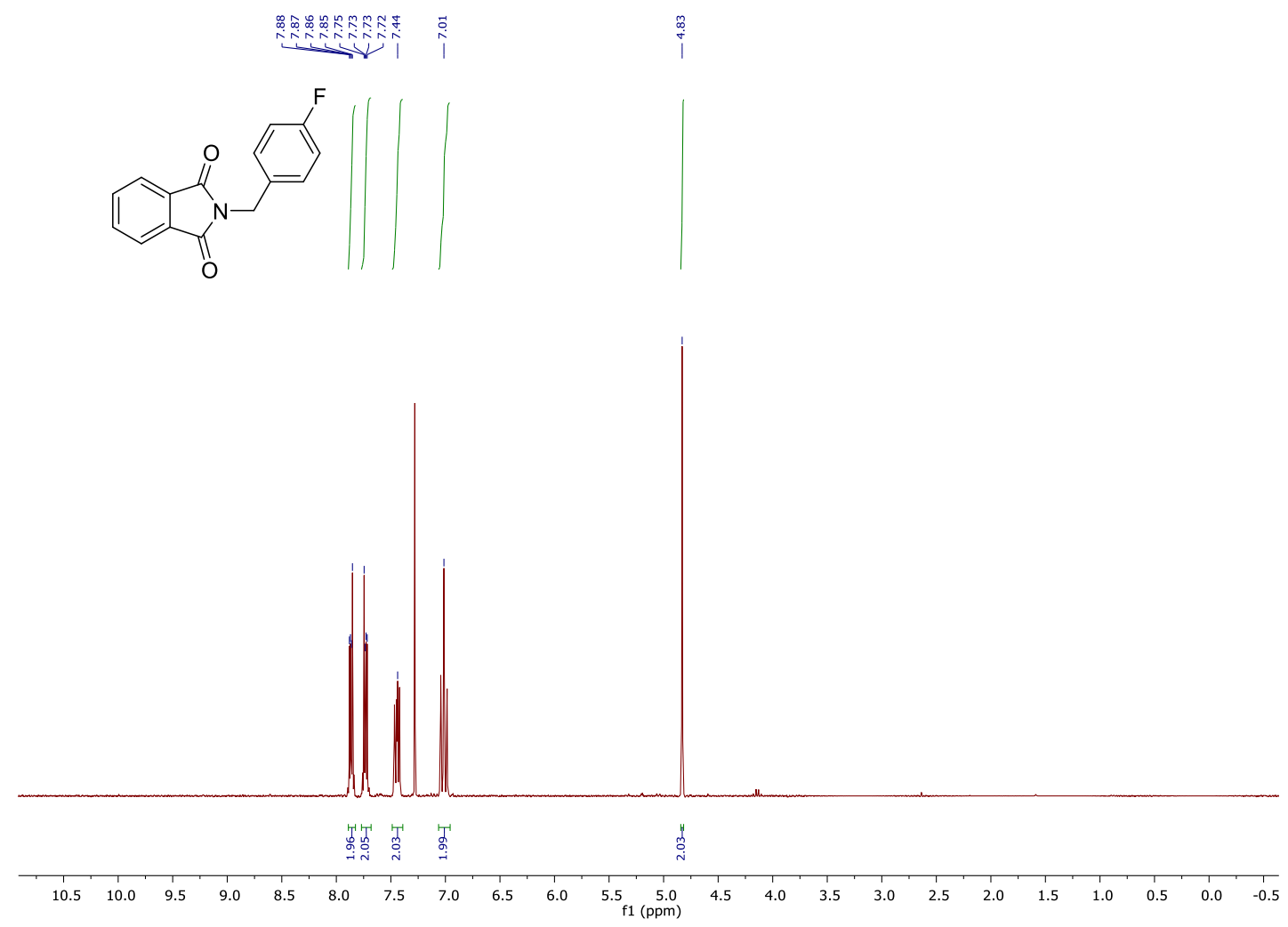

${ }^{1} \mathrm{H}$ NMR spectrum in $\mathrm{CDCl}_{3}$.
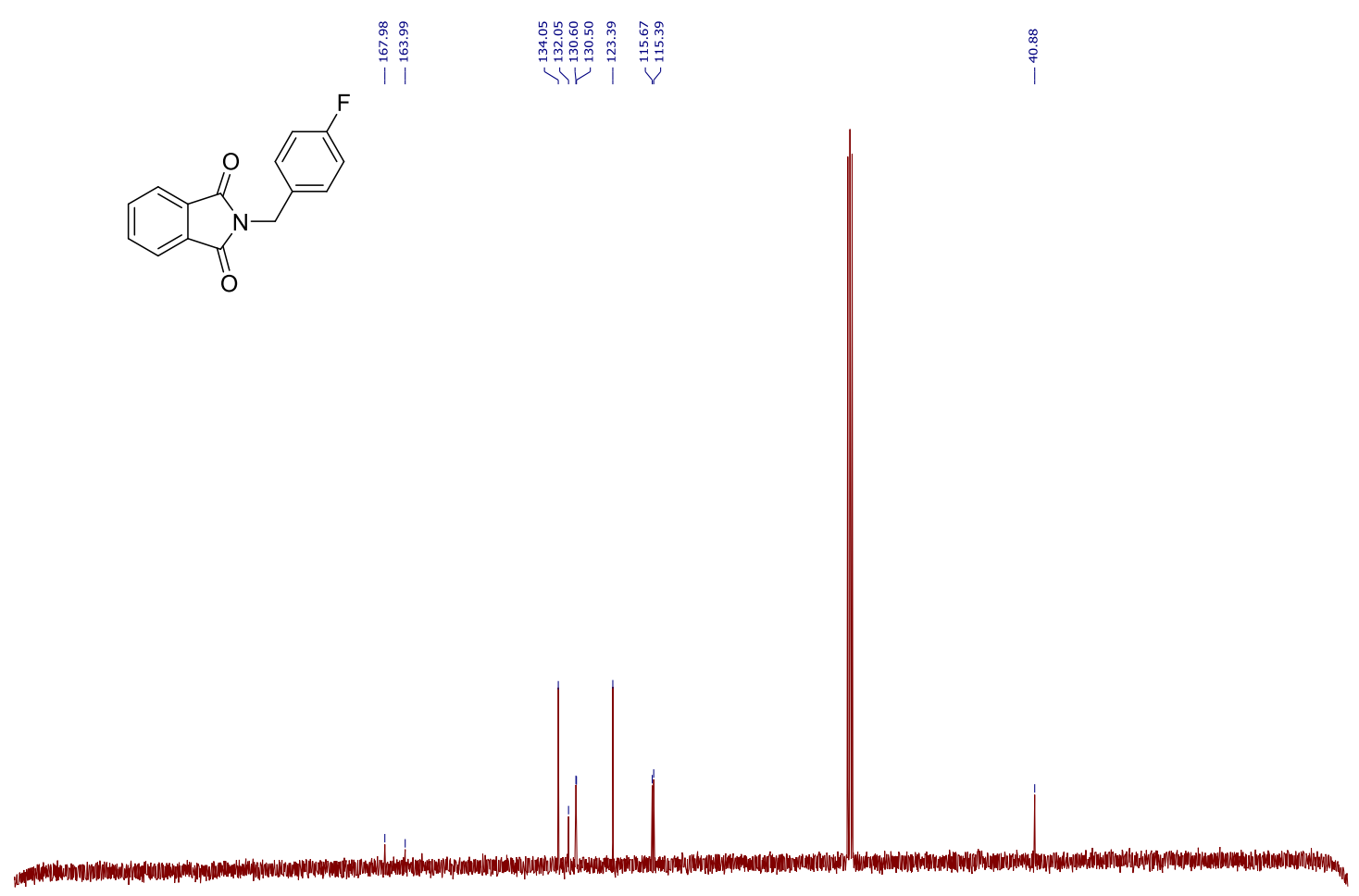

\begin{tabular}{llllllllllllllllllllllllllll}
\hline 40 & 230 & 220 & 210 & 200 & 190 & 180 & 170 & 160 & 150 & 140 & 130 & 120 & $\underset{\mathrm{f} 1}{(\mathrm{ppm})}$ & 100 & 90 & 80 & 70 & 60 & 50 & 40 & 30 & 20 & 10 & 0 & -10 & -4
\end{tabular}

${ }^{13} \mathrm{C}$ NMR spectrum in $\mathrm{CDCl}_{3}$. 
$152 b$
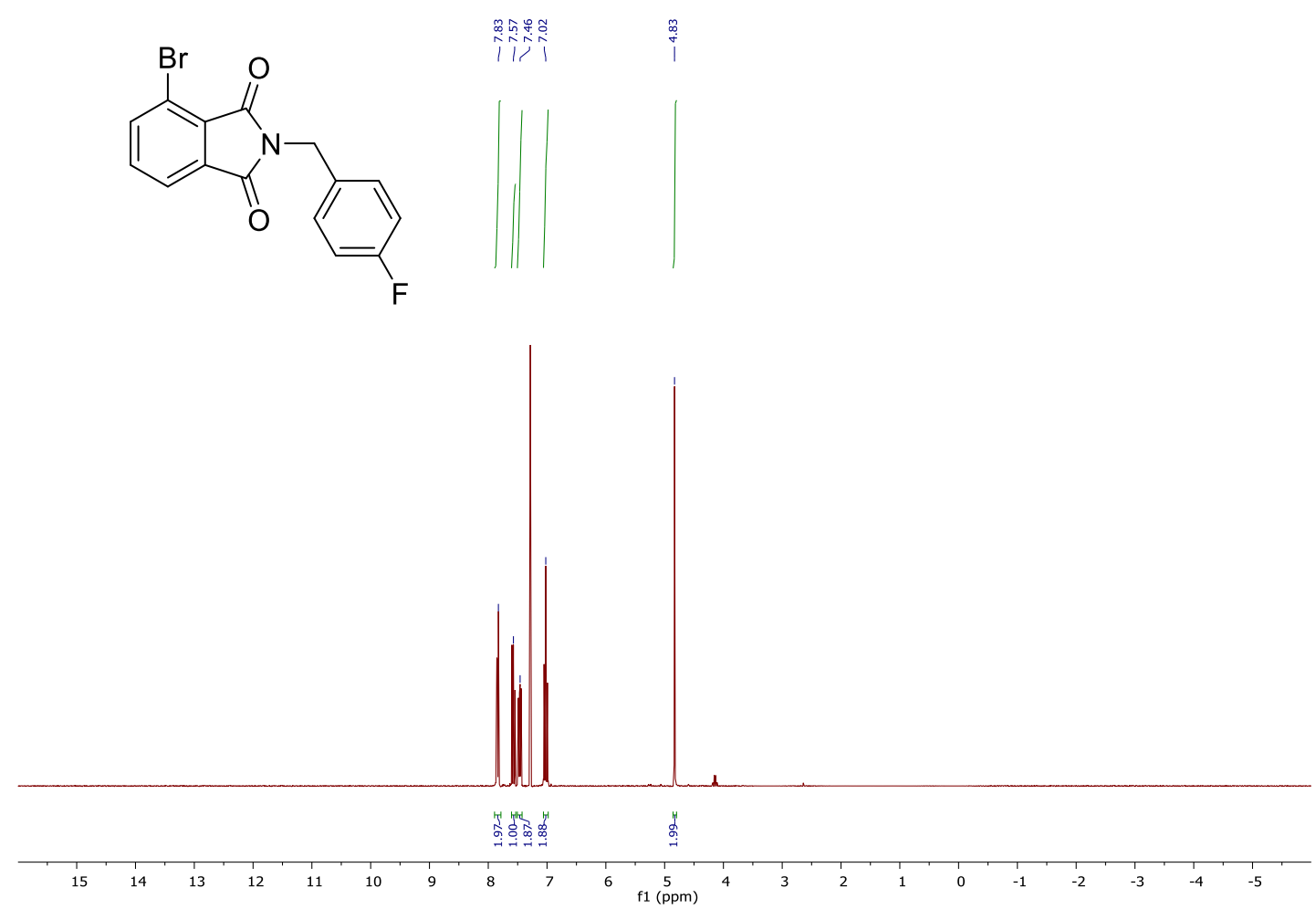

${ }^{1} \mathrm{H}$ NMR spectrum in $\mathrm{CDCl}_{3}$.<smiles>O=C1c2cccc(Br)c2C(=O)N1Cc1ccc(F)cc1</smiles>

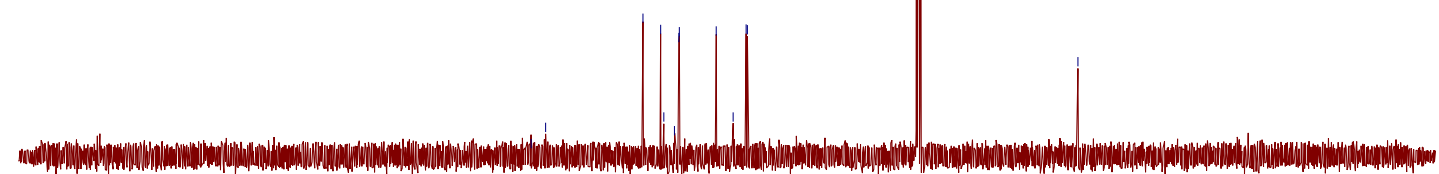

$\begin{array}{lllllllllllllllllllllllllllllllll}270 & 260 & 250 & 240 & 230 & 220 & 210 & 200 & 190 & 180 & 170 & 160 & 150 & 140 & 130 & 120 & 110 & 100 & 90 & 80 & 70 & 60 & 50 & 40 & 30 & 20 & 10 & 0 & -10 & -20 & -30\end{array}$ ${ }^{13} \mathrm{C}$ NMR spectrum in $\mathrm{CDCl}_{3}$. 
153b

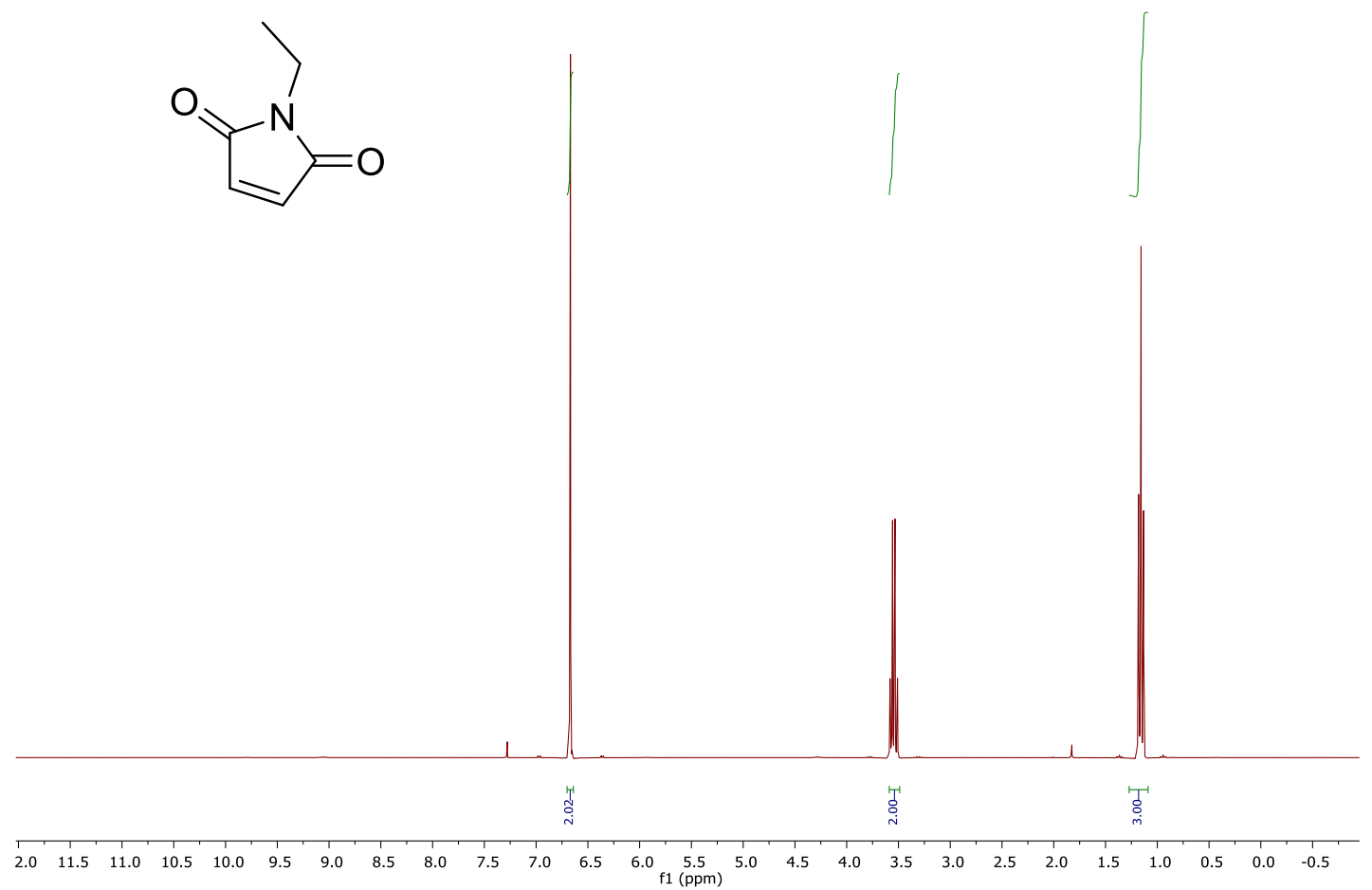

${ }^{1} \mathrm{H}$ NMR spectrum in $\mathrm{CDCl}_{3}$.<smiles>CCN1C(=O)C=CC1=O</smiles>

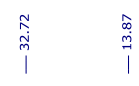

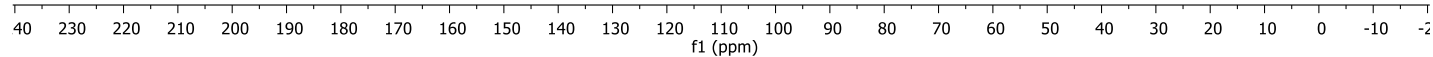

${ }^{13} \mathrm{C}$ NMR spectrum in $\mathrm{CDCl}_{3}$. 
154b

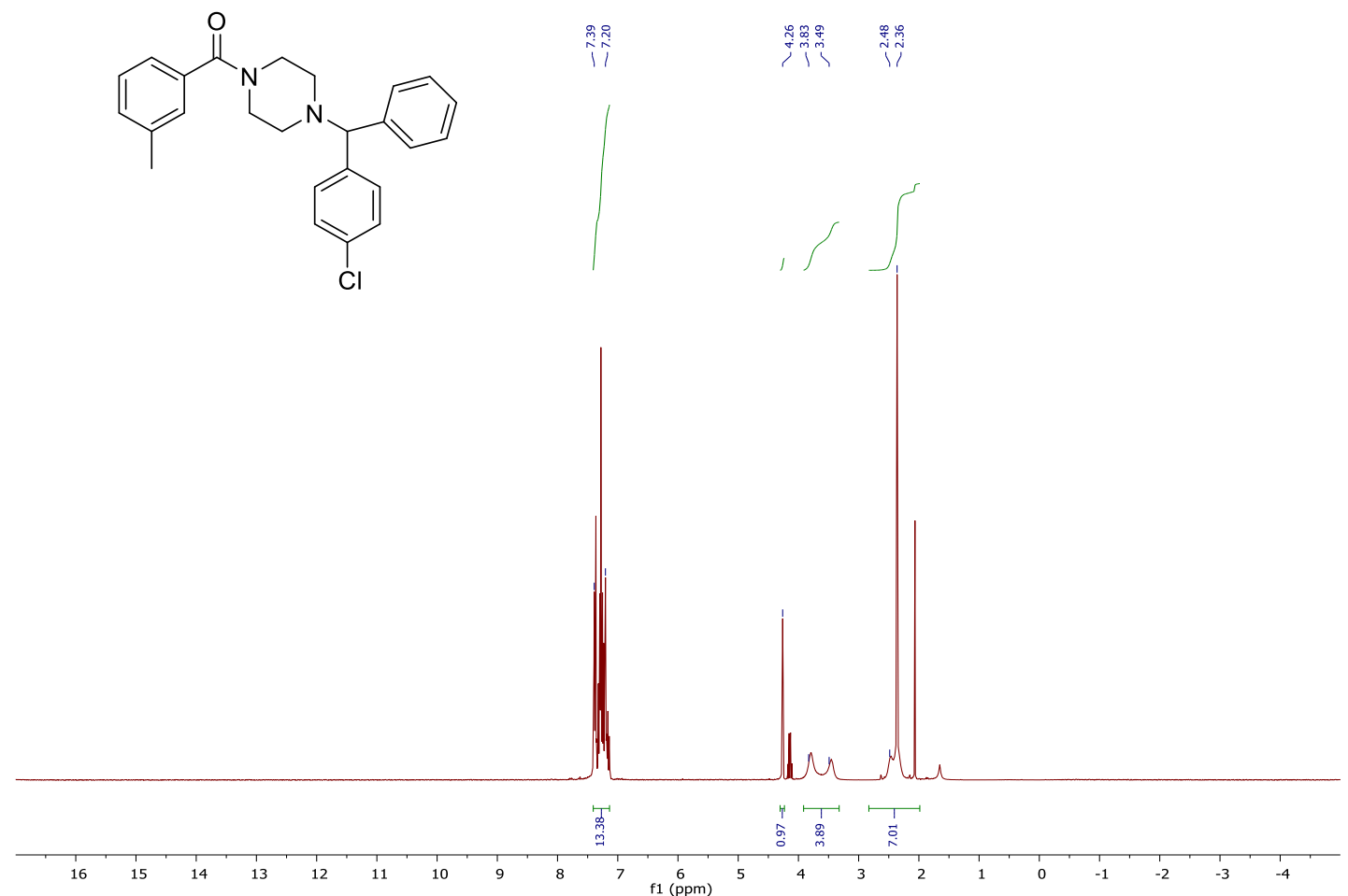

${ }^{1} \mathrm{H}$ NMR spectrum in $\mathrm{CDCl}_{3}$.
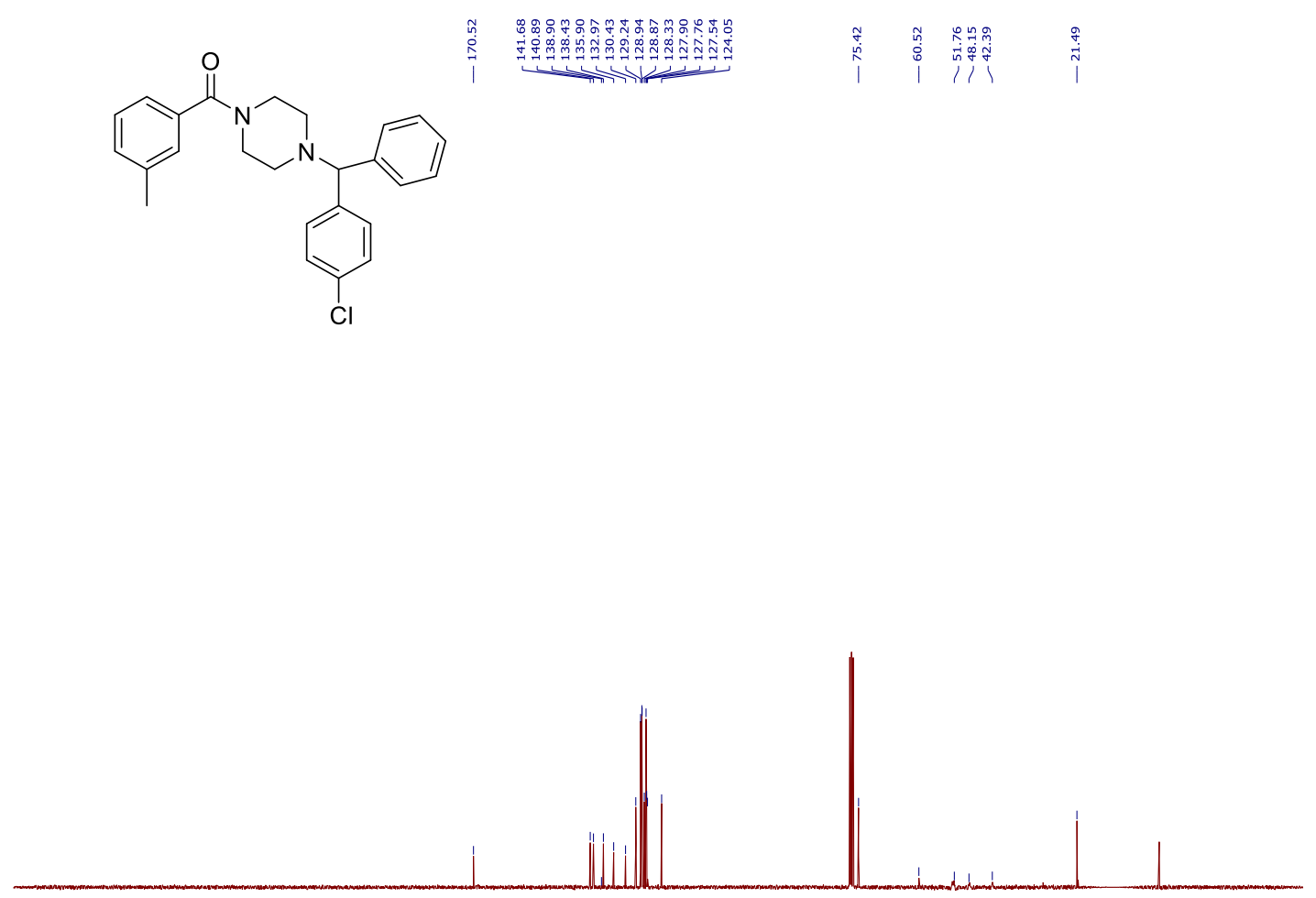

\begin{tabular}{lllllllllllllllllllllllllllllllllllllllllllll}
\hline 280 & 270 & 260 & 250 & 240 & 230 & 220 & 210 & 200 & 190 & 180 & 170 & 160 & 150 & 140 & 130 & 120 & 110 & 100 & 90 & 80 & 70 & 60 & 50 & 40 & 30 & 20 & 10 & 0 & -10 & -20 & -30
\end{tabular} ${ }^{13} \mathrm{C}$ NMR spectrum in $\mathrm{CDCl}_{3}$. 
$155 b$<smiles>COc1cc2c(cc1OC)C(CC1CCN(C(=O)c3ccccc3)CC1)CC2=O</smiles>

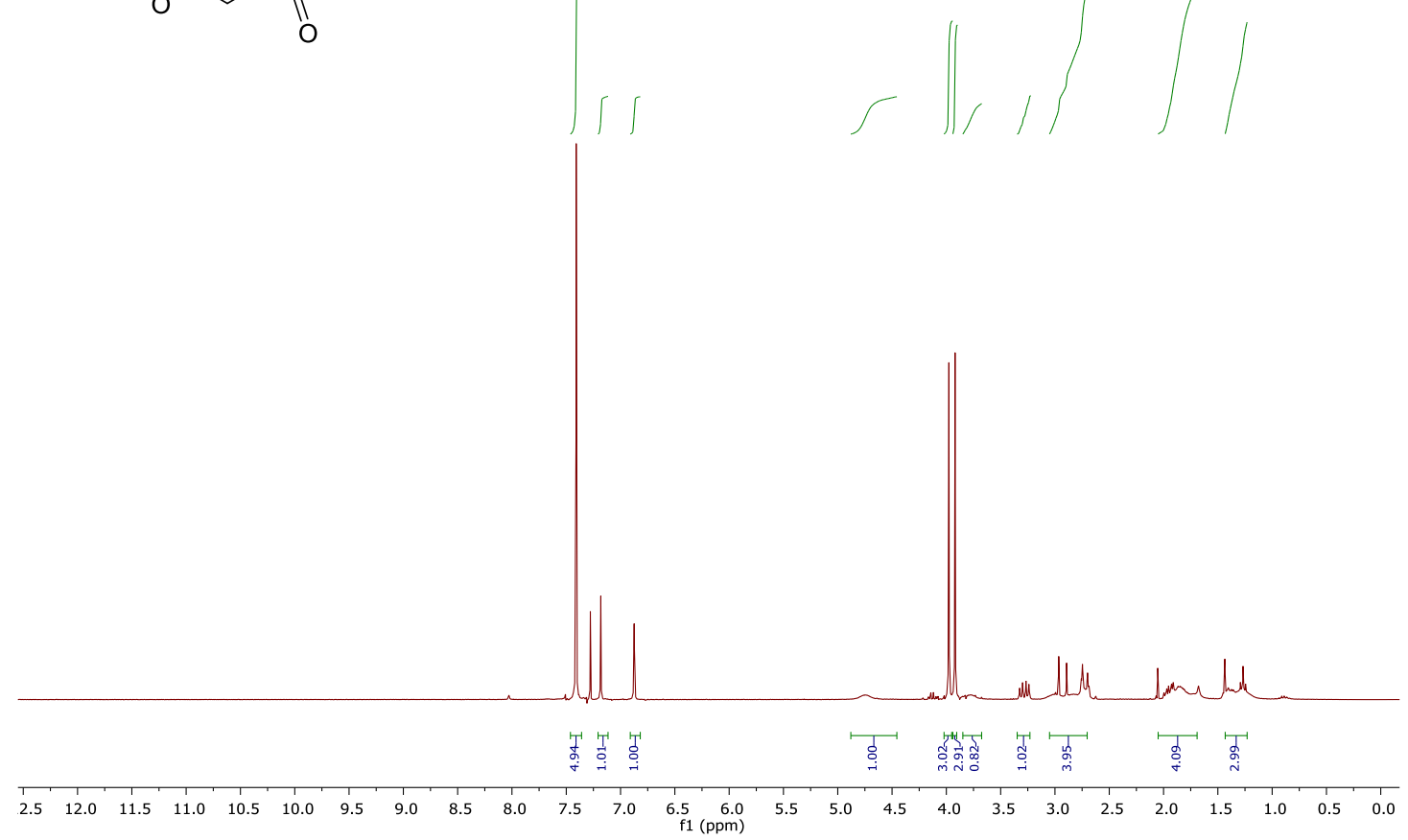

${ }^{1} \mathrm{H}$ NMR spectrum in $\mathrm{CDCl}_{3}$.
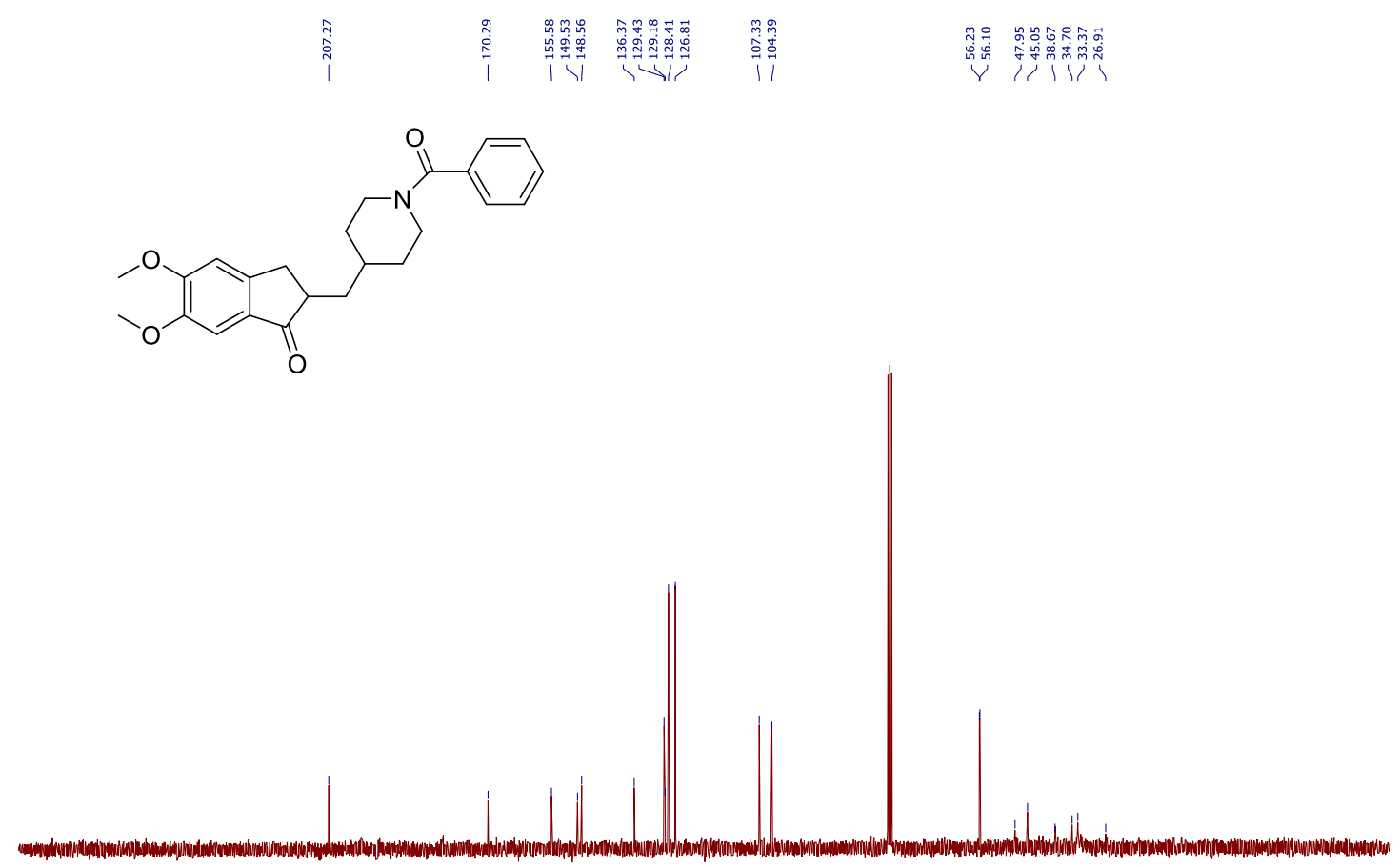

$\begin{array}{lllllllllllllllllllllllllllllllllllllllllll}270 & 260 & 250 & 240 & 230 & 220 & 210 & 200 & 190 & 180 & 170 & 160 & 150 & 140 & 130 & 120 & 110 & 100 & 90 & 80 & 70 & 60 & 50 & 40 & 30 & 20 & 10 & 0 & -10 & -20 & -30\end{array}$ ${ }^{13} \mathrm{C}$ NMR spectrum in $\mathrm{CDCl}_{3}$. 
$156 b$<smiles>COc1cc2c(cc1OC)[C@H]1Cc3ccc(OC)c(OC)c3C(=O)N1CC2</smiles>

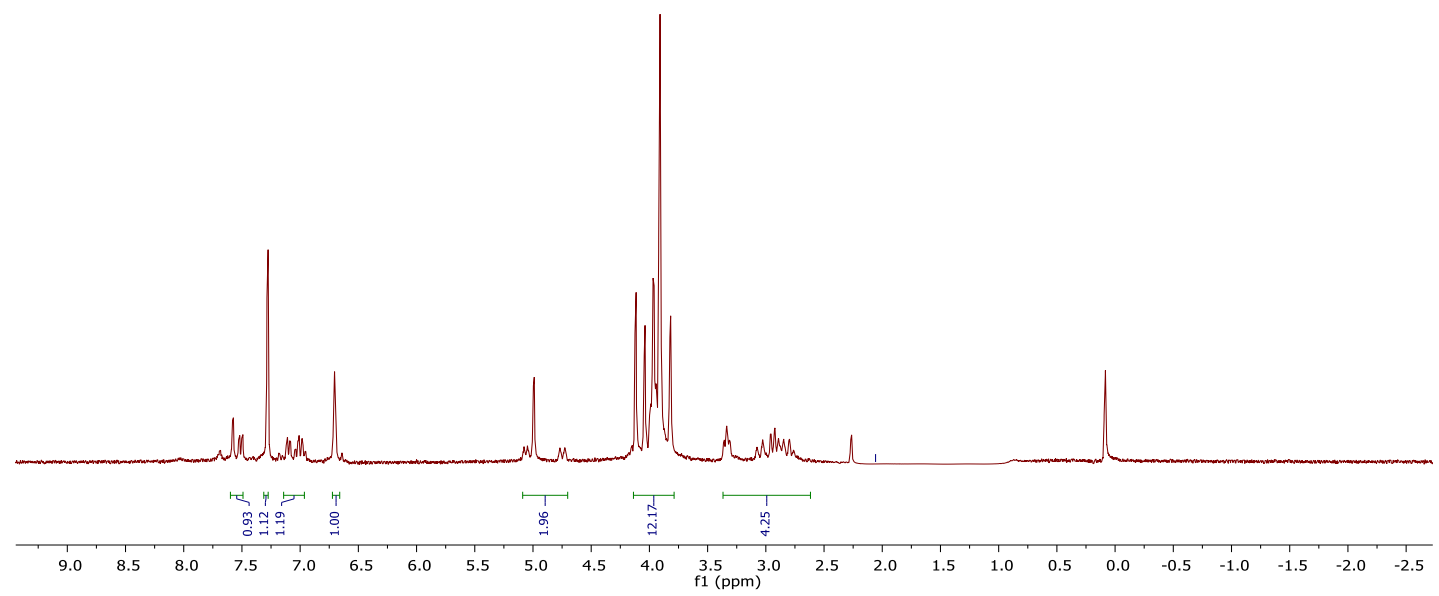

${ }^{1} \mathrm{H}$ NMR spectrum in $\mathrm{CDCl}_{3}$.

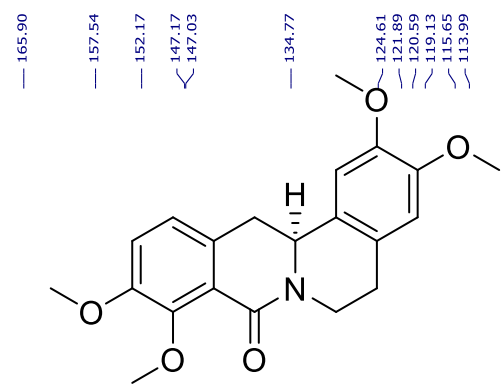

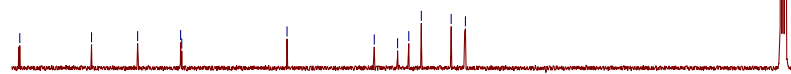

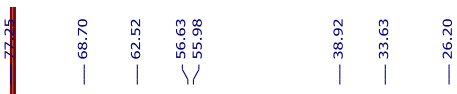

$\begin{array}{lllllllllllllllll}160 & 150 & 140 & 130 & 120 & 110 & 100 & 90 & \begin{array}{r}80 \\ \mathrm{f} 1(\mathrm{ppm})\end{array} & 70 & 60 & 50 & 40 & 30 & 20 & 10 & 0\end{array}$

${ }^{13} \mathrm{C}$ NMR spectrum in $\mathrm{CDCl}_{3}$. 
157b

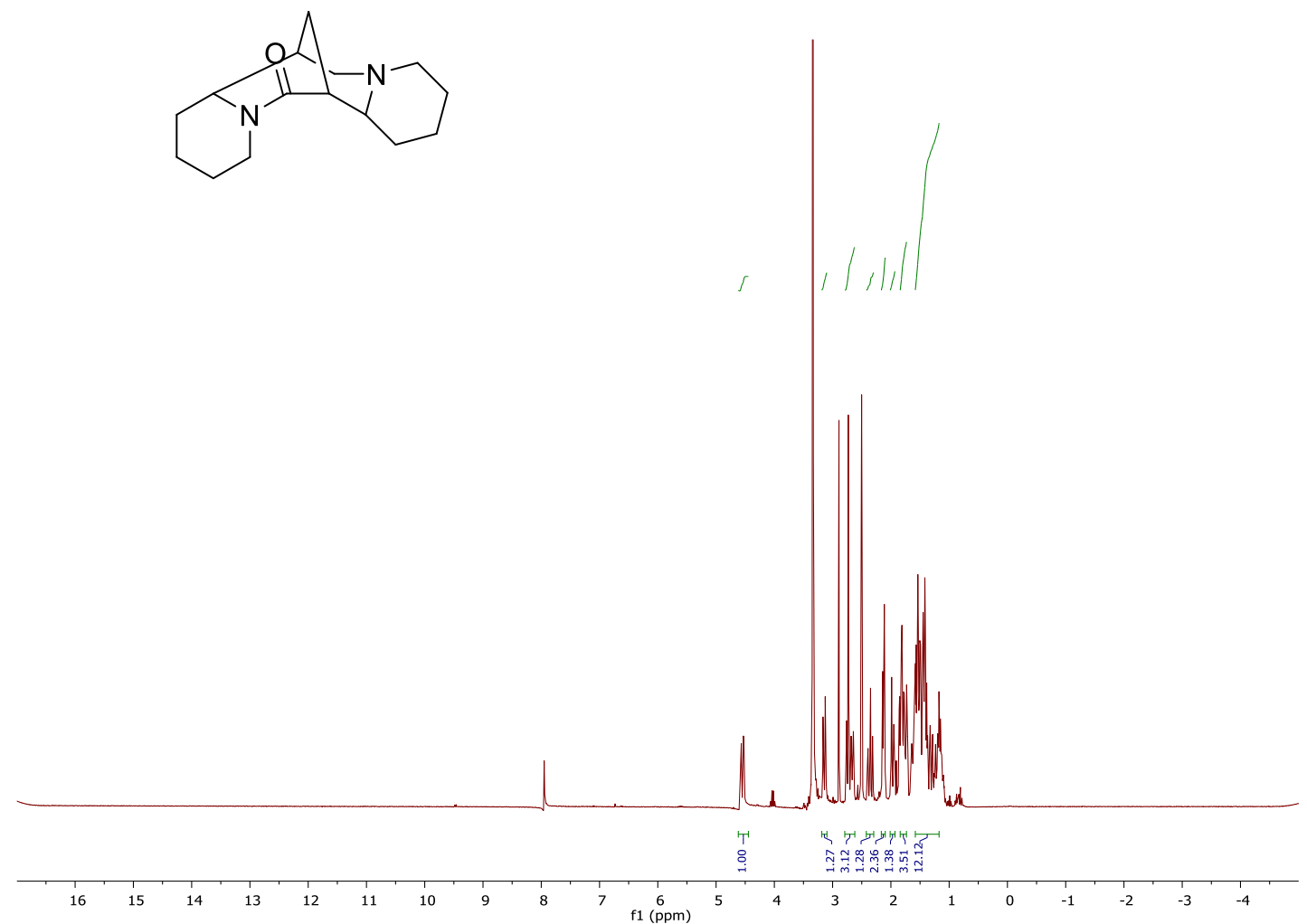

${ }^{1} \mathrm{H}$ NMR spectrum in DMSO- $d_{6}$.
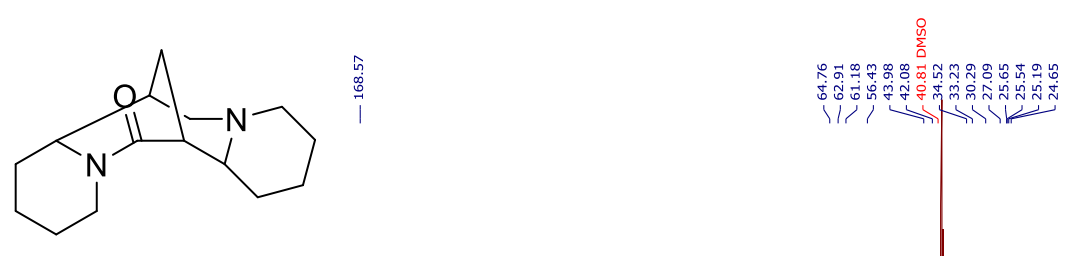

$\begin{array}{rllllllllllllllllllllllllllllllllllll}270 & 260 & 250 & 240 & 230 & 220 & 210 & 200 & 190 & 180 & 170 & 160 & 150 & 140 & 130 & 120 & 110 & 100 & 90 & 80 & 70 & 60 & 50 & 40 & 30 & 20 & 10 & 0 & -10 & -20 & -30\end{array}$

${ }^{13} \mathrm{C}$ NMR spectrum in DMSO- $d_{6}$. 

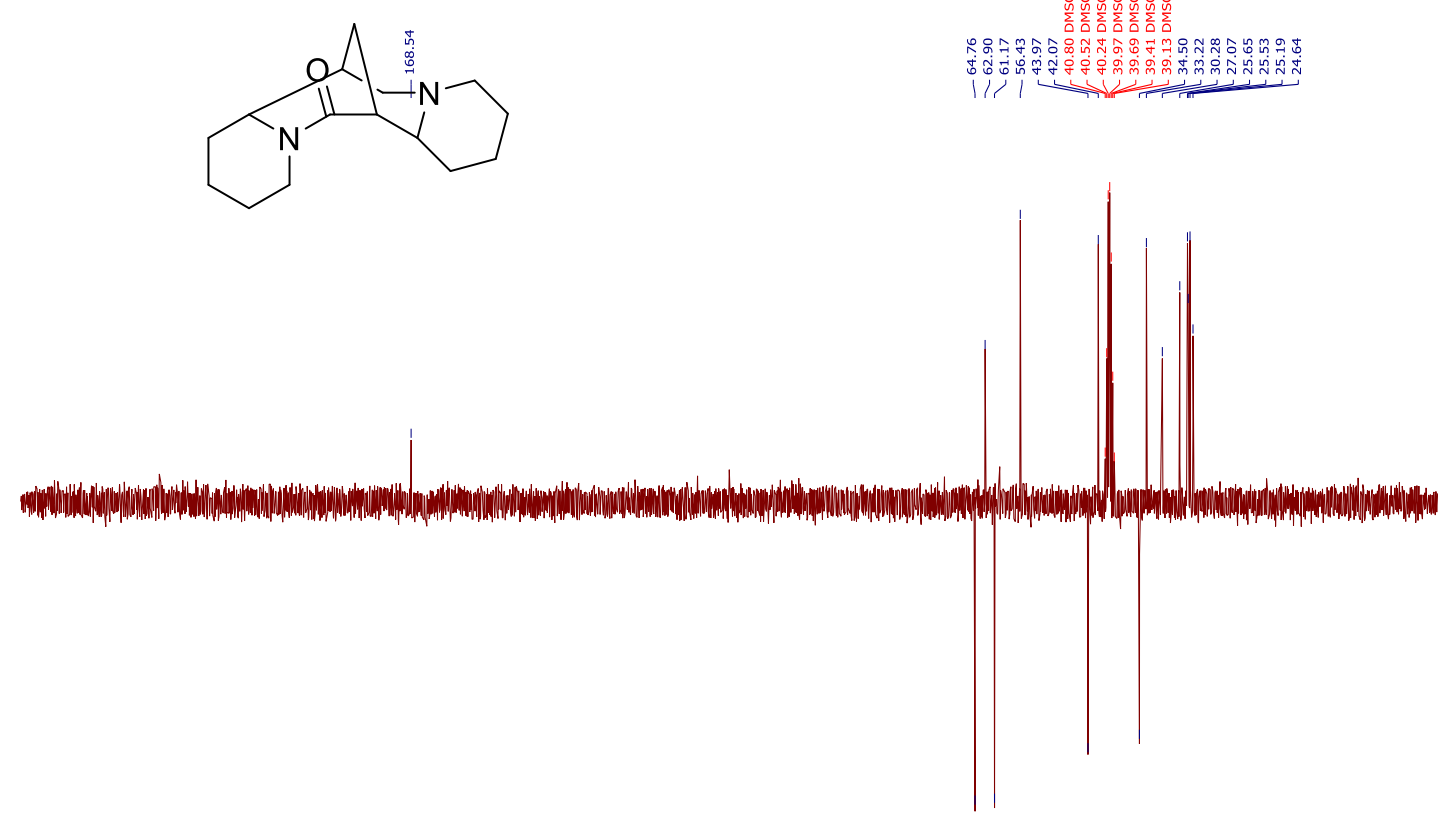

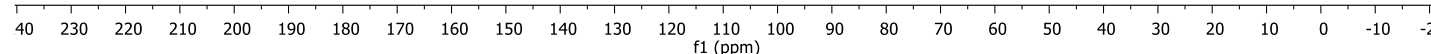

DEPT spectrum in DMSO- $d_{6}$.

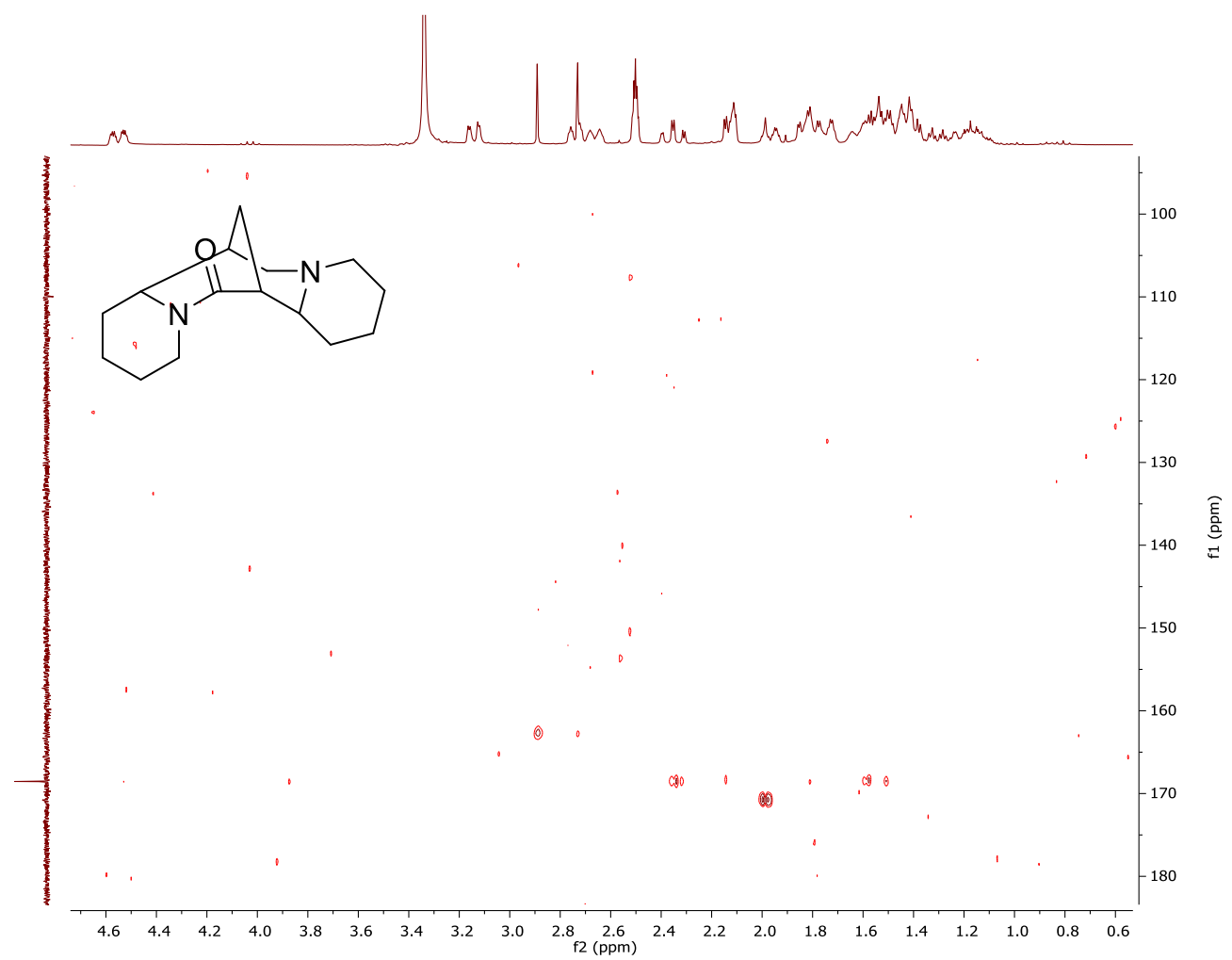

$\mathrm{HMBC}$ spectrum in DMSO- $d_{6}$. 


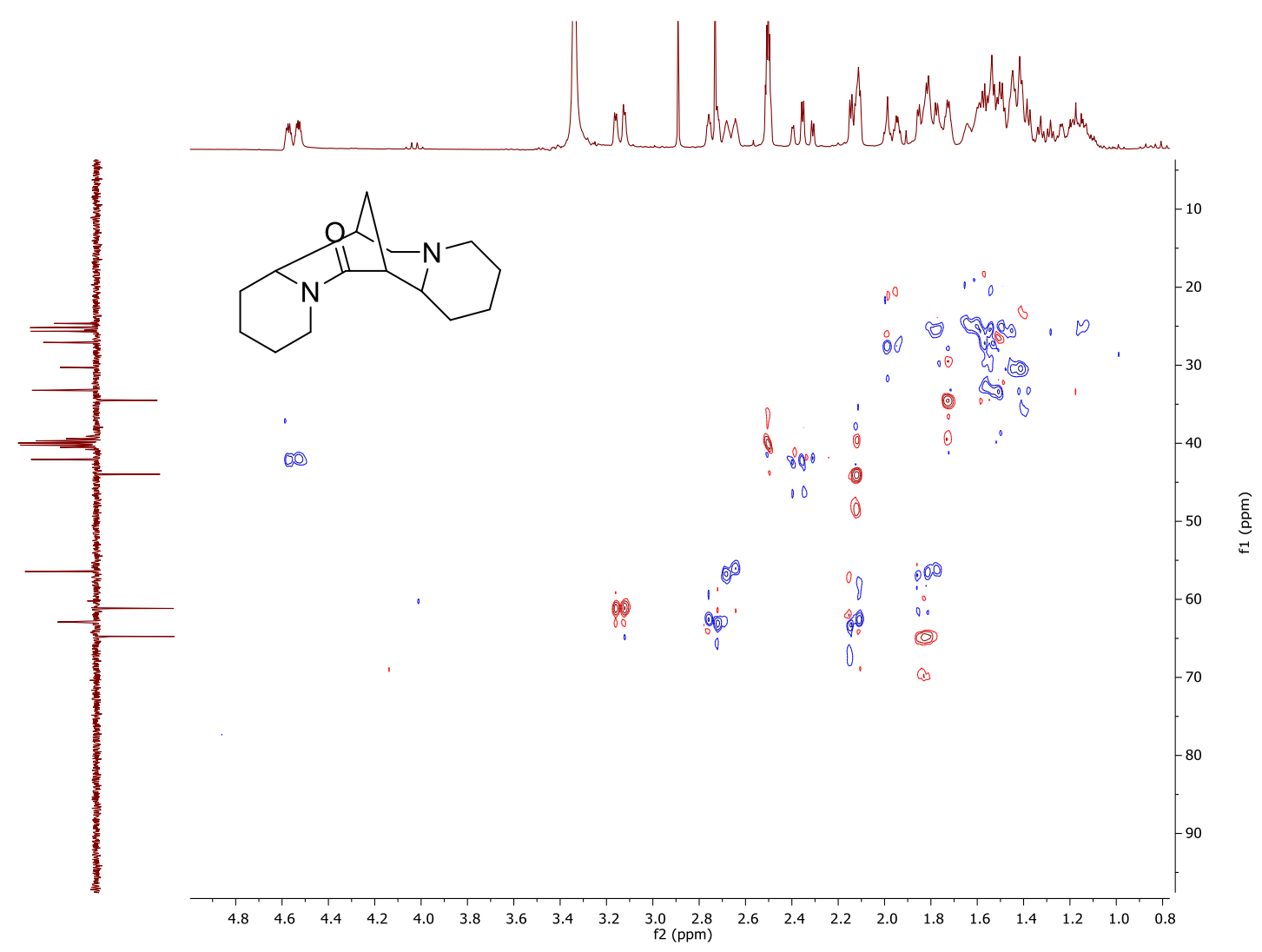

HSQC spectrum in DMSO- $d_{6}$. 
$160 b$

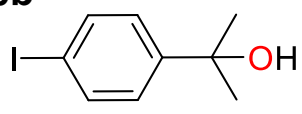

$\stackrel{8}{i} \stackrel{\text { กิ }}{i}$

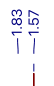

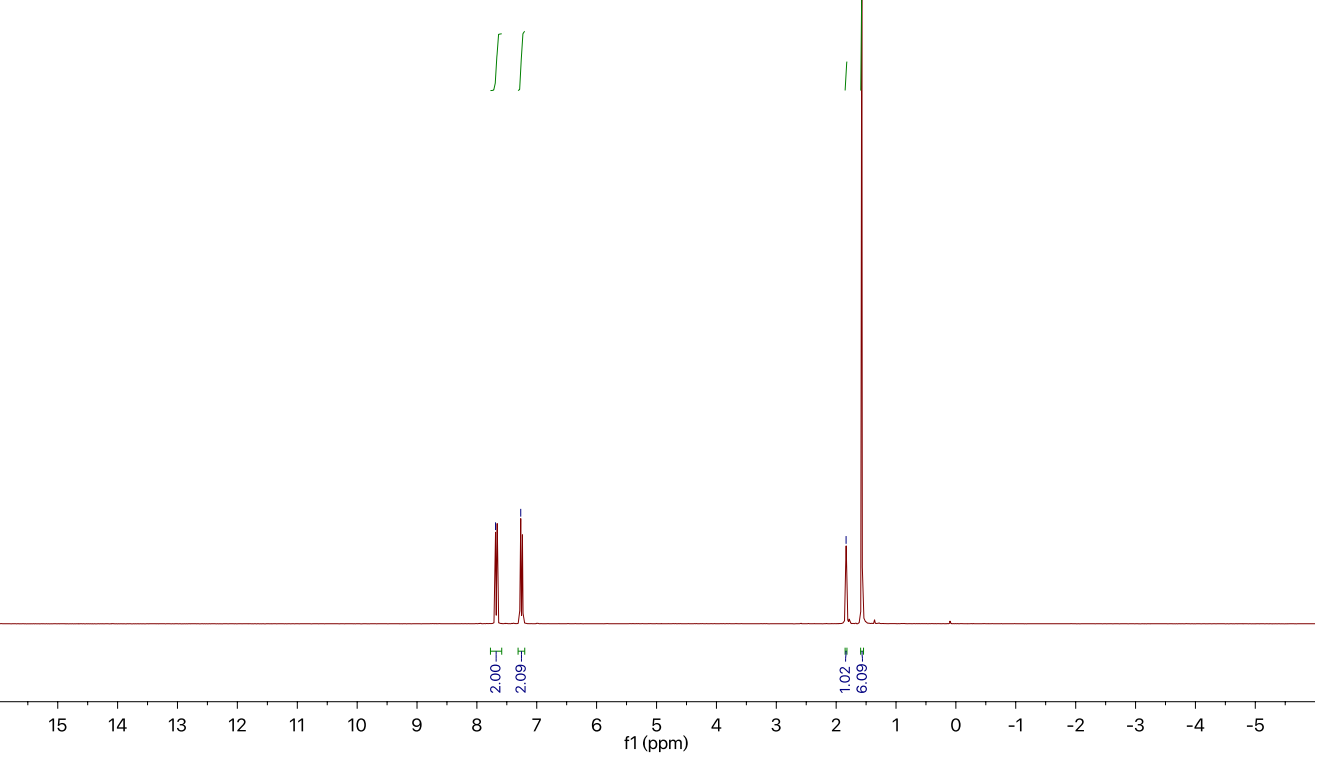

${ }^{1} \mathrm{H}$ NMR spectra in $\mathrm{CDCl}_{3}$

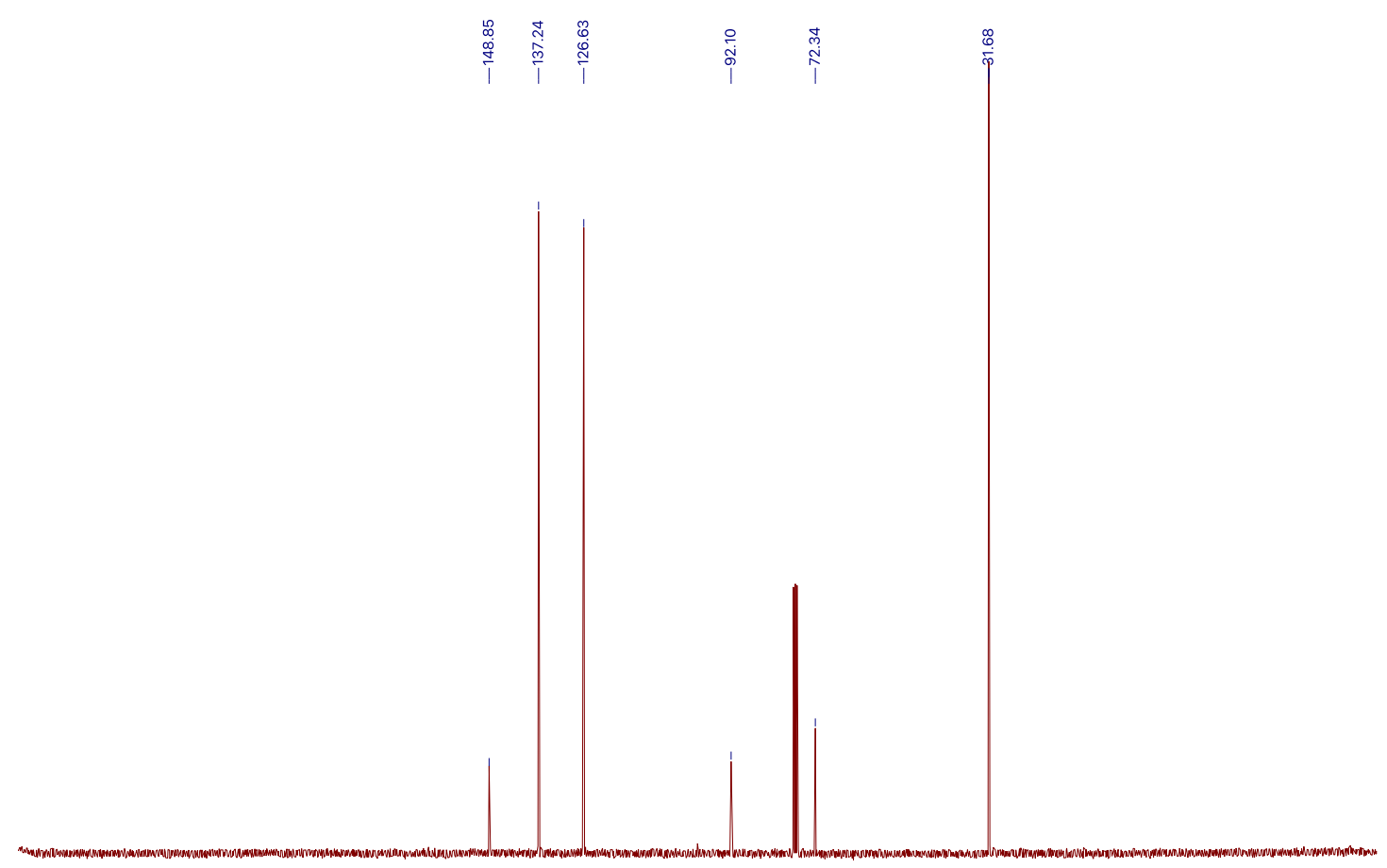

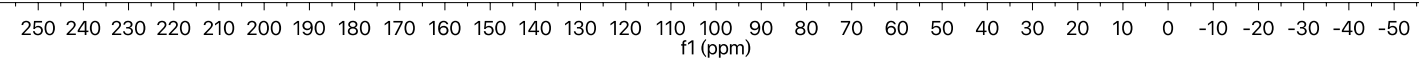

${ }^{13} \mathrm{C}$ NMR spectra in $\mathrm{CDCl}_{3}$. 
161b<smiles>CC(C)(O)c1ccc(Br)cc1</smiles>

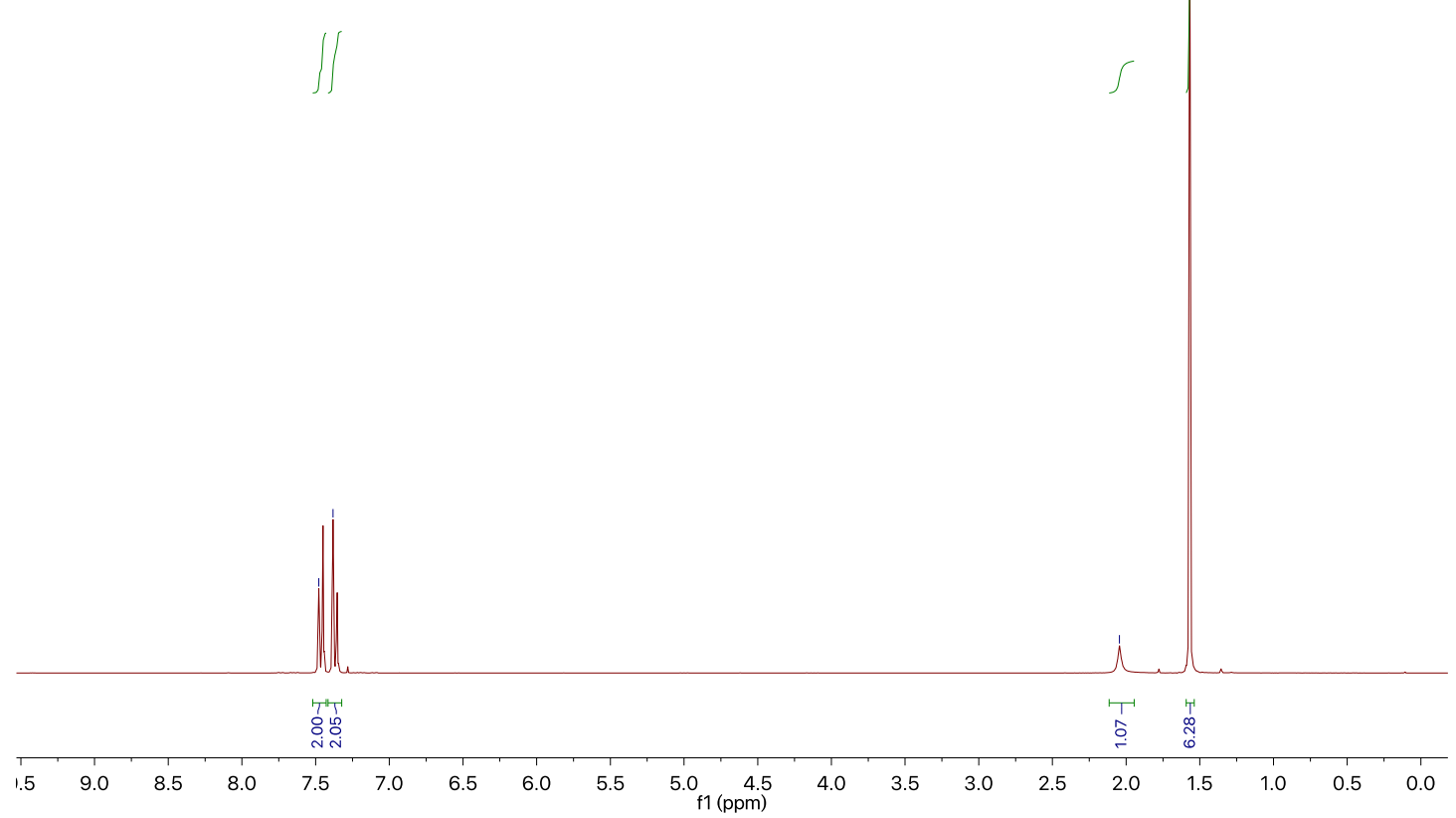

${ }^{1} \mathrm{H}$ NMR spectra in $\mathrm{CDCl}_{3}$
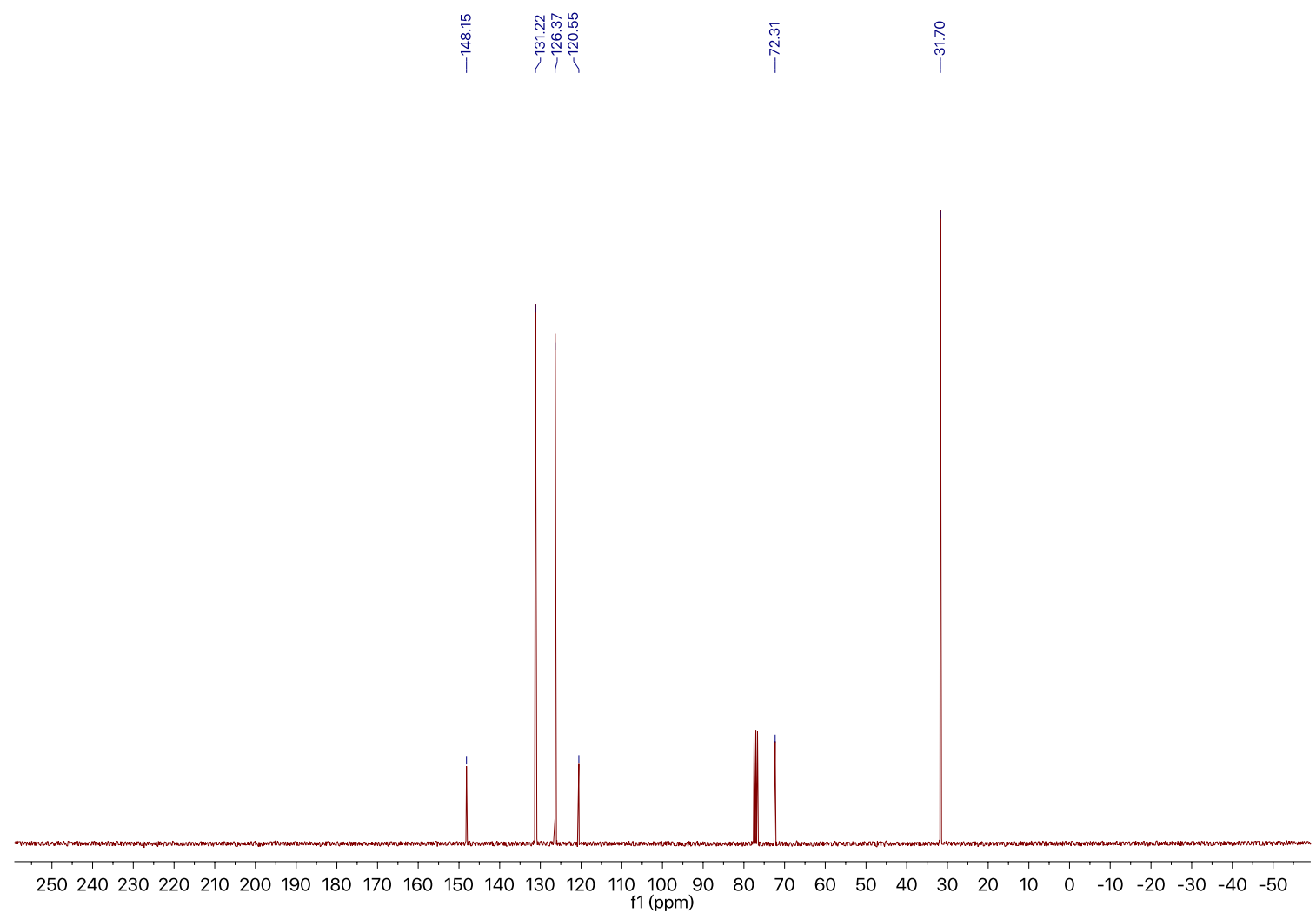

${ }^{13} \mathrm{C}$ NMR spectra in $\mathrm{CDCl}_{3}$. 
$162 b$

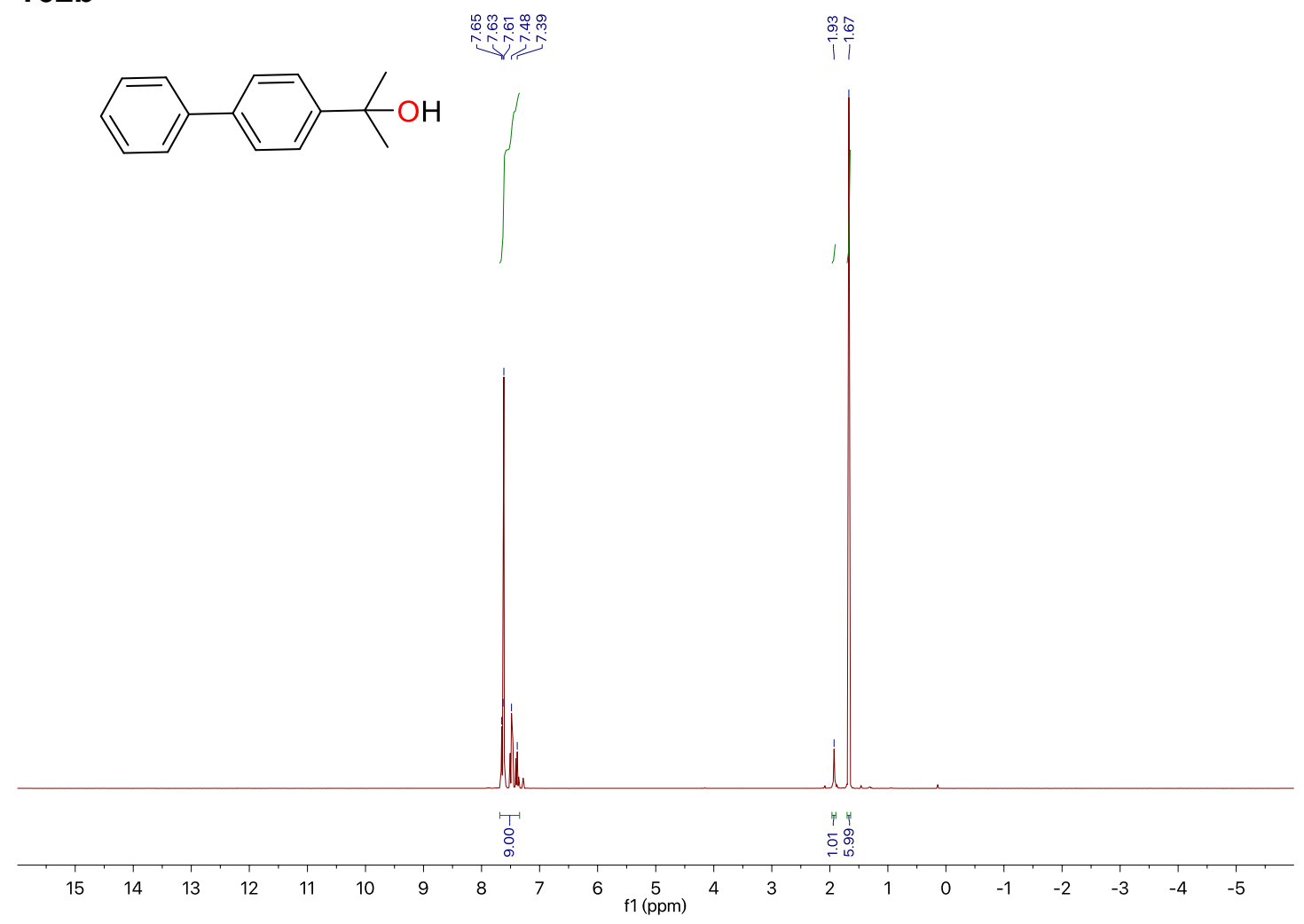

${ }^{1} \mathrm{H}$ NMR spectra in $\mathrm{CDCl}_{3}$

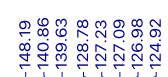
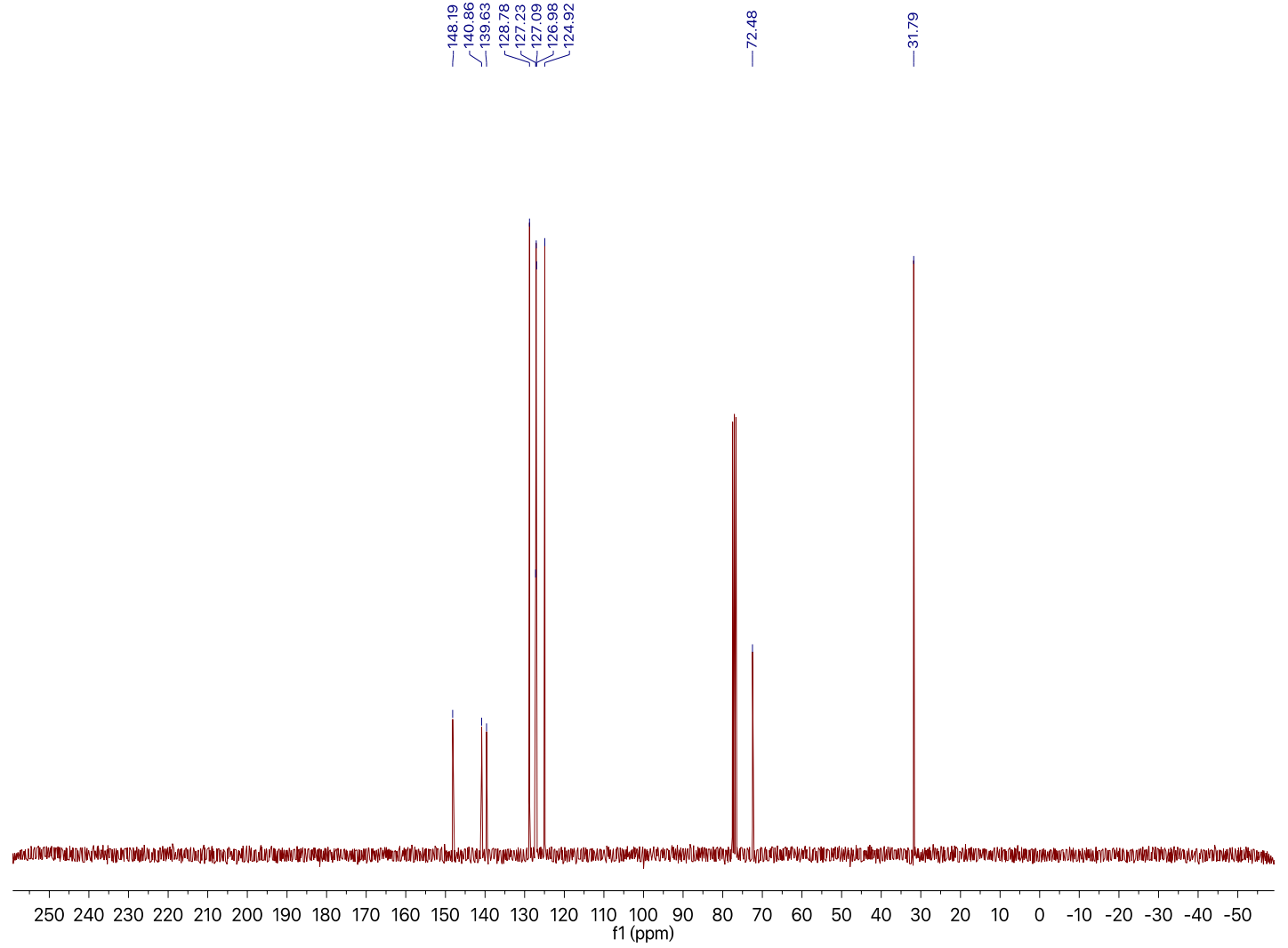

${ }^{13} \mathrm{C}$ NMR spectra in $\mathrm{CDCl}_{3}$. 
$163 b$

$\mathrm{HO}>\mathrm{H}_{\mathrm{O}}$

$\stackrel{\mathscr{B}}{i} \stackrel{\substack{0 \\ i}}{i}$
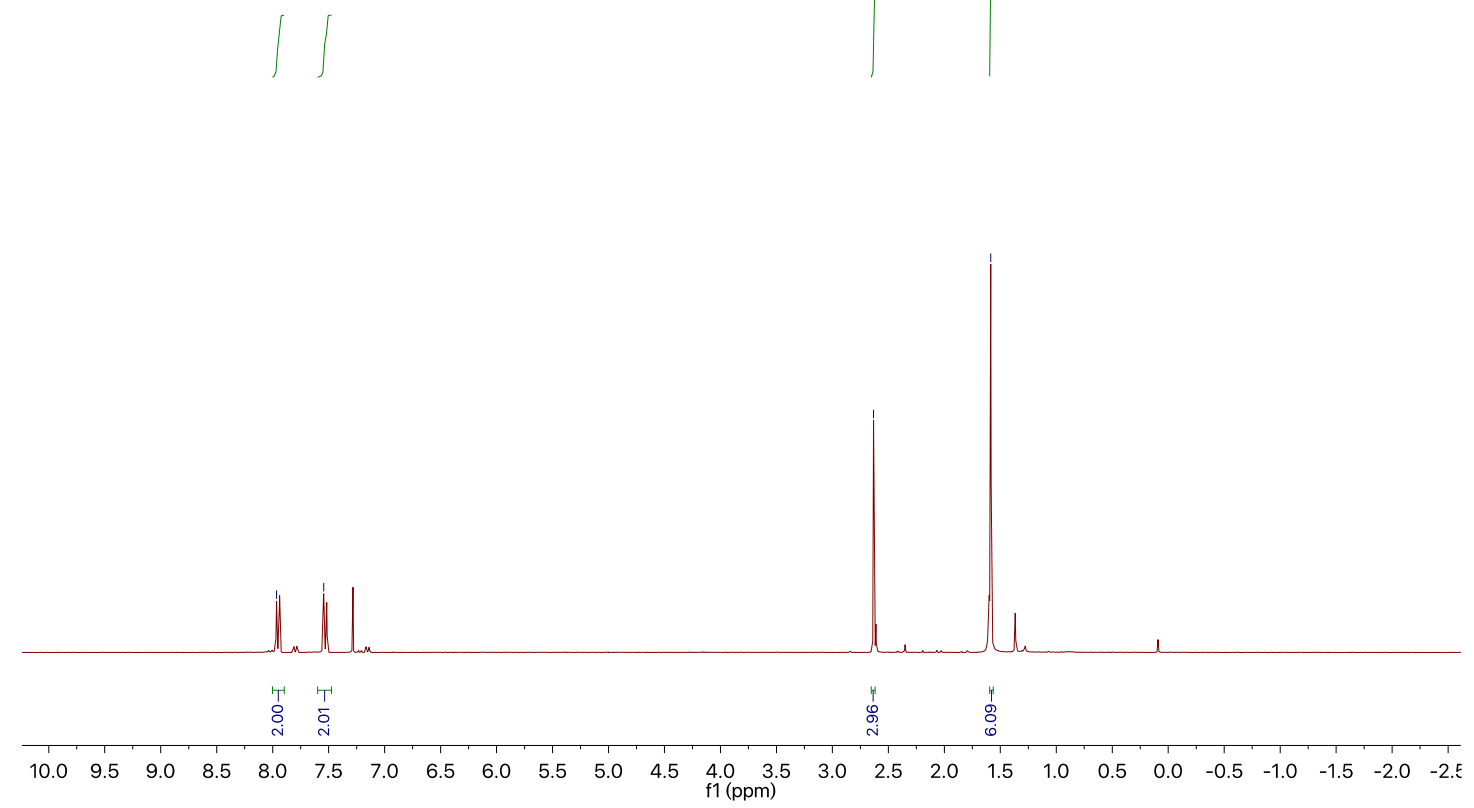

${ }^{1} \mathrm{H}$ NMR spectra in $\mathrm{CDCl}_{3}$

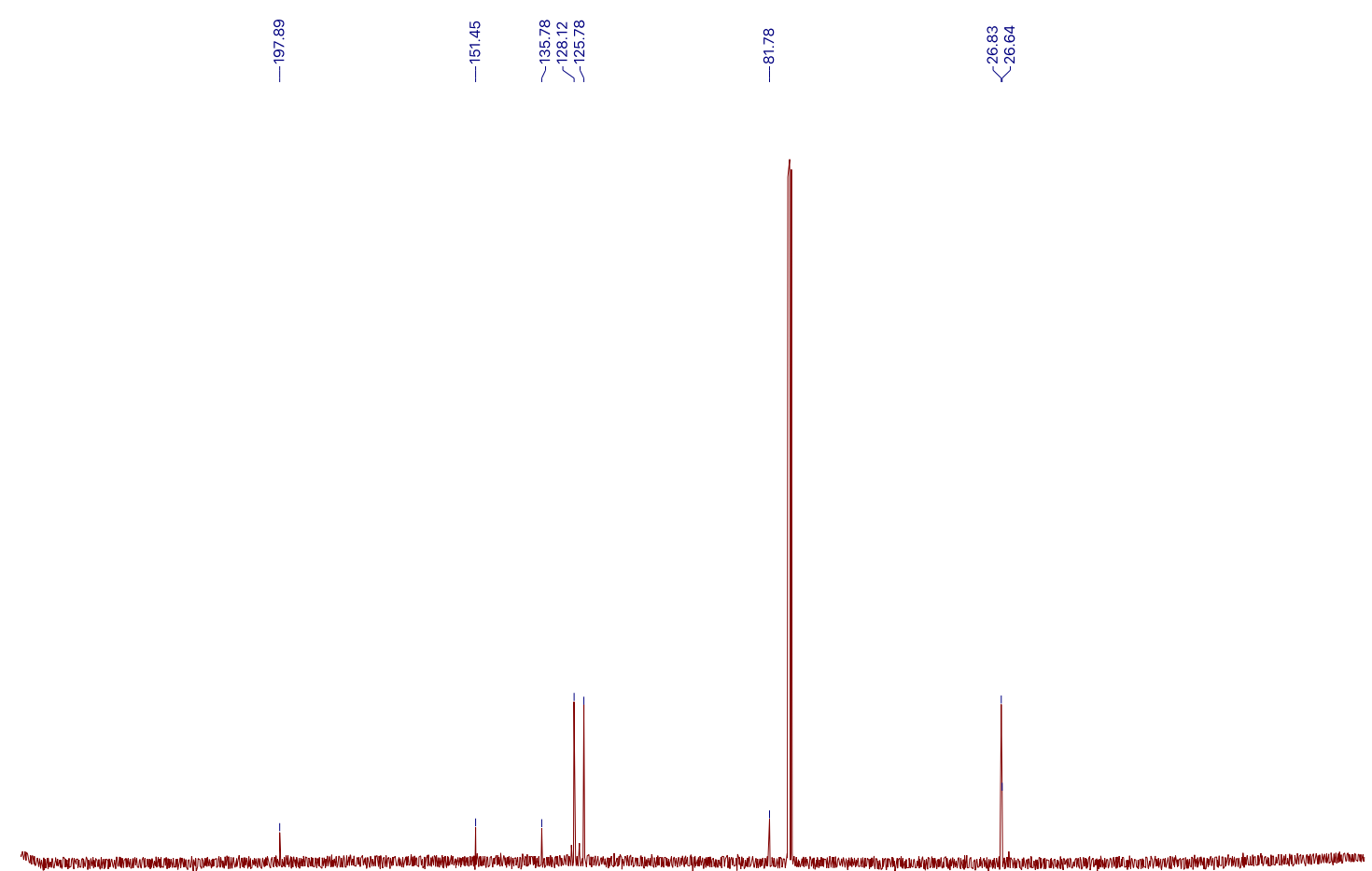

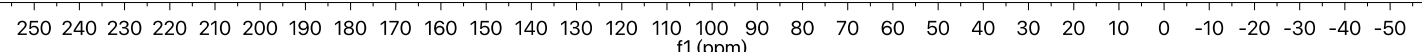

${ }^{13} \mathrm{C}$ NMR spectra in $\mathrm{CDCl}_{3}$. 


\section{$164 b$}

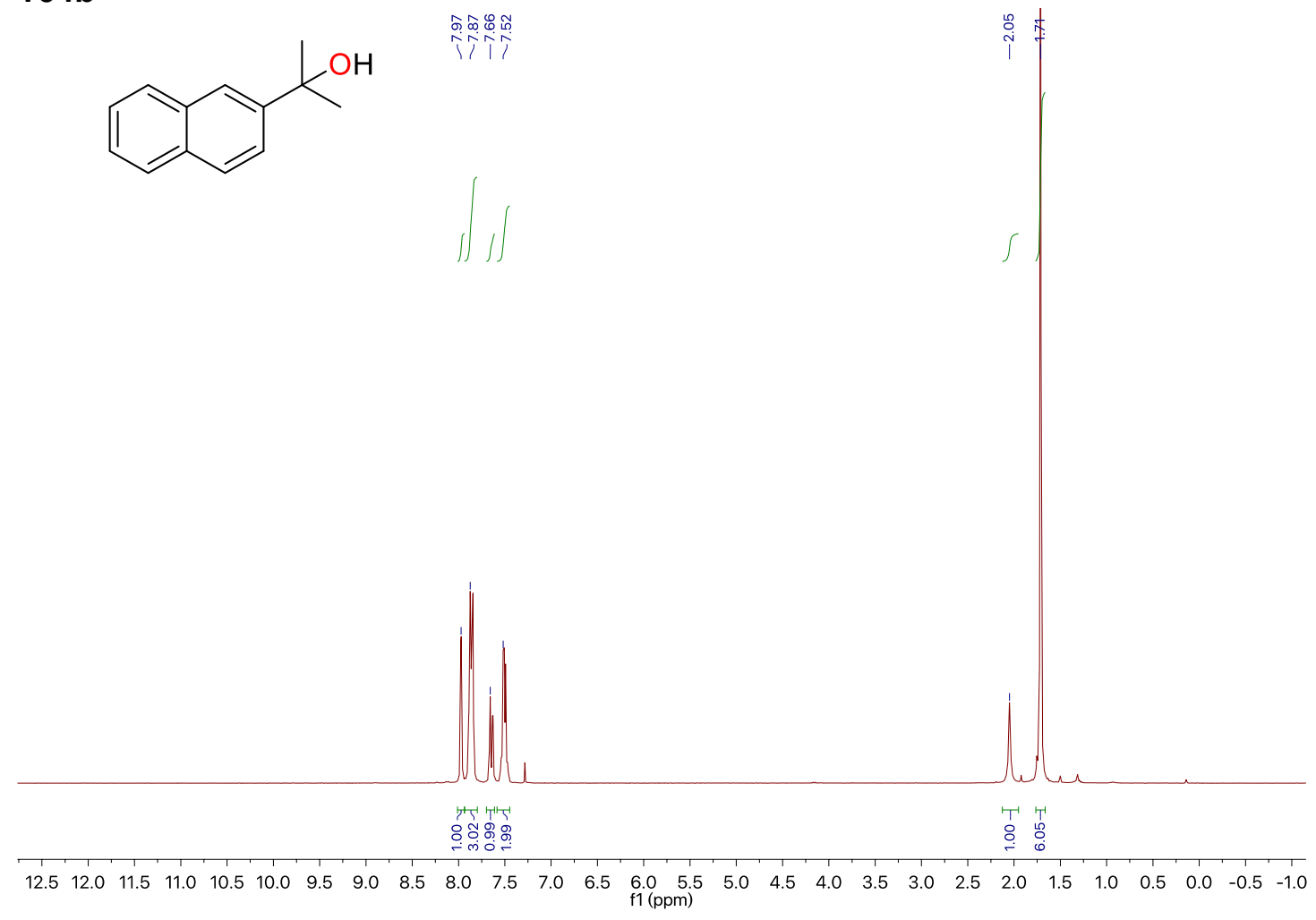

${ }^{1} \mathrm{H}$ NMR spectra in $\mathrm{CDCl}_{3}$

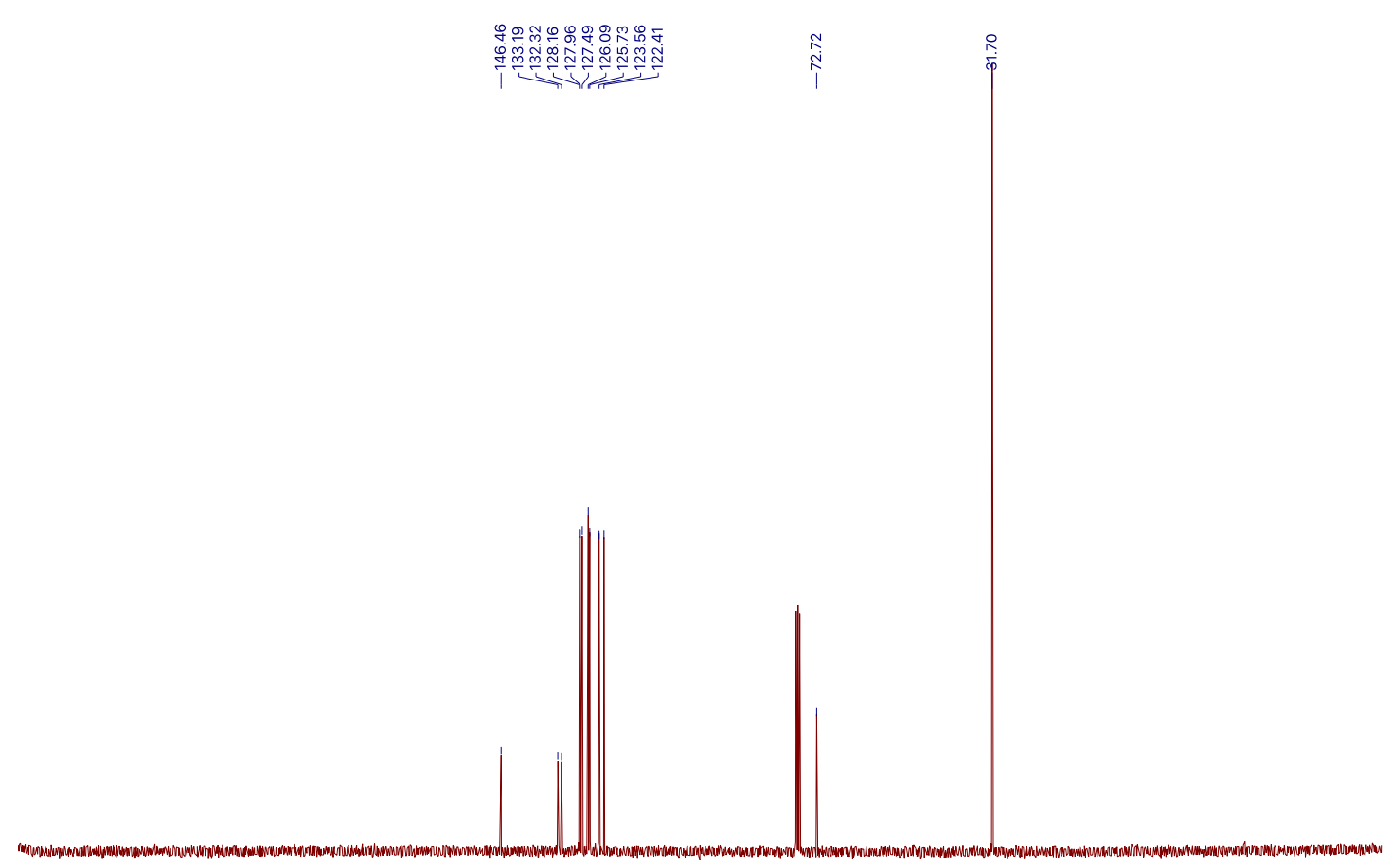

$250240230220210200190180170160150140130120 \begin{gathered}110 \quad 100 \\ f 1(p p m)\end{gathered}$ ${ }^{13} \mathrm{C}$ NMR spectra in $\mathrm{CDCl}_{3}$. 
$165 b$

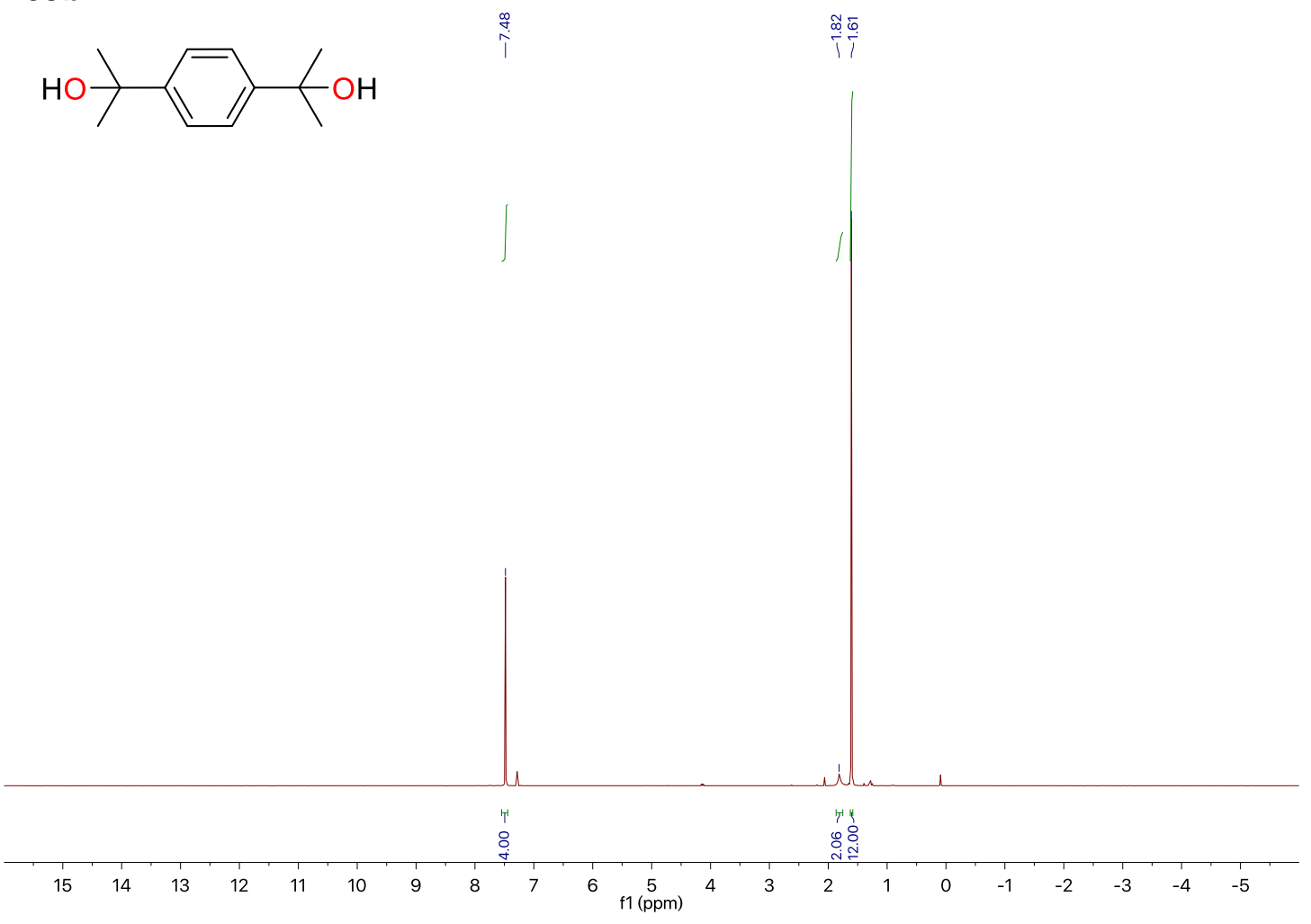

${ }^{1} \mathrm{H}$ NMR spectra in $\mathrm{CDCl}_{3}$

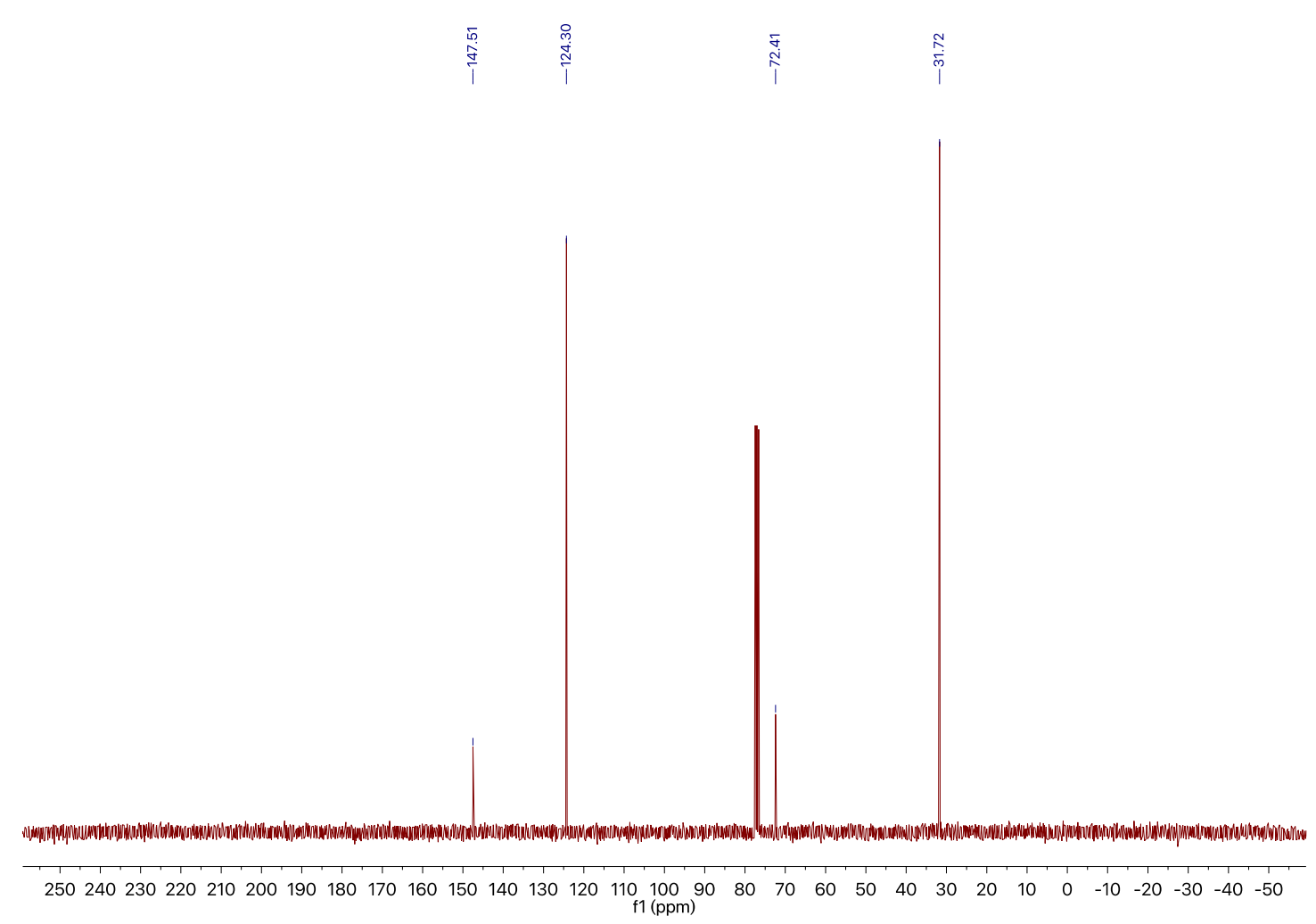

${ }^{13} \mathrm{C}$ NMR spectra in $\mathrm{CDCl}_{3}$. 
$166 b$

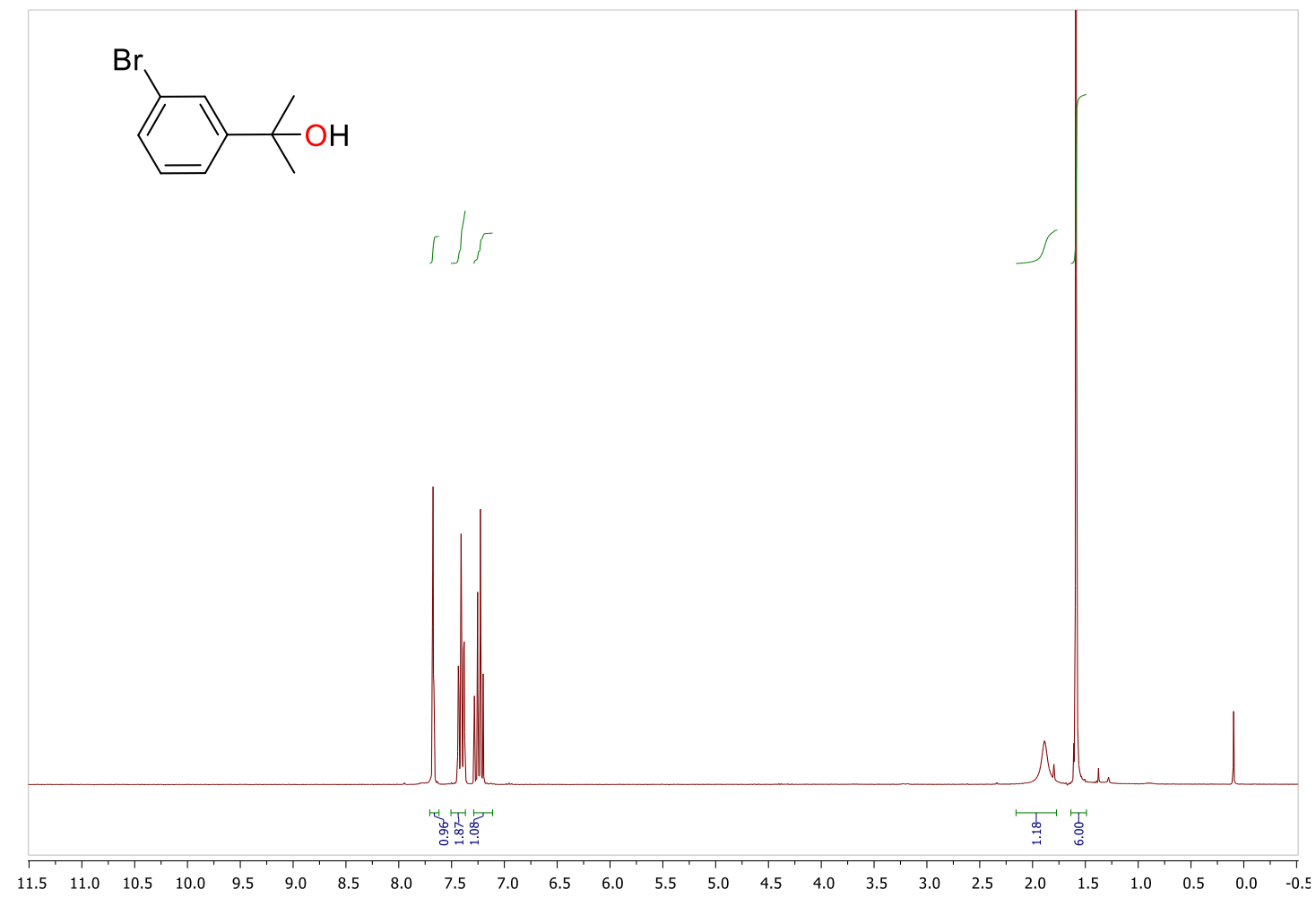

${ }^{1} \mathrm{H}$ NMR spectra in $\mathrm{CDCl}_{3}$

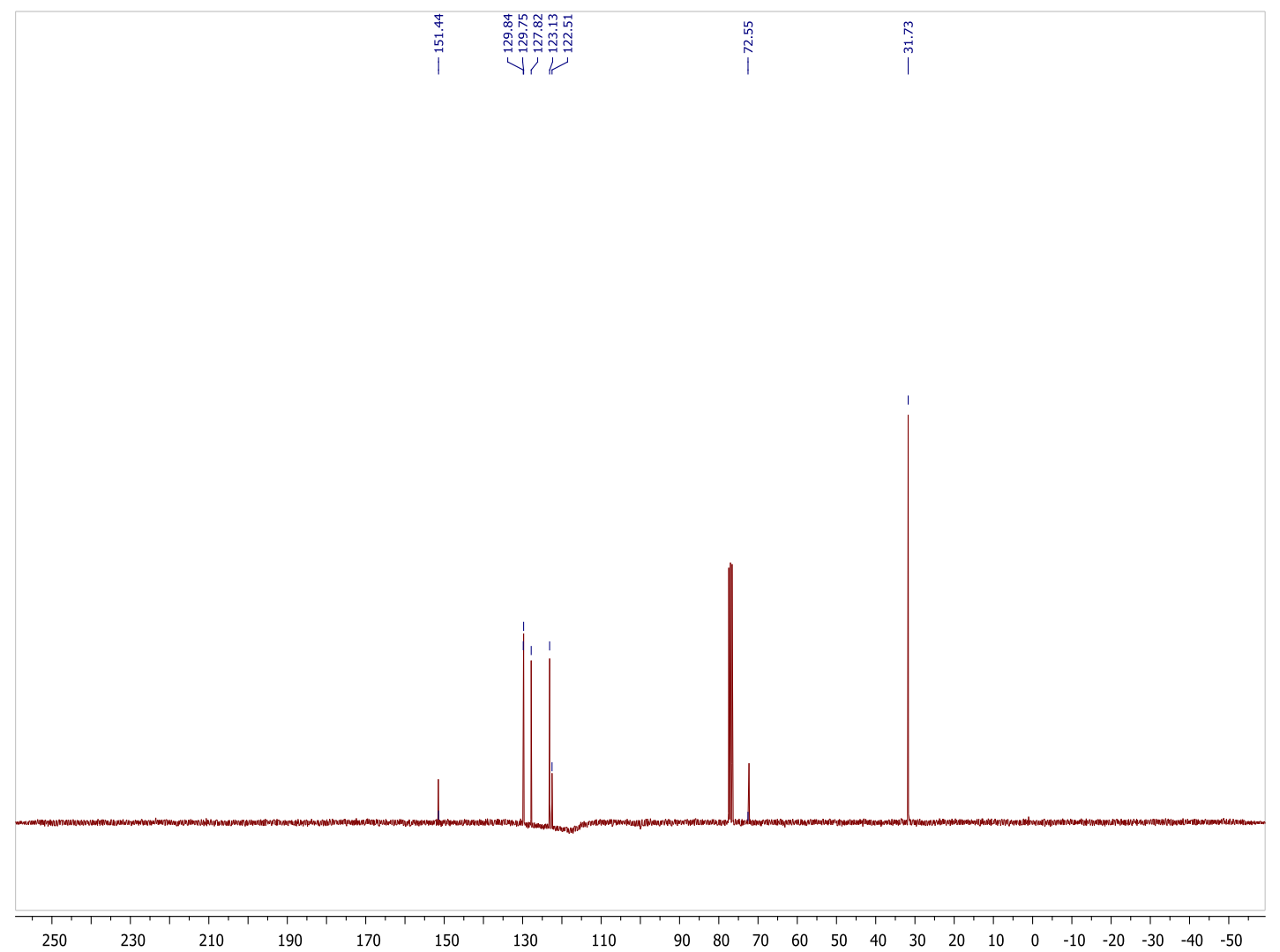

${ }^{13} \mathrm{C}$ NMR spectra in $\mathrm{CDCl}_{3}$. 
Experimental Section

$167 b$
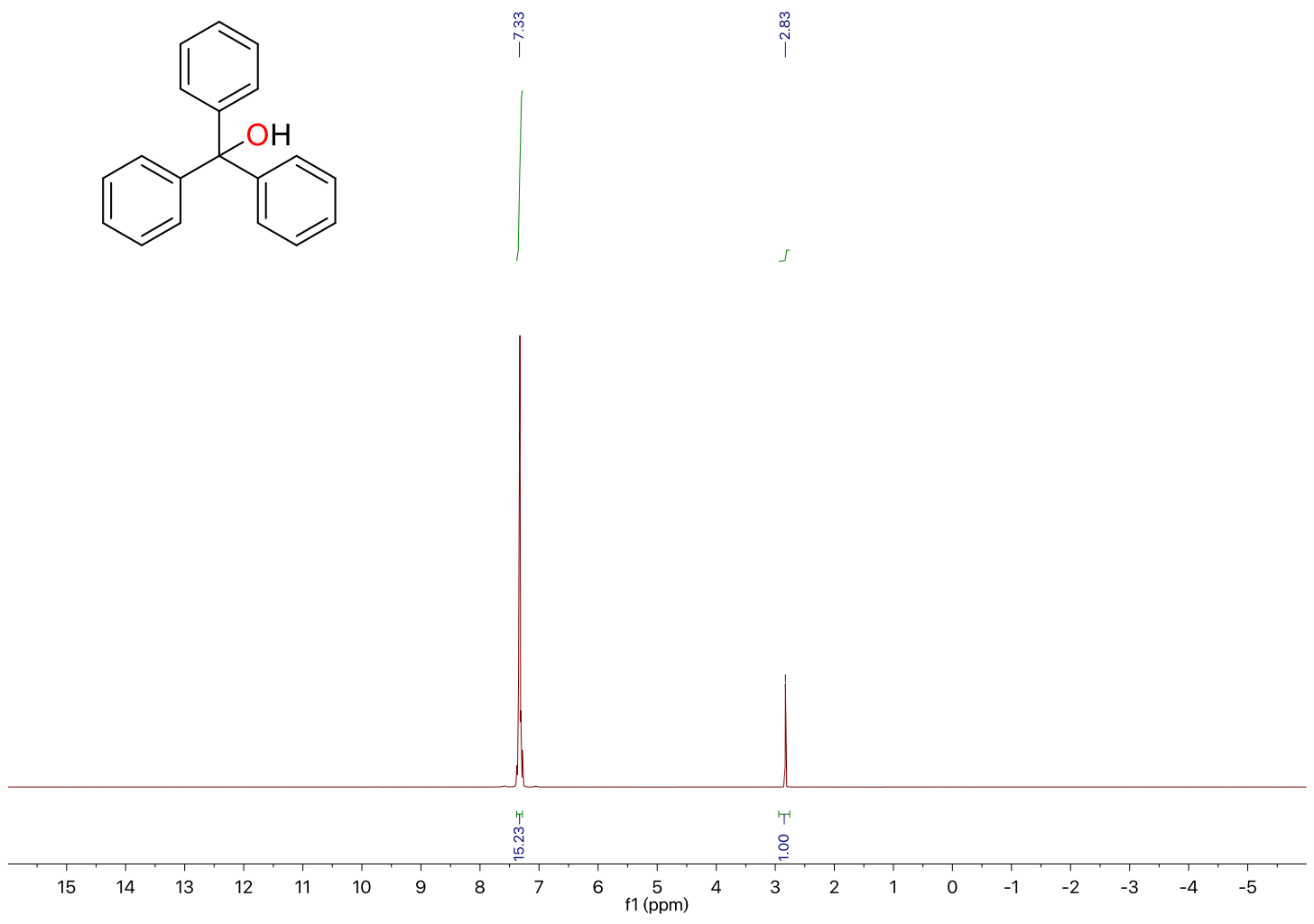

${ }^{1} \mathrm{H}$ NMR spectra in $\mathrm{CDCl}_{3}$

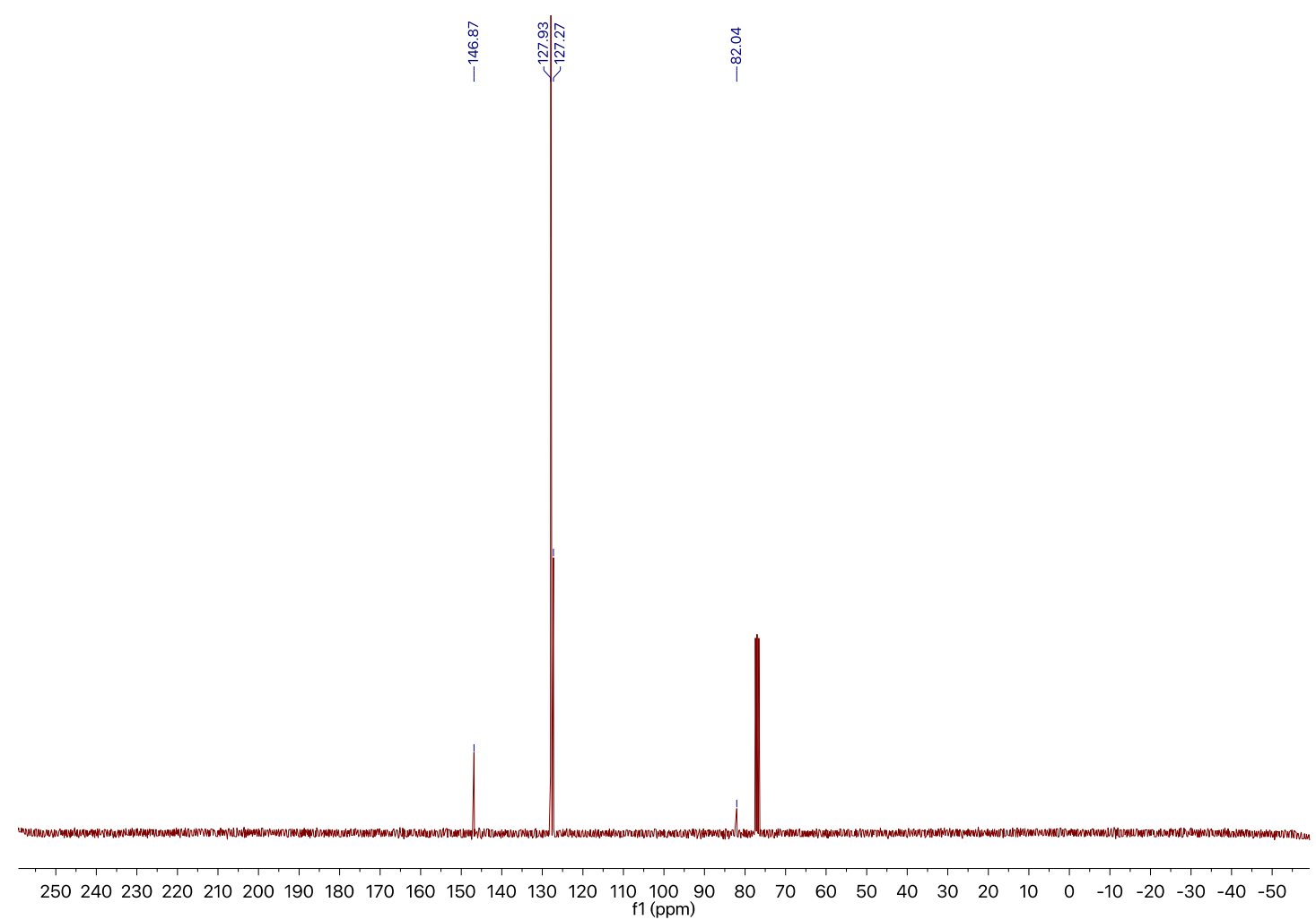

${ }^{13} \mathrm{C}$ NMR spectra in $\mathrm{CDCl}_{3}$.

209 
$168 b$

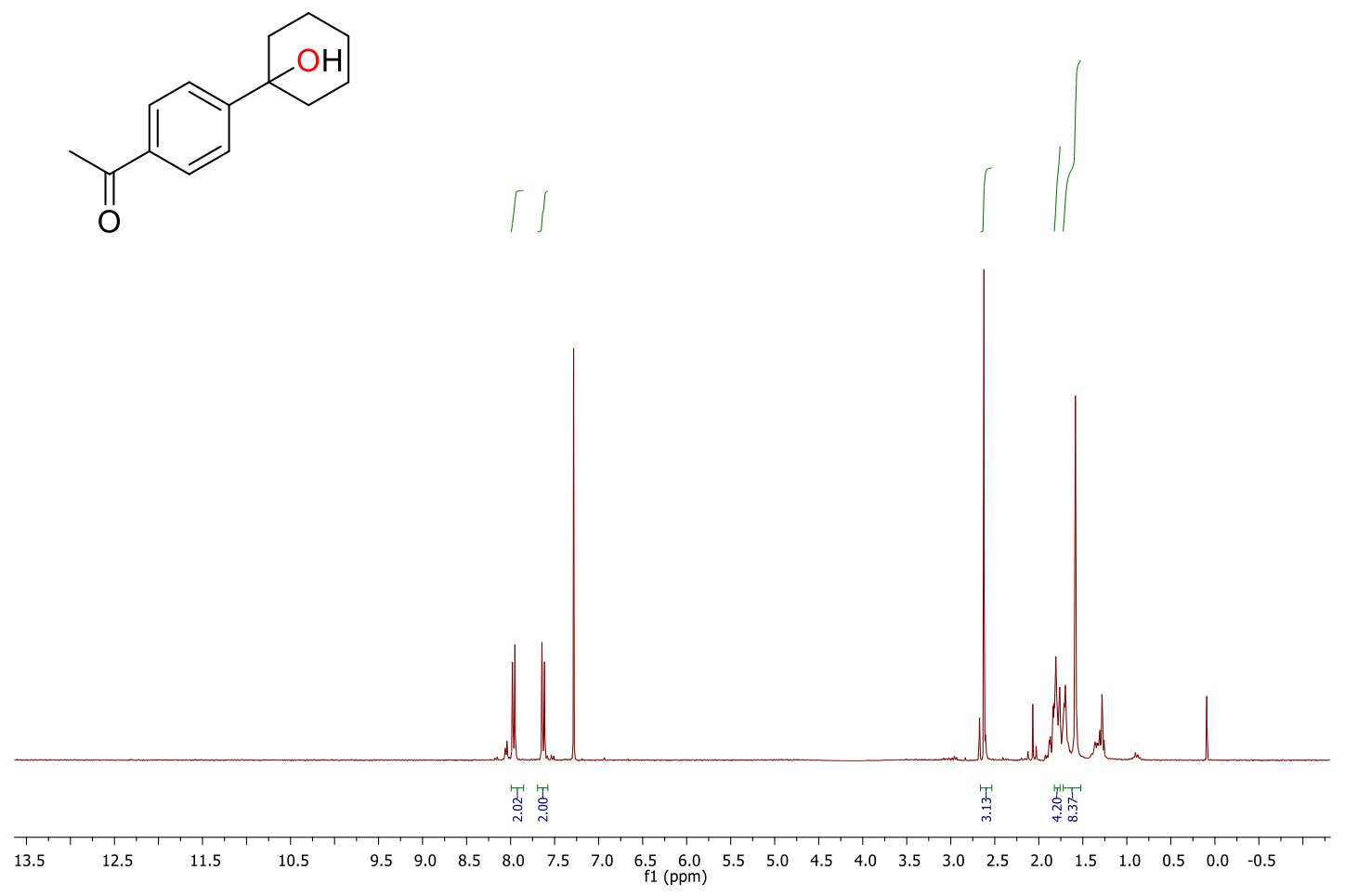

${ }^{1} \mathrm{H}$ NMR spectra in $\mathrm{CDCl}_{3}$

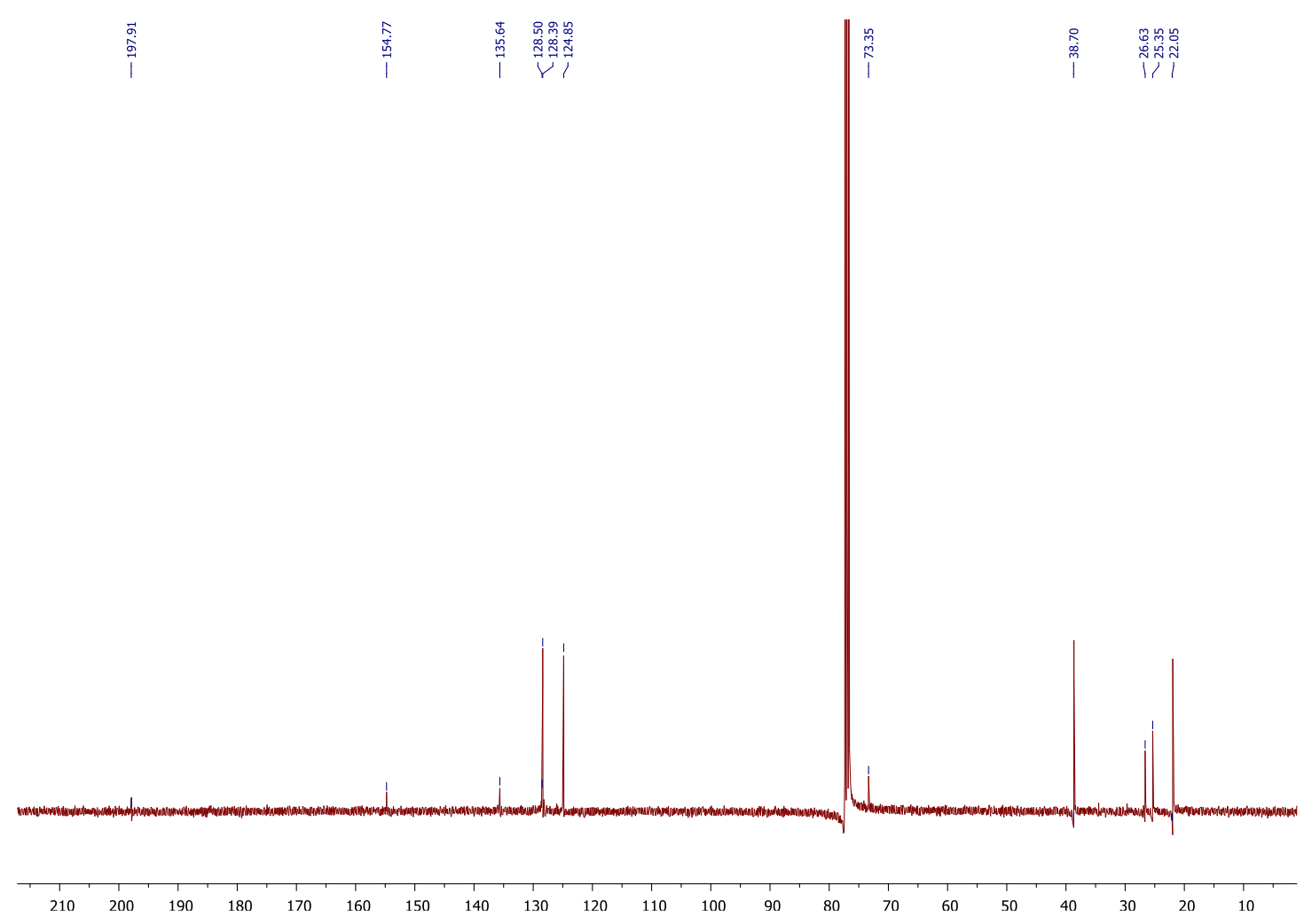

${ }^{13} \mathrm{C}$ NMR spectra in $\mathrm{CDCl}_{3}$. 


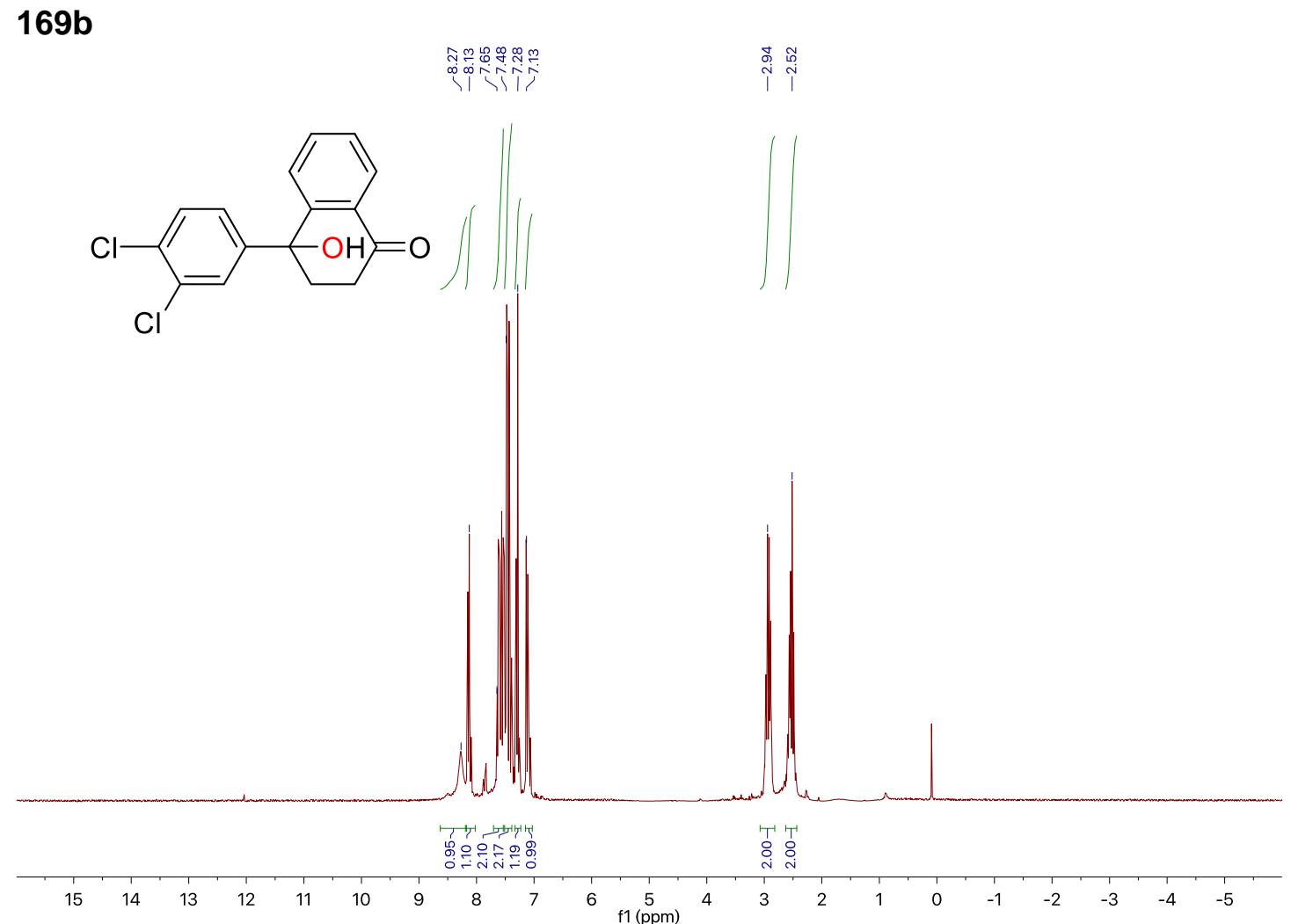

${ }^{1} \mathrm{H}$ NMR spectra in $\mathrm{CDCl}_{3}$

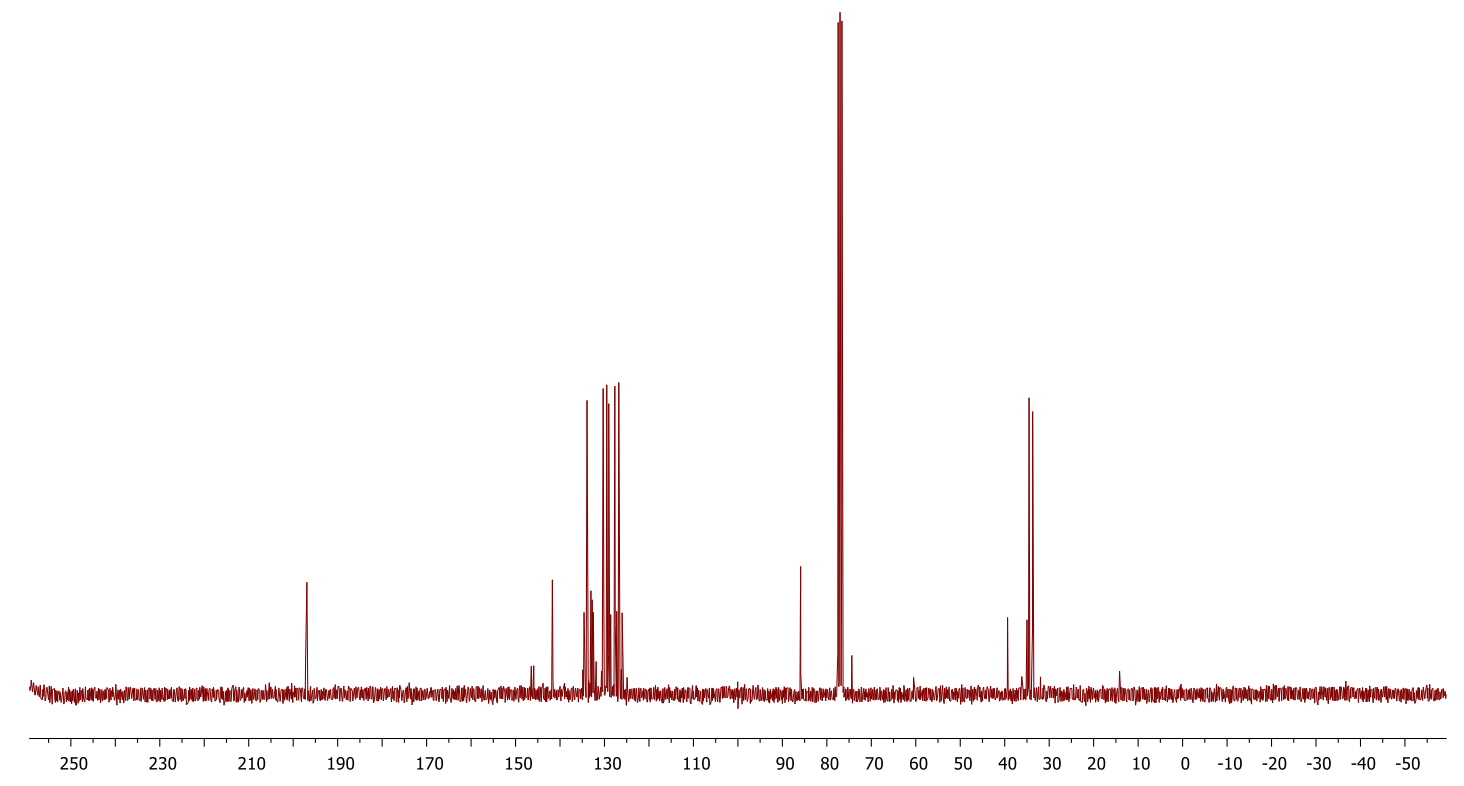

${ }^{13} \mathrm{C}$ NMR spectra in $\mathrm{CDCl}_{3}$. 
170b
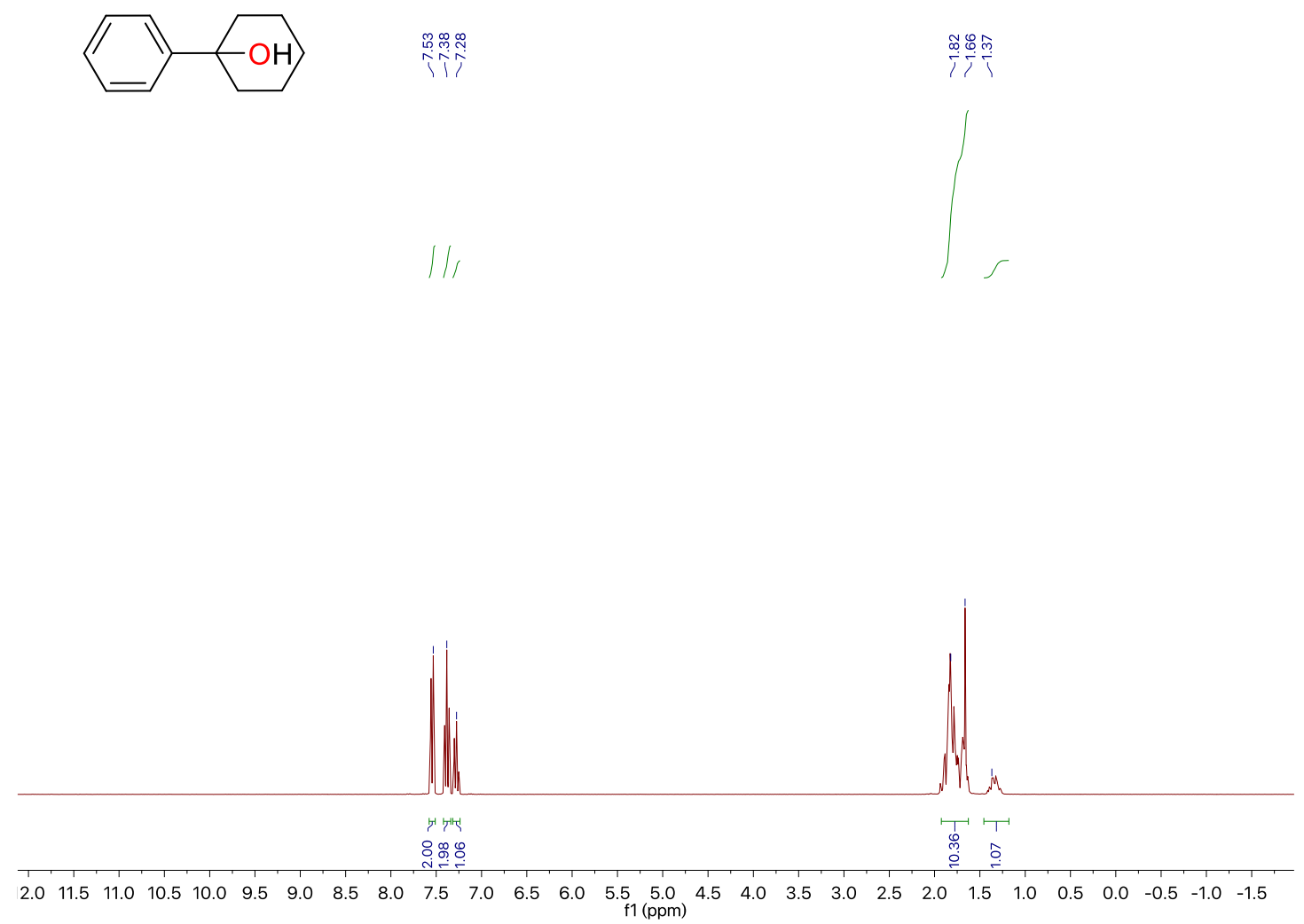

${ }^{1} \mathrm{H}$ NMR spectra in $\mathrm{CDCl}_{3}$

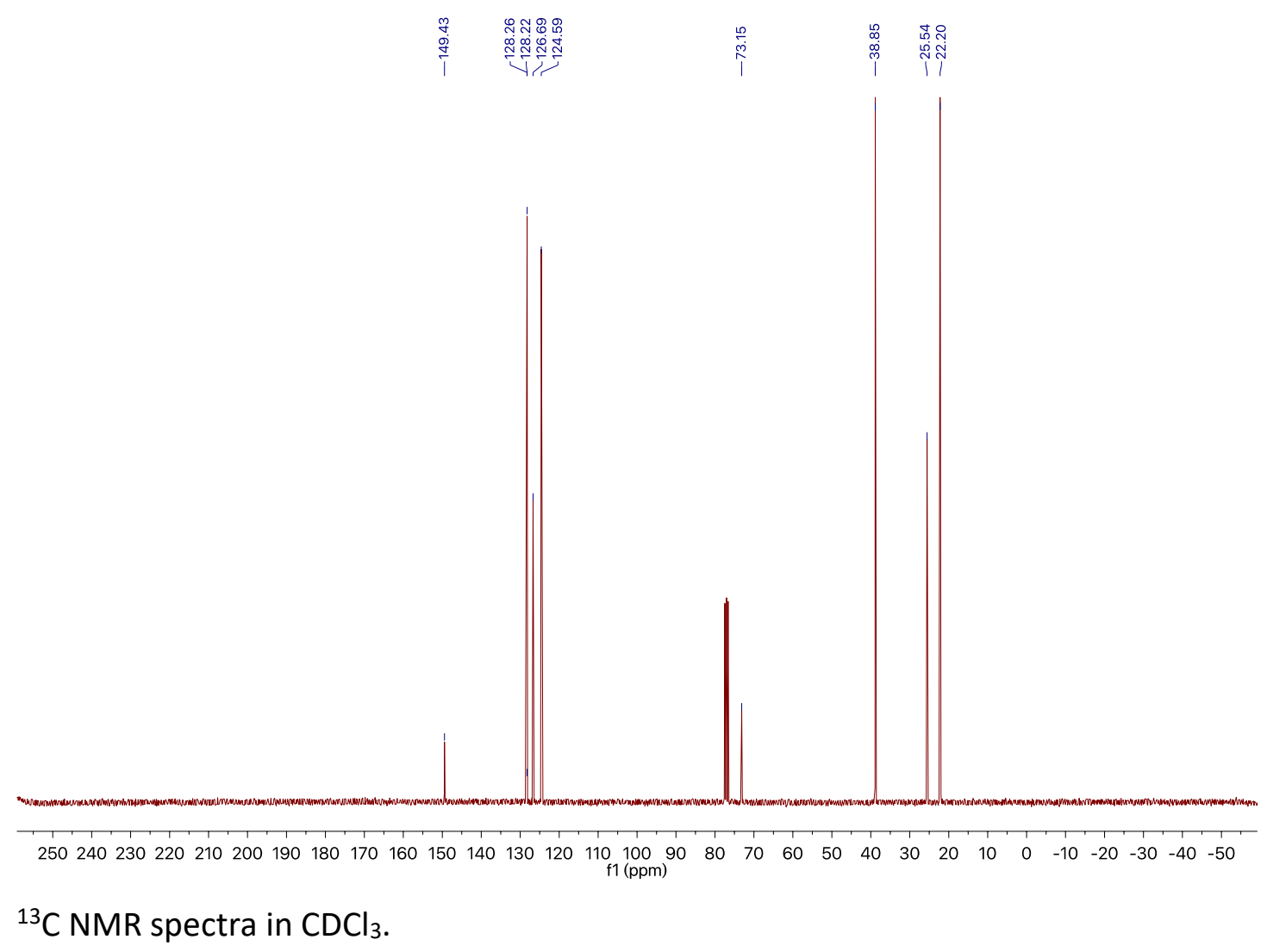


$172 b$<smiles>CC(C)(O)c1ccc(-c2ccc(C#N)cc2)cc1</smiles><smiles>[13CH3][13CH3]</smiles>

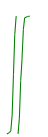

$$
\begin{array}{|l|}
\hline B(9) \\
7.59 \\
\hline A(9) \\
7.69 \\
\hline H-1
\end{array}
$$

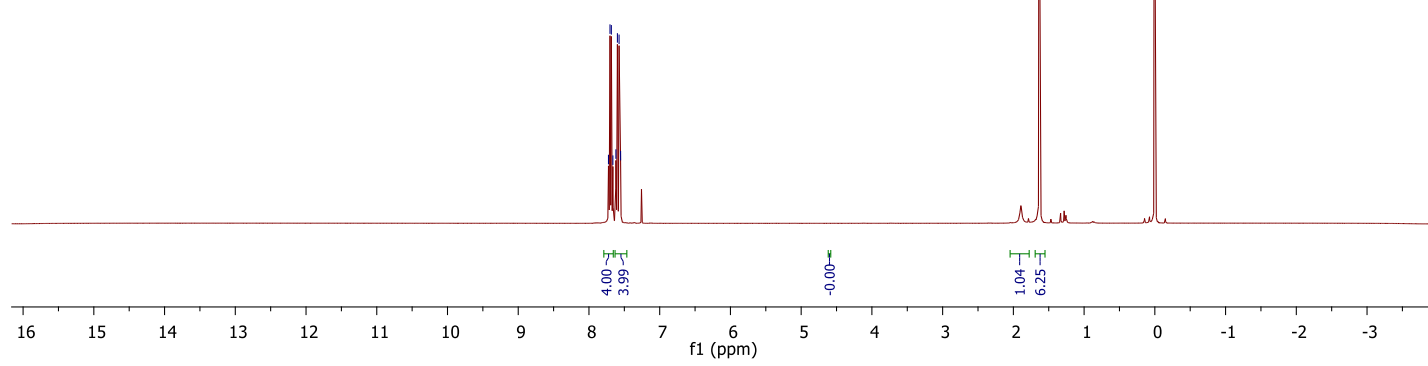

${ }^{1} \mathrm{H}$ NMR spectra in $\mathrm{CDCl}_{3}$

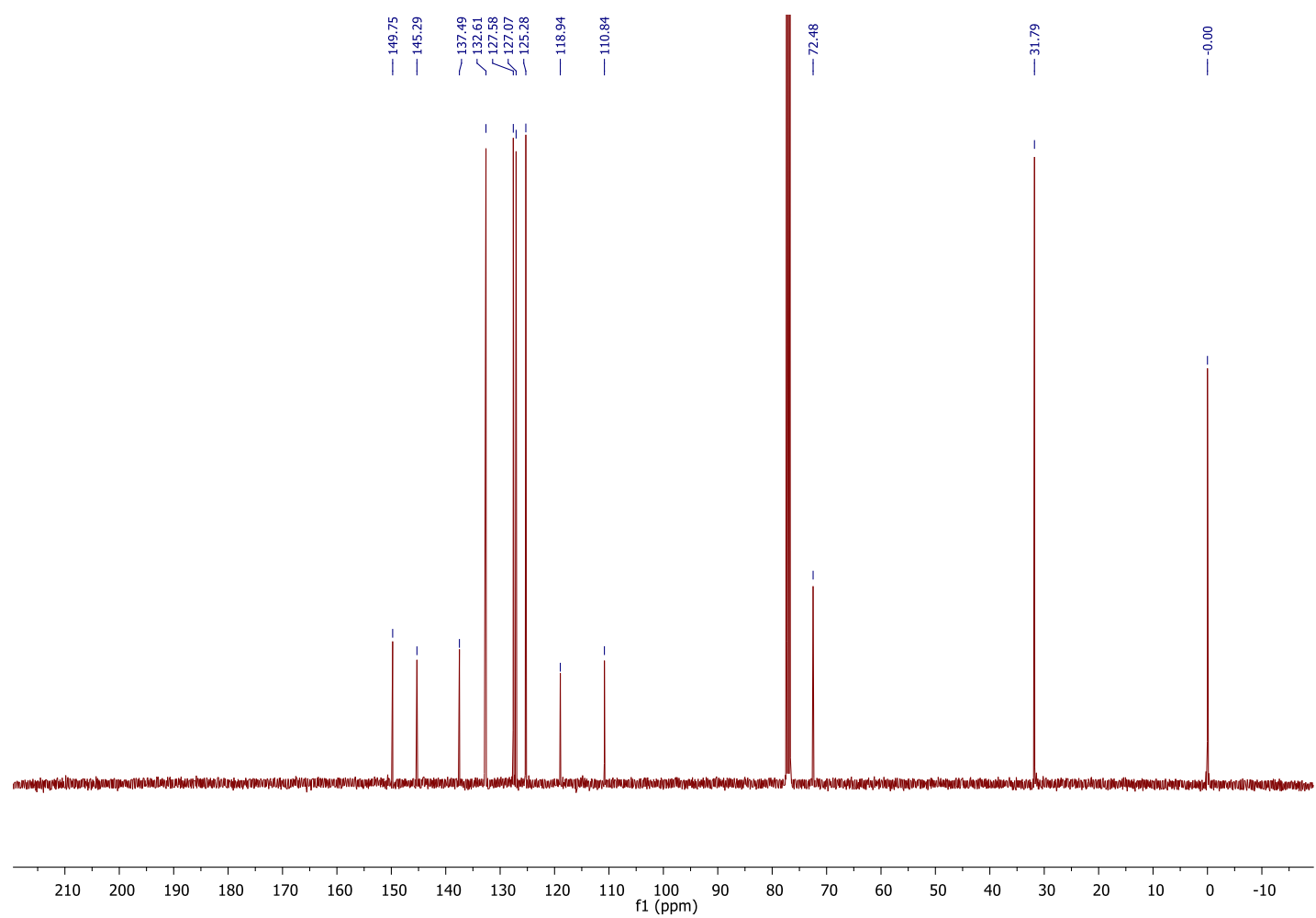

${ }^{13} \mathrm{C}$ NMR spectra in $\mathrm{CDCl}_{3}$. 
$173 b$

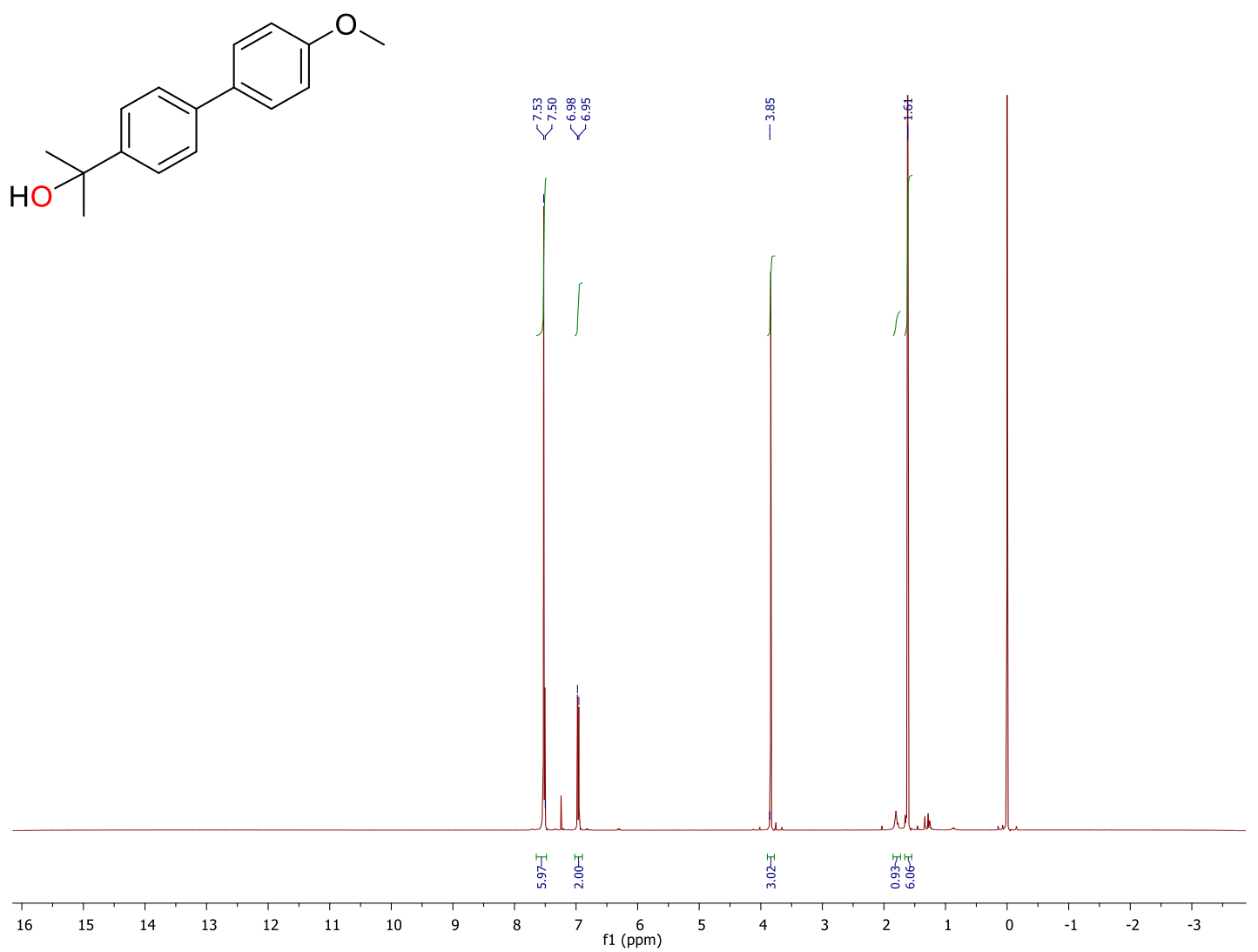

${ }^{1} \mathrm{H}$ NMR spectra in $\mathrm{CDCl}_{3}$

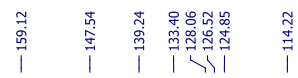

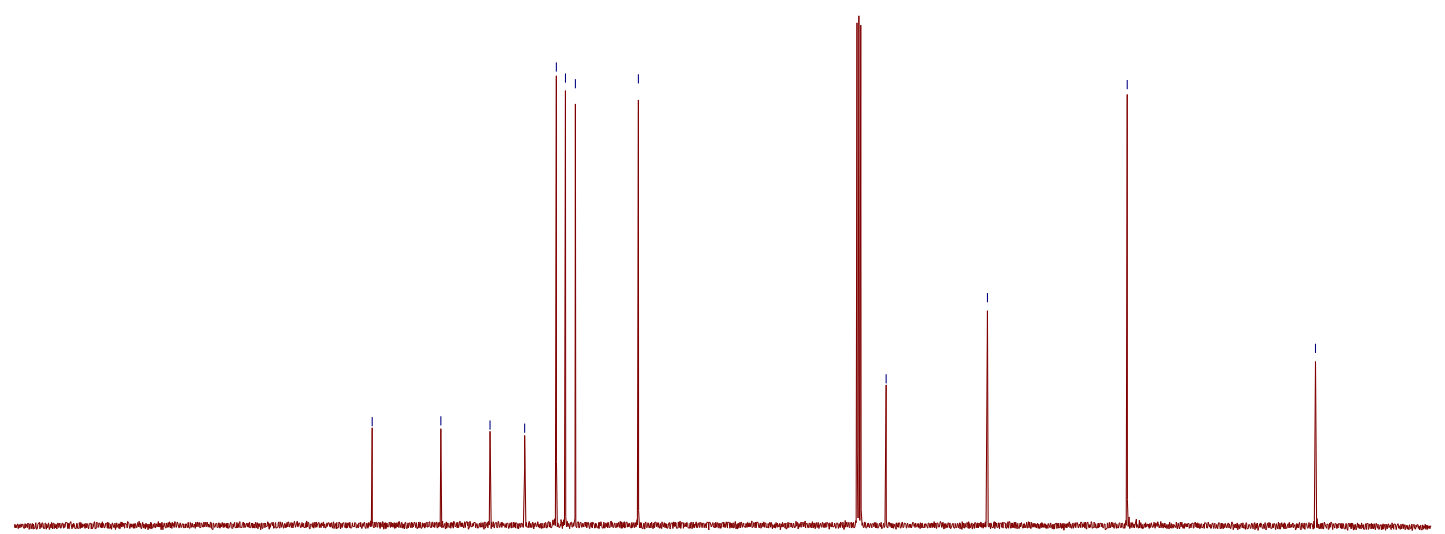

$\begin{array}{lllllllllllllllllllllllllll}210 & 200 & 190 & 180 & 170 & 160 & 150 & 140 & 130 & 120 & 110 & 100 & 90 & 80 & 70 & 60 & 50 & 40 & 30 & 20 & 10 & 0 & -10\end{array}$

${ }^{13} \mathrm{C}$ NMR spectra in $\mathrm{CDCl}_{3}$. 
174b<smiles>CC(C)(O)c1ccc(-c2cc3ccccc3c3ccccc23)cc1</smiles>

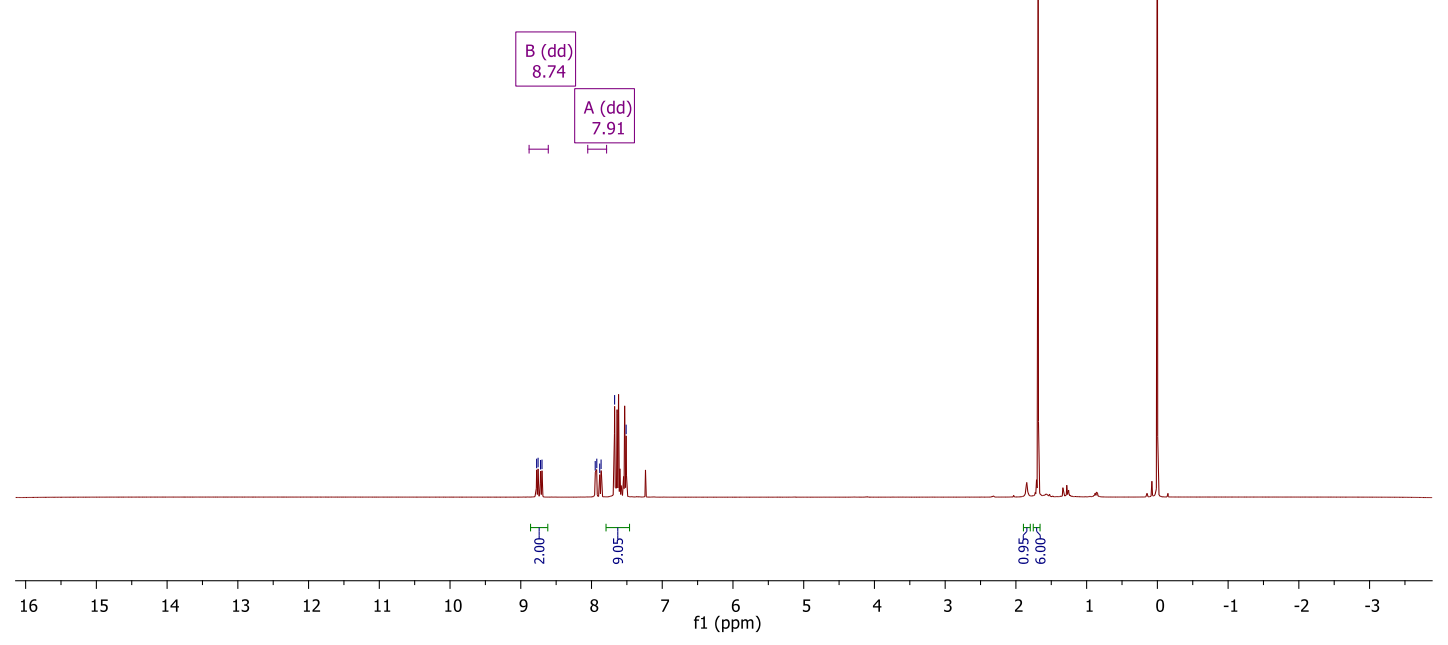

${ }^{1} \mathrm{H}$ NMR spectra in $\mathrm{CDCl}_{3}$

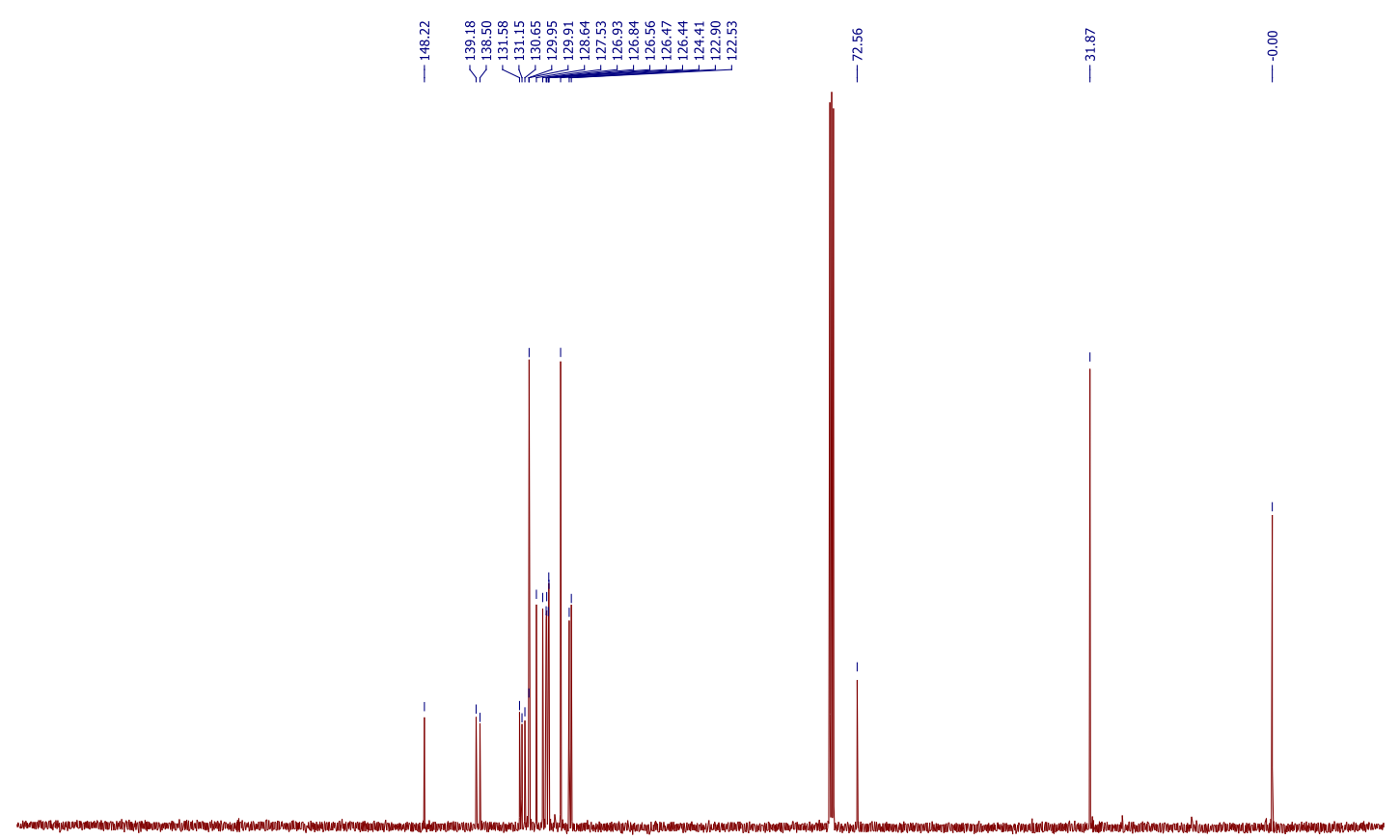

$\begin{array}{lllllllllllllllllllllll}210 & 200 & 190 & 180 & 170 & 160 & 150 & 140 & 130 & 120 & 110 & \underset{\mathrm{f} 1}{100}(\mathrm{ppm}) & 90 & 80 & 70 & 60 & 50 & 40 & 30 & 20 & 10 & 0 & -10\end{array}$

${ }^{13} \mathrm{C}$ NMR spectra in $\mathrm{CDCl}_{3}$. 
175b

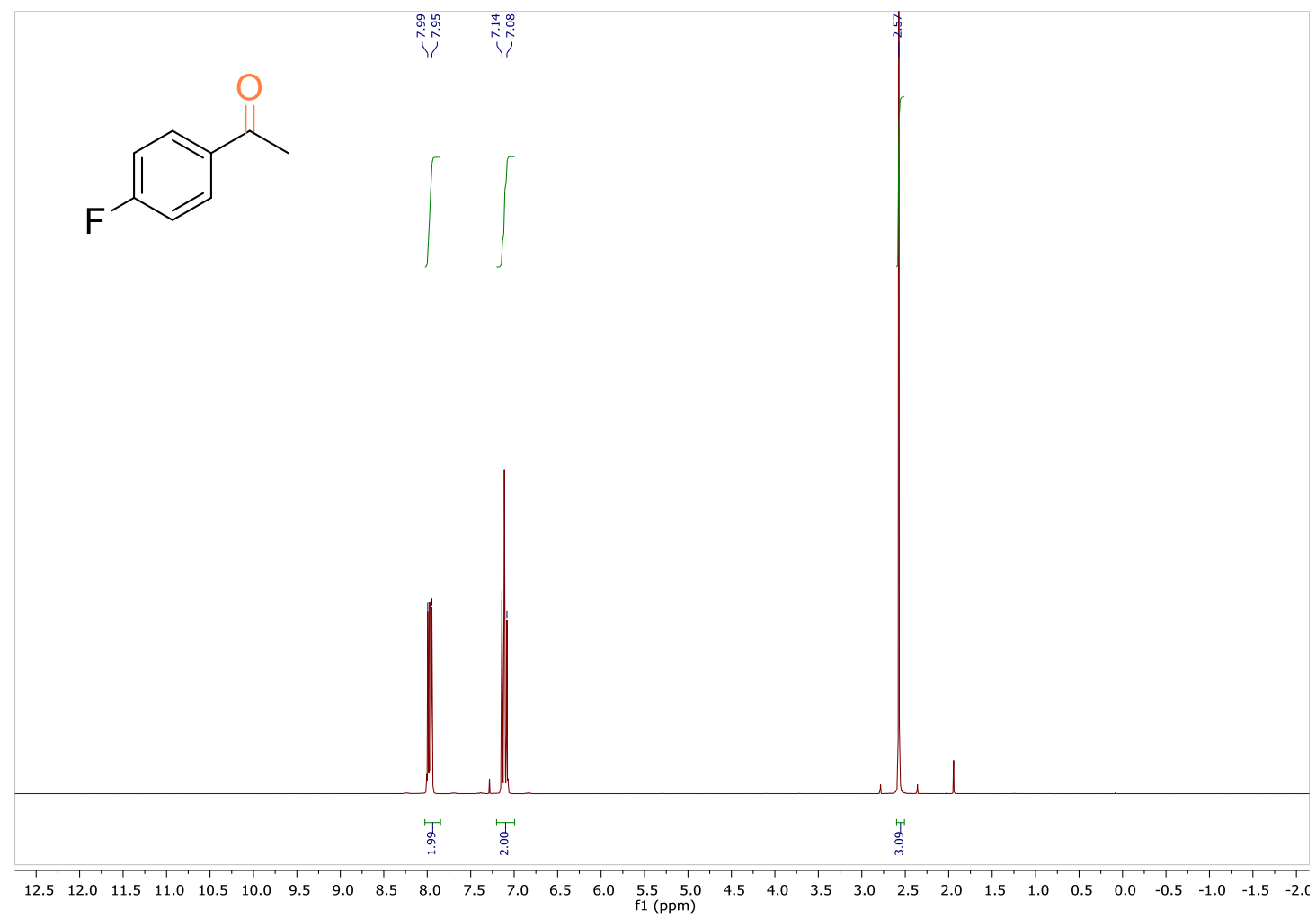

${ }^{1} \mathrm{H}$ NMR spectrum in $\mathrm{CDCl}_{3}$.

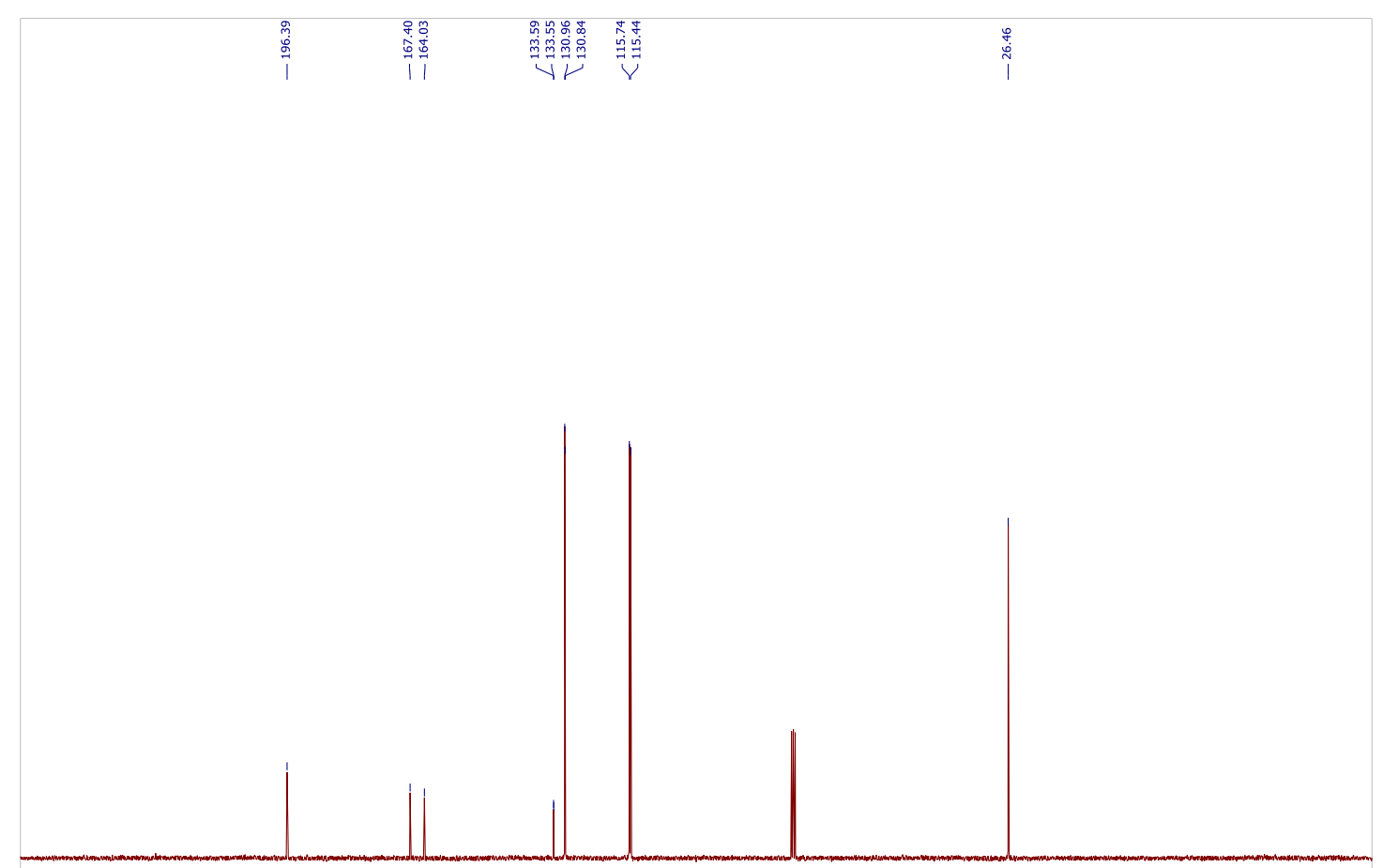

$\begin{array}{llllllllllllllllllllllllllllllllllll}250 & 240 & 230 & 220 & 210 & 200 & 190 & 180 & 170 & 160 & 150 & 140 & 130 & 120 & 110 & 100 & 90 & 80 & 70 & 60 & 50 & 40 & 30 & 20 & 10 & 0 & -10 & -20 & -30 & -40 & -50\end{array}$

${ }^{13} \mathrm{C}$ NMR spectrum in $\mathrm{CDCl}_{3}$. 
F57<smiles>O=C1OCCc2ccccc21</smiles><smiles>CCC1C#CC#C1</smiles>

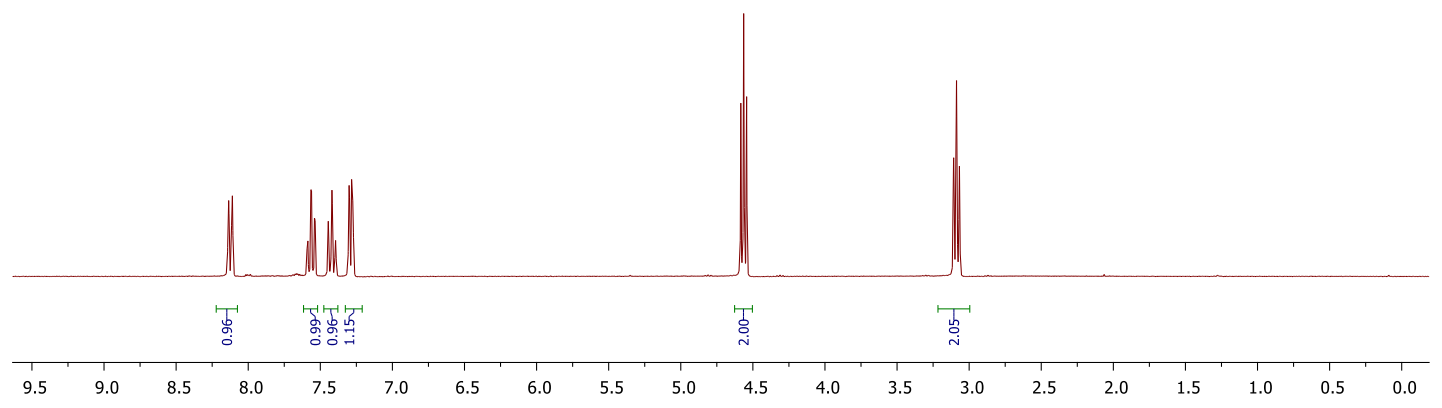

${ }^{1} \mathrm{H}$ NMR spectra in $\mathrm{CDCl}_{3}$

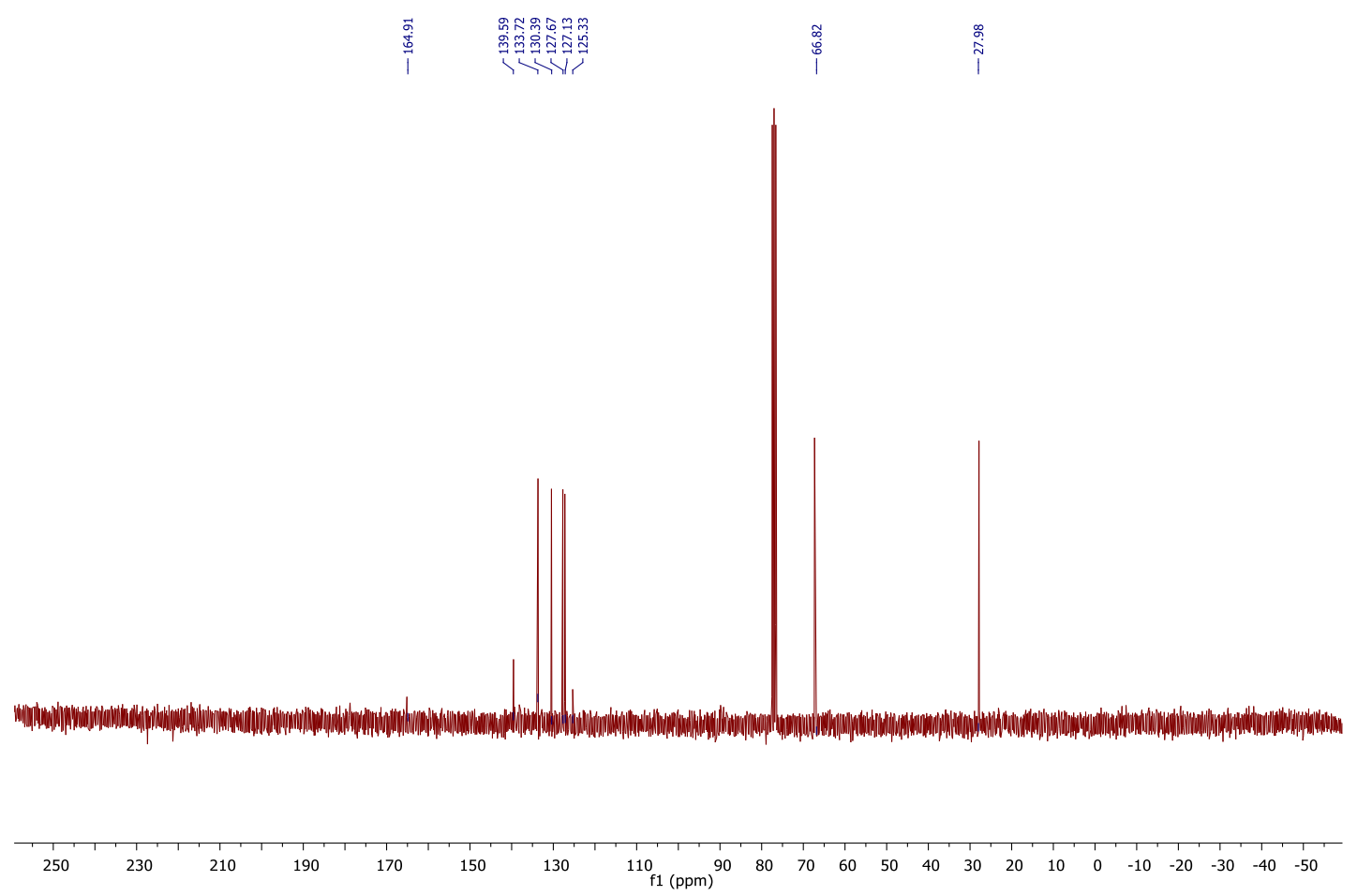

${ }^{13} \mathrm{C}$ NMR spectra in $\mathrm{CDCl}_{3}$. 
F58

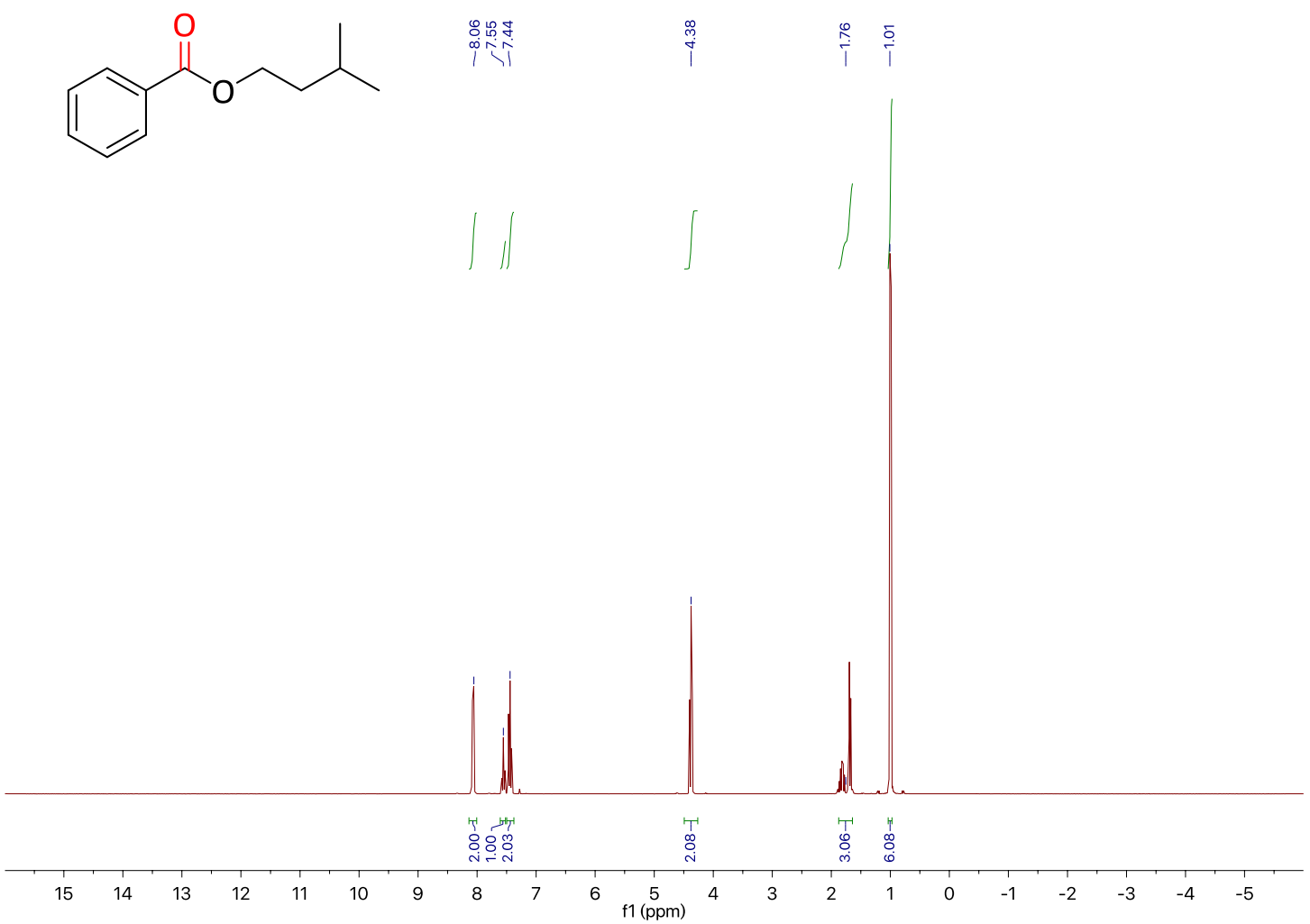

${ }^{13} \mathrm{C}$ NMR spectra in $\mathrm{CDCl}_{3}$.

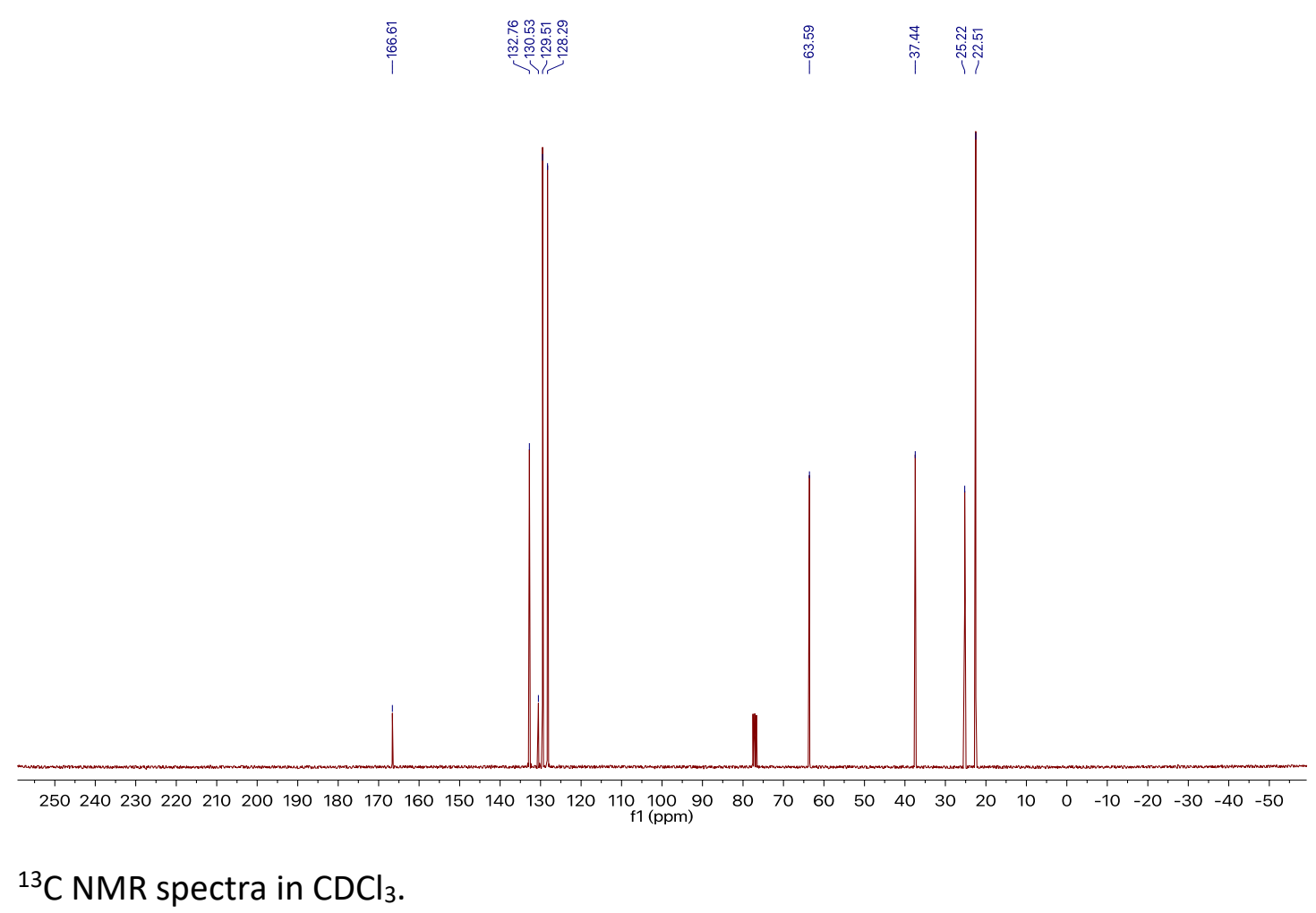


F59

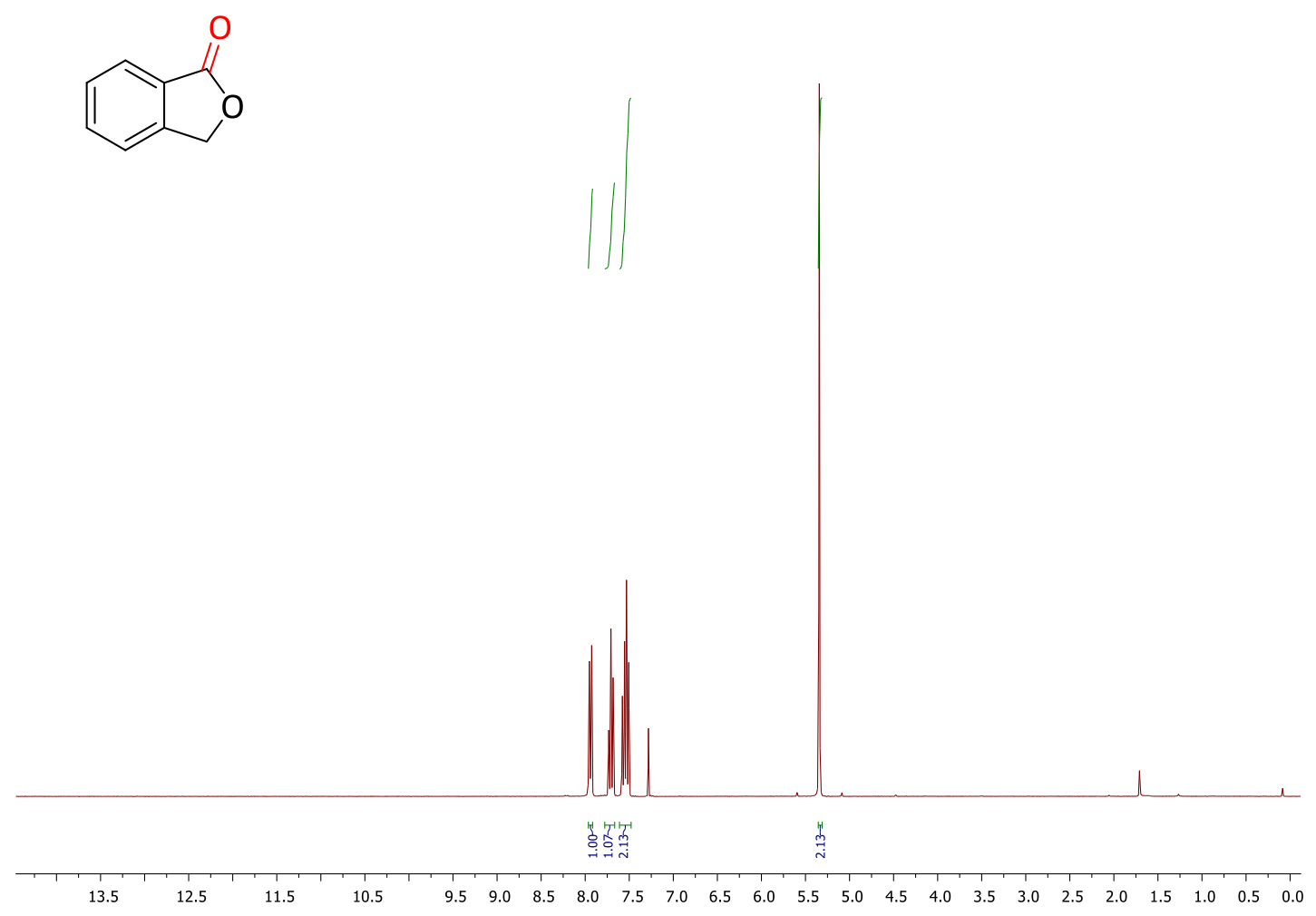

${ }^{1} \mathrm{H}$ NMR spectra in $\mathrm{CDCl}_{3}$
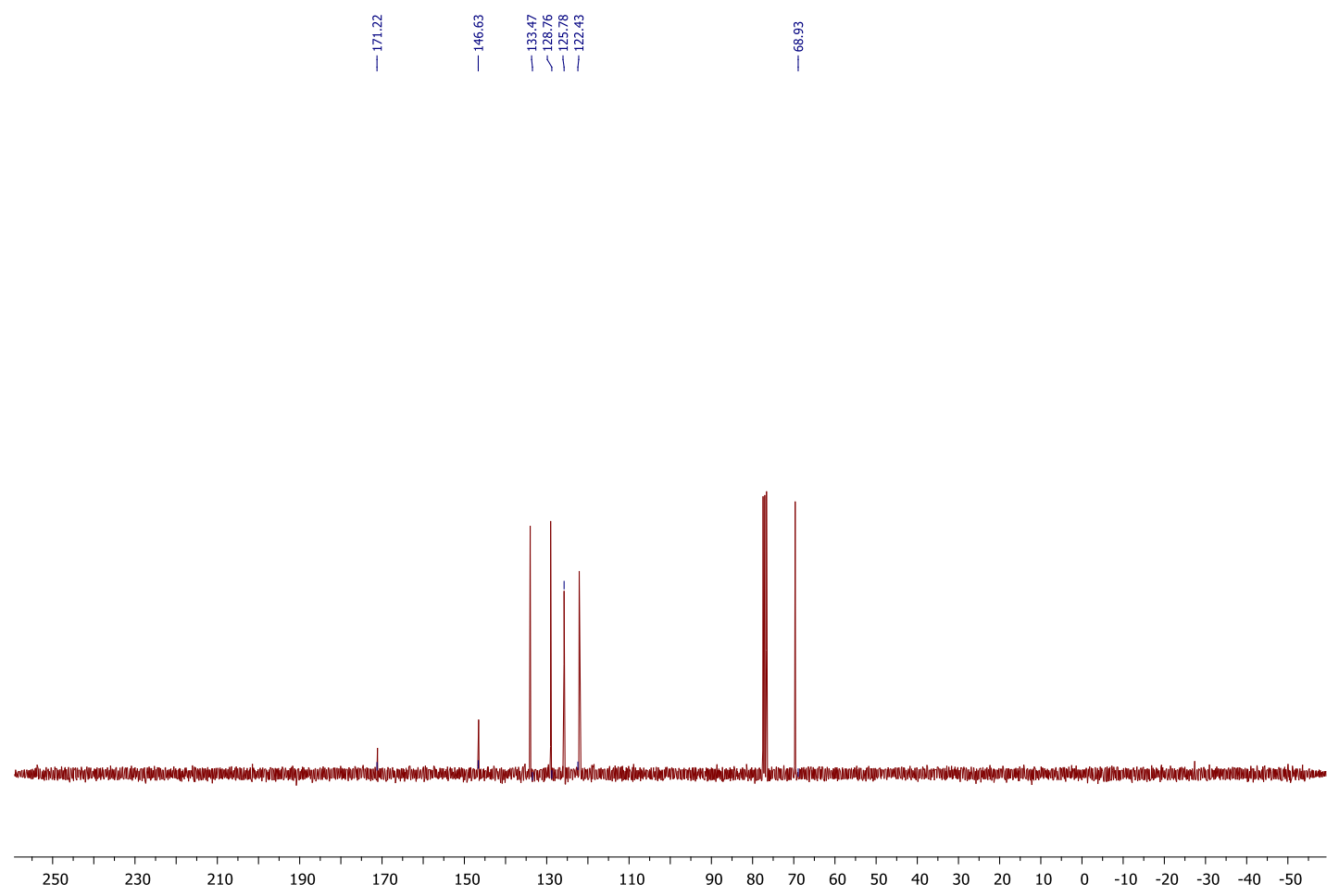

${ }^{13} \mathrm{C} \mathrm{NMR} \mathrm{spectra} \mathrm{in} \mathrm{CDCl}_{3}$. 
F60

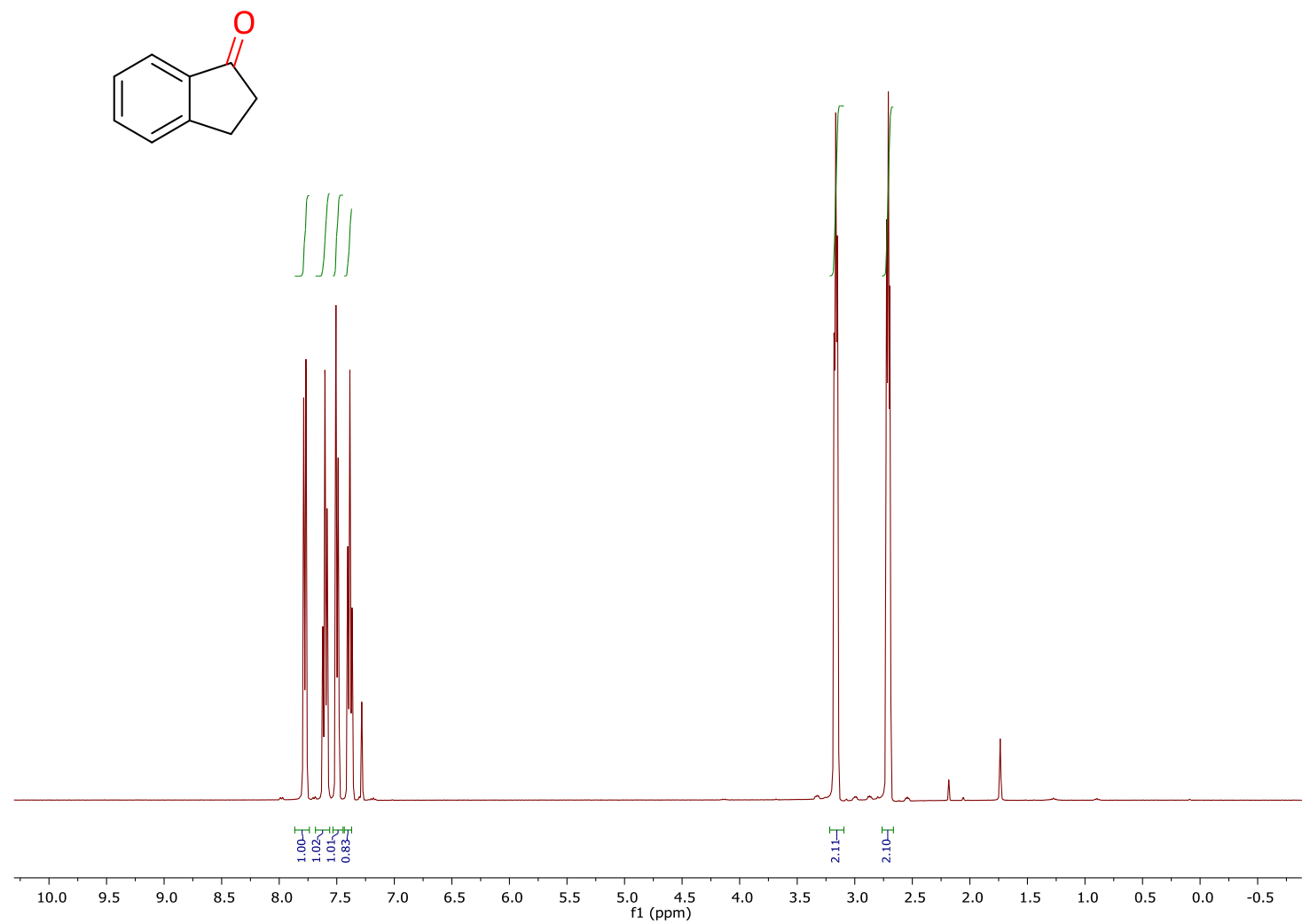

${ }^{1} \mathrm{H}$ NMR spectra in $\mathrm{CDCl}_{3}$

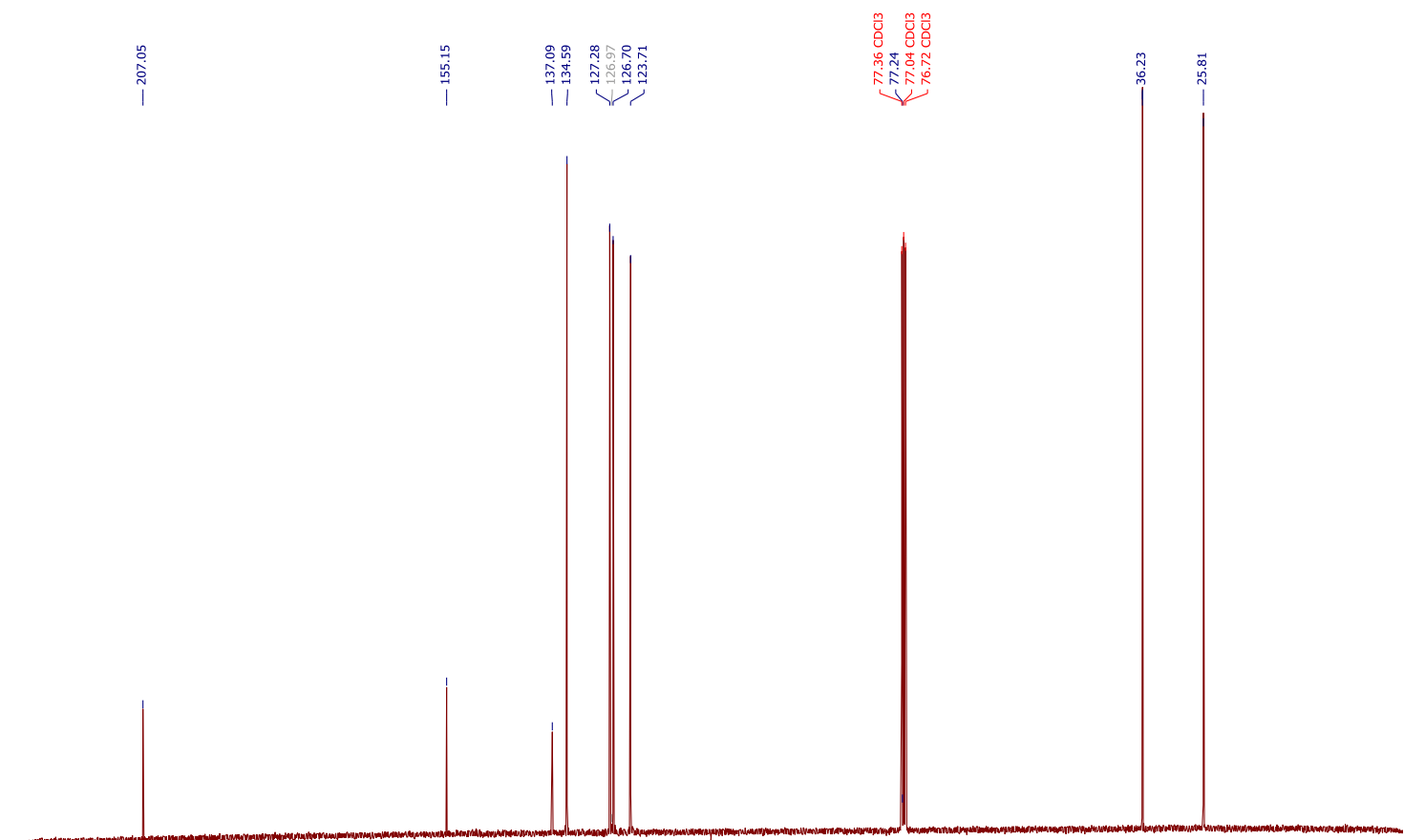

$\begin{array}{lllllllllllllllllllllllllllllllllll}220 & 210 & 200 & 190 & 180 & 170 & 160 & 150 & 140 & 130 & 120 & 110 & 100 & 90 & 80 & 70 & 60 & 50 & 40 & 30 & 20 & 10 & 0\end{array}$

${ }^{13} \mathrm{C}$ NMR spectra in $\mathrm{CDCl}_{3}$. 
F61<smiles>O=C1CCCc2ccccc21</smiles><smiles>C1=CCCC1</smiles>

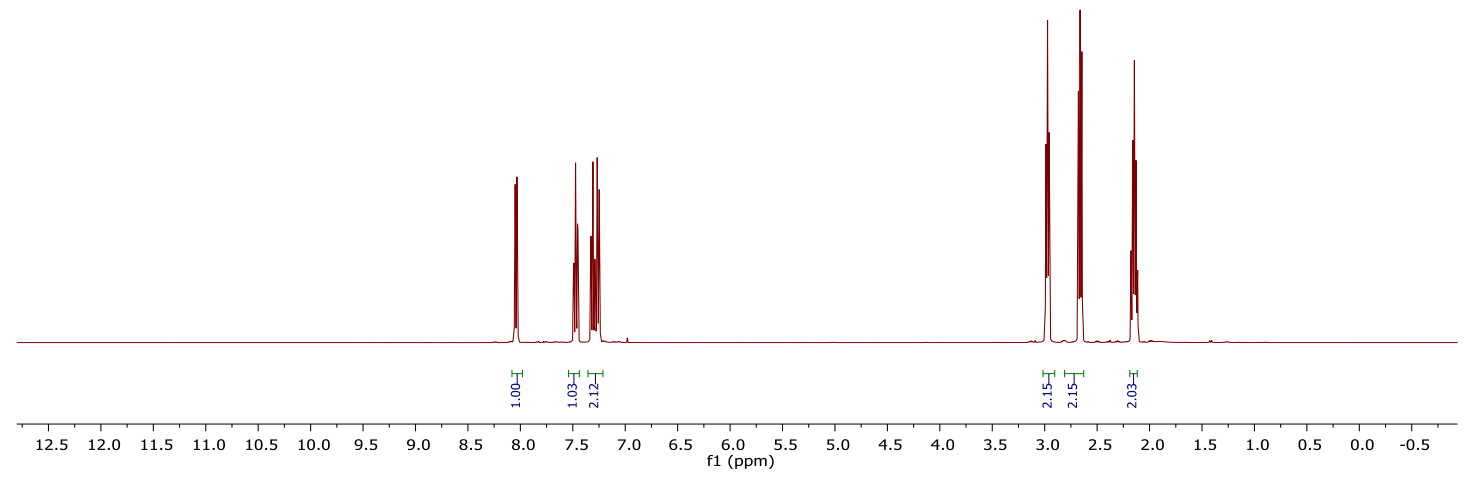

${ }^{1} \mathrm{H}$ NMR spectra in $\mathrm{CDCl}_{3}$

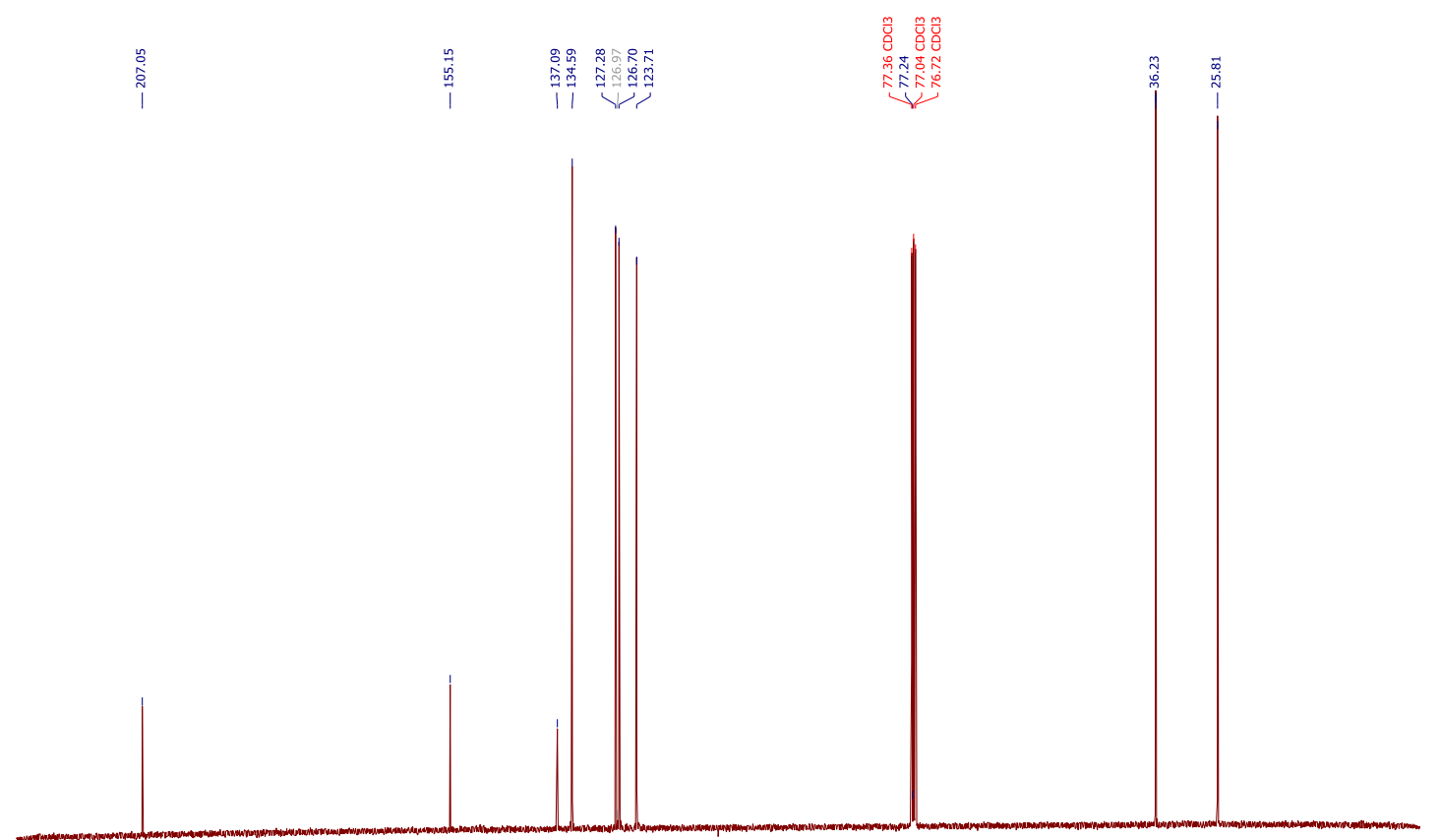

$\begin{array}{llllllllllllllllllllllllll}1 & 120 & 210 & 200 & 190 & 180 & 170 & 160 & 150 & 140 & 130 & 120 & \underset{f 1}{110} & 100 & 90 & 80 & 70 & 60 & 50 & 40 & 30 & 20 & 10 & 0\end{array}$

${ }^{13} \mathrm{C}$ NMR spectra in $\mathrm{CDCl}_{3}$. 
F62<smiles>O=C1Cc2cccc3cccc1c23</smiles>
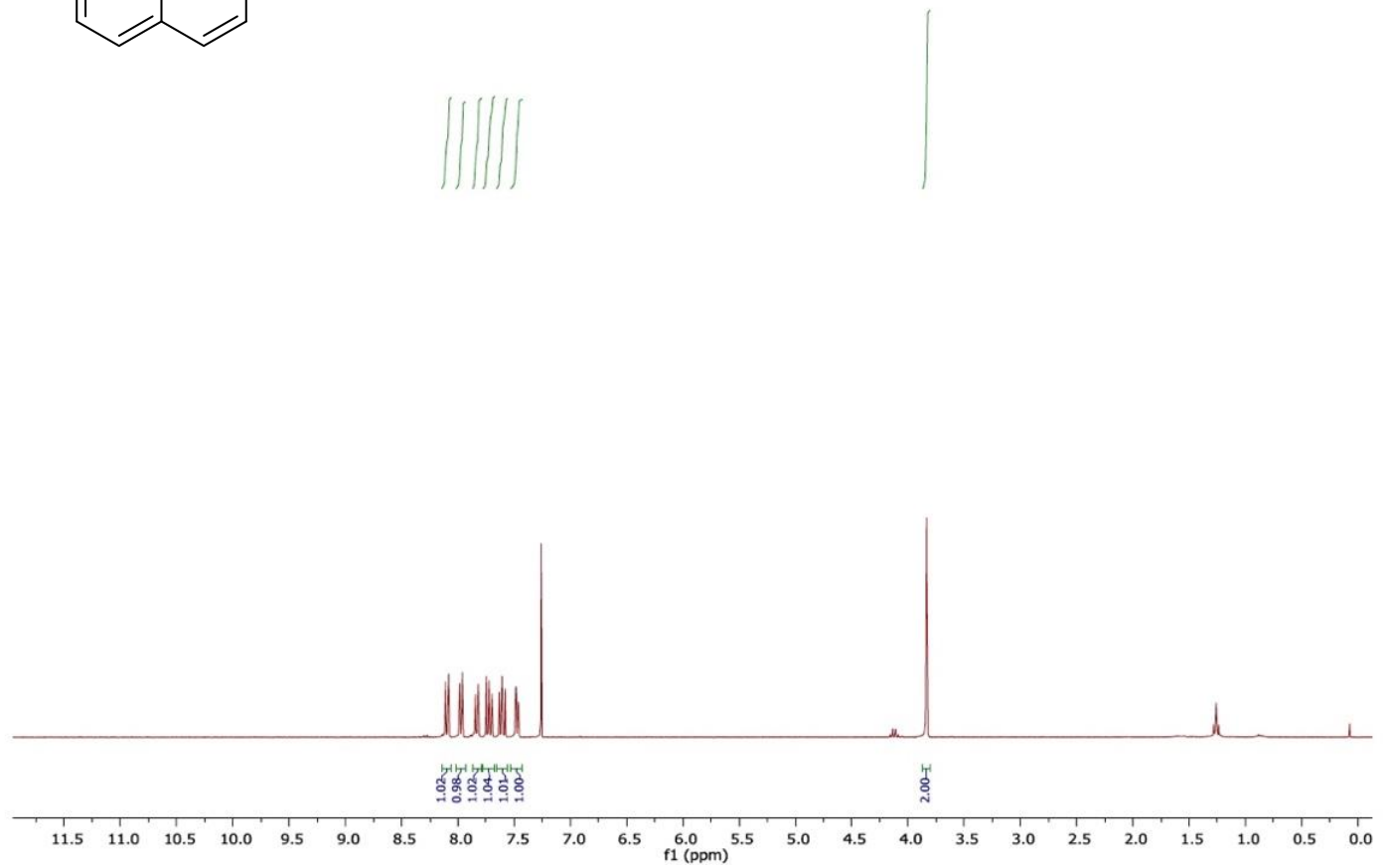

${ }^{1} \mathrm{H}$ NMR spectra in $\mathrm{CDCl}_{3}$

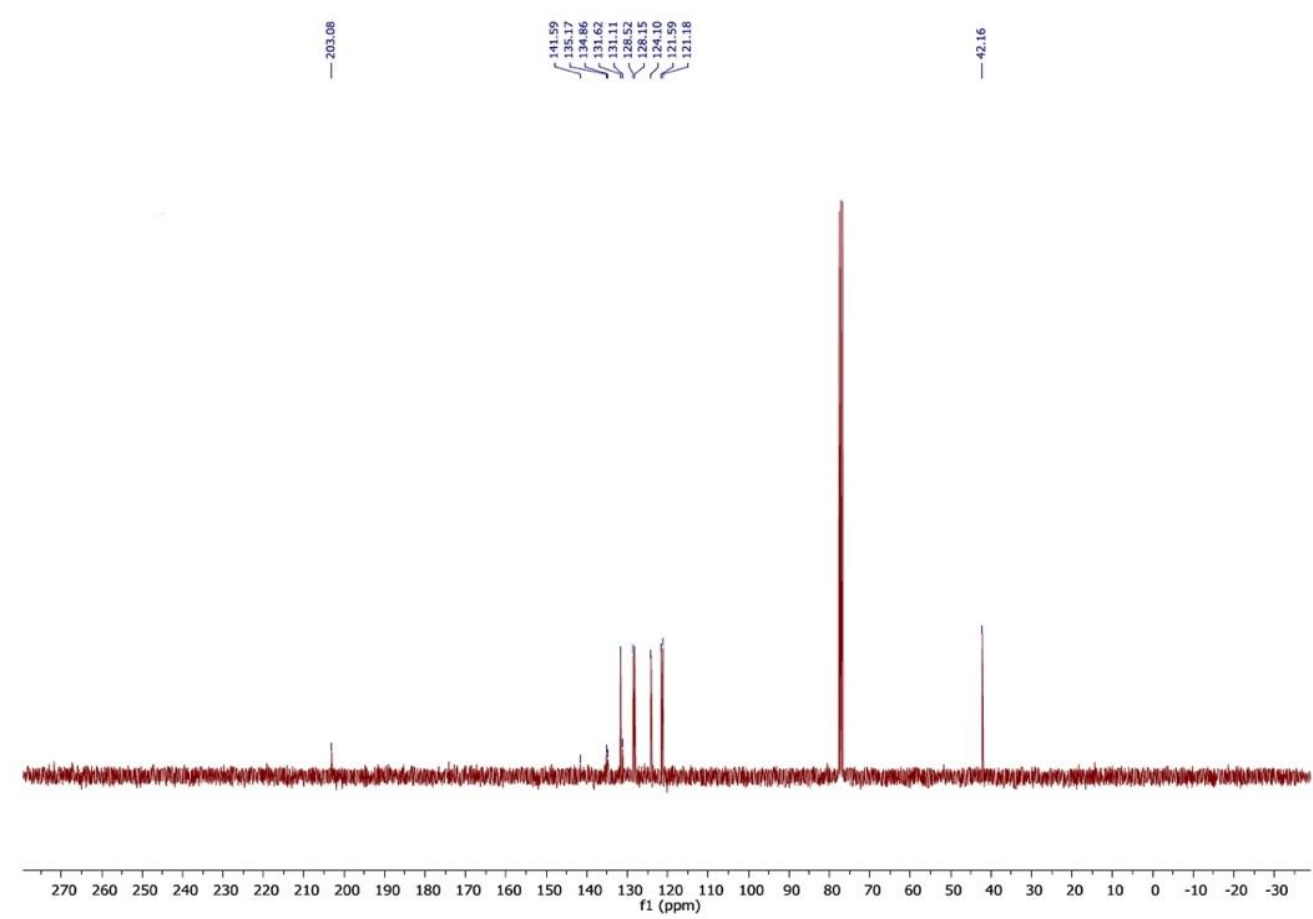

${ }^{13} \mathrm{C}$ NMR spectra in $\mathrm{CDCl}_{3}$. 
F63

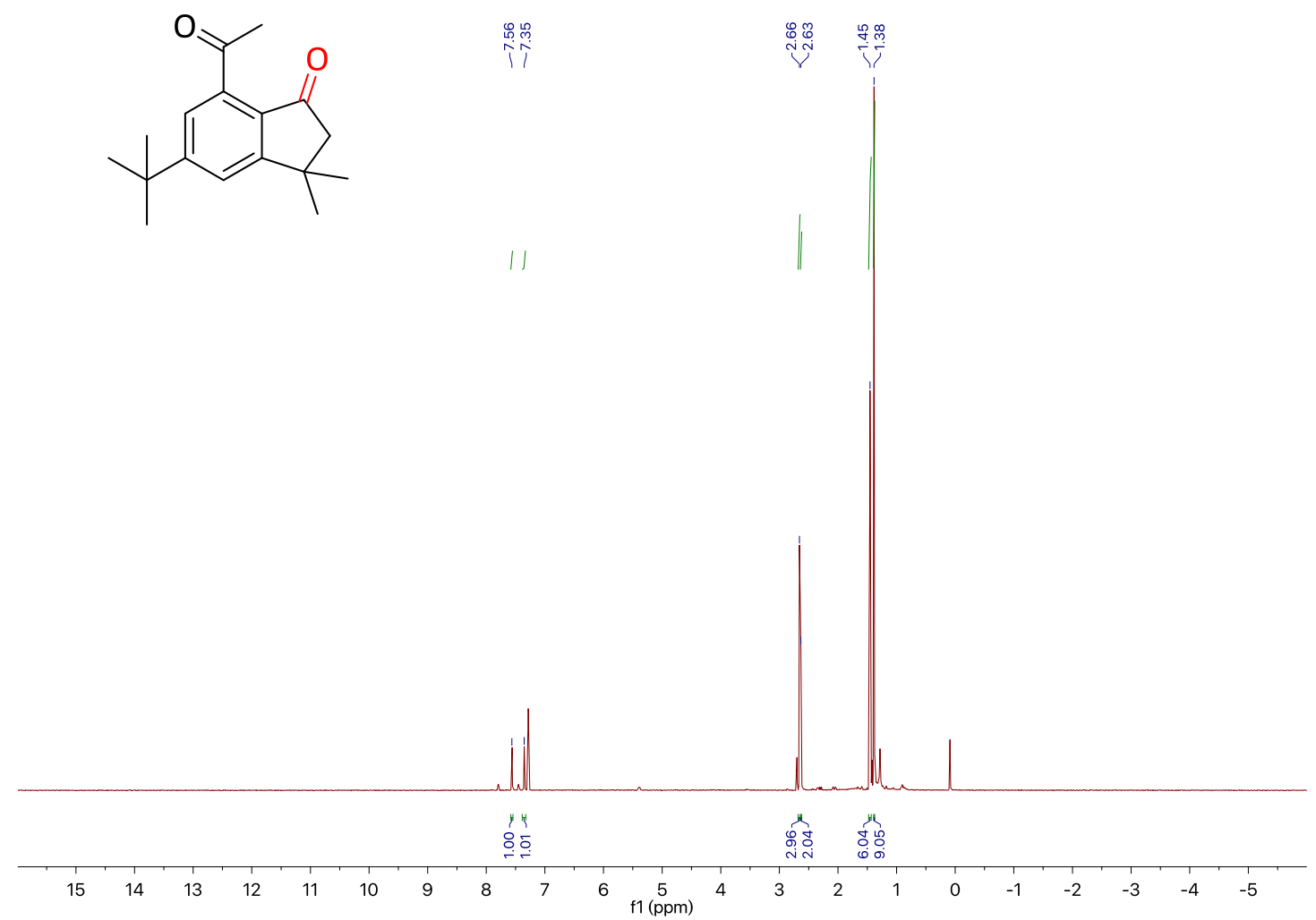

${ }^{1} \mathrm{H}$ NMR spectra in $\mathrm{CDCl}_{3}$

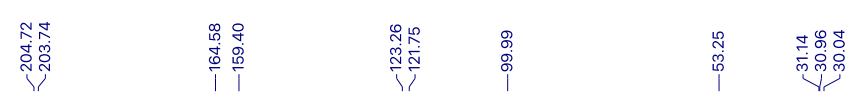

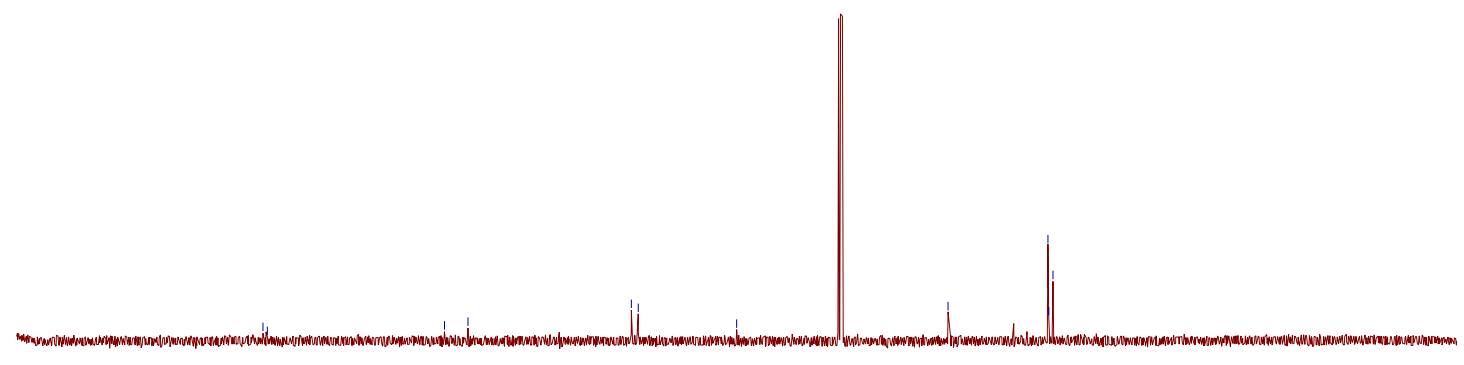

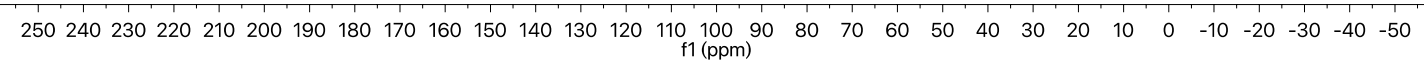

${ }^{13} \mathrm{C}$ NMR spectra in $\mathrm{CDCl}_{3}$. 


\section{$70 b$}

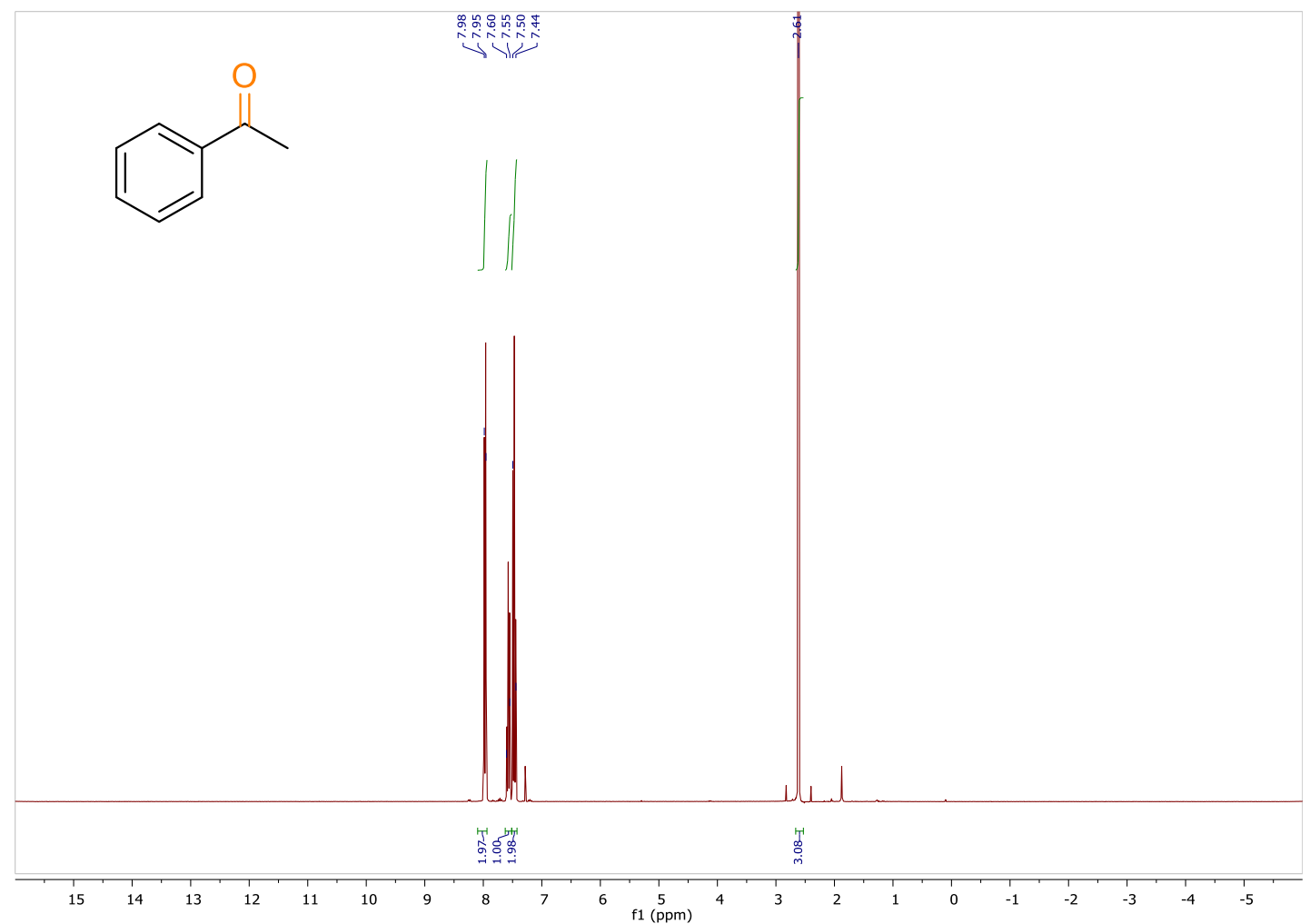

${ }^{1} \mathrm{H}$ NMR spectrum in $\mathrm{CDCl}_{3}$

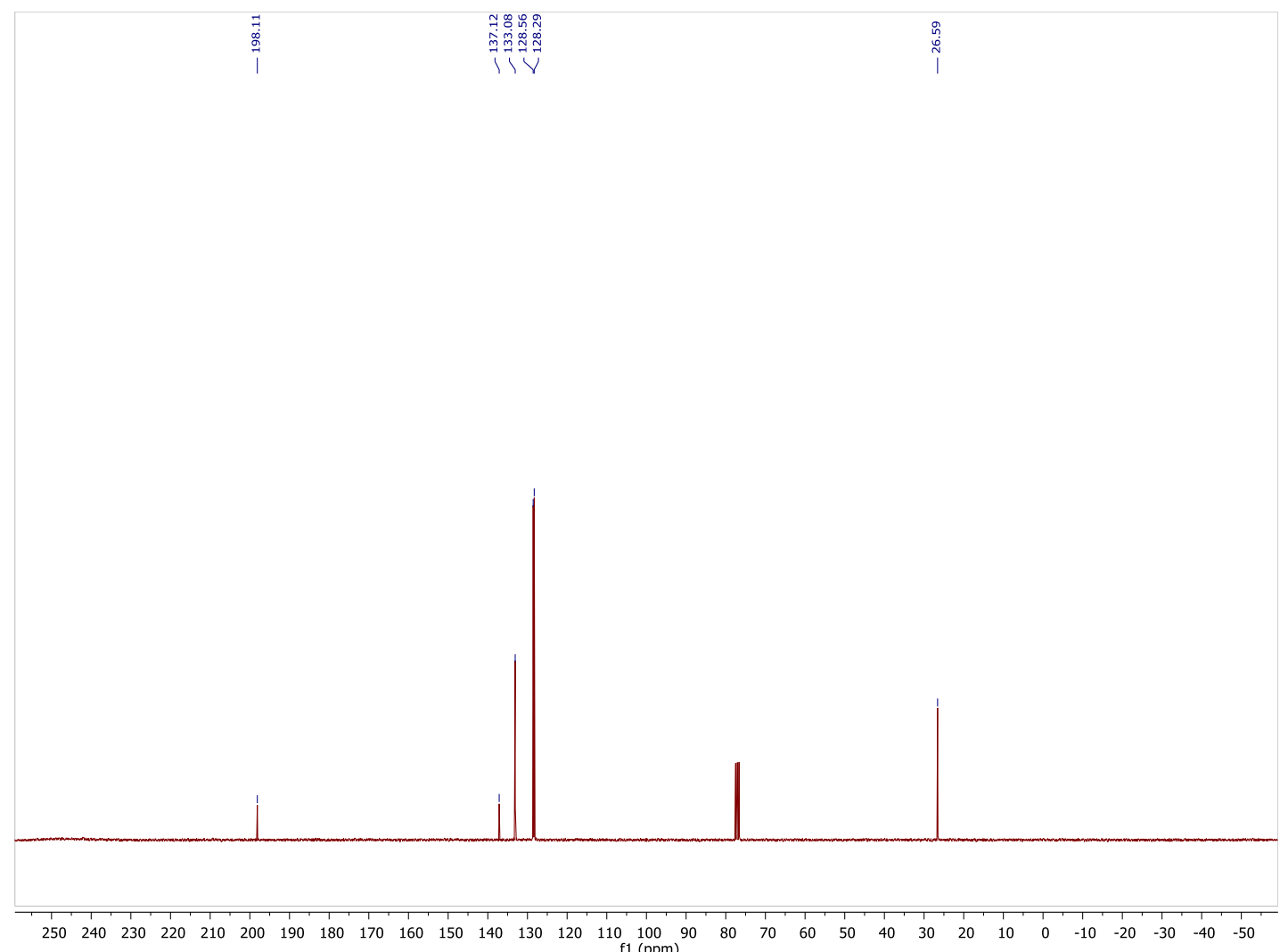

${ }^{13} \mathrm{C}$ NMR spectrum in $\mathrm{CDCl}_{3}$. 
$79 b$

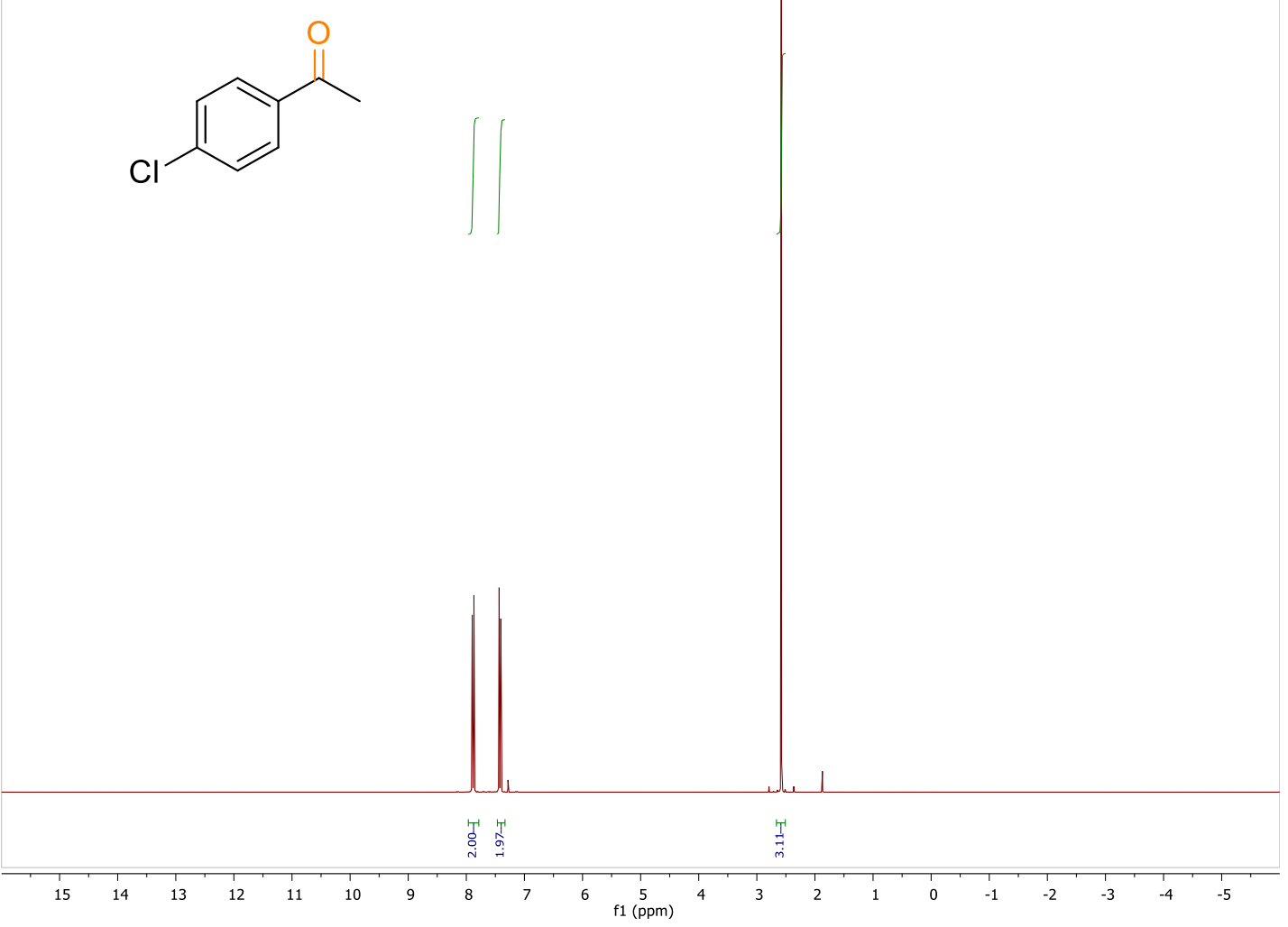

${ }^{1} \mathrm{H}$ NMR spectrum in $\mathrm{CDCl}_{3}$.

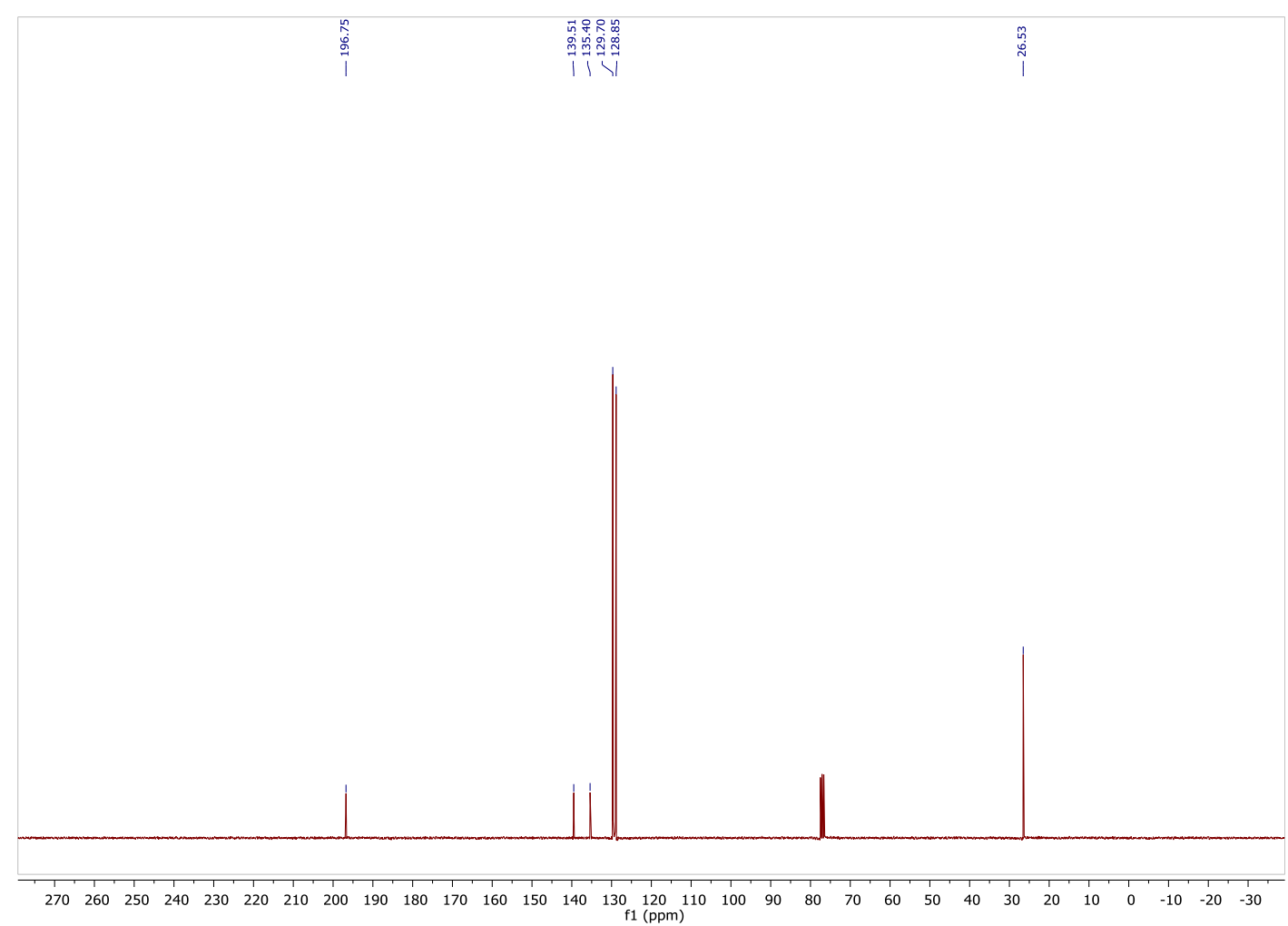

${ }^{13} \mathrm{C}$ NMR spectrum in $\mathrm{CDCl}_{3}$. 
176b

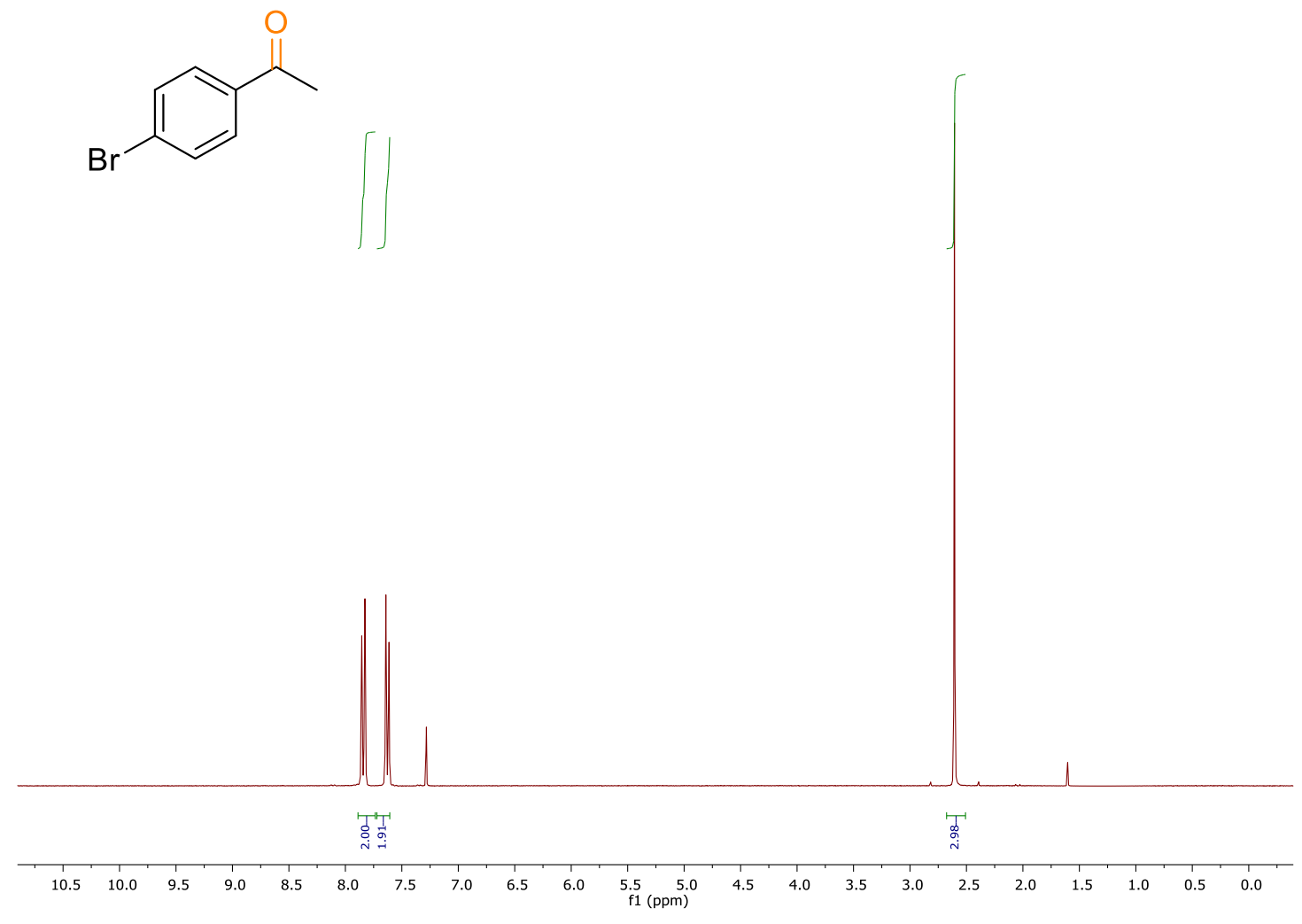

${ }^{1} \mathrm{H}$ NMR spectrum in $\mathrm{CDCl}_{3}$.

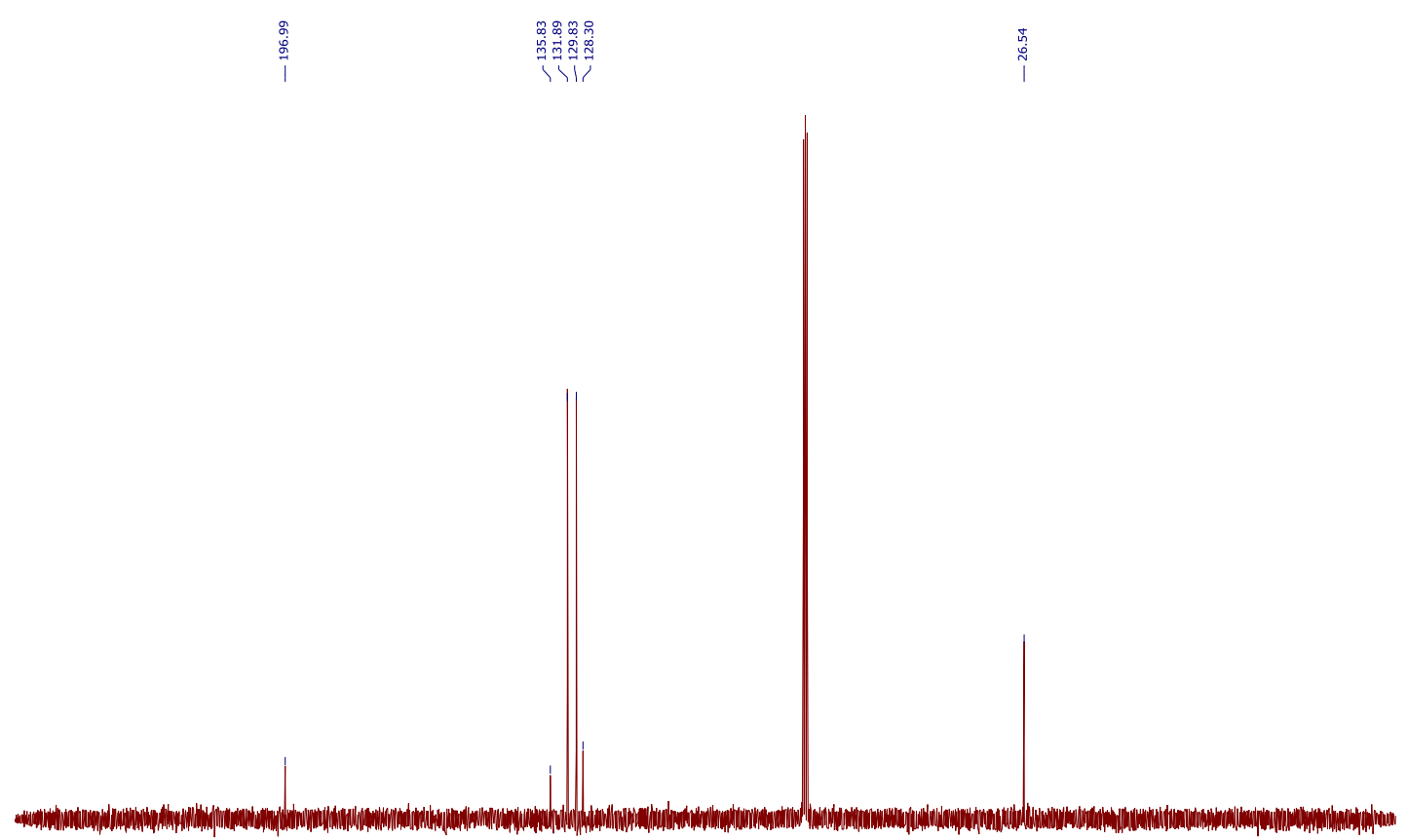

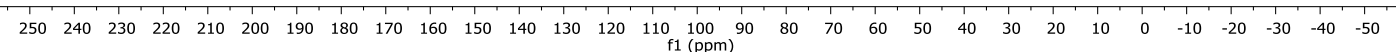

${ }^{13} \mathrm{C} \mathrm{NMR}$ spectrum in $\mathrm{CDCl}_{3}$. 


\section{7b}

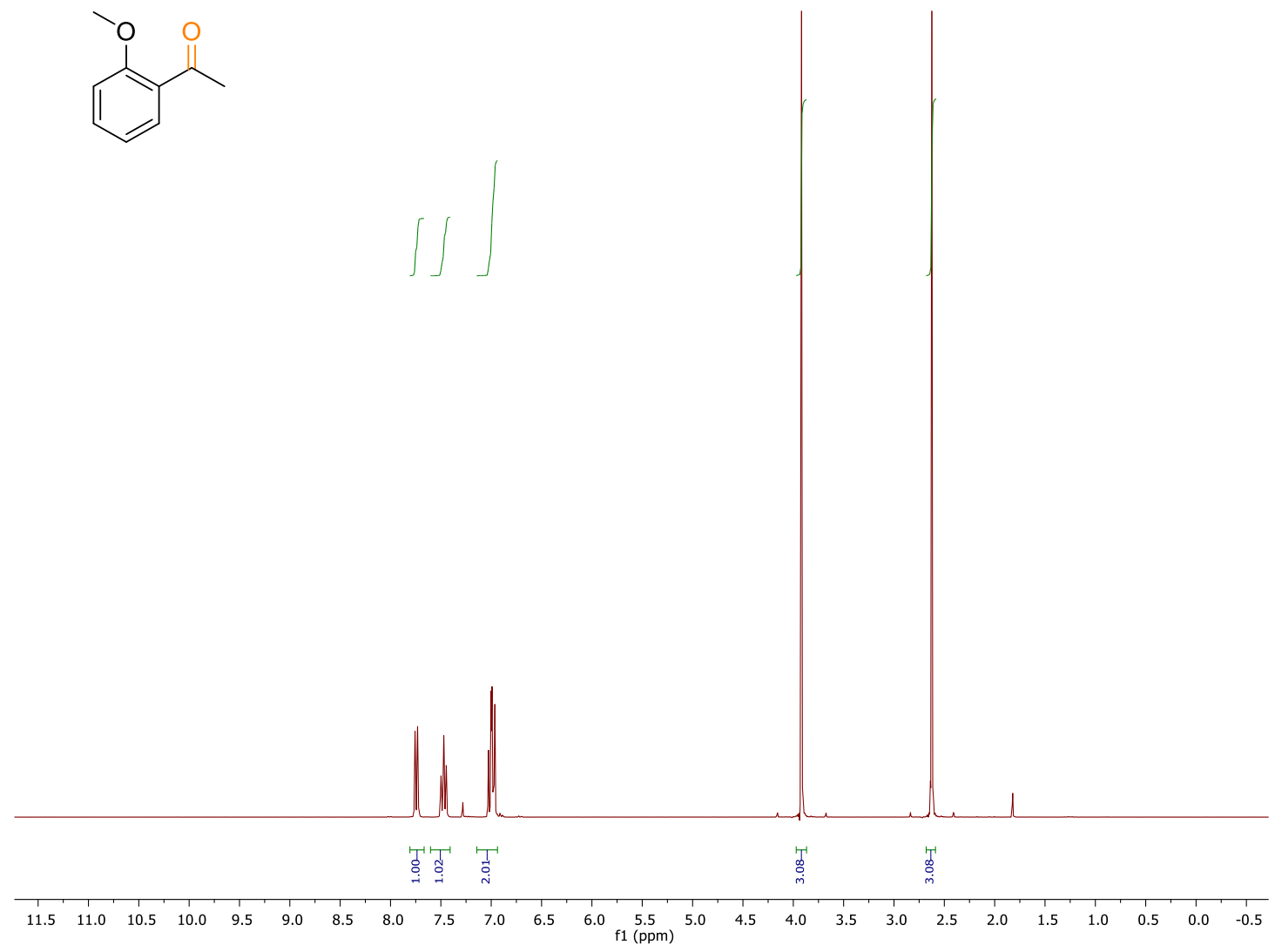

${ }^{1} \mathrm{H}$ NMR spectrum in $\mathrm{CDCl}_{3}$.

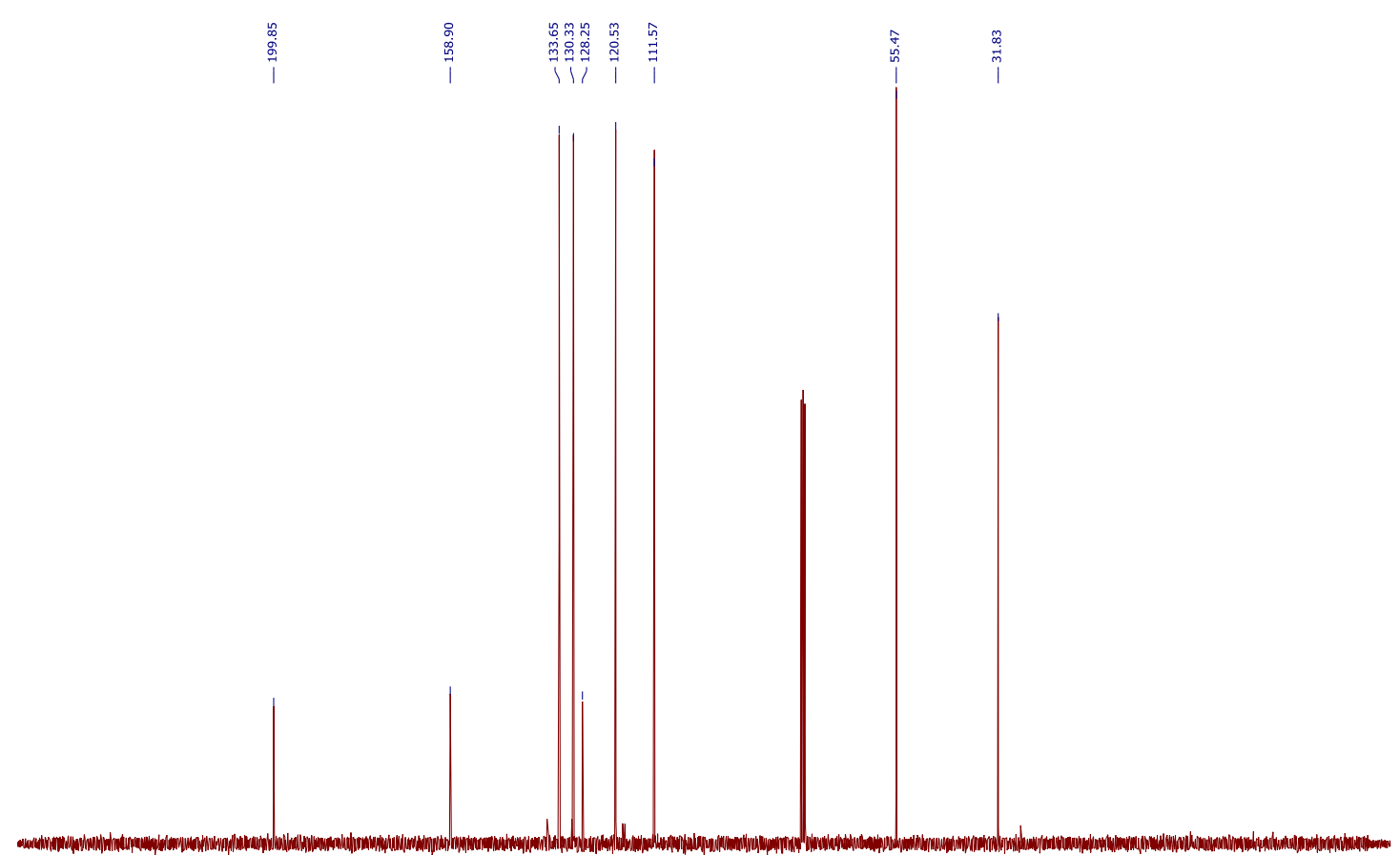

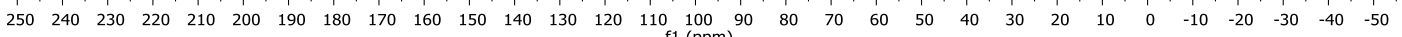

${ }^{13} \mathrm{C}$ NMR spectrum in $\mathrm{CDCl}_{3}$. 
$79 b$

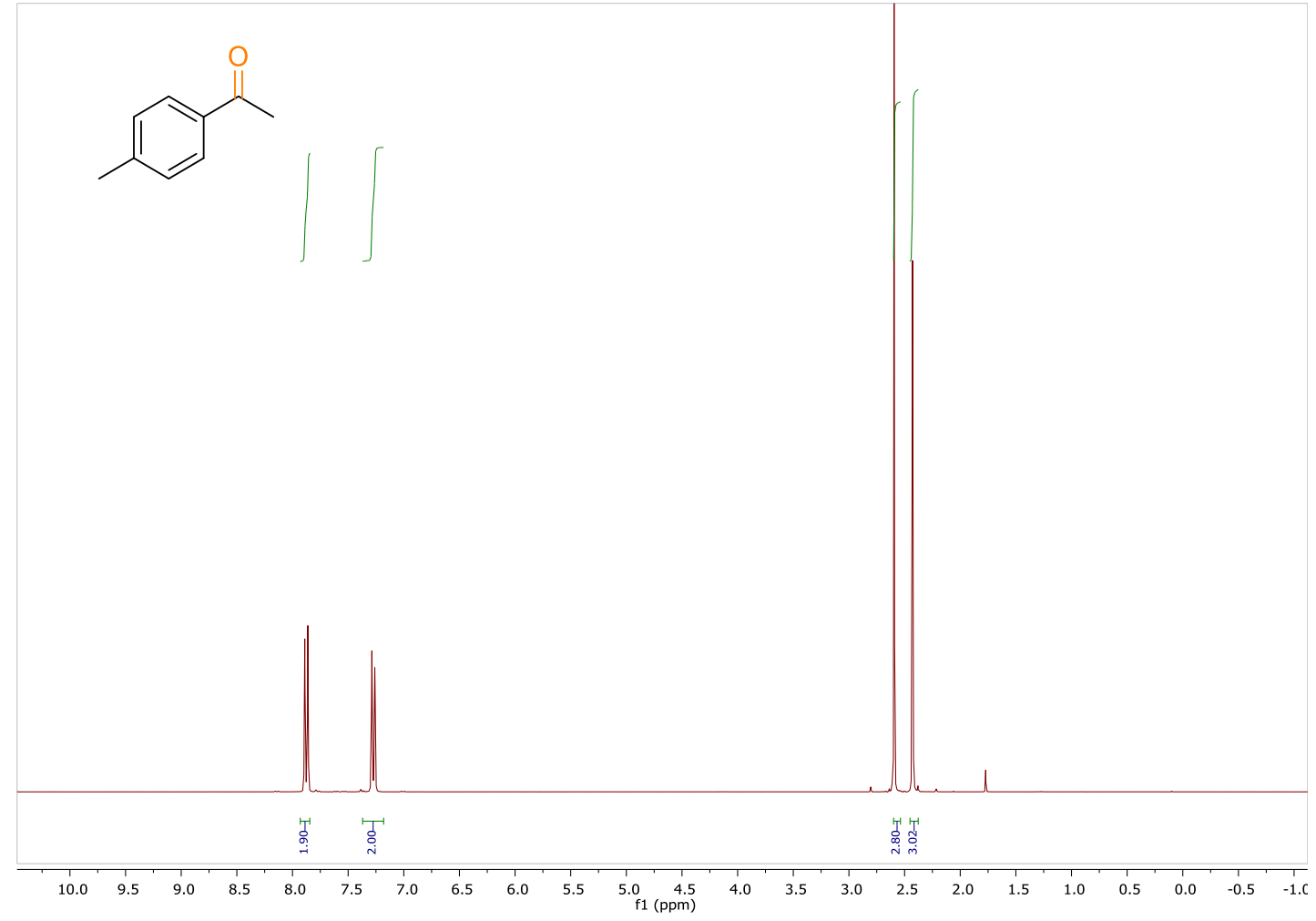

${ }^{13} \mathrm{C}$ NMR spectrum in $\mathrm{CDCl}_{3}$.

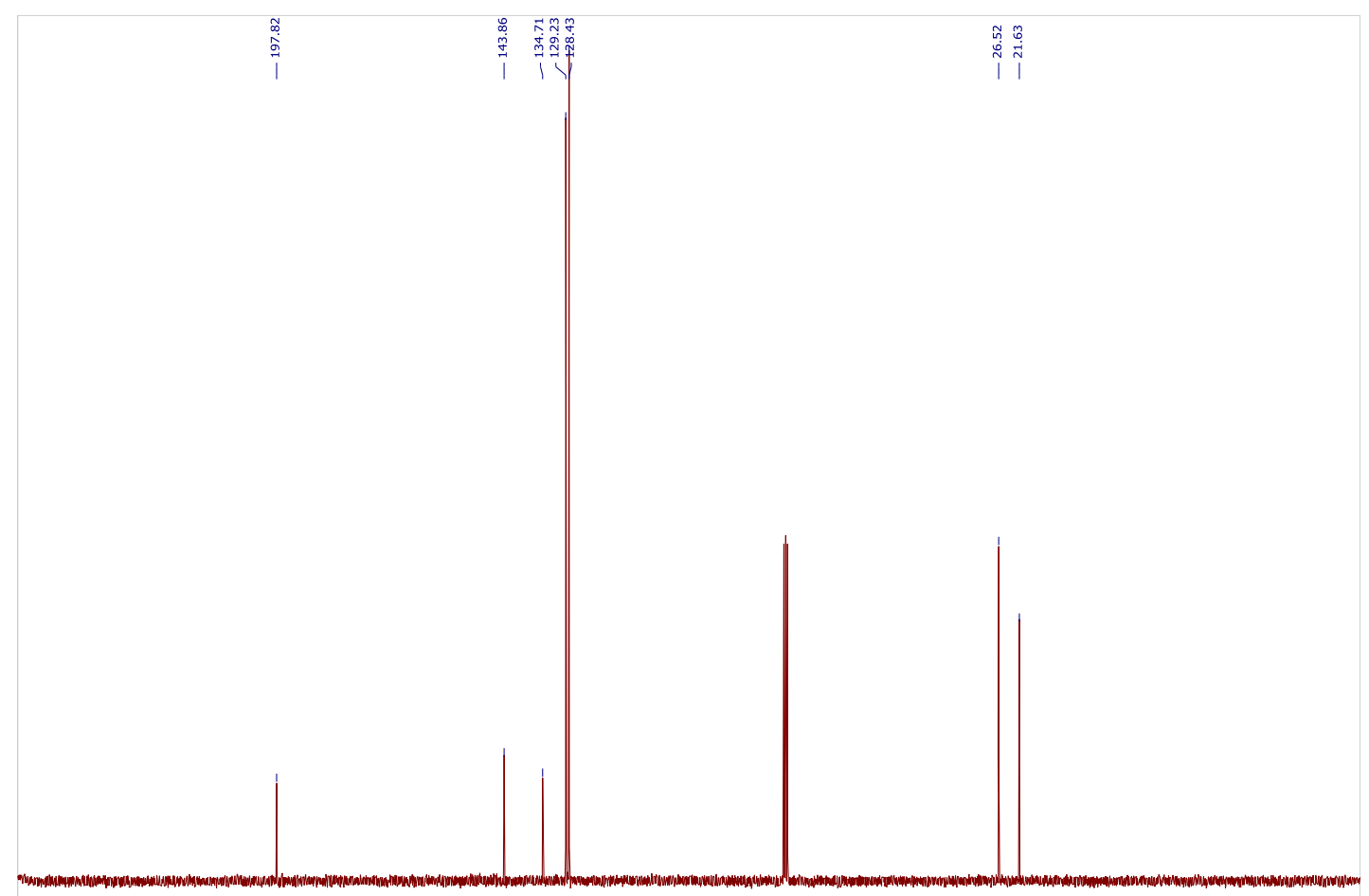

$\begin{array}{lllllllllllllllllllllllllllllllllllllllllllll}250 & 240 & 230 & 220 & 210 & 200 & 190 & 180 & 170 & 160 & 150 & 140 & 130 & 120 & 110 & 100 & 90 & 80 & 70 & 60 & 50 & 40 & 30 & 20 & 10 & 0 & -10 & -20 & -30 & -40 & -50\end{array}$

${ }^{1} \mathrm{H}$ NMR spectrum in $\mathrm{CDCl}_{3}$. 


\section{$78 b$}

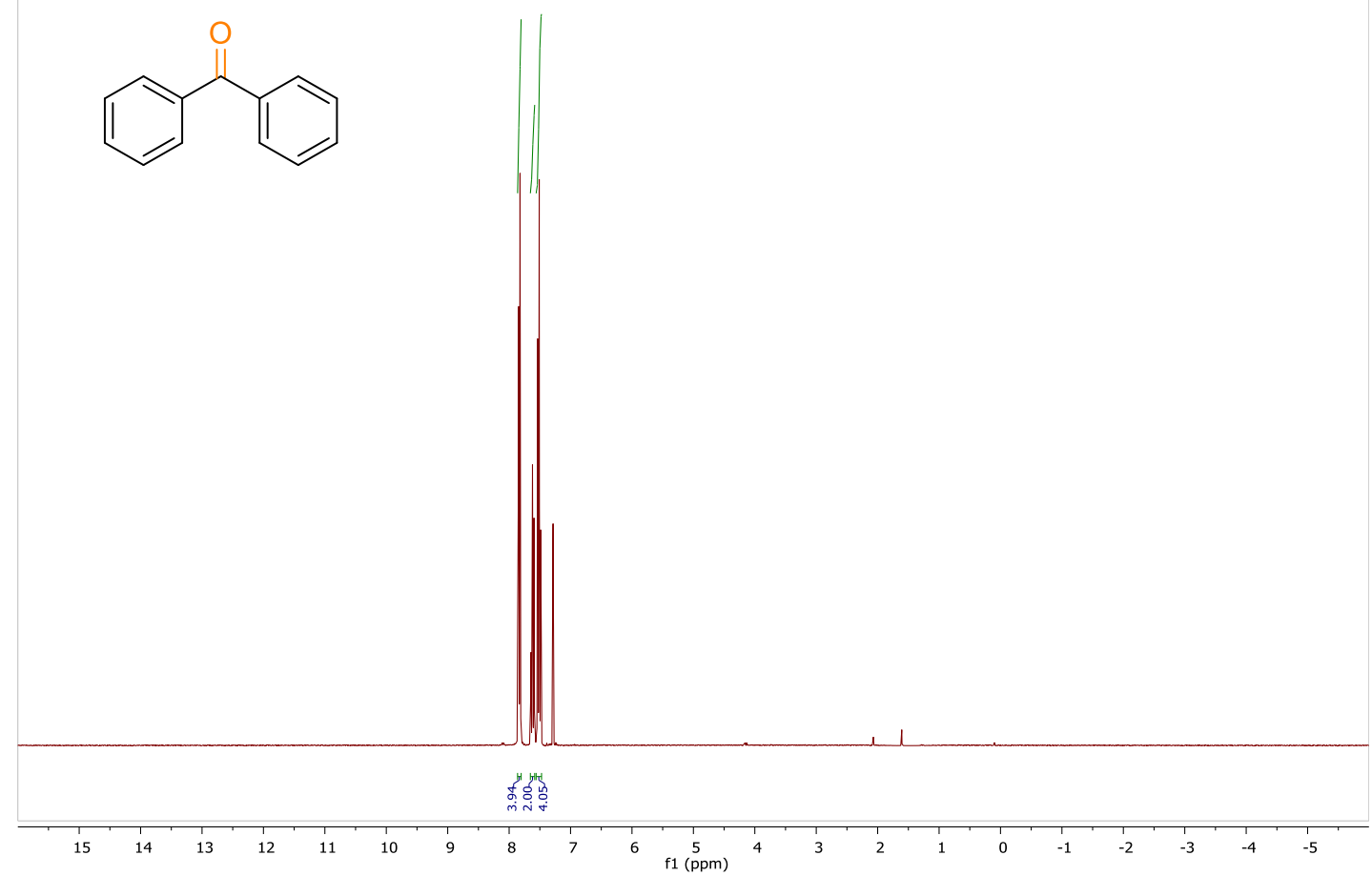

${ }^{1} \mathrm{H}$ NMR spectrum in $\mathrm{CDCl}_{3}$

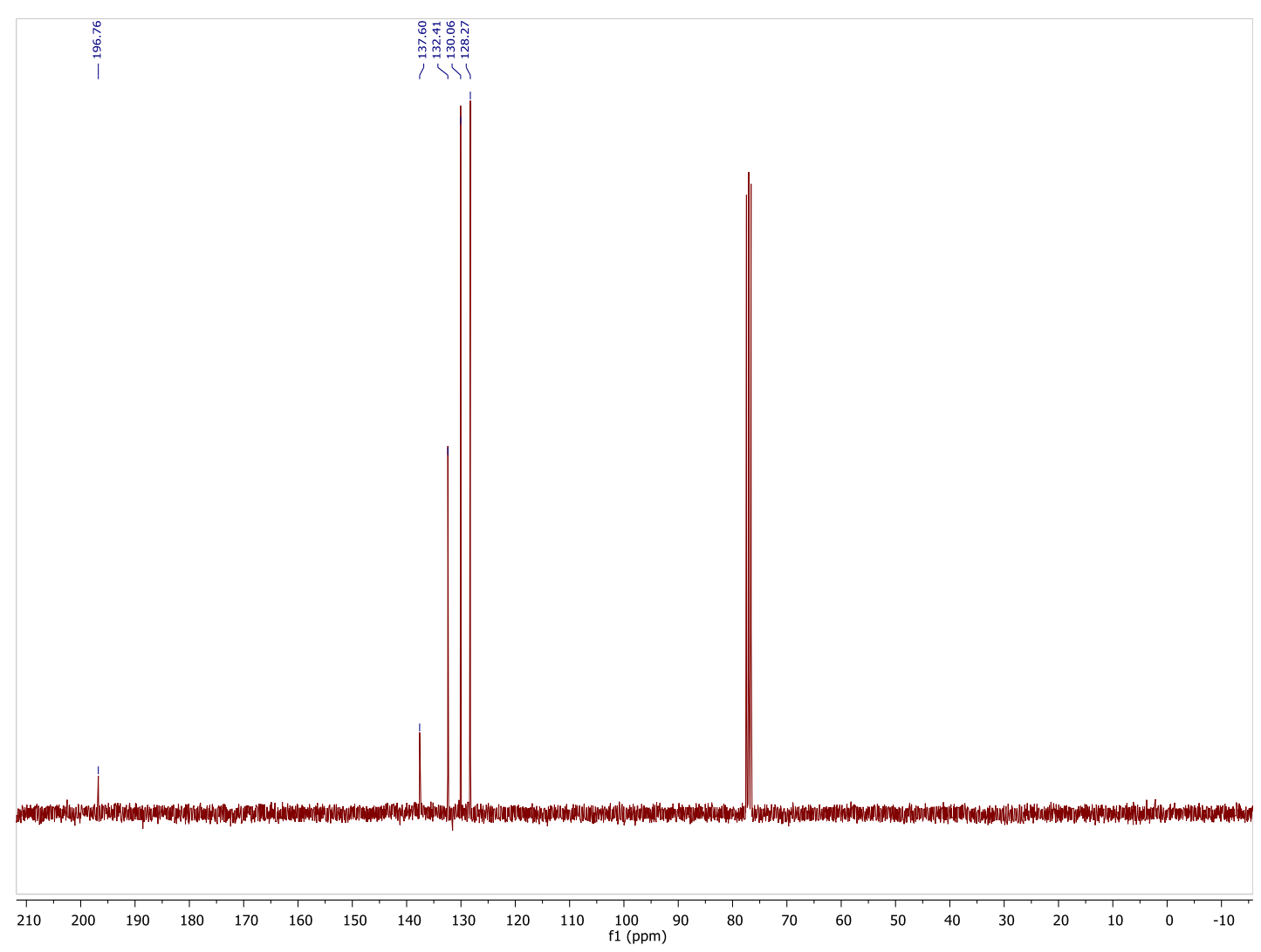

${ }^{13} \mathrm{C}$ NMR spectrum in $\mathrm{CDCl}_{3}$. 
Experimental Section

$178 b$
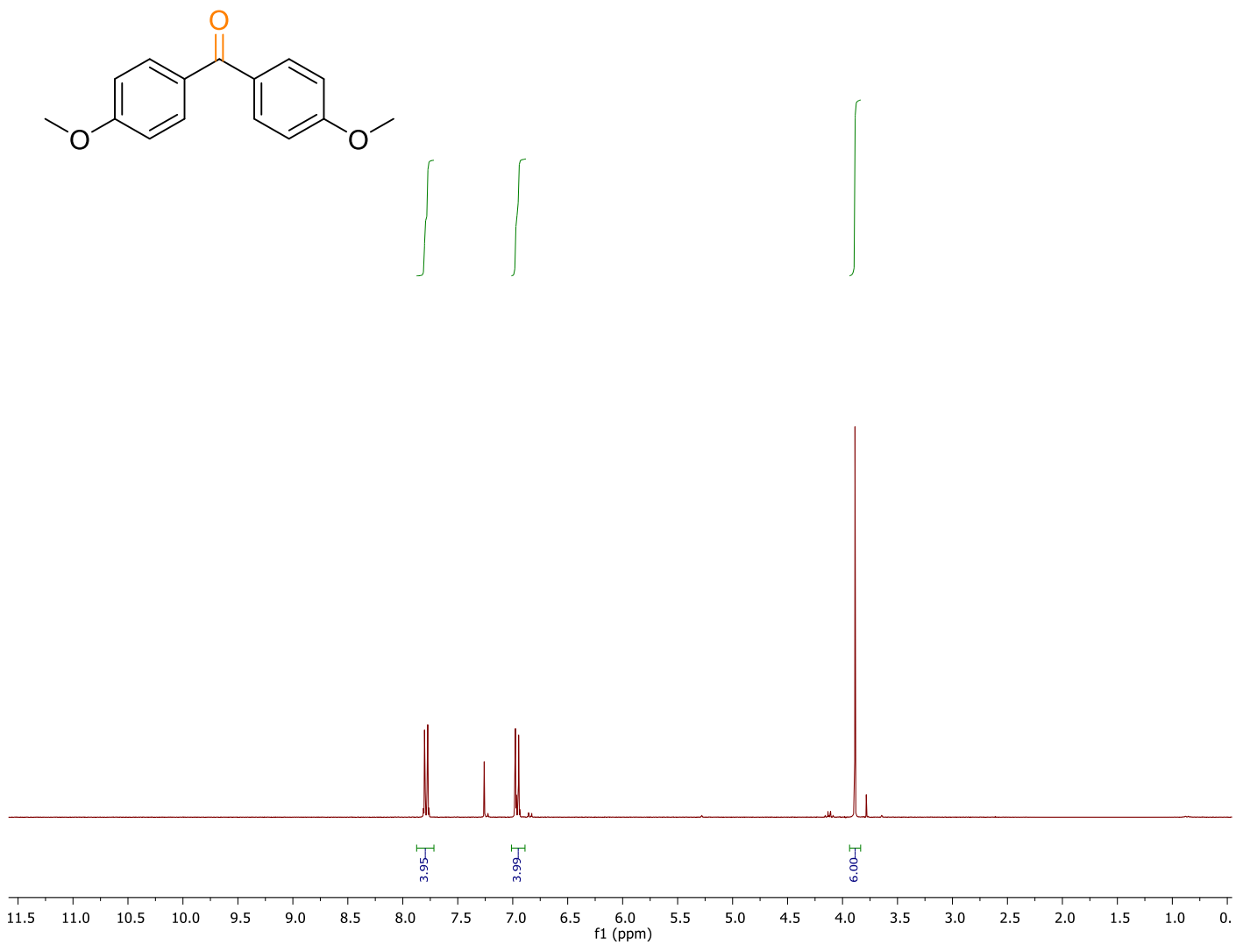

${ }^{1} \mathrm{H}$ NMR spectrum in $\mathrm{CDCl}_{3}$.

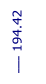

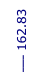

ํㅗำ

$\stackrel{\substack{q \\ \text { mat }}}{1}$

㐌
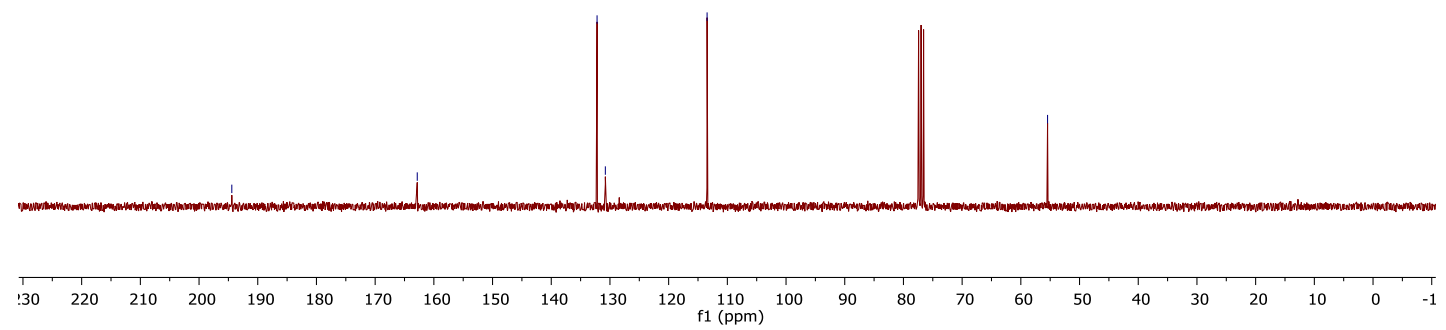

${ }^{13} \mathrm{C}$ NMR spectrum in $\mathrm{CDCl}_{3}$.

230 
Experimental Section

$179 b$
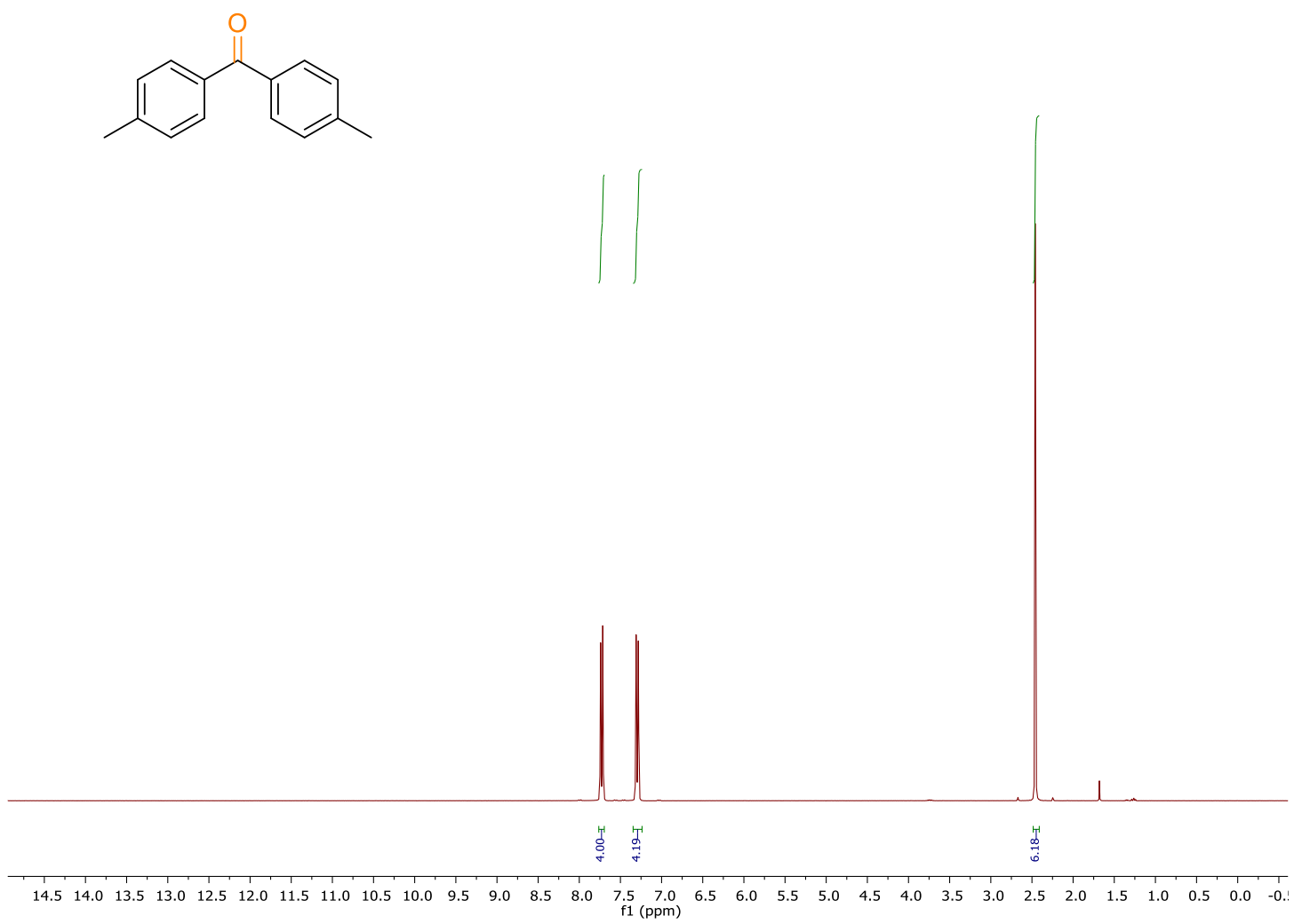

${ }^{1} \mathrm{H}$ NMR spectrum in $\mathrm{CDCl}_{3}$.
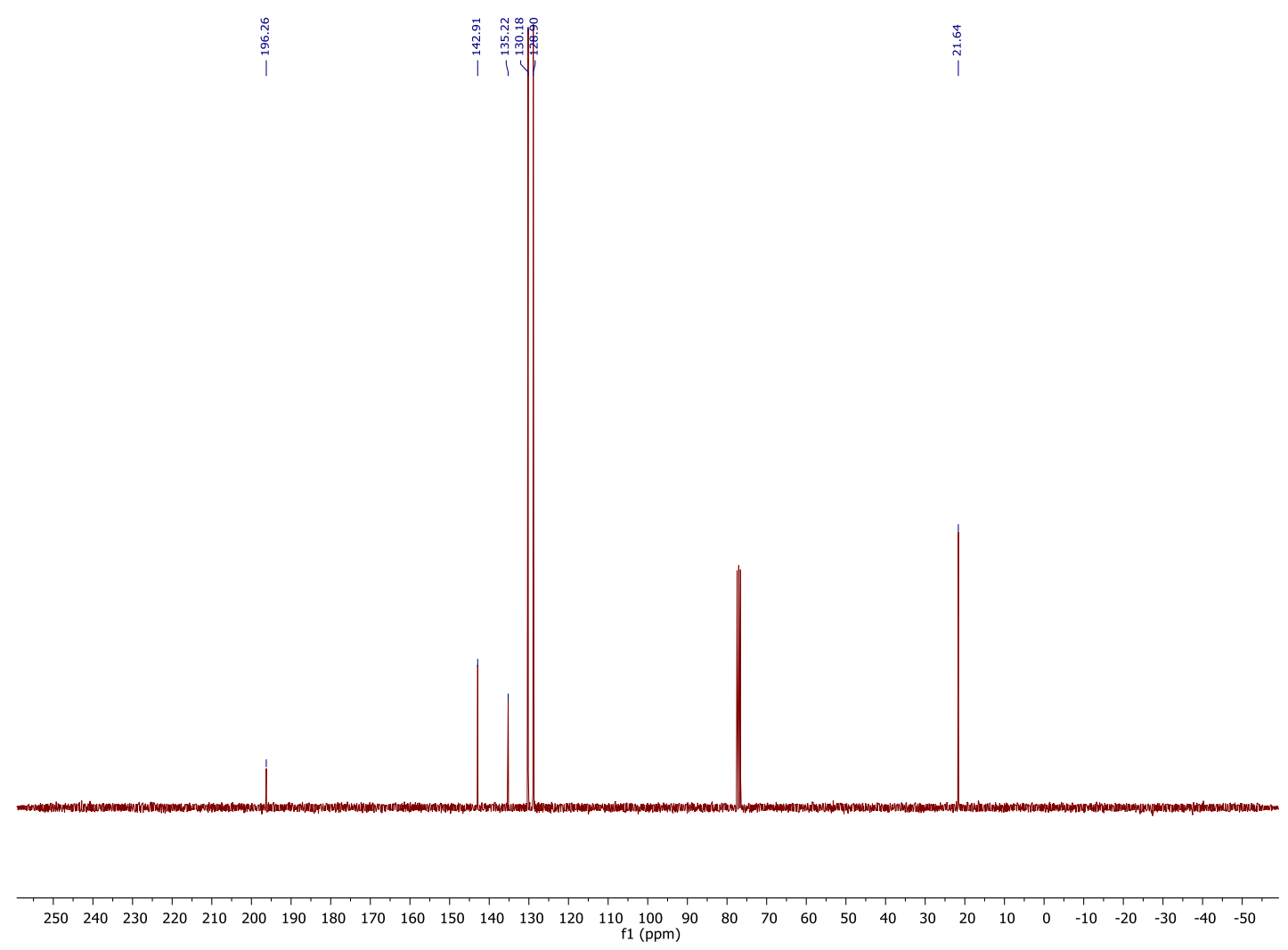

${ }^{13} \mathrm{C}$ NMR spectrum in $\mathrm{CDCl}_{3}$.

231 
180b

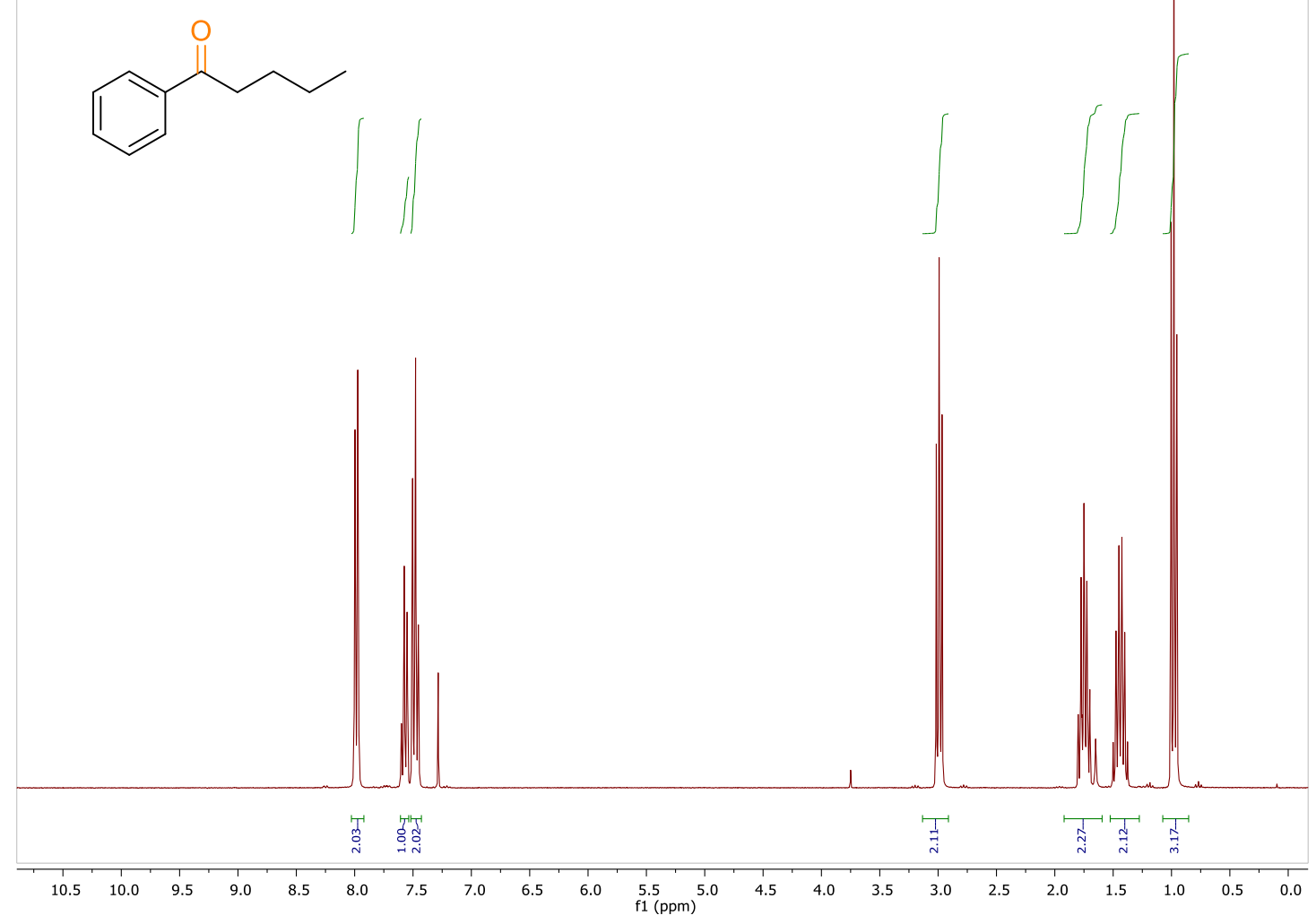

${ }^{1} \mathrm{H}$ NMR spectrum in $\mathrm{CDCl}_{3}$.

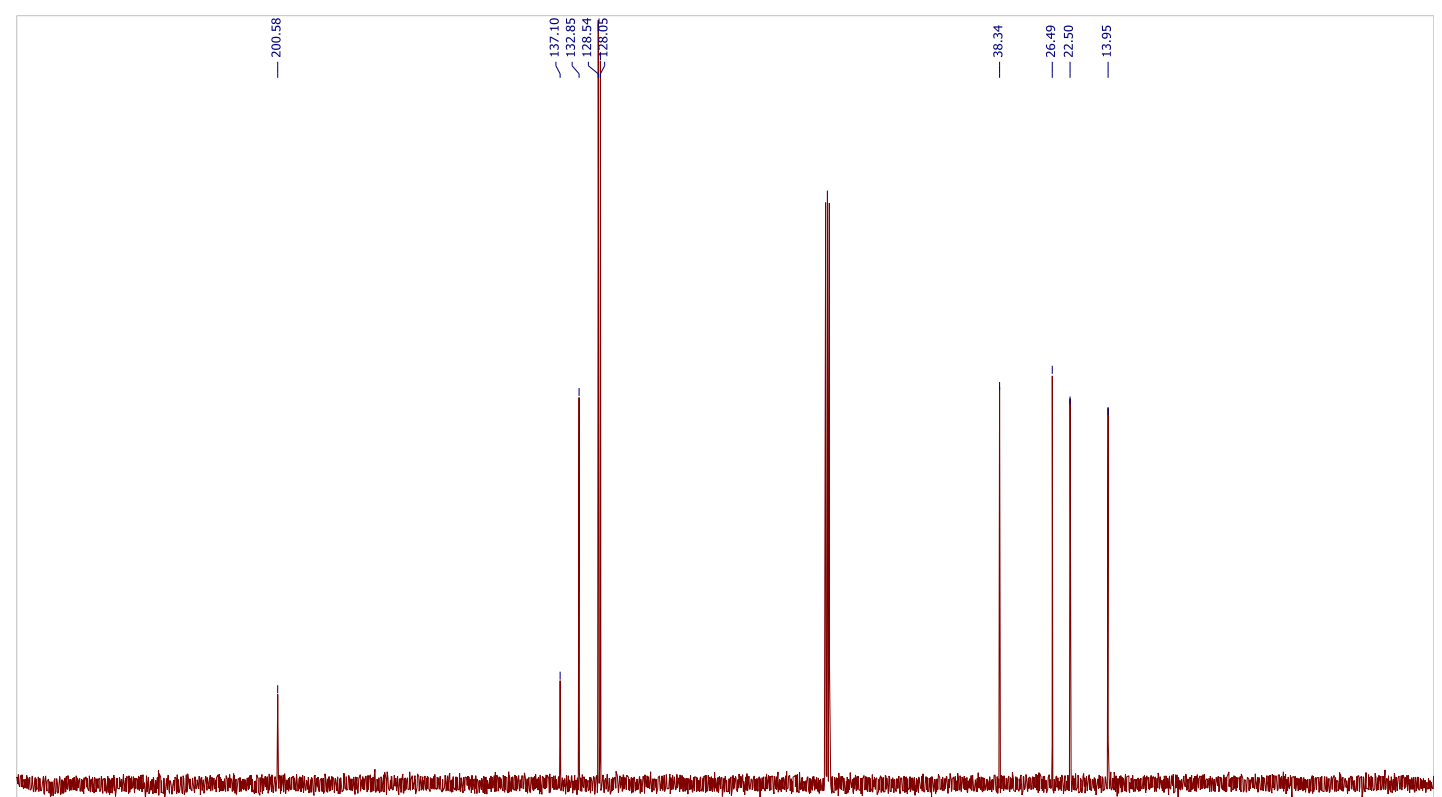

$\begin{array}{lllllllllllllllllllllllllllllllllllllllllllllllll}250 & 240 & 230 & 220 & 210 & 200 & 190 & 180 & 170 & 160 & 150 & 140 & 130 & 120 & 110 & 100 & 90 & 80 & 70 & 60 & 50 & 40 & 30 & 20 & 10 & 0 & -10 & -20 & -30 & -40 & -50\end{array}$

${ }^{13} \mathrm{C}$ NMR spectrum in $\mathrm{CDCl}_{3}$. 
$181 b$<smiles>CC#CCCCCC(=O)c1ccccc1</smiles><smiles>C1=CC2CCC1C2</smiles>

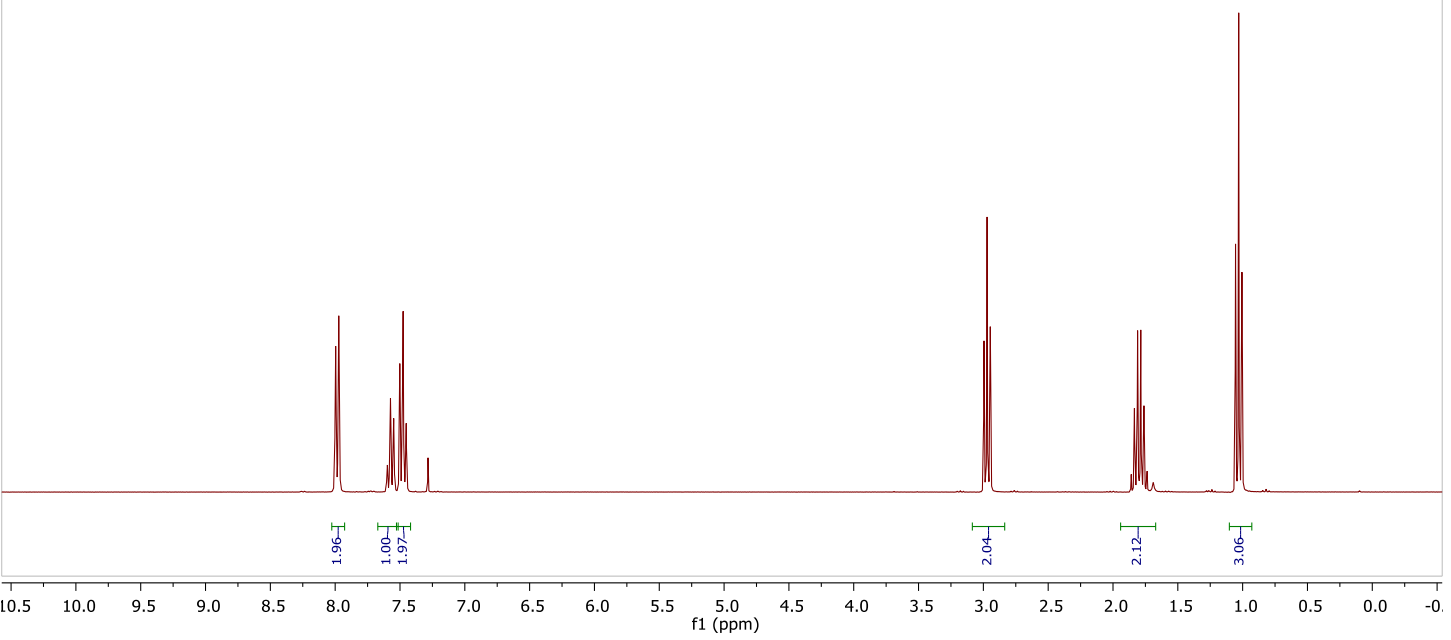

${ }^{1} \mathrm{H}$ NMR spectrum in $\mathrm{CDCl}_{3}$.

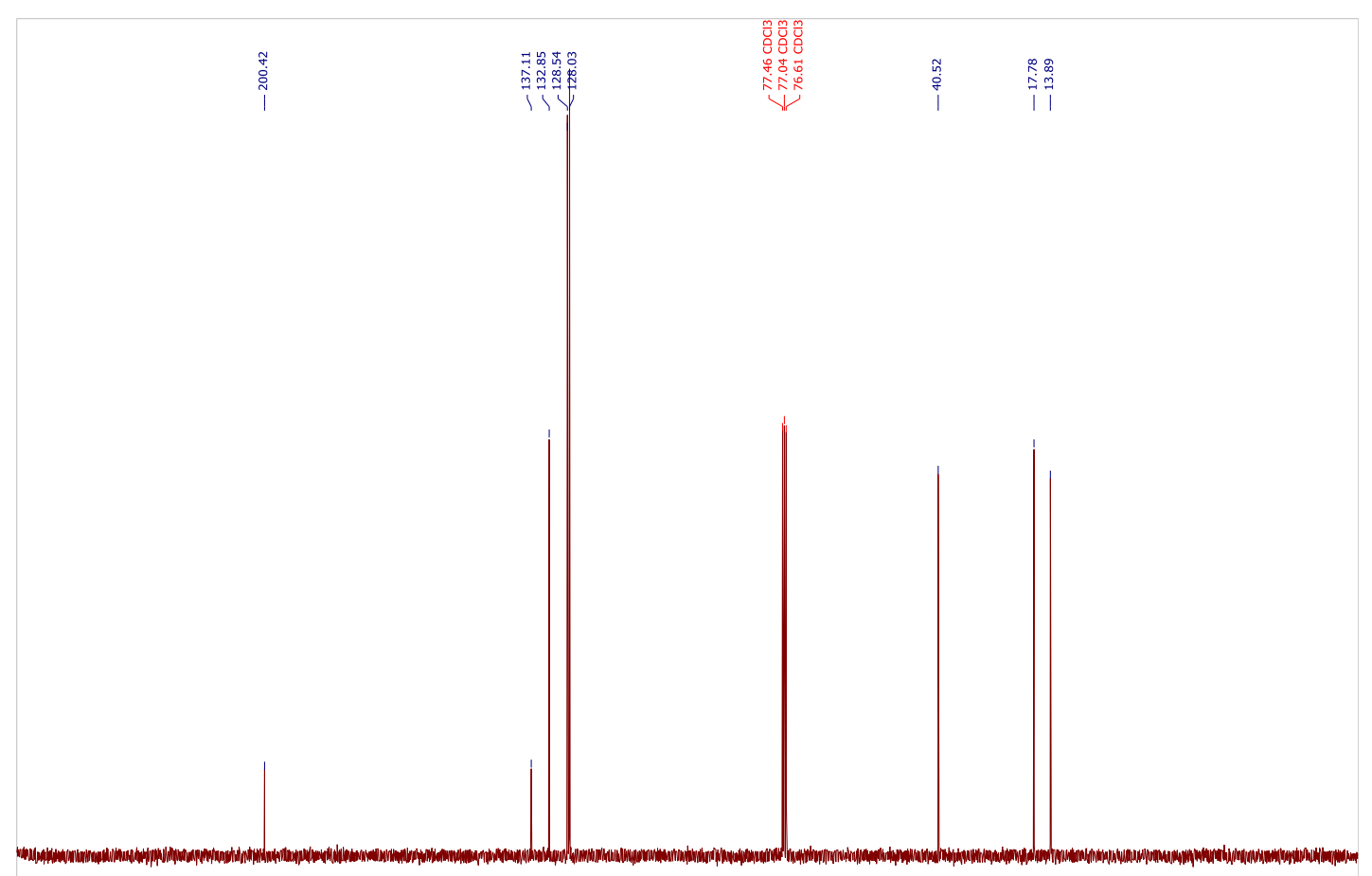

$\begin{array}{lllllllllllllllllllllllllllllllllll}250 & 240 & 230 & 220 & 210 & 200 & 190 & 180 & 170 & 160 & 150 & 140 & 130 & 120 & 110 & 100 & 90 & 80 & 70 & 60 & 50 & 40 & 30 & 20 & 10 & 0 & -10 & -20 & -30 & -40 & -50\end{array}$

${ }^{13} \mathrm{C}$ NMR spectrum in $\mathrm{CDCl}_{3}$. 
Experimental Section

$182 b$

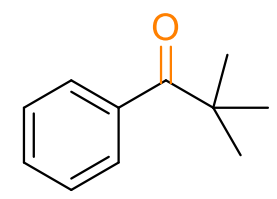

$\int$

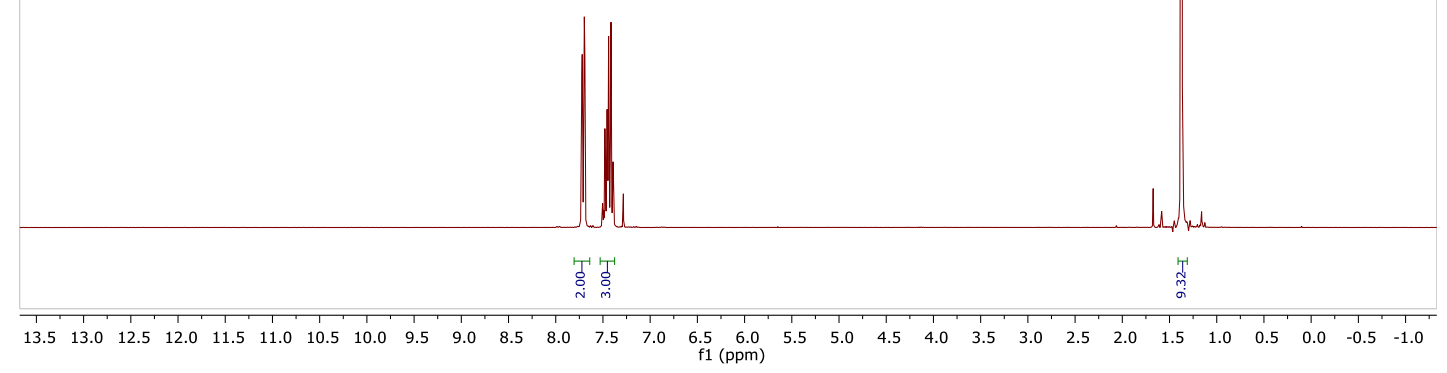

${ }^{1} \mathrm{H}$ NMR spectrum in $\mathrm{CDCl}_{3}$.

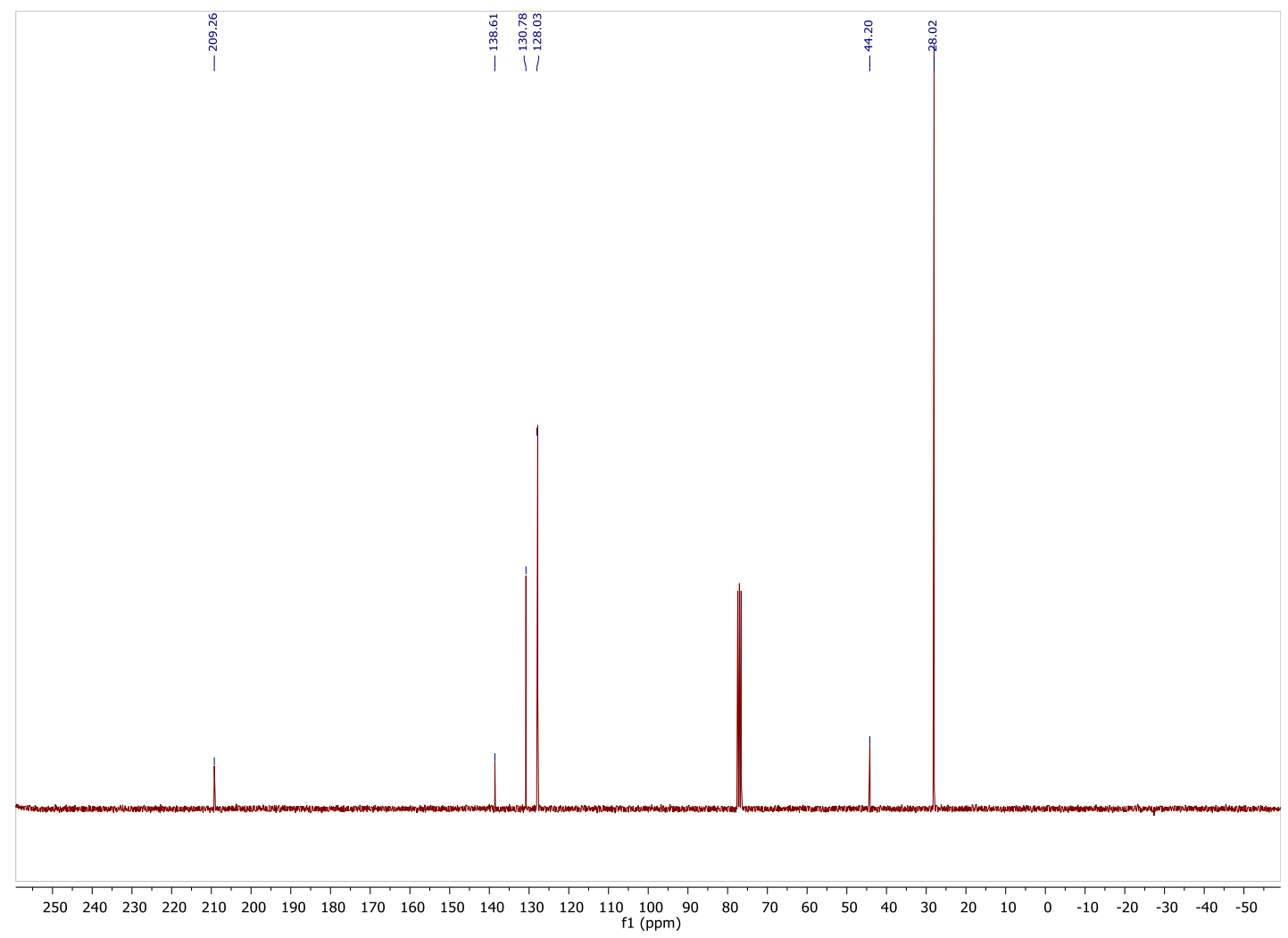

${ }^{13} \mathrm{C}$ NMR spectrum in $\mathrm{CDCl}_{3}$.

234 
Experimental Section

$183 b$

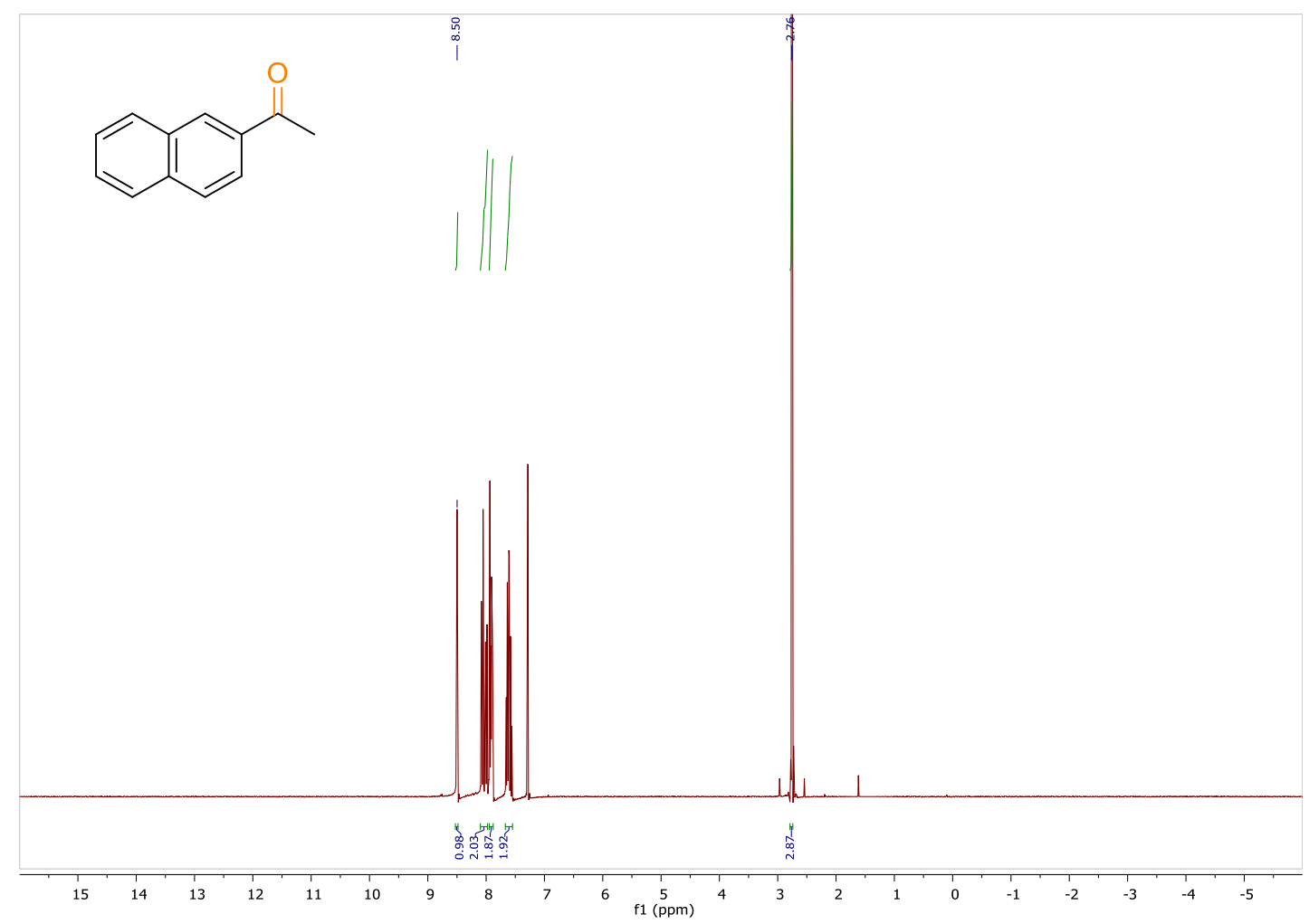

${ }^{1} \mathrm{H}$ NMR spectrum in $\mathrm{CDCl}_{3}$.

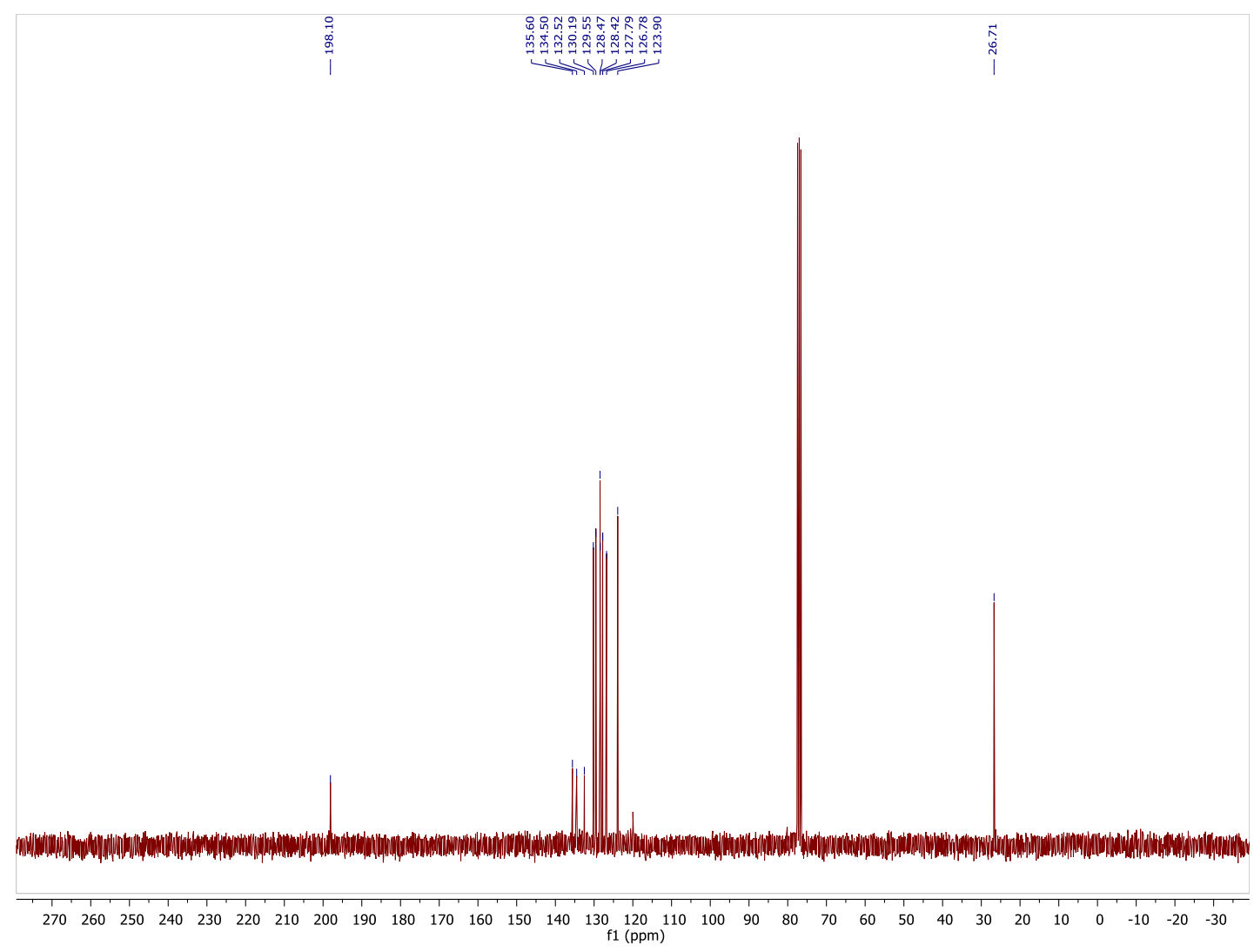

${ }^{13} \mathrm{C}$ NMR spectrum in $\mathrm{CDCl}_{3}$.

235 
Experimental Section

$184 b$
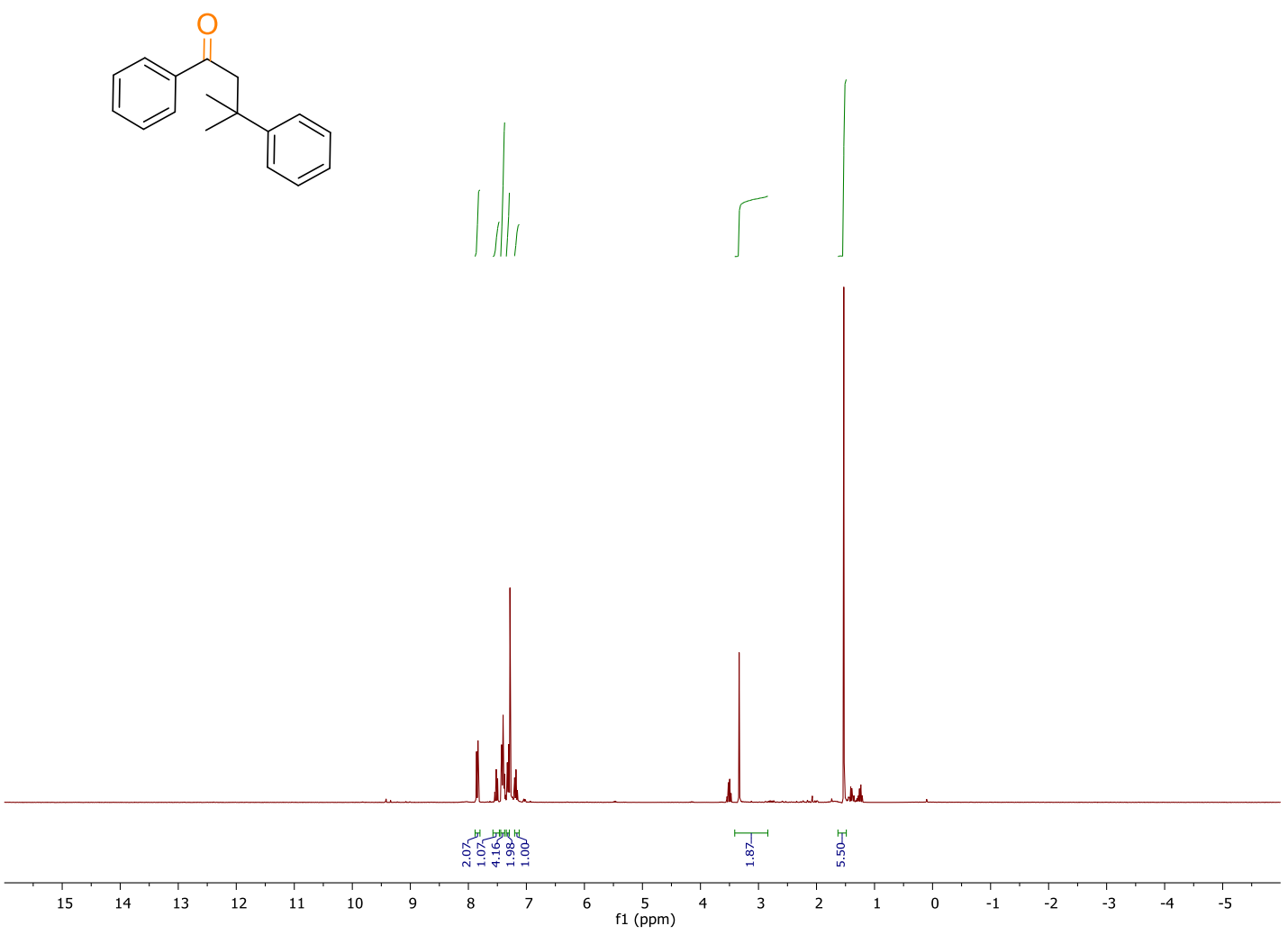

${ }^{1} \mathrm{H}$ NMR spectrum in $\mathrm{CDCl}_{3}$.

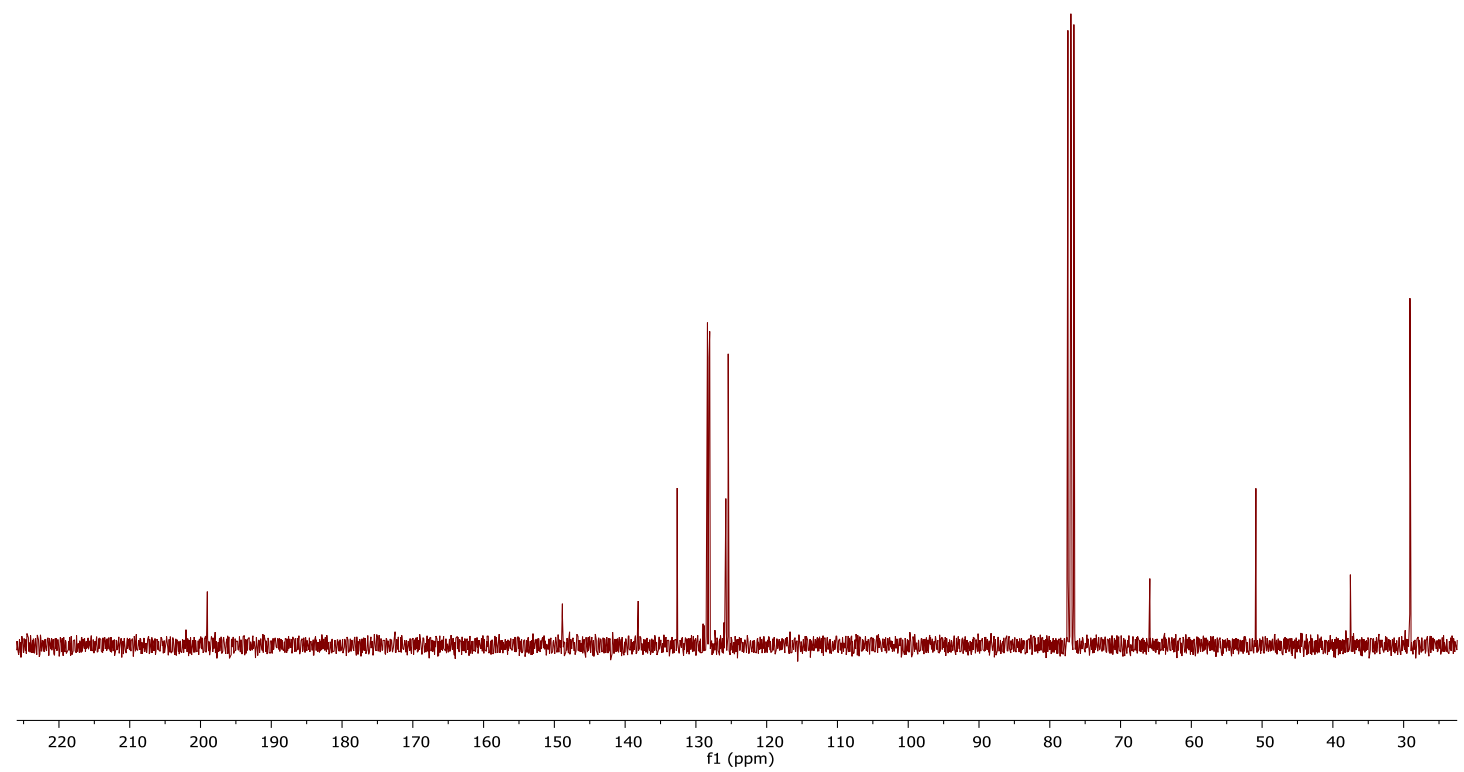

${ }^{13} \mathrm{C}$ NMR spectrum in $\mathrm{CDCl}_{3}$.

236 
185b

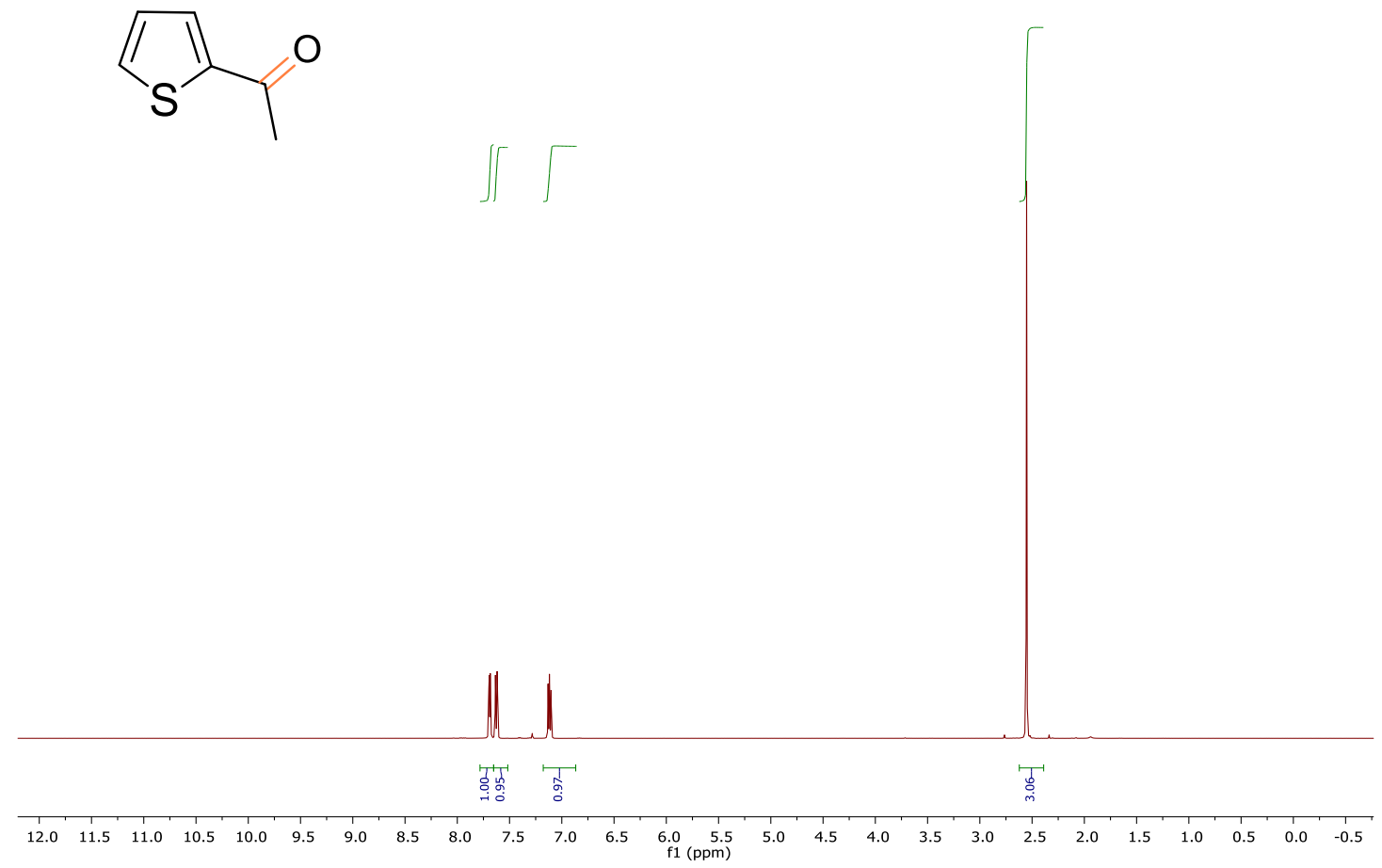

${ }^{1} \mathrm{H}$ NMR spectrum in $\mathrm{CDCl}_{3}$.

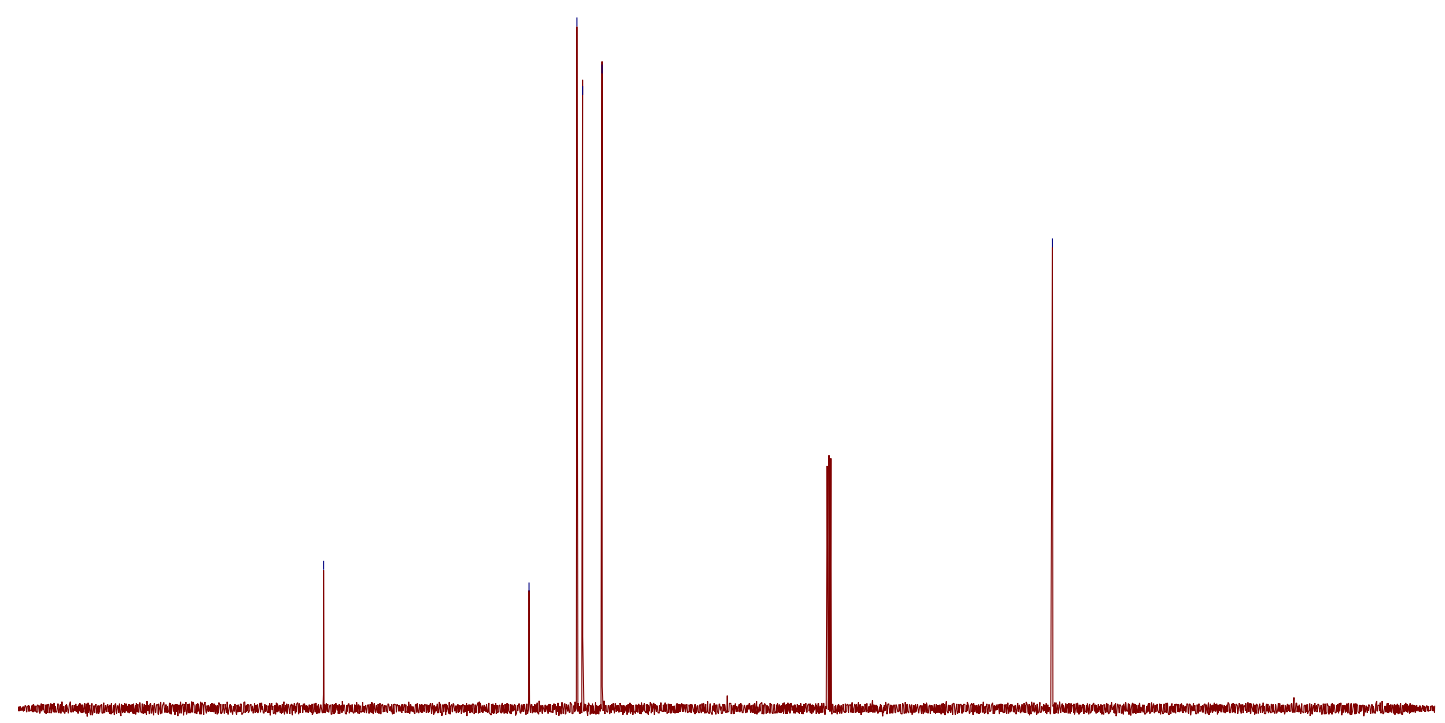

$\begin{array}{llllllllllllllllllllllllllllllllllllllllll}250 & 240 & 230 & 220 & 210 & 200 & 190 & 180 & 170 & 160 & 150 & 140 & 130 & 120 & 110 & 100 & 90 & 80 & 70 & 60 & 50 & 40 & 30 & 20 & 10 & 0 & -10 & -20 & -30 & -40 & -50\end{array}$

${ }^{13} \mathrm{C}$ NMR spectrum in $\mathrm{CDCl}_{3}$. 
$186 b$

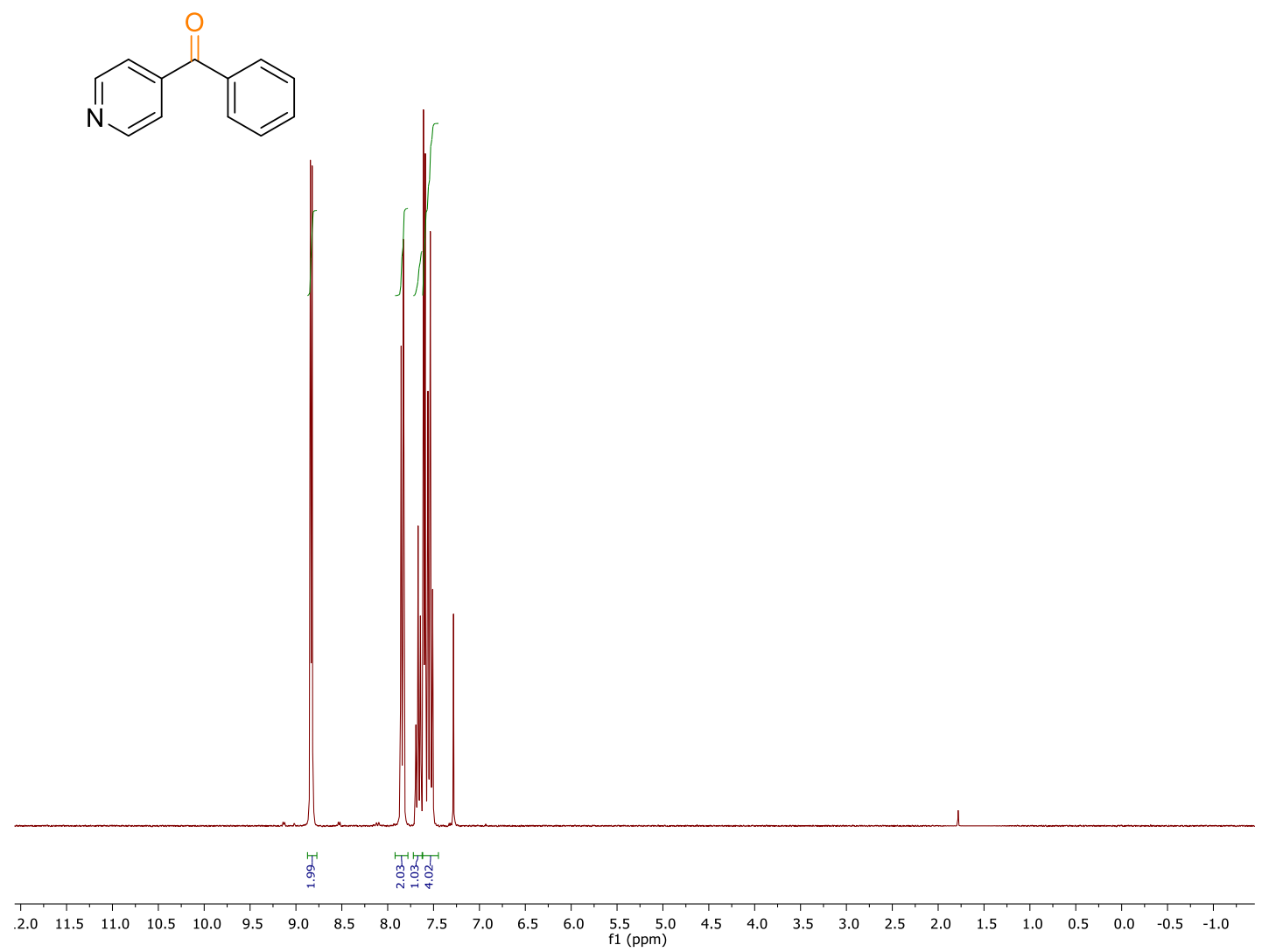

${ }^{1} \mathrm{H}$ NMR spectrum in $\mathrm{CDCl}_{3}$.

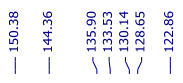

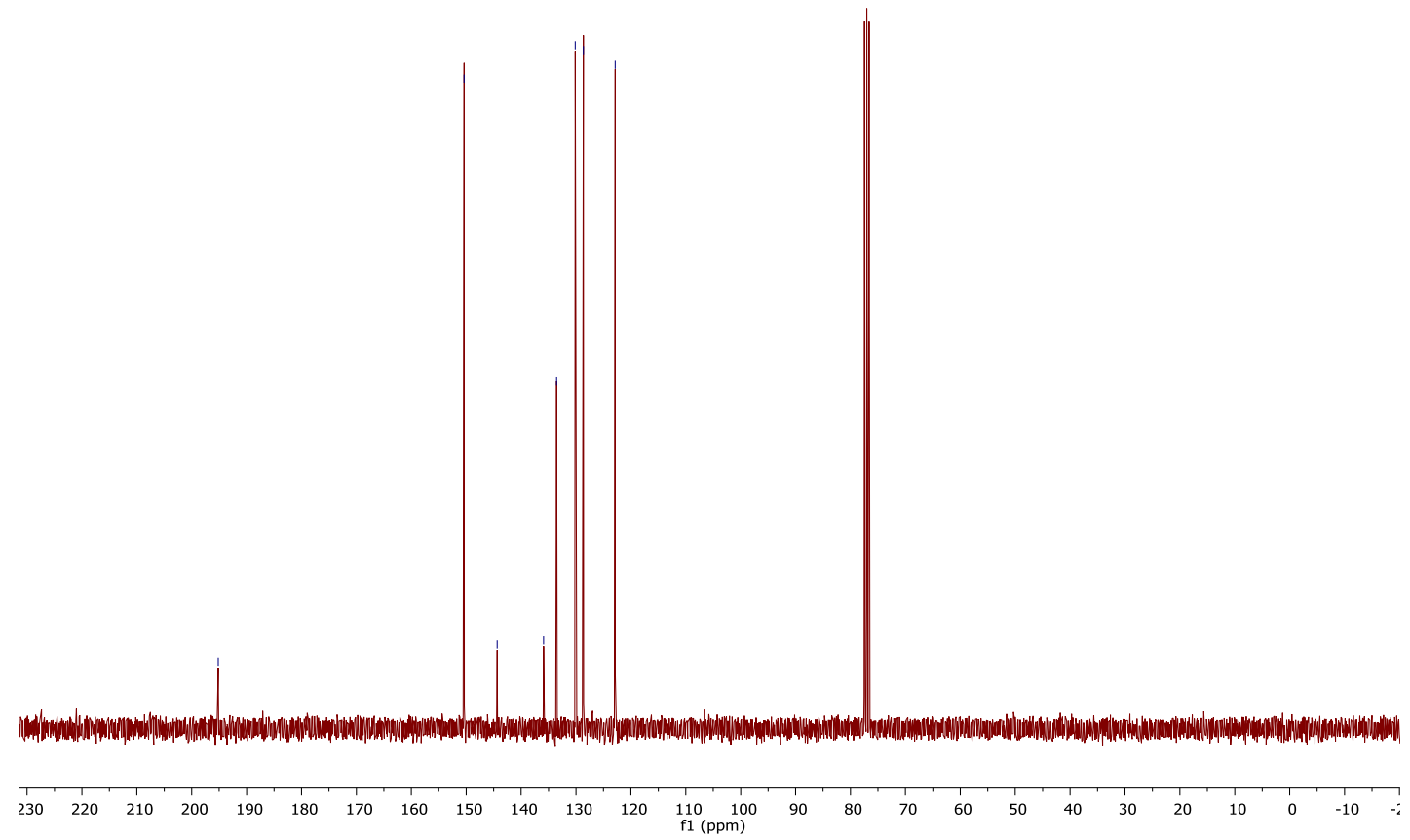

${ }^{13} \mathrm{C} \mathrm{NMR}$ spectrum in $\mathrm{CDCl}_{3}$. 
$187 b$

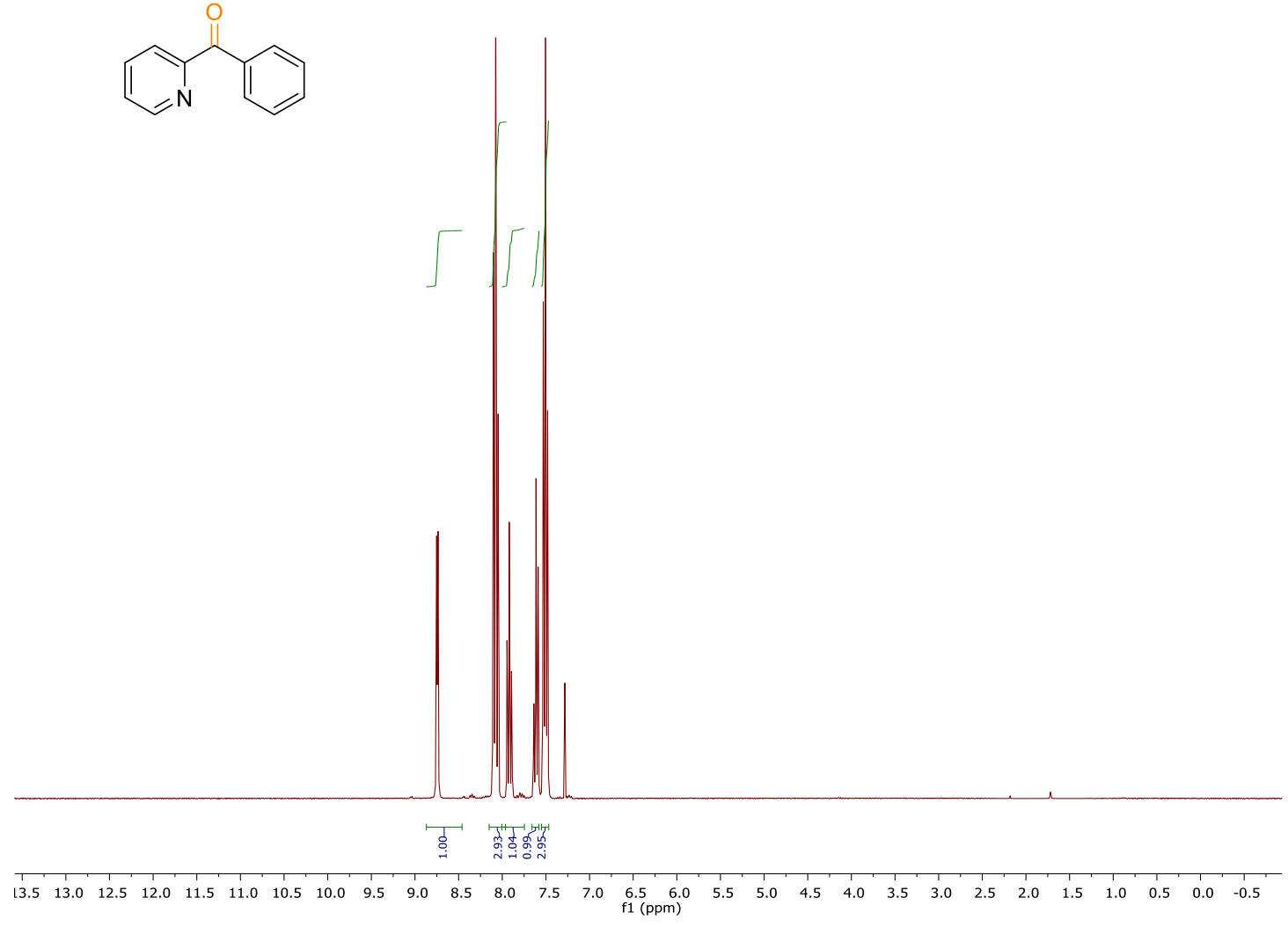

${ }^{1} \mathrm{H}$ NMR spectrum in $\mathrm{CDCl}_{3}$.

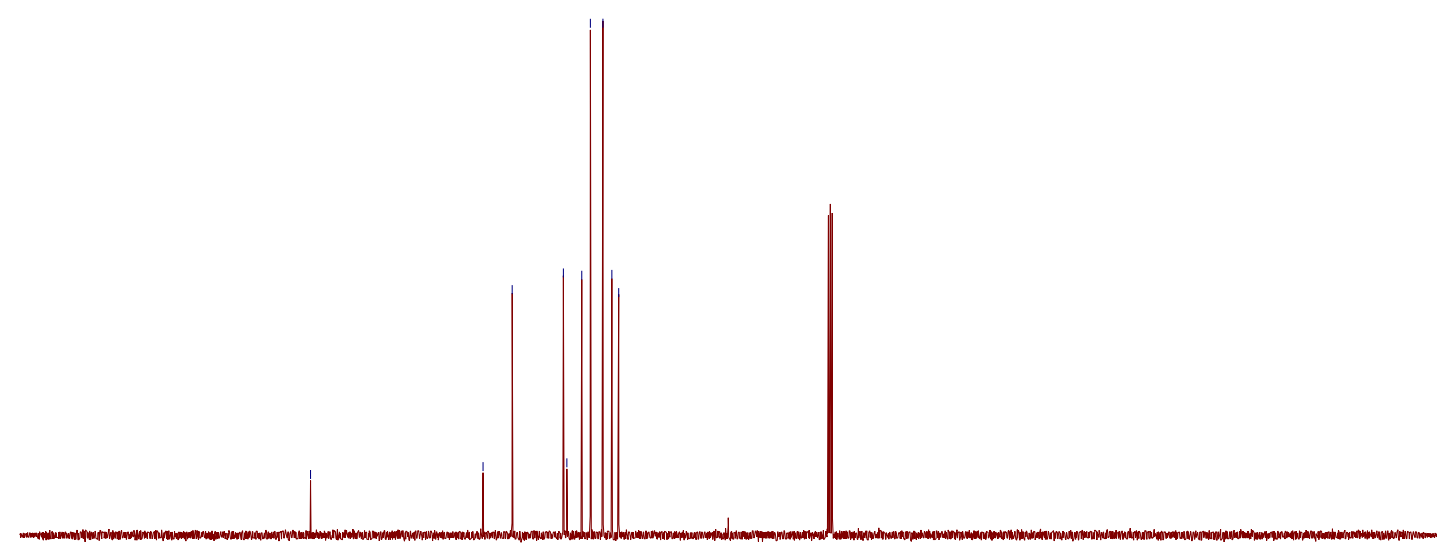

$\begin{array}{lllllllllllllllllllllllllllllllllllllllllllllllll}250 & 240 & 230 & 220 & 210 & 200 & 190 & 180 & 170 & 160 & 150 & 140 & 130 & 120 & 110 & 100 & 90 & 80 & 70 & 60 & 50 & 40 & 30 & 20 & 10 & 0 & -10 & -20 & -30 & -40 & -50\end{array}$

${ }^{13} \mathrm{C}$ NMR spectrum in $\mathrm{CDCl}_{3}$. 
$188 b$<smiles>Cc1ccc(C=O)cc1C</smiles>

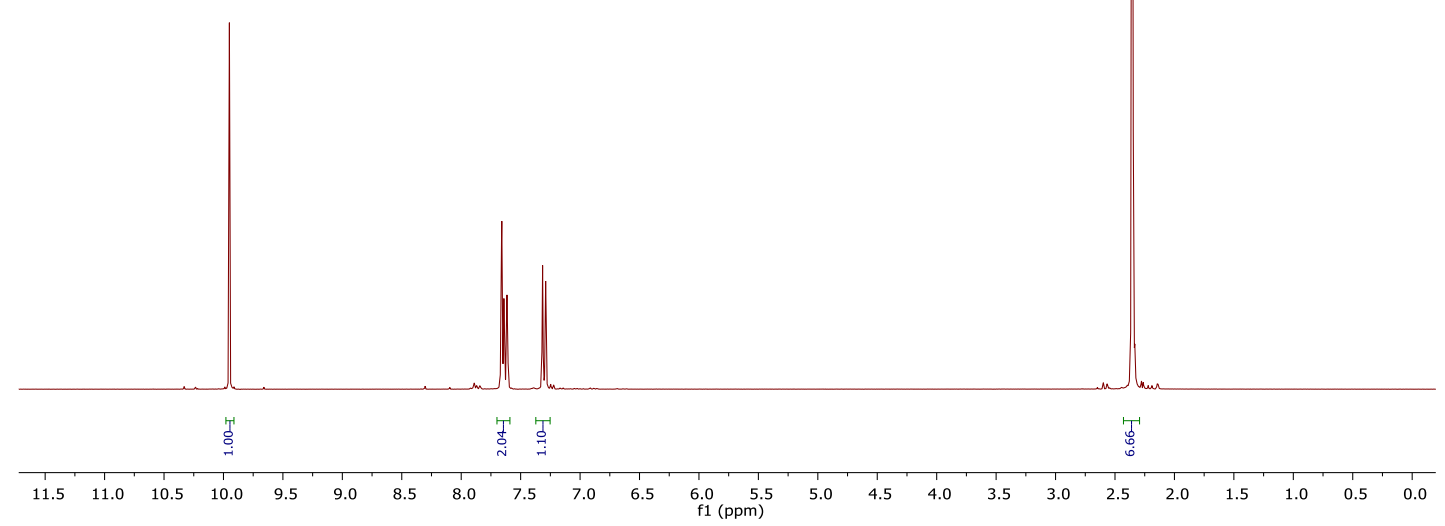

${ }^{1} \mathrm{H}$ NMR spectrum in $\mathrm{CDCl}_{3}$.
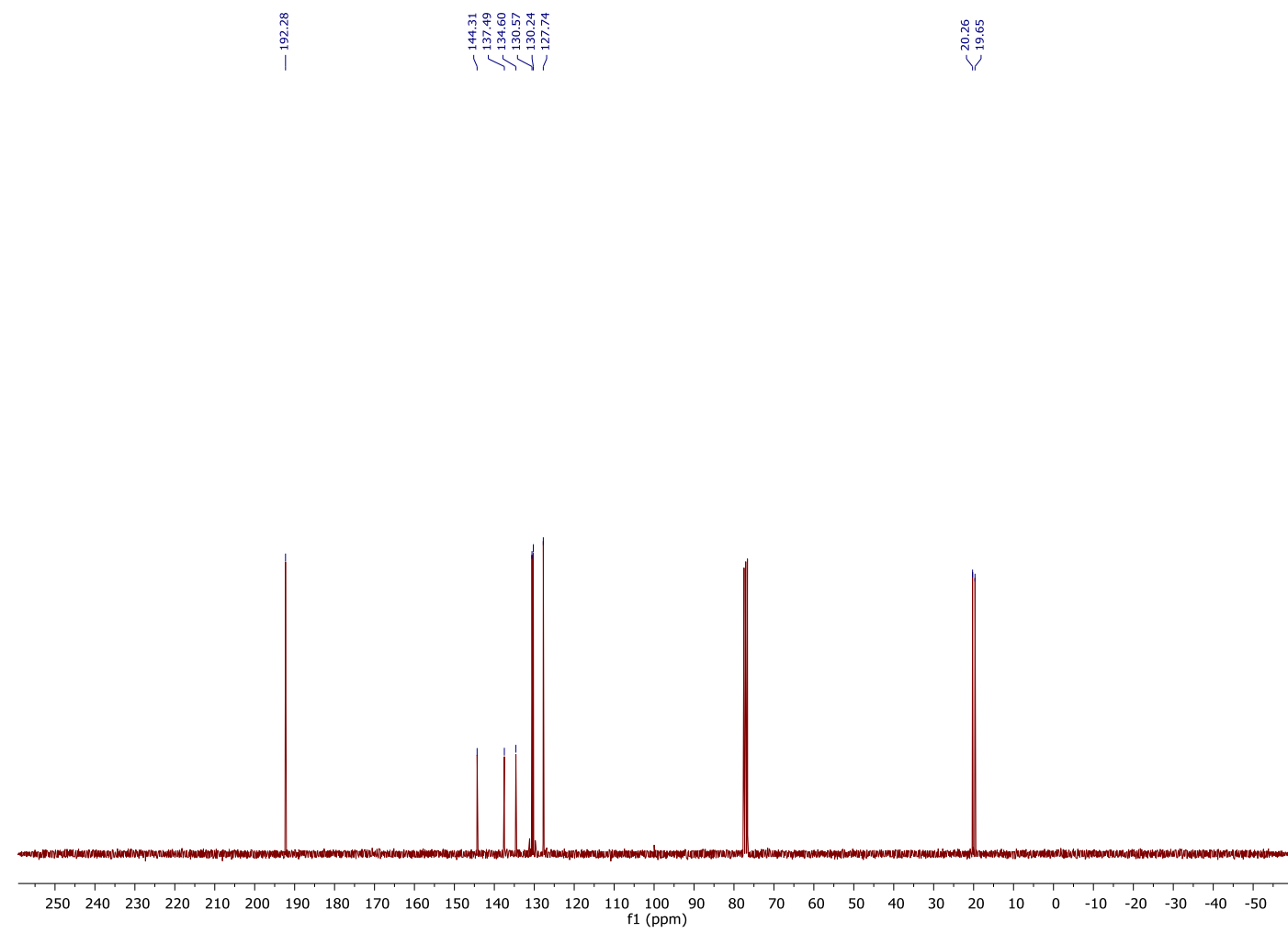

${ }^{13} \mathrm{C}$ NMR spectrum in $\mathrm{CDCl}_{3}$. 
189b<smiles>COc1cc(OC)c(OC)cc1C=O</smiles>

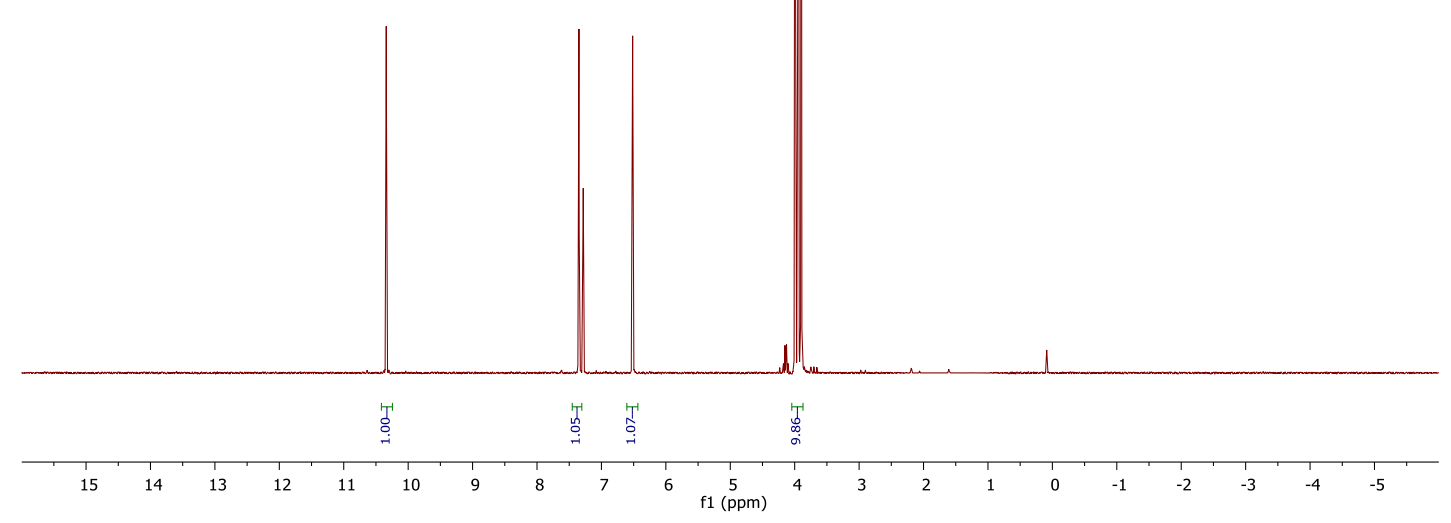

${ }^{1} \mathrm{H}$ NMR spectrum in $\mathrm{CDCl}_{3}$.

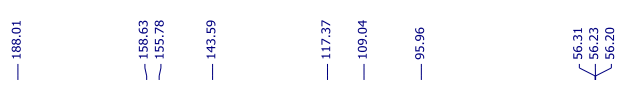

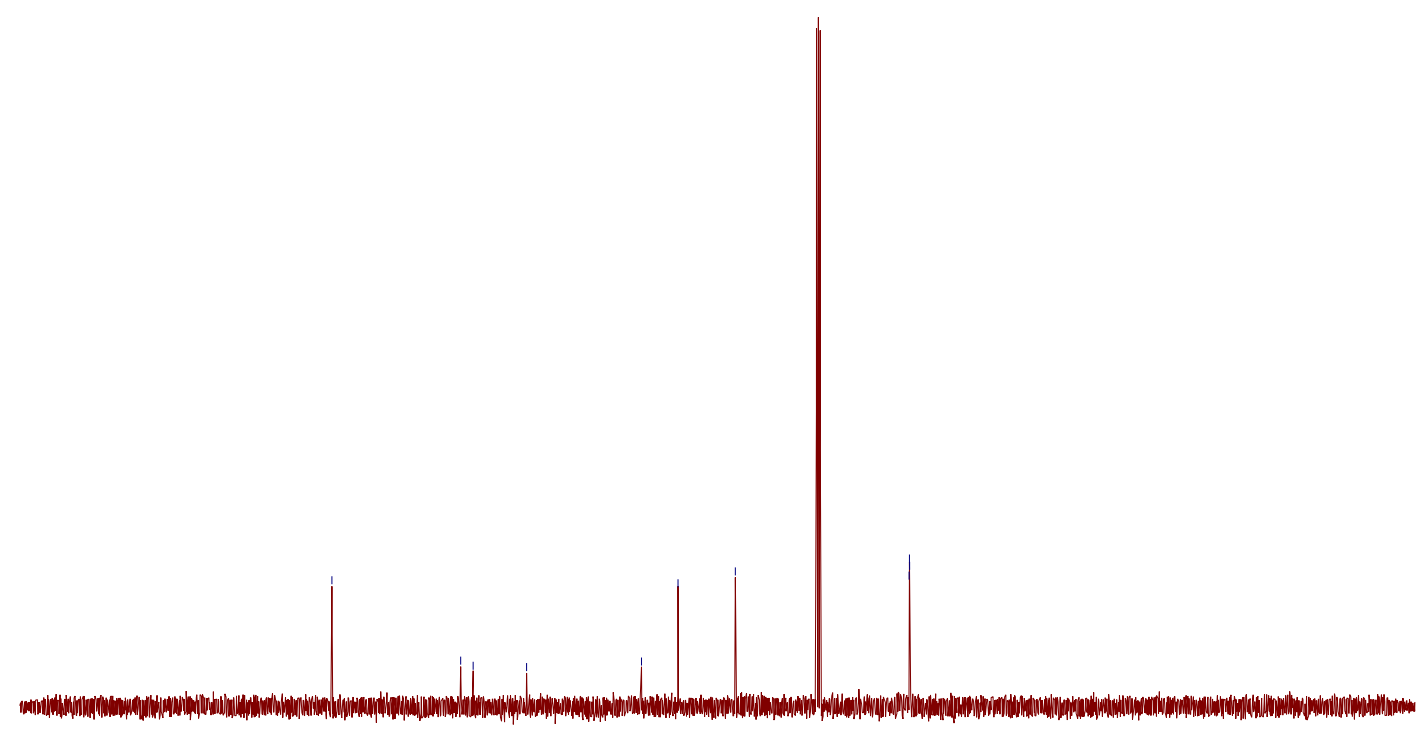

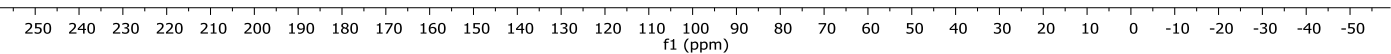

${ }^{13} \mathrm{C} \mathrm{NMR}$ spectrum in $\mathrm{CDCl}_{3}$. 
190b

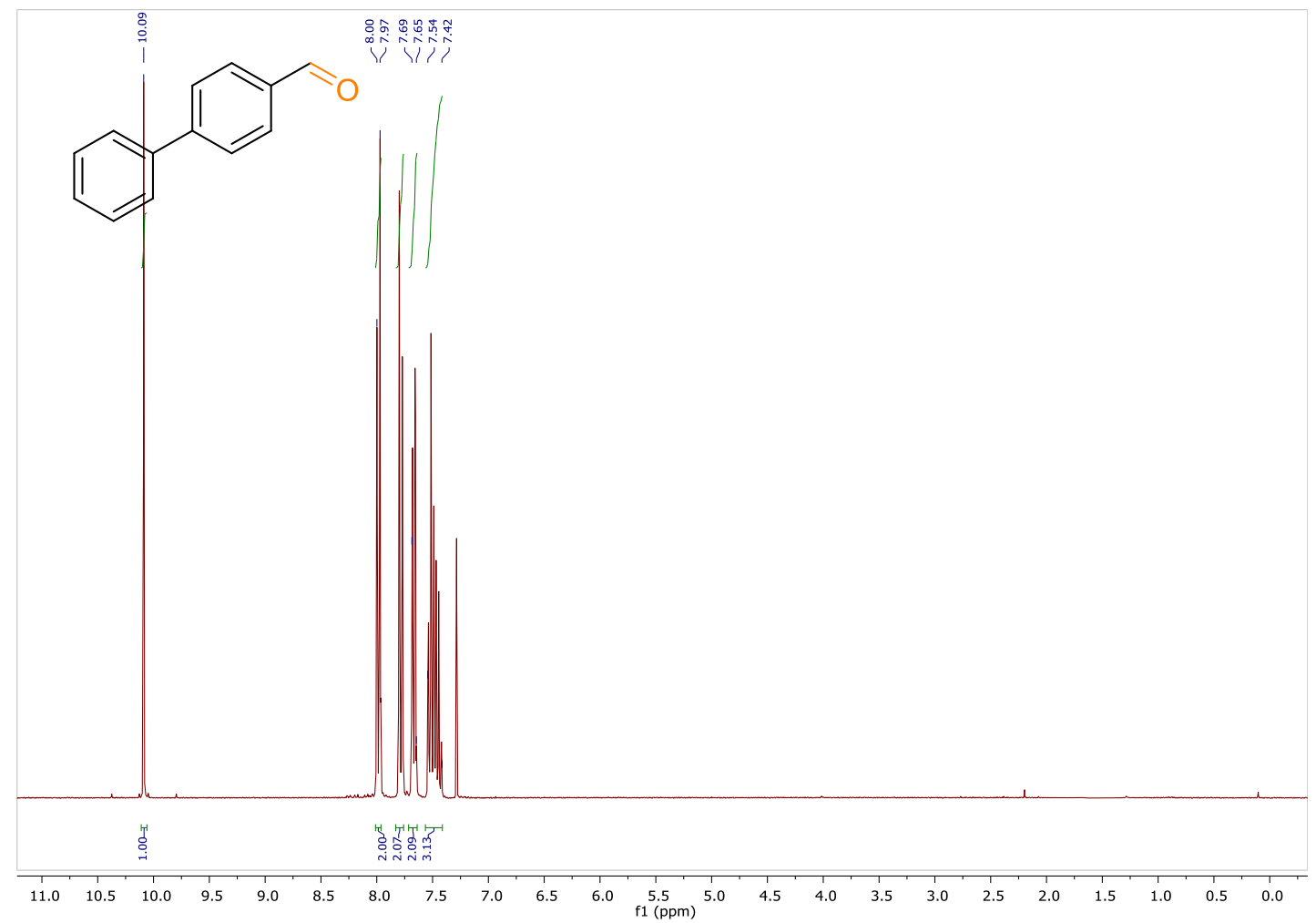

${ }^{1} \mathrm{H}$ NMR spectrum in $\mathrm{CDCl}_{3}$.

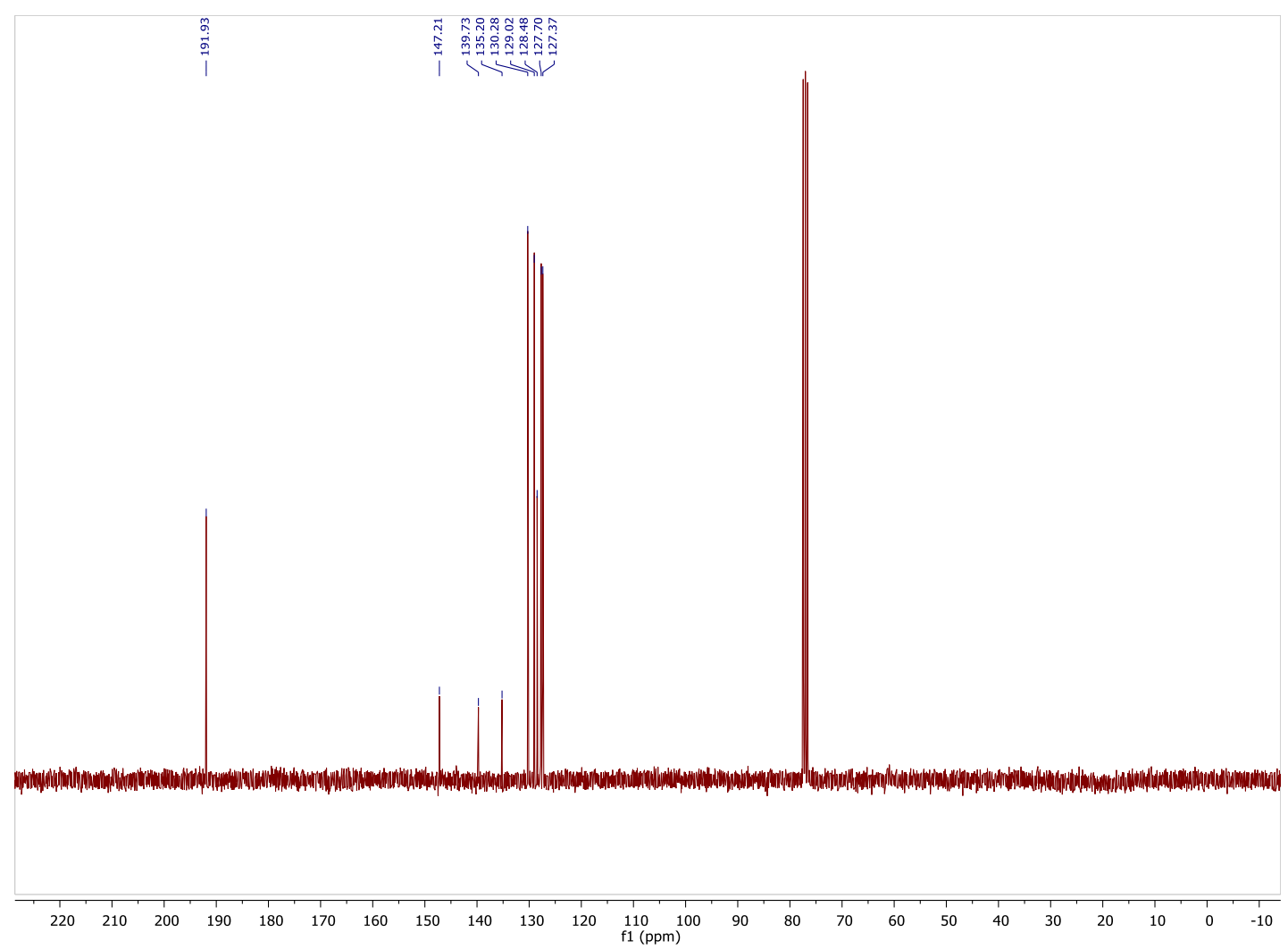

${ }^{13} \mathrm{C}$ NMR spectrum in $\mathrm{CDCl}_{3}$. 
191b<smiles>COc1ccc(C=O)cc1OC</smiles>
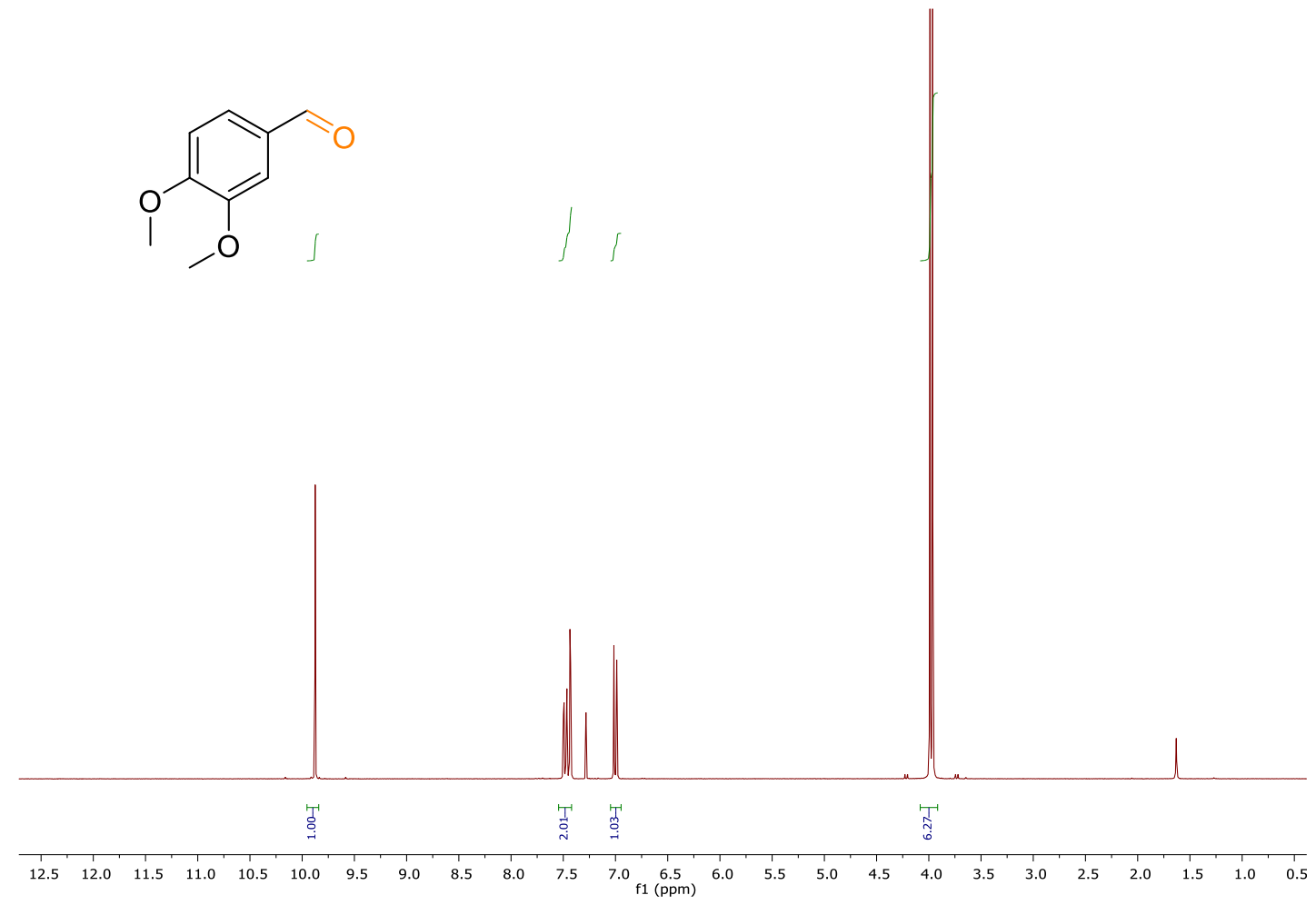

${ }^{1} \mathrm{H}$ NMR spectrum in $\mathrm{CDCl}_{3}$.

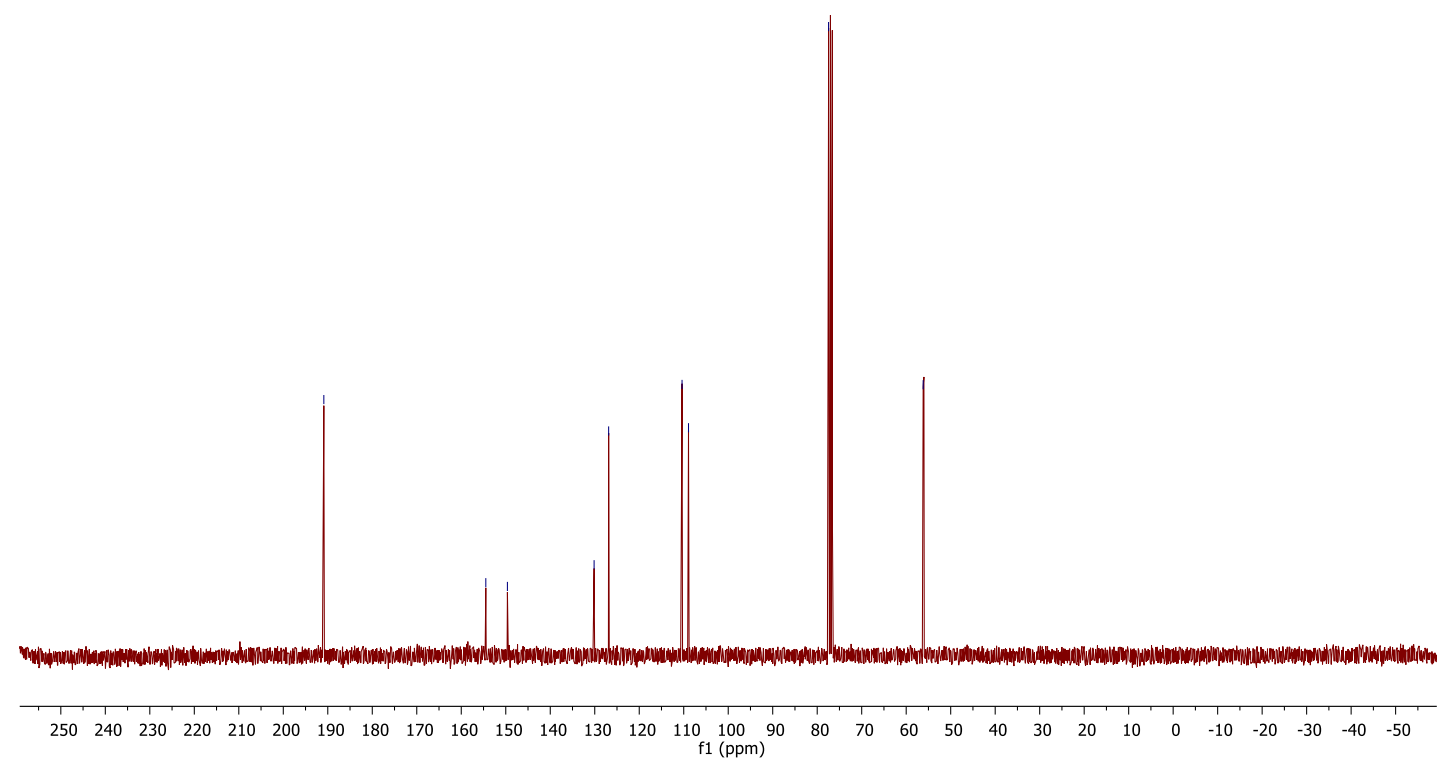

${ }^{13} \mathrm{C}$ NMR spectrum in $\mathrm{CDCl}_{3}$. 
$192 b$

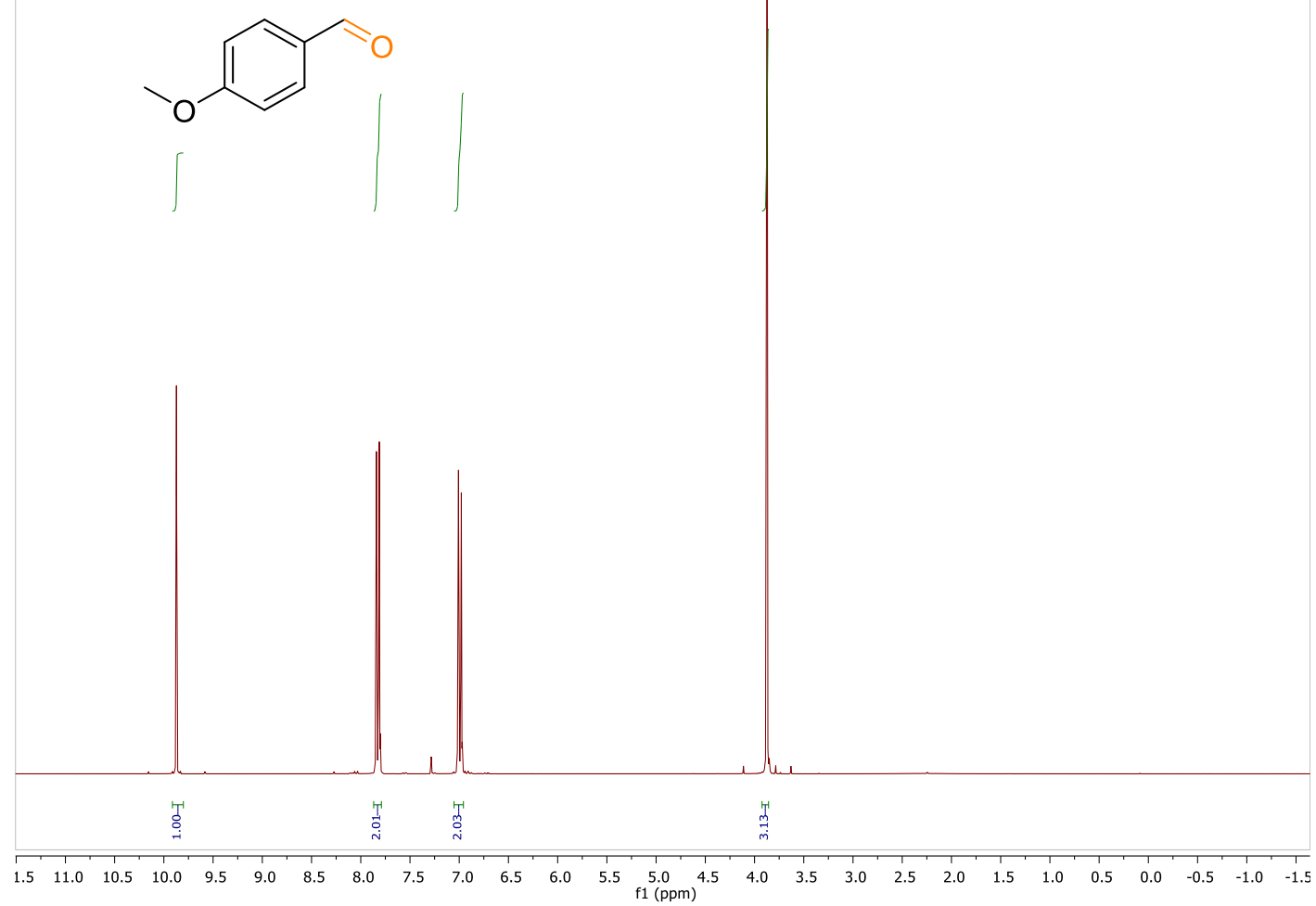

${ }^{1} \mathrm{H}$ NMR spectrum in $\mathrm{CDCl}_{3}$.

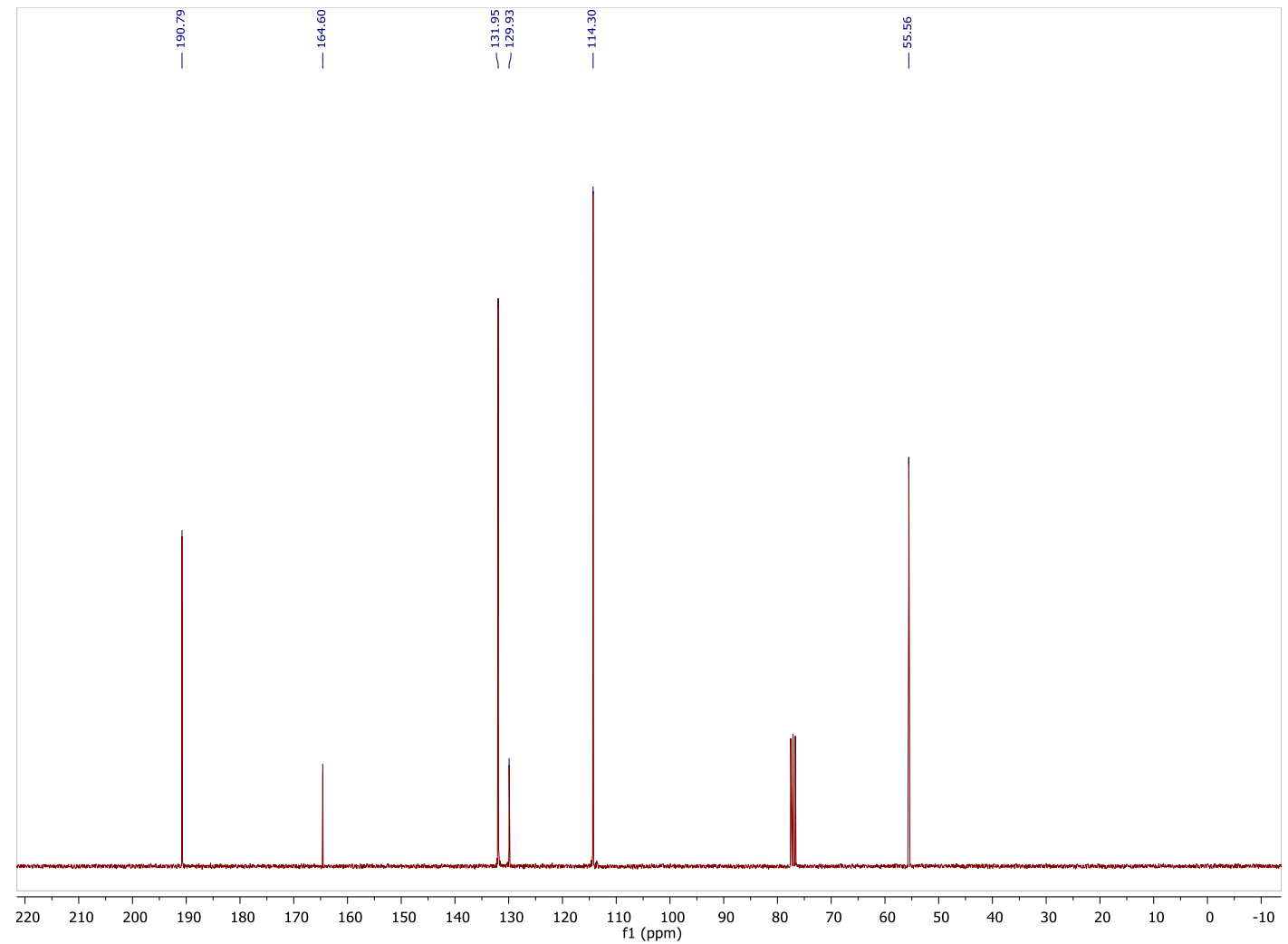

${ }^{13} \mathrm{C}$ NMR spectrum in $\mathrm{CDCl}_{3}$. 
193b
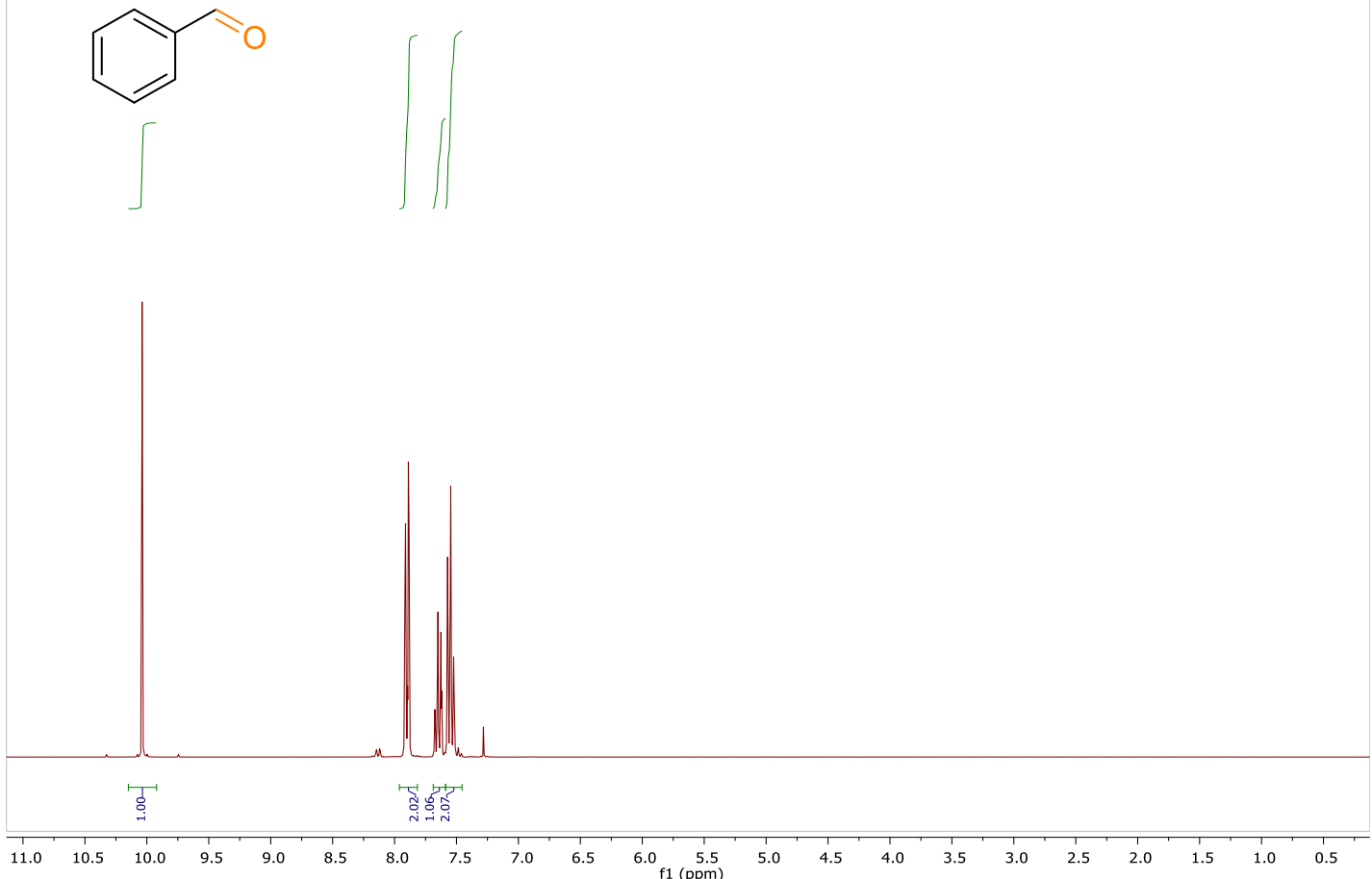

${ }^{1} \mathrm{H}$ NMR spectrum in $\mathrm{CDCl}_{3}$.

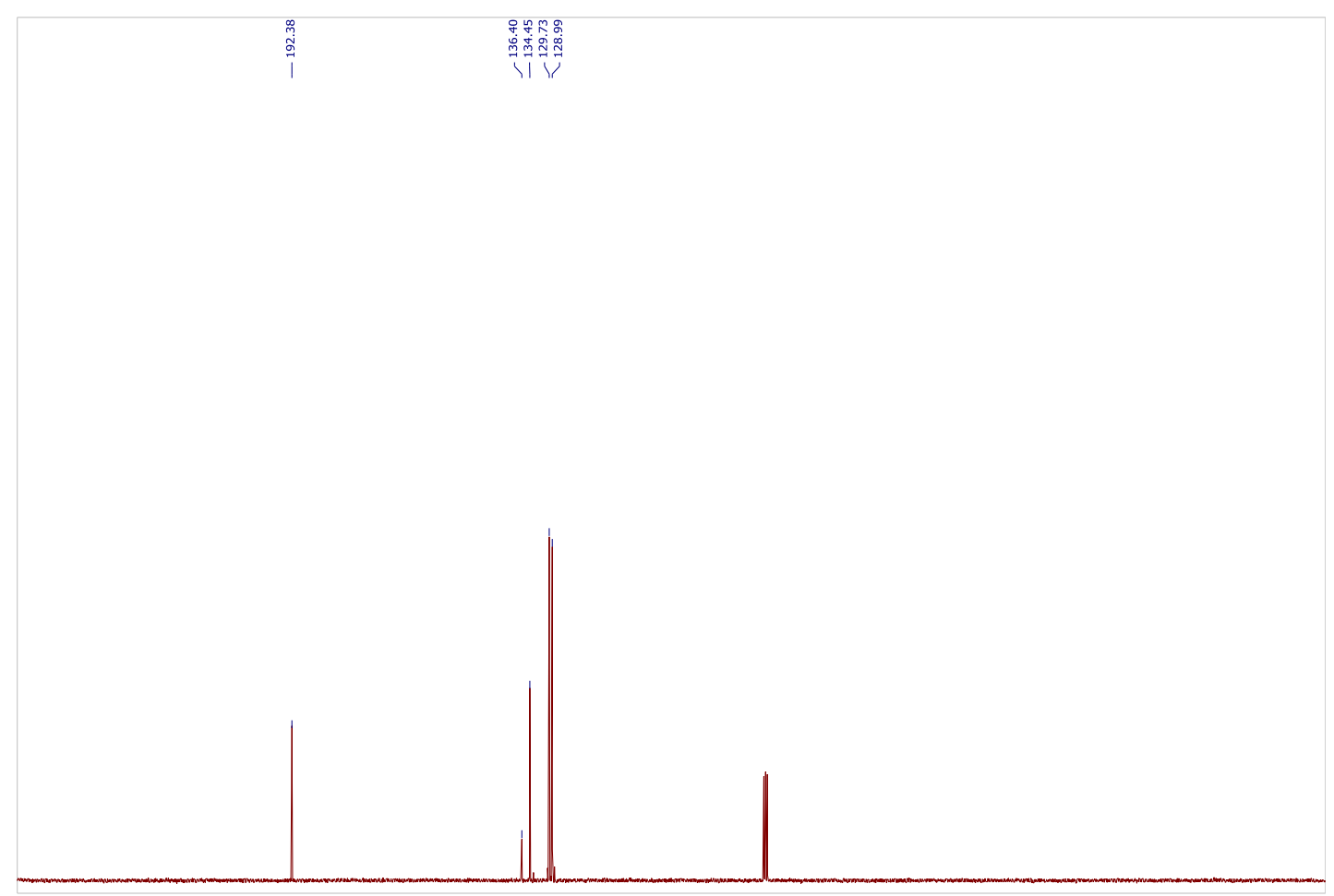

$\begin{array}{lllllllllllllllllllllllllllllllll}250 & 240 & 230 & 220 & 210 & 200 & 190 & 180 & 170 & 160 & 150 & 140 & 130 & 120 & 110 & 100 & 90 & 80 & 70 & 60 & 50 & 40 & 30 & 20 & 10 & 0 & -10 & -20 & -30 & -40 & -50\end{array}$

${ }^{13} \mathrm{C}$ NMR spectrum in $\mathrm{CDCl}_{3}$. 
291b

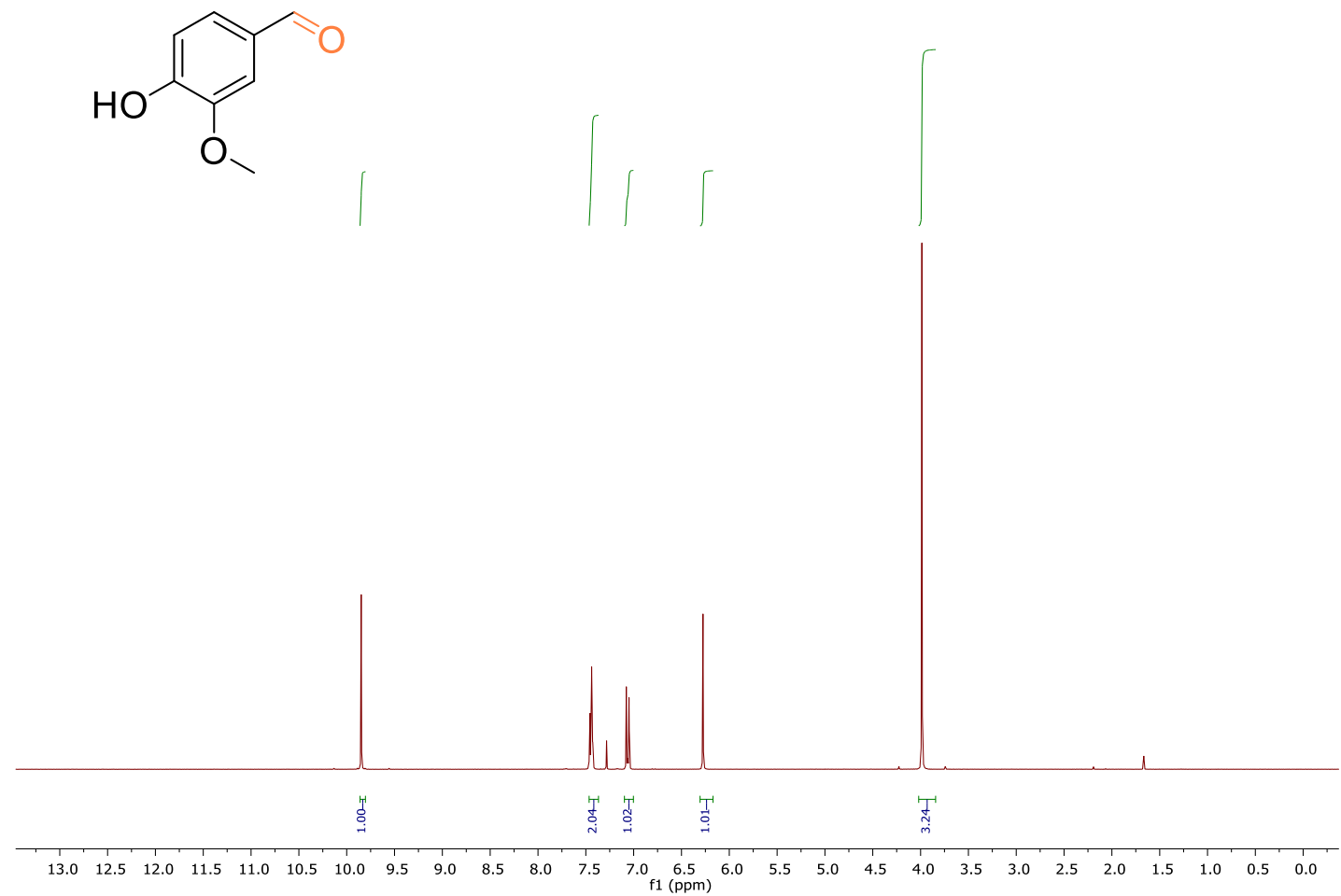

${ }^{1} \mathrm{H}$ NMR spectrum in $\mathrm{CDCl}_{3}$.

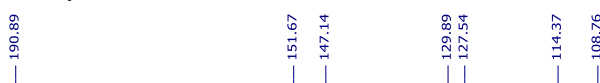

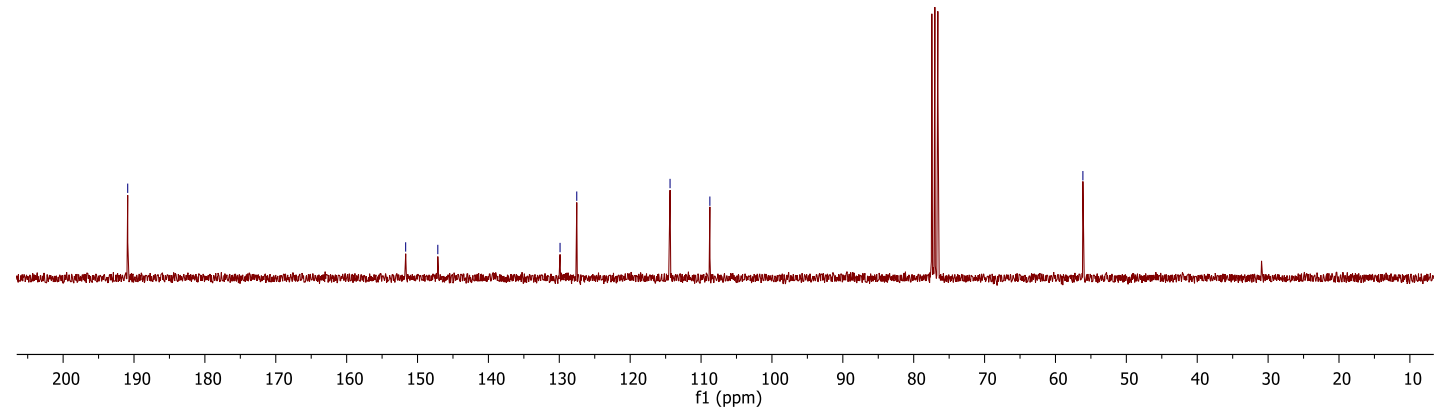

${ }^{13} \mathrm{C}$ NMR spectrum in $\mathrm{CDCl}_{3}$. 
196b<smiles>COc1cc(C=O)ccc1OC(C)=O</smiles>

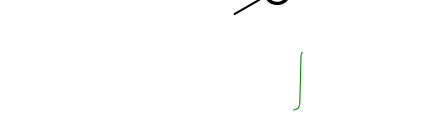

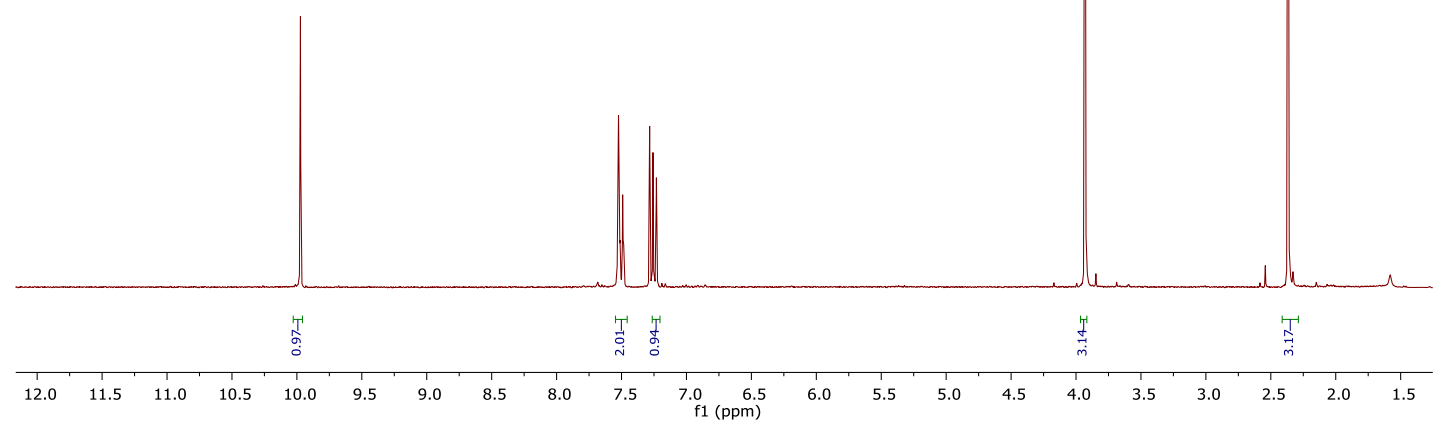

${ }^{1} \mathrm{H}$ NMR spectrum in $\mathrm{CDCl}_{3}$.

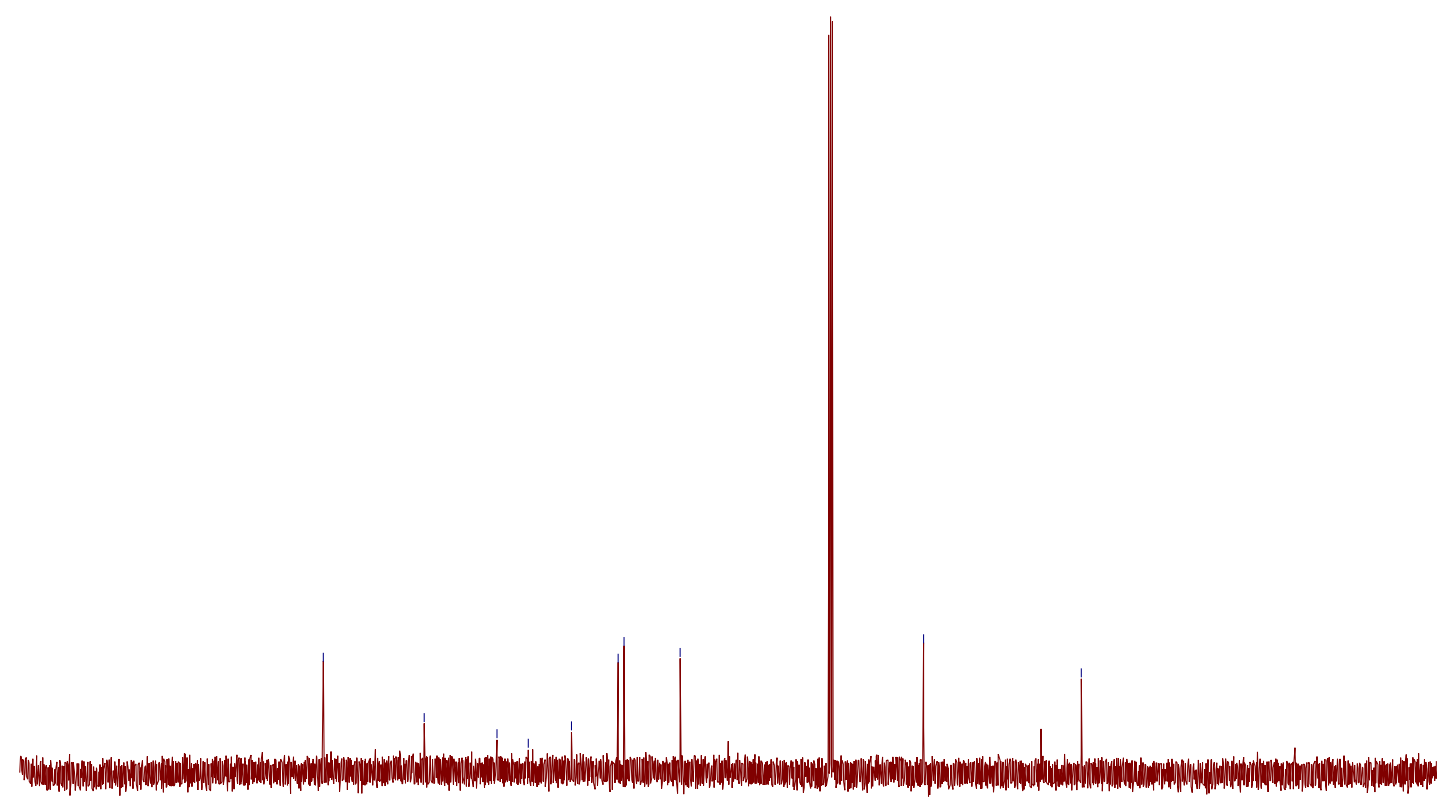
$\begin{array}{llllllllllllllllllllllllllllllllllll}250 & 240 & 230 & 220 & 210 & 200 & 190 & 180 & 170 & 160 & 150 & 140 & 130 & 120 & 110 & 100 & 90 & 80 & 70 & 60 & 50 & 40 & 30 & 20 & 10 & 0 & -10 & -20 & -30 & -40 & -50\end{array}$

${ }^{13} \mathrm{C}$ NMR spectrum in $\mathrm{CDCl}_{3}$. 
197b

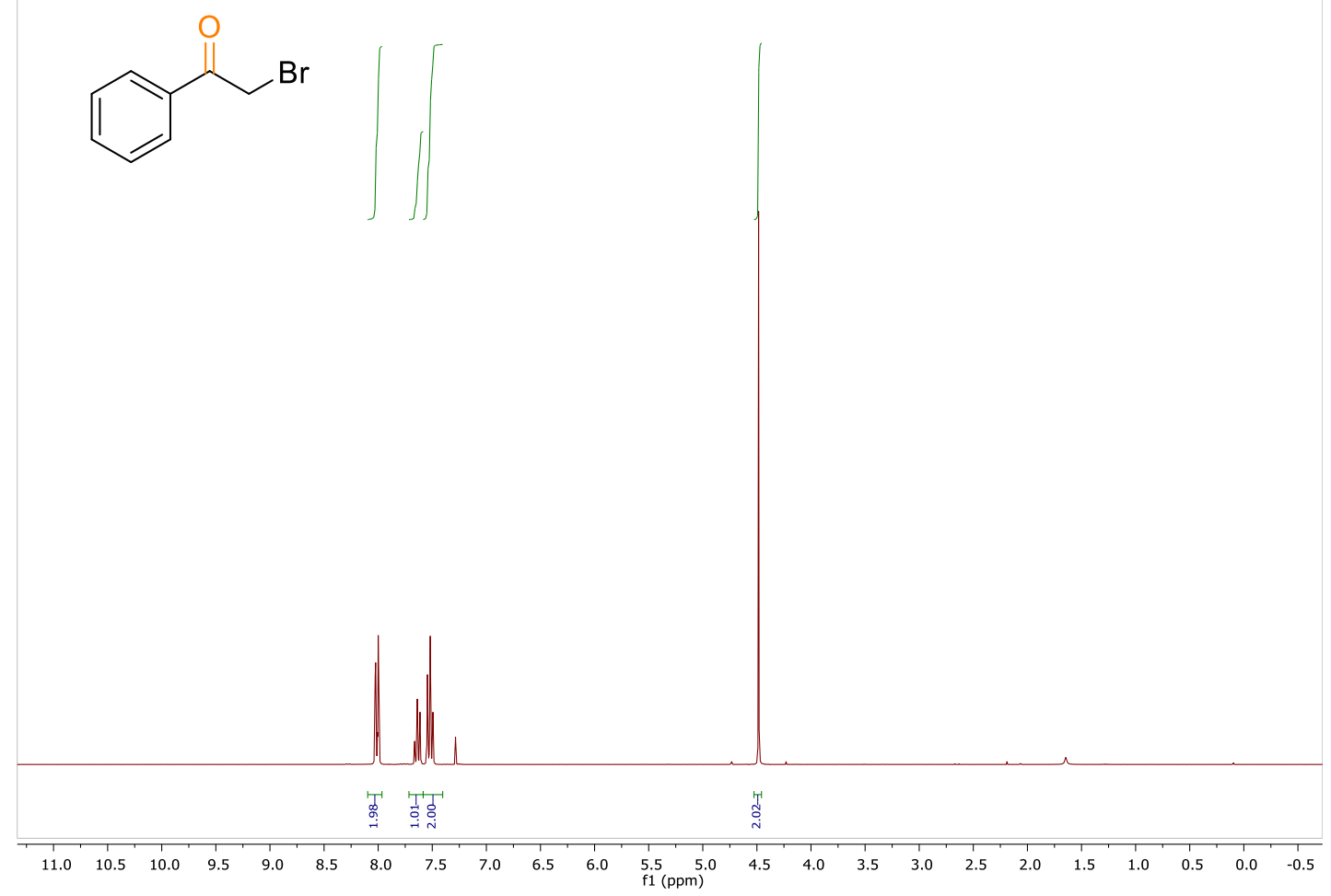

${ }^{1} \mathrm{H}$ NMR spectrum in $\mathrm{CDCl}_{3}$.

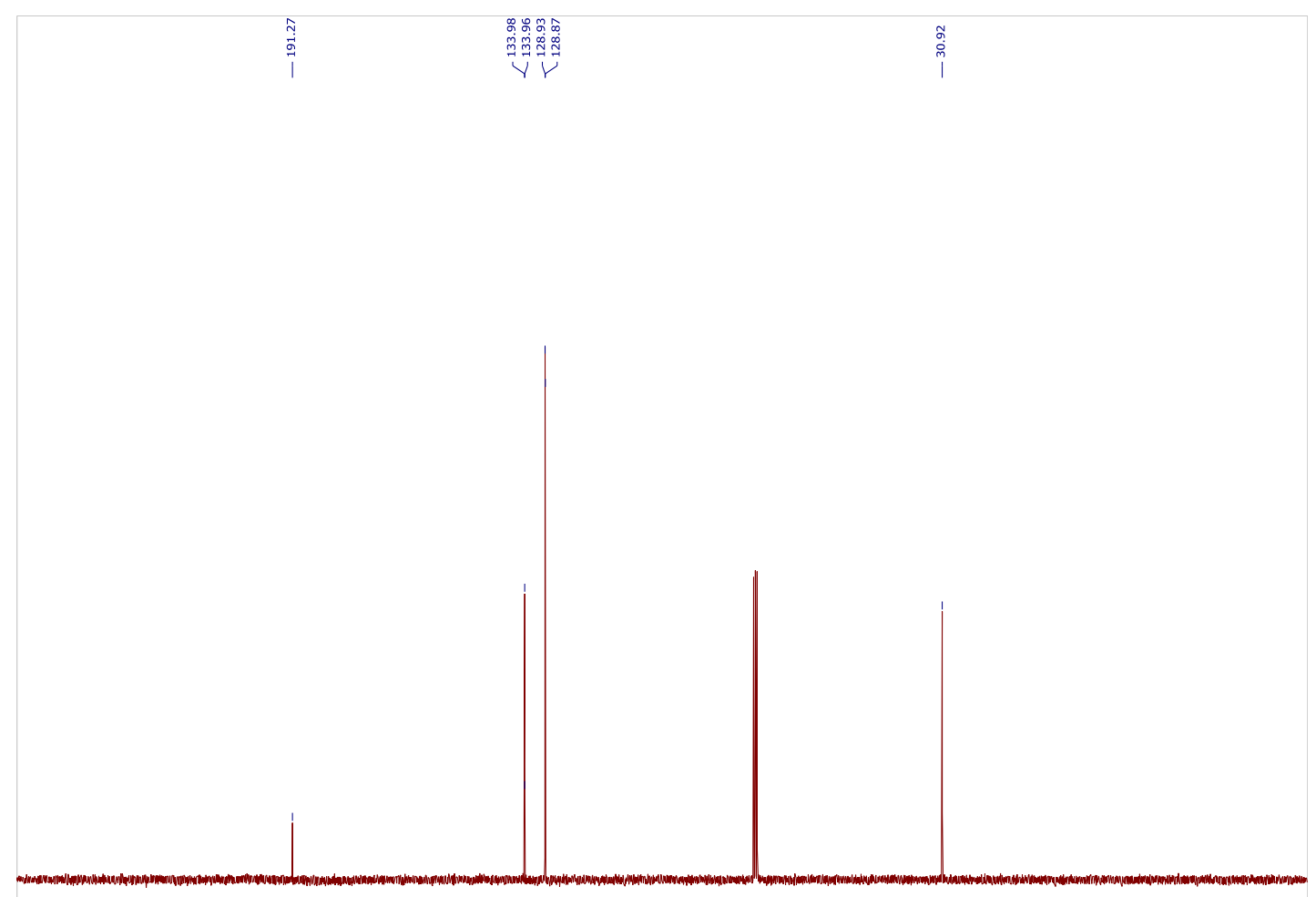

$\begin{array}{lllllllllllllllllllllllllllllllllllllllllllllllll}250 & 240 & 230 & 220 & 210 & 200 & 190 & 180 & 170 & 160 & 150 & 140 & 130 & 120 & 110 & 100 & 90 & 80 & 70 & 60 & 50 & 40 & 30 & 20 & 10 & 0 & -10 & -20 & -30 & -40 & -50\end{array}$

${ }^{13} \mathrm{C}$ NMR spectrum in $\mathrm{CDCl}_{3}$. 
198b

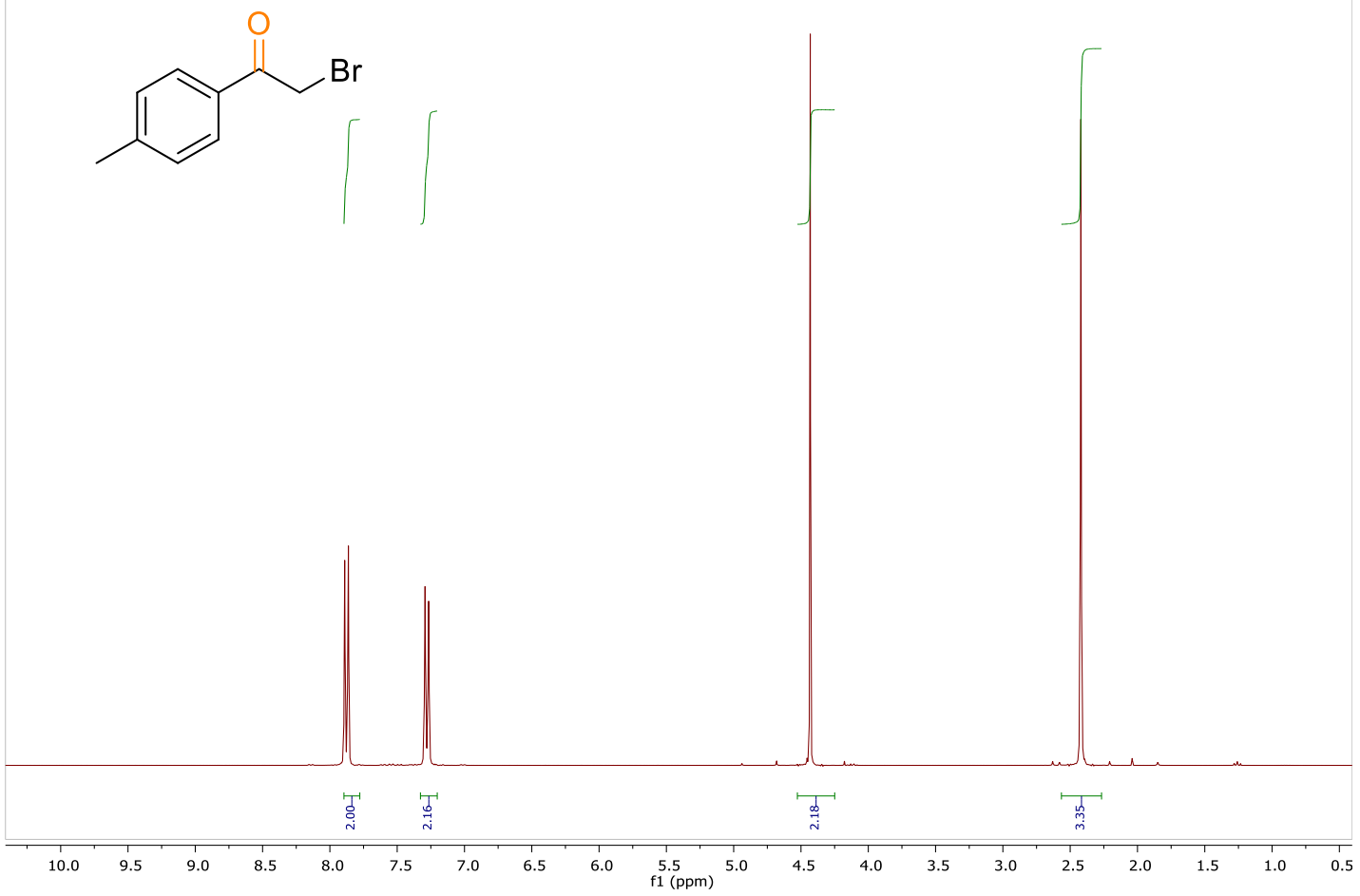

${ }^{1} \mathrm{H}$ NMR spectrum in $\mathrm{CDCl}_{3}$.

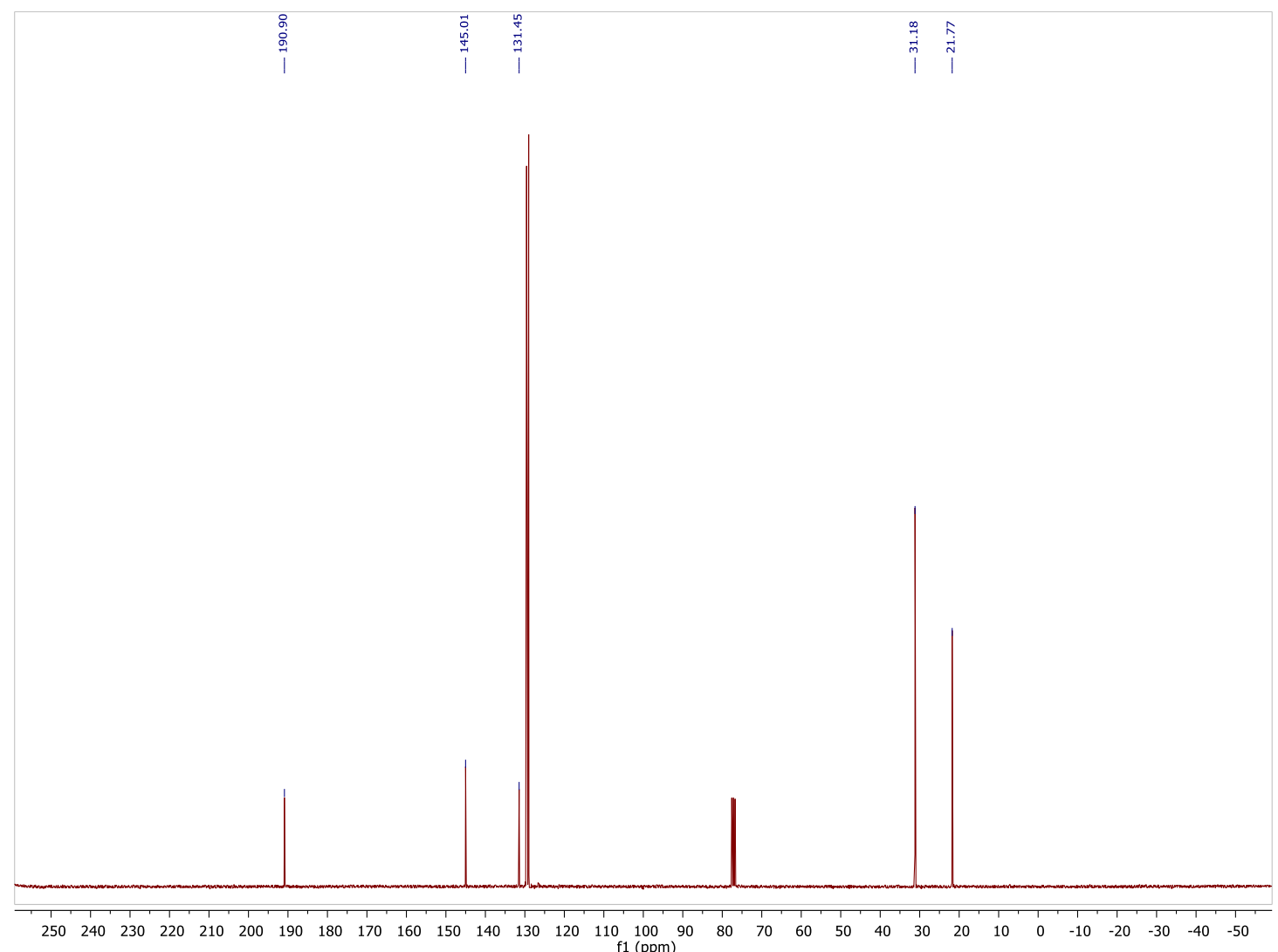

${ }^{13} \mathrm{C}$ NMR spectrum in $\mathrm{CDCl}_{3}$. 
199b

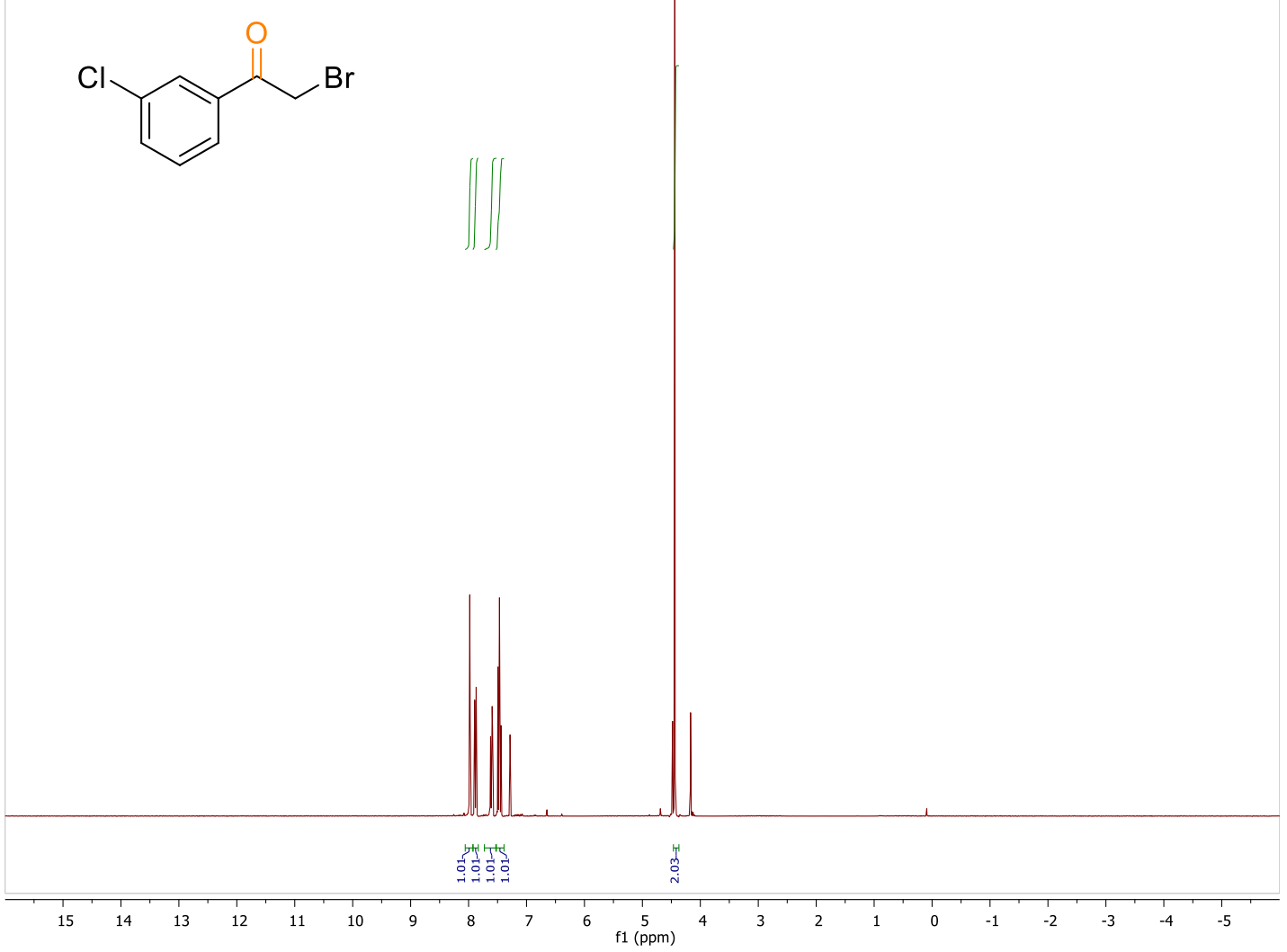

${ }^{1} \mathrm{H}$ NMR spectrum in $\mathrm{CDCl}_{3}$.

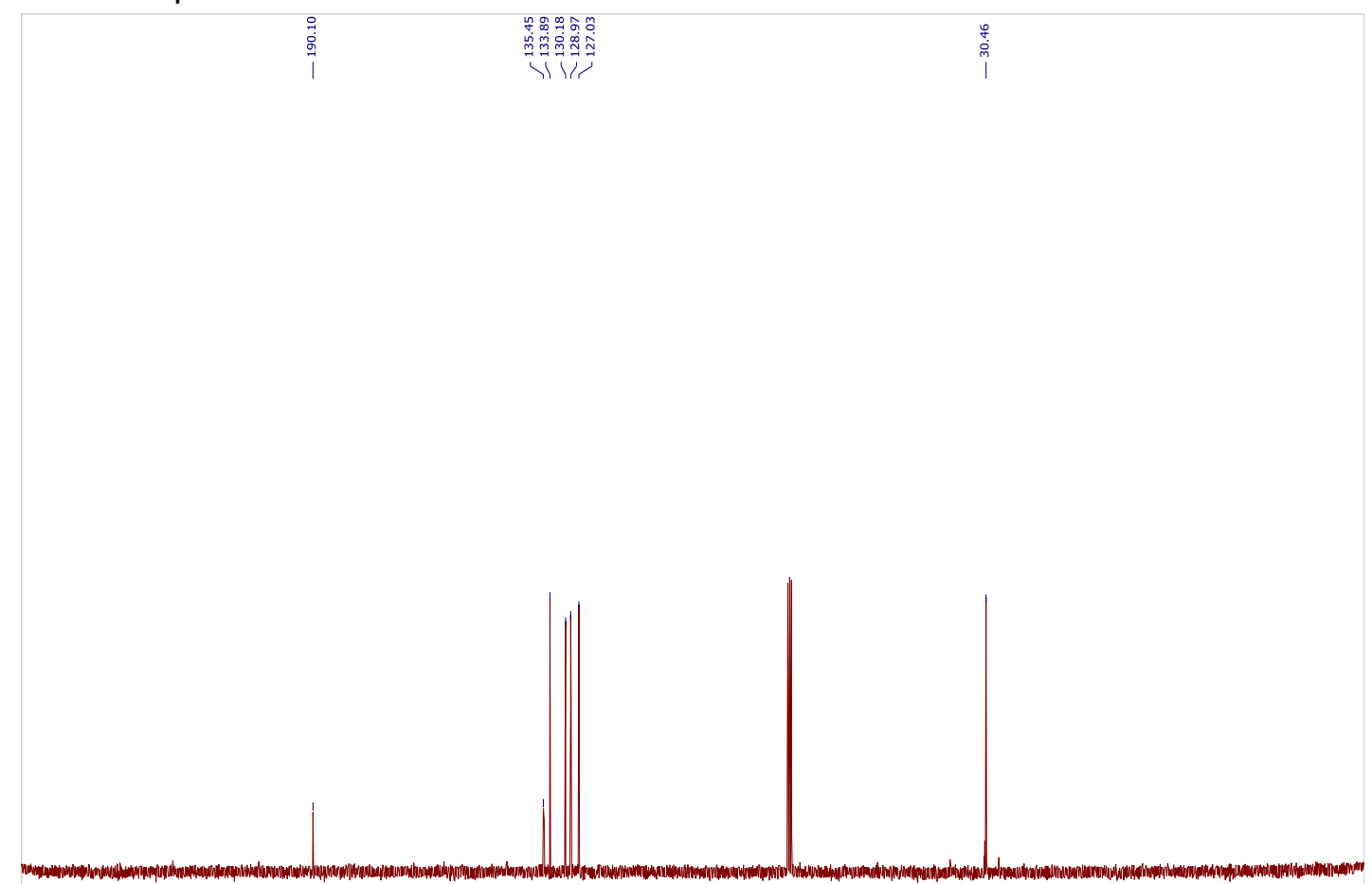

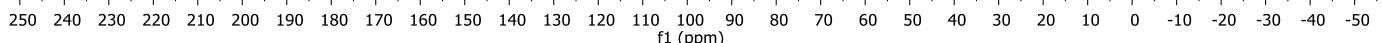

${ }^{13} \mathrm{C}$ NMR spectrum in $\mathrm{CDCl}_{3}$. 
200b-1

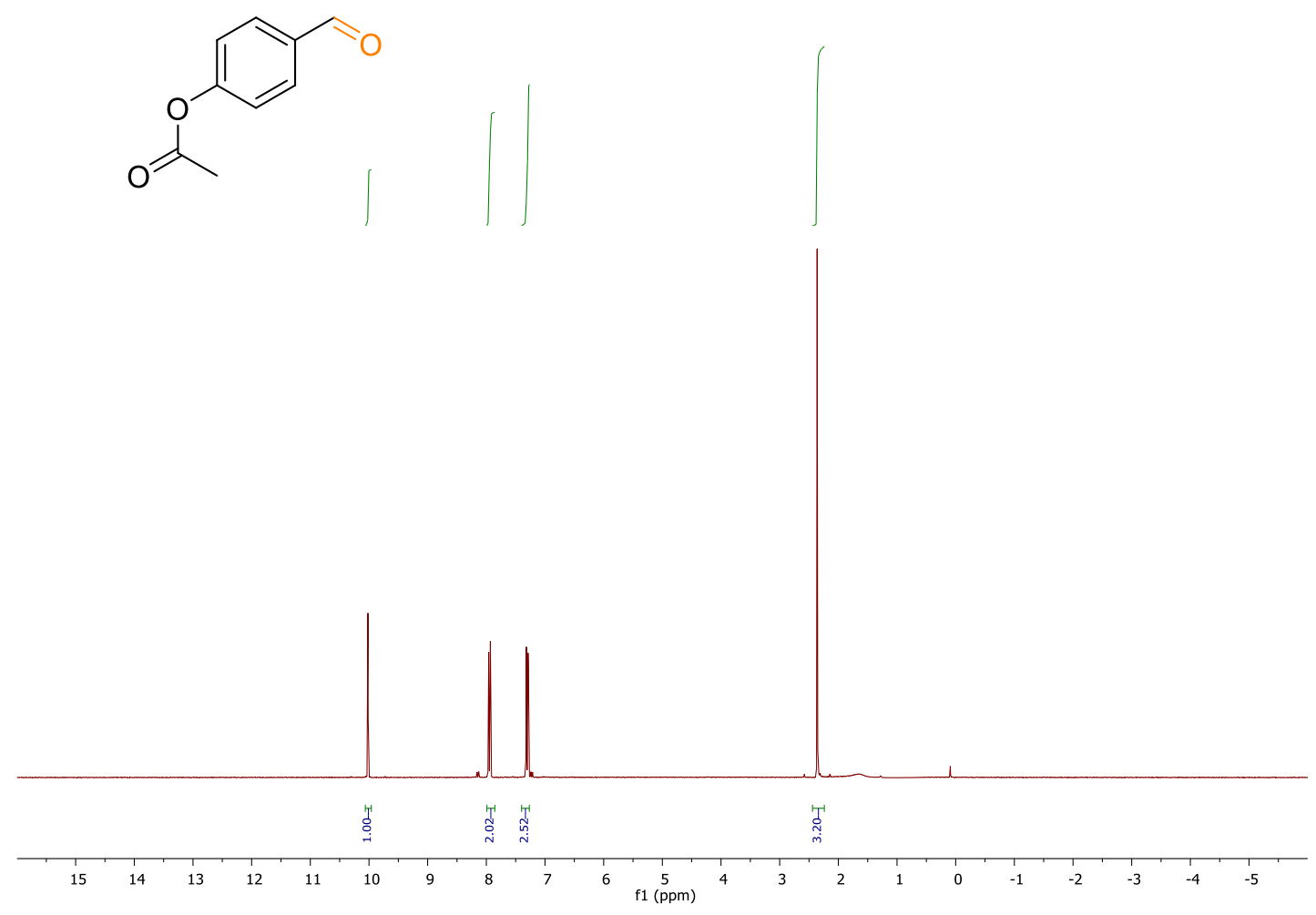

${ }^{1} \mathrm{H}$ NMR spectrum in $\mathrm{CDCl}_{3}$.

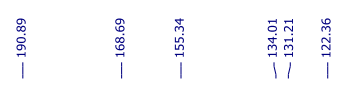

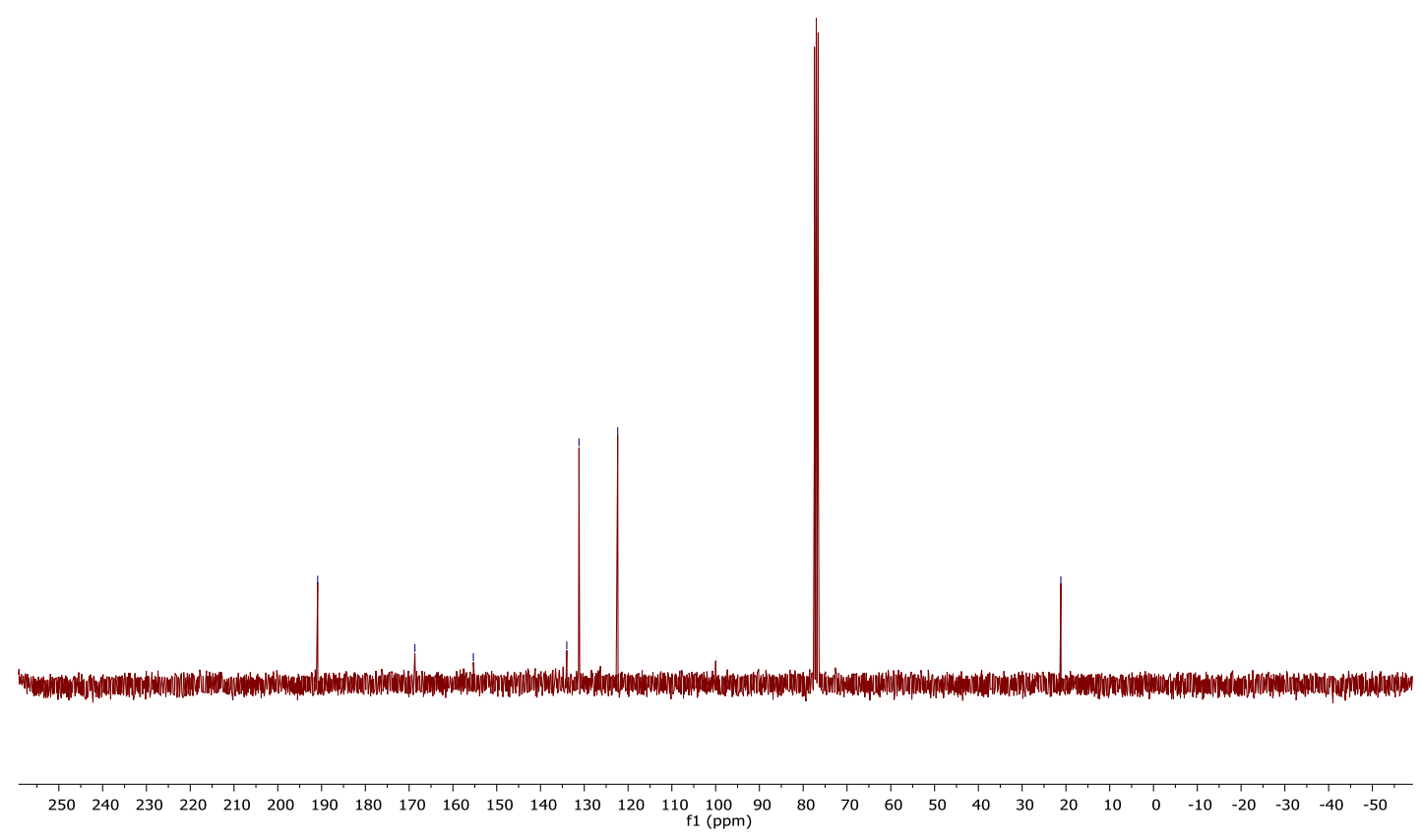

${ }^{13} \mathrm{C}$ NMR spectrum in $\mathrm{CDCl}_{3}$. 


\section{0b-2}<smiles>CC(=O)Oc1ccc(C=O)cc1OC(C)=O</smiles>
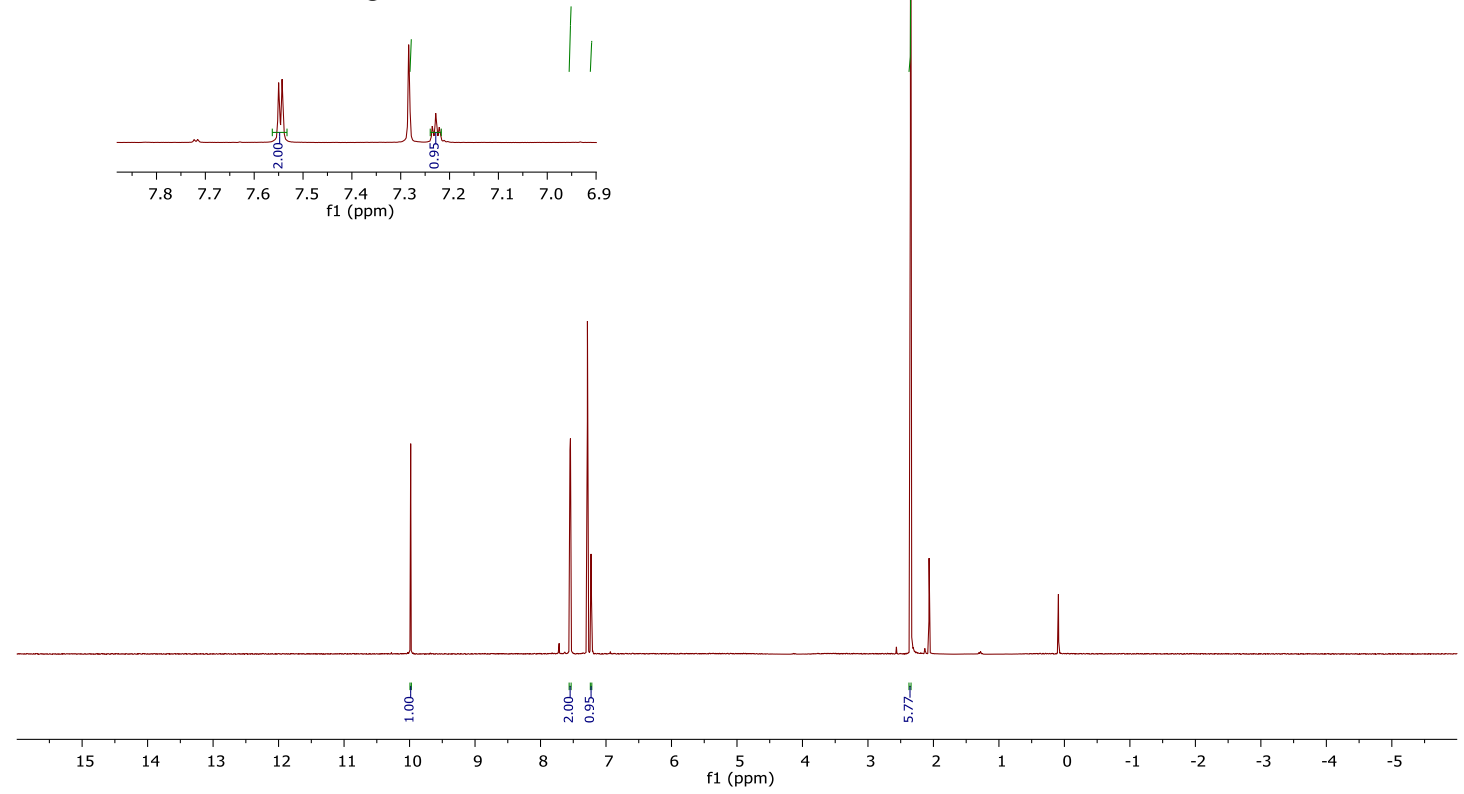

${ }^{1} \mathrm{H}$ NMR spectrum in $\mathrm{CDCl}_{3}$.

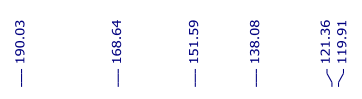

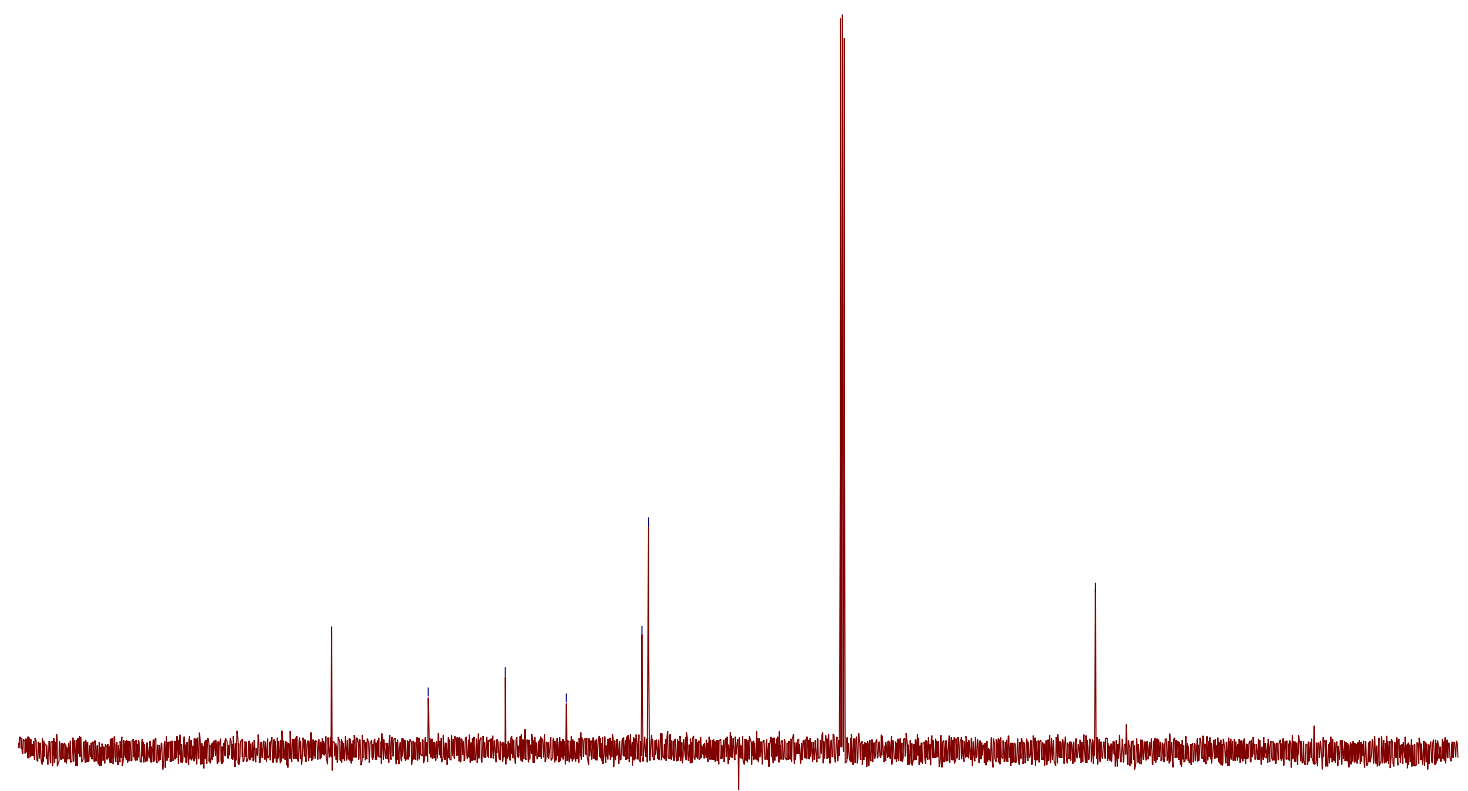

$\begin{array}{llllllllllllllllllllllllllllllllllllllllllll}250 & 240 & 230 & 220 & 210 & 200 & 190 & 180 & 170 & 160 & 150 & 140 & 130 & 120 & 110 & 100 & 90 & 80 & 70 & 60 & 50 & 40 & 30 & 20 & 10 & 0 & -10 & -20 & -30 & -40 & -50\end{array}$

${ }^{13} \mathrm{C}$ NMR spectrum in $\mathrm{CDCl}_{3}$. 
201b<smiles>CSc1cccc2sc3cc(Cl)cc(c2=O)c3c1</smiles>

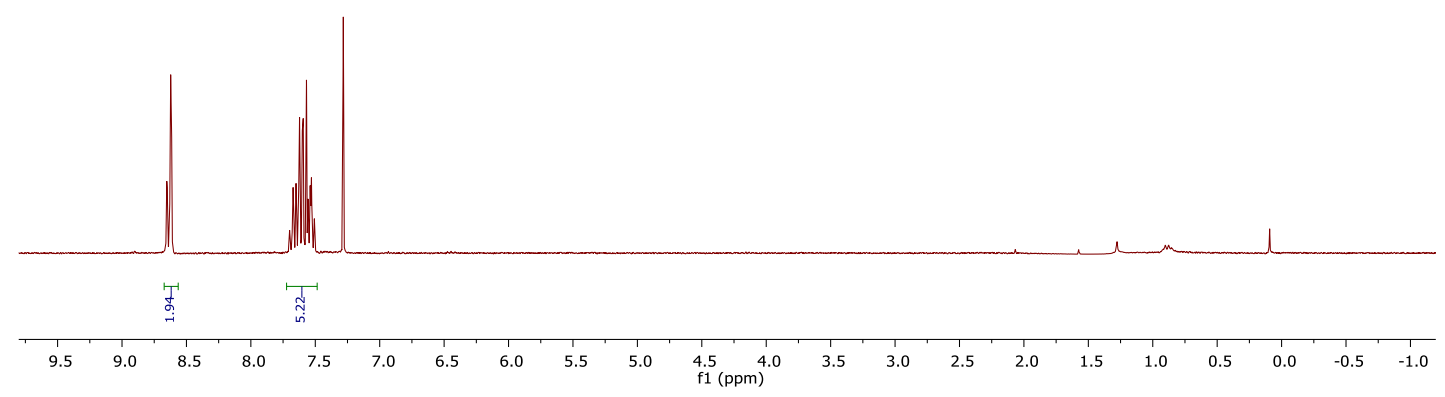

${ }^{1} \mathrm{H}$ NMR spectrum in $\mathrm{CDCl}_{3}$.

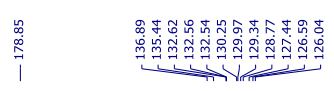

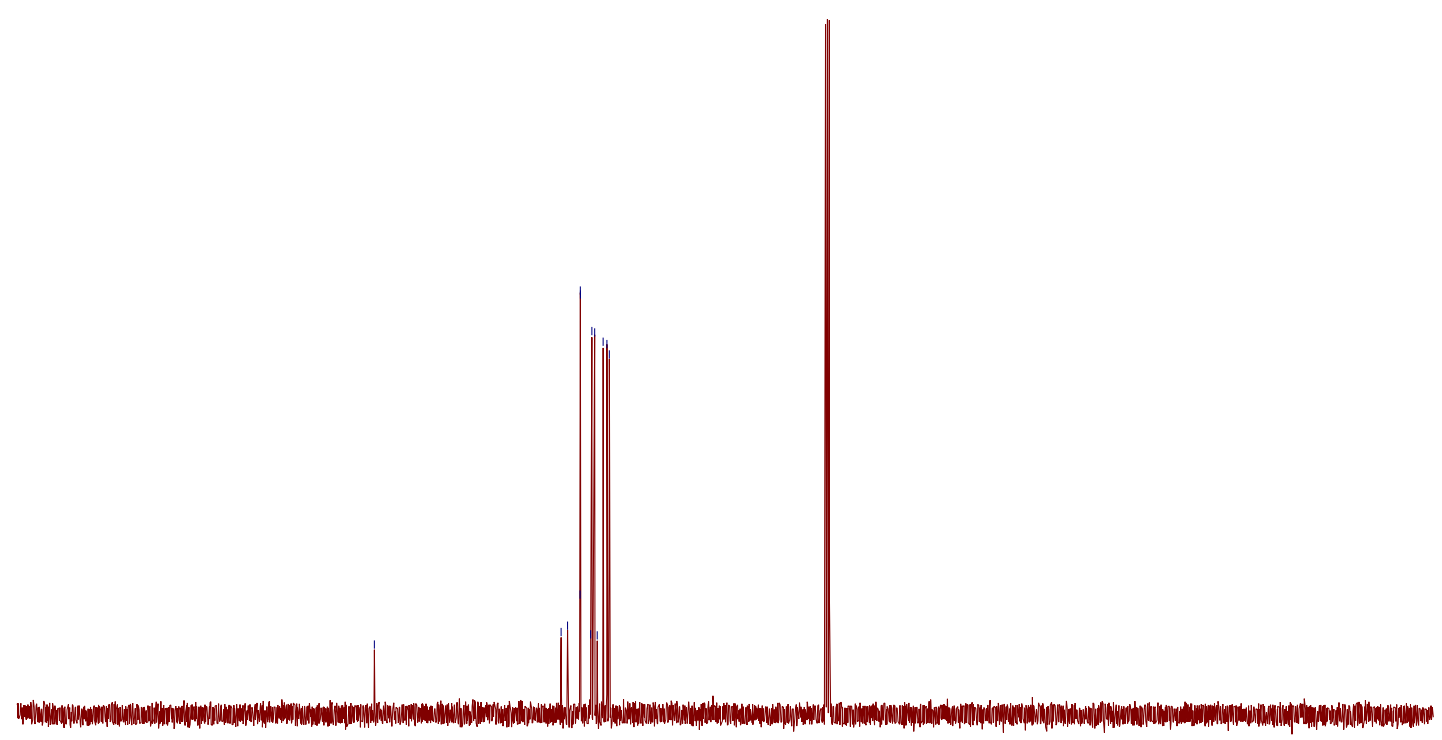
$\begin{array}{lllllllllllllllllllllllllllllllllllllll}250 & 240 & 230 & 220 & 210 & 200 & 190 & 180 & 170 & 160 & 150 & 140 & 130 & 120 & 110 & 100 & 90 & 80 & 70 & 60 & 50 & 40 & 30 & 20 & 10 & 0 & -10 & -20 & -30 & -40 & -50\end{array}$

${ }^{13} \mathrm{C} \mathrm{NMR}$ spectrum in $\mathrm{CDCl}_{3}$ 
202b

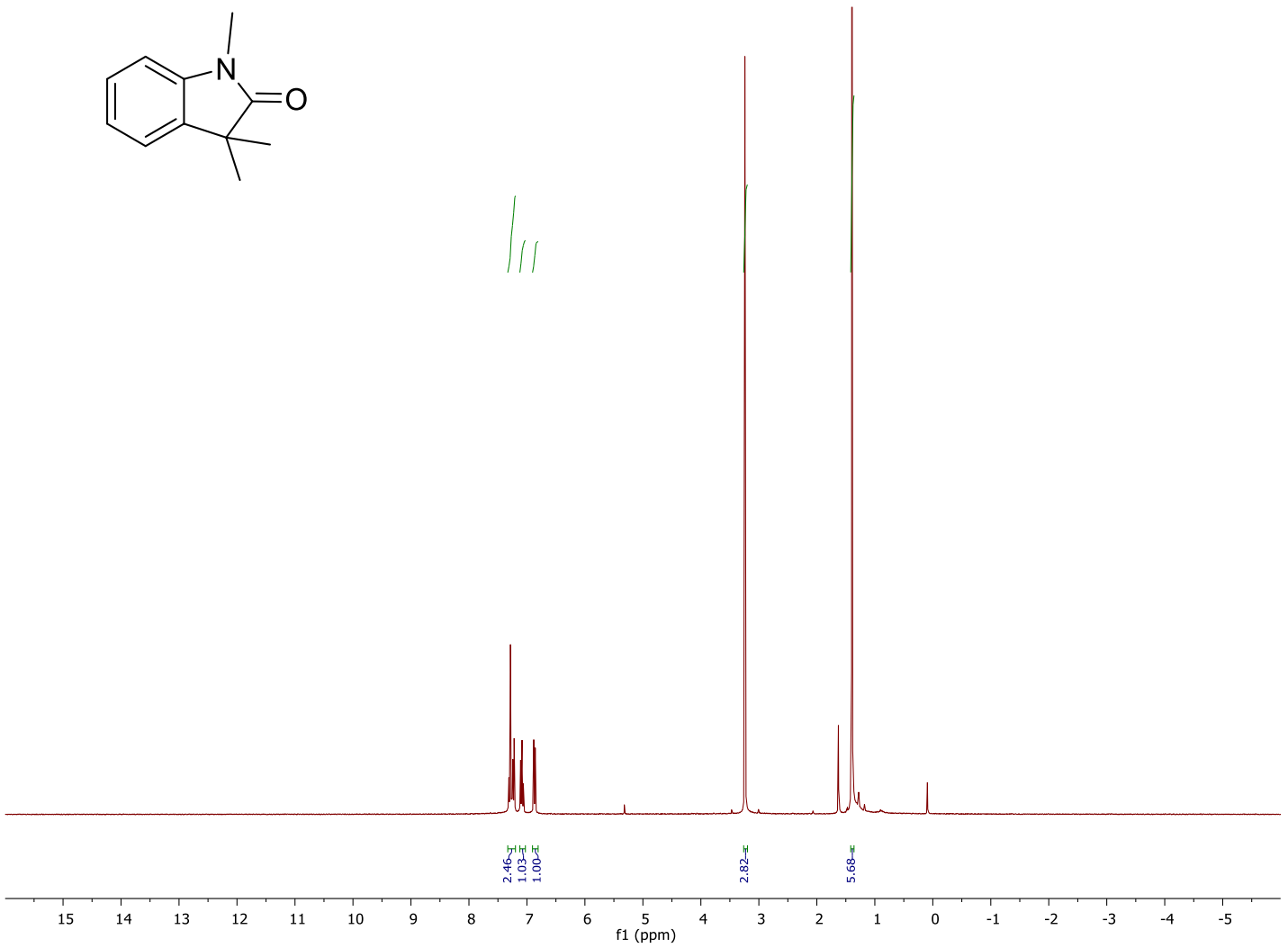

${ }^{1} \mathrm{H}$ NMR spectrum in $\mathrm{CDCl}_{3}$.
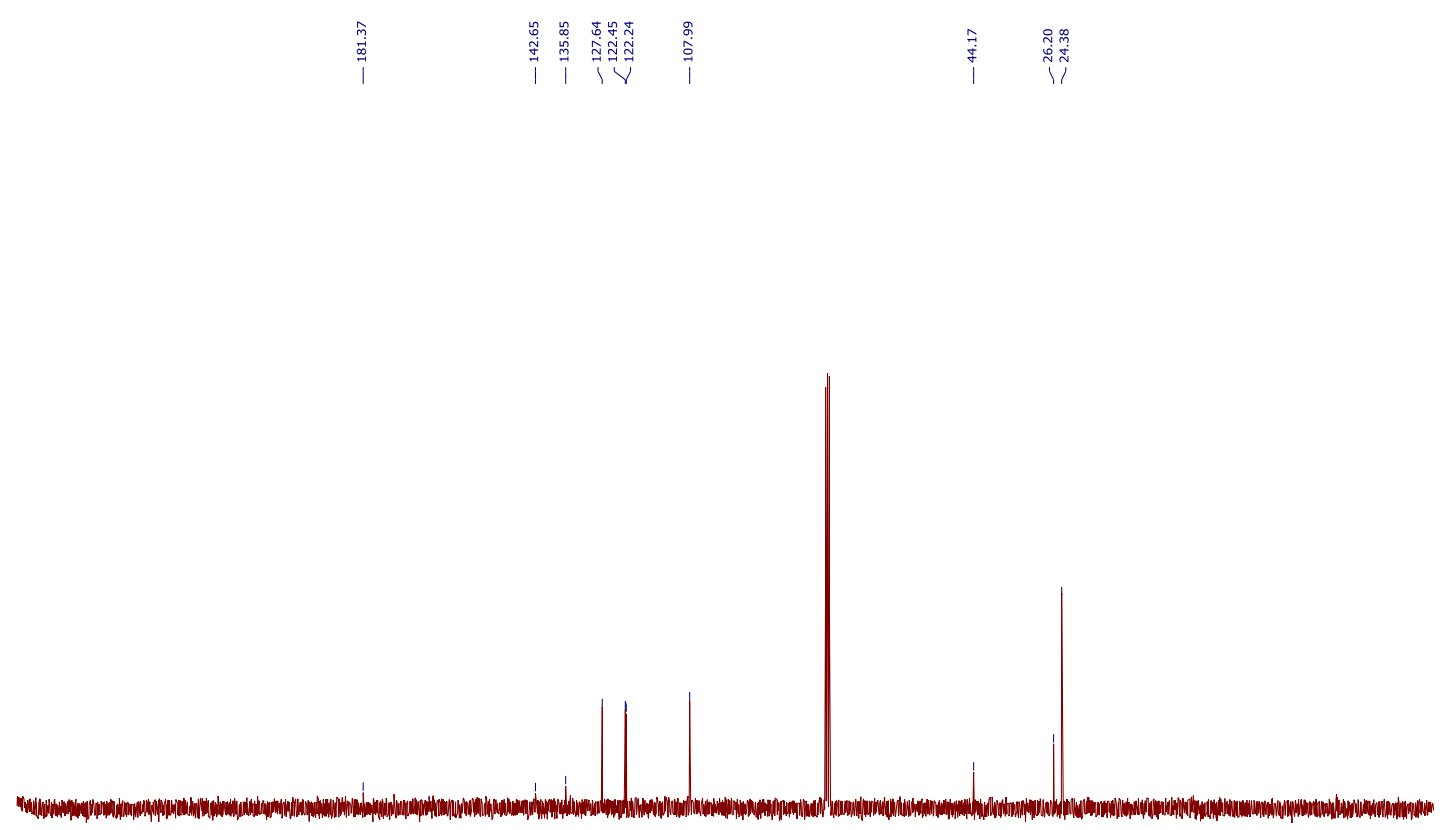

$\begin{array}{llllllllllllllllllllllllllllllllllll}250 & 240 & 230 & 220 & 210 & 200 & 190 & 180 & 170 & 160 & 150 & 140 & 130 & 120 & 110 & 100 & 90 & 80 & 70 & 60 & 50 & 40 & 30 & 20 & 10 & 0 & -10 & -20 & -30 & -40 & -50\end{array}$

${ }^{13} \mathrm{C} \mathrm{NMR}$ spectrum in $\mathrm{CDCl}_{3}$. 
203b

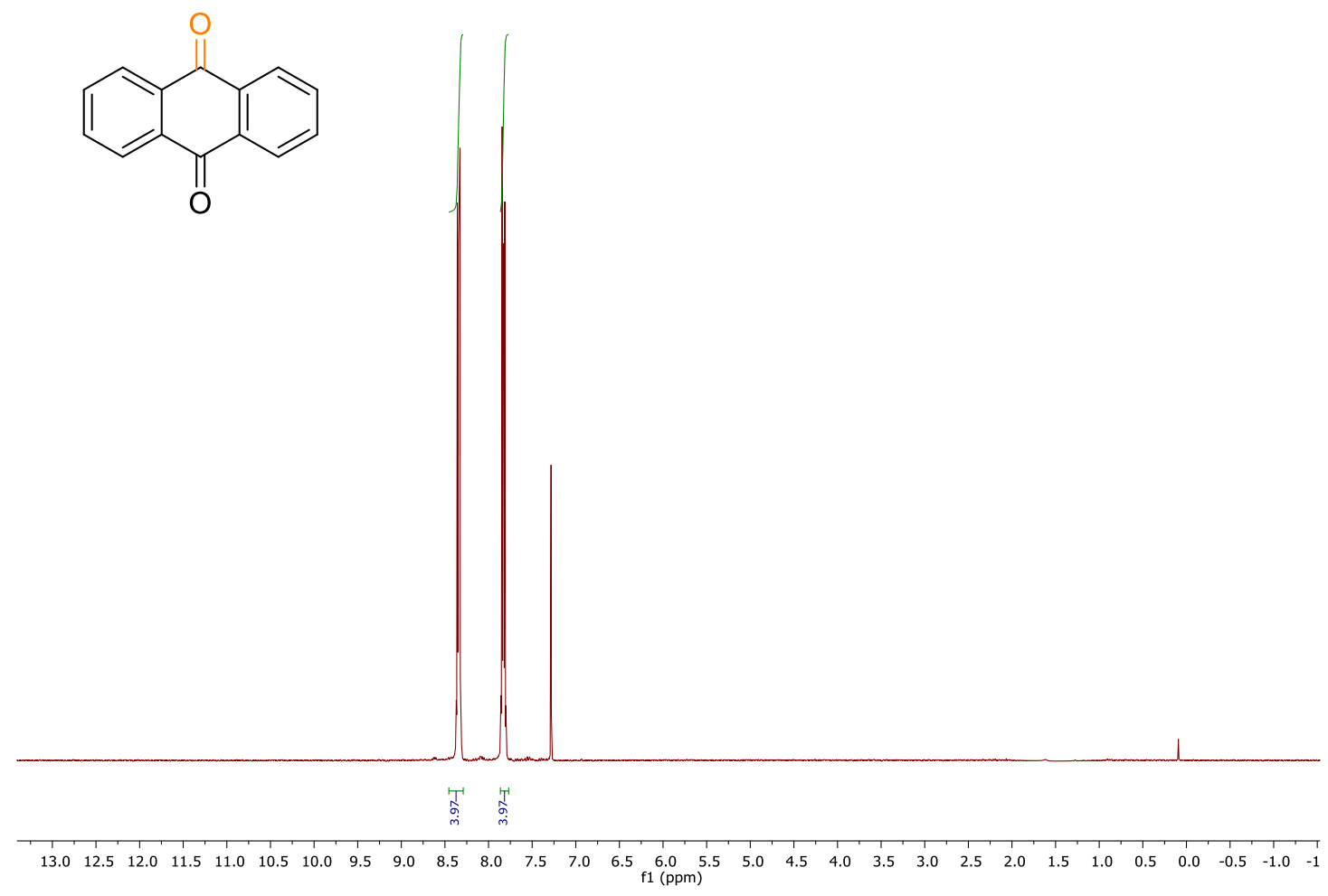

${ }^{1} \mathrm{H}$ NMR spectrum in $\mathrm{CDCl}_{3}$.
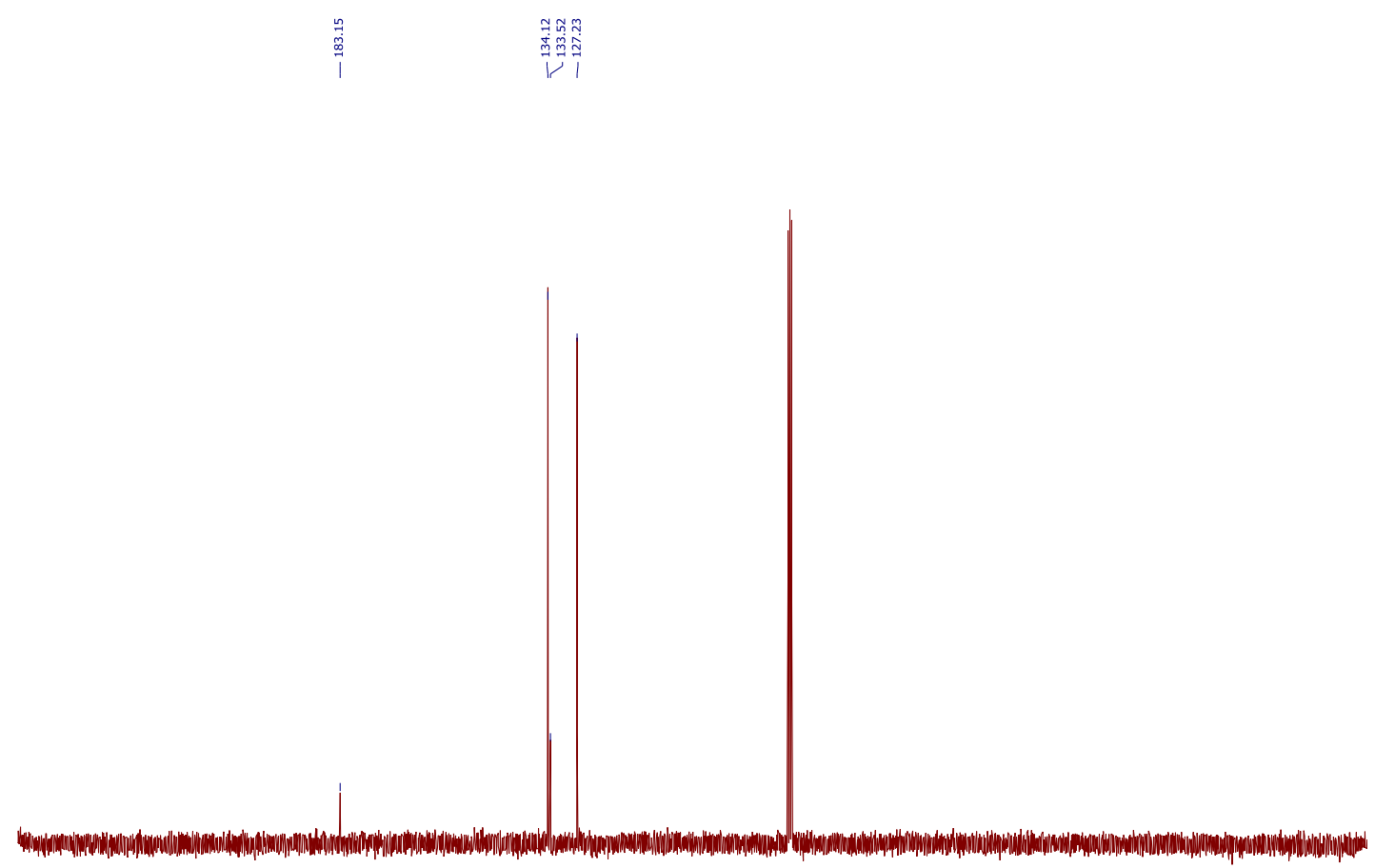

$\begin{array}{llllllllllllllllllllllllllllllllllllll}250 & 240 & 230 & 220 & 210 & 200 & 190 & 180 & 170 & 160 & 150 & 140 & 130 & 120 & 110 & 100 & 90 & 80 & 70 & 60 & 50 & 40 & 30 & 20 & 10 & 0 & -10 & -20 & -30 & -40 & -50\end{array}$

${ }^{13} \mathrm{C} \mathrm{NMR}$ spectrum in $\mathrm{CDCl}_{3}$. 


\section{5b}<smiles>CC(C)OC(=O)C(C)(C)Oc1ccc(C(=O)c2ccc(Cl)cc2)cc1</smiles>
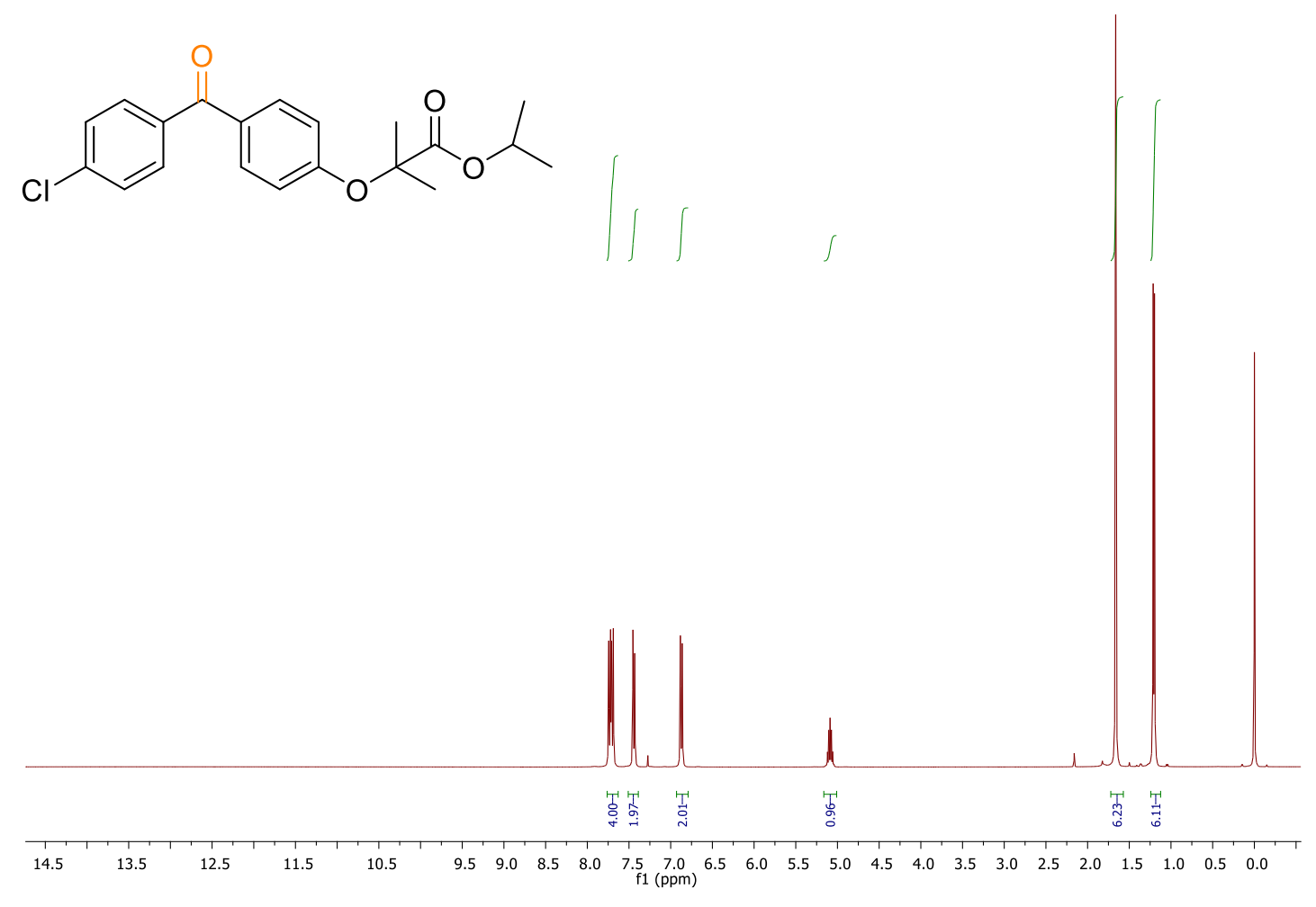

${ }^{1} \mathrm{H}$ NMR spectrum in $\mathrm{CDCl}_{3}$.

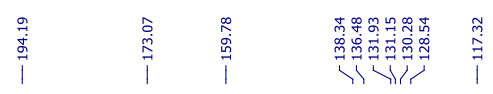

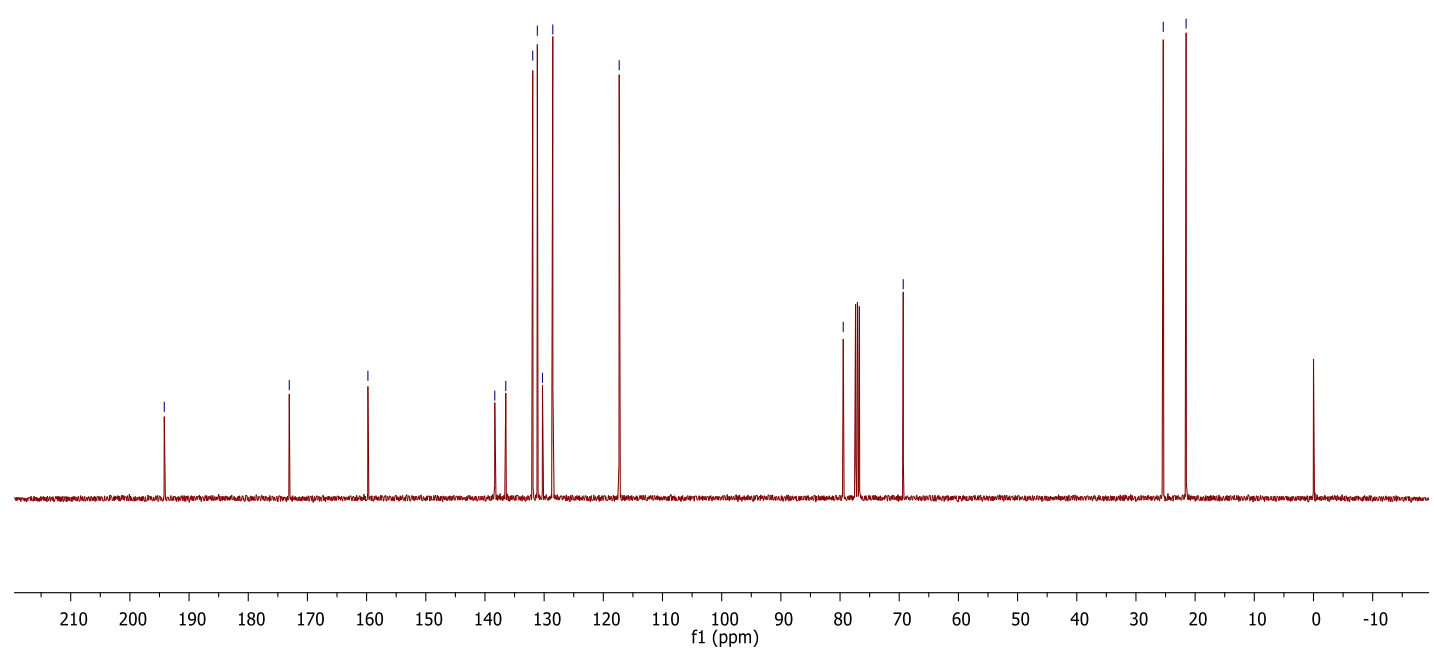

${ }^{13} \mathrm{C}$ NMR spectrum in $\mathrm{CDCl}_{3}$ 


\section{6b}

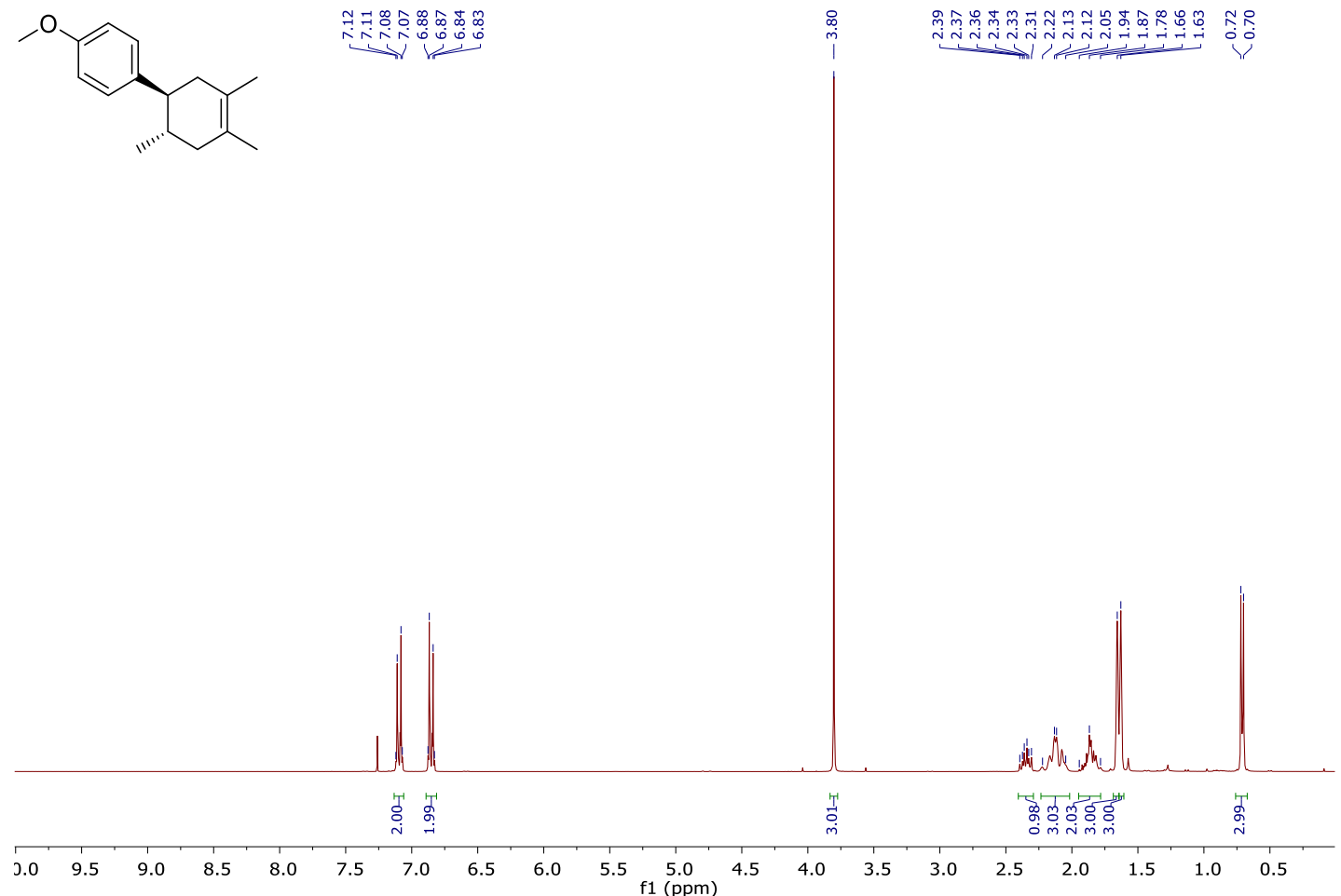

${ }^{1} \mathrm{H}$ NMR spectrum in $\mathrm{CDCl}_{3}$.

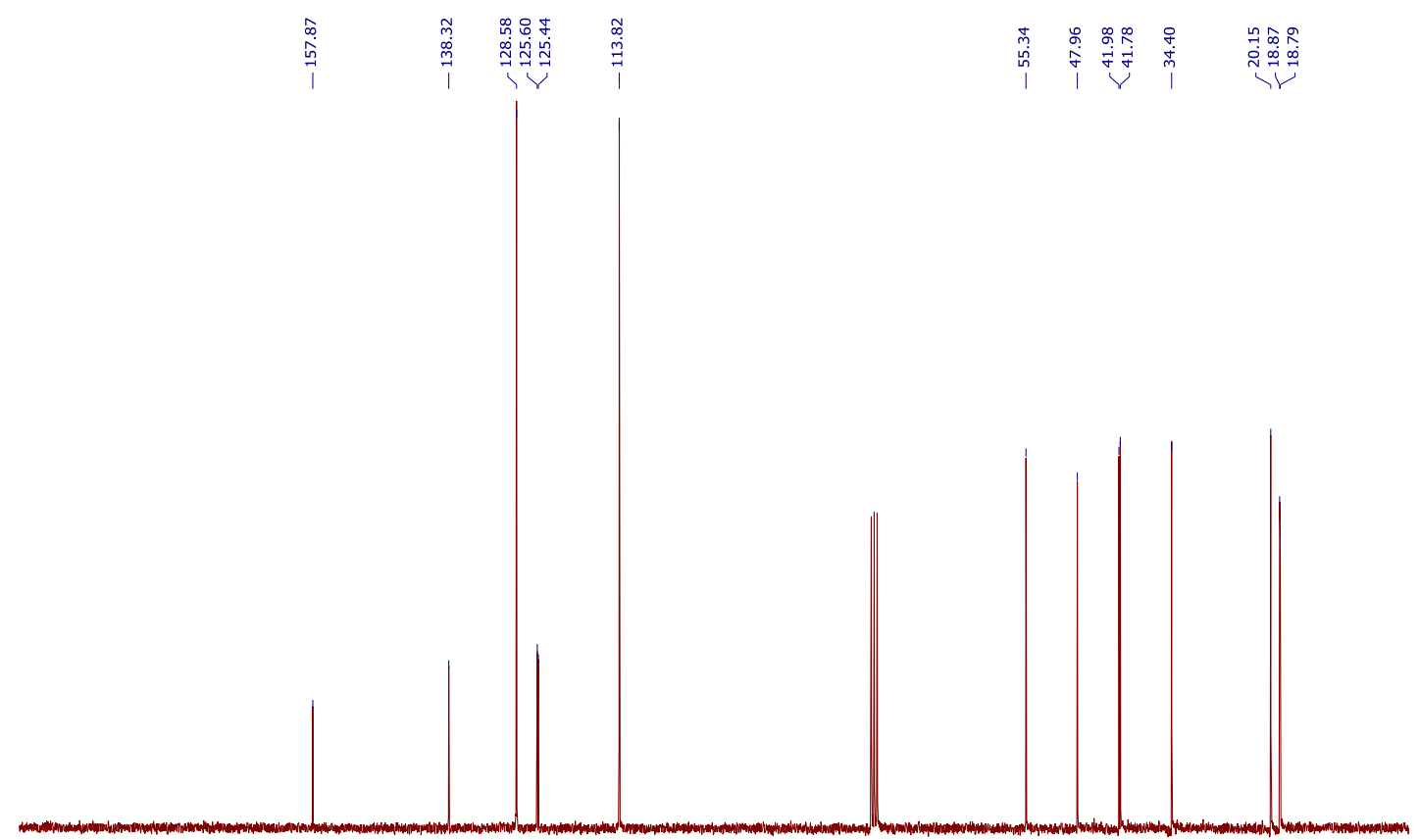

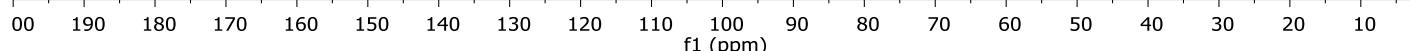

${ }^{13} \mathrm{C}$ NMR spectrum in $\mathrm{CDCl}_{3}$. 
207b

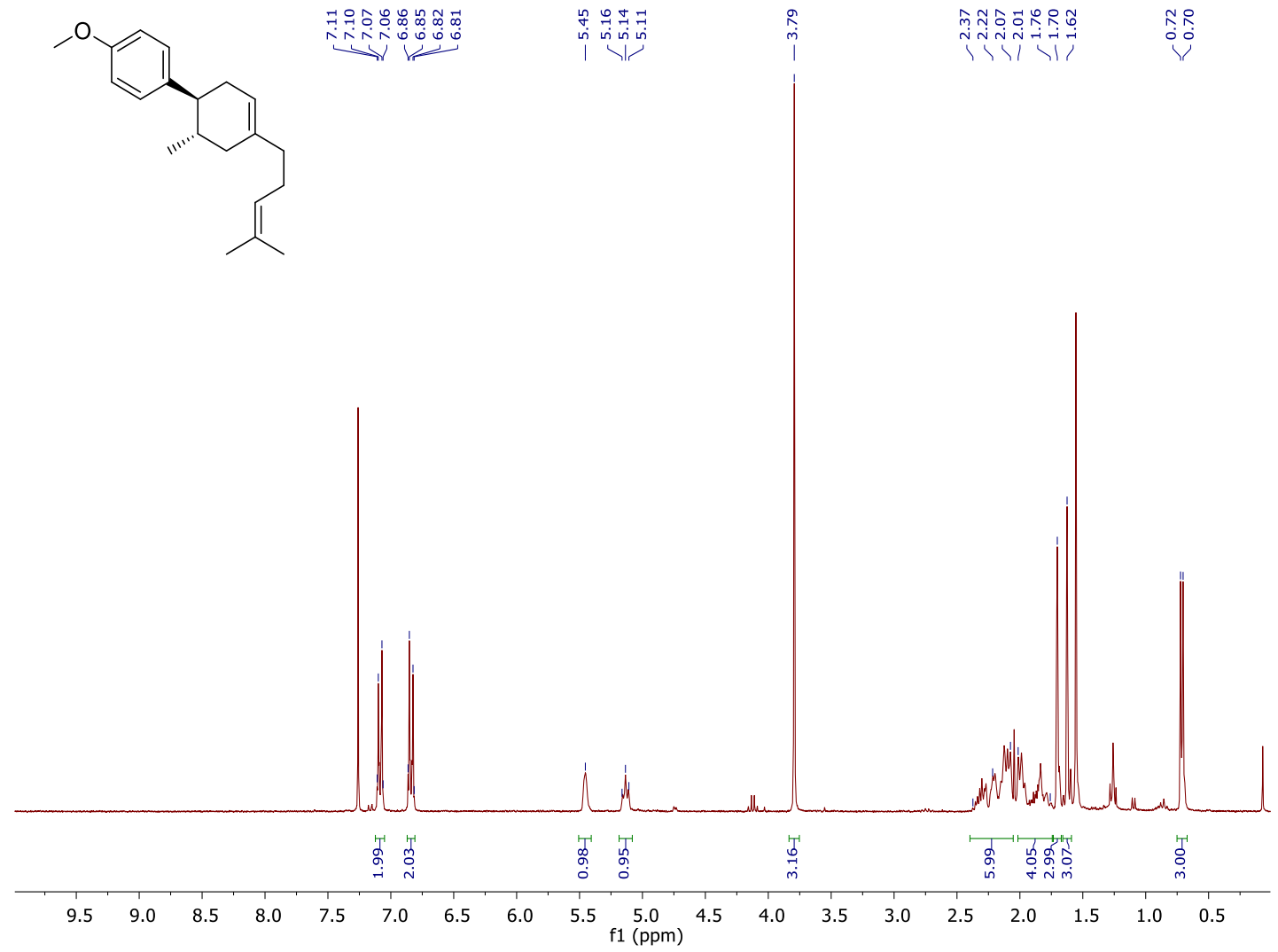

${ }^{1} \mathrm{H}$ NMR spectrum in $\mathrm{CDCl}_{3}$.

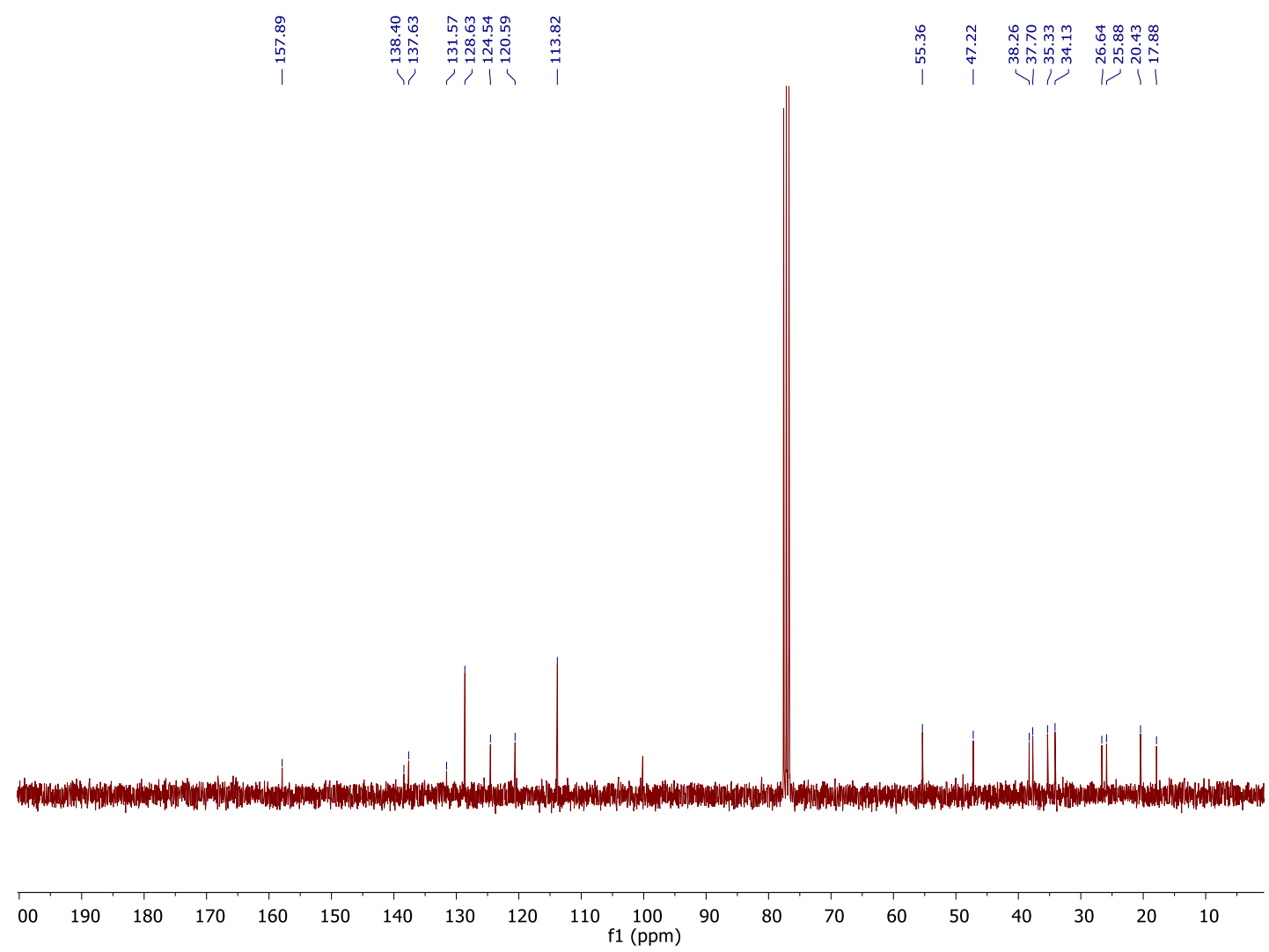

${ }^{13} \mathrm{C}$ NMR spectrum in $\mathrm{CDCl}_{3}$. 
208b

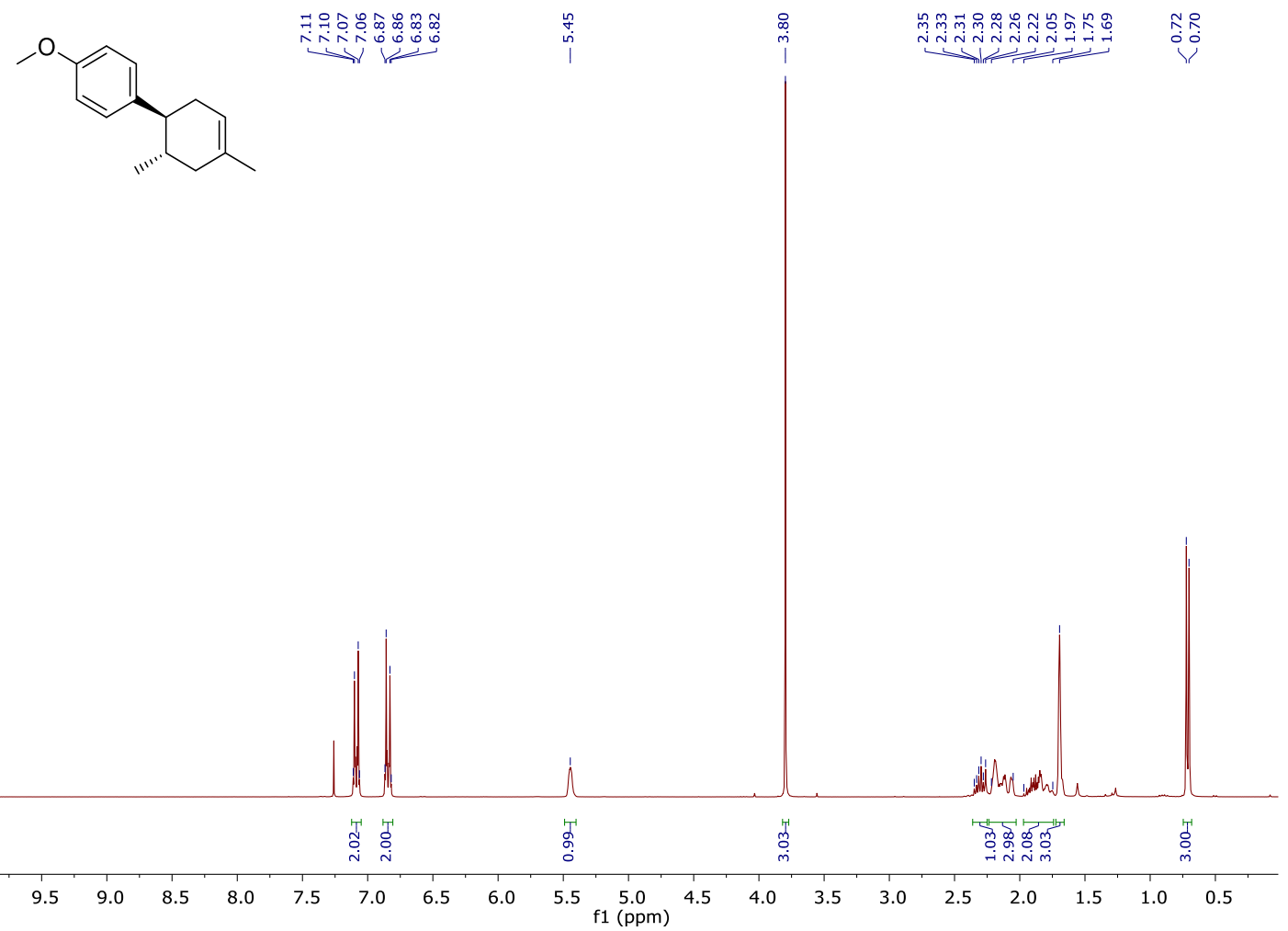

${ }^{1} \mathrm{H}$ NMR spectrum in $\mathrm{CDCl}_{3}$.

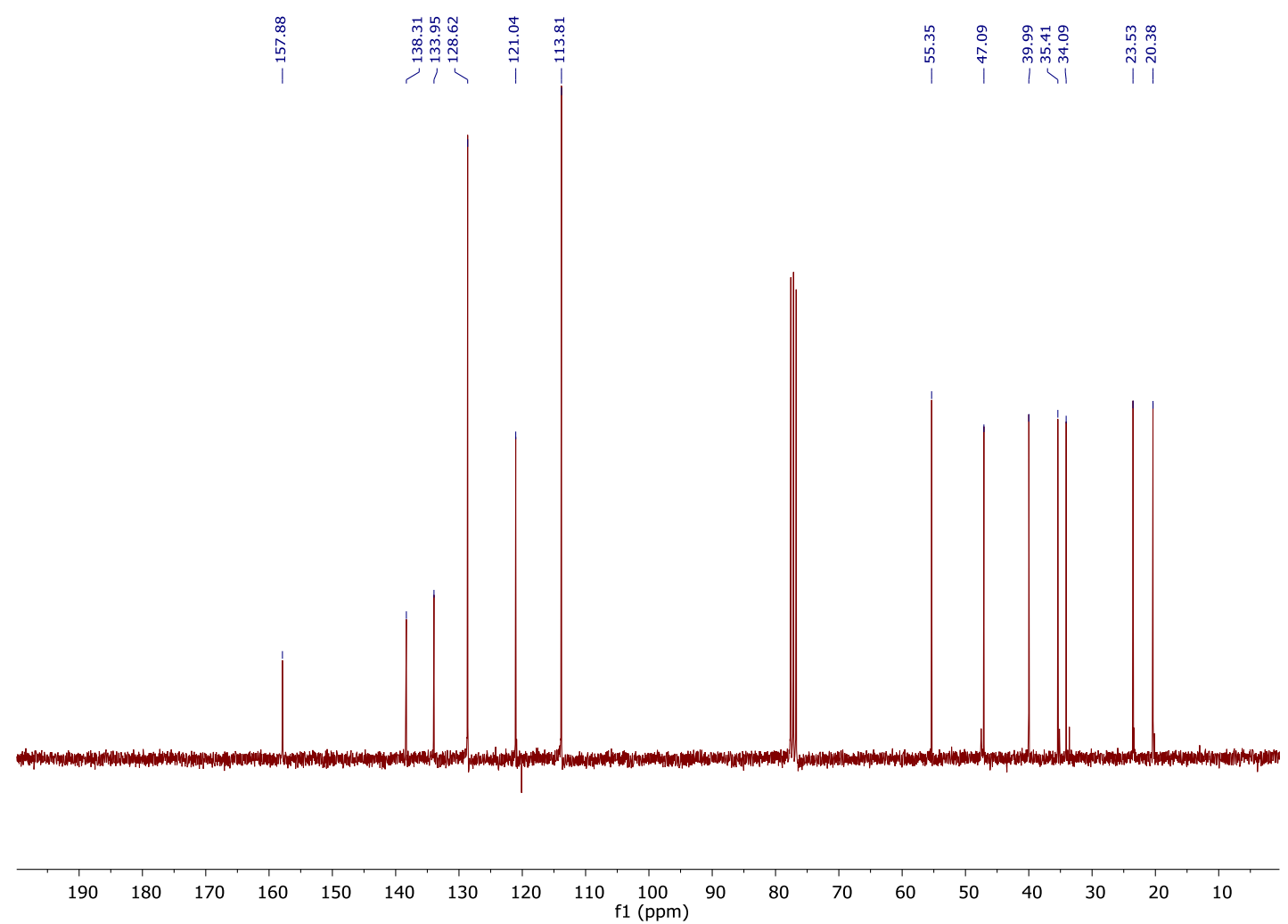

${ }^{13} \mathrm{C}$ NMR spectrum in $\mathrm{CDCl}_{3}$. 
209b
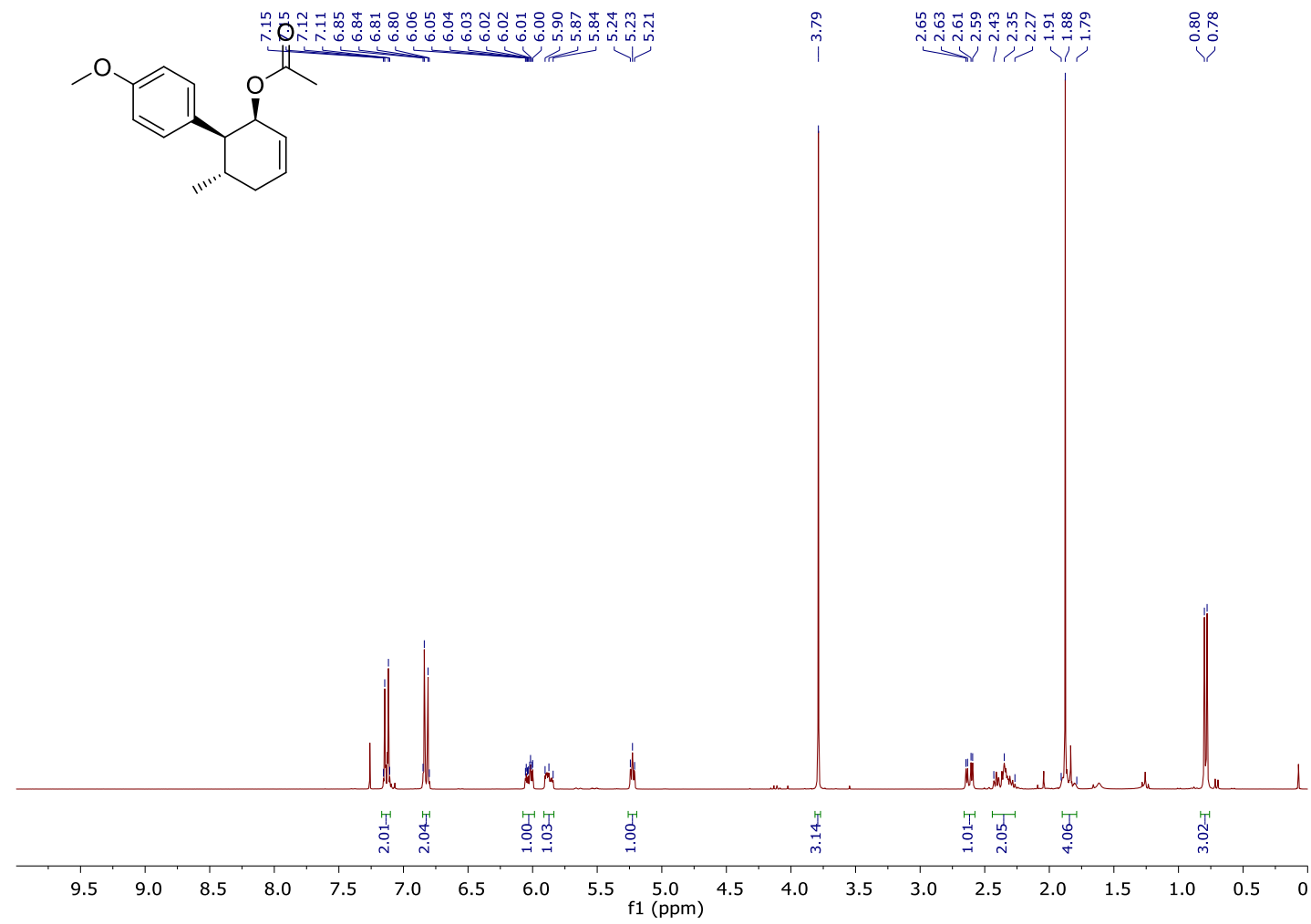

${ }^{1} \mathrm{H}$ NMR spectrum in $\mathrm{CDCl}_{3}$.

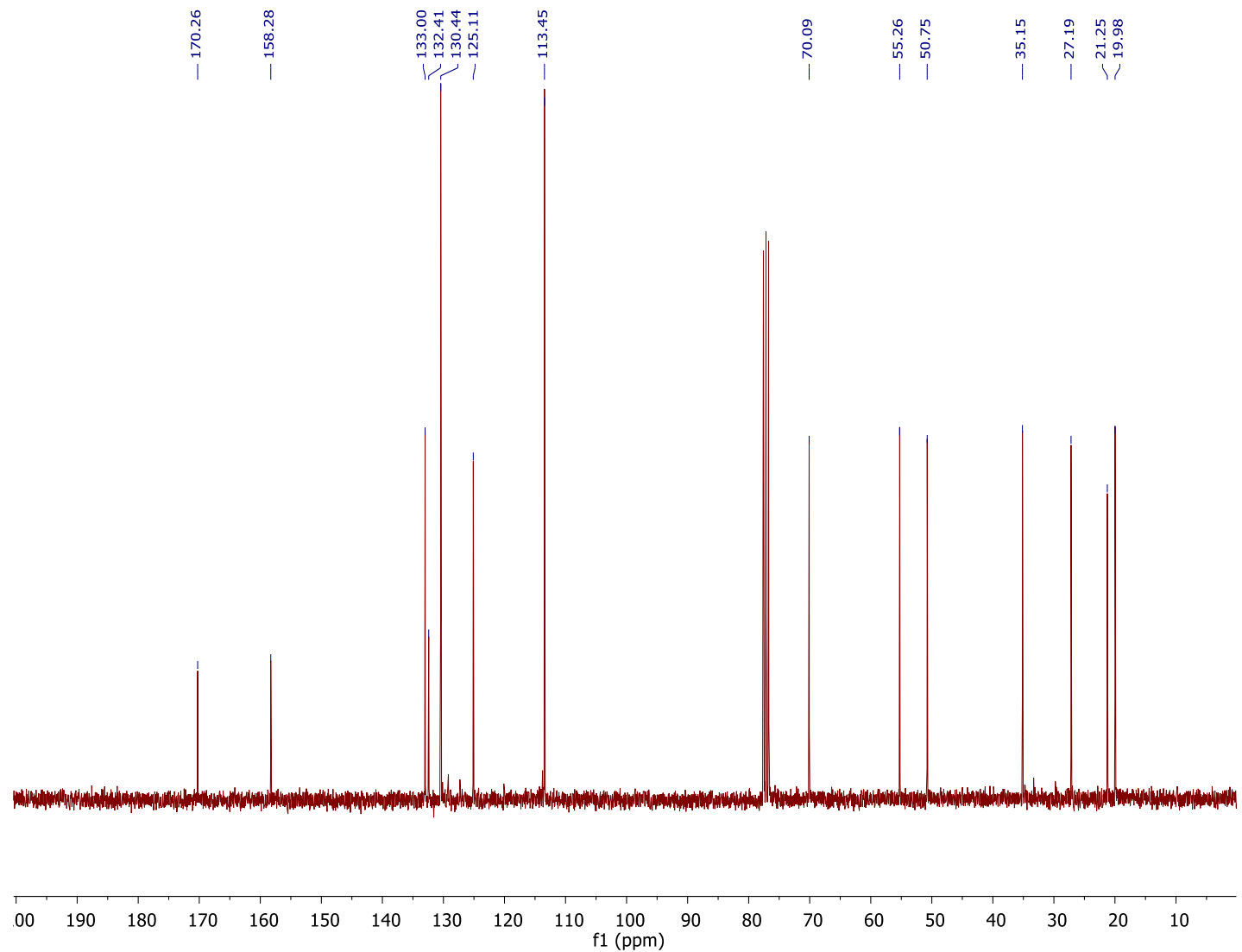

${ }^{13} \mathrm{C}$ NMR spectrum in $\mathrm{CDCl}_{3}$. 
210b<smiles>COc1ccc([C@@H]2CC(C)=C(C)C[C@H]2C)cc1OC</smiles>

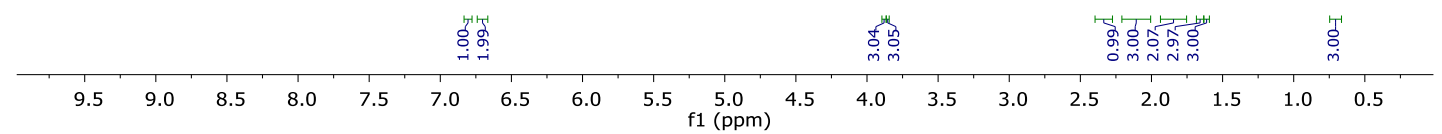

${ }^{1} \mathrm{H}$ NMR spectrum in $\mathrm{CDCl}_{3}$.

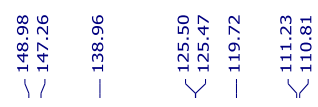

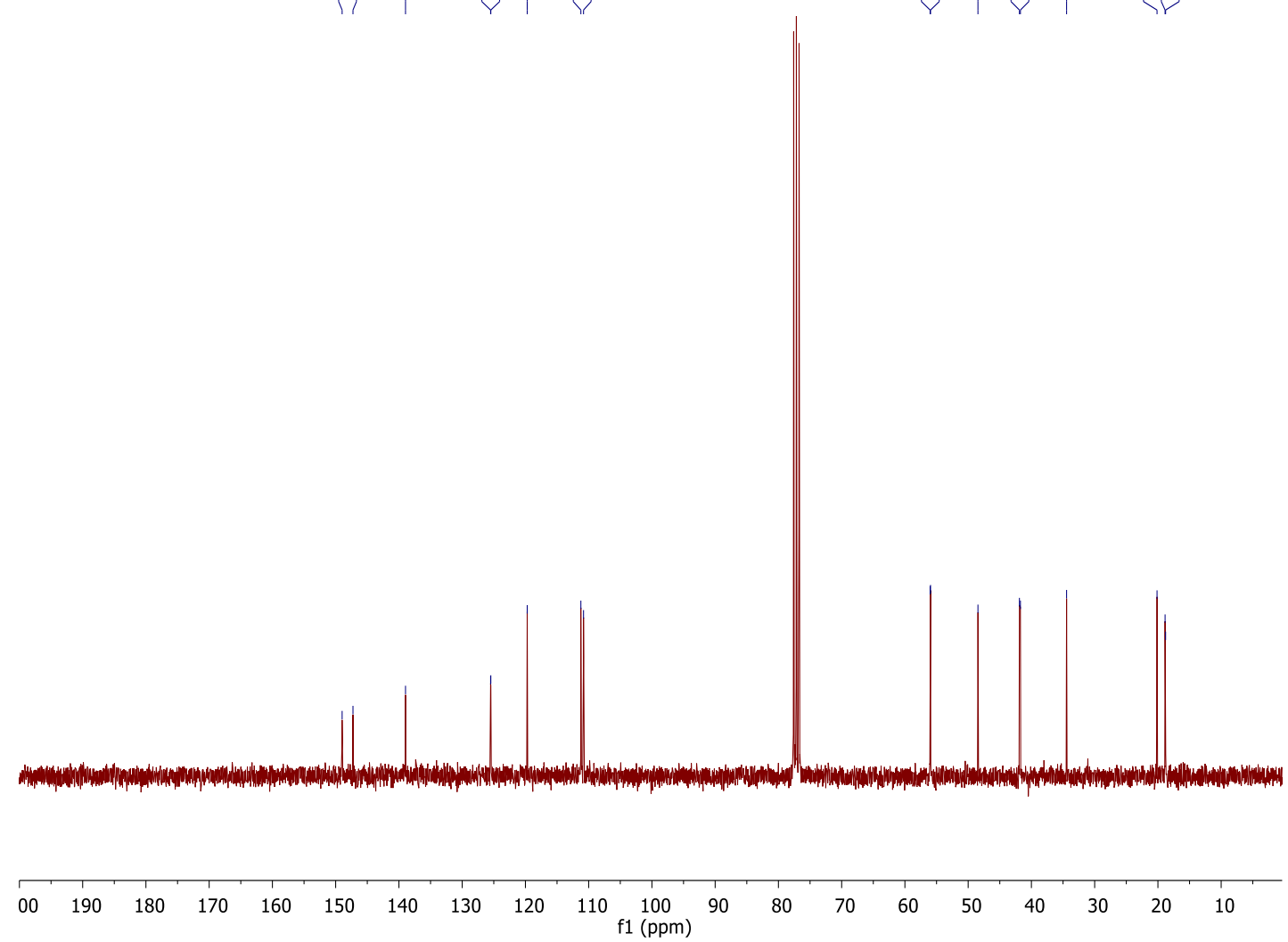

${ }^{13} \mathrm{C}$ NMR spectrum in $\mathrm{CDCl}_{3}$. 
211b
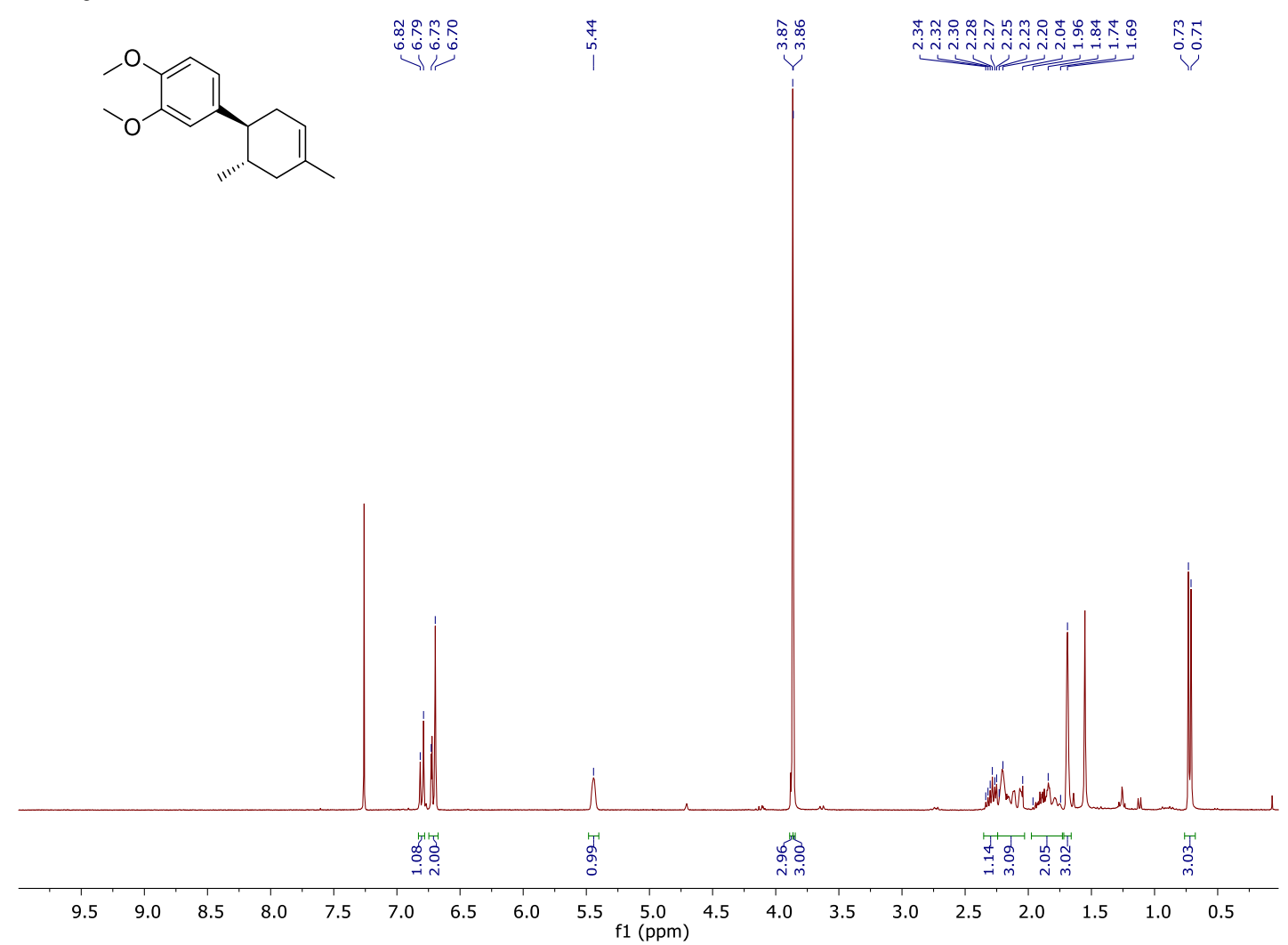

${ }^{1} \mathrm{H}$ NMR spectrum in $\mathrm{CDCl}_{3}$.

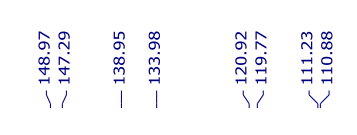

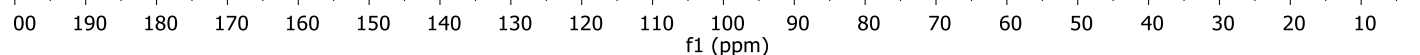

${ }^{13} \mathrm{C}$ NMR spectrum in $\mathrm{CDCl}_{3}$. 
212b<smiles>COc1ccc([C@H]2[C@@H](OC(C)=O)C=CC[C@H]2C)cc1OC</smiles>

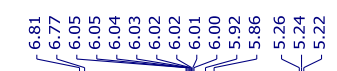

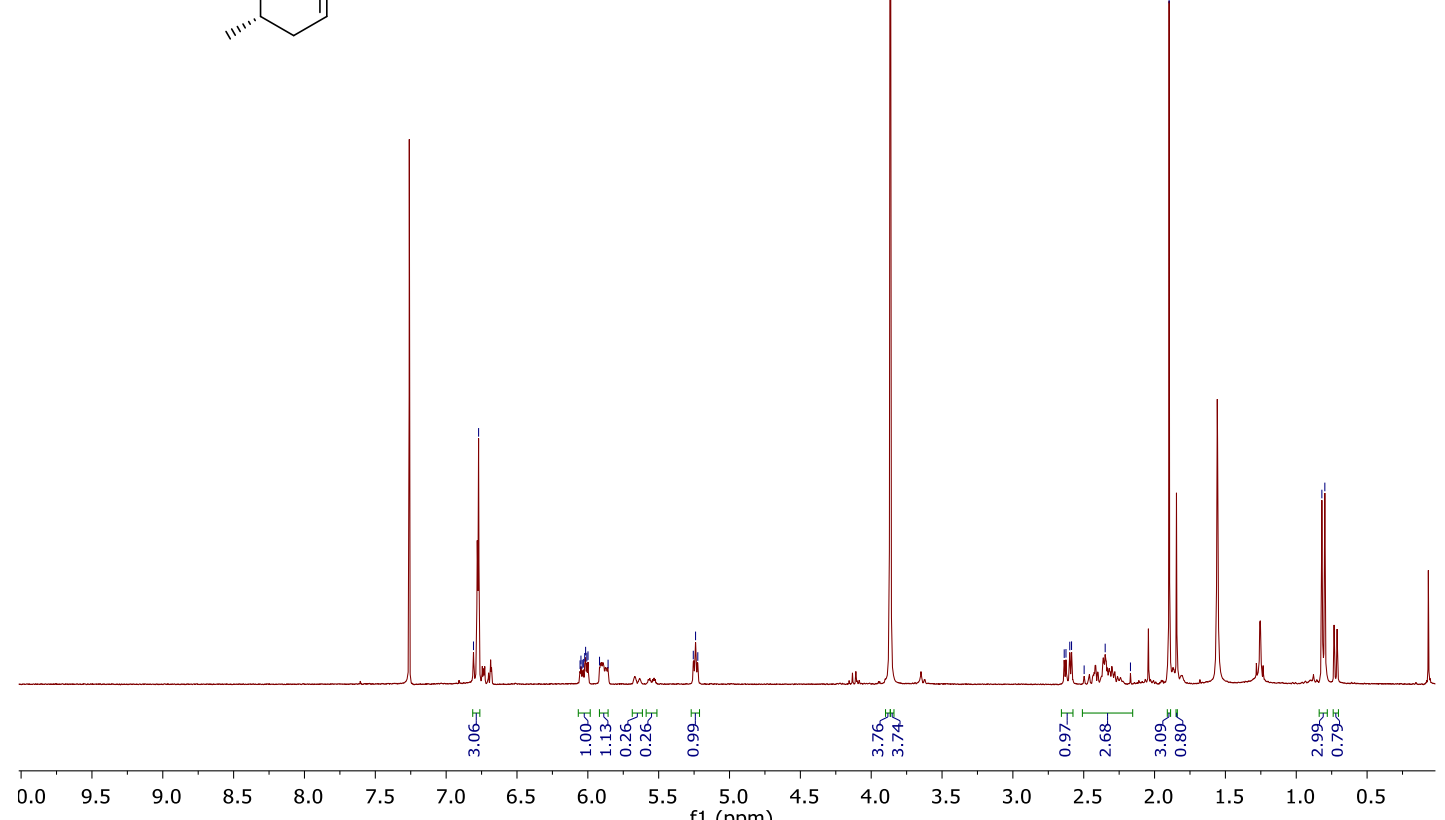

${ }^{1} \mathrm{H}$ NMR spectrum in $\mathrm{CDCl}_{3}$.

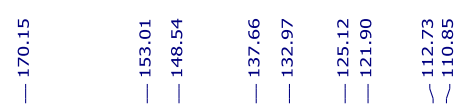

${ }^{13} \mathrm{C}$ NMR spectrum in $\mathrm{CDCl}_{3}$. 
213b<smiles>CC1=CC[C@H]2C(=O)OC(=O)[C@H]2C1</smiles>

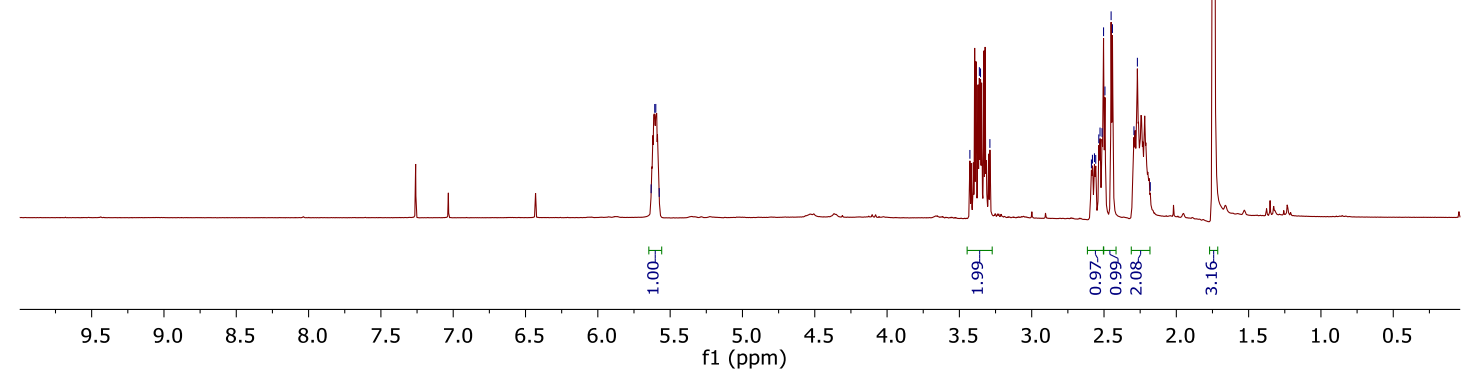

${ }^{1} \mathrm{H}$ NMR spectrum in $\mathrm{CDCl}_{3}$.

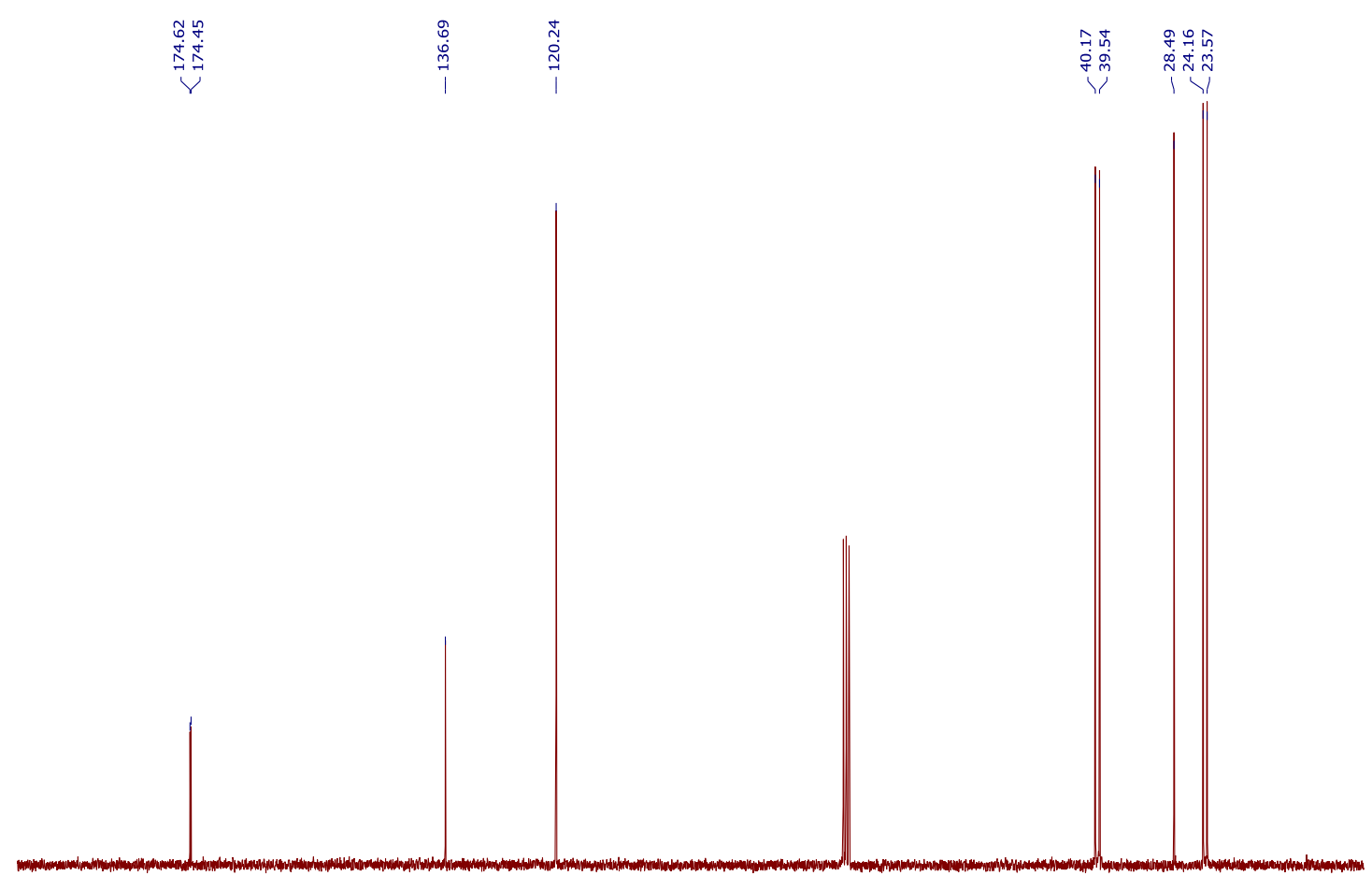

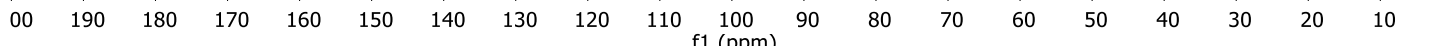
${ }^{13} \mathrm{C}$ NMR spectrum in $\mathrm{CDCl}_{3}$. 
214b<smiles>CC1=C(C)CC2C(=O)OC(=O)C2C1</smiles>

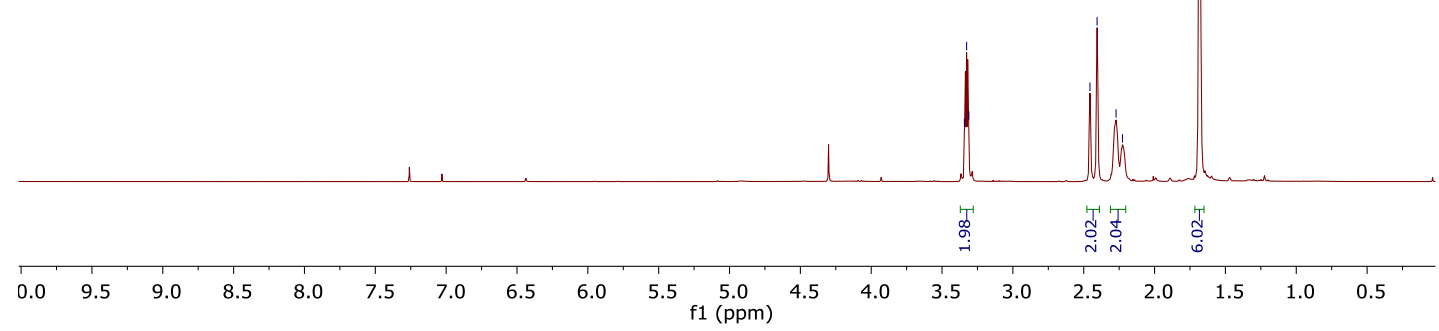

${ }^{1} \mathrm{H}$ NMR spectrum in $\mathrm{CDCl}_{3}$.

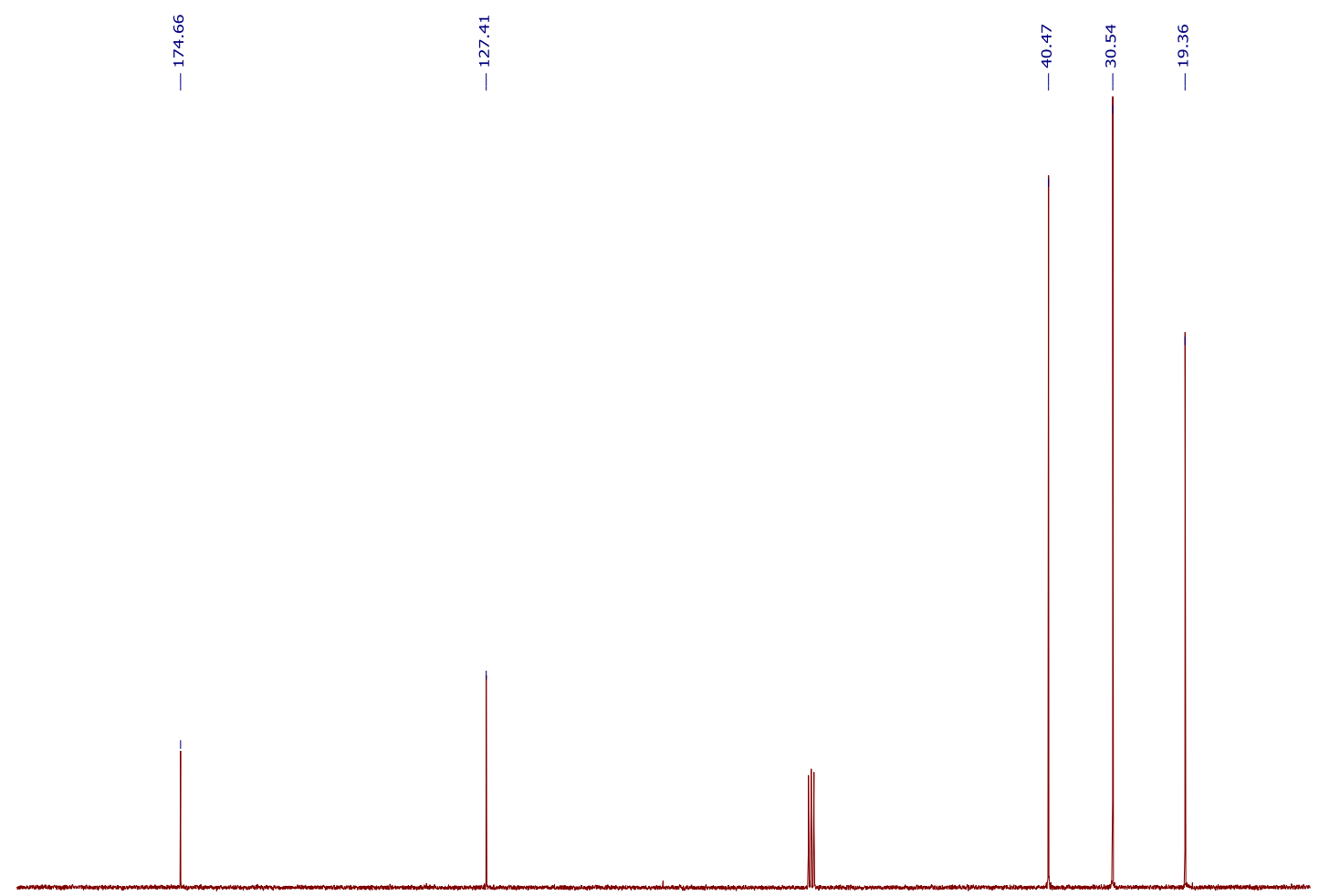

$\begin{array}{lllllllllllllllllll}190 & 180 & 170 & 160 & 150 & 140 & 130 & 120 & 110 & 100 & 90 & 80 & 70 & 60 & 50 & 40 & 30 & 20 & 10\end{array}$

${ }^{13} \mathrm{C} \mathrm{NMR}$ spectrum in $\mathrm{CDCl}_{3}$. 
215b<smiles>CCN1C(=O)C2CC(C)=C(C)CC2C1=O</smiles>

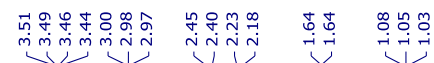

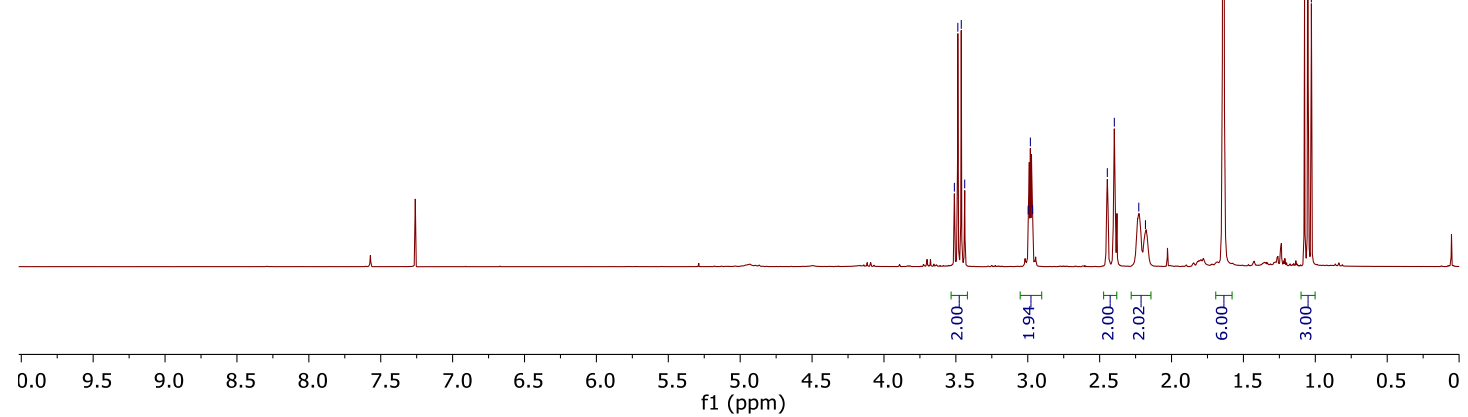

${ }^{1} \mathrm{H}$ NMR spectrum in $\mathrm{CDCl}_{3}$.

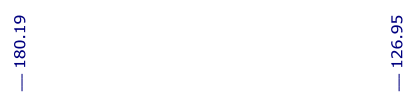



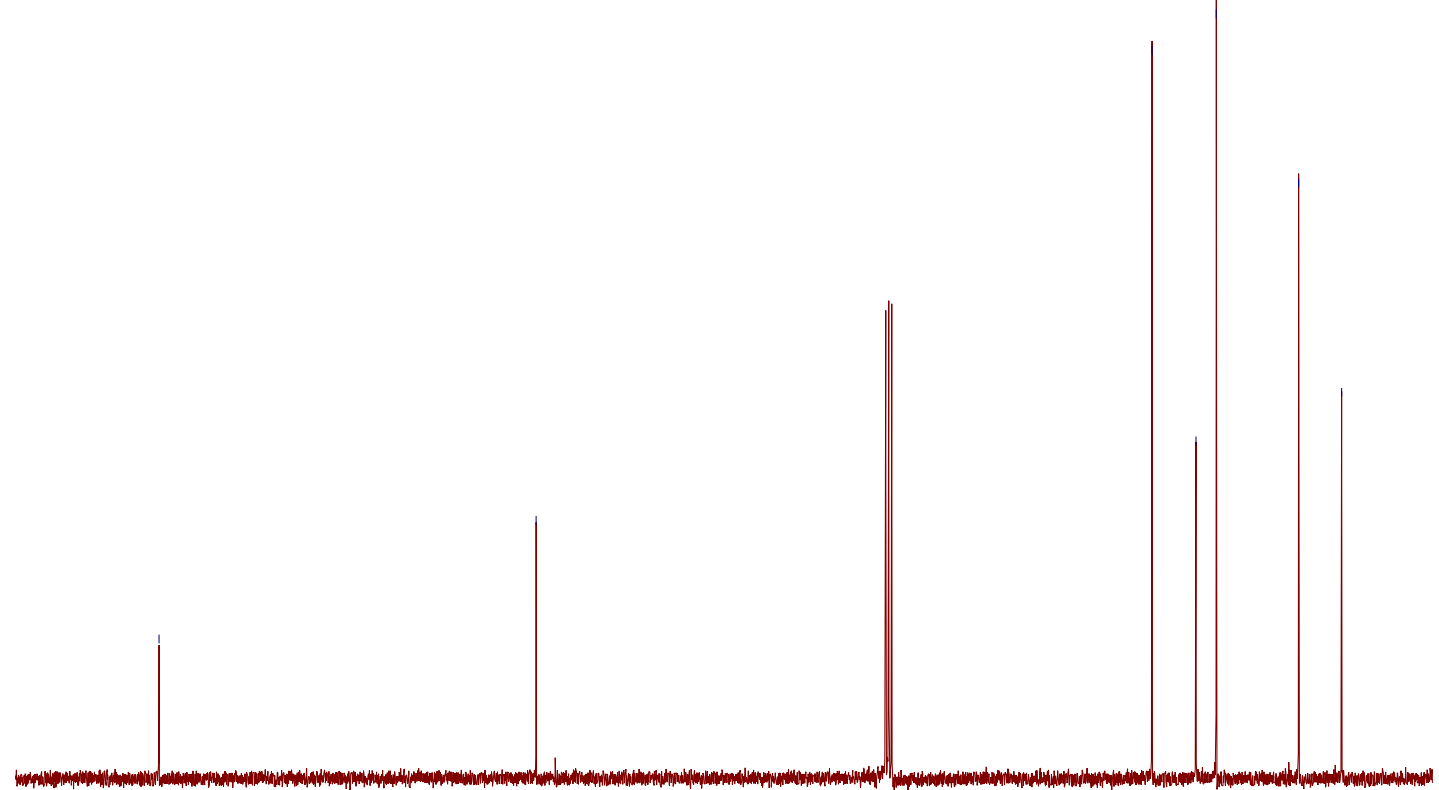

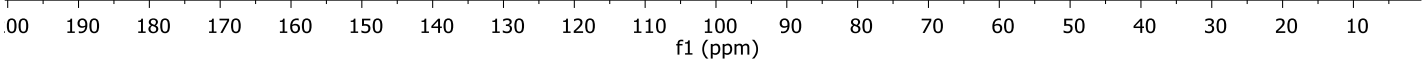

${ }^{13} \mathrm{C}$ NMR spectrum in $\mathrm{CDCl}_{3}$. 


\section{6b}
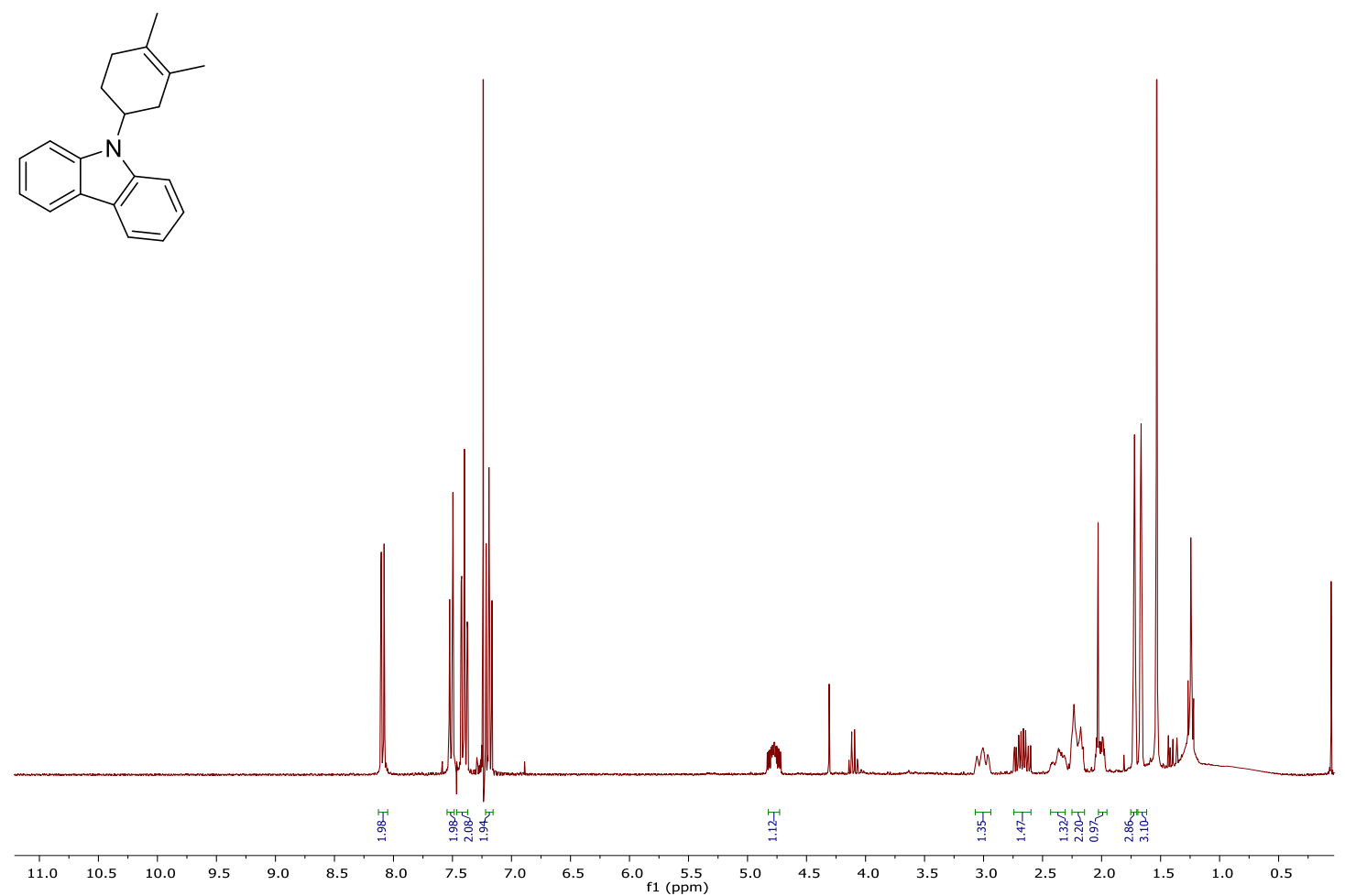

${ }^{1} \mathrm{H}$ NMR spectrum in $\mathrm{CDCl}_{3}$.

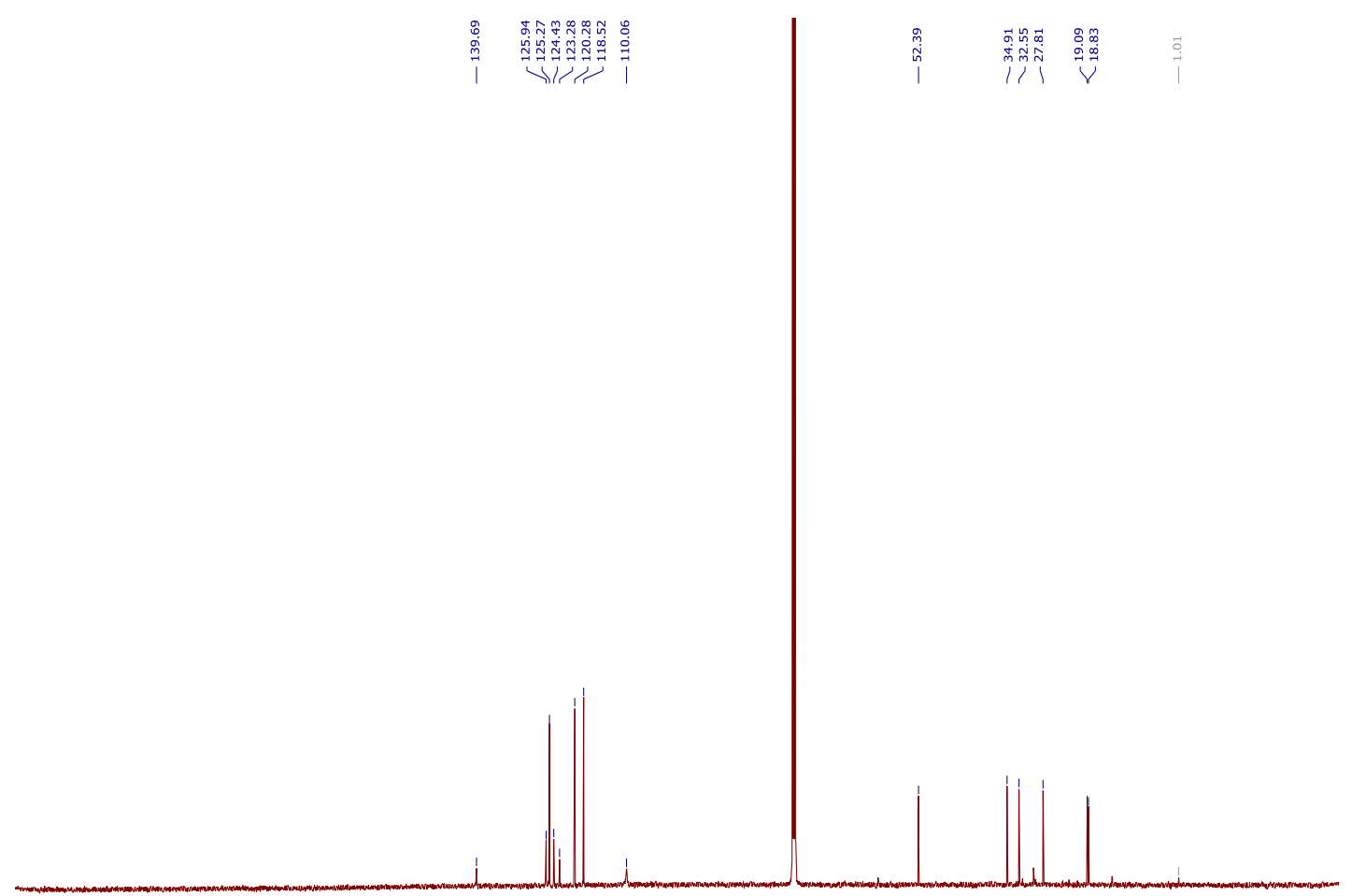

$\begin{array}{lllllllllllllllllllllllllllll}30 & 220 & 210 & 200 & 190 & 180 & 170 & 160 & 150 & 140 & 130 & 120 & 110 & 100 & 90 & 80 & 70 & 60 & 50 & 40 & 30 & 20 & 10 & 0 & -10 & -20 & -3\end{array}$

${ }^{13} \mathrm{C}$ NMR spectrum in $\mathrm{CDCl}_{3}$ 


\section{7b}

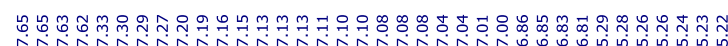

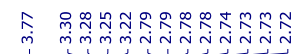<smiles>COc1ccc(C2CC(=O)C=CN2c2ccccc2)cc1</smiles>

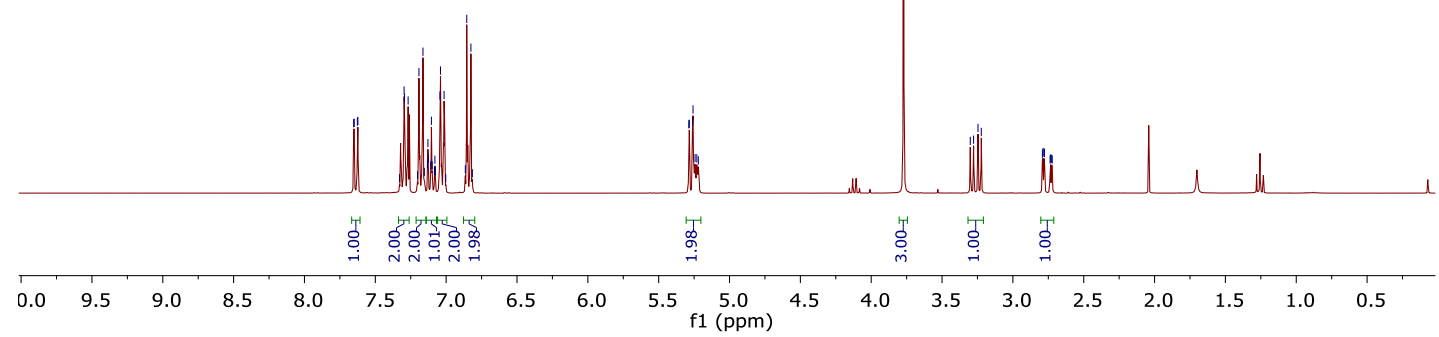

${ }^{1} \mathrm{H}$ NMR spectrum in $\mathrm{CDCl}_{3}$.

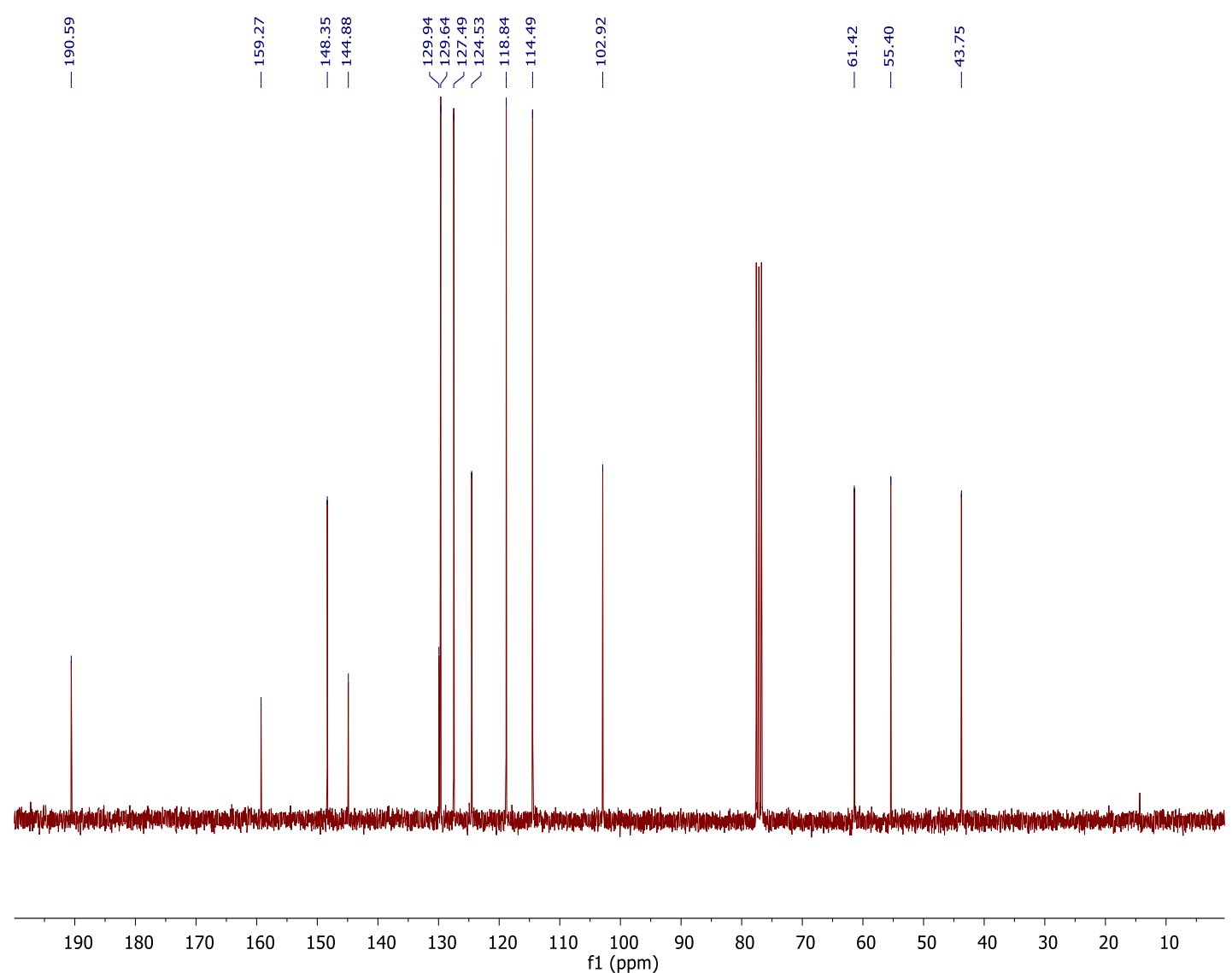

${ }^{13} \mathrm{C}$ NMR spectrum in $\mathrm{CDCl}_{3}$. 
218b
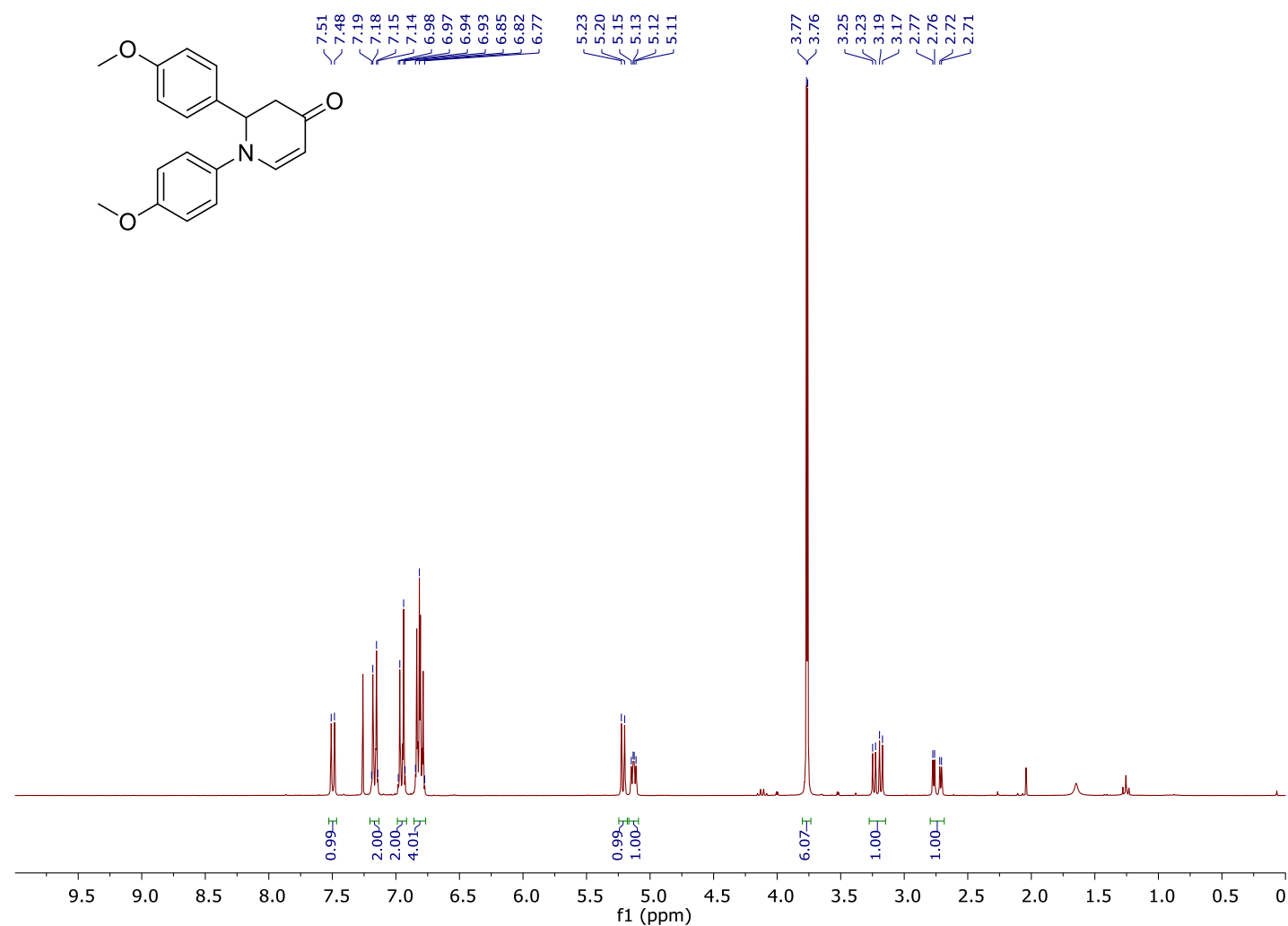

${ }^{1} \mathrm{H}$ NMR spectrum in $\mathrm{CDCl}_{3}$.

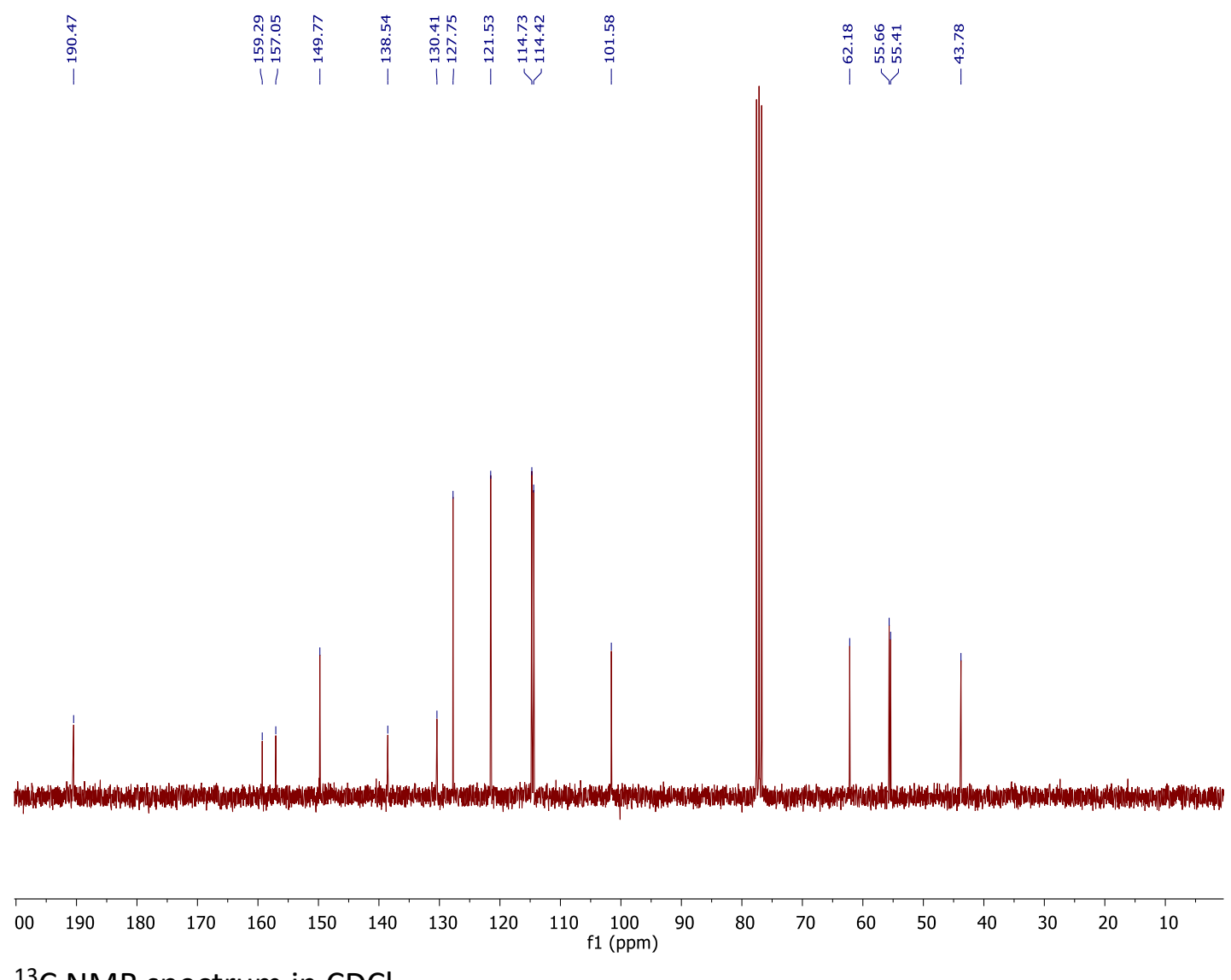

${ }^{13} \mathrm{C}$ NMR spectrum in $\mathrm{CDCl}_{3}$. 
$219 b$

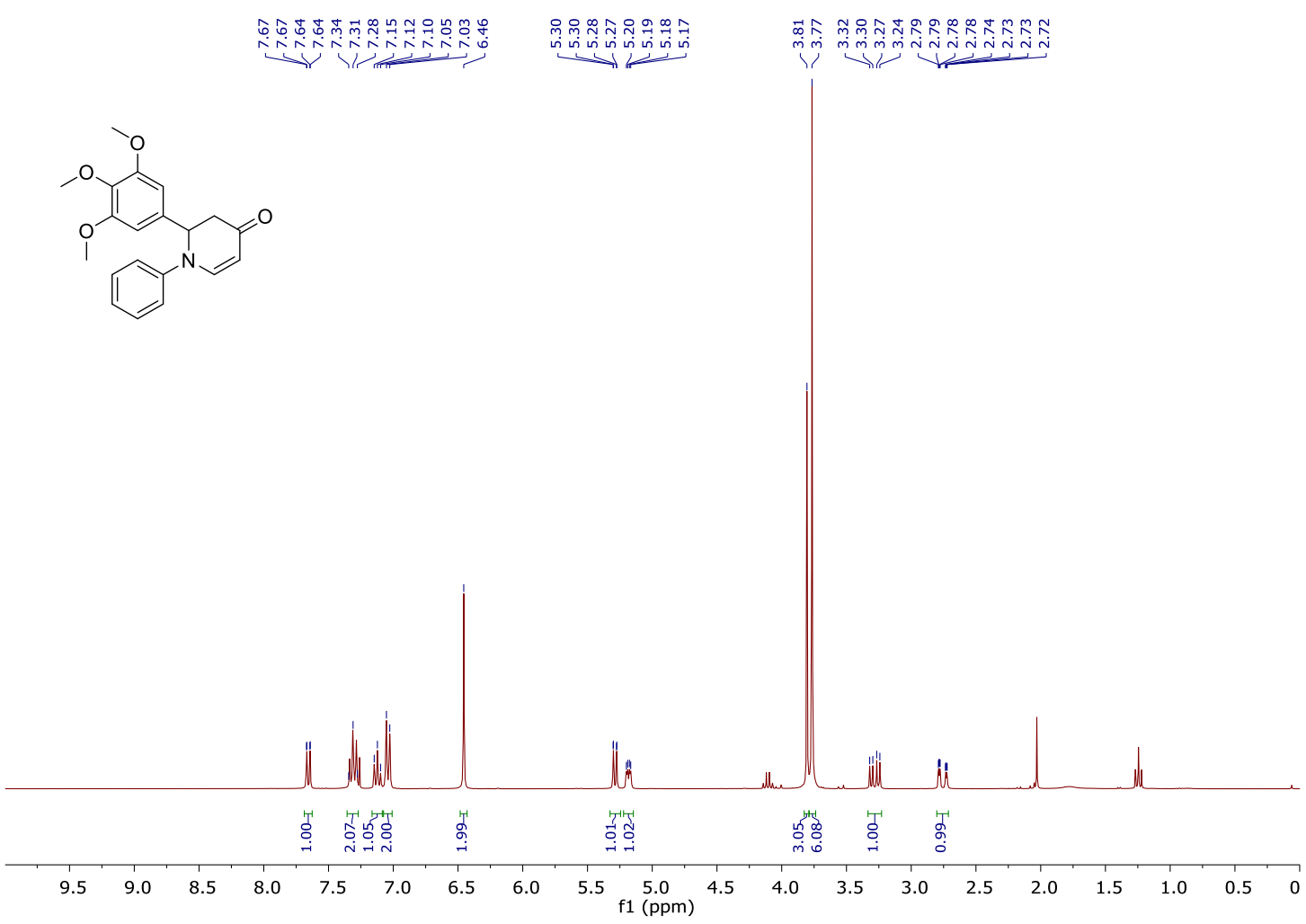

${ }^{1} \mathrm{H}$ NMR spectrum in $\mathrm{CDCl}_{3}$.

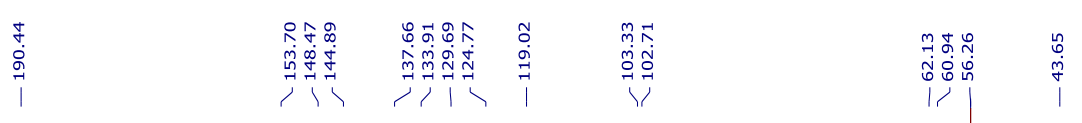

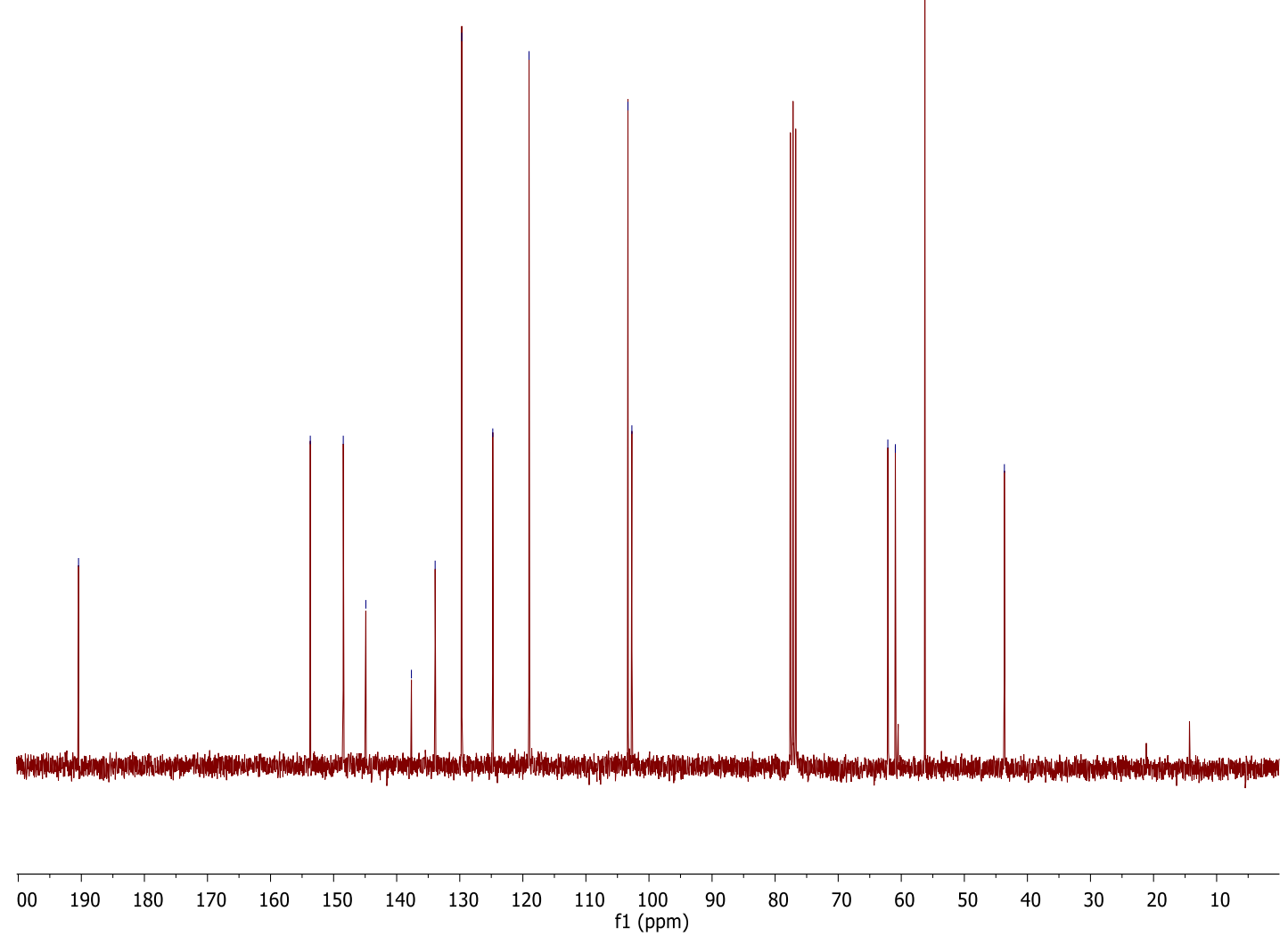

${ }^{13} \mathrm{C}$ NMR spectrum in $\mathrm{CDCl}_{3}$. 
220b

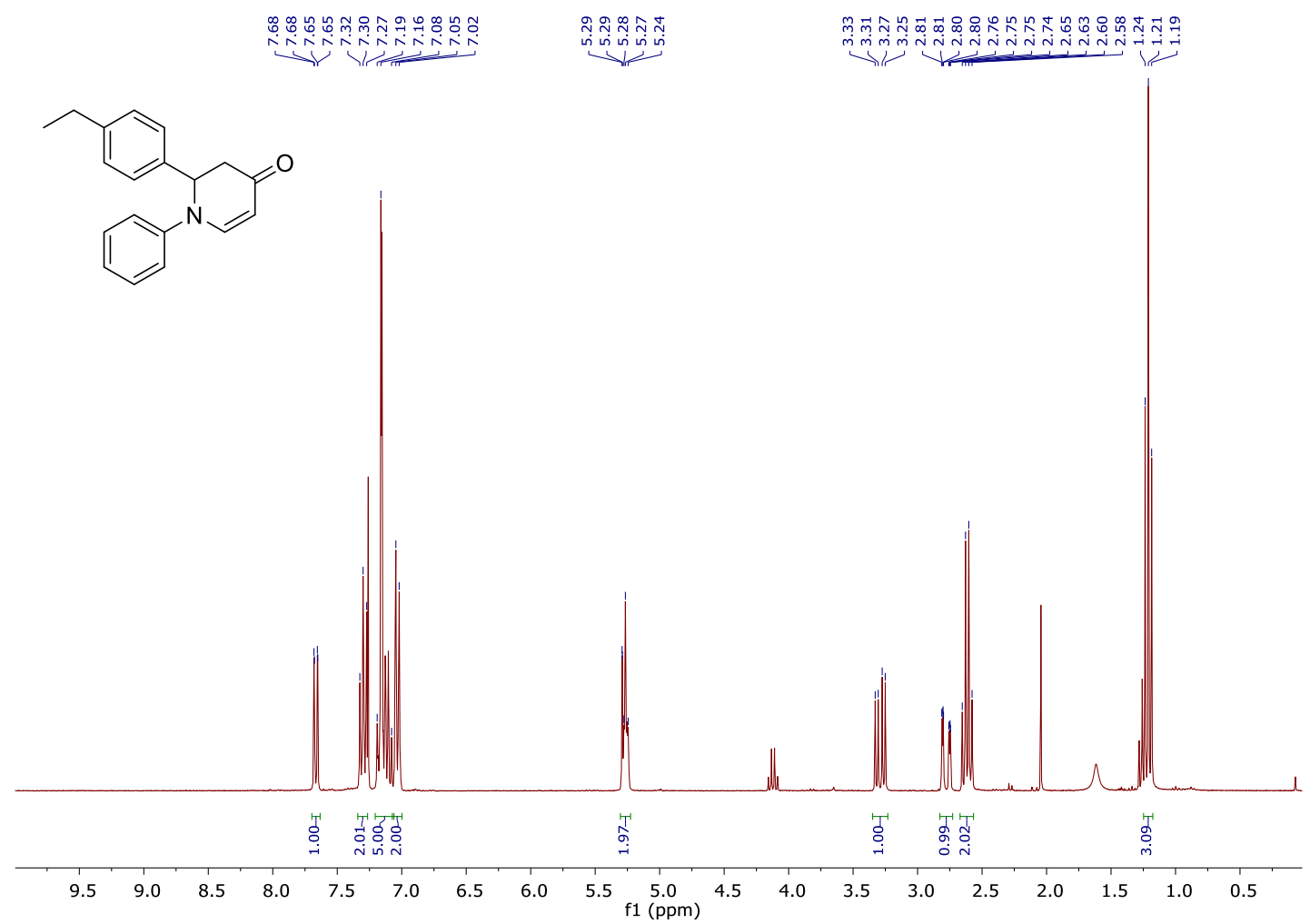

${ }^{1} \mathrm{H}$ NMR spectrum in $\mathrm{CDCl}_{3}$.

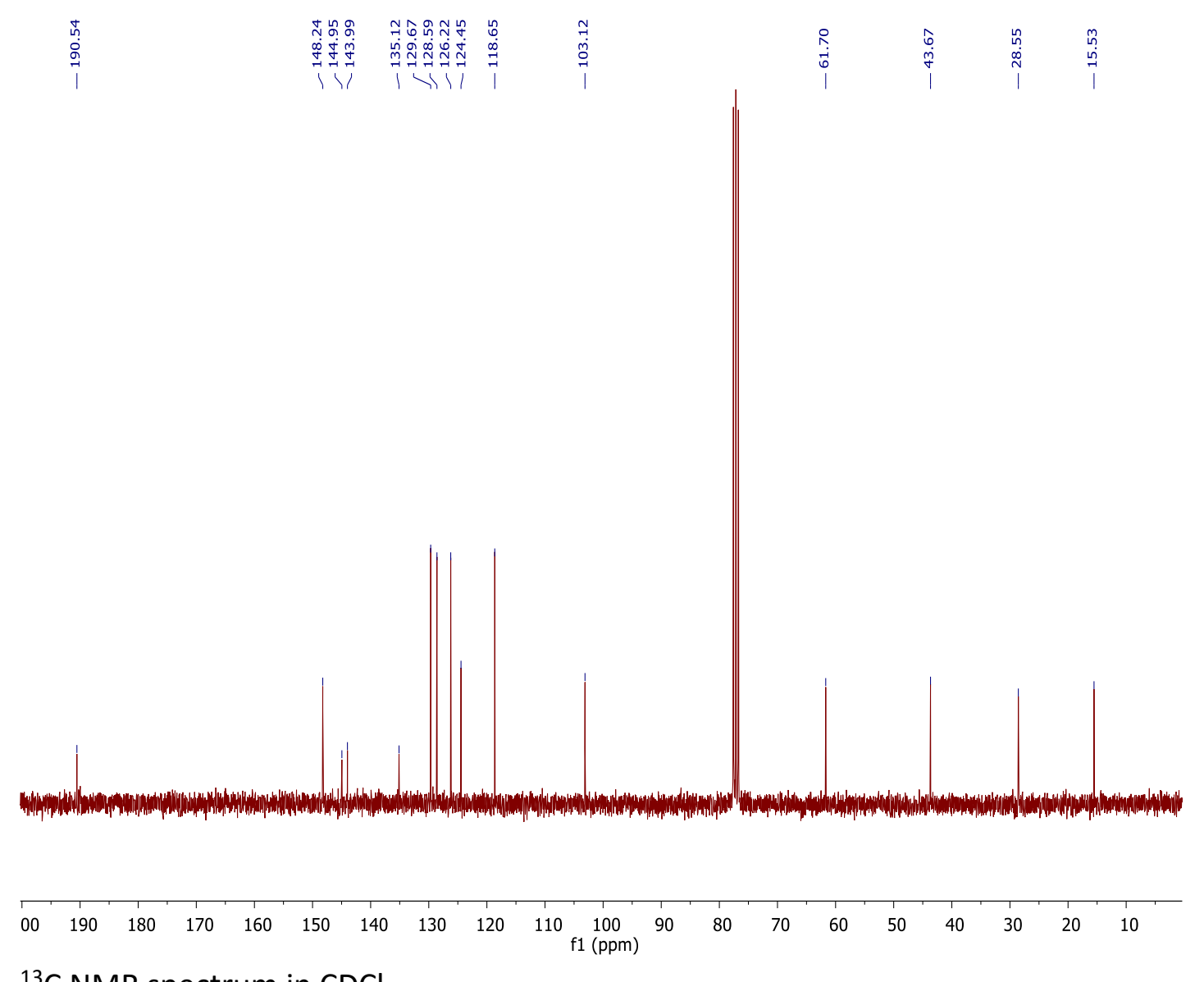

${ }^{13} \mathrm{C}$ NMR spectrum in $\mathrm{CDCl}_{3}$. 


\section{1b}

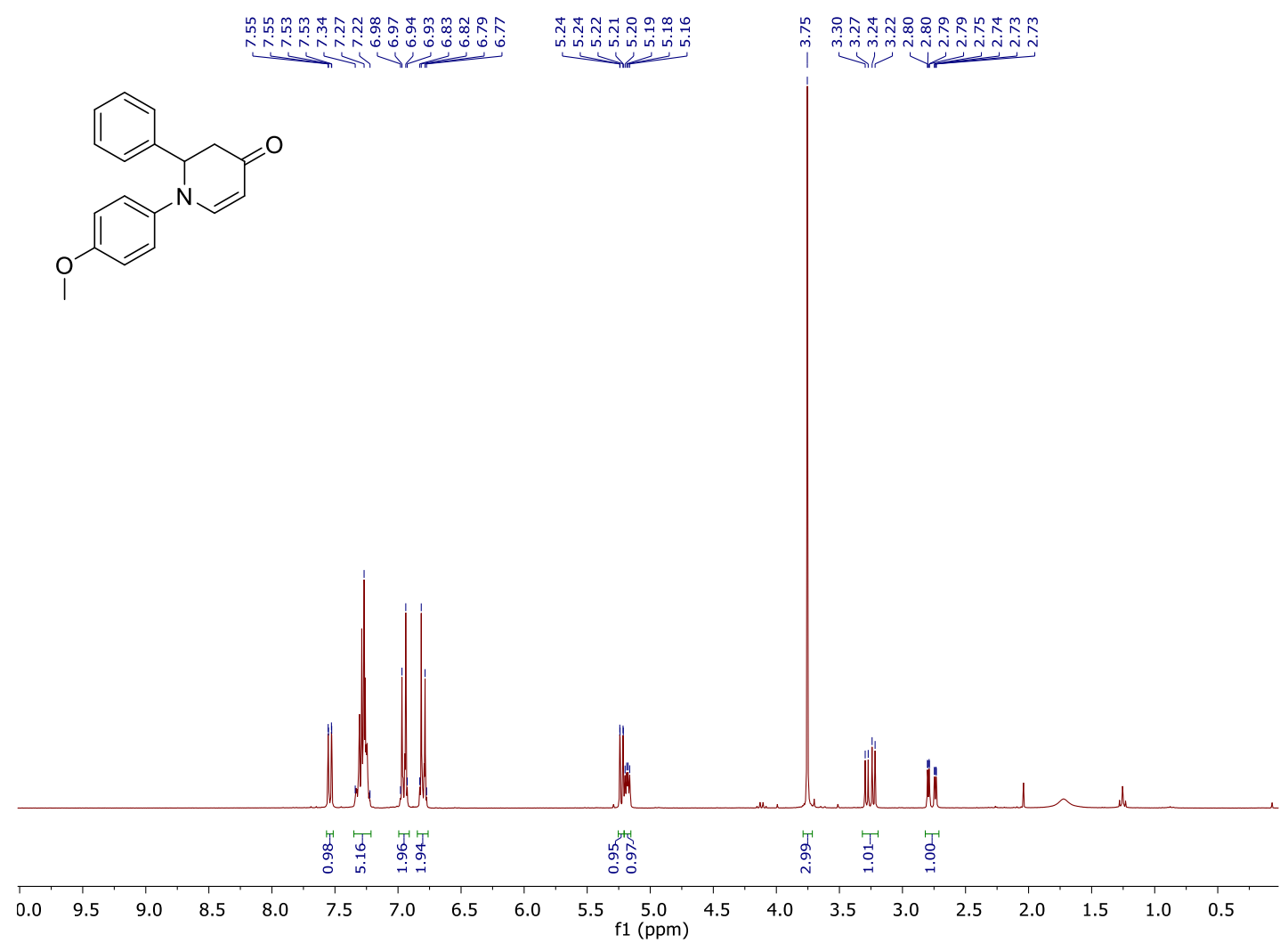

${ }^{1} \mathrm{H}$ NMR spectrum in $\mathrm{CDCl}_{3}$.

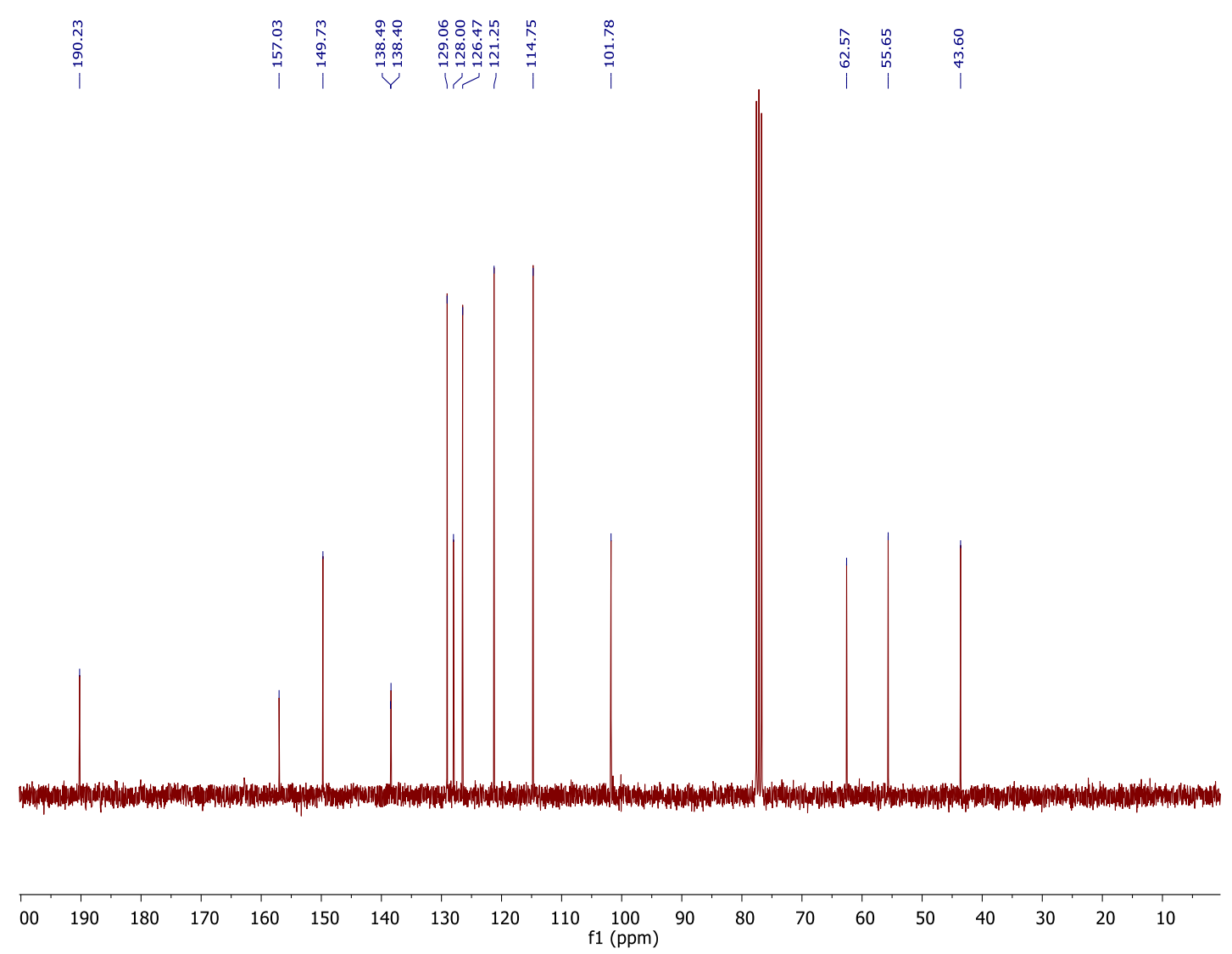

${ }^{13} \mathrm{C}$ NMR spectrum in $\mathrm{CDCl}_{3}$. 
222b<smiles>CN1CCC(=O)CC1c1ccccc1</smiles>

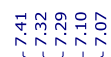

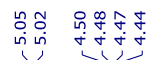

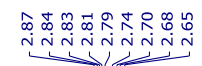

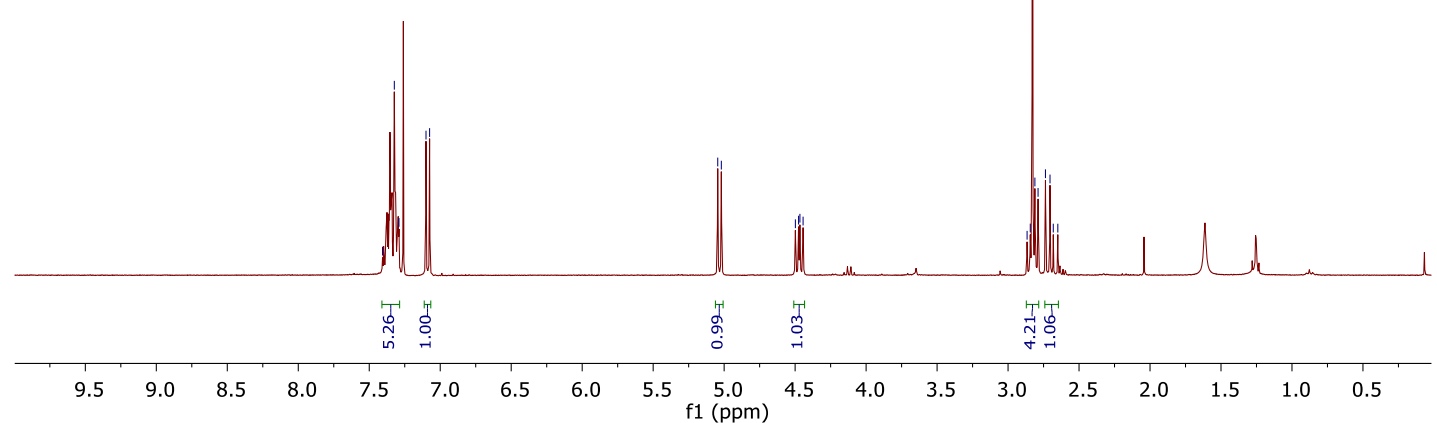

${ }^{1} \mathrm{H}$ NMR spectrum in $\mathrm{CDCl}_{3}$.

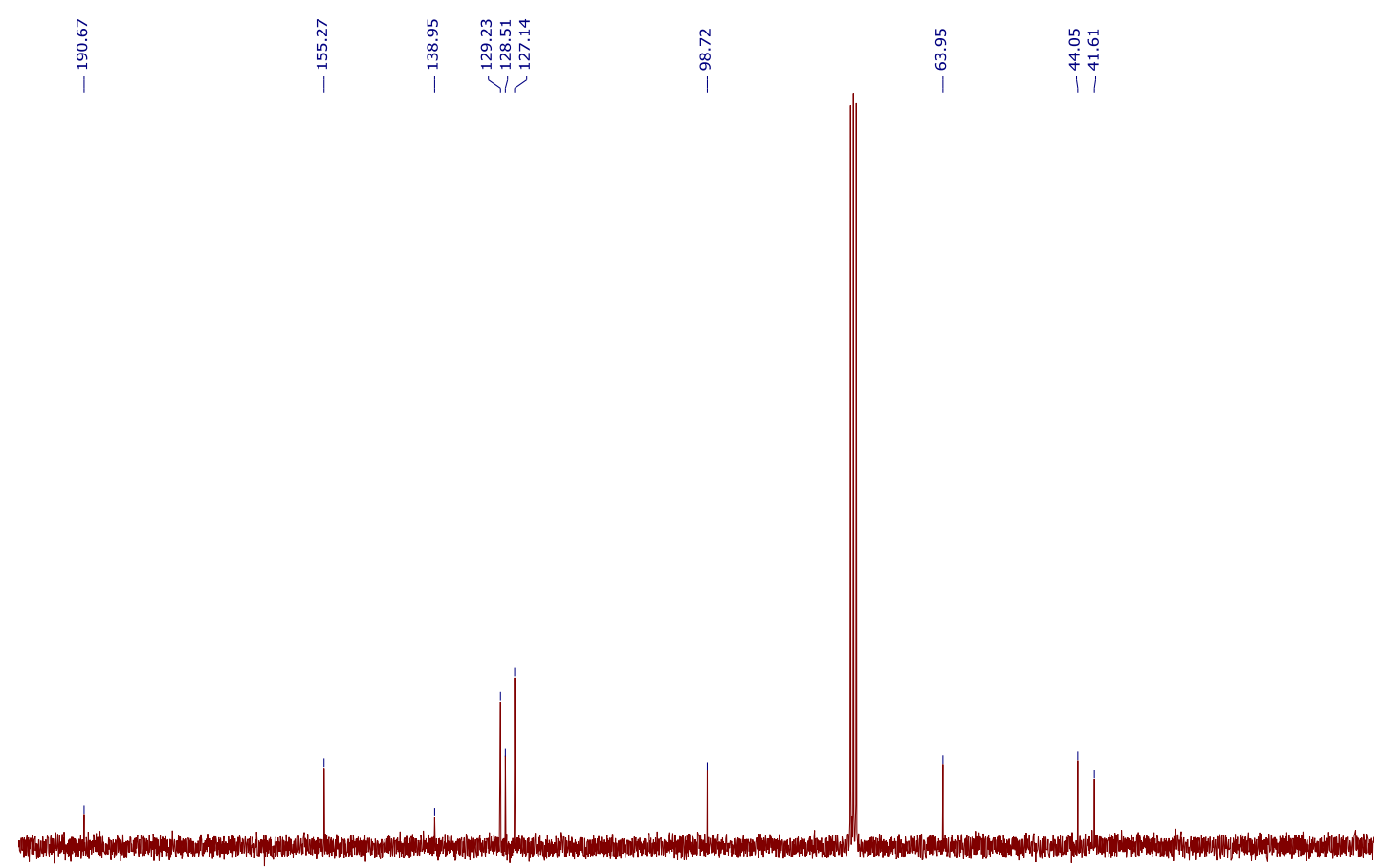

\begin{tabular}{llllllllllllllllllll}
\hline 00 & 190 & 180 & 170 & 160 & 150 & 140 & 130 & 120 & 110 & $\begin{array}{l}100 \\
\mathrm{f} 1(\mathrm{ppm})\end{array}$ & 90 & 80 & 70 & 60 & 50 & 40 & 30 & 20 & 10
\end{tabular}

${ }^{13} \mathrm{C}$ NMR spectrum in $\mathrm{CDCl}_{3}$. 
223b<smiles>CCCCN1C=CC(=O)CC1c1ccc([N+](=O)[O-])cc1</smiles>

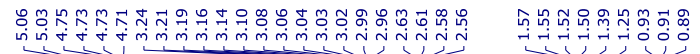

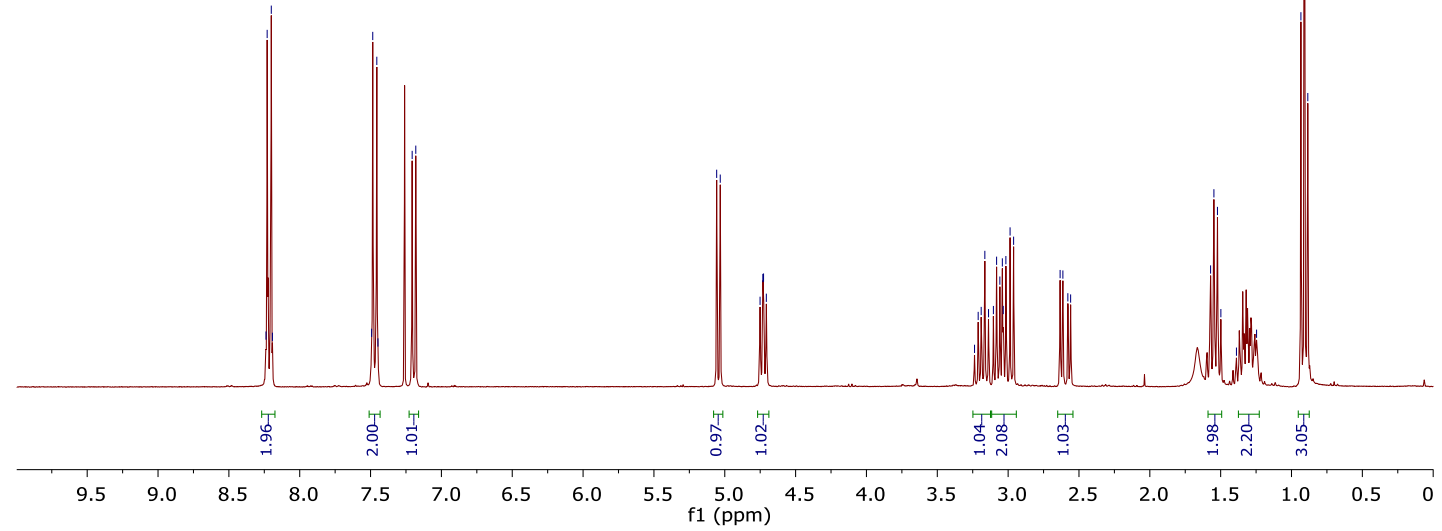

${ }^{1} \mathrm{H}$ NMR spectrum in $\mathrm{CDCl}_{3}$.

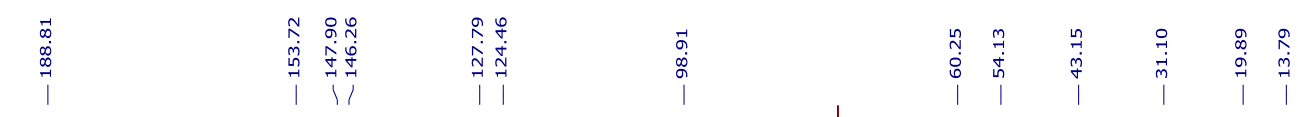

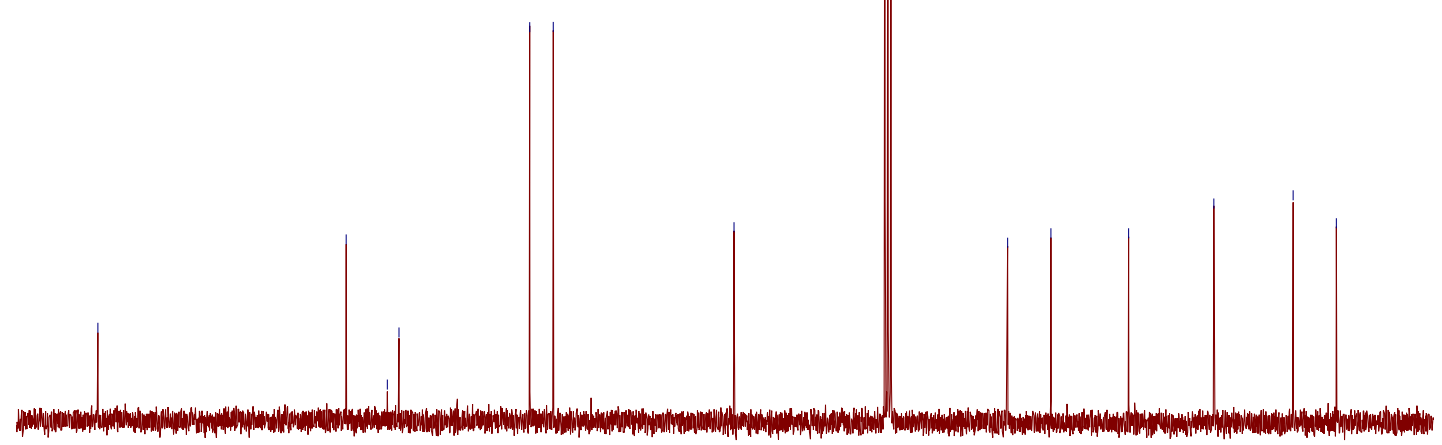

$\begin{array}{lllllllllllllllllll}00 & 190 & 180 & 170 & 160 & 150 & 140 & 130 & 120 & 110 \underset{\mathrm{f} 1(\mathrm{ppm})}{100} & 90 & 80 & 70 & 60 & 50 & 40 & 30 & 20 & 10\end{array}$

${ }^{13} \mathrm{C}$ NMR spectrum in $\mathrm{CDCl}_{3}$. 
224b

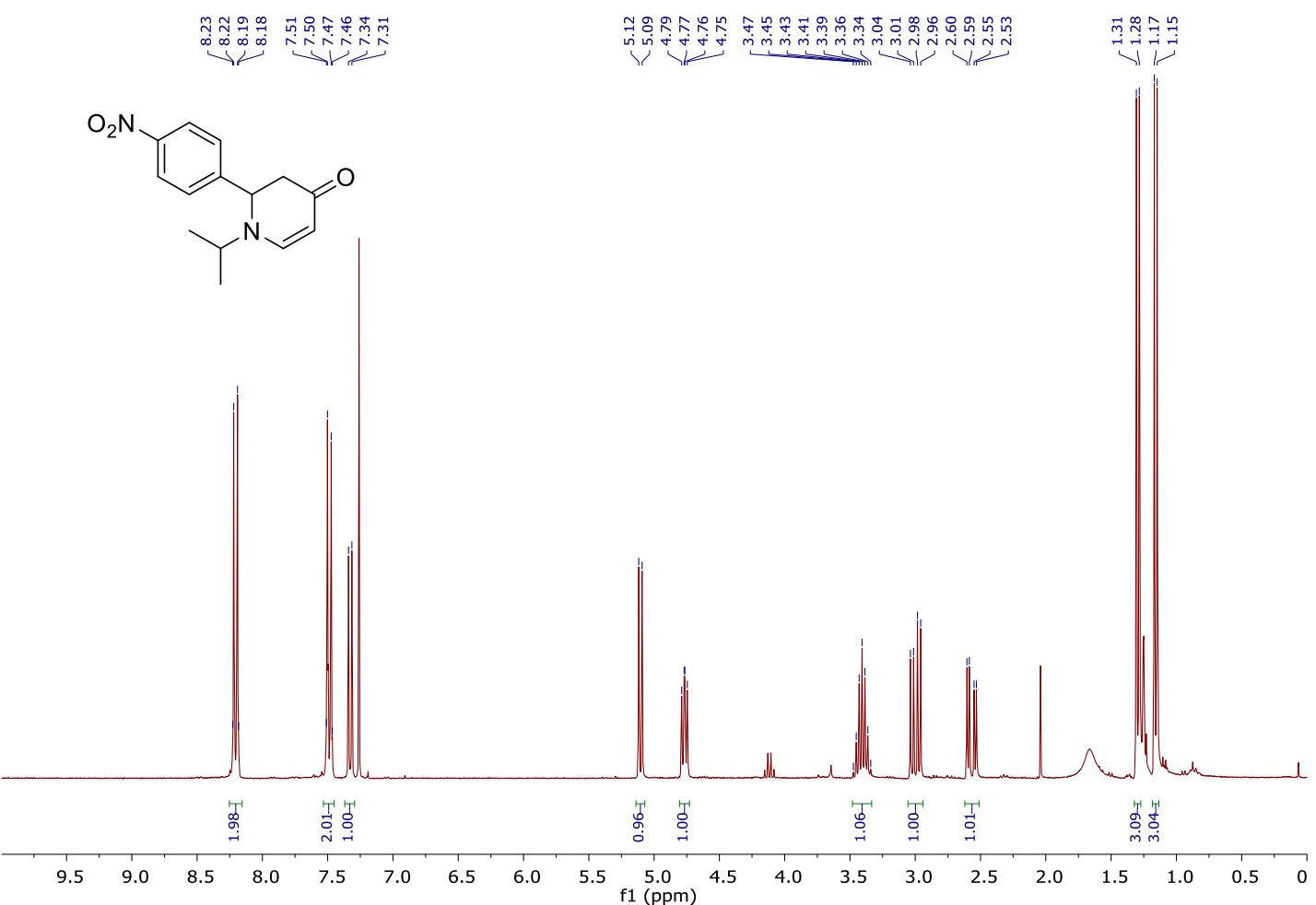

${ }^{1} \mathrm{H}$ NMR spectrum in $\mathrm{CDCl}_{3}$.

\begin{tabular}{|c|c|c|c|}
\hline 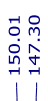 & 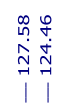 & $\begin{array}{l}o \\
\dot{0} \\
o \\
1 \\
1\end{array}$ & \\
\hline
\end{tabular}

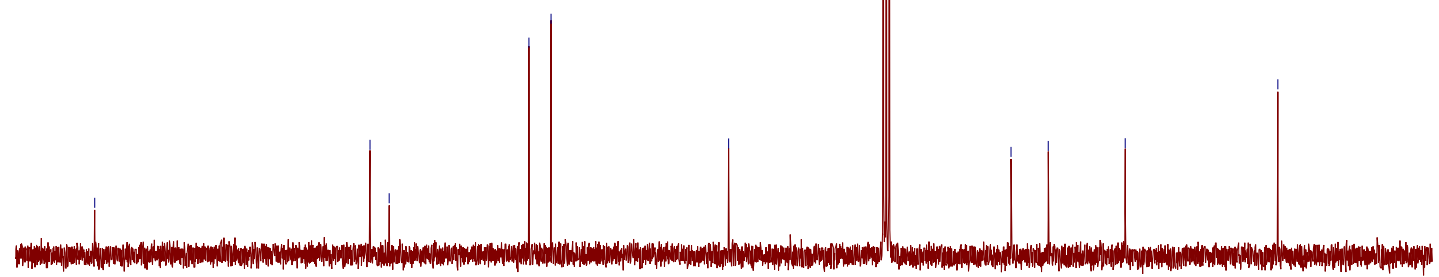

$\begin{array}{llllllllllllllllllll}00 & 190 & 180 & 170 & 160 & 150 & 140 & 130 & 120 & 110 \underset{\mathrm{f} 1(\mathrm{ppm})}{100} & 90 & 80 & 70 & 60 & 50 & 40 & 30 & 20 & 10\end{array}$ ${ }^{13} \mathrm{C}$ NMR spectrum in $\mathrm{CDCl}_{3}$. 
225b<smiles>CC(C)(C)N1C=CC(=O)CC1c1ccccc1</smiles>

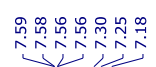

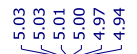

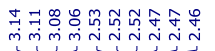

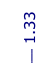

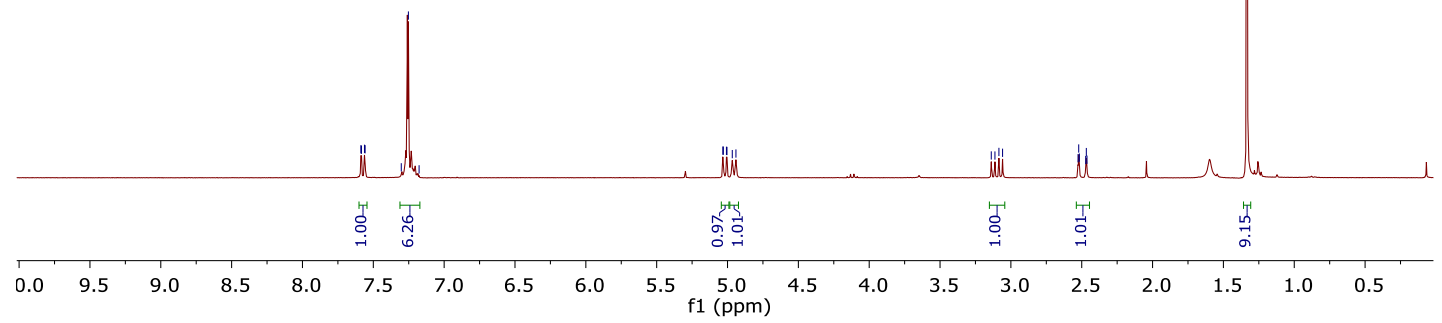

${ }^{1} \mathrm{H}$ NMR spectrum in $\mathrm{CDCl}_{3}$.

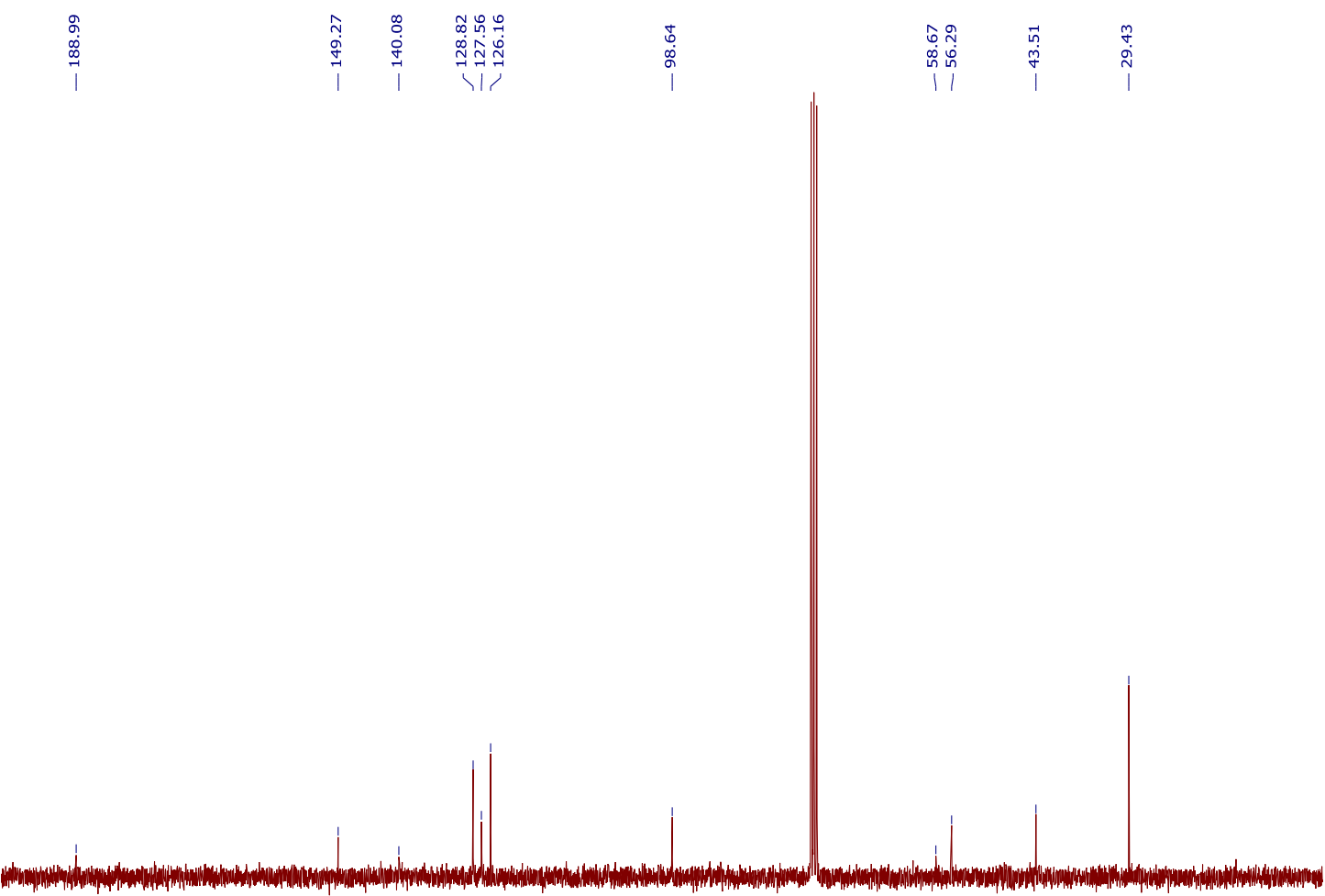

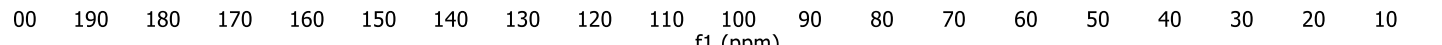

${ }^{13} \mathrm{C}$ NMR spectrum in $\mathrm{CDCl}_{3}$. 


\section{6b}

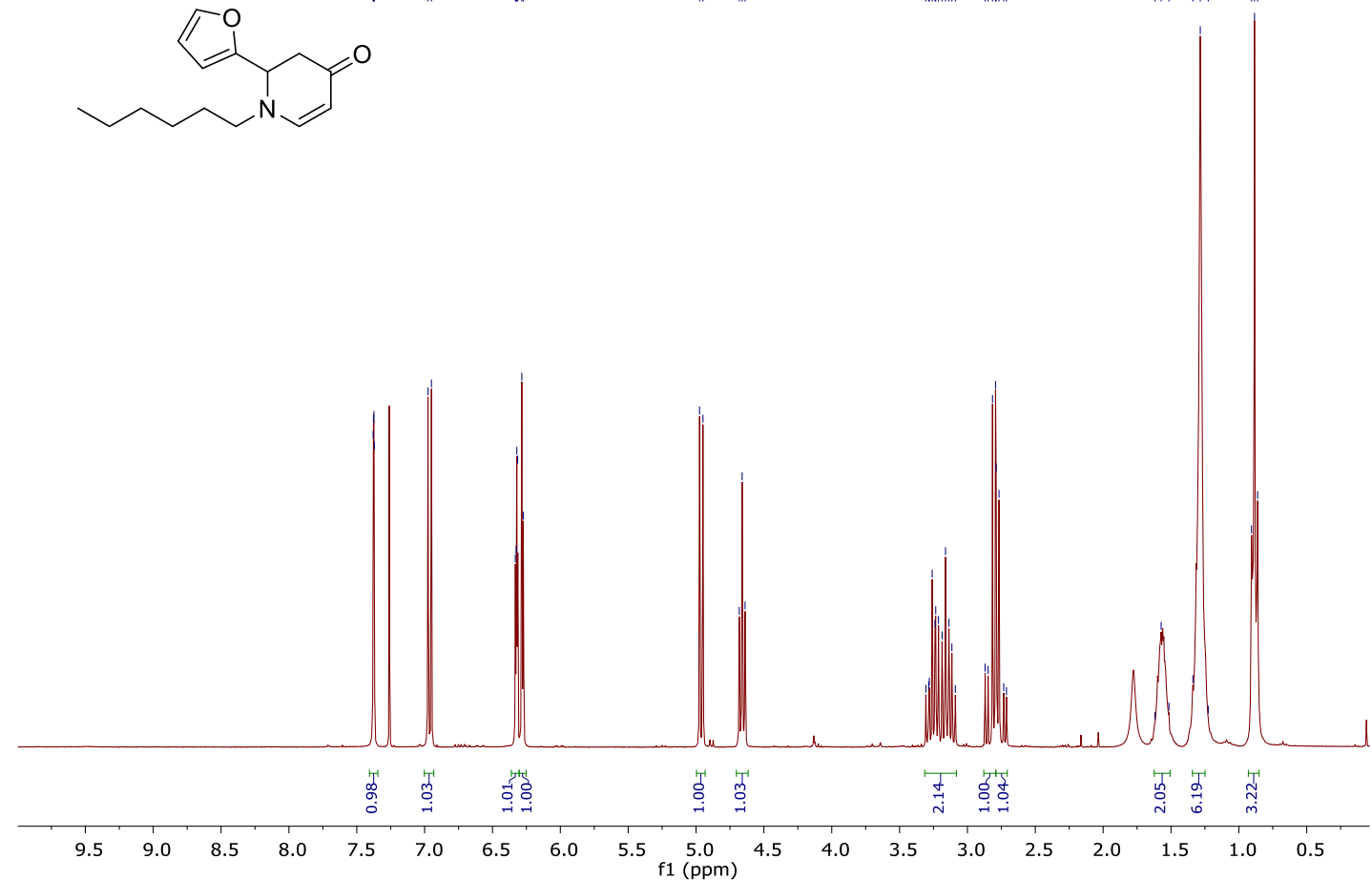

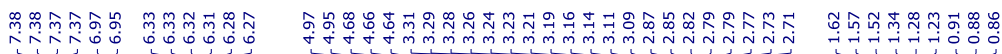

${ }^{1} \mathrm{H}$ NMR spectrum in $\mathrm{CDCl}_{3}$.

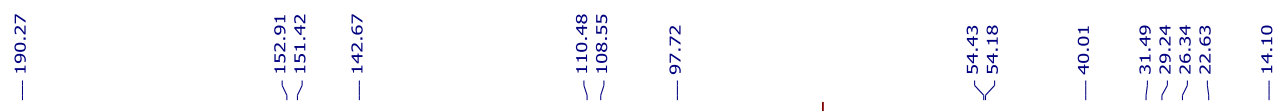

$\begin{array}{llllllllllllllllllll}00 & 190 & 180 & 170 & 160 & 150 & 140 & 130 & 120 & 110 & 100 & 90 & 80 & 70 & 60 & 50 & 40 & 30 & 20 & 10\end{array}$

${ }^{13} \mathrm{C}$ NMR spectrum in $\mathrm{CDCl}_{3}$. 
227b

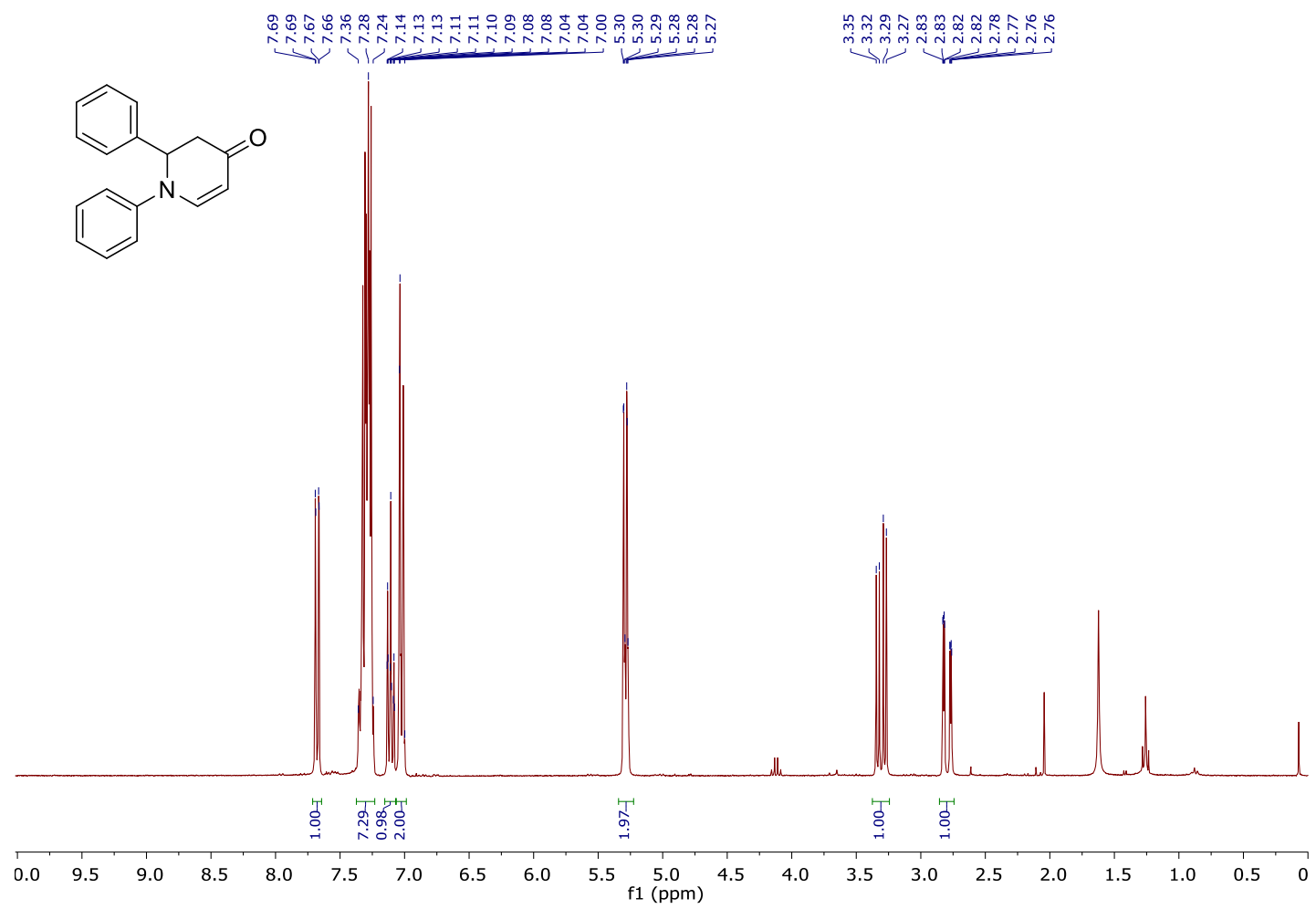

${ }^{1} \mathrm{H}$ NMR spectrum in $\mathrm{CDCl}_{3}$.

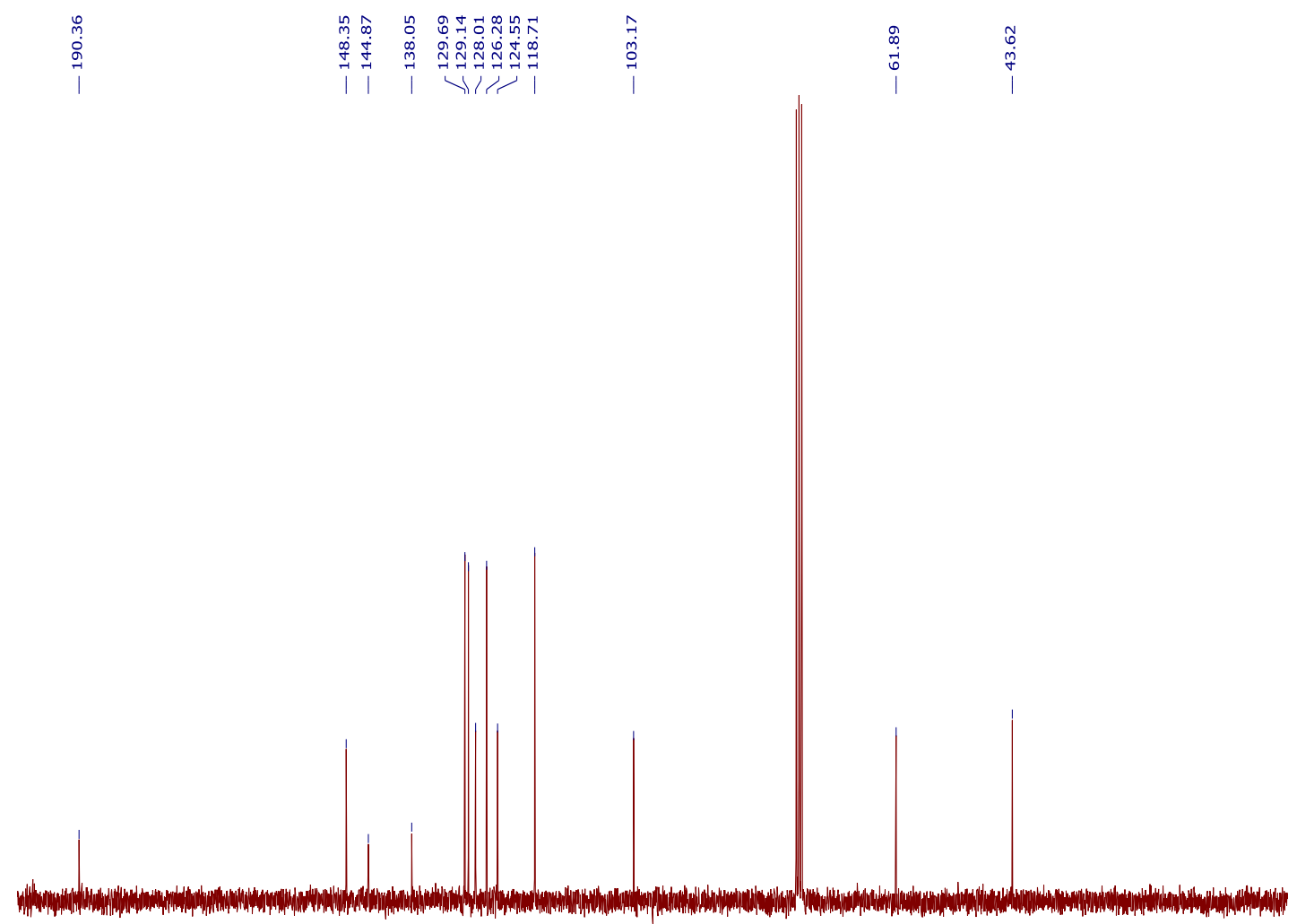

$\begin{array}{llllllllllllllllllll}00 & 190 & 180 & 170 & 160 & 150 & 140 & 130 & 120 & 110 & \begin{array}{c}100 \\ \mathrm{f} 1(\mathrm{ppm})\end{array} & 90 & 80 & 70 & 60 & 50 & 40 & 30 & 20 & 10\end{array}$

${ }^{13} \mathrm{C}$ NMR spectrum in $\mathrm{CDCl}_{3}$. 
$228 b$<smiles>COc1ccc(C2CC(=O)C=CN2c2ccc([N+](=O)[O-])cc2)cc1</smiles>

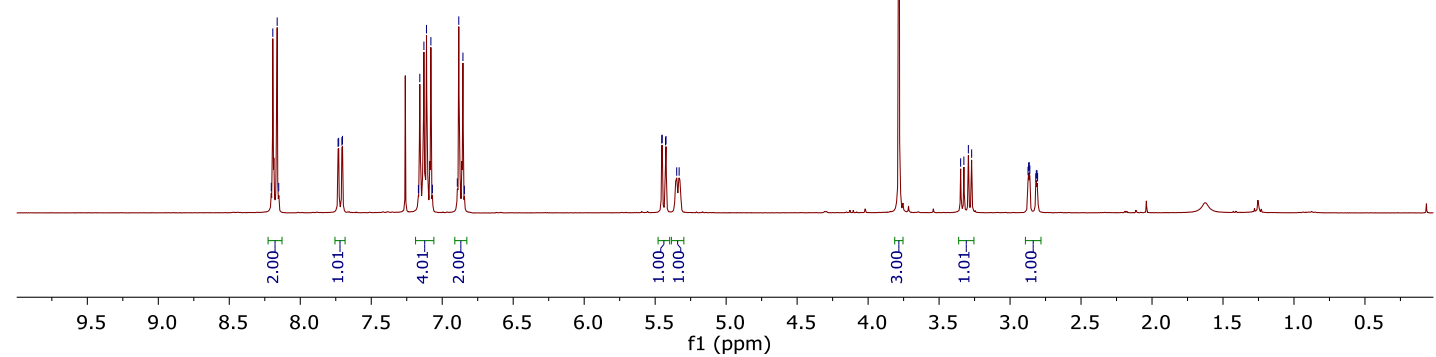

${ }^{1} \mathrm{H}$ NMR spectrum in $\mathrm{CDCl}_{3}$.

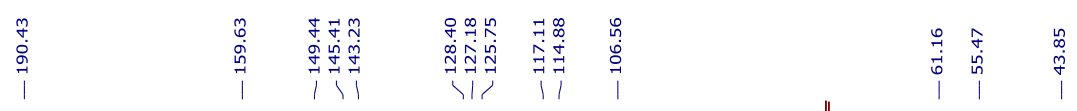

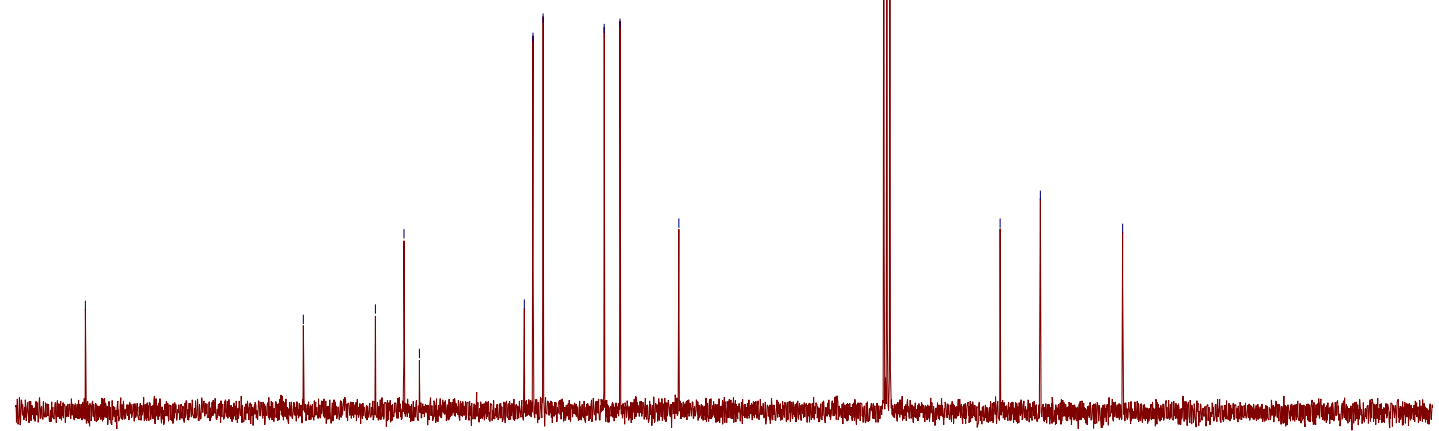

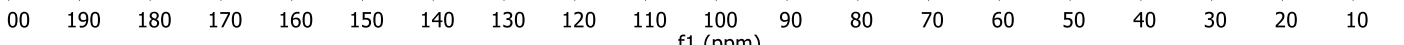

${ }^{13} \mathrm{C}$ NMR spectrum in $\mathrm{CDCl}_{3}$. 
$229 b$

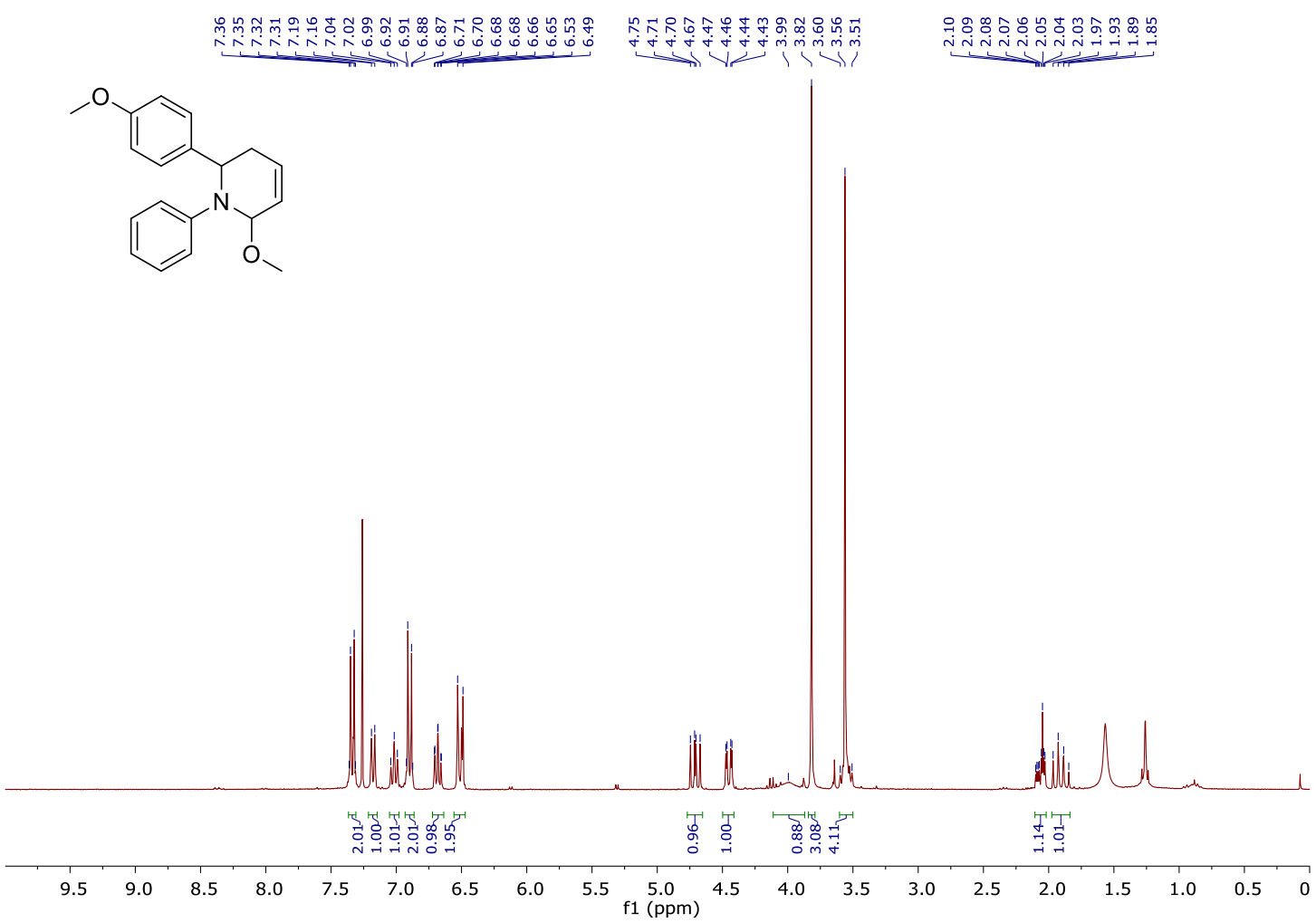

${ }^{1} \mathrm{H}$ NMR spectrum in $\mathrm{CDCl}_{3}$.

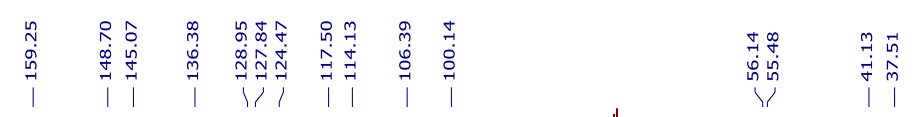

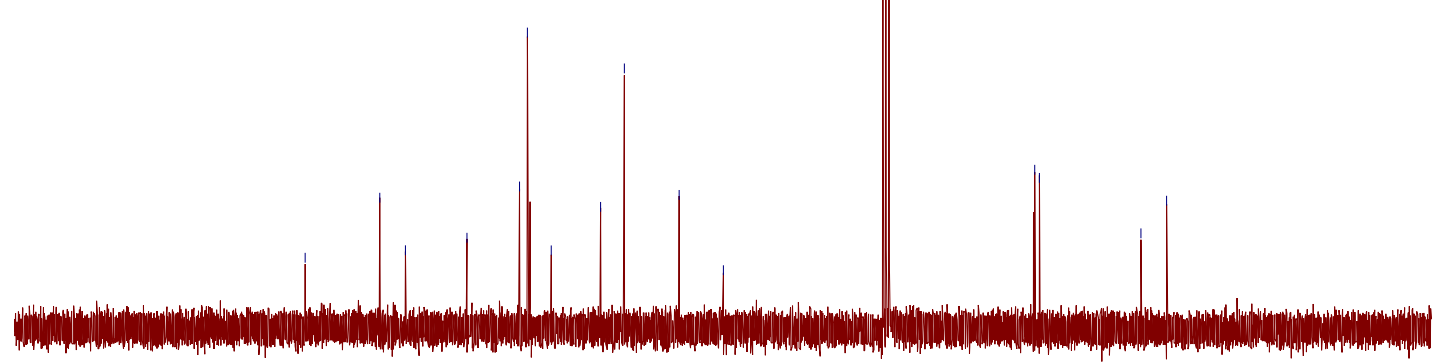

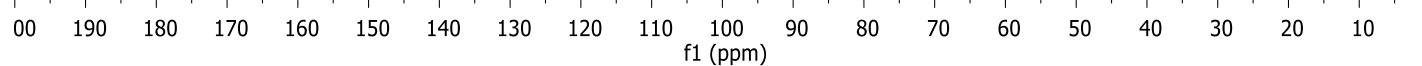

${ }^{13} \mathrm{C}$ NMR spectrum in $\mathrm{CDCl}_{3}$. 
230b

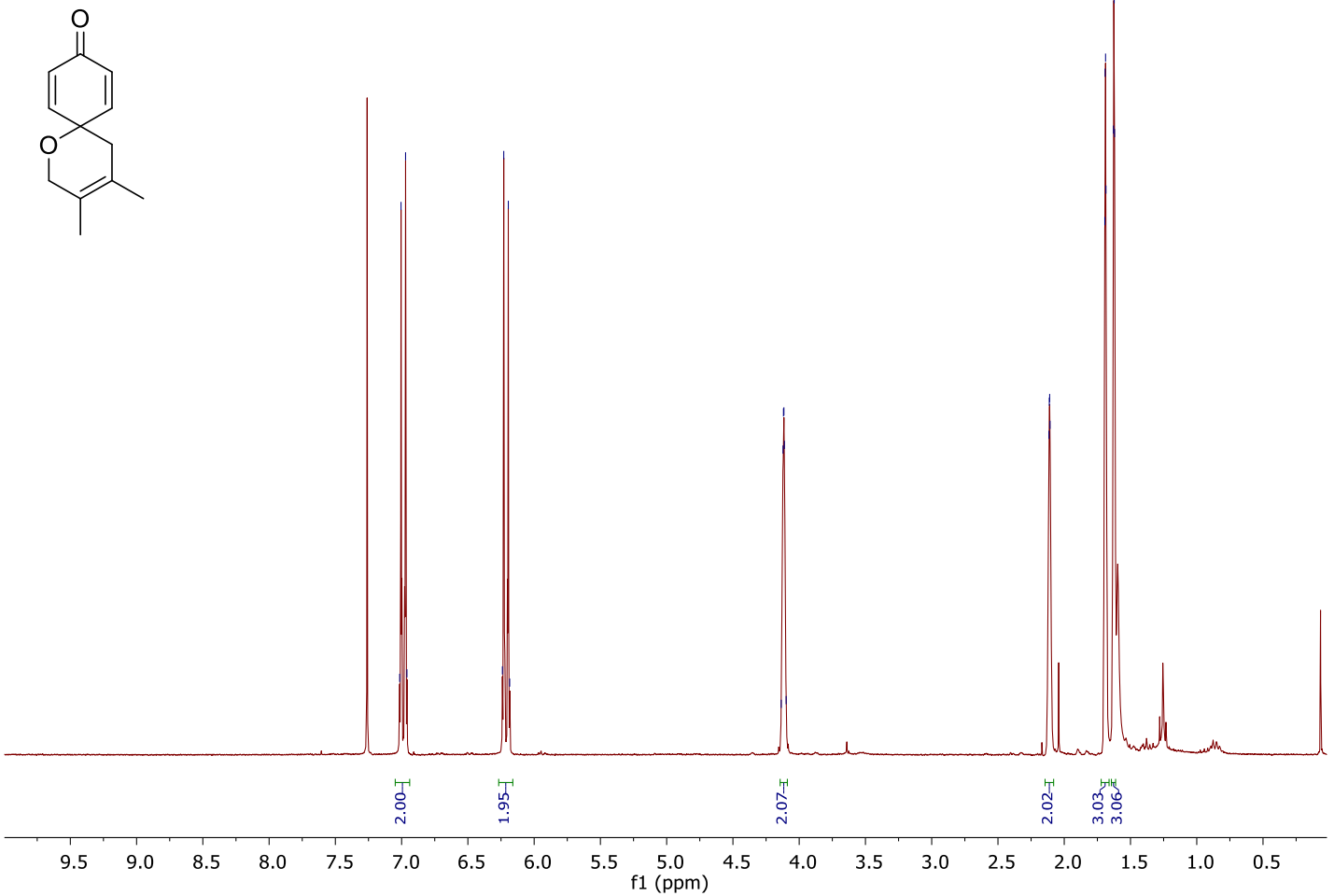

${ }^{1} \mathrm{H}$ NMR spectrum in $\mathrm{CDCl}_{3}$.

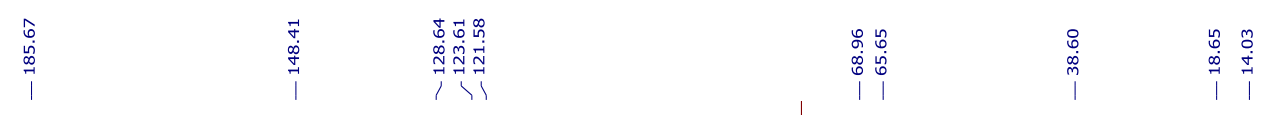

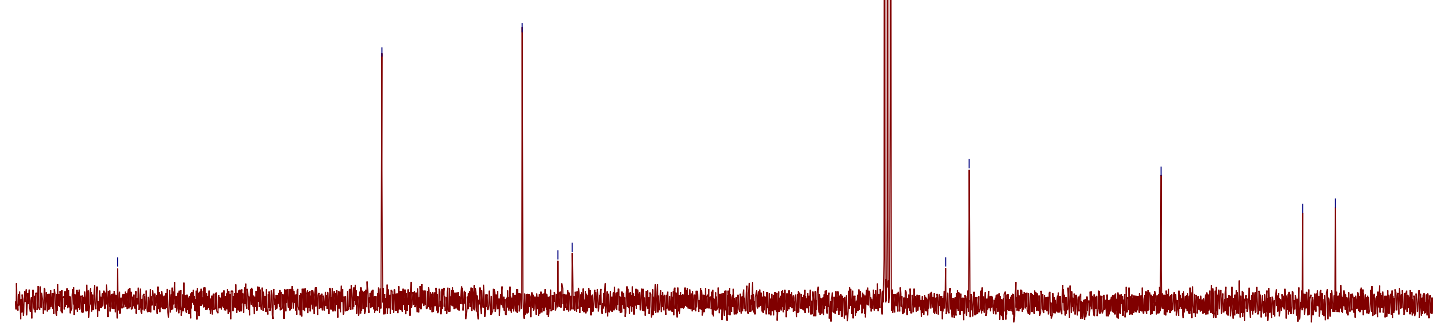

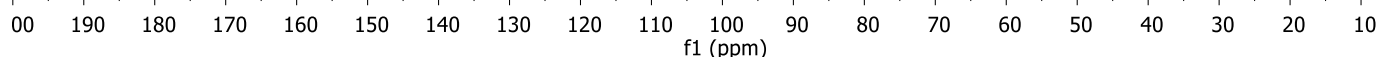

${ }^{13} \mathrm{C}$ NMR spectrum in $\mathrm{CDCl}_{3}$. 


\section{3b}
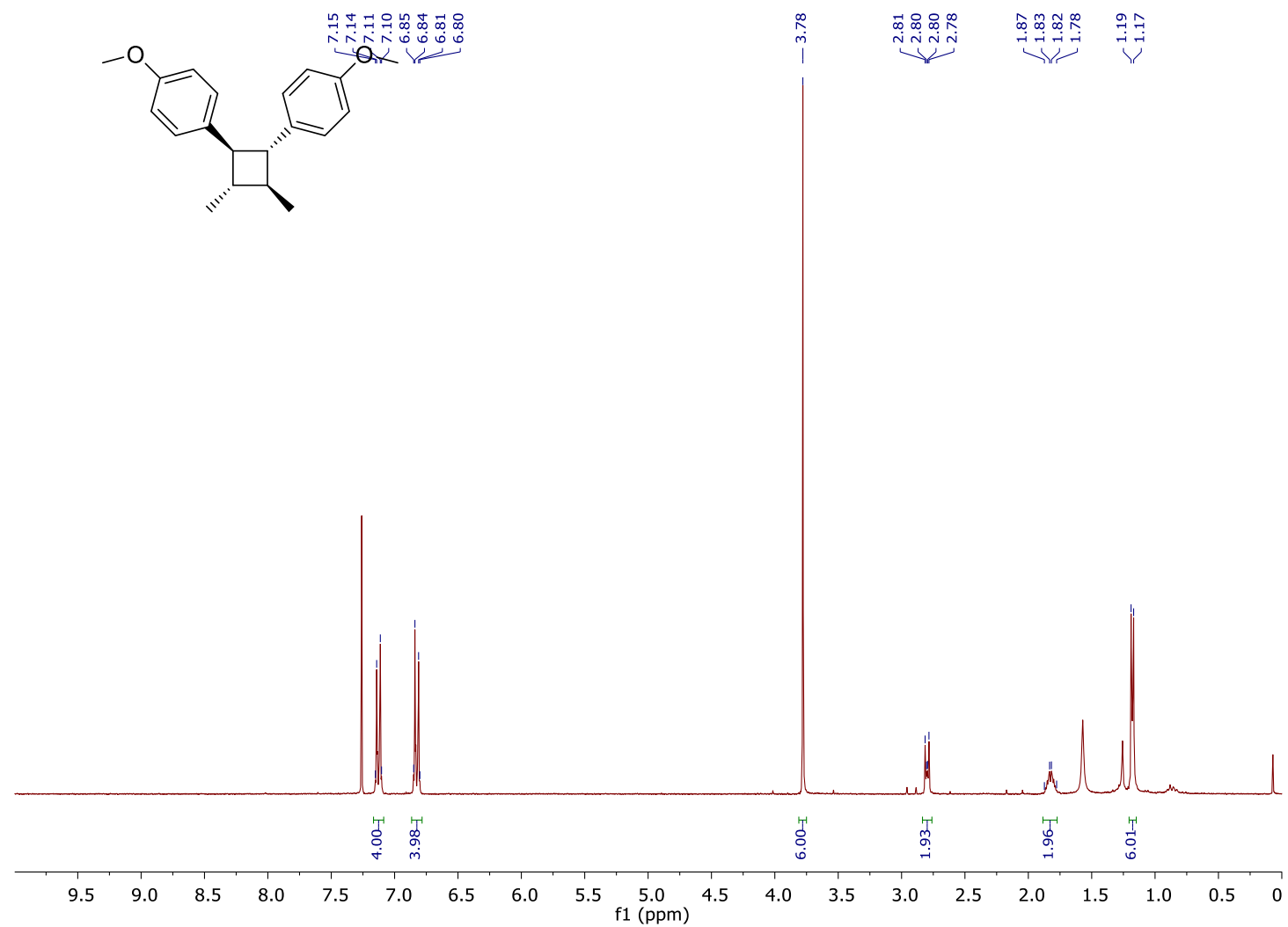

${ }^{1} \mathrm{H}$ NMR spectrum in $\mathrm{CDCl}_{3}$.

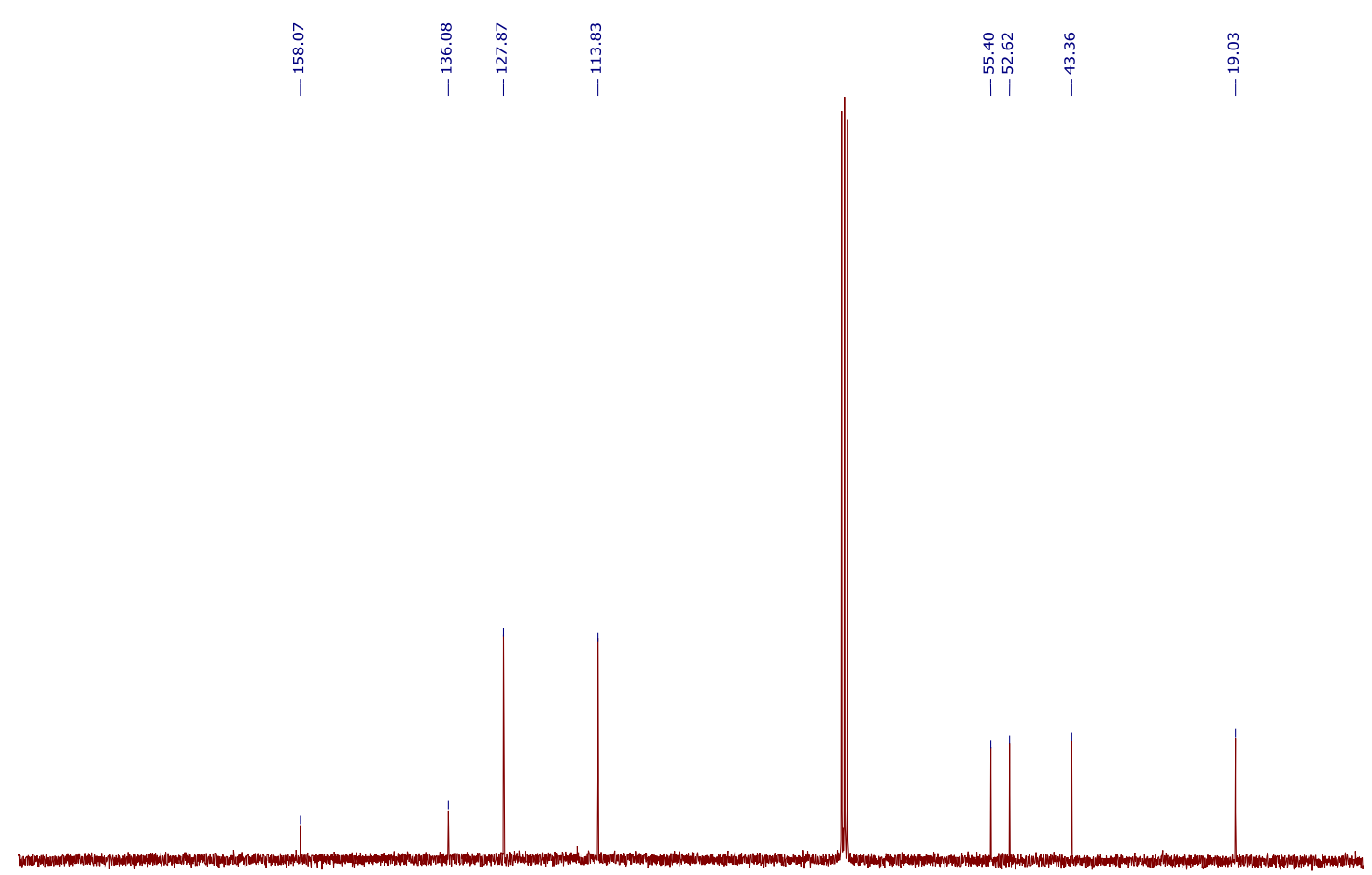

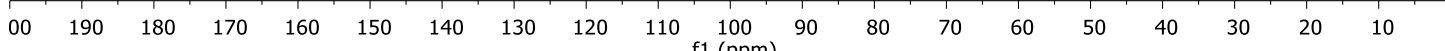

${ }^{13} \mathrm{C}$ NMR spectrum in $\mathrm{CDCl}_{3}$. 
234b

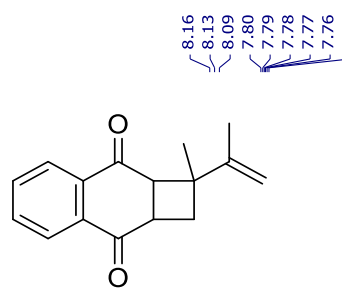

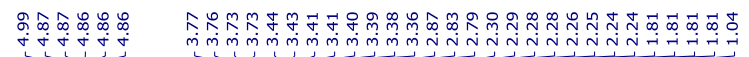

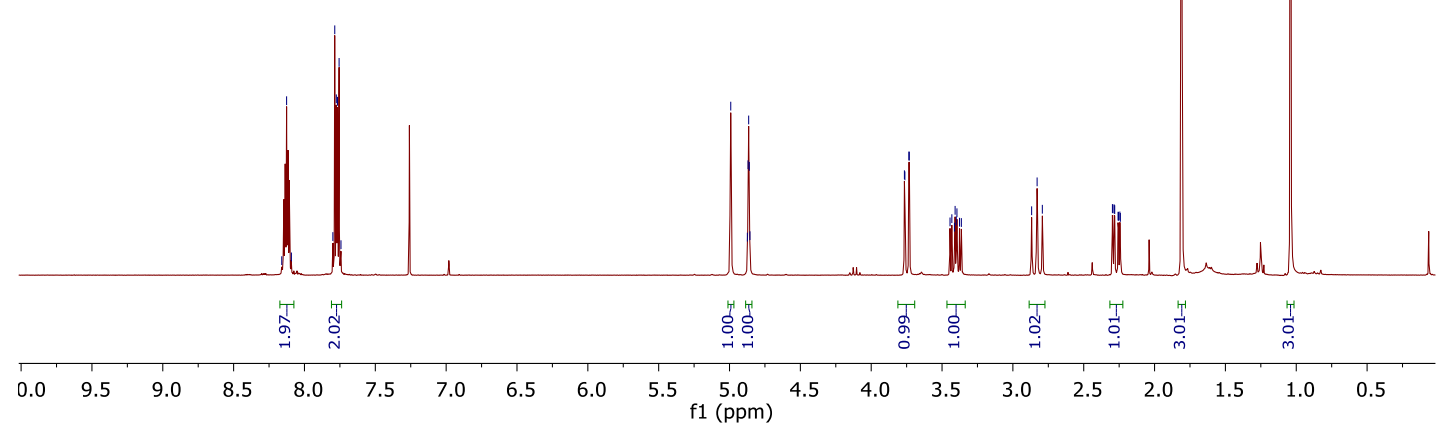

${ }^{1} \mathrm{H}$ NMR spectrum in $\mathrm{CDCl}_{3}$.

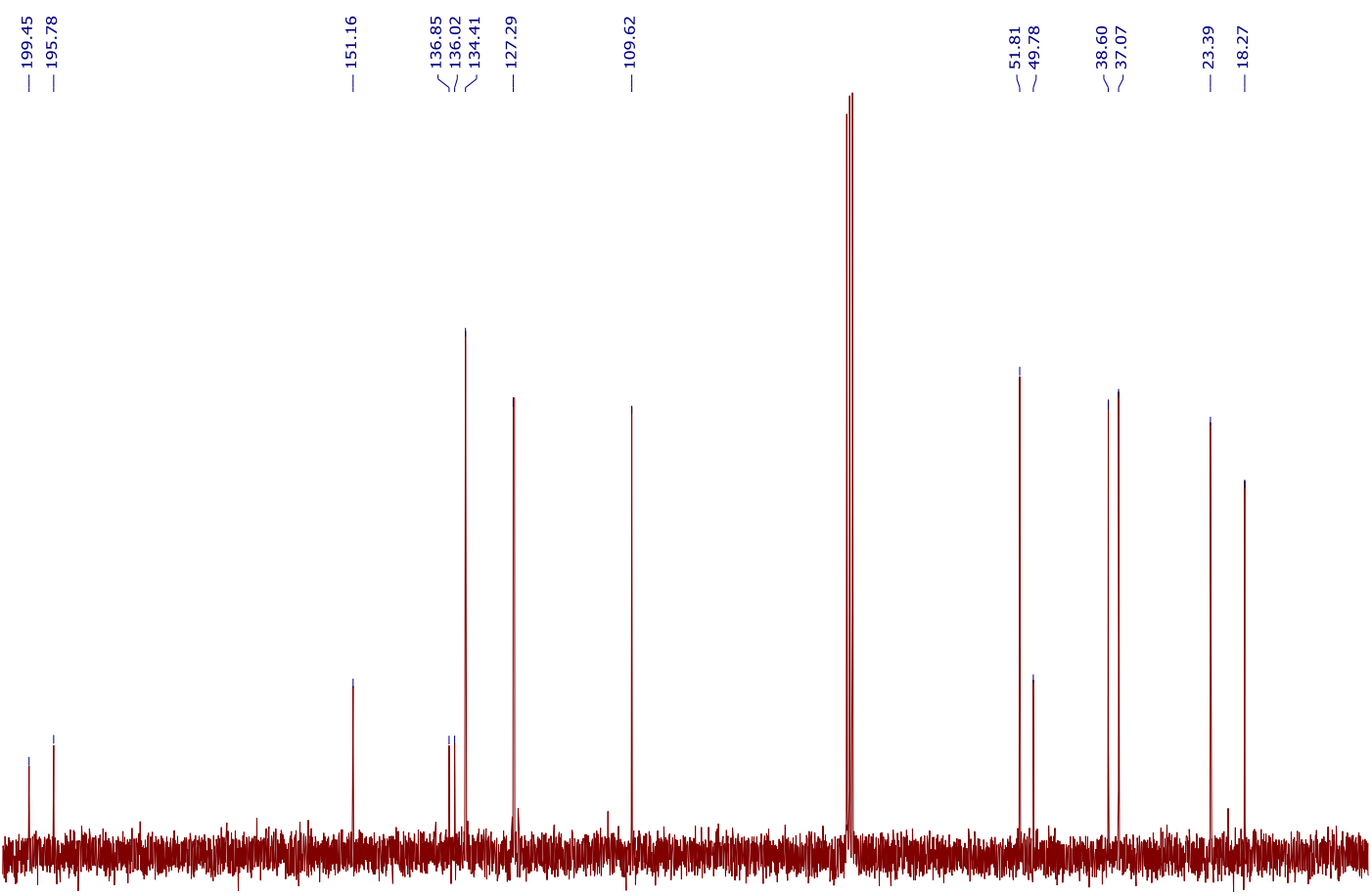

$\begin{array}{llllllllllllllllllllll}200 & 190 & 180 & 170 & 160 & 150 & 140 & 130 & 120 & 110 \begin{array}{l}100 \\ \mathrm{f} 1(\mathrm{ppm})\end{array} & 90 & 80 & 70 & 60 & 50 & 40 & 30 & 20 & 10 & 1\end{array}$

${ }^{13} \mathrm{C}$ NMR spectrum in $\mathrm{CDCl}_{3}$. 


\section{References}

[1] (a) P. T. Anastas, J. C. Warner, Green Chemistry: Theory and Practice, Oxford University Press, 1998; (b) J. H. Clark, Green Chem. 1999, 1, 1-8.

[2] (a) P. Anastas, N. Eghbali, Chem. Soc. Rev. 2010, 39, 301-312; (b) M. Poliakoff, P. Licence, P. Nature 2007, 450, 810-812; (c) P. T. Anastas, M. M. Kirchhoff, Acc. Chem. Res. 2002, 35, 686-694.

[3] H. C. Erythropel, J. B. Zimmerman, T. M. de Winter, L. Petitjean, F. Melnikov, C. H. Lam, A. W. Lounsbury, K. E. Mellor, N. Z. Janković, Q. Tu, L. N. Pincus, Green Chem. 2018, 20, 1929-1961.

[4] (a) J. Lalevée, S. Telitel, P. Xiao, M. Lepeltier, F. Dumur, F. Morlet-Savary, D. Gigmes, J.-P. Fouassier, Beilstein J. Org. Chem. 2014, 10, 863-876; (b) C. K. Prier, D. A. Rankic, D. W. C. MacMillan, Chem. Rev. 2013, 113, 5322-5363; (c) V. Balzani, A. Credi, M. Venturi, ChemSusChem 2008, 1, 26-58; (d) R. H. Crabtree, Chem. Rev. 1985, 85, 245.

[5] (a) D. A. Nagib, M. E. Scott, D. W. C. MacMillan, J. Am. Chem. Soc. 2009, 131, 10875-10877; (B) H.-W. Shih, M. N. Vander Wal, R. L. Grange, D. W. C. MacMillan, J. Am. Chem. Soc. 2010, 132, 13600-13603; (c) J. M. R. Narayanam, C. R. J. Stephenson, Chem. Soc. Rev. 2011, 40, 102-113; (d) M. H. Shaw, J. Twilton, D. W. C. MacMillan, J. Org. Chem. 2016, 81, 6898-6926.

[6] (a) N. A. Romero, D. A. Nicewicz, Chem. Rev. 2016, 116, 10075-10166; (b) M. Neumann, S. Fuldner, B. König, K. Zeitler, Angew. Chem. Int. Ed. 2011, 50, 951954.

[7] D. M. Hedstrand, W. M. Kruizinga, R. M. Kellogg, Tetrahedron Lett. 1978, 19, 1255-1258.

[8] (a) A. Savateev, M. Antonietti, ACS Catal. 2018, 8, 9790-9808; (b) Y. Zheng, L. Lin, B. Wang, X. Wang, Angew. Chem. Int. Ed. 2015, 54, 12868-12884.

[9] (a) A. Bard, M. Fox, Acc. Chem. Res. 1995, 28, 141-145; (b) X. Chen, S. Shen, L. Guo, S. S. Mao, Chem. Rev. 2010, 110, 6503-6570; (c) C. Chen, W. Ma, J. Zhao, Chem. Soc. Rev. 2010, 39, 4206-4219; (d) H. Zhang, G. Chen, D. W. Bahnemann, J. Mater. Chem. 2009, 19, 5089-5121; (e) M. Pelaez, N. T. Nolan, S. C. Pillai, M. K. 
Seery, P. Falaras, A. G. Kontos, P. S. M. Dunlop, J. W. J. Hamilton, J. A. Byrne, K.

O'Shea, M. H. Entezari, D. D. Dionysiou, Appl. Catal. B: Environ. 2012, 125, 331349; (f) W. Tu, Y. Zhou, Z. Zou, Adv. Mater. 2014, 26, 4607-4626; (g) H. Park, Y. Park, W. Kim, W. Choi, J. Photochem. Photobiol. C 2013, 15, 1-20.

[10] (a) X. Lang, X. Chen, J. Zhao, Chem. Soc. Rev. 2014, 43, 473-486; (b) H. Kisch, Angew. Chem. Int. Ed. 2013, 52, 812-847.

[11] (a) N. Hoffmann, Chem. Rev. 2008, 108, 1052-1103; (b) G. Ciamician, Science, 1912, 36, 385-394; (c) A. Albini, M. Fagnoni, ChemSusChem, 2008, 1, 63-66.

[12] D, M. Schultz, T. P. Yoon, Science 2014, 343, 1239176.

[13] F. Strieth-Kalthoff, M. J. James, M. Teders, L. Pitzer, F. Glorius, Chem. Soc. Rev. 2018, 47, 7190-7202.

[14] (a) O. S. Wenger, Acc. Chem. Res. 2013, 46, 1517-1526; (b) D. R. Weinberg, C. J. Gagliardi, J. F. Hull, C. F. Murphy, C. A. Kent, B. C. Westlake, A. Paul, D. H. Ess, D. G. McCafferty, T. J. Meyer, Chem. Rev. 2012, 112, 4016-4093.

[15] J. C. Gonzalez-Gomez, N. P. Ramirez, T. Lana-Villarreal, P. Bonete, Org. Biomol. Chem. 2017, 15, 9680-9684.

[16] C. Pac, M. Ihama, M. Yasuda, Y. Miyauchi, H. Sakurai, J. Am. Chem. Soc. 1981, $103,6495-6497$.

[17] W. J. Blaedel, R. G. Haas, Anal. Chem. 1970, 42, 918-927.

[18] J. M. R. Narayanam, J. W. Tucker, C. R. J. Stephenson, J. Am. Chem. Soc. 2009, $131,8756-8757$.

[19] (a) A. G. Condie, J. C. Gonzalez-Gomez, C. R. J. Stephenson, J. Am. Chem. Soc. 2010, 132, 1464-1465. (b) C.-J. Li, Acc. Chem. Res. 2009, 42, 335-344.

[20] J. W. Beatty, C. R. J. Stephenson, Acc. Chem. Res. 2015, 48, 1474-1484.

[21] (a) Y. Miyake, K. Nakajima, Y. Nishibayashi, J. Am. Chem. Soc. 2012, 134, 33383341. (b) P. Kohls, D. Jadhav, G. Pandey, O. Reiser, Org. Lett. 2012, 14, 672-675.

[22] Y. Xi, H. Yi, A.-W. Lei, Org. Biomol. Chem. 2013, 11, 2387-2403.

[23] Y. Cheng, J. Yang, Y. Qu, P. Li, Org. Lett. 2012, 14, 98-101.

[24] D. P. Hari, P. Schroll, B. König, J. Am. Chem. Soc. 2012, 134, 2958-2961. 
[25] (a) A. Wetzel, V. Ehrhardt, M. R. Heinrich, Angew. Chem. Int. Ed. 2008, 47, 9130-9133; (b) A. Wetzel, G. Pratsch, R. Kolb, M. R. Heinrich, Chem. Eur. J. 2010, $16,2547-2556$.

[26] J. W. Verhoeven, Pure Appl. Chem. 1996, 68, 2223-2286.

[27] (a) J. B. Metternich, R. Gilmour, J. Am. Chem. Soc. 2015, 137, 11254-11257; (b) J. B. Metternich, R. Gilmour, J. Am. Chem. Soc. 2016, 138, 1040-1045; (c) K. Gadde, P. Mampuys, A. Guidetti, H. Y. V. Ching, W. A. Herrebout, S. Van Doorslaer, K. A. Tehrani, B. U. W. Maes, ACS Catal. 2020, 10, 8765-8779; (d) E. Brachet, T. Ghosh, I. Ghosh, B. König, Chem. Sci. 2015, 6, 987-992; (e) M. A. Ischay, M. E. Anzovino, J. Du, T. P. Yoon, J. Am. Chem. Soc. 2008, 130, 12886-12887; (f) S. Lin, M. A. Ischay, C. G. Fry, T. P. Yoon, J. Am. Chem. Soc. 2011, 133, 19350-19353. (g) S. Lin, C. E. Padilla, M. A. Ischay, T. P. Yoon, Tetrahedron Lett. 2012, 53, 30733076.

[28] (a) A. E. Hurtley, M. A. Cismesia, M. A. Ischay, T. P. Yoon, Tetrahedron, 2011, 67, 4442-4448; (b) E. L. Tyson, E. P. Farney, T. P. Yoon, Org. Lett. 2012, 14, 11101113; (c) J. D. Parrish, M. A. Ischay, Z. Lu, S. Guo, N. R. Peters, T. P. Yoon, Org. Lett. 2012, 14, 1640-1643.

[29] Z. Lu, M. Shen, T. P. Yoon, J. Am. Chem. Soc. 2011, 133, 1162-1164.

[30] (a) M. A. Ischay, Z. Lu, T. P. Yoon, J. Am. Chem. Soc. 2010, 132, 8572-8574; (b) M. A. Ischay, M. S. Ament, T. P. Yoon, Chem. Sci. 2012, 3, 2807-2811.

[31] Z. Lu, T. P. Yoon, Angew. Chem. Int. Ed. 2012, 51, 10329-10332.

[32] M. S. Lowry, J. I. Goldsmith, J. D. Slinker, R. Rohl, R. A. Pascal, G. G. Malliaras, S. Bernhard, Chem. Mater. 2005, 17, 5712-5719.

[33] C.-J. Wallentin, J. D. Nguyen, P. Finkbeiner, C. R. J. Stephenson, J. Am. Chem. Soc. 2012, 134, 8875-8884.

[34] (a) A. A. Lamola, G. S. Hammond, J. Chem. Phys. 1965, 43, 2129-2135; (b) T. Ni, R. A. Caldwell, L. A. Melton, J. Am. Chem. Soc. 1989, 111, 457-464.

[35] (a) J. Twilton, C. Le, P. Zhang, M. H. Shaw, R. W. Evans, D. W. C. MacMillan, Nature Rev. Chem. 2017, 1, 1-19; (b) Z.-W. Zuo, D. T. Ahneman, L.-L. Chu, J. A. Terrett, A. G. Doyle, D. W. C. MacMillan, Science 2014, 345, 437-440. 
[36] (a) B. Sahoo, M. N. Hopkinson, F. Glorius, J. Am. Chem. Soc. 2013, 135, 55055508; (b) Y.-D. Ye, M. S. Sanford, J. Am. Chem. Soc. 2012, 134, 9034-9037; (c) A. Noble, S. J. McCarver, D. W. C. MacMillan, J. Am. Chem. Soc. 2015, 137, 624-627; (d) J. A. Terrett, J. D. Cuthbertson, V. W. Shurtleff, D. W. C. MacMillan, Nature 2015, 524, 330-334.

[37] D. Kalyani, K. B. McMurtrey, S. R. Neufeldt, M. S. Sanford, J. Am. Chem. Soc. 2011, 133, 18566-18569.

[38] J. Jin, D. W. C. MacMillan, Nature 2015, 525, 87-90.

[39] D. A. DiRocco, T. Rovis, J. Am. Chem. Soc. 2012, 134, 8094-8097.

[40] (a) M. T. Pirnot, D. A. Rankic, D. B. C. Martin, D. W. C. MacMillan, Science 2013, 339, 1593; (b) J. A. Terrett, M. D. Clift, D. W. C. MacMillan, J. Am. Chem. Soc. 2014, 136, 6858-6861; (c) J. A. Terrett, F. R. Petronijević, D. W. C. MacMillan, J. Am. Chem. Soc. 2015, 137, 8404-8407; (d) E. R. Welin, A. A. Warkentin, J. C. Conrad, D. W. C. MacMillan, Angew. Chem. Int. Ed. 2015, 54, 9668-9672.

[41] D. A. Nicewicz, D. W. C. MacMillan, Science 2008, 322, 77-80.

[42] (a) S. Yurdakal, G. Palmisano, V. Loddo, V. Augugliaro, L. Palmisano, J. Am. Chem. Soc. 2008, 130, 1568-1569. (b) N. Li, X. J. Lang, W. H. Ma, H. W. Ji, C. C. Chen, J. C. Zhao, Chem. Commun. 2013, 49, 5034-5036.

[43] (a) G. Kim, W. Choi, Appl. Catal. B 2010, 100, 77-83. (b) F. Parrino, V. Augugliaro, G. Camera-Roda, V. Loddo, M. J. Lopez-Munoz, C. Marquez-A'Ivarez, G. Palmisano, L. Palmisano and M. A. Puma, J. Catal. 2012, 295, 254-260.

[44] S. Linic, P. Christopher, D. B. Ingram, Nat. Mater. 2011, 10, 911-921.

[45] Y.-T. Gong, M.-M. Li, H.-R. Li, Y. Wang, Green Chem. 2015, 17, 715-736

[46] C. Q. Chen, W. D. Zhang, Mol. Catal. 2018, 453, 85-92.

[47] (a) F. Goettmann, A. Fischer, M. Antonietti, A. Thomas, Angew. Chem. Int. Ed. 2006, 45, 4467-4471; (b)F. Goettmann, A. Fischer, M. Antonietti, A. Thomas, Chem. Commun. 2006, 4530-4532.

[48] (a) P. Zhang, Y. Wang, J. Yao, C. Wang, C. Yin, M. Antonietti, H. Li, Adv. Synth. Catal. 2011, 353, 1447-1451; (b) P.-X. Geng, Y.-R. Tang, G.-L. Pan, W.-T. Wang, J.C. Hu, Y.-F. Cai, Green Chem. 2019, 21, 6116-6122. 
[49] (a) Y.-B. Zhao, M. Antonietti, Angew. Chem. Int. Ed. 2017, 56, 9336-9340; (b) C. Yang, R. Li, K. A. I. Zhang, W. Lin, K. Landfester, X.-C Wang, Nature Commun. 2020, 11, 1239.

[50] (a) K. Yamaguchi, N. Mizuno, Angew. Chem. Int. Ed. 2003, 42, 1480-1483; (b) J. S. M. Samec, A. H. Ell, J.-E. B ckvall, Chem. Eur. J. 2005, 11, 2327-2334; (c) B. L. Zhu, M. Lazar, B. G. Trewyn, R. J. Angelici, J. Catal. 2008, 260, 1-6.

[51] F.-Z Su, S. C. Mathew, L. Moehlmann, M. Antonietti, X.-C. Wang, S. Blechert, Angew. Chem. Int. Ed. 2011, 50, 657-660.

[52] (a) Y.-F. Cai, Y. R. Tang, L.-L Fan, Q. Lefebvre, H. Hou, M. Rueping, ACS Catal. 2018, 8, 9471-9476.

[53] I. Ghosh, J. Khamrai, A. Savateev, N. Shlapakov, M. Antonietti, B. König, Science 2019, 365, 360-366.

[54] (a) A. Sheldon, Chem. Ind. 1992, 23, 903-906; (b) Z. Guo, Q. Zhang, W. Deng, Y. Wang, Y. Yang, Chem. Soc. Rev. 2014, 43, 3480-3524; (c) S. D. McCann, S. S. Stahl, Acc. Chem. Res. 2015, 48, 1756-1766; (d) A. E. Wendlandt, S. S. Stahl, Angew. Chem. Int. Ed. 2015, 54, 14638-14658; (e) S. Murahashi, Angew. Chem. Int. Ed. 1995, 34, 2443-2465.

[55] (a) E. Romero, J. R. G. Castellanos, G. Gadda, M. W. Fraaije, A. Mattevi, Chem. Rev. 2018, 118, 1742-1769; (b) K. J. Tidgewell, J. Nat. Prod. 2016, 79, 2762-2762; (c) M. Siskin, A. R. Katritzky, J. Anal. Appl. Pyrolysis 2000, 54, 193-198.

[56] (a) G. Urgoitia, R. SanMartin, M. T. Herrero, E. Dominguez, Catalysts 2018, 8, 640-660; b) A. A. Ghogare, A. Greer, Chem. Rev. 2016, 116, 9994-10034.

[57] Modern Oxidation Methods (Ed.: J. E. Bckvall), Wiley VCH, Weinheim, 2004.

[58] (a) V. A. Vavilin, Biotechnol. Bioeng. 1982, 24, 1721-1723; (b) B. E. Rittmann, Environ. Sci. Technol. 1987, 21, 128-136.

[59] (a) H. Taube, J. Gen. Physiol. 1965, 49, 29-52; (b) N. Gunasekaran, Adv. Synth. Catal. 2015, 357, 1990-2010.

[60] (a) G. Evano, J. Wang, A. Nitelet, Org. Chem. Front. 2017, 4, 2480-2499; (b) Y. Qin, L. Zhu, S. Luo, Chem. Rev. 2017, 117, 9433-9520; (c) R. H. Crabtree, A. Lei, Chem. Rev. 2017, 117, 8481-8482. 
[61] (a) D. Munz, T. Strassner, Inorg. Chem. 2015, 54, 5043-5052; (b) V. S. Thirunavukkarasu, L. Ackermann, Org. Lett. 2012, 14, 6206-6209; (c) F. Zhang, K. Rauch, K. Kettelhoit, L. Ackermann, Angew. Chem. Int. Ed. 2014, 53, 1128511288; Angew. Chem. 2014, 126, 11467-11470; (d) M. S. Chen, M. C. White, Science 2007, 318, 783-787; (e) H. Sterckx, B. Morel, B. U. W. Maes, Angew. Chem. Int. Ed. 2019, 58, 7946-7970.

[62] (a) Y.-F. Liang, N. Jiao, Acc. Chem. Res. 2017, 50, 1640-1653; (b) T. Punniyamurthy, S. Velusamy, J. Iqbal, Chem. Rev. 2005, 105, 2329-2363; (c) C. Dçbler, G. Mehltretter, M. Beller, Angew. Chem. Int. Ed. 1999, 38, 3026-3028; Angew. Chem. 1999, 111, 3211-3212; (d) F. Shi, M. K. Tse, Z. Li, M. Beller, Chem. Eur. J. 2008, 14, 8793-8797; (e) D. Wang, A. B. Weinstein, P. B. White, S. S. Stahl, Chem. Rev. 2018, 118, 2636-2679; (f) D. P. Hruszkewycz, K. C. Miles, O. R. Thiel, S. S. Stahl, Chem. Sci. 2017, 8, 1282-1287.

[63] (a) J. Christoffers, A. Baro, T. Werner, Adv. Synth. Catal. 2004, 346, 143-151; (b) J. Christoffers, J. Org. Chem. 1999, 64, 7668-7669; (c) X. Baucherel, E. Levoirier, J. Uziel, S. Juge, Tetrahedron Lett. 2000, 41, 1385-1387.

[64] (a) M.-A. Légaré, M.-A. Courtemanche, P. Rochette, F.-G. Fontaine, Science 2015, 349, 513-516; (b) L. Dai, Y. Xue, L. Qu, H.-J. Choi, J.-B. Baek, Chem. Rev. 2015, 115, 4823-4892; (c) D. Koziakov, A. Jacobi von Wangelin, Org. Biomol. Chem. 2017, 15, 6715-6719.

[65] (a) V. Srivastava, P. P. Singh, RSC Adv. 2017, 7, 31377-31392; (b) Z.-C. Shen, P. Yang, Y. Tang, J. Org. Chem. 2016, 81, 309-317; (c) Y. Osaka, A. Y. Nosaka, Chem. Rev. 2017, 117, 11302-11336; (d) D. P. Hari, B. König, Chem. Commun. 2014, 50, 6688-6699; (e) L. Marzo, S. K. Pagire, O. Reiser, B. König, Angew. Chem. Int. Ed. 2018, 57, 10034-10072; (f) K. L. Skubi, T. R. Blum, T. P. Yoon, Chem. Rev. 2016, $116,10035-10074$.

[66] (a) D. A. Nicewicz, M. T. Nguyen, ACS Catal. 2014, 4, 355-360; (b) N. A. Romero, K. A. Margrey, N. E. Tay, D. A. Nicewicz, Science, 2015, 349, 1326-1330; (c) W. Schilling, D. Riemer, Y. Zhang, N. Hatami, S. Das, ACS Catal. 2018, 8, 5425-5430.

[67] (a) Q. Yan, L.-H. Zhu, S.-Z Luo. Chem. Rev. 2012, 117. 9433-9520; (b) X. Zhang, K. P. Rakesh, L. Ravindar, H.-L. Qin, Green Chem. 2018, 20, 4790-4833. (c) X. Jun, 
W.-J. Xiao, Angew. Chem. Int. Ed. 2012, 51. 6828-6838; (d) C.-L. Bian, A. K. Singh,

L.-B. Niu, H. Yi, A.-W. Lei, Asian J. Org. Chem. 2017, 6, 386-396.

[68] L. Robert, S. Kümmel, B. König. Photochem. Photobio. Science 2010, 9, 13671377.

[69] Y. Jin, L. Ou, H. Yang, H. Fu, J. Am. Chem. Soc. 2017, 139, 14237-14243.

[70] K. C. C. Agandaa, B. Hongband, A. Lee, Adv. Synth. Catal. 2019, 361, 11241129.

[71] (a) M. K. Sahoo, G. Jaiswal, J. Rana, E. Balaramn, Chem. Eur. J. 2017, 23, 1416714172; (b) D. Riemer, W. Schilling, A. Goetz, Y. Zhang, S. Gehrke, I. Tkach, O. Holloczki, S. Das, ACS Catal. 2018, 8, 11679-11687.

[72] K. Ohkubo, K. Mizushima, R. Iwata, K. Souma, N. Suzukib, S. Fukuzumi, Chem. Commun. 2010, 46, 601-603.

[73] H. Yi, C. Bian, X. Hu, L. Niu, A. Lei, Chem. Commun. 2015, 51, 14046-14049.

[74] (a) J.-P. Wan, Y. Gao, L. Wei, Chem. Asian J. 2016, 11, 2092-2102; (b) A. Rajagopalan, M. Lara, W. Kroutil, Adv. Synth. Catal. 2013, 355, 3321-3335.

[75] (a) L. F. Tietze, M. Bratz, Org. Synth. 1993, 71, 214; (b) R. Pappo, D. S. Allen, R. U. Lemieux, W. S. Johnson, J. Org. Chem. 1956, 21, 478-479; (c) Z. Hua, W. Yu, Z. Jin, Org. Lett. 2004, 6, 3217-3219.

[76] M. B. Smith, J. March, Marchs' Advanced Organic Chemistry, $5^{\text {th }}$ ed., Wiley, New York, 2001.

[77] A. Gonzalez-de-Castro, J. Xiao, J. Am. Chem. Soc. 2015, 137, 8206-8218.

[78] B.-J. Xiong, X.-Q. Zeng, S.-H. Geng, S. Chen, Y. He, Z. Feng, Green Chem. 2018, $20,4521-4527$.

[79] Y.-X. Liu, D. Xue, C.-Q. Li, J.-L, Xiao, C. Wang, Catal. Sci. Technol. 2017, 7, 55105514.

[80] Y. Deng, X. J. Wei, H. Wang, Y. Sun, T. Noël, X. Wang, Angew. Chem. Int. Ed. 2017, 56, 832-836.

[81] (a) K.-J. Jiao, Y.-K. Xing, Q.-L. Yang, H. Qiu, T.-S. Mei, Acc. Chem. Res. 2020, 53, 300-310; (b) T. H. Meyer, J. C. A. de Oliveira, D. Ghorai, L. Ackermann, Angew. Chem. Int. Ed. 2020, 59, 10955-10960; (c) Y. Qiu, C. Tian, L. Massignan, T. Rogge, L. Ackermann, Angew. Chem. Int. Ed. 2018, 57, 5818-5822. 
[82] Y. Imada, Y. Okada, K. Noguchi, K. Chiba, Angew. Chem. Int. Ed. 2019, 58, 125129.

[83] (a) J. G. Martin, R. K. Hill, Chem. Rev. 1961, 61, 537-562; (b) K. C. Nicolaou, S. A. Snyder, T. Montagnon, G. Vassilikogiannakis, Angew. Chem. Int. Ed. 2002, 41, 1668-1698; (c) R. B. Woodward, F. Sondheimer, D.Taub, K. Heusler, W. M. McLamore, J. Am. Chem. Soc. 1952, 74, 4223-4251.

[84] (a) G. Desimoni, G. Faita, P. Quadrelli, Chem. Rev. 2015, 115, 9922-9980; (b) R. Rossin, S. M. J. van Duijnhoven, T. Lappchen, S. M. van den Bosch, M. S. Robillard, Mol. Pharmaceutics 2014, 11, 3090-3096; (c) E. J. Corey, Angew. Chem. Int. Ed. 2002, 41, 1650-1667.

[85] X. Jiang, R. Wang, Chem. Rev. 2013, 113, 5515-5546

[86] (a) M. T. Crimmins, T. L. Reinhold, Org. React. 1993, 44, 297-588; (b) J. D. Winkler, C. M. Bowen, F. Liotta, F. Chem. Rev. 1995, 95, 2003-2020; (c) J. C. Namyslo, D. E. Kaufmann, Chem. Rev. 2003, 103, 1485-1537; (d) D. I. Schuster, G. Lem, N. A. Kaprinidis, Chem. Rev. 1993, 93, 3-22.

[87] G. Ciamician, Science 1912, 36, 385-394.

[88] (a) T.-G. Baik, A. L. Luis, L,-C. Wang, M. J. Krische, J. Am. Chem. Soc. 2001, 123, 6716-6717; (b) L,-C. Wang, H.-Y. Jang, Y. Roh, V. Lynch, A. J. Schultz, X.-P. Wang, M. J. Krische, J. Am. Chem. Soc. 2002, 124, 9448-9453.

[89] J.-N. Du, T. P. Yoon, J. Am. Chem. Soc. 2009, 131, 14604-14605.

[90] E. L. Tyson, E. P. Farney, T. P. Yoon, Org. Lett. 2012, 14, 1110-1113.

[91] (a) F. A. Bell, R. A. Crellin, N. Fugii, A. J. Ledwith, Chem. Commun. 1969, 251252; (b) R. A. Carruthers, R. A. Crellin, A. J. Ledwith, Chem. Commun. 1969, 252253.

[92] (a) T. Mukai, K. Sato, Y. Yamashita, J. Am. Chem. Soc. 1981, 103, 670-672; (b) C. Pac, T. Ohtsuki, T. Fukunaga, S. Yanagida, H. Sakurai, Chem. Lett. 1985, 18551858.

[93] Y. Kawanishi, N. Kitamura, Y. Kim, S. Tazuke, Sci. Pap. Inst. Phys. Chem. Res. (Jpn.), 1984, 78, 212-219. 
[94] S. Morris, T. Nguyen, N. Zheng, 2018. Visible Light Mediated Cycloaddition Reactions. Visible Light Photocatalysis in Organic Chemistry (Chapter 5).

[95] S. M. Stevenson, M. P. Shores, E. M. Ferreira, Angew. Chem. Int. Ed. 2015, 54, 6506-6510.

[96] Bode Research Group, OC VI (HS 2015), http://www.bode.ethz.ch/.

[97] Y. Zhang, D. Riemer, W. Schilling, J. Kollmann, S. Das, ACS Catal. 2018, 8, 66596664.

[98] (a) R. J. D'Amato, M. S. Loughnan, E. Flynn, J. Folkman, Proc. Natl. Acad. Sci. 1994, 91, 4082-4085; (b) M. C. Chung, P. Malatesta, P. L. Bosquesi, P. R. Yamasaki, J. L. D. Santos, E. O. Vizioli, Pharm. 2012, 5, 1128-1146.

[99] (a) M. K. Hidau, N. K. Mehra, S. Kolluru, S. Palakurthi, Drug Deliv. 2017, 7, 5461; (b) C. T. G. King, Science 1963, 141, 353-355; (c) R. C. Petersen, G. R. Thomas, R. M. Grundman, D. Bennett, R. Doody, S. Ferris, E. Pfeiffer, New Engl. J. Med. 2005, 352, 2379-2388.

[100] (a) F. M. Al-Saffar, R. C. Brown, Org. Lett. 2017, 19, 3502-3504; (b) D. N. Garad, S. B. Mhaske, Org. Lett. 2016, 18, 3862-3865.

[101] J. A. R. Salvador, S. M. Silvestre, V. M. Moreira, Curr. Org. Chem. 2006, 10, 2227-2257.

[102] (a) D. A. Nicewicz, M. T. Nguyen, ACS Catal. 2014, 4, 355-360; (b) N. A. Romero, K. A. Margrey, N. E. Tay, D. A. Nicewicz, Science, 2015, 349, 1326-1330; (c) W. Schilling, D. Riemer, Y. Zhang, N. Hatami, S. Das, ACS Catal. 2018, 8, 5425-5430.

[103] T. Shen, Z.-G. Zhao, Q. Yu, H. Xu, J. Photochem. Photobiol. 1989, 47, 203-212.

[104] G.-Y. Liu, R.-R. Tang, Z. Wang, Catal. Lett. 2014, 144, 717-722.

[105] M. A. Buckingham, W. Cunningham, S. D. Bull, A. Folli, D. M. Murphy, F. Marken, Electroanalysis 2018, 30, 1698-1705.

[106] Y. Zhang, N. Hatami, N. Lange, E. Ronge, W. Schilling, C. Jooss, S. Das, Green Chem. 2020, 22, 4516-4522.

[107] E. A. Dikusar, N. A. Zhukovskaya, K. L. Moiseichuk, E. G. Zalesskaya, P. V. Kurman, O. G. Vyglazov, Russ. J. Appl. Chem. 2008, 81, 643-646.

[108] A. P. Gollucke, O. Aguiar Jr, L. F. Barbisan and D. A. Ribeiro, J. Med. Food 2013, $16,199-204$. 
[109] K. F. Gey, A. Pletscher, J. Pharmacol. Exp. Ther. 1961, 133, 18-24.

[110] J. A. Balfour, D. McTavish, R. C. Heel, Drugs 1990, 40, 260-290.

[111] G. Xin, Y. Meng, J. Chem. 2012, 2013, 187912

[112] E. W. McFarland, Energy Environ. Sci. 2014, 7, 846-854.

[113] N. Koshino, B. Saha, J. H. Espenson, J. Org. Chem. 2003, 68, 9364-9370.

[114] J. Kollmann, Y. Zhang, W Schilling, T. Zhang, D. Riemer S. Das. Green Chem. 2019, 21, 1916-1920.

[115] (a) K. Mori, T. Hara, T. Mizugaki, K. Ebitani, K. Kaneda, J. Am. Chem. Soc. 2003, 125, 11460-11461; (b) Y. Ogasawara, S. Uchida, K. Yamaguchi, N. Mizuno, Chem. - Eur. J. 2009, 15, 4343-4349.

[116] (a) A. Peduto, A. Massa, A. D. Mola, P. de Caprariis, P. L. Colla, R. Loddo, S. Altamura, G. Maga, R. Filosa, Chem. Biol. Drug Des. 2011, 77, 441-449; (b) J. Sun, E.-Y. Xia, Q. Wu, C.-G. Yan, ACS Comb. Sci. 2011, 13, 421-426; (c) A. R. Ranade, G. I. Georg, J. Org. Chem. 2014, 79, 984-992.

[117] (a) M. Ali, S. H. Ansari, J. S. Qadry, J. Nat. Prod. 1991, 54, 1271-1278; (b) J. Matsuo, R. Okado, H. A. Ishibashi, Org. Lett. 2010, 12, 3266-3268.

[118] (a) N. S. Josephsohn, M. L. Snapper, A. H. Hoveyda, J. Am. Chem. Soc. 2003, 125, 4018-4019; (b) S. Kobayashi, M. Ueno, S. Saito, Y. Mizuki, H. Ishitani, Y. Yamashita, Proc. Natl. Acad. Sci. U. S. A. 2004, 101, 5476-5481; (c) S. Kaneko, Y. Kumatabara, S. Shimizu, K. Maruoka, S. Shirakawa, Chem. Commun. 2017, 53, $119-122$.

[119] M. Riener, D. A. Nicewicz, Chem. Sci. 2013, 4, 2625-2629.

[120] (a) A. E. Hurtley, Z. Lu, T. P. Yoon, Angew. Chem. Int. Ed. 2014, 53, 8991-8994;

(b) D. Bryce-Smith, E. H. Evans, A. Gilbert, H. S. McNeill, J. Chem. Soc., Perkin Trans. 1992, 1, 485-489.

[121] Metal-free photocatalyst for cycloadditions, master thesis of Jiri Kollmann, Georg-August-Universität Göttingen.

[122] Y. Zhang, W. Schilling, S. Das. Nature Protocols 2020, 15, 822-839.

[123] Y. Zhang, W. Schilling, S. Das. ChemSusChem 2019, 12, 2898-2910.

[124] T. Zhang, Y. Zhang, S. Das. ChemCatChem 2020, https://doi.org/10.1002/cctc.202001195. 
[125] T. Zhang, W. Liang, Y. Huang, X. Li, Y. Liu, B. Yang, C. He, X.-C. Zhou, J. Zhang, Chem. Commun. 2017, 53, 12536-12539.

[126] (a) Huang, J. Zhao, Chem. Commun. 2013, 49, 3751-3753; (b) S. Ramkumar, S. Anandan, Dyes Pigm. 2013, 97, 397-404.

[127] (a) N. J. Taylor, E. Emer, S. Preshlock, M. Schedler, M. Tredwell, S. Verhoog, J. Mercier, C. Genicot, V. Gouverneur, J. Am. Chem. Soc. 2017, 139, 8267-8276; (b) P. W. Tan, M. Haughey, D. J. Dixon, Chem. Commun. 2015, 1, 4406-4409.

[128] W.-L. Xie, M.-D. Zhao, C.-M. Cui. Organometallics 2013, 32, 7440-7444.

[129] Y. Hu, L. Liang, W.-T. Wei, X. Sun, X.-J. Zhang, M. Yan, Tetrahedron 2015, 71, 1425-1430.

[130] (a) N. Miyaura, T. Yanagi, A. Suzuki, Synth. Commun. 1981, 11. 513-519; (b) Journal of Organic Chemistry, 77(15), 6608-6614.

[131] (a) S. C. Yan, Z. S. Li, Z. G. Zou, Langmuir 2009, 25, 10397-10401; (b) M. Shalom, S. Inal, C. Fettkenhauer, C. Neher, D. M. Antonietti, J. Am. Chem. Soc. 2013, 135, 7118-7121.

[132] W. P. Gallagher, M. Marlatt, R. Livingston, S. Kiau, J. Muslehiddinoglu. Org. Process Res. Dev. 2012, 16, 1665-1668.

[133] M. Vayer, S. P. Morcillo, J. Dupont, V. Gandon, C. Bour. Angew. Chem. Int. Ed. 2018, 57, 3228-3232.

[134] N. Iranpoor, H. Firouzabadi, Z. T. Rizi, S. Erfan, RSC Adv. 2014, 4, 4317-4323.

[135] O. O. Kovalenko, A. Volkov, H. Adolfsson. Org. Lett. 2015, 17, 446-449.

[136] M. Zhu, K. I. Fujita, R. Yamaguchi, J. Org. Chem. 2012, 77, 9102-9109.

[137] Y. Liu, C. Wang, D. Xue, M. Xiao, J. Liu, C. Li, J. Xiao, Chem. Eur. J. 2017, 23, 3062-3066.

[138] M.-H. So, Y. Liu, C. M. Ho, M. Che, Chem. Asian J. 2009, 4, 1551-1561.

[139] R. Tank, U. Pathak, M. Vima, S. Bhattacharyya, L. K. Pandey, Green Chem. 2011, $13,3350-3354$.

[140] Y. Liu, S. Shi, M. Achtenhagen, R. Liu, M. Szostak. Org. Lett. 2017, 19, 16141617.

[141] S. De, J. Yin, D. Ma, Org. Lett. 2017, 19, 4864-4867.

[142] S. D. Friis, T. Skrydstrup, S. L. Buchwald, Org. Lett. 2014, 16, 4296-4299. 
[143] X.-F. Liu, X.-Y. Li, C. Qiao, H.-C. Fu, L.-N. He, Angew. Chem. Int. Ed. 2017, 129, 7533-7537.

[144] K. S. Srinivas, S. Badigenchala, G. Sekar, Adv. Synth. Catal. 2015, 357, 14371445.

[145] S. L. Zultanski, J. Zhao, S. S. Stahl, J. Am. Chem. Soc. 2016, 138, 6416-6419.

[146] G. Ding, B. Lu, Y. Li, J. Wan, Z. Zhang, X. Xie, Adv. Synth. Catal. 2015, 357, 10131021.

[147] M. Wang, J. Lu, J. Ma, Z. Zhang, F. Wang, Angew. Chem. Int. Ed. 2015, 54, 14061-14065.

[148] N. S. Birudukota, R. Franke, B. Hofer, Org. Biomol. Chem. 2016, 14, 3821-3837.

[149] B. T. Smith, J. A. Wendt, J. Aubé, Org. Lett. 2002, 4, 2577-2579.

[150] G. Manolikakes, M. A. Schade, C. M. Hernandez, H. Mayr, P. Knochel, Org. Lett. 2008, 10, 2765-2768.

[151] B. Zhao, W. J Brittain, Macromolecules 2000, 33, 342-348.

[152] G. Manolikakes, C. Hernandez, M. A. Schade, A. Metzger, P. Knochel, J. Org. Chem. 2008, 73, 8422-8436.

[153] J. B. C. Mack, J. D. Gipson, J. D. Bois, M. S. Sigman, J. Am. Chem. Soc. 2017, 139, 9503-9506.

[154] Wolfgang Robien, University of Vienna, spectral data (with id: CNMRMHKO009022) were obtained from Wiley Subscription Services, Inc.

[155] K. Moriyama, Y. Nakamura, H. Togo, Org. Lett. 2014, 16, 3812-3815.

[156] T. Korenaga, A. Ko, K. Uotani, Y. Tanaka, T. Sakai, Angew. Chem. Int. Ed. 2011, 50, 10703-10707.

[157] N. Basarić, C. Nikola, B. Damir, M.-M. Kata, U. Lidija, K. Marijeta, W. Peter, Photochem. Photobiol. Sci. 2011, 10, 1910-1925.

[158] S. Mukherjee, B. Maji, A. Tlahuext-Aca, F. Glorius, J. Am. Chem. Soc. 2016, 138, 16200-16203.

[159] B. H. Min, D. S. Kim, H. S. Park, C. H. Jun, Chem. Eur. J. 2016, 22, 6234-6238.

[160] N. R. Vautravers, D. D. Regent, B. Breit, Chem. Commun. 2011, 47, 6635-6637.

[161] B. Xu, J. P. Lumb, B. A. Arndtsen, Angew. Chem. Int. Ed. 2015, 54, 4208-4211.

[162] G. Yang, Q. Zhang, H. Miao, X. Tong, J. Xu, Org. Lett. 2005, 7, 263-266. 
[163] J. A. Marko, A. Durgham, S. L. Bretz, W. Liu, Chem. Commun. 2019, 55, 937940.

[164] A. Fryszkowska, K. Fisher, J. M. Gardiner, G. M. Stephens, J. Org. Chem. 2008, 73, 4295-4298.

[165] D. Ganapathy, G. Sekar, Org. Lett. 2014, 16, 3856-3859.

[166] G. Cahiez, O. Gager, V. Habiak, Synthesis 2008, 16, 2636-2644.

[167] J. Stec, E. Thomas, S. Dixon, R. J. Whitby, Chem. Eur. J. 2011, 17, 4896-4904.

[168] J.-M. Liu, H. Yi, X. Zhang, C. Liu, R. Liu, G.-T. Zhang, A.-W. Lei, Chem. Commun. 2014, 50, 7636-7638.

[169] B.-J. Xiong, X.-Q. Zeng, S.-S. Geng, S. Chen, Y. He, Z. Feng, Green Chem. 2018, 20, 4521-4527.

[170] (a) H. F. Koch, G. Ladder, J. G. Koch, D. J. Bodgman, G. H. Brown, C. A. Carlson, A. B. Dean, R. Hage, P. Han, J. C. P. Hopman, L. A. James, P. M. Knape, E. C. Roos, M. L. Sardina, R. A. Sawyer, B. O. Scott, C. A. Testa, S. D. Wickman, J. Am. Chem. Soc. 1997, 119, 9965-9974; (b) K. Tanaka, A. Hosokama, K. Yoshida, Synthesis 1999, 2, 249-253.

[171] Y. Liu, Y. L. Steve Tse, A. Di Mola, F. Y. Kwong, Y. Y. Yeung, ACS Catal. 2017, 77, 4435-4440.

[172] S.-L. Xu, Z. Yun, T. Tang, Z.-X. Fang, T.-D. Tang, RSC Adv. 2016, 6, 69822-69827.

[173] D. Riemer, B. Mandaviya, W. Schilling, A. C. Götz, T. Kühl, M. Finger, S. Das, ACS Catal. 2018, 8, 3030-3034.

[174] S. Seth, S. Jhulki, J. N. Moorthy, Eur. J. Org. Chem. 2013, 12, 2445-2452.

[175] R. Guo, X.-X. Zheng, D.-Y. Zhang, G.-Z. Zhang, Chem. Sci. 2017, 8, 3002-3006.

[176] C.-Y. Guo, Y. H. Zhang, Y. Zhang, J.-D. Wang, Chem. Commun. 2018, 54, 37013714.

[177] S. Nakai, T. Uematsu, Y. Ogasawara, K. Suzuki, K. Yamaguchi, N. Mizuno, ChemCatChem 2018, 10, 1096-1106.

[178] G.-F. Zhang, X.-W. Han, Y.-X. Luan, Y. Wang, X. Wen, L. Xu, C.-R. Ding, J.-R. Gao, RSC Adv. 2013, 3, 19255-19258.

[179] J.-A. Jiang, C. Chen, Y. Guo, D.-H. Liao, X.-D. Pan, Y.-F. Ji, Green Chem. 2014, 16, 2807-2814. 
[180] Y. Itoha, R. Kitaguchia, M. Ishikawa, M. Naitob, Y. Hashimoto, Bioorg. Med. Chem. 2011, 19, 6768-6778.

[181] J.-Z. Chen, D.-L. Liu, N. Butt, C. Li, D.-Y. Fan, Y.-G. Liu, W.-B. Zhang, Angew. Chem. Int. Ed. 2013, 52, 11632-11636.

[182] C.-H. Huang, N. Ghavtadze, B. Chattopadhyay, V. Gevorgyan, J. Am. Chem. Soc. 2011, 133, 17630-17633.

[183] D. Pan, Y. Wang, M. Li, X. Hu, N. Sun, L.-Q. Jin, B.-X. Hu, Z.-L. Shen, Synlett 2019, $30,218-224$.

[184] K. Lu, X.-W. Han, W.-W. Yao, Y.-X. Luan, Y.-X. Wang, H. Chen, X.-T. Xu, K. Zhang, M. Ye, ACS Catal. 2018, 8, 3913-3917.

[185] M. Klaper, P. Wessig, T. Linker, Chem. Commun. 2016, 52, 1210-1213.

[186] D. J. Lippincott, P. J. Trejo-Soto, F. Gallou, B. H. Lipshutz, Org. Lett. 2018, 20, 5094-5097.

[187] F. Fringuelli, R. Girotti, F. Pizzo, L. Vaccaro, Org. Lett. 2006, 8, 2487-2489.

[188] N. Windmon, V. Dragojlovic, Green Chem. Lett. Rev. 2008, 1, 155-163.

[189] H. Lü, X. An, J. Yu, X. Song, J. Phys. Org. Chem. 2012, 25, 1210-1216.

[190] Y. Park, E. Park, H. Jung, Y.-J. Lee, S. Jew, H. Park, Tetrahedron 2011, 67, 11661170.

[191] F. Bellezza, A. Cipiciani, U. Costantino, F. Fringuelli, M. Orrù, O. Piermatti, F. Pizzo, Catal. Today 2010, 152, 61-65.

[192] J. Revuelta, S. Cicchi, A. Brandi, J. Org. Chem. 2005, 70, 5636-5642.

[193] A. Massa, V. de Sio, R. Villano, M. Acocella, L. Palombi, G. Sellitto, A. Peduto,

R. Filosa, P. De Capraris, A. Scettri, Synthesis 2009, 4, 643-649

[194] P. J. Alaimo, R. O'Brien, A. W. Johnson, S. R. Slauson, J. M. O'Brien, E. L. Tyson, A.-L. Marshall, C. E. Ottinger, J. G. Chacon, L. Wallace, C. Y. Paulino, S. Connell, Org. Lett. 2008, 10, 5111-5114.

[195] J. A. Barltrop, B. Hesp, J. Chem. Soc. C 1967, 1625-1635.

[196] R. Li, B. C. Ma, W. Huang, L. Wang, D. Wang, H. Lu, K. Landfester, K. A. I. Zhang, ACS Catal. 2017, 7, 3097-3101. 
